\title{
THE 100 YEARS OF THE FUNGUS COLLECTION MUCL 1894-1994
}
Fungal Taxonomy and Tropical Mycology: Quo vadis?

Taxonomy and Nomenclature of the Fungi

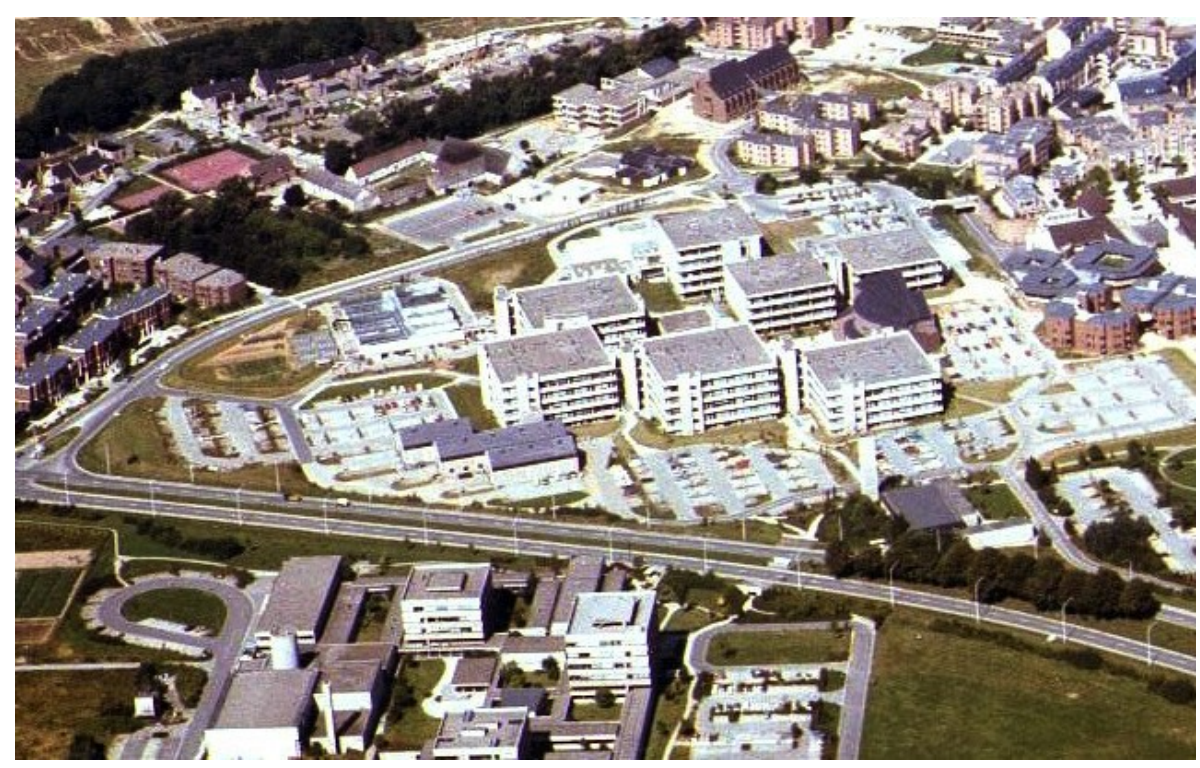

Grégoire L. Hennebert

Catholic University of Louvain, Belgium 
Notice of the editor

This document is now published as an archive

It is available on www.Mycotaxon.com

It is also produced on CD and in few paperback copies

G. L. Hennebert ed.

Published by Mycotaxon, Ltd.

Ithaca, New York, USA

December 2010

ISBN 978-0-930845-18-6 (www pdf version)

ISBN 978-0-930845-17-9 (paperback version)

DOI 10.5248/2010MUCL. pdf 


\section{4-1994 MUCL Centenary}

CONTENTS

Lists of participants

Forword

John Webser

\section{PLENARY SESSION}

The 100 Year Fungus Culture Collection MUCL, June 29th, 1994

G.L. Hennebert, UCL

Mycothèque de l'Université Catholique de Louvain (MUCL)

D. Hawksworth, IMI, U.K.

Fungal genetic resource collections and biodiversity.

D. van der Mei, CBS, MINE, Netherlands

The fungus culture collections in Europe.

J. De Brabandere, BCCM, Belgium

The Belgian Coordinated Collections of Microorganisms.

Fungal Taxonomy and tropical Mycology

G.L. Hennebert, UCL

Introduction. Fungal taxonomy and tropical mycology: Quo vadis?

C.P. Kurtzman, NRRL, USA

Molecular taxonomy in the yeast fungi: present and future.

M. Blackwell, Louisiana State University, USA

Phylogeny of filamentous fungi deduced from analysis of molecular characters: present and future.

J. Rammeloo, National Botanical Garden, Belgium

Importance of morphological and anatomical characters in fungal taxonomy. 57

M.F. Roquebert, Natural History Museum, France

Possible progress of modern morphological analysis in fungal taxonomy.

A.J. Masuka, Forest Research Center, Zimbabwe

Mycofloristic studies in South Central Africa: status, constraints,

opportunities and strategies.

A. Peerally, University of Mauritius, Mauritius Strategies for the development of tropical mycology.

E.J. Da Silva, MIRCEN, UNESCO, France

The role of the Microbiological Resources Centers (MIRCEN) in the development of mycology in the tropics. 


\section{POSTERS}

\section{Fungus Collections and Services}

The Mycothèque de l'Université Catholique de Louvain (MUCL) 100 years 1894-1994 - Grégoire L. Hennebert

MUCL, a Public Service Collection. - C. Decock, Ch. Moulliard, L. Nélissen, P. Massart, P. Evrard, N. Jamin, P. Charue and G.L. Hennebert

MUCL, a Fungus Culture Collection, service to African fundamental and applied mycology. - Grégoire L. Hennebert

The activities and mycological collections of the Belgian National Botanical Garden (BR) - André Fraiture

\section{Classical Taxonomy of the Fungi}

Anamorph-teleomorph connectionsin the Xylariaceae. - M.A. Whalley, G.P. Sharples and A.J.S. Whalley

Anamorphs and the classification of xylariaceous fungi.- K. Van Der Gucht 133

First occurrence in nature of the teleomorph of Cochliobolus . - R. Raemaekers and J. Coosemans

Pyxidiophora, a possible teleomorph of Pleurocatera acicularis. - Günter R.W. Arnold

Illustrations of the cultural features of Gyrodontium boveanum (Montagne) Maas Geesteranus - Cony Decock and Anxious J. Masuka

The Lactarius gymnocarpus complex in tropical Africa - Annemieke Verbeken (abstract)

An interesting species of Puccinia on Gentiana - Evangelia Kapsanaki-Gotsi and I. Vovou (abstract)

Cultural characteristics and their taxonomic potential in Lentinellus species - Zapi Gonou-Zagou and Maria Pantidou (abstract)

Responses to the thermal stress in the inter-sterility groups of Ganoderma australe in Taiwan - Zuei-Ching Chen (abstract) 


\section{Molecular Taxonomy}

18S rRNA and the fungal tree: evolutionary relationships among ascomycetes, basidiomycetes, zygomycetes, and chytridiomycetes. - Yves Van de Peer, Grégoire L. Hennebert and Rupert De Wachter

A rapid molecular technique to distinguish Cylindrocladium species. - P.W.

Crous, A. Korf and W.H. Van Zyl

Identification of basidiomycetous yeasts using whole-cell protein electrophoresis

Marc Vancanneyt, Grégoire L. Hennebert and Karl Kersters

Phytopathogenic filamentous (Ashbya, Eremothecium) and dimorphic fungi (Nematospora) with falcate ascospores as new members within the Saccharomycetaceae. H. Prillinger, R. Messner, M. Breitenbach, P. Briza, E.

Staudacher, O. Molnør, F. Weigang, K. Lopandic, M. Ibl \& G. Himmler. 162

Genetic Organization of the Yeast Yarrowia lipolytica. - Serge Casaregola, Huu Vang Nguyen, Chantal Feynerol, Monique Diez and Claude Gaillardin 168

Taxonomic revision of Saccharomyces sensu stricto strains of NCAIM. - J. Tornai-Lehoczki., D. Dlauchy.and G. Peter

Phylogenic relationships within the Saccharomyces cerevisiae complex species. -

Dilnora E. Gouliamova and Grégoire L. Hennebert

What about the "species group" concept in Aspergillus flavus ? - Joelle Dupont, Marie-France Roquebert and Evelyne Guého (abstract)

A comparative study of electrophoretic karyotypes in the yeast genera Torulaspora Lindner and Zygosaccharomyces Barker - Guido Capriotti and Ann Vaughan-Martini (abstract)

\section{Fungal biodiversity}

Biodiversity of Cylindrocladium on water-lilies at SSR Pamplemousses Botanic Garden in Mauritius. - Abed Peerally

The occurrence of wood decaying fungi in Belgium houses - Cony Decock and Grégoire L. Hennebert

Symbiotic fungi in the galleries of the striped bark beetle, Trypodendron lineatum) (Coleoptera, Scolytidae). - G. Babuder and F. Pohleven

Cercosporoid Fungi associated with the Eyespot Disease of graminicolous Hosts. G.F. Campbell, B. Robbertse, P.W. Crous and B.J.H. Janse 
Diversity of Colletotrichum gloeosporioides pathogenic to Stylosanthes sp. in tropical areas. - F. Munaut, N. Hamaide and H. Maraite

Diversity in non-specific foliar pathogens of wheat from non-traditional warm areas. - Di Zinno T., Duveiller E., Longrée H. and H. Maraite

Pathogenic diversity in Magnaporthe grisea, the rice blast pathogen. - J.B.

Bahama, J.L.Notteghem and H.Maraîte

Characterization of Polymyxa graminis involved in the transmission of Peanut Clump Virus in tropical areas. - A. Legreve, B. Vanpee, P. Delfosse, D. V. R.

Reddy and H. Maraite

\section{Practical Mycology}

Practical Advice for collecting wood-rotting Fungi in the Tropics. - Erast Parmasto

The influence of preparation on ascospore measurements. - Bellis Kullman, Aivo Jakobson and Mart Rahi

Microplate technique of physiological tests for Yeast identification - Pierre

Evrard and Grégoire L. Hennebert (abstract)

Cryopreservation of Aspergillus repens - David Smith (abstract)

\section{Cultivation of the fungi}

Activated charcoal-mediated sporophore initiation in Agaricus and Tricholoma Abed Peerally.

In vitro Culture of endomycorrhizal Fungi: why hat interest? - S. Declerck, T.

Diop, C. Plenchette and D.G. Strullu.

Enhancement of carpophore production and protein content of Pleurotus spp. by cultivation on grass hay. - Kandanda Tshinyangu (abstract)

Is the rust of the Creeping Thistle (Cirsium arvense) the microsymbiont of its VAM? - Hartmut Heilmann (abstract)

Morphological studies of mycelia from ectomycorhizian fungi cultivated in vitro -

J. Jeanfils and J.-J. Cuvelier (abstract)

\section{Fungal Biotechnology}

Yeast fungi and alcohol from bamboos. - Francis S.S. Magingo

Growth of Fusidium coccineum on solid Media. - Helen E. Smith and Glyn Hobbs 
Strain polymorphism in PDR1, a transcription regulator of pleiotropic drug resistance genes in Saccharomyces cerevisiae. - E. Carvajal, E. Balzil and A. Goffeau (abstract)

Structural identification and biochemical mode of action of a mycotoxine isolated from Cochliobolus sativus MUCL 18528. - D. Vilret, P. Goblet, J.

Hutschemackers, N. Cosyns, M. Noël and M. Briquet (abstract)

Characterization of the carbohydrate moiety on proteins secreted from different fungi. - M. Maras, W. Laroy, W. Fiers, G.L Hennebert and R. Contreras (abstract)

Specific and non-specific interactions in yeast cell flocculation. - P.B. Dengis, L.R. Nélissen, and P.G. Rouxhet (abstract)

Modification of support surface properties to improve microorganism adhesion -

P.A. Gerin, M.-N. Bellon-Fontaine, M. Asther \& P.G. Rouxhet (abstract) 249

\section{Bacteriology}

Identification of Lactic Acid Bacteria using RAPD-PCR. - A. Noël and J. Decallonne

Gas Chromatographic Analysis of Cellular Fatty Acids in the Identification of

Microorganisms. - P. Wauthoz, M. El Lioui and J. Decallonne

\section{LAST LECTURE, 5 December 1995}

Taxonomy and Nomdenclature of the fungi:Reasons for a Mycotheque. Grégoire L. Hennebert 


\section{LIST OF PARTICIPANTS}

Ainsworth A. Martyn, Applied Mycology Department, Xénova Ltd, Ipswich Road 545, Slough, SL1 4EQ, Bucks, United Kingdom.

Antoine Raymond, "Le Sous-Bois", Chemin des Vignerons 32, B-5100, Wépion, Belgium.

Aptroot André, Centraalbureau voor Schimmelcultures, P.O. Box 273, 3740, AG Baarn, Netherlands.

Arnold Günter, Friedrich-Schiller-Universität Jena, Biologische Fakultät, Pilzkulturensammlung, Freiherr-vom-Stein-Allee 2, 99425, Weimar, Germany.

Babuder Gorazd, Biotechnical Faculty, Department of Wood Science and Technology, University of Ljubljana, Rozna dolina Cesta VIII/34, Vecna pot 2, 61000, Ljubljana, Slovenia.

Bahama Jean-Baptiste, Clinique des Plantes, Unité de Phytopathologie - FYMY, Département BAPA, Faculté des Sciences Agronomiques, UCL, Place Croix du Sud 2 bte 3, B-1348, Louvain-laNeuve, Belgium.

Beblowska Marta, Zaklad Systematyki i Gaeografii Roslin, Warsaw University, Aleje Ujazdowskie 4, 00-478, Warsaw, Poland. Email: beblowsk@orion.astrouw.edu.pl

Belloch Carmela, Coleccion Espanola de Cultivos Tipo - CECT, Facultad de Ciencias Biologicas, Departamento de Microbiologia, Universidad de Valencia, C/Dr. Moliner 50, 46100, Burjassot, Spain. Email: Belloch@evalun11.bitnet

Blackwell Meredith, Department of Botany, Louisiana State University, Life Sciences Building 502, Baton Rouge, 70803-1705, Louisiana, United States. Email: btblac@1suvm.bitnet

Bonneff Eric, Laboratoire de Microbiologie Industrielle, Université de Reims / Champagne-Ardenne, U.R.C.A., UFR Sciences Exactes et Naturelles, Moulin de la Housse B.P. 347, 51062, Reims Cédex, France.

Briquet Michel, Unité de Biochimie Physiologique FYSA, Département CABI, Faculté des Sciences Agronomiques, UCL, Place Croix du Sud 2 Bte 20, B-1348, Louvain-la-Neuve, Belgium.

Buffin de Chosal Nathalie, Guido Bezellestraat 7, B-8970, Poperinge, Belgium.

Caeymaex Louis, Bureau Marcel van Dijk, Avenue Louise 250 Boîte 14 , B-1050, Bruxelles, Belgium.

Cahagnier Bernard, Laboratoire de Microbiologie et Technologie Céréalières, Centre de Recherches de Nantes, Institut National de la Recherche Agronomique, B.P. 527, 44026, Nantes Cédex, France.

Casaregola Serge, Laboratoire de Génétique Moléculaire et Cellulaire, Collection de Levures d'Intérêt Biotechnologique, Institut National de la Recherche Agronomique, 78850, Thiverval-Grignon, France.

Castillo Cabello Gabriel, Dép. de Botanique (B.22), Université de Liège, B-4000, Liège, Belgium.

Cerisier Yvanne, Collection Nationale de Cultures de Microorganismes, Institut Pasteur, Rue du Docteur Roux 25, 75015, Paris Cédex 15, France.

Chasseur Camille, Institut d'Hygiène et d'Epidémiologie, IHE, Rue Juliette Wijtsman 14, B-1050, Bruxelles, Belgium

Chen Zuei-ching, The Mycological Society of the Republic of China, Mycology Laboratory, Department of Botany, National Taiwan University, Roosevelt Road, Section 4 N¹, Taipei, 10764, Taiwan, China.

Cimerman Aleksa, Culture Collection of Fungi, Boris Kidric Institute of Chemistry, Hajdrihova 19, P.O. Box 30, 61115, Ljubljana, Slovenia. Email: aleksa.cimerman@ki.si

Contreras Roland, Laboratorium voor Moleculaire Biologie, Rijksuniversiteit Gent - RUG, K.L. Ledeganckstraat 35, B-9000, Gent, Belgium. Email: Roland@lmb1.rug.ac.be

Coosemans Jozef, Laboratorium Fytopathologie en Plantenbescherming, Faculteit der Landbouwwetenschappen der Leuven, Katholieke Universiteit Leuven, Willem De Croylaan 42, B-3001, Heverlee, Belgium.

Crous Pedro W., Department of Plant Pathology, University of Stellenbosch, Private bag X5018, 7600, Stellenbosch, South Africa. Email: pwc@maties.sun.ac.za

Cuvelier Jean-Jacques, Faculté de Médecine, Université Mons-Hainaut, Avenue du Champ de Mars 8, B-7000, Mons, Belgium

Danckaert Jan, Kabinet Wetenschapbeleid, Wetstraat 155/16, B-1040, Brussels, Belgium.,

De Brabandere Jan, Services Fédéraux des Affaires Scientifiques, Techniques et Culturelles, Rue de la Science 8, B-1040, Bruxelles, Belgium.

De Bruyne Erik, SES Europe s.a., Industriepark 15, B-3300, Tienen, Belgium.

De Wachter Rupert, Departement Biochemie, Fakulteit Wetenschappen, Universitaire Instelling Antwerpen - UIA, Universiteitsplein 1, Bâtiment T, B-2610, Antwerpen, Belgium. Email: dwachter@reks.uia.ac.be

Decallonne Jacques, Laboratoire de Bactériologie, Unité de Microbiologie MBLA, Département CABI, Faculté des Sciences Agronomiques, UCL, Place Croix du Sud 2 Bte 12, B-1348, Louvain-laNeuve, Belgium. 
Declerck Stéphane, Laboratoire d'Agronomie, Institut National de la Recherche Agronomique - INRA, B.P. 1540, Rue Sully 17, 21034, Dijon, France.

Decock Cony, MUCL, 2 Place Croix du Sud, B-1348 Louvain-la-Neuve, Belgique, E-mail : decock@mbla.ucl.ac.be

Degreef Jérôme, Laboratoire d'Ecologie, Faculté des Sciences Agronomiques de Gembloux, Passage des Déportés, B-5030, Gembloux, Belgium.

Delvaux Bruno, Unité des Sciences du Sol PEDO, Département MILA, Faculté des Sciences Agronomiques, UCL, Place Croix du Sud 2 Bte 10, B-1348, Louvain-la-Neuve, Belgium.Phone: 010-473686,Email: Delvaux@pedo.ucl.ac.be

Di Zinno Tonio, Clinique des Plantes, Unité de Phytopathologie - FYMY, Département BAPA, Faculté des Sciences Agronomiques, Université Catholique de Louvain - UCL, Place Croix du Sud 2 bte 3, B-1348, Louvain-la-Neuve, Belgium., Email: dizinno@fymy.ucl.ac.be

Diederich Paul, Rue de la Solidarité 22, 8020, Strassen, Luxembourg.

Doyle Alan, European Collection of Animal Cell Cultures, Pathology Division, PHLS Centre for Applied Microbiology and Research, Porton Down, Salisbury, SP4 0JG, Wiltshire, United Kingdom.

Dupont Joëlle, Laboratoire de Cryptogamie, Muséum National d'Histoire Naturelle, Rue de Buffon 12, F75005, Paris, France

Fattori Maria, SES Europe s.a., Industriepark 15, B-3300, Tienen, Belgium

Fisch Colette, Laboratoire Intercommunal de Chimie et Bactériologie, Rue du Maelbeek 3, B-1040, Bruxelles, Belgium.

Fraiture André, Jardin Botanique National de Belgique, Domaine de Bouchout, Brusselsesteenweg 8-26, B-1860, Meise, Belgium

Gabor Peter, National Collection of Agricultural \& Industrial Microorganisms, Department of Microbiology and Biotechnology, University of Horticulture and Food Industry, Somloi ut 14-16, 1118, Budapest, Hungary.

Gams Walter, Centraalbureau voor Schimmelcultures, P.O. Box 273, 3740, AG Baarn, Netherlands. Email: walter.gams@orange.nl

Gigi Jacques, Cliniques Universitaires St-Luc, Laboratoire de Microbiologie, Université Catholique de Louvain - UCL, Avenue Hippocrate 10, B-1200, Bruxelles, Belgium.

Girlanda Mariangela, Centro di Studio sulla Micologia del Terreno, Dipartimento di Biologia Vegetale dell'Universita, Consiglio Nazionale delle Ricerche - CNR, Viale Mattioli 25, 10125, Torino, Italy.

Godden Bernard, Laboratoire de Microbiologie, Unité de Physiologie et d'Ecologie Microbienne, Faculté des Sciences, Université Libre de Bruxelles - ULB, Institut Pasteur du Brabant, Rue Engeland 642, B-1180, Bruxelles, Belgium.

Gonou Zagou Zapi, Section of Ecology \& Systematics, Department of Biology, University of Athens, Panepistimiopolis, 15784, Athens, Greece.

Gouliamova Dilnora, MUCL, 2 Place Croix du Sud, B-1348 Louvain-la-Neuve, Belgique,

Gürler Bülent, Centre for Research and Application of Culture Collections of Microorganisms, Microbiology Department, Istanbul Faculty of Medicine, Temel Bilimler Binasi, 34390, CapaIstanbul, Turkey.

Halama Patrice, Institut Supérieur d'Agriculture, Rue du Port 41, 59046, Lille Cédex, France.

Hawksworth David L., International Mycological Institute - IMI, Bakeham Lane, Englefield Green, Egham, TW20 9TY, Surrey, United Kingdom.

Hennebert Alexis-Jean, Clos des Salanganes 15, B-1150, Bruxelles, Belgium.

Hennebert Gregoire Laurent, MUCL, 2 Place Croix du Sud, B-1348 Louvain-la-Neuve, Belgique, Phone 010-473742, E-mail : hennebert@mbla.ucl.ac.be. Privé : 32 Rue de l'Elevage, 1340 0ttignies-LLN, Belgique

Hennebert Paul, Faculté de Médecine, UCL, Ophemstraat 81, B-3050, Oud-Heverlee, Belgium.

Hughes Stanley J., 360 Hamilton Avenue, Ottawa, K1Y 1C6, Ontario, Canada.

Innicka-Olejniczak Olga, Technical Microbiology and Biochemistry Department, Institute of Agro-Food Biotechnology, Rakowiecka Street 36, 02-532, Warszawa, Poland.

Janssens Danielle, Laboratorium voor Microbiologie en Microbiële - LMG, Rijksuniversiteit Gent - RUG , K.L. Ledeganckstraat 35, B-9000, Gent, Belgium., Email: Danielle.Janssens@rug.ac.be

Jeanfils Joseph, Faculté de Médecine, Université Mons-Hainaut, Avenue du Champ de Mars 8, B-7000, Mons, Belgium.

Jones Gareth E.B., School of Biological Sciences, University of Portsmouth, King Henry Building, King Henry 1 Street, PO1 2DY, Portsmouth, Hants, United Kingdom.

Kapsanaki-Gotsi Evangelia, Department of Biology, Section of Ecology and Systematics, University of Athens, Panepistimiopolis, 15784, Athens, Greece. 
Kersters Karel, Laboratorium voor Microbiologie en Microbiele Genetica - LMG, Faculteit Wetenschappen, Rijksuniversiteit Gent - RUG, K.L. Ledeganckstraat 35, B-9000, Gent, Belgium.

Knuyt Walter, Services Fédéraux des Affaires Scientifiques, Techniques et Culturelles, Rue de la Science 8, B-1040, Bruxelles, Belgium.

Korf Richard P., College of Agriculture and Life Sciences, Plant Pathology Herbarium, Cornell University, Plant Science Building 401, Ithaca, 14853-4203, New York, United States. Email: rpk1@cornell.edu

Kost Gerhard, Fachbereich Biologie, Botanik/Mykologie, Philipps Universität Marburg, 35032, Marburg, Germany.

Kracht Manfred, Deutsche Sammlung von Mikroorganismen - DSM, Mascheroder Weg 1B, 3300, Braunschweig, Germany.

Kullman Bellis, Institute of Zoology and Botany, Estonian Academy of Sciences, Vanemuise St. 21, EE2400, Tartu, Estonia. Email: bkullman@iozb.tartu.ee

Kurtzman Cletus P., United States Department of Agriculture, Northern Regional Research Center U.S., Midwest Area, 1815 North University Street, Peoria, 61604, Illinois, United States

Laurent Serge, Fast Net sa, Avenue du Bempt 41, B-1190, Bruxelles, Belgium.

Legrève Anne, Clinique des Plantes, Unité de Phytopathologie - FYMY, Département BAPA, Faculté des Sciences Agronomiques, UCL, Place Croix du Sud 2, B-1348, Louvain-la-Neuve, Belgium. Email: legreve@fymy.ucl.ac.be

Lehoczki Tornai Judit, National Collection of Agricultural \& Industrial Microorganisms, Department of Microbiology and Biotechnology, University of Horticulture and Food Industry, Somloi ut 14-16, 1118, Budapest, Hungary

Lierneux Pierre, Musée Royal de l'Armée et d'Histoire Militaire, Parc du Cinquantenaire 3, B-1040, Bruxelles, Belgium.

Locci Romano, Dipartimento di Biologia Applicata alla Difesa delle Piante, Universita degli Studi di Udine, Via delle Scienze 208, Area Rizzi, 33100, Udine, Italy.

Macau J., Research and Development Department, Citrique Belge, Pastorijstraat 249, B-3300, Tienen, Belgium.

Magingo Francis S.S., Applied Microbiology Unit, Department of Botany, University of Dar Es Salaam, P.O. Box 35060, Dar Es Salaam, Tanzania.

Maraite Henri, Clinique des Plantes, Unité de Phytopathologie - FYMY, Département BAPA, Faculté des Sciences Agronomiques, UCL, Place Croix du Sud 2, B-1348, Louvain-la-Neuve, Belgium., Email: Maraite@fymy.ucl.ac.be

Maras Marleen, Laboratorium voor Moleculaire Biologie, Rijksuniversiteit Gent - RUG, K.L. Ledeganckstraat 35, B-9000, Gent, Belgium. n

Marin Bernard, Laboratoire de Biotechnologie, ORSTOM, Avenue Agropolis 911, B.P. 5045, F-34032, Montpellier Cedex 1, France.

Marson Guy, Musée National d'Histoire Naturelle, Marché aux Poissons, 2345, Luxembourg, Luxembourg.

Martini-Vaughan Ann, Collezione del Lieviti Industriali, Dipartimento di Biologia Vegetale, Universita degli Studi di Perugia, Borgno XX Giugno 74, 06121, Perugia, Italy.

Marvel Danielle, Rue Saint-Henri 65, 1200 Bruxelles.

Masuka Anxious J., Forest Research Centre, Biotechnology Kutsaga Research Station, Tobacco Research Board Zimbabwe, P.O. Box HG 595, Highlands, Harare, Zimbabwe.

Melcion Dominique, Laboratoire de Microbiologie et Technologie Céréalières, Centre de Recherches de Nantes, Institut National de la Recherche Agronomique, B.P. 527, 44026, Nantes Cédex, France.

Mencinicopschi Gheorghe, Department of Biotechnology, Institute for Food Chemistry, Gârlei Street 1, 71576, Bucharest, Romania;

Messner Robert, Universität für Bodenkultur, Nussdorfer Lände 11, 1190, Wien, Austria. Email: messner@star3.boku.ac.at

Mibey Richard K., Department of Botany, University of Nairobi, Chiromo, P.O. Box 30197, Nairobi, Kenya.

Michiels Chris, Katholieke Universiteit Leuven - KUL, Kardinaal Mercierlaan 92, B-3001, Heverlee, Belgium.

Miller Liliane, Somycel, Z.I. Sud, Route de Tours, B.P. 25, 37130, Langeais, France.

Montemartini Corte Aurora, Istituto botanico "Hanbury" ed Orto botanico dell'Universita, Universita di Genova, Corso Dogali 1c, 16136, Genova, Italy.

Mouchacca Jean, Laboratoire de Cryptogamie, Muséum National d'Histoire Naturelle, Rue Buffon 12, 75005, Paris, France.

MUCL, Laboratoire de Mycologie systématique et appliquée, Faculté des Sciences Agronomiques UCL, 2 Place Croix du Sud, B-1348 Louvain-la-Neuve, Belgium. Pr.Grégoire L. Hennebert, Dr Cony 
Decock, Dr Dilnora Gouliamova, Dr .Kandanda Tshinyangu, Laurence Nélissen, Pascale Massart; Ch. Mouilliard, Ph. Charue, P. Evrard, N. Jamin.

Munaut Françoise, Clinique des Plantes, Unité de Phytopathologie - FYMY, Département BAPA, Faculté des Sciences Agronomiques, UCL, Place Croix du Sud 2 bte 3, B-1348, Louvain-laNeuve, Belgium.

Naveau Henry, Unité de Génie Biologique - GEBI, Département CABI, Faculté des Sciences Agronomiques, Université Catholique de Louvain - UCL, Place Croix du Sud 2 Bte 19, B-1348, Louvain-la-Neuve, Belgium. Phone: 010-473646, Email: Naveau@gebi.ucl.ac.be.

Nguyen Huu-Vang, Laboratoire de Génétique Moléculaire et Cellulaire, Collection de Levures d'Intérêt Biotechnologique, Institut National de la Recherche Agronomique, 78850, Thiverval-Grignon, France. Email: clib@grignon.inra.fr

Noël Anne, Laboratoire de Bactériologie, Unité de Microbiologie MBLA, Département CABI, Faculté des Sciences Agronomiques, UCL, Place Croix du Sud 2 Bte 12, B-1348, Louvain-la-Neuve.

Nolard Nicole, Ministère de la Santé Publique, Institut d'Hygiène et d'Epidémiologie - IHEM, Rue Juliette Wytsman 14, B-1050, Bruxelles, Belgium.

Noordeloos Machiel E., Mycological Department, Rijksherbarium/Hortus Botanicus, P.O. Box 9514, Schelpenkade 6, 2300, RA Leiden, Netherlands.

Oxenboell Karen M. , Novo Nordisk A/S, Novo Allé, 2880, Bagsvaerd, Denmark.

Parmasto Erast, Laboratory of Mycology, Institute of Zoology and Botany, 21 Vanemuise Street, 2400, Tartu, Estonia. Email: erast@park.tartu.ee

Peerally Abed, School of Agriculture, Department of Natural Sciences, University of Mauritius, Reduit, Mauritius.

Peloille Michèle, Laboratoire d'Ecologie Parasitaire, Centre de Recherches Tours-Nouzilly, Institut National de la Recherche Agronomique, 37380, Monnaie, France.

Penninckx Michel, Laboratoire de Microbiologie, Unité de Physiologie et d'Ecologie Microbienne, Faculté des Sciences, Université Libre de Bruxelles - ULB, Institut Pasteur du Brabant, Rue Engeland 642, B-1070, Bruxelles, Belgium.

Poppe Jozef, Centrum voor de studie van de Sierplantenteelt, I.W.O.N.L. Sect, Labo Fytopathologie, Fakulteit van de Landbouwwetenschappen, Rijksuniversiteit Gent - RUG, Coupure Links 653, B9000, Gent, Belgium.

Poswick Pierre, Fast Net sa, Avenue du Bempt 41, B-1190, Bruxelles, Belgium.

Prieels Anne-Marie, Tech Know, Avenue de l'Observatoire 2, B-1180, Bruxelles, Belgium .

Prillinger Hansjörg, Institut für Angewandte Mikrobiologie - IAM, Universität für Bodenkultur, Nussdorfer Lände 11, 1190, Wien, Austria. Email: iam@mail.boku.ac.at

Raemaekers Romain, Belgian Administration for Developement Cooperation - BADC, Marsveldplein 5/57, B-1050, Bruxelles, Belgium.

Rammeloo Jan, Jardin Botanique National de Belgique, Domaine de Bouchout, Brusselsesteenweg 8-26, B-1860, Meise, Belgium.

Risede Jean-Michel, CIRAD, B.P. 153, 97202, Fort de France, Martinique

Roquebert Marie-France, Laboratoire de Cryptogamie, Muséum National d'Histoire Naturelle, Rue de Buffon 12, 75005, Paris, France.

Rosentuler Leon, Foreign Relations Department, Institute for Food Chemistry, Gârlei Street 1, 71576, Bucharest, Romania.

Rouxhet Paul, Unité de Chimie des Interfaces - CIFA, Département CABI, Faculté des Sciences Agronomiques, UCL, Place Croix du Sud 1, B-1348, Louvain-la-Neuve, Belgium. Email: Rouxhet@cifa.ucl.ac.be

Sedeyn Patrick, Mycoblank Bvba, Oude Gaversesteenweg 70, B-9820, Merelbeke, Belgium.

Simonart Paul, Guido Gezellelaan 10, B3090, Overijse, Belgium

Smith David, International Mycological Institute, Bakeham Lane, Englefield Green, Egham, TW20 9TY, Surrey, United Kingdom.

Spencer-Martins Isabel, Portuguese Yeast Culture Collection, Instituto Gulbenkian de Ciencia, Rua da Quinta Grande 6, Apartado 14, 2781, Oeiras Codex, Portugal Email: ispencer@gulbenkian.pt

Stalpers Joost, Centraalbureau voor Schimmelcultures - CBS, Oosterstraat 1, P.O. Box 273, 3740, AG Baarn, Netherlands., Email: Stalpers@pc.cbsc.nl

Stegehuis Gerrit, Centraalbureau voor Schimmelcultures, P.O. Box 273, 3740, AG Baarn, Netherlands.

Subramanian C.V., Plot 885, Ramaswami Salai 62, K.K. Nagar, 600078, Madras, India.

Sugiyama Junta , Institute of Molecular and Cellular Biosciences, IAM Culture Collection, University of Tokyo, Yayoi 1-1-1, Bunkyo-ku 113, Tokyo, Japan. Email: jsugi@tansei.cc.u-tokyo.ac.jp

Suihko Maija-Liisa, VTT Collection of Industrial Microorganisms, VTT Biotechnical Laboratory, P.O. Box 1504, 02044, Espoo, Finland. Email: Maija-Liisa.Suihko@vtt.fi 
Sulten Evelyne, Laboratoire de Bactériologie, Institut Royal des Sciences Naturelles de Belgique IRSNB, Rue Vautier 29, B-1040, Bruxelles, Belgium.

Swinne Danielle, Département de Mycologie, Institut de Médecine Tropicale, Nationalestraat 155, B2000, Antwerpen, Belgium.

Symoens Françoise, Institut d'Hygiène et d'Epidémiologie - IHEM, Rue Juliette Wytsman 14, B-1050, Bruxelles, Belgium.

Thiran Jean-Philippe, Laboratoire de Télécommunications et Télédétection, Département ELEC, Faculté des Sciences Appliquées, Université Catholique de Louvain - UCL, Place du Levant 2, B-1348, Louvain-la-Neuve, Belgium.

Tshinyangu Kandanda, MUCL, 2 Place Croix du Sud, B-1348 Louvain-la-Neuve, Belgique,

Uruburu Federico, Coleccion Espanola de Cultivos Tipo - CECT, Facultad de Ciencias Biologicas, Departamento de Microbiologia, Universidad de Valencia, C/Dr. Moliner 50, 46100, Burjassot, Spain. Email: Belloch@evalun11.bitnet

Vancanneyt Marc, Laboratorium voor Microbiologie en Microbiële Genetica - LMG, Faculteit Wetenschappen, Rijksuniversiteit Gent - RUG, K.L. Ledeganckstraat 35, B-9000, Gent, Belgium.

Van Cutsem Jan M.P., Department of Bacteriology and Mycology, Janssen Research Foundation, Zenithlaan 17, B-2340, Beerse, Belgium.

Vandenput Olivier, Services Fédéraux des Affaires Scientifiques, Techniques et Culturelles, Rue de la Science 8, B-1040, Bruxelles, Belgium.

Van de Peer Yves, Universitaire Instelling Antwerpen - UIA, Universiteitsplein 1, B-2610, Wilrijk, Belgium. Email: yvdp@reks.uia.ac.be

Van der Gucht Katleen, Laboratorium voor Morfologie Systematiek en Ecologie van de Planten, Rijksuniversiteit Gent, K.L. Ledeganckstraat 35, B-9000, Gent, Belgium.

Van der Mei Dirk, Centraalbureau voor Schimmelcultures, Oosterstraat 1, P.O. Box 273, 3740, AG Baarn, Netherlands.

Vanderveken J. Professor, Laboratorium voor Morfologie Systematiek en Ecologie van de Planten, Rijksuniversiteit Gent, K.L. Ledeganckstraat 35, B-9000, Gent, Belgium.

Vandewyer Paul H., Research and Development Department, Citrique Belge, Pastorijstraat 249 , B-3300, Tienen, Belgium.

Van Gestel Jef, Janssen Pharmaceutica n.v., Plant Protection Division, Turnhoutseweg 30, B-2340, Beerse, Belgium.

Van Heetvelde Ingeborg, Research \& Development Center, Vandemoortele n.v., Prins Albertlaan 79, Postbus 40, B-8770, Izegem, Belgium.

Vanhonacker Katrien, Laboratorium voor Microbiologie en Microbiële - LMG, Rijksuniversiteit Gent RUG, K.L. Ledeganckstraat 35, B-9000, Gent, Belgium. Email: Danielle.Janssens@rug.ac.be

Vanhoucke Martine, Laboratory of Molecular Biology - Plasmid Collection, Rijksuniversiteit Gent RUG, K.L. Ledeganckstraat 35, B-9000, Gent, Belgium. Email: Martine@lmbp.rug.ac.be

Van Langenhove Luk, Kabinet Wetenschapbeleid, Wetstraat 155/16, B-1040, Brussels, Belgium.

Verbeken Annemieke, Faculteit Wetenschappen, Rijksuniversiteit Gent - RUG, K.L. Ledeganckstraat 35, B-9000, Gent, Belgium.

Verhoyen Michel, Laboratoire de Phytovirologie, Faculté des Sciences Agronomiques, Université Catholique de Louvain - UCL, Place Croix du Sud 3, B-1348, Louvain-la-Neuve, Belgium. Email: Verhoyen@fymy.ucl.ac.be

Viteri Ricardo, Xénova Ltd, Ipswich Road 545, Slough, SL1 4EQ, Bucks, United Kingdom.

Vivegnis Jacques, Laboratoire de Bactériologie, Unité de Microbiologie MBLA, Département CABI, Faculté des Sciences Agronomiques, UCL, Place Croix du Sud 2 Bte 12, B-1348, Louvain-laNeuve, Belgium.

Vrijmoed Lilian L.P., Department of Biology and Chemistry, City Politechnic of Hong Kong, Tat Chee Avenue 83, Kowloon, Hong Kong, Hong Kong. Email: bhlilian@cphkvx.cphk.hk

Wauthoz Pascale, Laboratoire de Bactériologie, Unité de Microbiologie MBLA, Département CABI, Faculté des Sciences Agronomiques, UCL, Place Croix du Sud 2 Bte 12, B-1348, Louvain-laNeuve, Belgium.

Webster John, Hatherly Laboratories, Department of Biological Sciences, University of Exeter, Prince of Wales Road, EX4 4PS, Exeter, United Kingdom.

Whalley Anthony J.S., Department of Biomolecular Sciences, Laboratory of Microbiology, Liverpool John Moores University, Byrom Street, L3 3AF, Liverpool, England, U.K. 


\title{
Forword
}

\section{Centenary Celebration of the Mycotheque de l'Université Catholique de Louvain, Belgium, 1894-1994}

\author{
JOHN WEBSTER
}

University of Exeter, United Kingdom

MUCL is a fungal culture collection founded in Louvain in 1894 by Philibert Biourge. The centenary of the inauguration of this important collection is celebrated at a special symposium attended by some 150 guests from many countries at the Catholic University at Louvain-la-Neuve on Wenesday $29^{\text {th }}$ June 1994. There are two sessions of presented papers, followed by a discussion, a poster presentation and an exhibition tracing the history of the collecton.

The morning sesssion is openend by addresses of welcome from Dr $\mathrm{H}$. Naveau, Head of Applied Chemistry and Bioindustry Department of the Faculty of Agricultural Sciences and Dr P. Rouxhet, Prorector of UCL. Dr Rouxhet explains the philosophy behind the setting up of the University at Louvain-la-Neuve. It is an integrated community of academic staff and their families, students, residents, shops, private bisinesses and a science and industry park. Priority is given to scientific research and development, advanced technology, etc., with spin-offs likely to be benefit to the University. The Faculty of Agricultural Sciences, which receives state support, includes graduate school forming chemists and engineers able to apply their skills to the living world. The Prorector paid a large tribute to the dedication and hard work of the present director of the culture collection, Professor Grégoire Laurent Hennebert, and assured him that the collection would continue to receive University support. Professor Hennebert then traced the history of the culture collection over the past century since its foundation by Biourge, who had studied with Pasteur. A large number of distinguished scientists had been on the staff including Dierckx and Biourge who laid the foundation of the classification of Penicillium, Vandendries who worked on sexuality of Basidiomycetes, Wieldiers who discovered the "bios", a growth factor for yeast, later found composed of the B vitamins, Cappuyns whose work with the strains of Penicillium species and Aspergillus niger from the Biourge's collection established the industrial process of citric acid p-fermentation, Simonart, former director of the collection and present at the celebration, who worked with Raistrick of Oxford on the chemistry of Penicillium griseofulvum and discovered the first antifungal antibiotic the griseofulvin. The rest of the morning session is also devoted to papers on the significance of fungus culture collections. Prof. D.L. Hawksworth (Director of the International Mycolgical Institute, IMI) speaks about "Fungal resource collections and biodiversity", Dr. D. van der Mei (Director of the Centraalbureau voor Schimmelcultures, CBS) "The fungus culture collections in Europe", and Dr J. De Brabandere (Federal Service for Scientific Affairs, Belgium) "The Belgian Co-ordinated Collections of Microorganisms".

The afternoon session is a workshop entitled "Fungal taxonomy and tropical mycology: Quo vadis?" After an introduction by Pr. Hennebert, Dr C.P. Kurtzman 
(NRRL, USA) and Dr. M. Blackwell (President MSA, USA) express the benefits of phylogenetic analysis for fungal taxonomy, Dr. J. Rammeloo, (National Botanical Garden, Belgium) and Dr. M.-F. Roquebert (National Natural History Museum, France) show the both the historical foundation up to the development of new means of the morphological approach in fungal taxonomy, Dr. A.J. Masuka (Forest Research Centre, Zimbabwe), Dr. A. Peerally (University of Mauritius) and Dr. J. Mouchaca, in name of E.J. daSilva (MIRCEN, UNESCO, France) develop the strategies in the development of mycological research and of fungus collections in the Tropics. In the subsequent discussion much concern is expressed about the lack of funding for taxonomic research on fungi and in the training of future generations of fungal taxonomists not only in developing tropical countries but also in developed temperate areas.

The exhibition of the history of MUCL was very interesting, including the notesbooks of Biourge, some of the original apparatus and microscopes used in his first laboratory, portraits, photographs, manuscripts, paintings and drawings, and publications, and a most attractive display of colourful and stricking fungal cultures.

The celebration ended with a reception in the grounds of a ruined Cistersian monastery, followed by a banquet in the adjacent Hotel des Ruines. The guests were entertained during the banquet by singers and dancers who were refugees from Rwanda. The British Mycological Society President, Professor A. Walley, presented Professor Hennebert with a ceramic sculpture of Polyporus squamosus on behalf of the BMS Council, thanked him for his hospitality and for organizing such a splendid celebration and gave the MUCL good wishes and continuing success for the next century of their activities. 


\section{PLENARY SESSION}




\title{
PART 1
}

\section{MYCOTHEQUE DE L'UNIVERSITE CATHOLIQUE DE LOUVAIN (MUCL): 100 YEARS OF EXISTENCE AND ACTIVITY OF THE FUNGUS CULTURE COLLECTION}

\author{
Grégoire L. HENNEBERT
}

Mycothèque of the Catholic University of Louvain, Place Croix du Sud, 1348 Louvain-la-Neuve, Belgium

The Mycotheque of the Catholic University of Louvain is a fungal culture collection of filamentous fungi and yeasts.

It was started by Professor Philibert Biourge, microbiologist at the Brewery High School of the University in 1892. From contaminated beers, malts and barleys he purified many strains of beer yeasts and isolated wild yeasts, filamentous fungi and bacteria that he maintained in the collection. The collection was publicly inaugurated on July 8th, 1894.

After a well-known preliminary study of Penicillium Link by his assistant F. Dierckx (1901), Biourge prepared a full monograph of the genus Penicillium (1920, 1923). Later Professor P. Simonart made biochemical investigations on the Penicillium species in the collection and discovered the first antimycotic antibiotic griseofulvin from Penicillium griseofulvum Dierckx (1937). After Biourge's death in 1942, Pr. Simonart named the collection "Mycothèque Philibert Biourge".

In 1968, Pr. Simonart brought the collection together with the fungal collection begun in 1956 by G.L. Hennebert, and which already included 11000 strains and herbarium specimens. In $1968 \mathrm{Pr}$. J. Meyer's fungus culture collection of Hyphomycetes from Central Africa, and in 1984, the collection of beer yeasts initiated by Biourge and maintained by the UCL Laboratory of Brewery Sciences were also incorporated.

In 1968 the Collection was officially recognized as the "Mycothèque de l'Université Catholique de Louvain" (MUCL) by the University, in 1969 by the International Association of Plant taxonomy (IAPT), in 1972 by the World Federation of Culture Collections (WFCC), and registered at the World Data Center (WDC). Since 1983, the MUCL Collection has been financially supported by the Science Policy Office of Belgium. It is a member of the Belgian Coordinated Collections of Microorganisms (BCCM), together with the collection of medical fungi at the Institute of Hygiene and Epidemiology (IHEM) in Brussels, the one of bacteria from the Laboratorium for Microbiology, University of Ghent (LMG) and the one of plasmids at the Laboratory for Molecular Biology, University of Ghent (LMB). From 1983 MUCL also has been a member of the European Culture Collections' Organization (ECCO) and from 1985, a partner in the Microbial Information Network Europe (MINE).

\section{The MUCL culture collection}

The MUCL Collection currently holds 24000 strains from all groups of filamentous and yeast fungi, including Oomycetes, Zygomycetes, Ascomycetes, Basidiomycetes, Blastomycetes Hyphomycetes and Coelomycetes. Most of the living 
strains originated from fungal specimens collected throughout the world and kept in the MUCL mycological herbarium which includes 38000 specimens of which 24000 are as living strains: 22000 filamentous fungi and 2000 yeast fungi, representing 3500 species of fungi and yeasts. The second edition of the catalogue was issued in 1992 with about 8000 strains $^{1}$.

The MUCL strains are maintained in at least two different ways, depending on the taxonomic group and on their effective sporulation. All strains are maintained on agar slants under mineral (parafin) oil. Strains producing spores in culture are lyophilized. Delicate and important strains are also maintained frozen at $-80^{\circ} \mathrm{C}$.

MUCL is a research-based culture collection. Its staff is currently carrying on research in both systematic and applied mycology.

Systematic mycology:

- Collection and isolation of fungi in unexplored sites and substrates throughout the world, particularly in extreme environments and in Africa.

- Morphotaxonomy, chemotaxonomy and nomenclature of yeasts and filamentous fungi, particularly Blastomycetes, Hyphomycetes and Aphyllophorales.

- Morphogenesis and morphology of sexual and asexual reproduction.

- Floristics and ecology of fungi in their natural habitats (thermal waters, burned sites, sea sand, rocks, dung, resins, leathers, dried fish, tar, all kinds of industrial products and residues).

- Physiological, biochemical and biomolecular characterization of fungi and yeasts for taxonomy and biotechnology, implemented into a new expert system of identification.

- Biochemical and biomolecular taxonomy of yeasts and filamentous fungi: protein and enzymic profiles, coenzyme Q, RFLP, RAPD mapping, rRNA and rDNA sequencing, phylogenetic analysis.

- Development of a fungal data base on Macintosh (software 4D) following the MINE formats.

Applied Mycology:

- Food contamination. Post-harvest deterioration in storage of cereals and legumes. Flour and dairy contamination. Food fermentation and starter production (cheese, kefirs, sourdoughs, tempeh, fermented cassava, beers and wines).

- Cultivation of edible mushrooms. Production of spawn. Improvement of production and nutritional value. Domestication of edible non-cultivated tropical species.

- Industrial, agricultural and domestic waste biodegradation, agricultural composting and industrial recycling.

- Characterization of wood-decaying fungi. Deterioration of material by fungi (wood, paints, plastics, textiles, etc). Fungal resistance testing of materials. Bioassays of fungicides. Diagnoses in industries and buildings. - Association of termites and fungi.

- Monitoring of product contamination in agricultural and industrial environments. Improvement of production and storage conditions.

- Characterization and production of mycorrhizae for reforestation in the tropics. Production of starters for inoculation.

- Screening of fungi in search of particular properties for biosynthesis.

\footnotetext{
${ }^{1}$ The Mycotheque preserves presently over 51.000 herbarium specimens and 35.000 living strains of fungi
} 
- Improvement of preservation techniques of living strains of fungi (freeze-drying and cryopreservation).

\section{References}

Biourge Ph. 1920. Les moisissures du groupe Penicillium Link, étude monographique (Conference, Louvain, 3 April 1916) Bull. Ass. Anc. El. Ec. Sup. Brass. Univ. Louv., 20(3): 99-127.

Biourge Ph. 1923. Les moisissures du groupe Penicillium Link. Etude monographique. La Cellule, 33: 5331.

Dierckx F. 1901. Essai de revision du genre Penicillium Link. Note préliminaire. Annales Soc. Scient. Brux., 25: 83-89.

Hennebert G.L. 1979. Philibert Biourge, microbiologiste et mycologue, 1864-1942. In Les Sciences Exactes et Naturelles à l'Université de Louvain de 1835 à 1940. IIIe Colloque d'Histoire des Sciences, Louvain-la-Neuve, 17 Mars 1977. Recueil de Travaux d'Histoire et de Philologie, 6e Série, 15: 61-98, 3pl. Louvain.

Hennebert G.L. 1985. Dierckx' Contribution to the Genus Penicillium. In Advances in Penicillium and Aspergillus Systematics, Samson, R.A. and J.I. Pitt, eds., 9-21., 4 fig. Plenum Press N.Y. and London. 


\section{MUCL 1894-1994 step by step}

1891-1892 Philibert Biourge, born on April 8, 1864, is Dr in Botany UCL, and is awarded a scientifi research stay at the Pasteur Institute in Paris, under the direction of Louis Pasteur and of Drs Roux and Duclaux on the pure culture of microorganisms.

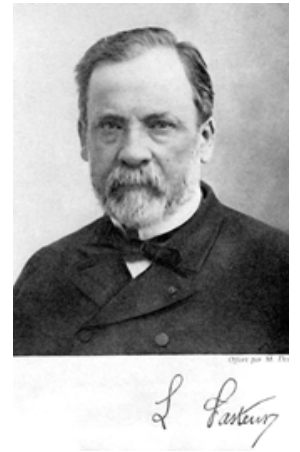

1892 On return from Paris $\mathrm{Ph}$. Biourge is nominated assistent of Prof. Jean-Baptiste Carnoy , Professor of Botany and Microbiology, UCL. (photo). Carnoy was author of "Recherches morphologiqueset physiologiques sur les champignons" (Bull. Soc. Roy. Bot. Belg.9-2:157$311,1870)$.

$1892 \quad$ Ph. Biourge makes a second study stay in Denmark by E. Christian Hansen wo was applying the monospore technique of Pasteur for

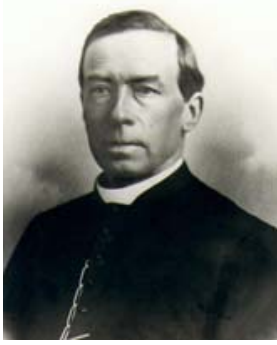
the purification of Saccharomyces cereviviae from the wild yeasts.

1892-1894 $\mathrm{Ph}$. Biourge initiates a Culture Collection of Microorganisms, yeasts, filamentous fungi and bacteria from beers and cereals, at the Laboratory of Zymotechny of the Brewery High School, Catholic University of Louvain, in Louvain.

1894 Public inauguration of the Biourge's Culture Collection with exhibition of living cultures of yeasts, filamentous fungi and bacteria, at the Brewers Congress, Antwerp, 8 July 1894 (Journal du Petit Brasseur, 2 (19) suppl., p.7-11, July 8, 1894, photos).

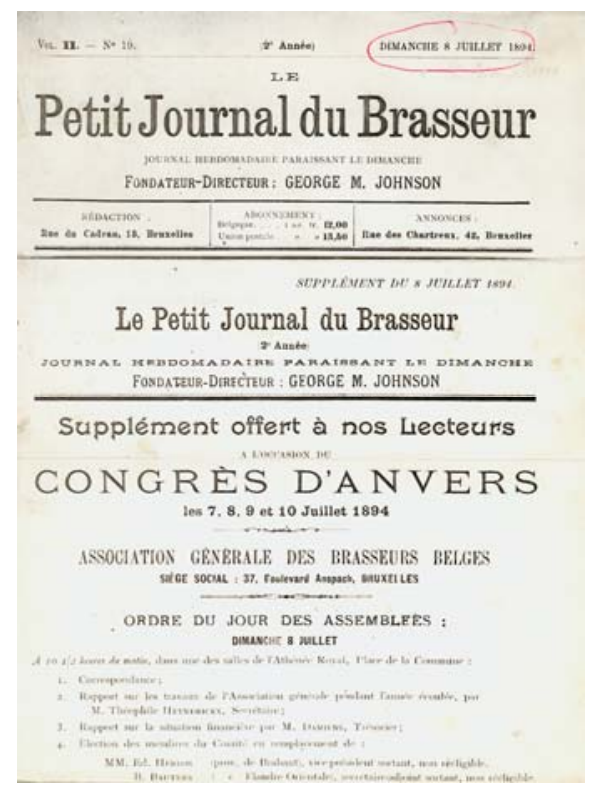

\section{Exposition de l'Ecole de Brasserie DE LOUVAIN.}

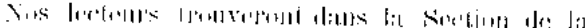

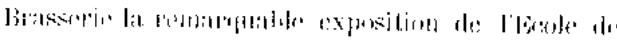

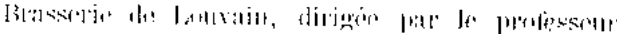

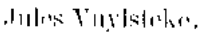

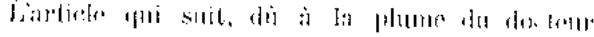

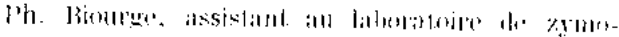

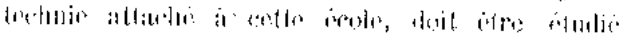

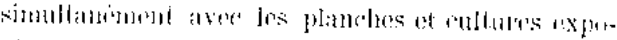
rive :

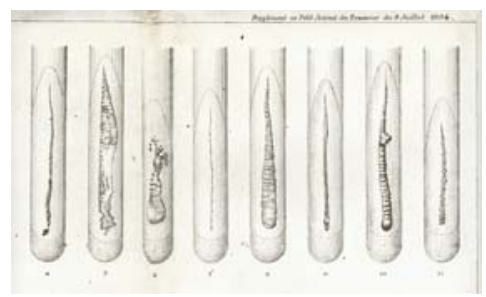



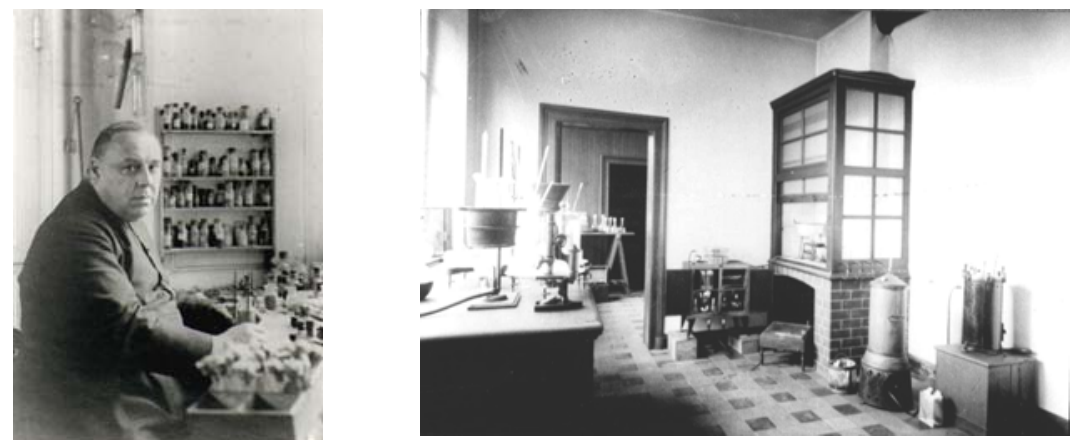

1895 Biourge studies the microbial alterations of beers and of the products of alcoholic fermentation at the Laboratoire de zymologie de l'Ecole Supérieure de Brasserie de l'UCL (photos) (Ph. Biourge, "Les maladies microbiennes de la bière" Bull. Assoc. Anc. El. Ec. Sup. Brass. Univ. Louv., 1(1): 1-4 "Recherches sur la fermentation alcoolique" La Cellule, 11: 93-109). Applying single cell culture method from Pasteur and Hansen, Ph. Biourge is purifying the Belgian beer yeasts for breweries.

1896 Ph. Biourge is nominated Professor UCL.

1897 Ph. Biourge develops the culture collection of moulds, Penicillium and others, from malts. Oscar Semal study fungal production of ammonium (La Cellule, 13(2): 284-312, 1897). René Vandendries presents a $\mathrm{PhD}$ thesis on the "Matières colorantes azotées chez les Champignons".Vandendries will be one pioneer in the sexual mechanism in the Basdiomycetes from 1923 to 1937 ,

1898-1901 François Dierckx (1863-1937) makes a taxonomic study of Penicillium species of the Biourge's collection.

1899 Death of Prof. Jean-Baptiste Carnoy (1836-1899).

Prof. Ph. Biourge becomes head of the Laboratory of Microbiology, Faculty of Sciences, UCL.

1900 F. Dierckx (photo) presents a $\mathrm{PhD}$ thesis on 26 new species of Penicillium with drawings and publishes "Essai de revision du genre Penicillium Link. Note préliminaire", Anns. Soc. scient. Brux., 25(1): 83-89, 1901. He showed his type cultures to P.A. Saccardo, on his way to Indonésia. but lost all his belongings including the cultures in that country. Only one

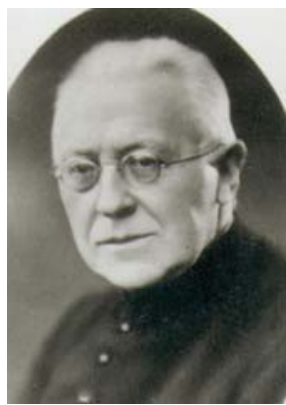
duplicate type culture left in Louvain, Penicillium griseo-roseum Dierckx, could be recovered by Biourge.

1901 Ernest Wieldiers, Md Dr, another Biourge's collaborator, discovers the "Bios", an extract of yeast that contains the required vitamins B, K and others for the growth of yeast. Wieldiers E. "Nouvelle substance indispensable au développement de la levure" La Cellule, 18(2): 311-333, 1901.

1902 The "Question of Bios" is a matter of dispute among scientists. A. Amand, collaborator of Biourge, answers the dispute (La Cellule, 20(2): 223-251, 1902).

1903-1914 $\mathrm{Ph}$. Biourge develops his collection of Penicillium strains. He collected again the species described by Dierckx on basis of Dierckx drawings left in Louvain. He also gathers other Penicillium types from Zaleski, Whemer, Thom, Bainier and Franz Krâl Collections (Krâl started in 1884 a private collection of several hundreds of bacteria, yeasts and filamentous fungi at the Institute of Hygiene of the Faculty of Medecine at the Prague University. It was one of the earliest collection in the world. After Krâl's death in 1911, the collection went to Vienna from where Ersnst Pribam got it in 1915 and published a first catalogue of it in1919).

1910-1914 Alphonse Cappuyns (1887-1936) starts biochemical researches on bacteria and fungi. 
1914-1918 World war: $\mathrm{Ph}$. Biourge retires in his landhome where he works on a Penicillium monograph. A. Cappuyns retires in a studio at Bruxelles where he selects a citric acid producing Penicillium strain from Biourge's collection.

1919 First industrial production of citric acid from a Penicillium strain by A. Cappuyns in "s.a. Les Produits Organiques de Tirlemont", Belgium, after the failure of such a production by the Fabrique de Produits Chilmiques de Thann et Mulhouse, France. (Mazé P. \& Perrier A. 1904 Anns. Inst. Pasteur, 18:553575)

$1920 \mathrm{Ph}$. Biourge publishes a summary of his forthcoming monograph "Les moisissures du groupe Penicillium Link, étude monographique", Bull. Assoc. Anc. El. Ec. Sup. Brass. Univ. Louv., 20(3): 99127, 1920.

1923 Ph. Biourge publishes his Penicillium monograph with 126 species "Les moisissures du groupe Penicillium Link, étude monographique", La Cellule, 33: 3331, 1923.

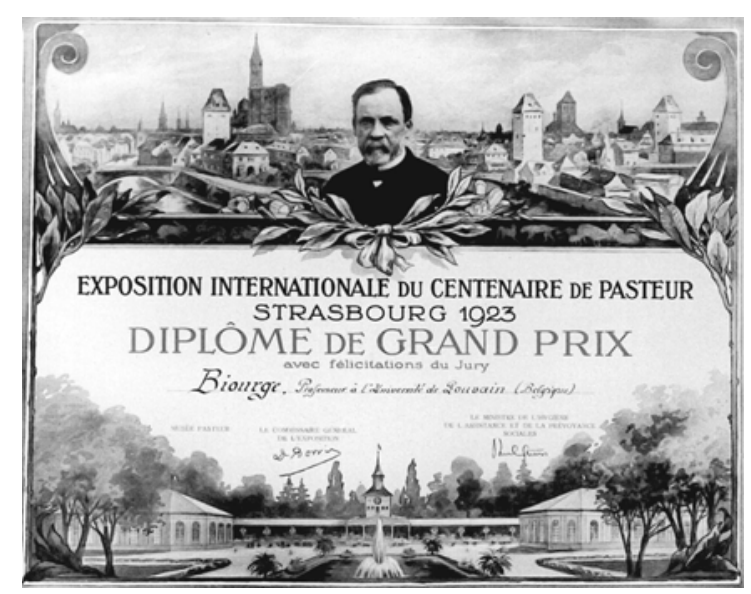

Ph. Biourge receives the Grand Prix de l'Exposition International du Centenaire de Pasteur à Strasbourg (photo) acremonioides)

A collaborator, Victor Estienne presents a $\mathrm{PhD}$ thesis on Aspergillus atro-ruber (Harzia

1925 Ph. Biourge is also devoting much time to the study of the Dutch Elm disease: "La maladie des ormes. J. Soc. cent. Agric. Belg., 73: 22-44, 1925.

1926 A. Cappuyns started a new selection of an acid-resistant citric acid producing strain to substitute it to the too instable Penicillium strain in use: "Sur la formation d'acide sulfurique libre dans les cultures de certains microorganismes.", Anns. Soc. scient. Bruxelle, 45: 177-183, 1926).

1927 Biourge is working on the manuscript of a monogrraph of the Aspergillus as "Brevis Conspectus generis Aspergillus Link" and a preliminary paper entitled "Monographie culturale macroscopique des Aspergillus" with 13 colour plates describing the colours of 208 strains on two media, of which three original plates are preserved at MUCL.

1929 New and successful start of "La Citrique Belge" in Tirlemont by

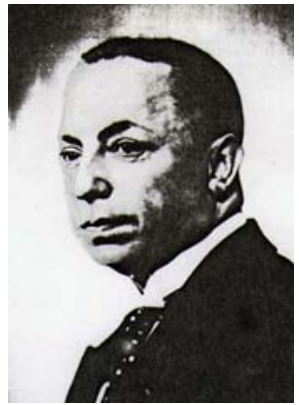
A. Cappyuns (photo), from a selected strain of Aspergillus group niger from the Biourge's Collection.

Paul Simonart (1907-1998), collaborator of Biourge, starts biochemical studies of the lactic fermentation.

1931-1934 P. Simonart is awarded with Prof. H. Raistrick in London. They publish the discovery of the gentisic acid in the biochemistry of Penicillium griseofulvum Dierckx: Raistrick H \& Simonart P. Studies in the biochemistry of microorganisms 29. 2:5-Dihydroxybenzoic acid (gentisic acid) a new product of the metabolism of glucose by Penicillium griseofulvum Dierckx. Biochem. J. 27(3): 628-633, 1933);

1932 Raoul Mosseray makes physiological and morphological studies on the Aspergillus group niger for PhD: "Influence du zinc sur les Aspergillus de la série niger et sur quelques autres." La Cellule 41: 11128

1933 Biourge provides in his paper "Sur les champignons dits moisissures. A quoi bon leur étude et comment la faire.", Rev. Quest. scient., 52(103): 53-78, a justification of a fundamental and taxonomic study of the fungi, in view of their large physiological and biochemical potentialities. 
1934 P. Simonart defends his $\mathrm{PhD}$ thesis on "The biochemistry of Penicillium griseofulvum Dierckx" at the University of London.

From the Aspergillus strains collected by $\mathrm{Ph}$. Bourge and collected by himself in Africa, Raloul Mosseray publishes the monographic study of "Les Aspergillus de la Section Niger Thom et Churh." La Cellule, 43: 201-286. also Ann. Soc. scient. Brux. B, 54: 72-85. He also studies the "Races naturelles et variations de culture chez divers Aspergillus." Ann. Soc. scient. Brux. B, 54: 161-189.

René Vandendries develops his study of the sexuality in Basidiomycetes

Paul Henrard develops a PhD study on the sexuality of Aspergillus nidulans and Eurotium species, "Polarité, hérédité et variation chez diverses souches d'Aspergillus." La Cellule, 43(3): 350-324.

1935 P. Henrard studies further the sexuality of ascomycetes in "Observation sur le comportement de souches monascosporées de Xylaria polymorpha Pers. (Grev.)." Broteria 14(31): 79-83.

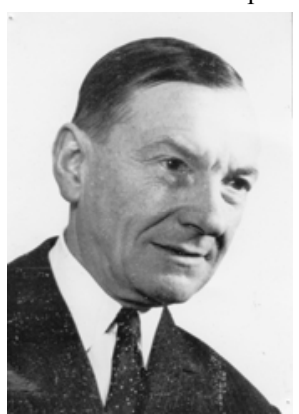

P. Simonart, in collaboration with A.E. Oxford and H. Raistrick publishes the discovery of the "Fulvic acid, a new crystalline yellow pigment, a metabolic product of Penicillium griseofulvum Dierckx, P. flexuosum Dale and P. brefeldianum Dodge.and other species." Biochem. J. 29(5) 1102-1115.

$1936 \mathrm{Ph}$. Biourge publishes his observations on Ophiostoma ulmi "Le cycle du champignon de la maladie de l'orme." Ann. Soc. scient. Brux. 56 130-193.

P. Simonart (photo) is nominated Professor UCL, and awarded Advanced Fellow of the Belgian American Educational Foundation. With Dr. Ch. Thom, in Washington, he is confronting the Biourge's Penicillium Collection to that of Thom.

$1938 \mathrm{Ph}$. Biourge is Emeritus Professor. Prof. P. Simonart is responsible of the Laboratory of Microbiology and takes the Fungus Culture Collection in charge, but the Biourge's yeast collection is kept under care at the Brewery School.

1939 Ph. Biourge, G. Van Cutsem \& E. Bredo put forwards Ophistoma ulmi as the cause of a humain infection, "Une mycose nouvelle: la graphiomycose." Rev. Belg. Scienc. Médic., 11(5): 217-236.

P. Simonart, in collaboration with A.E. Oxford and H. Raistrick publishes the discovery of the "Griseofulvin, $\mathrm{C}_{17} \mathrm{H}_{17} \mathrm{O}_{6} \mathrm{Cl}$, a metabolic product of Penicillium griseofulvum Dieckx." Biochem. J. 33(2): 240-248, the first antimycotic antibiotic.

The Fungus Culture Collection moves, together with the Laboratory of Microbiology, from Carnoy Institute of Botany, Faculty of Sciences, to the new Institute of Agronomy. Prof. Simonart denominates the Collection "Mycothèque Philibert Biourge".

1940-1944 Second world war.

1942 Prof. Ph. Biourge passed away on April 19, 1942.

1945 The Mycothèque $\mathrm{Ph}$. Biourge within the Laboratory of Microbiology, moves from the old Institute in Louvain city to the new Institut Agronomique in the Parc van Arenberg in Heverlee near Louvain.

1949 Raymond Lambert (1923-1992), UCL assistant, then workleader in Prof. Simonart's Microbiology laboratory, takes care of the Mycothèque Philibert Biourge up to 1960.

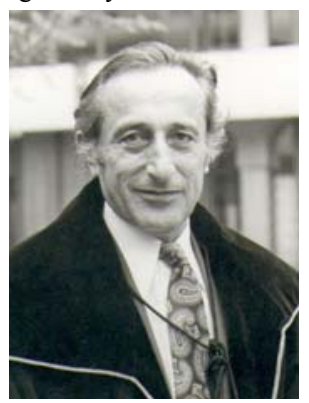

1950-1960 Joseph Meyer (1924-), Dr in Botany, studies soil microfungi, Hyphomycetes and others, of Central Congo, in Yangambi, Congo, establishing a herbarium and a culture collection. (photo)

1956 Grégoire L. Hennebert (1929-), FNRS research fellow at UCL, initiates a personal Fungus Culture Collection in the Plant Pathology Laboratory, UCL Faculty of Agronomy, and starts a research on Botrytis and the Sclerotiniaceae with Prof. V. Estienne as promotor.

1960 Joseph Meyer, collaborator in Prof. Simonart's Laboratory, takes care of the Mycothèque Philibert Biourge, until 1969, as well as of his collection of African Hyphomycetes.

G.L. Hennebert presents the thesis "Recherches morphologiques sur le genre Botrytis Persoon", for obtention of the PhD graduation at UCL. 


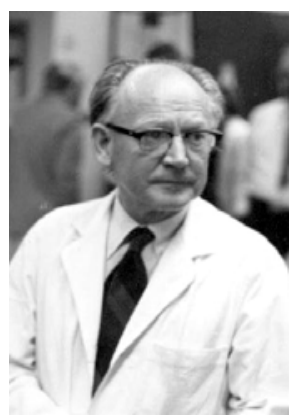

1960-1962 G.L. Hennebert is awarded by the Canadian National Research Council a two-year postdoctoral fellowship at the Mycology Unit of the Plant Research Institute, Ottawa, with Dr Stanley J. Hughes (photo).

1962 G.L. Hennebert Visiting Research Mycologist, Plant Pathology \& Mycology Dept., Cornel Univerity, Ithaca, NY.

1962-1965 G.L. Hennebert is awarded FNRS qualified researcher and appointed UCL teaching assistant in Plant pathology, developing the Fungus Culture Collection, at the Faculty of Agriculture, Heverlee-Leuven. (photo)

1963-1975 MUCL develops a close collaboration with the Central Bureau voor Schimmelcultures CBS in Baarn.

1964 Visite of Dr.C.J. Alexopoulos from to the Collection (24.08.1964).

1965 Visit of Pr. Dr. C.V. Subramanian from Madras to the Collection (23.06.1965) Professors.

G.L. Hennebert and J. Meyer are nominated UCL

1966 Visit of Pr. DR. R.P. Korf from Cornell University,

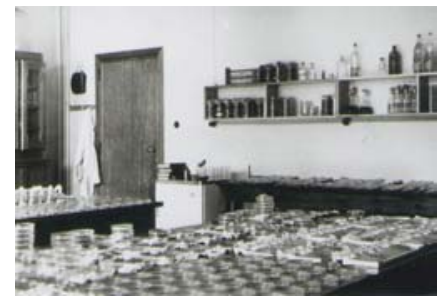
Ithaca, NY. to the Collection (13.06.1966)

1967 G.L. Hennebert, visiting lecturer, University Lovanium, Kinshasa, Congo.

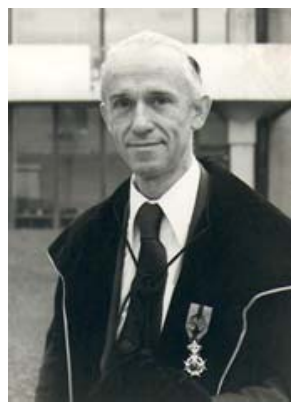

1969).

1968 The G.L.H. Collection is composed of 12,000 herbarium specimens and 7,000 living strains (Anonymous 1968. Centre d'Etudes: le Laboratoire de Mycologie Systématique et Appliquée. Nouvelles Brèves, REUL, Rel. ext. Univ. Louvain, 18 Juin: 3-4)

Prof. Simonart transfers the Mycothèque Philibert Biourge to the G.L. Hennebert Fungus culture Collection. The Mycoth.èque Biourbe consists of 683 Penicillium strains and 122 Aspergillus strains, a number of them having been returned to Pr. Simonart by Thom (USA) as earlier received from Biourge.

The UCL authorities agrees upon the new denomination of the integrated Collection of Fungi as the "Mycothèque de l'Université Catholique de Louvain (MUCL)", which becomes internationally recognized by the International Association for Plant Taxonomy (IAPT Regnum vegetabile

1969 Pr. J. Meyer contributed his Collection of African soil fungi to the Mycothèque.

Visit of DR. J.A. von Arx, CBS, Baarn, The Netherlands (19.04.1969)

1969-1986 G.L. Hennebert is effective member of the Special Committee for the Nomenclature of Fungi and Lichens of the IAPT-N.

1970 G.L. Hennebert is visiting Professor, Laval Univerity, Québec, and invited Professor Tchechoslovakian Academy of Sciences, Praha.

1970-1994 MUCL is member of the International Biodeterioration Research Group (IBRG) and of the Internationa Association for Wood Preservation (IRGWP)

Second Visit of Pr. Dr. C.V. Subramanian, India (28.09.1971).

1972 MUCL becomes affiliate of the World Federation of Culture Collections (WFCC Directory ed. 1972). A first catalogue of the included species represented by living strains is then provided. 


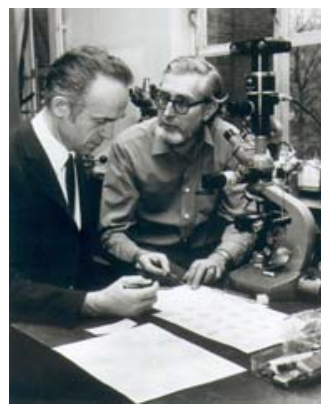

1972-1973

Prof. Richard P. Korf is awarded Fulbright-Hays Senior Research Scholar and stay six months at MUCL and the UCL Faculty of Agriculture (photo). He delivered a special conference on "A new classification of the Operculate Discomycetes"

1973 Visit of Dr. Stanley J. Hughes at MUCL (photo) for discussion on the classification of Fungi Imperfecti and collaborative work on Sporoschismopsis (317.05.1973).

1974 Creation and first issues of MYCOTAXON, an international journal for the taxonomy of fungi and lichens, by Dr R.P. Korf and G.L. Hennebert.

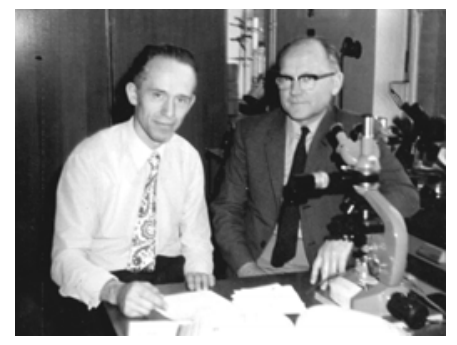

1975 Move of the MUCL Fungus Culture Collection to new laboratories of the Faculty of Agricultural Sciences on the new UCL campus in Louvain-la-Neuve, $30 \mathrm{~km}$ south-east of Brussels.

1977 Publication of "Anamorph, teleomorph and holomorph, terms for forms of fungi, their names and types", defined by Hennebert and Weresub, Mycotaxon 6: 207-211.

1981-1990 Creation of the Laboratory of Microbiology at the University of Burundi, Faculty of Agronomical Sciences, Bujumbura, Burundi and establishment of the LMUB Culture Collection.

1981 G.L. Hennebert, Invited Professor at the Potchefstroom Univerity, South Africa

1982-1990 G.L. Hennebet, Visiting Professor in Microbiology, University of Burundi, Bujumbura.
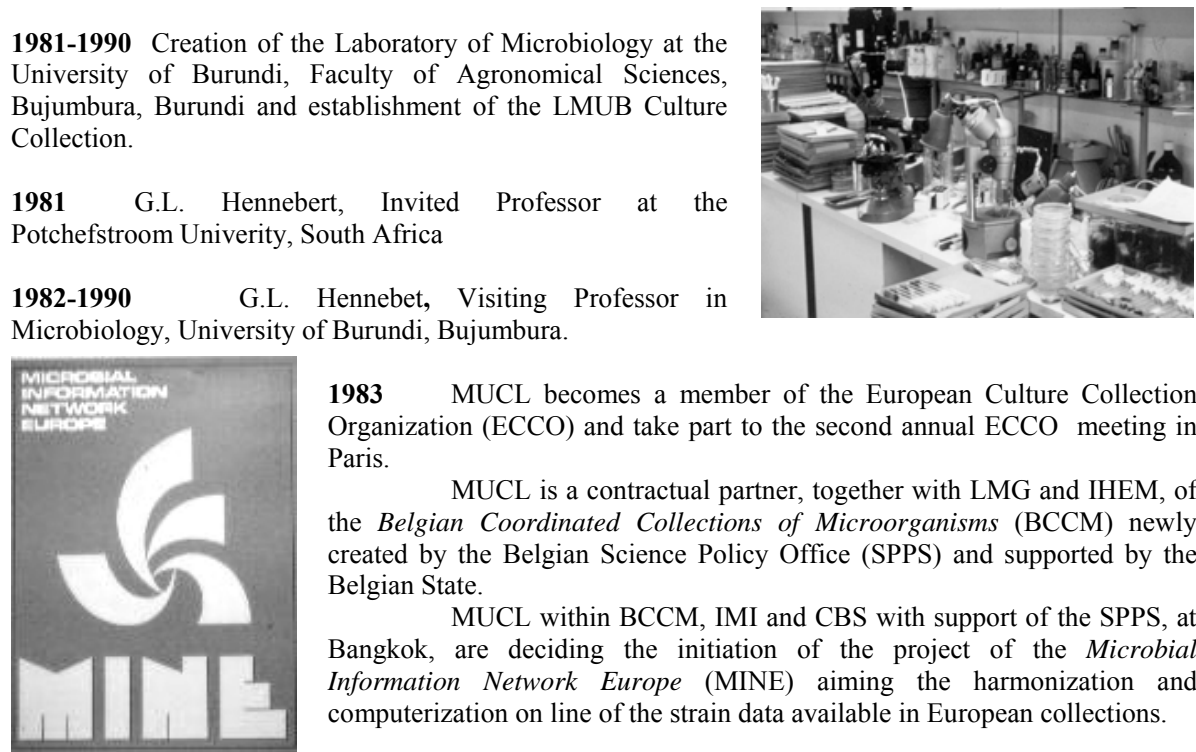

1983 MUCL becomes a member of the European Culture Collection Organization (ECCO) and take part to the second annual ECCO meeting in Paris.

MUCL is a contractual partner, together with LMG and IHEM, of the Belgian Coordinated Collections of Microorganisms (BCCM) newly created by the Belgian Science Policy Office (SPPS) and supported by the Belgian State.

MUCL within BCCM, IMI and CBS with support of the SPPS, at Bangkok, are deciding the initiation of the project of the Microbial Information Network Europe (MINE) aiming the harmonization and computerization on line of the strain data available in European collections.

1985 The project MINE is approuved by the CEC with MUCL, LMG and ICP in BCCM, CBS, CMI, DSM, IP, LCP as first partners. Among them MUCL, LMG and CBS elaborate the standard format for the database and published catalogue (photo) of the fungi and bacteria.

1986 G.L. Hennebert, Visiting Professor, Institut Agronomique et Vétérinaire d'Agadir, Maroc.

1987 Visit of Dr. M. van Uden (IGC, Portugal), Dr. S. Udagawa (NHL, Japan), DR. R.P. Korf (CUP, USA), Dr. L. Blaine (ATCC, USA); Dr. R. Haruenkit (TISTR, Thailand) to MUCL in Louvain-la-Neuve.

1989 First edition of the "MUCL List of Cultures Fungi-Yeasts 1989" of almost 5000 strains (photo).

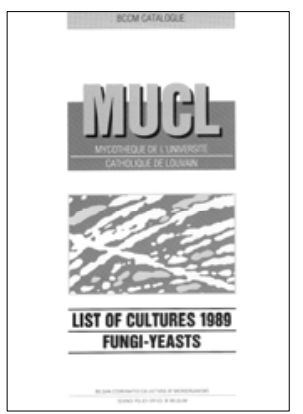


1990 MUCL is elected responsible of the MINE Committee for the harmonization of the computerized strain data on Fungi and Yeasts, under the BRIDGE EEC Programme.

Publication of "La Mérule, Science Technique et Droit" par G.L. Hennebert, Ph. Boulenger et Fr. Balon, Ed. Ciaco, 198 p. over the dry rot fungus problem.

1992 Second edition of the "MUCL Catalogue Fungi-Yeasts 1992" containing almost 8000 strains.(photo)

MUCL, and the other BCCM Collections, are elected as International Depository Authority (IDA) for the deposit of fungus and yeast strains for patent purposes under the Budapest Treaty by Belgian state decision..

1994 July 8. The Mycothèque de l'Université Catholique de Louvain is since 100 Years a public collection.

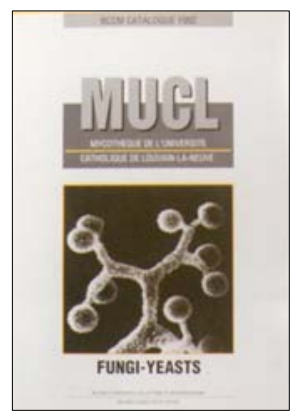




\title{
FUNGAL GENETIC RESOURCE COLLECTIONS AND BIODIVERSITY
}

\author{
David L. HAWKSWORTH \\ International Mycological Institute, Bakeham Lane, Egham, Surrey TW20 9TY, UK
}

\begin{abstract}
Institutions or activities must reconsider and reinterpret their objectives in the language of the day; failure may endanger their survival. The single act of relabelling "Culture Culture Collections" as "Microbial Genetic Resource Collections" immediately makes a link with agendas of the 156 government signatories to the Convention on Biological Diversity. In addition, collections must be poised to answer fundamental questions: Who needs the collections and why? How effective is the present system? How many strains of a species are needed ? In what ways should collections collaborate to maximize effectiveness? And how can national and regional collections contribute to the sustainable use of the Earth's resources? In order to realize the potential of the collections, a fuller integration in which national, regional, and international facilities have complementary roles must be sought.
\end{abstract}

\section{Introduction}

In order to prosper, an institution or activity must repeatedly reconsider and reinterpret its objectives in the language of the day. And also in accordance with the agendas of its users and funding sources. However much a service is seen as invaluable and essential to those intimately involved in it, failure to be seen to relate to topical issues risks increasing marginalization, and a falling away of the very constituency essential to its survival. Microbial collections have no special exemption, and heed should be taken of the experience of some of our major museums and botanical herbaria.

Founded in 1894, the Mycothèque de l'Université Catholique de Louvain, whose centenary we are celebrating, is the oldest collection of fungal cultures still operating. If the Mycothèque had not been able to adapt to changing policy requirements, we would not be here today.

In the mid-1990s, demands for justification and accountability have become universal. In this climate, it is imperative that those involved in culture collection development and management are poised to present their case in a manner which is in harmony with the issues of today. Here, I pose questions that need to be addressed at levels from the individual collection to the national, regional, and international.

\section{What should culture collections be called?}

The label "culture collection" is a barrier to communication. It has hindered the forging of links with the rise of interest and funding devoted to the ex-situ conservation of plants and animals during the last two decades. Interdisciplinary meetings and reports on "genetic resources" have only exceptionally considered microorganisms, and it is proving a slow process to change the perception that fungi and microorganisms are something apart from "genetic resources".

Article 2 of the Convention on Biological Diversity defines genetic resources as "genetic material of actual or potential value"; "genetic material" being "any material of plant, animal and microbial or other origin containing functional units of heredity" (UNEP 1992). Article 9 (b) directs the contracting parties to the Convention to "Establish and maintain facilities for ex-situ conservation of and research on plants, animals and micro-organisms, preferably in the country of origin of genetic resources". A dictionary definition of a resource is "a source or possibility 
of help"; that immediately conveys a positive message in contrast to "culture" which makes no value statement.

The phrase "culture collection" does not appear in the Convention. The single act of relabelling "culture collections" as "microbial genetic resource collections" makes an instant link with the agendas of the 156 governments that are signatories to the Convention; and also to other programmes starting to be developed as the ripples from Rio extend ever wider (e.g. Kapoor-Vijay 1992).

\section{Who needs the collections and why?}

Fungal genetic resource collections have a diverse customer base. However, while they are crucial at different points in time in many areas, a single customer will often use their services only rarely. The key needs are:

- to provide named material for industry and both pure and applied research in a timely and cost effective manner.

- to safeguard genetic resources as an insurance policy through holding stocks in long-term storage for future needs.

- for comparative purposes to make identifications and maintain type cultures ${ }^{*}$ that fix the application of names.

- to maintain patent and industrial strains of microorganisms.

- to use in education and training.

Common myths must also be dispelled. There is a frequent misconception that fungi and other microorganisms are everywhere and just wait to be isolated. Were that true, desired fungi could be isolated from nature on demand. While that thesis may hold for opportunistic moulds associated with humans and their products, and perhaps some elements of the soil mycobiota (Gams 1992), such statements have to be kept in perspective. Quotations such as "everything is everywhere and the environment selects" (Baas-Becking 1934) can be profoundly damaging if not placed in context. In reality, around $70 \%$ of the fungi are obligately associated with particular host plants or animals (including insects) as pathogens, commensals, mutualists, or specialized saprobes. Their distributions will thus tend to be closely correlated with those of their hosts.

In the case of fungi which are not visible to the unaided eye or with a hand lens, and which are isolated from nature by dilution-plate or similar methods (Gams 1992), the probability of refinding a species the spores of which occur at low frequencies is so remote as to be impractical.

Further, many fungi which can be seen by eye, forming macroscopic fruit-bodies or otherwise visible colonies, have only once or exceptionally been successfully brought into culture. In some cases this may not be technically difficult provided that isolations are conducted while the material is in a fresh condition - for example, isolated close to the point of collection before the specimen dries out. An example of what can be achieved by developing particular isolation strategies is a recent project involving lichen-forming fungi. Although around one fifth of all fungi have this biology, lichen-forming fungi are scarcely present in microbial collections. Yet 427

\footnotetext{
${ }^{*}$ Although living cultures of fungi are not acceptable as nomenclatural types under the International Code of Botanical Nomenclature (ICBN), the latest edition of the Code (Greuter et al. 1994) makes clear by an example that cultures "permanently preserved in a metabolically inactive state", such as by lyophilization or storage in liquid nitrogen are acceptable; the Code recommends that cultures resurrected from such permanently preserved cultures are referred to as ex-holotype, ex-isotype, etc.
} 
(42\%) of 1021 lichen-forming and lichen-inhabiting species attempted were successfully isolated, including representatives of many genera, families, and even orders never previously obtained in culture (Crittenden et al. in prep.).

Fungal genetic resource collections can maintain such rarely or not otherwise cultured strains so that they are available for both research and evaluation for potential applications. Pragmatically, the collections are the only source of pure living isolates of many fungi.

\section{How effective is the present system?}

One measure of the effectiveness of the present system is the number of species of fungi available in the world's service collections. In 1990 the collective holdings were estimated at 11500 fungi, about $17 \%$ of the 69000 then described (Hawksworth 1991) ${ }^{*}$. If the conservative working estimate of 1.5 million fungi on Earth is accepted, something for which there is growing evidence (Hawksworth 1993), that means only about $0.8 \%$ of those actually existing are currently in a service collection.

Fungi, as catalogued in the Index of Fungi, are currently described at the rate of about 1700 species per year. Precise data are not available, but only about $30 \%$ of these are known in culture. As the number of known species increases, on current trends the proportion represented in the service collections can be forecast to decrease rather than to increase. This situation could be at least partly rectified by mycologists endeavouring to prepare cultures immediately after collection, as strongly advocated by Huhtinen (1994), although major targeted isolation programmes (see above) may be necessary to make a significant impact on the current situation.

\section{How many strains of a species are needed?}

At 351263 , the number of individual strains of fungi available in the world's service collections at first seems impressive; on average that is 17 strains under each name indexed (Sugawara et al. 1993). However, this statistic does not automatically imply an enormous duplication in the genetic resource held. The seed banks of major cereals count their accessions in 100 000s, for example, with 333413 accessions for Oryza sativa in 1991 (Groombridge 1992) in order to preserve the range of the genetic resources within a single species. Fungi also exhibit substantial variation within a species. The extent of infraspecific variation in a particular fungus is often unclear, due to the lack of investigation, but well-studied examples show that this can be expected to be considerable.

The mycelia from morphologically defined species are often unable to fuse together, forming "vegetative incompatability groups" in both sexually and asexually reproducing species (Brasier 1987). For example, Ploetz (1990) found 11 incompatability groups in 96 strains of Fusarium oxysporum sp. f. cubense. Stable aneuploids and polyploids may be a key factor in some cases, but chromosomes

${ }^{*} *^{*}$ The most recent edition of the World Directory of Collections of Cultures of Microorganisms (Sugawara et al. 1994) lists 19392 names of fungi implying that a much higher proportion of species is held. Further, of the names of fungi listed, 9539 are indicated as held in only one. However, these figures are inflated as they do not allow for synonyms, separately named anamorphs, alternative taxonomies, nor different spelling variants used in the collections submitting data. 
receive scant attention from mycologists; indeed, aneuploidy was recognized in Penicillium only recently (Bridge et al. 1986).

The extent of variation at the molecular level can also be exemplified by $F$. oxysporum, where seven mtDNA RFLP patterns may occur within a single special form (Jacobsen \& Gordon 1991). In the particularly well-studied Neurospora crassa, genetic stock collections maintain some 3000 different genetic variants of this single species (Fungal Genetics Stock Center 1988).

Against the background of this genetic variation, it is sobering to note that a study of the holdings of the various living fungal collections demonstrates that a considerable number of fungi are represented only by single isolates. An indication of this is provided by data compiled in the World Directory of Collections of Microorganisms (Sugawara et al. 1993) where $49 \%$ of the taxa are listed as represented in only a single collection. In the case of the International Mycological Institute (IMI) collection, $2541(56 \%)$ of the 4541 species maintained are represented by only one isolate.

There is at present no general answer to the question "How many strains of a species are needed?" in fungi, but we can be confident that the figure is considerable. Were a modest 10 strains to be maintained for all 72000 known fungi, that 720000 would require a doubling of the collective holdings of all the world's service collections.

\section{In what ways should collections collaborate to maximize effectiveness?}

The World Directory of Collections of Cultures of Microorganisms lists 246 collections of fungi (including yeasts) world-wide (Sugawara et al. 1993). While this work does not include industrial and other research collections, this number approximates to those actively involved in the preservation and supply of fungus cultures today. These collections vary enormously in size, from a few hundred to more than 45000 strains. Some are general in scope, but many specialize in fungi relevant to particular applied areas, for example plant pathology, medical mycology, genetic stocks, edible fungi, or brewing.

The collections are not evenly distributed geographically. Only 39 of the 142 countries wholly or partly located in the tropics have collections registered with the World Directory; while 107 collections are involved, 52 (49\%) are in one of two countries, Australia and India (Hawksworth \& Ritchie 1993).

It has to be accepted that: (a) no single collection is ever likely to achieve the level of funding necessary to serve as a genetic resource collection for all groups of fungi; and (b) many countries are likely to remain without national collections for the foreseeable future unless appropriate funds can be released. If an adequate service to the world is to be provided, one which is able to fulfil all the criteria identified above, and further satisfy national needs, collaborative mechanisms must be sought.

The need for collaboration was foreseen in the Convention, the signatory governments agreeing under Article 9 (e), to "Cooperate in providing financial and other support for ex-situ conservation". This call was not a new one; the UN Conference on the Human Environment, meeting in Stockholm in 1972, had already recommended action "in respect of micro-organism germ plasms, [to] co-operatively establish and properly fund a few large regional collections" (United Nations 1972). Co-operative action on the level required has not taken place, and nor will it now 
unless the collections themselves develop a workable strategy in discussion with the donor community and national governments.

An indication of what could be achieved is provided by the International Plant Genetic Resources Institute (IPGRI; formerly the International Board for Plant Genetic Resources, IBPGR). IPGRI, founded in 1974, established a network of centres specializing in storing the seeds of particular crops in order to further the collection, documentation and use of germplasm for crop species (Williams 1988).

In the case of microbial collections, organizations and networks exist which can be used to ascertain what strains are held, including the European Culture Collection Organization (ECCO), the World Federation for Culture Collections (WFCC), Microbial Strain Data Network (MSDN), Microbial Information Network Europe (MINE), and various national organizations. The Biodiversity Information Network 21 (BIN 21; Canhos et al. 1992) also promises to ease the exchange of data on holdings. However, these bodies do not have the resources or mandate to control or coordinate their member collections. The UNESCO/UNEP-sponsored Microbiological Resource Centres (MiRCENs) scheme, establishing centres, especially in less developed countries (Da Silva 1991), is very much on the lines required but has been undersourced and will need to adapt to the changed situation with regard to property rights.

\section{How can national and regional collections contribute to the sustainable use of the earth's resources?}

Article 10 of the Convention on Biological Diversity places emphasis on "the sustainable use of components of biological diversity" and not only on its conservation. In addition to the general roles of genetic resource collections of fungi, they can contribute to sustainability at the national and regional level by:

- safeguarding the resource, especially from disappearing habitats.

- making the resource available for screening and use on the terms of the country of origin.

- utilizing the resource for biocontrol, waste degradation, and food production.

- maintaining mycorrhizal mutualists important for forest re-establishment.

- providing a source of reference strains to speed the diagnosis of plant pathogens.

Making the fungal resource available in a controlled way for screening purposes which will bring maximum income into a country is of especial significance. The issues are complex (Reid et al. 1993), and national legislations have yet to evolve. However, payments on a per strain basis linked to royalty agreements are possible. When the question of how to use a particular hitherto uncut forest in a sustainable way for the benefit of the local population is posed, isolating and selling on fungi is an option not to be discounted. In cases where an individual country cannot provide a national facility, agreements on a regional basis should be sought.

\section{Conclusion}

Microbial resource collections are the way of making fungal biodiversity available for exploitation in support of sustainable development. They are the catalyst required to turn potential into reality. 
However, fulfilment of that goal will require both collaboration and the development of mechanisms to enhance the complementarity between collections. Collaborative networks at a variety of levels are emerging as the logical approach to the provision of biosystematic services in speciose groups (Janzen 1993, Jones 1994). Genetic resource collections have demonstrated, through MINE and other initiatives, that they can successfully combine and exchange data. We should not be complacent, and recognize the need to move to a second level of co-operation beyond that of networking data; to a fuller integration in which national, regional, and international facilities have complementary roles. As such a proposal would be in accord with current concerns as to the conservation and sustainable use of biodiversity, we should not be afraid of making major funding proposals. "Big science" funds with no immediate benefits to humankind are secured because of a unanimous clamour from the enthusiastic scientists involved; we need to follow suit and convey our vision of the potential to the donor community.

\section{Acknowledgement}

I am indebted to Dr David Smith for his contribution to the statistics and also discussion and comments during the preparation of this article, and to Dr H. Sugawara for a detailed analysis of some of the data in the World Directory.

\section{References}

Baas-Becking L.G.M. 1934. Geobiologie of inleiding tot de milieukunde. The Hague: Van Stockhum. [Not seen.]

Brasier C.M. 1987. The dynamics of fungal speciation. In Evolutionary Biology of the Fungi, Rayner A.D.M., Brasier C.M. and Moore D. eds., Cambridge: Cambridge University Press. pp. 231-260

Bridge P.D., Hudson L., Hawksworth D.L. and Bridge D.A. 1986. Variation in nuclear DNA content in an ex-type isolate of Penicillium measured by continuous flow microfluorimetry. FEMS Microbiology Letters 37: 241-244

Canho V., Lange D., Kirsop B.E., Nandi S. and Ross E. 1992. Needs and Specifications of a Biodiversity Information Network. Nairobi: United Nations Environment Programme.

Crittenden P.D., David J.C., Hawksworth D.L. and Campbell F.S.1995. Isolation and culturing success in a broad spectrum of lichen-forming and lichenicolous fungi. Mycol. Res. 106: 788-795.

Fungal Genetics Stock Center. 1988 Catalogue of Strains. Second edition. Kansas City, Mo.: University of Kansas Medical Center.

Da Silva E.J. 1991. Biotechnologies, microbes and the environment. Nature and Resources 27(3): 23-29.

Gams W. 1992. The analysis of communities of saprophytic microfungi with special reference to soil fungi. In Fungi in Vegetation Science Winterhoff W. ed., Handbook of Vegetation Science, 19(1): 183-223.. Dordrecht: Kluwer Academic Publishers.

Greuter W., Barrie F., Burdet H.M., Chaloner W.G., Demoulin V., Hawksworth D.L., Jørgensen, P.M. Nicolson D.H., Silva P.C., Trehane P. and McNeill J. eds. 1994 International Code of Botanical Nomenclature (Tokyo Code). Regnum Vegetabile No. 131. Königstein: Koeltz Scientific Books.

Groombridge B. ed. 1992. Global Biodiversity. Status of the Earth's Living Resources. London: Chapman and Hall.

Hawksworth D.L. 1991. The fungal dimension of biodiversity: magnitude, significance, and conservation. Mycological Research 95: 641-655.

Hawksworth D.L. 1993. The tropical fungal biota: census, pertinence, prophylaxis, and prognosis. In Aspects of Tropical Mycology. Isaac S., Frankland J.C., Watling R. and Whalley A.J.S. eds. Cambridge: Cambridge University Press. pp. 265-293.

Hawksworth D.L. and Ritchie, J.M. 1993. Biodiversity and Biosystematic Priorities: Microorganisms and Invertebrates. $120 \mathrm{pp}$. Wallingford: CAB International.

Huhtinen S. 1994. Traditional discomycete taxonomy: should we also shift to a second gear. In Ascomycete Systematics: Problems and Prospects in the Nineties. Hawksworth D.L. ed.: New York: Plenum Press. pp. 295-302. 
Jacobsen D.J. and Gordon, T.R. 1991. Fusarium oxysporum f.sp. melonis: a case study of diversity within a forma specialis. Phytopathology 81: 1064-1067.

Janzen D.H. 1993. Taxonomy: universal and essential infrastructure for development and management of tropical wildland biodiversity. In Proceedings of the Norway/UNEP Expert Conference on Biodiversity, Trondheim, Norway. Sandland O.T. and Schei P.J. eds. Oslo: NINA, pp. 100-113.

Jones T. 1994. BioNET-INTERNATIONAL: a global network for biosystematics of arthropods, nematodes, and microorganisms. In The Identification and Characterization of Pest Organisms. Hawksworth D.L. ed. Wallingford: CAB INTERNATIONAL, pp. 81-91

Kapoor-Vijay P. 1992. Biological Diversity and Genetic Resources. The Programme of the Commonwealth Science Council. London: Commonwealth Secretariat.

Ploetz R.C. 1990. Variability in Fusarium oxysporum f. sp. cubense. Canadian Journal of Botany 68 : 1357-1363.

Reid W.V., Laird S.A., Meyer C.A., Gámex R., Sittenfeld A., Janzen D.H., Gollin M.A. and Juma C. 1993. Biodiversity Prospecting. Washington, D.C.: World Resources Institute.

Sugawara H., Ma, J., Miyazaki S., Shimura J. and Takishima Y. 1993. World Directory of Collections of Cultures of Microorganisms. Fourth Edition. Saitama: WFCC World Data Center on Microorganisms.

United Nations. 1972. Report of the United Nations Conference on the Human Environment, Stockholm, 5-16 June 1972. New York: United Nations.

United Nations Environment Programme. 1992. Convention on Biological Diversity. June 1992. Nairobi: United Nations Environment Programme.

Williams J.T. 1988. Identifying and protecting the origins of our food plants. In Biodiversiy. Wilson E.O. ed. Washington, D.C.: National Academy Press, pp. 240-247 


\title{
THE FUNGAL CULTURE COLLECTIONS IN EUROPE: CHANCES, CHANGES AND CHALLENGES
}

\author{
D. VAN DER MEI \\ Centraalbureau voor Schimmelcultures, Baarn, the Netherlands
}

The celebration of the centennial of the "Mycothèque" of the Catholic University of Louvain-la-Neuve is in itself reason enough to congratulate the University and the "Mycothécaire", Gregoire Hennebert. I could leave it at this.

This centennial jubilee, however, concerns an Institution within a University, and a collection of fungi. And these two aspects ask for a somewhat deeper reflection.

To-day's universities are characterized by rapid changes, triggered by financial problems and short-term policies more often than by long-term considerations in which sciences should be embedded. This holds for most disciplines, but particularly so for systematic biology with as its main component taxonomy and as its major instrument the collections. Most universities, as a consequence, have in the recent history been not very accommodating media for these two.

In the last two decades the number of taxonomic staff in universities all over Europe and world-wide has dwindled, collections in particular being considered as questionable assets.

One of the reasons is that collections are rather expensive to keep, while the revenues, mostly measured as numbers of publications in highly esteemed international journals, are only to be seen in the long term and are seldom directly retraceable to the collection and the university that pays for it.

More important is that the scientific scene for many years has been dominated in biology by the so-called "reductionistic approaches", with as main components molecular and cell-biology. The great break-through, originating from these disciplines, has grasped the imagination of the public domain, masterminding the direction of research in virtually all biological subjects. The holistic approach, in which the organisms themselves in relation to their surrounding environment, living and dead, are the issue, has lagged behind.

The fact is that these two mainstreams, reductionism and holism, are still not well-balanced, making biology a divided house. This is in contrast to physics and chemistry. The holistic approach, of which collections - whether they contain living or dead material - are to be seen as a major component, has to gain a lot of lost ground in order to compete successfully for national and international shares of the budget.

Well over 100 years ago the Swiss author Rudolph Töpfer created his "Monsieur Cryptogame", internationalized as "Herrn Steckelbein" in Germany and as "Mijnheer Prikkebeen" in the Netherlands. He personified the "Collectioner" as a man deeply satisfied by the pleasure of sampling in Nature, and deeply unhappy with womanhood, expressed by his pathological fear for his sister Ursula. A caricature he was.

But when discussing collections and taxonomy with those who decide upon my budget for next year, I still encounter the deep-felt suspicion that the only reason for our existence is in playing Monsieur Cryptogame, the fear for sister Ursula maybe replaced by the fear for bureaucrats, still mainly motivated by gathering as many 
organisms as possible for the pleasure we get from them and not bothering about the sense of it all.

The taxonomist, at the time of birth of Monsieur Cryptogame, stood at the cradle of the unifying concept in biology, the theory of evolution. And he still stands in the centre of evolutionary biology, looking for the roots and the history of biodiversity as it presents itself to-day. And he has grasped the opportunities that are offered by new techniques, especially in molecular biology, to tackle the problems more adequately. In taxonomy, and directly in relation to it, in collections, a major effort is undertaken to bridge the gap between reductionism and holism, for the benefit of biology as a whole.

I hope that in the near future the taxonomy, within the context of systematic and evolutionary biology, of life on the planet earth will reach on the scale of issues worthwhile investigating the same level as the taxonomy of our stellar system, for which billions of dollars are attributed easily in the construction and salvation of Hubble-telescopes and the like.

The fact that Biodiversity has appeared on the agenda of the United Nations is a milestone, as is the fact that taxonomy nowadays is firmly linked to the mainstream in biology.

Let's not be over-optimistic and self-confident. There are some major hurdles ahead, to mention a few:

1. Taxonomy and collections lack a strong organizational structure, that can act as a sparring partner speaking with a voice that is clearly heard.

In the Netherlands, within the framework of the graduate school of biodiversity, botanists and zoologists are always to be reminded that there are also microorganisms and collections, besides botanical gardens and zoos, where living organisms are kept in perfect shape. There seem to be three taxonomies, that of animals, that of plants and that of microorganisms.

2. The expectations of those who put biodiversity on the political agenda may differ greatly from insights, expectations, possibilities and capacities of taxonomists and collections.

3. Biodiversity has the danger of becoming a fashion. Fashions in science may lead to a proliferation of activities, based upon status and "getting a slice of the pie", rather than upon sound scientific strategies and ideas. The fact that taxonomy is at the basis of this fashionable term is often overlooked.

Nevertheless, there is a revival of taxonomy, and together with that, a keen interest in what collections are and what they can do. It is up to us to show that we are up to the challenges, national, European and world-wide.

A recent report of the Royal Netherlands Academy of Arts and Sciences, in which its scientific priorities are reconsidered, still does not mention taxonomy as one of the main issues.

For survival of the bacterial collections in the Netherlands money has to be found exclusively on the free market and in contract-research.

These are also facts. Structural support has to come from reshuffling of budgets invested in medicine, chemistry and physics, disciplines which have well-structured organizations and lobbies. Without a fight we will not succeed.

My first words in this part of my lecture were to praise the Universities of Leuven and Louvain-la-Neuve for fostering the "Mycothèque" through the first 100 years. They have shown wisdom in doing so, and it is my sincere wish that they continue to support the Mycothèque for the next 100 years, as the only 
"mycothèque" among many just plain European fungal "collections". And we even do not ask them to ensure that the "mycothécaire" should be allowed to go on till he can celebrate his centennial. We know him well enough that - apart from any official position - he will continue as the taxonomist in heart and soul that he has always been.

The main theme given to me to talk about is.

\section{Fungal collections in Europe.}

My expertise on this subject should not be exaggerated. On first sight it seems to be quite appropriate for someone who has been the coordinator of the Microbial Information Network Europe (MINE) project, aiming at bringing together some 40 collections in 12 European countries in the endeavour to set up a common format for the data on bacteria and fungi and to bring these data together at a Central Data Node. I have to admit that my view on this matter is still a bit blurred, MINE not always being illuminating in this respect. Notwithstanding the rather limited financial support from EC, and an underestimation of the complexity of the project, it is clear that:

1. There is great willingness to cooperate between collections.

2. The framework for optimal cooperation still has to be established.

In order to improve on this second factor, the good framework for cooperation, a number of facts and conditions should be kept in mind.

1. Collection policy is mainly determined at the national level, not in Brussels. Any cooperative approach should be based on a "Europe of Nations" not on a "Federal Europe". In retrospect, without having participated during the whole preparatory phase of MINE, I am convinced that this aspect has been neglected in the onset of the MINE project. After all, we must realize that, however important Brussels has become on the playground of subsidizing institutions in Europe, a role recently illustrated by the acceptance of the $4^{\text {th }}$ Framework in which some 12.3 billion ECU's are to be spent on research for the next four years, it still does not cover more than $5 \%$ of the total input from public means in the countries of the European Union. The major impact of Europe is in cooperation and in stimulation, the necessary infrastructure still being a matter of the national governments.

2. Without cooperation and presentation of initiatives that are considered worthwhile and challenging in the pursuit of biotechnology, a sustainable use of natural resources, and in the competition with Asian and American counterparts, major inputs from the European Union in taxonomy and collections are, at least, questionable. This support is needed to cope with expected technical and methodological changes in collections.

3. Without cooperation and coordination, the diversity in European collections, which can be considered a great asset, both in the variety of organisms kept and in the expertise they represent, will become a hampering factor, not to be used to the maximum in the world's biodiversity programs.

4. Any future cooperation must include Eastern Europe. The borders of the European Union are not to be considered limitative.

5. Collections are fiercely competing organizations, not only in assets and their scientific value, but also - and increasingly so - on the free market. 
This summary of statements proves that any framework for cooperation should accommodate the deeply felt necessity for working together bearing in mind that there are a number of hampering factors in this respect. There is a certain reluctance to be encountered when discussing input from taxonomy and collections in biodiversity research with EU officials. This was the case with MINE, and it can be clearly seen in the cautious approach in the concerted action for microbial biodiversity. And the underlying factor is mistrust in the potential for collections and taxonomists to come forth with coherent and well-defined proposals. Maybe not rightfully so, but the fact has to be acknowledged.

An overview of the major actors, professional groups as well as organizations, in the fields of taxonomy, collections and biodiversity research is represented here in the form of a human figure, the caption 'Quo Vadis?' indicating that the direction it will take is not certain yet.

The trunk consists of the scientific community and it financing bodies. The arms represent the major international organizations, the United Nations and the Unions of the International Council of Scientific Unions.

The head, becoming rather large, represents the politicians, civil servants, managers and lawyers.

The legs, symbolizing the European Culture Collections Organization (ECCO) and MINE are in contrast rather thin.

The driving factors are, of course, to be found at the heart: the taxonomist and the curator. They are supplied, symbolized by blood vessels and lungs, by enthralling ideas from many other scientific disciplines such as molecular biology and biotechnology, which in turn acknowledge the mighty impulses they get from the heart, recently revived by the bypasses biodiversity and evolutionary biology.

The discussions between these groups are not always easy, as illustrated by some imaginary conversations on biodiversity.

The molecular biologist has just received a DNA sample for identification from a zoo in some faraway country. He compares it with some DNA obtained from his colleague, the taxonomist. After one day he exclaims that in the country concerned they have the atrocity to keep human beings in zoos, as the DNA sample matches rather perfectly the DNA from the taxonomist. The taxonomist, who by nature is a curious man, travels to the zoo, and returns quite disappointed: the sample was that of a chimpanzee. Every one of us in this case is an expert: quite clearly the chimpanzee and the human being are different species, one bothering about his origins, the other, as far as we know, not.

However, when we are talking about microorganisms, the claim of the molecular biologist that he is the one who can unequivocally discriminate between species by applying his DNA trick is often acknowledged without dispute.

The biotechnologist and the taxonomist discuss biodiversity in a quantitative way, the taxonomist stating that there are over six million species on earth. The biotechnologist is hardly impressed. His biodiversity concept is governed by the enzymes and he knows that in evolution, through all kingdoms, from archaebacteria to the animals, has resulted in about 5000 different enzymes, subdivided in 60 groups and 6 classes. His biodiversity concept is much simpler, interested as he is in regulatory systems that lead to the production of enzymes in higher quantities and that do the tricks he wants them to perform, better. And then again he is primarily interested in strains, not in species. The direct surrounding of the taxonomist thus is 
one in which different paradigms are amalgamated in an enriching way. The least that should come of it is a reconsideration of species concepts.

The discussions with the organizations constituting the rest of the body are quite different, but not less important in character.

They have to do with selling ideas in such a way that the product, of which we only are at the onset aware of its value, becomes attractive to those who should pay for it.

This process takes place at an increasing number of levels and is to a certain degree masterminded by politicians, managers and lawyers. It starts at the level of universities and research councils and ends at conferences in Mexico and Nairobi. It is sometimes tempting to look at these levels with some disdain, but fact is that all of us are devoting more and more time to travel around the world and participate in the circuit of buying and selling science. Without clear research-strategies the danger that the outcome is mainly determined by the buyer, is evident. When looking at biodiversity, the four main topics are: the scale, where? what? and how?

\section{The scale}

It is the world, but also the own back garden.

The most far-fetched proposal, the All Taxon Biodiversity Investigation may raise severe doubts about feasibility and scientific value; it has the scale that can grasp the mind of policy-makers, which surely can boost the impact of taxonomy, together with collections as the main actors in the biodiversity field, a role that seemingly cannot be attained by smaller projects.

\section{Where?}

The focus on the tropics is evident. There are, however, many other habitats that are of great interest and about which much is still unknown.

They should not be overgrown by over-emphasizing, strongly supported by public concern about its destination, the Rain Forest. The soil, the tundra, the sea are habitats in the temperate zones that ask for special attention of taxonomists and of collections in Europe too.

\section{What?}

Besides taxonomic issues about inventory, isolation and identification, evaluation of species concepts, and nomenclature, the collections in particular are confronted with questions on how to preserve, in situ and ex situ, what to preserve, and where.

This is accompanied by international questions about ownership of microorganisms, resulting form the Biodiversity Convention.

\section{How?}

There is a limited number of taxonomists, and a rather large number of collections, different in character and quality. Without good cooperation between collections, resulting in well-defined forms of quality control and division of labour and fields of activity, I am afraid that collections will find it even more difficult to present themselves in the field of biodiversity research than the taxonomists in general.

It is important to indicate strong - and consequently also weak - points in the European collections, and this should be done by collections themselves. 
I have already stated that the legs are in bad shape: we have to worry about their capacity to carry the whole body steadily. The message is clear: the legs have to be strengthened.

Rather then finding new formulas, I am of the opinion that in principle the existing organizational structures, ECCO for more conceptual thinking, MINE for more technical matters should be adequate, on condition that they are well-equipped for their supporting roles. And this asks for a rather fundamental discussion and reshaping of both organizations.

ECCO should evolve from a yearly meeting of curators into an organization that can speak up for collections and is not afraid to raise issues that may be of some concern to the collections themselves.

MINE should be transferred into a consortium.

Most important is to find, for both organizations, a constitution that is recognized by all collections as fair, with boards that are equipped with a clear mandate to work with.

There is nothing new, only the form has changed and things are now more complicated. CBS originated in 1903 as a result from a concerted action by the international community of scientists to start something that is to the benefit of that community.

I am sure that we also will celebrate our centennial as proof that international cooperation pays off in the long run.

And if things become boring or too complicated we have always our inspiration from the organism that governs our daily lives, the Fungus. In itself it is beautiful and far more complicated than the structures I have been talking of. 


\title{
THE BELGIAN COORDINATED COLLECTIONS OF MICROORGANISMS BCCM
}

\author{
J. DE BRABANDERE \\ SSTC, Rue de la Science 8, 1040 Bruxelles
}

The 'Belgian coordinated Collections of Microorganisms, BCCM' form a consortium of four complementary research-based culture collections at the service of the international scientific and industrial community.

The Belgian Science Policy Office launched the BCCM-action in 1983 with the following mission.

1. The characterization, inventory and preservation of the pool of available isolates, reference strains and plasmids (over 41.000 items), to be expanded along the lines of the specializations of each collection:

- the LMG bacteria-collection (Director: Prof. Dr. K. Kersters, Curator: Dr. D. Janssens) holding over 13000 strains;

- the MUCL collection for fungi/yeasts of biodiversity and (agro)industrial interest

(Director/Curator: Prof. Dr. Ir. G.L. Hennebert) holding over 22000 strains;

- the IHEM collection for fungi/yeasts of biomedical interest (Director/Curator: Dr. N. Nolard) holding over 5000 strains;

- the LMBP plasmid-collection (Director: Prof. Dr. Ir. W. Fiers, Curarator: Prof. Dr. E, Remaut) holding over 1000 plasmids for use in bacteria, filamentous fungi, yeasts and animal cells.

2 The distribution of the authenticated biological material and the corresponding information to interested scientific and industrial circles.

3 The acceptance of patent deposits valid within the framework of the international Budapest Treaty (N.B. The BCCM received in 1992 - and this as a consortium status of International Depositary Authority, IDA);

4 The valorization of the available skills in the field of both fundamental and applied (micro)biology by providing professional and confidential ad hoc services and contract-research to third parties (e.g. molecular characterization and typing of industrial and medical strains, safe deposits of propriety strains, training, screening for primary and secondary metabolites etc.).

All BCCM collections are active members of the "World Federation for Culture Collections, WFCC" and the "European Culture Collections Organization, ECCO". Moreover, the BCCM are founding-members of the "Microbial Information Network Europe, MINE". As such, they host the "Data Integrating Node" for bacteria at Ghent and chair the "Responsible Committees" for bacteria and fungi/yeasts

The organization of the BCCM-consortium constitutes a rather unique model that distinguishes itself from foreign examples by the stable funding from one common financier that is also in charge of the central coordination and support of the main policy issues (client approach, informatics, quality management etc.). 


\title{
PART 2
}

FUNGAL TAXONOMY AND TROPICAL MYCOLOGY: QUO VADIS ? An Introduction

\author{
Grégoire L. HENNEBERT
}

Mycotheca of the Catholic University of Louvain, 2 Place Croix du Sud, 1348 Louvain-la-Neuve, Belgium

Modern taxonomy, including mycotaxonomy, relies in an increasing degree on new molecular approaches.

As such, one can observe that the traditional morphotaxonomic approach is more and more complemented - or sometimes even substituted - by chemotaxonomic methods or genome-based analyses. Questioning the value and mutual complementarities of all available tools - both classical and modern - is essential to be able to handle the systematic classification problem in an integrated and effective way.

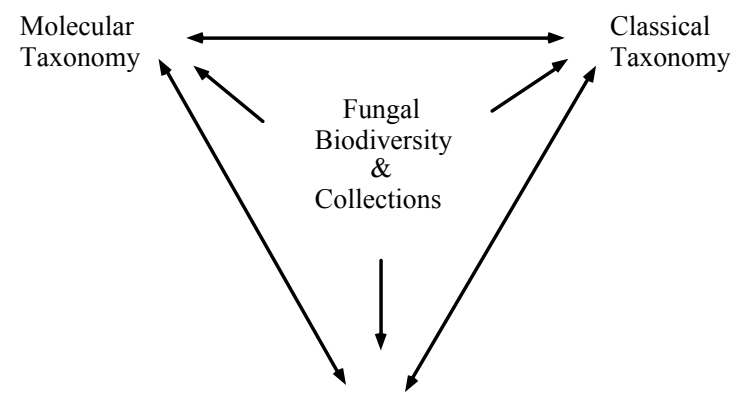

Taxonomy in the Tropics

On the other hand, the greater part of the fungal biodiversity has still to be discovered and described especially in the tropical regions. As these are mainly situated in developing countries, one has to discuss - in the spirit of the Rio de Janeiro earth summit - key topics such as training, technology transfer and the set-up of local 'ex situ' collections.

During such an exercise, special attention has to be given to taxonomic and preservation methods that are not only effective but also widely applicable, userfriendly and robust. 


\title{
MOLECULAR TAXONOMY OF THE YEASTS: PRESENT AND FUTURE
}

\author{
Cletus P. KurtZman
}

Microbial Properties Research, National Center for Agricultural Utilization Research, Agricultural Research Service, U. S. Department of Agriculture, Peoria, Illinois 61604 U.S.A.

Key words. -- Yeast systematics, ribosomal RNA/DNA, molecular evolution, phylogeny

\section{Introduction}

The systematics of yeasts, as with other groups of fungi, is predominantly based on a hierarchy constructed from morphological differences among the taxa. The present system of classification relies heavily on types of budding or the occurrence of fission, presence or absence of hyphae, morphology of ascospores, and morphology of basidia and the manner in which basidiospores are produced. As a consequence, these characters often define orders, families, genera, and even species. Whether the characters reflect the evolutionary history of the yeasts is unknown, but if systematics is to be based on phylogeny, i.e., a natural classification, the genetic basis of currently used characters must be understood.

Application of molecular comparisons to questions in yeast classification offers an unprecedented opportunity to reevaluate current taxonomic schemes from the perspective of quantitative genetic differences. This review examines the impact of molecular comparisons, notably ribosomal RNA/ribosomal DNA (rRNA/rDNA) sequence divergence, on the current phenotypically defined classification of the yeasts.

\section{Placement of yeasts among the fungi}

The yeasts represent a unique group of fungi characterized by vegetative growth that is predominantly unicellular, and by the formation of sexual states which are not enclosed in fruiting bodies. Saccharomyces cerevisiae has been recognized as an ascomycete for well over a century, but it was not until much later that some yeasts were thought to be basidiomycetes (Kluyver and van Niel 1927). This supposition was confirmed by Banno (1967) with the description of the heterobasidiomycetous species Rhodosporidium toruloides. With this finding came the realization that the yeasts are phylogenetically quite divergent. In the following discussion, relationships among ascomycetous and basidiomycetous taxa are examined from the perspective of phylogeny determined from small and large (18S, 26S) subunit rRNA/rDNA sequence divergence. Methodologies have been discussed by Bruns et al. (1991), Kaltenboeck et al. (1992), Kurtzman (1992), O'Donnell (1992), and White et al. (1990).

\section{Ascomycetous yeasts}

The phylogeny of the ascosporogenous yeasts has been vigorously debated since the time of Guilliermond (1912) and before. Some have viewed the yeasts as primitive fungi while others perceived them to be reduced forms of more evolved taxa. Cain (1972) has been a proponent of this latter idea, arguing that hat (galeate)spored genera such as Pichia and Cephaloascus are likely to be reduced forms of the 
perithecial euascomycete genus Ceratocystis. Redhead and Malloch (1977) and von Arx and van der Walt (1987) accepted this argument and commingled yeasts and mycelial taxa in their treatments of the Endomycetales and Ophiostomatales.

Examination of rRNA/rDNA sequence divergence from a limited number of taxa indicated the ascosporogenous yeasts, with the exception of Schizosaccharomyces, to form a monophyletic group (clade) distinct from the filamentous species (Barns et al. 1991, Bruns et al. 1991, Hausner et al. 1992, Hendriks et al. 1992, Kurtzman 1993a, Nishida and Sugiyama 1993, Walker 1985, Wilmotte et al. 1993). Kurtzman and Robnett (1994a) analyzed rRNA sequence divergence from type species of all cultivatable ascomycetous yeasts and yeastlike taxa. This work demonstrated the yeasts, as well as yeastlike genera such as Ascoidea and Cephaloascus, to comprise a clade sister to the "filamentous" ascomycetes (euascomycetes). Eremascus, which forms asci unenclosed in a fruiting body, aligned with the euascomycete clade and may represent a genus close to the phylogenetic demarcation of the hemiascomycetes (excluding Schizosaccharomyces) and the euascomycetes. These results substantiate the long-held observation that yeasts cannot be defined solely on the basis of presence or absence of budding. Such members of the yeast clade as Ascoidea, Ashbya and Eremothecium show no typical budding, whereas Aureobasidium, Phialophora and certain other genera of euascomycetes are usually dimorphic. Budding is also a common mode of vegetative reproduction among many basidiomycetous genera. Similarly, vegetative reproduction by fission is shared by Dipodascus and Galactomyces, members of the yeast clade, as well as by the distantly related genus Schizosaccharomyces. Sexual states of all members of the yeast clade are characterized by asci unenclosed in a fruiting body. This feature is shared by only a few taxa outside the yeast clade such as Eremascus and Schizosaccharomyces. Myrioconium and Trichomonascus form unenclosed asci but may be euascomycetes as well.

Phylogenetic relationships among the ascomycetous yeasts, calculated from partial sequences of small and large subunit rRNAs, are depicted in Fig. 1. Although there are insufficient phylogenetically informative sites to resolve many of the genera, the comparison gives an overview of relationships. Tree topology is similar to that presented by Wilmotte et al. (1993), who examined fewer species, but used nearly complete $18 \mathrm{~S}$ sequences. In the comparison of partial sequences, most taxa having coenzyme $\mathrm{Q}$ with the same number of isoprene units tend to group, but this is not true of the closely related genera Ashbya, Eremothecium, Holleya and Nematospora which show a variation in coenzyme Q ranging from 5-9 isoprene units. Some congruence is found between location of taxa on the rRNA gene tree and the type of hyphal septal pore produced. The two known genera (Ambrosiozyma, Hormoascus) that form dolipore-like septa are closely associated. However, Arthroascus produces septa with a simple central pore whereas Guilliermondella has multiperforate septa, yet the two genera closely cluster. These incongruities may be resolved by sequencing the complete $18 \mathrm{~S}$ molecule and by including all known species for each of the genera under study.

rRNA/rDNA sequence comparisons have been quite helpful for understanding species relationships within genera. For example, Schwanniomyces occidentalis (Kurtzman and Robnett 1991), Wingea robertsii (Kurtzman and Robnett 1994b), and the Pichia species, P. carsonii and P. etchellsii (Yamada et al. 1992), were found to be members of the genus Debaryomyces on the basis of rRNA relatedness. The initial assignment of these species to other genera resulted from misinterpretation of 
the phylogenetic significance of ascospore morphology. Species originally placed in Debaryomyces are characterized by spheroidal ascospores that are roughened by wartlike or ridgelike outgrowths of wall material. An exception is D. marama which has ellipsoidal ascospores with wartlike outgrowths and spiral ridges. In contrast, Schwanniomyces forms spheroidal ascospores with surface projections and a prominent equatorial ring, Wingea has smooth, lenticular ascospores, and the two former Pichia species produce smooth, spheroidal ascospores. Furthermore, Saturnospora and Williopsis both form saturnoid ascospores, yet the two genera are only distantly related (Liu and Kurtzman 1991).

The impact of rRNA/rDNA comparisons on the taxonomy of ascomycetous yeasts is just being felt and will require additional work to fully realize its potential. Major findings to date include: 1) yeasts and yeastlike species are phylogenetically separate from the euascomycetes, 2) the fission yeast genus Schizosaccharomyces is phylogenetically distant from the "budding" yeast clade and from the euascomycetes, resulting in the reassignment of the fission yeasts to a separate order, the Schizosaccharomycetales (Eriksson et al. 1993, Kurtzman 1993a) and, 3) the demonstration that many phenotypic characters such as ascospore morphology are poor indicators of phylogeny.

\section{Basidiomycetous yeasts}

Anamorphic (asexual) basidiomycetous yeasts may be morphologically indistinguishable from anamorphic ascomycetous yeasts. The discovery that the inner cell walls of basidiomycetous yeasts are lamellar when viewed in thin section under the transmission electron microscope, in contrast to the uniform inner layer of ascomycetes, has provided a reliable means for separation of the two taxonomic classes when sexual states are not found (Kreger-van Rij and Veenhuis 1971). A second method of separation, more easily applied, is the Diazonium Blue B (DBB) staining technique (van der Walt and Hopsu-Havu 1976). Colonies of basidiomycetes stain a magenta color in the presence of DBB whereas colonies of ascomycetes remain unstained. From these findings, it has become apparent that basidiomycetes make up a large part of the yeast domain.

Basidiomycetous yeasts (Fell and Kreger-van Rij 1984, Boekhout et al. 1993). In the first, teliospores are formed and germinate to produce a basidium that bears basidiospores. This type of sexual cycle shows considerable similarity to the rust and smut fungi. The second type of sexual state lacks teliospores. Basidia develop on hyphae or yeast cells and give rise to basidiospores in a manner similar to the Tremellales (jelly fungi).

Several other characteristics are added to the dichotomy of sexual states. Some taxa produce carotenoids, and the presence of these pigments has been used as a criterion for genus assignment. Ballistoconidia, forcibly ejected vegetative cells, are common to some taxa and their presence is a defining character of genera. Additionally, the hyphal septal pore of basidiomycetous yeasts may be either simple or the ultrastructurally more complex dolipore. Finally, some taxa exhibit the presence of cellular xylose, evidently arising from extracellular polysaccharides (Golubev 1991), whereas other species do not.

Guého et al. (1989) presented an overview of the phylogeny of basidiomycetous yeasts from measurements of divergence among partial sequences of large and small subunit rRNAs. Three major groups were resolved; 1) teliospore formers with hyphae having simple septal pores, 2) teliospore formers with hyphae having 
dolipore septa and, 3) non-teliospore formers with hyphae having dolipore septa. On the basis of $18 \mathrm{~S}$ sequence comparisons, Suh and Sugiyama (1993) placed the smut fungus Ustilago maydis, a teliospore former, near the clade comprising representative genera of all basidiomycetous yeasts. In turn, the basidiomycetous yeasts appear to be a sister group to the Agaricales (Berbee and Taylor 1993).

At present, the most extensive phylogenetic comparison of basidiomycetous yeasts is that of Fell et al. (1992) who examined 117 species assigned to 23 genera. A 247-nucleotide segment in the D2 region was sequenced; this region resolves closely related species, but may have too few phylogenetically informative sites to accurately assess more distant relationships. The phylogram from that work (Fig. 2) shows the species to be divided between two major clades. The analysis generally supports the concept that taxa assigned to the Tremellales are characterized by dolipore septa and cellular xylose, whereas taxa placed in the Ustilaginales form teliospores, have simple septal pores, and lack cellular xylose. Some exceptions are apparent. The teleomorphic genus Erythrobasidium does not form teliospores as do other members of the clade. Cystofilobasidium and Mrakia, both members of the Tremellales, form teliospores. Another inconsistency concerns the genera located in the lower portion of the Tremellales clade (Fig. 2, B). From what is known of their septal pore structure and lack of cellular xylose, the genera would be expected to group with the Ustilaginales. If their placement is correct, this would suggest that the Tremellales arose from within an already highly diversified group that now represents the Ustilaginales.

Ballistoconidia and carotenoids are found among many genera of the basidiomycetous yeasts suggesting these traits to be ancestral, but not always expressed, thus rendering them of little value for defining taxa. These conclusions were also drawn by Nakase et al. (1993). Heterogeneity of coenzyme Q composition occurs in many currently defined genera and will require additional study before its taxonomic significance is fully understood. rRNA sequence analysis demonstrates Rhodotorula, Sporobolomyces, Cryptococcus, and Bensingtonia to be polyphyletic, further confirming that commonly used phenotypic characters are insufficient for defining anamorphic genera.

A strong start has been made to understand the phylogeny of basidiomycetous yeasts from rRNA/rDNA sequence comparisons, but additional sequencing must be done to better resolve taxa. This will include sequences of greater length as well as inclusion of all known species of a group. For example, the anamorphic genera Tseuchiyaea and Ballistosporomyces, which were recently defined from differences in partial sequences, may overlap with some earlier described genera (Fig. 2).

\section{Reliability of rRNA/rDNA gene trees to infer phylogeny}

With such great emphasis being placed on rRNA/rDNA gene trees to reconstruct phylogenies, it must be asked if there is other evidence to corroborate the conclusions drawn. There are presently only a few comparisons of other molecular sequences that allow this question to be addressed. Sequence analysis of orotidine 5'monophosphate decarboxylase by Radford (1993) demonstrated the budding yeasts and the euascomycetes to be sister groups as shown from rRNA/rDNA sequences. One unusual aspect of this work was placement of the Mucorales as a sister group to the basidiomycetes. Tsai et al. (1994) showed phylogenetic relationships among species of Epichloë (Clavicipitaceae) were the same when analyzed from either 
rDNA sequences or those from the ß-tubulin gene. Relationships among species of Dekkera and its anamorph Brettanomyces were essentially identical when analyzed from either nuclear rDNA sequences or from sequences of the mitochondrially encoded cytochrome oxidase subunit II gene (Boekhout et al. 1994).

Another aspect of gene tree reliability is the method used for its construction. Most investigators now analyze data using phylogeny inference programs based on cladistic principles. These programs often include a statistics package to test the robustness of competing phylogenetic trees. Several recent reviews address these important issues (Avise 1989, Felsenstein 1988, Hillis et al. 1994, Saitou and Imanishi 1989).

\section{Rapid molecular methods for yeast}

The specificity of nucleic acid sequences has prompted development of several methods for rapid species identification that are directly applicable to the strains maintained by culture collections. Because these techniques can detect single nucleotide changes, they should first be tested on a large variety of genetically defined strains to understand species variation because aberrant strains may represent different species.

\section{Restriction fragment length polymorphisms of rDNA}

rDNAs occur in multiple copies and lend themselves to analysis based on restriction fragment length polymorphisms (RFLP). Magee et al. (1987) treated rDNAs from several medically important Candida species with a variety of restriction endonucleases and concluded that Candida guilliermondii, C. tropicalis, and $C$. albicans produced sufficiently different digestion patterns to allow recognition of each species. Similar results were obtained by Vilgalys and Hester (1990) for several species of the genus Cryptococcus. Lachance (1990) used RFLP patterns to map the genetic profiles of 125 isolates of the cactus yeast Clavispora opuntiae that had been collected worldwide. Nearly all of the restriction sites that allowed discrimination of individual strains were located in the hypervariable intergenic spacer region.

Data from the preceding studies show that RFLP patterns allow recognition of individual species, as well as individual strains of a species. Consequently, the method has considerable diagnostic value. Estimates of evolutionary relationships from RFLP patterns have been reported for species assigned to Candida (Magee et al. 1987) and Cryptococcus (Vilgalys and Hester 1990). Such estimates would be expected to be less accurate than estimates derived from sequence comparisons because as evolutionary distances increase, the extent of pattern similarities becomes less certain. Bruns et al. (1991) listed some of the factors that require attention when using RFLPs.

\section{Random amplified polymorphic DNA (RAPD)}

RAPDs represent another methodology that promises widespread application. The technique is based on amplification of genomic DNA in the presence of one or more short (ca. 10-15-mers) oligonucleotide primers of random sequence. 


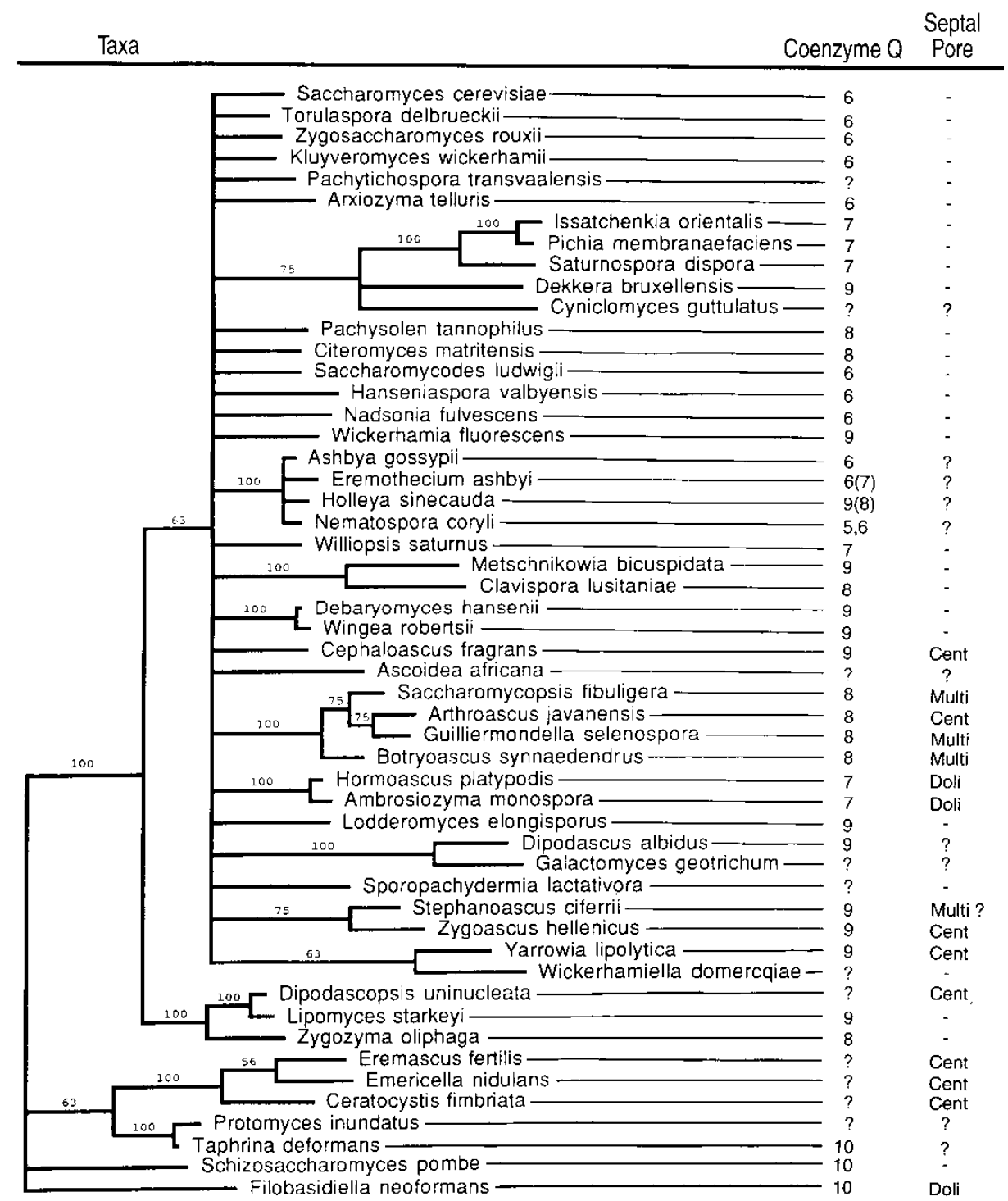

Fig. 1. A phylogenetic tree derived from maximum parsimony analysis depicting the ascomycetous yeasts, yeastlike fungi, and various reference species. The phylogram was calculated from combined small and large subunit rRNA partial sequences as described by Kurtzman and Robnett (1994a). Branch lengths are proportional to nucleotide differences, and the numbers given on branches are the percentage of frequencies with which a given branch appeared in 100 bootstrap replications. Branches without numbers had frequencies of less than 50\%. Coenzyme Q data are from the compilation of Barnett et al. (1990) and refer to the number of isoprene units in the sidechain on the parent molecule. Information on septal pores is from Kreger-van Rij and Kurtzman (1984) and Barnett et al. (1990).

Cent $=$ central pore, Multi $=$ multiperforate septum, Doli $=$ dolipore-like. 


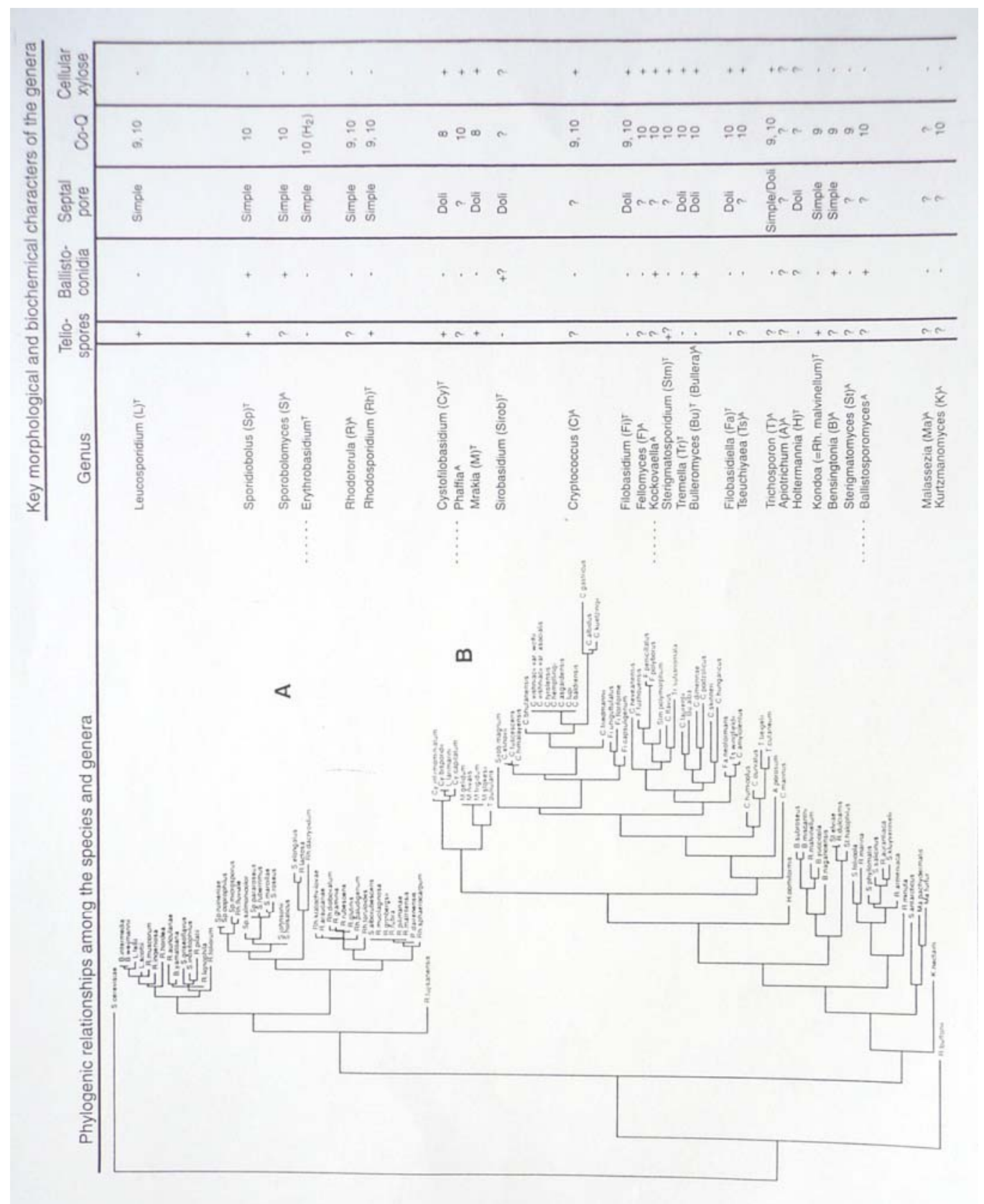

Fig. 2. A phylogenetic tree derived from maximum parsimony analysis depicting the basidiomycetous yeasts. The phylogram, which represents the most parsimonious tree, was calculated from rRNA sequences of the D2 region of the large subunit as described by Fell et al. (1992). Branch lengths are proportional to the number of nucleotide differences. Bootstrap values were not determined. Clade A is comprised of taxa placed in the Ustilaginales. Taxa in Clade B are predominantly assigned to the Tremellales, but see text for discussion. The strain of the outlying species Rhodotorula buffonii that was examined was shown to be a misidentified ascomycete. Genera preceded by a broken line were not in the original analysis and have been placed in the present phylogram on the basis of other studies (Guého et al. 1989, Nakase et al. 1993, 
Sugiyama and Suh 1993). Teleomorphic genus names are followed by the letter T and anamorphic genera are designated with the letter A. Morphological and biochemical characters are from Barnett et al. (1990), Boekhout et al. (1993) and Fell and Kreger-van Rij (1984). Simple = simple septal pore, Doli = dolipore septum.

The amplified products are visualized on an agarose gel and strains identified from matching band patterns. Hadrys et al. (1992) discussed details of this procedure and also noted points of technical difficulty.

\section{Species-specific PCR reactions}

Peterson and Kurtzman (1991) and Kurtzman (1993b) have shown that the 5' end of the large subunit rRNA/rDNA is sufficiently variable to detect even closely related yeast species. Fell (1993) used this region to identify individual yeast species by applying a three-primer, PCR-based technique. In this method, the reaction mix includes genomic DNA, two external primers for the D1/D2 region of large subunit rDNA, and a species-specific internal primer. The external primers allow amplification of the ca. 600-nucleotide D1/D2 region, but in the presence of a species-specific primer (third primer) the amplification product is shorter and easily detected on an agarose gel. There is currently considerable activity in the field of molecular probe development and rapid methods appear nearly ready for widespread use.

\section{Future prospects}

rRNA/rDNA sequence comparisons have provided us with a genetic perspective to the relationships among yeasts and other fungi. On the basis of limited comparisons, rRNA/rDNA gene trees are generally congruent with phylogenies from other molecular sequences, but additional studies should be done to verify this preliminary assessment. In many cases, molecular comparisons are supportive of relationships among the fungi determined from traditional morphological studies. In other cases, the molecules have led us to new perspectives. One of the greatest challenges facing the field of mycology is to use molecular data to develop a phylogenetic system of classification.

The unique nature of molecular sequences promises to allow development of species-specific molecular probes for rapid strain identification. Once this technology becomes widespread, a realistic assessment of the earth's biodiversity may finally be possible.

\section{References}

Avise J. 1989. Gene trees and organismal histories: a phylogenetic approach to population biology. Evolution 43: 1192-1208

Banno I. 1967. Studies on the sexuality of Rhodotorula. J. Gen. Appl. Microbiol. 13: 167-196.

Barnett J A., Payne, R.W. and Yarrow, D. 1990. Yeasts: characteristics and identification. 2nd ed. Cambridge University Press. Cambridge.

Barns S.M., Lane D.J., Sogin M. L., Bibeau C. and Weisburg W.G. 1991. Evolutionary relationships among pathogenic Candida species and relatives. J. Bacteriol. 173: 2250-2255.

Berbee M.L. and Taylor J.W. 1993. Ascomycete relationships: dating the origin of asexual lineages with $18 \mathrm{~S}$ ribosomal RNA gene sequence data. In The Fungal Holomorph: Mitotic, Meiotic and 
Pleomorphic Speciation in Fungal Systematics. Reynolds, D.R. and Taylor, J.W. eds. CAB International. Wallingford, UK, pp. 67-78.

Boekhout T., Fonseca A., Sampaio J.-P. and Golubev W.I. 1993. Classification of heterobasidiomycetous yeasts: characteristics and affiliation of genera to higher taxa of heterobasidiomycetes. Can. J. Microbiol. 39: 276-290.

Boekhout T., Kurtzman C.P., O'Donnell K. and Smith M.Th. 1994. Phylogeny of Hanseniaspora/Kloeckera, Dekkera/Brettanomyces and Eeniella inferred from partial 26S ribosomal DNA nucleotide sequences. Int. J. Syst. Bacteriol. 44: 781-786.

Bruns T.D., White T.J. and Taylor J.W. 1991. Fungal molecular systematics. Ann. Rev. Ecol. Syst. 22: 525-564.

Cain R.F. 1972. Evolution of the fungi. Mycologia 64: 1-14.

Cole G.T. and Samson R.A. 1979. Patterns of development in conidial fungi. Pitman London.

Eriksson O.E., Svedskog A. and Landvik S. 1993. Molecular evidence for the evolutionary hiatus between Saccharomyces cerevisiae and Schizosaccharomyces pombe. Systema Ascomycetum 11: 119-162.

Fell J.W. 1993. Rapid identification of yeast species using three primers in a polymerase chain reaction. Mol. Mar. Biol. Biotechnol. 2: 174-180.

Fell J.W. Statzell-Tallman A., Lutz M.J. and Kurtzman, C.P. 1992. Partial rRNA sequences in marine yeasts: a model for identification of marine eukaryotes. Mol. Mar. Biol. Biotechnol. 1: 175-186.

Fell J.W. and Kreger-van Rij N.J.W. 1984. Classification of the basidiomycetous yeasts. In The Yeasts - A Taxonomic Study. Kreger-van Rij N. J. W. ed., 3rd ed., Elsevier Science Publ., Amsterdam, pp. 3235.

Felsenstein J. 1988. Phylogenies from molecular sequences: inference and reliability. Ann. Rev. Genet. 22: 521-565.

Golubev W.I. 1991. Capsules. In The Yeasts, vol. 4. Rose A. H. and Harrison J. S. eds., 2nd ed., Academic Press, London, pp. 175-198.

Guého E., Kurtzman C.P. and Peterson S. W. 1989. Evolutionary affinities of heterobasidiomycetous yeasts estimated from $18 \mathrm{~S}$ and $25 \mathrm{~S}$ ribosomal RNA sequence divergence. Syst. Appl. Microbiol. 12: 230-236.

Guilliermond,A. 1912. Les levures. Encyclopédie Scientifique. O. Doin et Fils, Paris.

Hadrys H., Balick M. and Schierwater B. 1992. Applications of random amplified polymorphic DNA/RAPD in molecular ecology. Mol. Ecol. 1: 55-63.

Hausner G., Reid J. and Klassen G.R. 1992. Do galeate-ascospore members of the Cephaloascaceae, Endomycetaceae and Ophiostomataceae share a common phylogeny? Mycologia 84: 870-881.

Hendriks L., Goris A., Van de Peer Y., Neefs J.-M., Vancanneyt M., Kersters K., Berny J.-F., Hennebert G.L. and De Wachter R. 1992. Phylogenetic relationships among ascomycetes and ascomycete-like yeasts as deduced from small ribosomal subunit RNA sequences. Syst.. Appl. Microbiol. 15(1): 98104.

Hillis D.M., Huelsenbeck J.P. and Cunningham C.W. 1994. Application and accuracy of molecular phylogenies. Science 264: 671-677.

Kaltenboeck B., Spatafora J.W., Zhang X., Kousalas K.G., Blackwell M. and Storz J. 1992. Efficient production of single-stranded DNA as long as $2 \mathrm{~kb}$ for sequencing of PCR-amplified DNA. BioTechniques 12: 164-171.

Kluyver A.J. and van Niel C.B. 1927. Sporobolomyces: ein Basidiomyzet? Ann. Mycol. 25:389-394.

Kreger-van Rij N.J.W. and Kurtzman C. P. 1984. Classification of the ascoporogenous yeasts. In The Yeasts - A Taxonomic Study. Kreger-van Rij N.J.W. ed., 3rd ed.. Elsevier Science Pub. Amsterdam, pp. 25-32.

Kreger-van Rij N.J.W. and Veenhuis M. 1971. A comparative study of the cell wall structure of basidiomycetous and related yeasts. J. Gen. Microbiol. 68: 87-95.

Kurtzman C.P. 1992. rRNA sequence comparisons for assessing phylogenetic relationships among yeasts. Int. J. Syst. Bacteriol. 42: 1-6.

Kurtzman C.P. 1993a. Systematics of the ascomycetous yeasts assessed from ribosomal RNA sequence divergence. Antonie van Leeuwenhoek 63: 165-174.

Kurtzman C.P. 1993b. The systematics of ascomycetous yeasts defined from ribosomal RNA sequence divergence: theoretical and practical considerations. In The Fungal Holomorph: Mitotic, Meiotic and Pleomorphic Speciation in Fungal Systematic. Reynolds, D. R. and Taylor, J. W. eds. CAB International, Wallingford, Oxon, UK, pp. 271-279.

Kurtzman C.P. and Robnett C.J. 1991. Phylogenetic relationships among species of Saccharomyces, Schizosaccharomyces, Debaryomyces, and Schwanniomyces determined from partial ribosomal RNA sequences. Yeast 7: 61-72.

Kurtzman C.P. and Robnett C.J. 1994a. Orders and families of ascosporogenous yeasts and yeast-like taxa compared from ribosomal RNA sequence similarities. In Ascomycete Systematics: Problems and 
Perspectives in the Nineties. Hawksworth, D.L. ed., Plenum Press, New York, USA pp 249-258.

Kurtzman C.P. and Robnett C.J. 1994b. Synonymy of the yeast genera Wingea and Debaryomyces. Antonie van Leeuwenhoek 66(4): 337-342..

Lachance M.-A. 1990. Ribosomal DNA spacer variation in the cactophilic yeast Clavispora opuntiae. Mol. Biol. Evol. 7: 178-193.

Liu Z. and Kurtzman C.P. 1991. Phylogenetic relationships among species of Williopsis and Saturnospora gen. nov. as determined from partial rRNA sequences. Antonie van Leeuwenhoek 60: 21-30.

Magee B.B., D'Souza T.M. and Magee P.T. 1987. Strain and species identification by restriction fragment length polymorphisms in the ribosomal DNA repeat of Candida species. J. Bacteriol. 169: 16391643 .

Nakase T., Takematsu A. and Yamada Y. 1993. Molecular approaches to the taxonomy of ballistosporus yeasts based on the analysis of the partial nucleotide sequences of $18 \mathrm{~S}$ ribosomal ribonucleic acids. $J$. Gen. App. Microbiol. 39: 107-134.

Nishida H. and Sugiyama J. 1993. Phylogenetic relationships among Taphrina, Saitoella, and other higher fungi. Mol. Biol. Evol. 10: 431-436.

O'Donnell K. 1992. Ribosomal DNA internal transcribed spacers are highly divergent in the phytopathogenic ascomycete Fusarium sambucinum (Gibberella pulicaris). Curr. Genet. 22: 213220.

Peterson S.W. and Kurtzman C.P. 1991. Ribosomal RNA sequence divergence among siblings species of yeasts. Syst. Appl. Microbiol. 14: 124-129.

Radford A. 1993. A fungal phylogeny based upon orotidine 5'-monophosphate decarboxylase. J. Mol. Evol. 36::389-395.

Redhead S.A. and Malloch D.W. 1977. The Endomycetaceae: new concepts, new taxa. Can. J. Bot. 55: $1701-1711$.

Saitou N. and Imanishi T. 1989. Relative efficiencies of the Fitch-Margoliash, maximum-parsimony, maximum-likelihood, minimum-evolution, and neighbor-joining methods of phylogenetic tree construction in obtaining the correct tree. Mol. Biol. Evol. 6: 514-525.

Sugiyama J. and Suh S.-O. 1993. Phylogenetic analysis of basidiomycetous yeasts by means of $18 \mathrm{~S}$ ribosomal RNA sequences: relationship of Erythrobasidium hasegawianum and other basidiomycetous yeast taxa. Antonie van Leeuwenhoek 63: 201-209.

Suh S.-O. and Sugiyama J. 1993. Phylogeny among the basidiomycetous yeasts inferred from small subunit ribosomal DNA sequence. J. Gen. Microbiol. 139: 1595-1598.

Tsai H.-F., Liu J.-S., Staben C., Christensen, M.J., Latch, G.C.M., Siegel, M.R. and Schardl, C.L. 1994 Evolutionary diversification of fungal endophytes of tall fescue grass by hybridization with Epichloë species. Proc. Natl. Acad. Sci. USA 91: 2542-2546.

van der Walt J.P. and Hopsu-Havu V.K. 1976. A colour reaction for the differentiation of ascomycetous and hemibasidiomycetous yeasts. Antonie van Leeuwenhoek 42: 157-163.

Vilgalys R. and Hester M. 1990. Rapid genetic identification and mapping of enzymatically amplified ribosomal DNA from several Cryptococcus species. J. Bacteriol. 172: 4238-4246.

von Arx J.A. and van der Walt J.P. 1987. Ophiostomatales and Endomycetales. Stud. Mycol. 30: 167-176.

Walker W.F. 1985. 5S ribosomal RNA sequences from ascomycetes and evolutionary implications. Syst. Appl. Microbiol. 6: 48-53.

White T.J., Bruns T., Lee S. and Taylor J. 1990. Amplification and direct sequencing of fungal ribosomal RNA genes for phylogenetics. In PCR Protocols: A Guide to Methods and Applications. Innis N., Gelgand, D., Sninsky J. and White T. eds., Academic Press, Inc., New York, pp. 315-322.

Wilmotte A., Van de Peer Y., Goris A., Chapelle S., De Baere R., Nelissen B., Neefs J.-M., Hennebert G.L. and De Wachter R. 1993. Evolutionary relationships among higher fungi inferred from small ribosomal subunit RNA sequence analysis. Syst. Appl. Microbiol. 16: 436-444.

Yamada Y., Maede K., Banno I. and van der Walt J.P. 1992. An emendation of the genus Debaryomyces Lodder et Kreger-van Rij and the proposals of two new combinations, Debaryomyces carsonii and Debaryomyces etchellsii (Saccharomycetaceae). J. Gen. Appl. Microbiol. 38: 623-626. 


\title{
PHYLOGENY OF FILAMENTOUS ASCOMYCETES DEDUCED FROM ANALYSIS OF MOLECULAR CHARACTERS: PRESENT AND FUTURE
}

\author{
MeREDith BLACKWELL \\ Department of Plant Biology, Louisiana State University, Baton Rouge, LA 70803 USA
}

\begin{abstract}
Phylogenetic studies of ascomycetes dispersed by arthropods have benefited from the use of rDNA sequence analysis. At higher taxonomic levels it has been necessary to compare a variety of taxa that either lack corresponding morphological characters (Raffaelea, Ambrosiella, Laboulbeniales, yeasts) or have characters that may be the result of convergent evolution (Ceratocystis, Ophiostoma, Pyxidiophora). For example Ambrosiella has been found to be polyphyletic with one group of species related to Ceratocystis and the other group allied with Ophiostoma. Species of Ambrosiella are asexual and could not have been compared to sexual forms soley on the basis of morphology. Several genera with evanescent asci and arthropod associations previously have been placed in Ophiostomatales. We now know that Ophiostoma, Ceratocystis, and Pyxidiophora are not closely related, and, in fact, at least six lineages of such fungi have been identified. One other taxonomically problematical group, Laboulbeniales, lacks a number of morphological characters that can be compared with filamentous forms (including even the hyphal filaments!). The hypothesis of a relationship with Pyxidiophora based on few morphological characters now has support from rDNA sequence analysis. It is important to note that our results could not have been obtained so easily were it not for the previous studies of the morphology, life histories, and associations of the fungi. For the future it will be important for mycologists in distant localities using different techniques to cooperate in discovering and classifing the Earth's fungal diversity. In addition to the continuing development of phylogenetic techniques, another inovation is important. Worldwide collaboration already is enhanced at the close of the twentieth century by our ability to communicate rapidly and at will using computerized communication networks, and this technology must be available to all mycologists throughout the world.
\end{abstract}

Previous attempts at phylogenetic classification of ascomycetes have been confounded by the presence of convergent morphological features, the absence of characters that extend across all taxa, and an inability to determine character polarity. However, a non-morphological approach, specifically ribosomal DNA (rDNA) sequence analysis has provided new characters that circumvent the problems assciated with the use of morphological characters.

In our work with arthropod-associated fungi we have been particularly interested in the ascomycetes with long necked perithecia and evanescent asci and the possibility that these characters are the result of convergent evolution to facilitate ascopore dispersal by arthropods. Some of this work has been published and will be reviewed only briefly to provide an example of the usefulness of the technique. In this case nuclear-encoded rDNA sequence was applied to the study of ascomycetes previously placed in Ophiostomatales, or in some cases, never placed in an ordinal level taxon. The taxa are all characterized by evanescent asci and, in most cases, are arthropod dispersed.

The relationships hypothesized on the basis of rDNA analysis indicate that a divergence of the fungal lineage gave rise to the basidiomycetes and ascomycetes. Both groups presumably arose from a filamentous ancestor. An early ascomycete divergence, or perhaps several, produced such forms as Taphrina, Schizosaccharomyces, Protomyces, and Pneumocystis (Sugiyama and Nishida 1993, Nishida and Sugiyama 1994, Kurtzman 1993). These fungi are marked by yeast-like stages as well as mycelial growth. It is interesting that they often have been at the center of taxonomic controversy and all have been considered basidiomycetes at 
some time in their history.

The next major divergence of ascomycetes gave rise to two lineages, the yeasts of Saccharomycetales and the filamentous ascomycetes. It is these two groups that have numerous arthropod associations. In addition some of the species in both groups have morphological and biochemical characters in common. For example the galeate or hat-shaped ascopore morphology, yeast thallus, and various conidial stages found among species of Ophiostoma and Ceratocystis as well as some yeasts led to the appealing hypothesis that these forms might constitute a monophyletic group (Redhead and Malloch 1977, von Arx and van der Walt 1987). With most of the major groups of yeasts examined, it does not seem likely that any of them are derived from the filamentous ascomycetes, and the characters typical of arthropodassociated ascomycetes are convergent features.

One major accomplishment involving the filamentous ascomycetes has been the resolution of the relationship of Ceratocystis and Ophiostoma. At times species of both genera have been considered congeneric. We now have evidence that not only are the two genera distinct, but also they occur within different subclades of perithecial ascomycetes (Spatafora and Blackwell 1993). Other filamentous forms with evanescent asci including Pyxidiophora, Kathistes, and Subbaromyces lie outside of the perithecial ascomycetes, and each may be part of an independent lineage. The yeast-like Symbiotaphrina kochii also is in this part of the tree.

Several years ago Dr. David Malloch and I (Blackwell and Malloch 1989) used morphology and life cycle studies to propose a relationship between Pyxidiophora and Laboulbeniales. This hypothesis was tested using rDNA analysis and the evidence supports the previous conclusion of their close relationship (Blackwell 1994). However, the nearest relatives of the Pyxidiophora-Laboulbeniales, Kathistes, Subbaromyces, and Symbiotaphrina lineages have not been identified because this part of the tree is unstable. Clarification awaits addition of more taxa from the discomycetes and loculoascomycetes, and, perhaps sequencing of other DNA regions.

Our more recent work on arthropod-associated ascomycetes involves the analysis of more rapidly evolving gene regions to develop species concepts for Pyxidiophora and the asexual taxa associated with Ceratocystis and Ophiostoma. Of particular interest is the finding that Ambrosiella is not a monophyletic group, but consists of two groups of species, one related to Ceratocystis and the other allied with Ophiostoma, both in association with scolytid beetles. Convergence of conidial forms has followed the pattern of convergence in their sexual relatives (Cassar 1993). Another asexual lineage does appear to be monophyletic. Species of Raffaelea, however, have diverged from an ophiostomatalean lineage as associates of platypodid beetles.

I have discussed examples of the impact of recent technological advances in helping to define monophyletic groups and clarify the phylogenetic relationships of the ascomycetes. But there are other reasons why mycologists study fungi, and the same methods are useful in these cases as well. Molecular technology has become important to us in identifying particular fungi at a variety of taxonomic levels. From the practical point of view, a knowledge of species that attack plants and animals is necessary. Not only is an identification of species necessary, but it also has become important to identify populations among species of some fungi. 


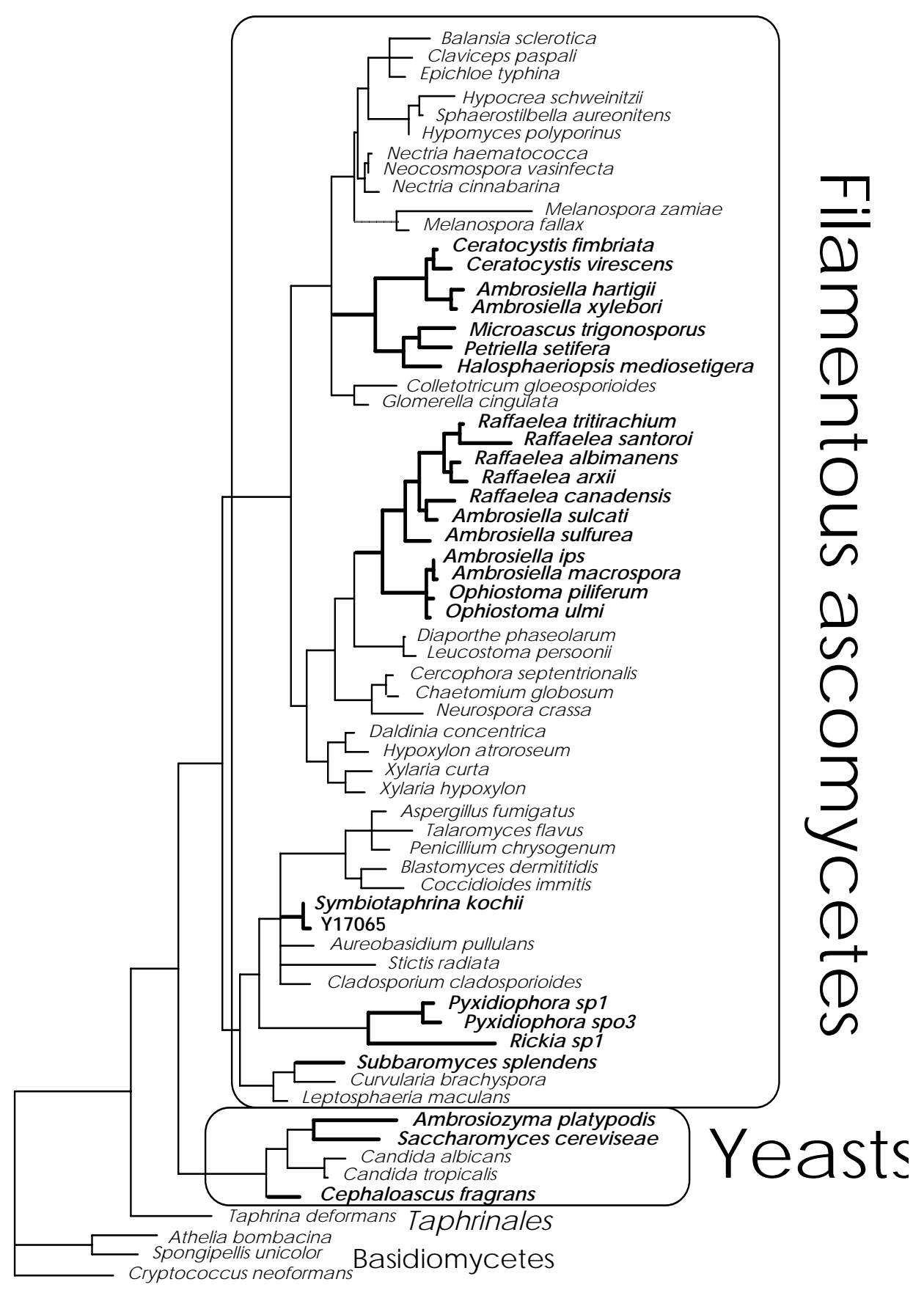

Fig. 1. Phylogenetic hypothesis of the relationships of certain arthropod-associated ascomycetes based on analysis of small subunit rDNA partial sequences using parsimony criteria. See Spatafora and Blackwell (1993) and Blackwell (1994) for details. 
The identification of ectomycorrhizal fungi usually has depended on collection of basidiocarps during seasonal fruiting periods to answer questions about the presence and prevelance of individual mycorrhizal species. However, it now is possible to use molecular methods to identify the fungi to family and, sometimes to species, from samples of field collected roots at any time of the year (Bruns and Gardes 1993). The technique already has shown that the most important mycorrhizal species in terms of frequency are not those that are discovered to be common on the basis of fruiting. The tools that are being developed soon will allow mycologists to determine the precise ecological relationships of the symbioses.

Molecular techniques applied to population studies of Phyphthophtora infestans (Drenth et al. 1993, Therrien et al. 1993), Ophiostoma ulmi (Brasier 1994), and Cryphonectria parasitica (Anagnostakis 1993) are helping to determine the origin and monitor the epidemiology of introduced pathogens, to determine the importance of different modes of reproduction in the development of aggressive populations and, perhaps, to discover the basis of speciation. All of this information depends upon the use of molecular techniques to provide more characters than mycologists have ever had available to them. However, in addition the characters are available at all taxonomic levels, extend across all taxa, and can be polarized.

The polymerase chain reaction (PCR) has lead to rapid progress in molecular methods. As the technology is simplified through continued improvements in automated DNA sequencers and more biologists can use the techniques, the associated costs will come down. In fact there are now rumors of automated sequences that will become available within a few years that will cost no more than a sophisticated light microscope.

It is important to recognize that our new-found ability as mycologists to address problems that previously eluded us, should be viewed as contributing to a worldwide revitalization of fungal studies that is even greater than the electron microscopy revolution of the 1960's and 1970's. It also is possible to use morphological characters with new confidence because their polarity and convergent nature can be inferred with the help of molecular characters. If one looks at previous phylogenetic hypothesis the inability to determine polarity and recognize the possibility of convergence has most often caused mistaken interpretations.

It is obvious to me that mycology is poised to make progress in all areas. Previous morphological studies have contributed to the basic body of knowledge that provides the context for molecular applications, and they will continue to be important in the discovery of the great fungal diversity (Hawksworth 1991). The importance of isolation and culture is increased by the knowledge of the genetic potential of diversity. In these endeavors all mycologists do not have to use all techniques. Some may not have the inclination or the technology to apply molecular methods. Those versed in molecular techniques may not have the means of acquiring the training in morphology that may require years of concentrated study. However, teams of mycologists with different backgrounds must cooperate, perhaps in formally organized groups in distant geographical regions. These collaborations already are practical because of another technological advance, the rapid communication available through computer communications networks. It is imperative that the mycologists of the world be united by "e-mail" before the end of the twentieth century so that all may participate fully in the task at hand. 


\section{Acknowledgements}

I thank Drs. Kevin Jones and Joseph Spatafora and Mr. Steven Cassar for the use of unpublished data and for many helpful discussions on fungi. Mss. Brandye Sawyer, Hester Johnson, and Aimée Kinney gave expert technical assistance. The National Science Foundation provided financial support (DEB 9208027) for the work reported.

\section{Literature Cited}

Anagnostakis S.L. 1992. Diversity within populations of fungal pathogens on perennial parts of perennial plants. In: The Fungal Community. Carroll G.C.and Wicklow D. T. eds, Dekker, NY, USA., pp. 183 192.

Blackwell M. 1994. Minute mycological mysteries: the influence of insects on the lives of fungi. Mycologia 86:1-17.

Blackwell M. and Malloch D.W.. 1989. Pyxidiophora: a link between the Laboulbeniales and the hyphal ascomycetes. Mem. N. Y. Bot. Gard. 49::23-32.

Brasier C.M. 1993. The genetic system as a fungal taxonomic tool: gene flow, molecular variation and sibling species in the 'Ophiostoma piceae-Ophiostoma ulmi' complex and its taxonomic and ecological significance. In: Ceratocystis and Ophiostoma. Taxonomy, Ecology, and Pathogenicity. Wingfield M. J., Seifert K.A., and Webber J.F. eds., APS Press, St. Paul, MN, USA, pp. 77-92.

Bruns T.D., and Gardes M. 1993. Molecular tools for the identification of ectomycorrhizal fungi: taxonspecific oligonucleotide probes for the suilloid fungi. Mol. Ecol. 2: 233-242.

Drenth A., Goodwin S. B., Fry W.E. and Davidse L.C. 1993. Genotypic diversity of Phytophthora infestans in The Netherlands revealed by DNA polymorphisms. Phytopathology 83: 1087-1092.

Kurtzman C.P. 1993. Systematics of the ascomycetous yeasts assessed from ribosomal RNA sequence divergence. Anton. v. Leeuw. 63: 165-174.

Nishida H. and Sugiyama J. 1993. Phylogenetic relationships among Taphrina, Saitoella, and other higher fungi. Mol. Biol. Evol. 10:431-436.

Redhead R.A. and Malloch D.W. 1977. The Endomycetaceae: new concepts, new taxa. Canad. J. Bot. 55 1701-1711.

Spatafora J.W. and Blackwell M. 1993. The polyphyletic origins of the ophiostomatoid fungi. Mycol. Res. 98: $1-9$.

Sugiyama J. and Nishida H. 1994. Phylogenetic divergence of taphrinalean fungi: evidence from molecules and morphology. International Mycological Congress (Abstract). Vancouver, Canada.

Therrien C.D., Tooley P.W., Spielman L.J., Fry W.E., Ritch D.L. and Shelly S.E. 1993. Nuclear DNA content, allozyme phenotypes and metalaxyl sensitivity of Phytophthora infestan $s$ from Japan. Mycol. Res. 97: 945-950.

von Arx J.A. and van der Walt J.P. 1987. Ophiostomatales and Endomycetales. Stud. Mycol. 30: 166-183. 


\title{
IMPORTANCE OF MORPHOLOGICAL AND ANATOMICAL CHARACTERS IN FUNGAL TAXONOMY
}

\author{
J. RAMMELOO \\ National Botanical Garden, Belgium
}

\section{Introduction}

Morphological and anatomical characters and their importance to the taxonomy of fungi must be seen in a historical context. The reason is obvious: when taxonomic classification started only macroscopical characters were taken into account (as it was the case for the study of other organisms). Later anatomical and microscopical characters were added as the science continued to develop. Today, physiology, molecular biology, genetics, enzymology, and ecology all contribute to an understanding of living organisms and their classification.

With this background in mind, I will discuss the following questions:

1. How has our knowledge at the most "basic level" of "species", the level used in taxonomy, evolved; and how important are morphology and anatomy in this conception?

2. How has "taxonomy" been interpreted over time?

3. What is the currrent situation, with an emphasis on morphology and anatomy?

4. Is the importance of morphology and anatomy similar for all groups of fungi?

5. With respect to this symposium's emphasis on tropical mycology: is the situation in the tropics different from the one in temperate regions?

\section{How has our knowledge at the most "basic level" of "species", the level used in taxonomy, evolved; and how important are morphology and anatomy in this conception?}

The often very sudden appearance on fruit-trees of larger fungi as well as the immediate impact of moulds on the everyday lives of human beings prompted the initial study of fungi. To commence such a study, however, it was necessary to begin the process of classifications.

In the case of fungi, the first observations were visual. This is apparent given that the eating of macrofungi was important. As such, the first classifications were very pragmatic. Classifying was done on the basis of edibility and morphology in the very broad sense of the word.

It is to be expected then that the oldest classification, Clusius (1601), which made the distinction between Fungi esculenti and Fungi noxii et perniciosi would be adopted by Bauhin (1623) and Van Sterbeeck (1675) who also made use of these criteria. Ray (1686-1704) added the morphological criterion of whether or not the fungal fruitbodies were lamellate, as well as other ecological criteria. Magnol (1689) continued in this line, though Dillenius (1718) added a phenological criterion, which was subsequently refined by Micheli (1729).

Linaeus developed his system of classification on the grounds that (Micheli's) system was not satisfactory. Gleditsch (1753) introduced, in turn, more morphological characters, while Person (1794 and following) made a classification 
system based on gross morphology that included very accurate descriptions of serveral common microfungi. The most important contribution was made by Fries who described 1860 species of agarics in twenty genera, using mainly macroscopical characters.

I could go on, but that is not the aim. The important thing to remember is that descriptions and classification schemes started as very empirical. From this simple beginning, gross morphology was introduced and shortly thereafter microscopy was added. In the beginning, microscopy was very basic, but it progessed to finer microscopy. Later, developmental characters were introduced. Later still, the results from experimental biology, ecology, physiology, and biochemistry were included as characters for classifying fungi.

Let us look at the actual situation. I have selected a few characters from recent publications, selecting them from different systematic groups. As a general conclusion, it must be said that it was rather difficult to find descriptions of new species which used characters other than morphology and anatomy. This situation is easy to observe through a short analysis of vols. 46, 47, and 48 of Mycotaxon. Only systematic treatments have been taken into account, and lichens were not included.

\begin{tabular}{lrrr}
\hline Volume & 46 & 47 & 48 \\
\hline Basidiomycetes & & & \\
& & & \\
morphology, anatomy, ecology: & 8 & 10 & 10 \\
morphology, anatomy, culture: & - & - & 1 \\
morphology, anantomy, experimental: & - & - & 1 \\
numeric: & - & - & 1 \\
& & & \\
Ascomycetes and deuteromycetes & & & \\
morphology, anatomy: & & & 2 \\
morphology, anatomy, culture: & 13 & 12 & 3 \\
morphology, anatomy, culture, developmental: & 3 & 4 & 1 \\
morphology, anatomy, culture, physiology: & - & - & 1 \\
morphology, anatomy, culture, genetics: & - & 1 & - \\
morphology, anatomy, culture, computer: & - & 2
\end{tabular}

It is obvious that even in today's practices morpholgy and anatomy remain the basic characters in systematic research and taxonomy.

I did the same for Mycological Research, but as the bulk of the work is experimental and not taxonomic, this exercise was not very valuable. Nevertheless, it is clear that while Mycological Research explores many new fields, its studies do not influence basic taxonomic research to a great extent. These fields of study are excellent, however, as they force taxonimists to think about biological phenomenon which they will have to account for eventually.

Descriptions and the delimitation of taxa remain very traditional and are based primarily on morphology and anatomy. Description, however, demonstrate an increasing degree of precision. The increased precision in describing morphological and anatomical characters can be assessed easily by examining the introduction of Singer's Agaricales in modern taxonomy (1975) or Kuhner's Les Hymenomycètes agaricoïdes (1980), for example. 
Within this context it should be stressed that often small differences in morphological or anatomical characters can be extrememly important for understanding the biology of a given species. Good examples can be found in experimental phytopathological work where it has been demonstrated that spore form and spore dimension play a major role in the place where a spore will land on a phanerogam in order to infect its host.

Still, morphology and anatomy will continue to play a major role in systematics because our knowledge, gathered from previous generations, has created a framework into which more recent observations are to be fitted. Such a framework put together by so-called modern techniques is lacking for a large number of species. Given the enormous investment in human and other resources needed to produce this framework, it cannot be expected that this situatiuon will change very fast. Still, the results from new techniques are very welcome. Quite often these recent inquiries derive from studies of species or species groups that are easy to cultivate or to collect, or from studies of fungi of high economic value.

\section{How has "taxonomy" been interpreted over time?}

Classification and taxonomy aim to order certain kinds of information about living organisms. Whereas, on the whole, species and infraspecific categories can be defined by the existence of the interbreeding groups of individuals, this is no longer the case for the supraspecific categories used in taxonomy.

Genera and more universally supraspecific categories can be approached in different ways:

- genera (and higher taxa) can be recognized in virtue of being objectively recognisable in nature from patterns of morphological similarity (Kornet, 1994: 5);

- genera exist in virtue of there being a historical entity, possessing cohesion over time as assured by relationships among its members (Kornet, 1994: 5).

In early classification systems, morphological and anatomical characters were absolutely predominant in ordering information about living organisms: phenetic similarity was the basis of these classification schemes. At that time, however, it was often impossible to eliminate all background considerations in the classification which were due to convergent or parallel evolution.

With the introduction of cladistic methods, this situation changed to a certain extent. However, one of the major obstacles in using cladistic methods is the lack of knowledge of most of the characters of organisms. In most instances the characters described in the past were those which were considered as taxonomically important, and it is these characters which have noted by ("phenetic") taxonomists.

What is the currrent situation, with an emphasis on morphology and anatomy?

From a theroretical point of view, a phylogenetic methodology can give far superior classifications than the phenetic approach. On the other hand, when looking at the results of cladistic analyses in mycology, it is obvious that the interpretations of the cladograms have been leading to interpretations which are cleary ambiguous. This lack of clarity is partly due to the characters which were readily employed in the studies. 
In order to address this ambiguity, then, if the group of species for which the analysis will be made has not been treated monographically, data should be included from a number of sources: different periods, different authors and different taxonomic traditions. And yet, as can be seen in even recent monographs, it can be difficult or even impossible to construct a solid data matrix as, for instance, phenetic taxonomists have preselected certain characters in their descriptions on the basis of their experience with the group. What this practical consideration highlights is that taxonomists can easily prejudice their analyses, if they do not assess their taxonomic characters adequately enough.

Taxonomist must use good descriptive terminology consistently, describing character states very precisely. Given the examples I have seen, I must stress that the collecting of basic data needs to be done more rigourously.

Going through the recent cladistic mycological literature, I have noticed that too often character sets have been accummulated from the literature without any real expertise in the group, which has led to the misinterpretation of characters and their significance. Unfortunately, the results from such analyses cannot be expected to improve our knowledge of systematics.

To avoid these sorts of problems from a practical point of view means that ecologists, phytopathologists, medical mycologists as well as amateur mycologists must have a common understanding of characters at the species level. Thus far, experience shows that easily observable morphological and anatomical characters have so far been the most readily available.

\section{Is the importance of morphology and anatomy similar in all groups of fungi?}

Considering fungi as a whole, it is obvious that classification and systematics have employed different starting philosophies. As a result, it can be expected that systematics developed along different lines in the case of yeasts and yeast-like fungi as compared to other groups of fungi.

The origin of systematics of yeasts and yeast-like fungi was in the first place an applied study, having its roots in the fermenation industry. From its practical point of view, selection of physiological properties was the most important character. As systematics progressed along these lines, a new study developed which we now know as bacteriology. In this new field, the use of morphological characters was hardly possible because of the lack of a well-differentiated thallus that would have been useful for distinguishing between groups of bacteria.

The systematics of the majority of fungi developed along the line of practice of systematics for higher plants. They began as primarily morphological and progressed to include anatomical characters. For a long time, systematic of both "types" of fungi developed on these lines, and it was thought that yeasts were a heterogeneous groups of organisms. But with the introduction of new techniques, links were made between both groups when it became obvious that yeasts are unicellular, ontogenetic stages of ascomycetes and basidiomycetes.

From this perspective, it is obvious that for the classification of yeasts and yeastlike fungi an important contribution will be made by mycologists who are acquainted with systematics of ascomycetes, basidiomycetes, endomycetes and ustomycetes. One result may be that the virtual non-exception to the general rule that classification is primarily based on morphology and anatomy will disappear. However, from a 
practical point of view, the artificial classification of yeasts and yeast-like fungi will continue to be valuable as an approach for specific purposes, though not as a general approach for basic systematics.

With respect to this symposium's emphasis on tropical mycology: is the situation in the tropics different from the one in temperate regions?

Basic knowledge of fungi from the tropics is extremely poor given the large number of species which require describing. On the other hand, this situation can be advantageous as mycologists can, by adapting their experience from temperate regions, collect and describe fungi from the tropics according to the specific requirements of new techniques, such as cladistics. Furthermore, since tropical mycology is not as encumbered by nomenclatorial problems as mycology conducted in temperate regions, this circumstance can be considered as an advantage. Systematic mycologists can profit from this situation, though they need to be as careful and consistent as possible when collecting and describing specimens. The need for care is certainly the case where, because new techniques are more limited in the tropics, systematics will continue to rely on morphology and anatomy.

\section{Conclusion}

Morphology and anatomy have been the primary characters used in the systematics of fungi. Other types of characters which have been considered outmoded have not always been adapted by taxonomists. At present, taxonomists have high performance computers at their disposal, which have greatly enhanced the possibility for networking and the exchange of data and results between scientists. Even though taxonomy has evolved considerably from its early beginnings, it is still very important that it continues to develop, to adapt new techniques and technologies as they arise. The critical issue is that taxonomy remains a central discipline in the study of fungi. This possibility is especially significant in view of the fact that systematists work to synthesize everything that is known about organisms. For it is through these syntheses that more complete schemes of relationships can be developed. Though there is a distinct possibility that the importance of morphology and anatomy will diminish with the development of these syntheses, from a practical point of view, and this is especially true for tropical mycology, they will remain the most important characters for separating adjacent taxa.

\section{References}

Bauhin J. 1620. Prodromus theatri botanici. Frankfurt, $4^{\circ}, 160 \mathrm{p}$.

Clusius C. 1601. Rariorum aliquot stirpium per Pannoniam, Austriam et vicinas. Fungi p.261-295.

Antwerp, $760 \mathrm{p}$.

Dillenius J.J. 1718. Catalogus plantarum circa Gissam sponte nascentium.

Gledistsch J.G. 1753. Methodus fungorum. Berlin 162 p.

Kornet D.J., Reconstructing species: demarcations in genealogical networks. PhD thesis. Institute for Theoretical Biology and Rijksherbarium - Hortus Botanicus Leiden, Leiden.

Kuhner R. 1980. Les Hymenomycètes agaricoïdes. Bull. mens. Soc. Linn. Lyon 49: 1-1027.

Magnol P. 1684 Botanicum Monspeliense. Hamburg.

Micheli P.A. 1729. Nova plantarum genera. Florence, $4^{\circ}, 234 \mathrm{p}$.

Persoon C.H. 1794 . Neuer versuch einer systematische eintleitung der Schamme. Römer's Neues Mag. f. d. Botanik, pp.63-128.

Persoon C.H. 1795. Observationes mycologiciae. Usteri's Annal. d. Botan. 15: 1-39; 16: 1-33.

Persoon C.H. 1796. Observationes mycologicae. Leipsig, 1: 115 p. 
Persoon C.H. 1799. Observationes mycologicae. Leipsig, 2: $106 \mathrm{p}$

Persoon C.H. 1797. Tentamen dispositionis methodical fungorum. Leipsig, $76 \mathrm{p}$.

Persoon C.H. 1801. Synopsis methodical fungorum. Götingen, 706 p.

Ray J. 1686. Historia plantarum. London, M. Clark, 1686: 1-983; 1688: 985-1944; 1704: 255p.

Singer R. 1975. The Agaricales in modern Taxonomy. 3d ed., J. Cramer, Vaduz, 912 p.

Van Sterbeek Fr. 1675. Theatrum fungorum. Antwerp. 


\title{
POSSIBLE PROGRESS OF MORPHOLOGICAL ANALYSIS IN FULGAL TAXONOMY
}

\author{
M.F. ROQUEBERT
}

Laboratoire de Cryptogamie, MNHN, 12 rue Buffon 7500,Paris

\section{Introduction}

From its beginning, fungal taxonomy was based on morphology, first by means of direct examination of the fungus and later by microscopical observations. Since the launching of the new techniques by the pioneer microscopists of the 17th Century such as Hook and Leeuwenhoek, optical microscopy progressed considerably to the end of the 19th Century, when the cultural study of fungi was introduced. This new perspective opened the possibility of a complementary developmental approach to fungi.

Much later, around 1950, the use of the Transmission Electron Microscopy enabled researchers to make comprehensive progress in the study of fungal structure and ontogeny, while the use of Scanning Electron Microscoopy permitted more detailed surface and three-dimensional observation of fungal specimens. Generally speaking, data obtained by electron mircoscopy (Pegler and Young 1971, Cole and Samson 1979) did not impair those obtained by good optical observations and descriptions, rather it improved them. Accordingly, both macroscopical and microscopical approaches have become increasingly useful and complementary.

Still later in the 1980's, the advent of molecular techniques was applied to the taxonomy of fungi. This development gave raise to the belief that the descriptive phase of taxonomy was drawing to a close (Hillis 1987). Nevertheless, it is becoming more evident that these new techniques do not modify substantially the results of traditional morphological taxonomy. Rather these techniques help to clarify questions suggested by the limited resolution of morphological studies, such as for example, the exact delimitation of taxa or the definition of holomorphic relationships (Kohn 1992). In point, it is important to stress that these various approaches should be considered as complementary and not exclusive of one another. As such, microscopists should be encouraged to assist molecular biologists in their efforts to order any new data in the context of taxonomy, and vice-versa (Reynolds and Taylor 1993).

In this respect, while morphological analysis may be understood to lie at one "end"of a phyllum, it remains still in a process of evolution. Given that such techniques will continue to evolve, two axes need to be considered:

1. the improvement and simplification of observational techniques

2. the interpretation of multiple data sets

\section{The improvement and simplification of observational techniques}

At present, morphological studies are generally performed simultaneously through direct observation in situ through optical and electron microscopical examination. Of course, each technique has its limits, its advantages and 
disadvantages. The performance and efficiency of optical microscopy does not need to be demonstrated. Still, it is a stumbling block for fungal identification. Although it is relatively inexpensive and thus readily available in all countries as well as easy to use, magnification (x 2000) and resolution $(0.2 \mu \mathrm{m})$ can be limiting factors despite the efficiency of this device.

Electron microscopy, on the other hand, permits high magnification (x 100000) and resolution (3-10 ̊m), but both transmission and scanning electron microscopes necessitate observation under vacuum conditions which requires special preparation of specimens (fixation, dehydration, sectioning, and staining). Recently, considerable progress has been made in these areas particularly with the development of low temperature fixations (Hock 1986). A low temperature scanning microscope (LTSEM) is now widely available and allows for morphological observation in hydrated states (Jeffree and Read, 1991).

Even if these various forms of electron microscopy are really useful for fungal taxonomists, however, these techniques require heavy and expensive equipment which is not always available to everyone in all parts of the world. An ideal situation for the future would be the development of less expensive equipment that allows an easy observation of the specimen at high magnification and resolution but does not require that the specimen undergo preparation, like the use of vacuum.

New techniques are progressing in this direction. The most promising for morphological analysis in fungal taxonomy is Confocal Microscopy. It bridges the gap betweeen conventional optical and electron microscopes. (Laurent et al. 1992, Shotton 1989). In this case, the image is produced by scanning a diffraction limited laser over the specimen, exposing an extremely small volume of it to the incident light, and thereby restricting sources of reflected light. The most novel imaging mode accounts for vertical sectioning. Instead of scanning the specimen along the $\mathrm{x}$ y planes, this mode generates images by scanning optical sections that are parallel to the axis of the microscope. This approach has the advantage of limiting out of focus information from the observed image.

Confocal microscopy does not need any preparation of the specimen except for fluorescent staining. With resolution down to $0.25 \mu \mathrm{m}$, the technique is used frequently in cytology, cytometry, and molecular biology (Shotton 1989). Preliminary results of morphological observation obtained with Penicilium chryogenum (Fig. 1) shows clearly the absence of out of focus blur in the confocal images. These results also show the fine resolution in successive vertical planes which allows for the exploration of thick structures (70-80 Åm).

Cell surfaces can also be investigated by other new kinds of microscopes: the Scanning Probe and Scanning Tunnelling Microscopes (STM) as well as the Atomic Force Microscope (AFM) (Guckenberger and Baumeister 1992). Research with STM is still focused on exploring type of experiments that might be more feasible in the future. At present, it is being tested principally on non-biological materials. In contrast, AFM has already been used quite successfully for obtaining images of biological specimens in aqueous solutions (Butt et al. 1990, Kasas 1992). The principle technique applied in AFM involves scanning the surface of the specimen with the tip of a very thin probe (diamond or silicium). The three dimensional scanned displacements are recorded and analysed by a computer. AFM combines a high resolution ( $80 \AA \mathrm{m}$ on blood cells) without the need for sample preparation as it works in aqueous environments. 


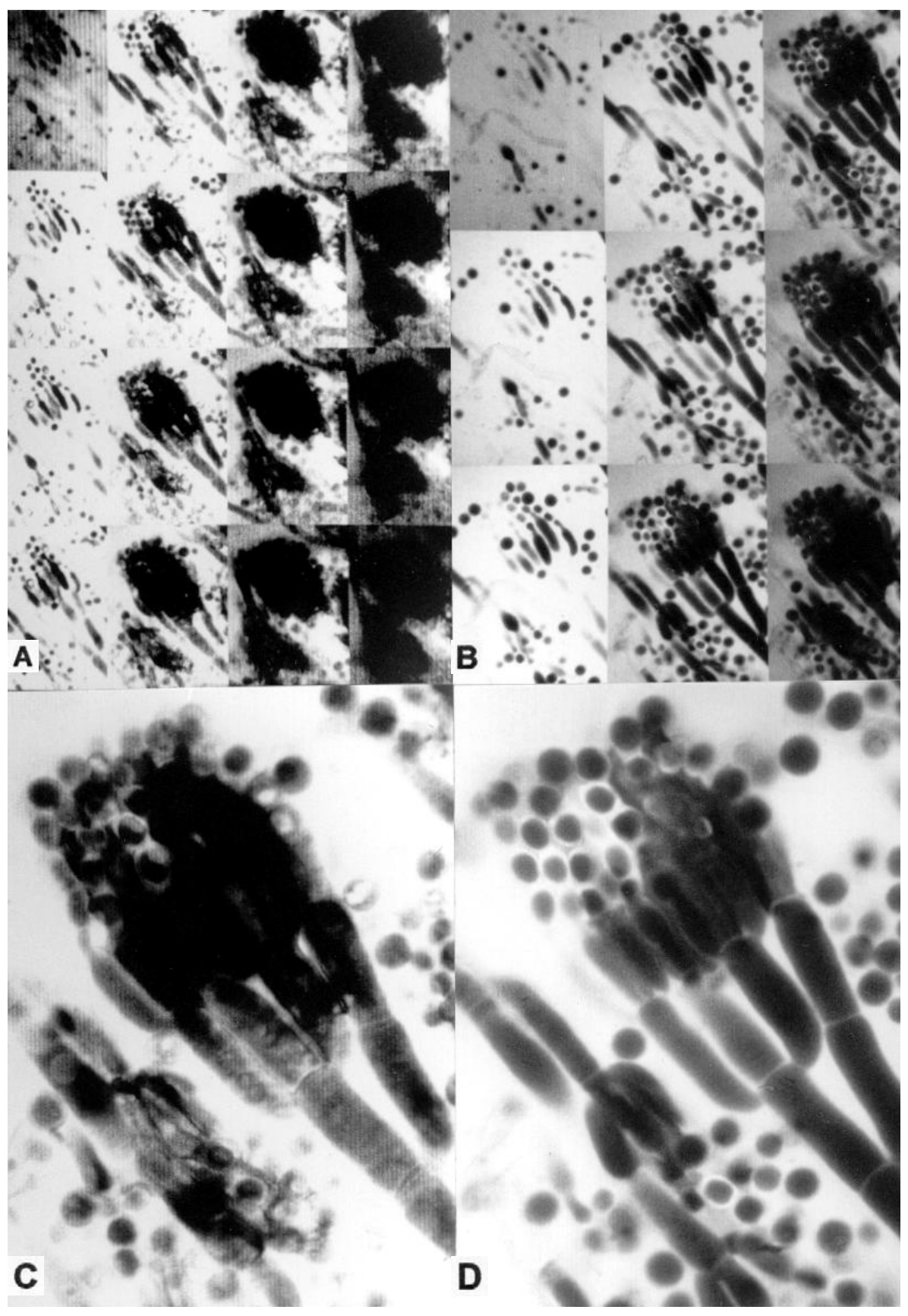

Figure 1. Penicillium chrysogenum Thom, Coloration: Fluorescein-dextran (negative coloration) Confocal Microscope Biorad MRC 1000. A-B: Optical sections at different focal planes separated by Ám. A: Non-confocal, B: Confocal optics. C-D: Detail of penicillus. C: Non-confocal, D: Confocal optics. 
It is currently used for studying molecules (proteins, phospholipids, and DNA) constituting organic matter. The advantage of the technique is that it permits observations in environmental conditions with good magnification and resolution. The lightness of this equipment also constitutes an advantage.

Even if these new microscopy tehniques are still in the research phase, we can safely assume that these techniques, in particular confocal and atomic force microscopy, will be used in the near future for fungal morphological observation and taxonomy.

\section{The interpretation of multiple data sets}

To be effective in taxonomy, observations must be intrepreted and represented as objectively as possible. Until now, line drawings and photographs have been very effective for providing clear visual representations of macro- and microscopical observations, but because they are man-made and interpretative, they are often very expressive and artistic, and thus quite subjective.

On the other hand, quantitative morphological analysis provides a means to translate visually apparent structures into numerical data that can be subjected to the powerful methodologies of mathematical analysis. (Wagener 1990, Steer 1991). Because of its capacity for decomposing and anaylsing a great number of images, image analysis may be a very effective tool for limiting subjective interpretations. The important point is to obtain the maximum information from visual images so that the data can be interpreted with the greatest possible confidence. This possibility may also be very helpful for taking into account the variability of specimens. One of the advantages of the technique is that image analysers can be integrated directly into the apparatus of microscope as they are already in confocal and scanning probe microscopes.

In addition to image analysis, another way to exploit a large number of morphological observations may be the use of morphometrics. The field of morphometrics is traditionally concerned with methods for the description and statistical analysis of shape variation within and among samples of organisms adjusted to a common size, measuring certain landmarks of an organism (length, width, etc.). An example of the impact that morphometric analysis can have on fungal taxonomy is evident in the recent publication by Vanev (1993) on the conidia of 14 type specimens of Discosia section Strobilina. Analyses of the shape as well as the length and width measurements of the conidia enabled the author to reach decisions about the taxonomic status of the specimens, that is, to gather data useful for separating and grouping taxa.

Many methods agree on the nature of the differences among a set of shapes, but fewer agree on the relative significance of the differences between these shapes. A new trend in morphometrics is to incorporate data on the geometry of the structure so as to distinguish changes due to mere differences of size from those which describe purely homogeneous features (Zelditch et al. 1992). The ideal aim would be to eliminate from the analysis, dimensional features that are due to the natural variability of biological material, so that phenetic or cladistic relationships can be estimated more accurately. 


\section{Conclusion}

Interesting progress is being made in the morphological observation and analysis of biological material. The methods and equipments used in these techniques are being simplified considerably without loss of performance. Even though the analysis and the objective interpretation of data remain at the research phase, in part because of the variety of biological material, great possibilities exist for these techniques as they are effectively adapted to fungal taxonomy. These technical and analytic approaches seem promising for the progress of fungal taxonomy, as they also are an interesting subject for research in themselves.

\section{References}

Butt H.J., Wolff E.K., Gould S.A.C., Dixon-Northern B., Peterson C.M. and Hansma P.K. 1990. Imaging cells with the atomic field miscroscope. Journal of Structural Biology 105: 54-61.

Cole G.T. and Samson R.A. 1979. Patterns of Development in Conidial Fungi. Pitman., London.

Guckenberger, R., Hartma n T., Wiegrabe W.and Baumeister. W. 1992. The Scanning tunnelling microscope in Biology. In: Scanning Tunnelling Microscopy II . Wisendanger R. and Guntherodt H.J. eds., Springer Series in Surface Science, 8: 51-98.

Hillis D.M. 1987. Molecular versus morpholical approaches to systemeatics. Ann. Review of Ecology and Systematics 18: 23-42.

Hoch H.C. 1986. Freeze substitution of fungi. In: Ultrqstructures techniques for microorganisms. Aldrich H.C. and Todd W.J.J. eds., Plenum. N.Y.

Jeffree, C.E. and Read N.D.. 1991. Ambient- and low-temperature scanning electron microscopy. In: Electron microscopy of plant cells. Hall J.L. and Hawes C. eds. Academic Press, London, pp. 313413..

Kasas S. 1992. La microscopie Ó force atomique dans les recherches en biologie. Médecine/Science 8: 140-148.

Kohn, L.M. 1992. Developing new characters for fungal systematics: an experimental approach for determining the rank of resolution. Mycologica 84: 139-153.

Laurent M., Johanin G., Le Guyader H. and Fleury A. 1992. Confocal scanning optical microscopy and three dimensional imaging. Biol. Cell 76: 113-124.

Pegler D.N. and Young T.W.K.. 1971. Basidiospore morphology in the Agaricales. Beih. Nova Hedwigia 35 .

Reynolds, D.R. and Taylor J.W. 1993. The fungal holomorph: mitotic, meiotic and pleomorphic speciation in fungal systematics. Wallingsford, CAB International, $375 \mathrm{pp}$.

Shotton D.M. 1989. Confocal scanning optical microscopy and its application for biological specimens. J. Cell Science 94: 175-206.

Steer M.W. 1991. Quantitative morphological analysis. In: Electron microscopy of plant cells. Hall J.L.and Hawes C. eds., Academic Press, London, pp. 85-104..

Vanev S.G. 1993. Morphological variability of the conidia of the fungi from the genus Discosia section Strobilina. Mycotaxon, 49: 199-207.

Wagener, M. 1990. Digitization in scanning electron microscopy. In: Electron microscopy of plant cells. Hall J.L. and Hawes C. eds., Academic Press, London., pp. 297-305.

Zelditch M.L. F.L. Bokkstein and Lundrigan B.L.. 1992. Ontogeny of integrated skull growth in the cotton rat Sigmodon fulviventer. Evolution 46: 1164-1180. 


\title{
MYCOFLORISTIC STUDIES IN SOUTH CENTRAL AFRICA: STATUS, CONSTRAINTS, OPPORTUNITIES AND STATEGIES
}

\author{
A.J. MASUKA \\ Forest Research Centre, P.O. Box HG 595, \\ Highlands, Harare, Zimbabwe
}

\begin{abstract}
Mycofloristic studies of the South Central African region, comprising Malawi, Zambia and Zimbabwe, have largely focussed on the identification of macrofungi, apart from fungi of agricultural importance. The only relatively well studied group is the Aphyllophorales, with over 200 known species. In addition to their studies on the Aphyllophorales, visiting scientists have contributed immensely to our present knowledge of the Agaricales, Boletales, Cantharellales, Russullales and other non-basidiomycete fungi. Termitomyces, Cantharellus species, edible boletes and species of Russula and Lactarius have a unique contribution to the diets of many people during the rainy season. This has prompted publication of country and regional guides to mushroom identification and mushroom poisoning. Most studies of the larger fungi have been of a taxonomic nature, though mushroom productivity studies have just begun. The major taxonomic approach has stressed morphological and anatomical characters; supplemented by chemical, ecological, cultural and other characteristics. We find the approach to be pragmatic under our circumstances, and applicable to a large range of fungi, though its shortcomings have been realised in recent collaborative studies on African Armillaria. The genetic basis of species differentiation has largely been of an academic interest. Unfortunately, taxonomic studies are still regarded as being peripheral to agricultural and forest pathology/mycology and most identifications of fungi are conducted by institutions outside Africa. There is a lack of qualified scientists, as well as adequate and continuous funding at some established research institutions. There is need to undertake more fungal inventories and ecological studies because of their importance to aspects of the conservation of fungal and ecosystem biodiversity. Furthermore, with an estimated annual deforestation rate of over 250000 hectares in the region, some species will eventually be lost before they are known. Assistance in training local scientists must be sought, and there should be a provision for suitable equipment and literature. Local, regional and international support for such activities should be mobilised. The importance of herbaria for educational, reference, conservation and other purposes should be publicised. These are our present challenges and obligations.
\end{abstract}

\section{Introduction}

\section{The South Central African Region}

The South Central African region comprises Malawi, Zambia and Zimbabwe. The region is bound by the latitudes $8^{\circ} 25^{\prime}$ and $22^{\circ} 30^{\prime}$ South and longitudes $23^{\circ}$ and $36^{\circ}$ East. The region is wholly land-locked, the nearest points to the oceans being over $100 \mathrm{~km}$ to the Mozambique Channel and $900 \mathrm{~km}$ to the Atlantic Ocean. The total land area is about 1.25 million $\mathrm{km}^{2}$. Climate is broadly divided into two distinct seasons - a summer rainfall season starting November and ending in April, and a prolonged dry winter season. This has a marked influence on the fruiting times of fungi and the timing of collection forays - which in our region are best productive in February and March. The mean annual rainfall is $635-1500 \mathrm{~mm}$ per annum. Mean temperatures are $18^{\circ}-21^{\circ} \mathrm{C}$ in areas of a latitude range of 900 to 1200 m.a.s.l. (about $70 \%$ of the land area). Wild and Barbosa's (1967) Vegetation Map of the Flora Zambesiaca Area presents a floristic and physiognomic classification of the region, in addition to Mozambique and Botswana, the area draining the Zambezi River.

The characteristic vegetation type is that of the Leguminoseae genera Brachystegia, Julbernardia and Isoberlina. Such dry deciduous forests are termed miombo - they are widespread and predominant, covering up to half of the area of the region, at medium to high altitude and rainfall areas, on soils derived from 
granite, schist, quartzite, gabbro or shale. The miombo ecozone - or miombo region also extends into southern Zaire, northern Mozambique and parts of Tanzania and Kenya. Other forest types found in the region are the Zambezi Teak Forests, in which Baikiaea plurijuga is the dominant species (on the Kalahari Sands in western Zimbabwe and south-western Zambia, where rainfall is generally low). These forests also occur in parts of Angola and Botswana. There are small relic swamp and evergreen forests in high rainfall areas, containing the only coniferous species of the region (Juniperus, Podocarpus and Widdringtonia). Typical savanna woodlands are associated with some impediment in drainage and species of Acacia, Combretum and Terminalia predominate, though there are several secondary associates. In the lower elevation areas Colophospermum mopane predominates, especially on calcareous soils. Forested areas are interspersed with shallow depressions or drainage canals that are seasonally waterlogged (dambo or vlei), forming grasslands. Considerable development of plantations with exotic species (mainly Acacia, Cupressus, Eucalyptus and Pinus) currently totalling $2500 \mathrm{~km}^{2}$ has taken place within the last 4-5 decades

The assemblage of fungi in South Central Africa is large and heterogenous. It is impractical and probably unadvisable, considering the variety of species and the constellations of characters they posses, for one to sufficiently and efficiently address aspects of their taxonomy, ecology, distribution etc. - floristics - in this presentation. I will therefore, focus on the macrofungi, a group I have also studied for some years now.

\section{Importance of fungi}

The importance of and uses of fungi are diverse, some being detrimental to mankind, crops and animals. The presence and importance of fungi in agricultural and forestry systems as pathogens, saprotrophs and symbionts is well recognised. Their use in wine-making and beer-brewing industries, as food, medicines and mycopesticides, and in other novel biotechnological applications have been tapped to a variable extent in the region (Subramanian, 1992, Hawksworth, 1993, for the general importance of mycology to development). The applied mycology aspects attract the bulk of funding from public and private institutions. Generally, studies on fungal taxonomy and ecology, are regarded as being of fundamental importance only, and have received limited attention.

\section{STATUS OF MYCOLOGY RESEARCH AND TAXONOMIC STUDIES}

Meaningful mycology research is conducted by Universities, Departments of Agriculture and Forestry, some parastatals and a few private organisations.

\section{Parasites}

Comprehensive lists of fungi of pathological importance in agriculture (Rothwell 1982, Angus 1962-66, Doidge 1950) and forestry (Masuka and Ryvarden 1992a) have been compiled. Macrofungi on living forest trees (Table 1) have only been collected for serious study recently. The fungi play an important role in tree succession and hence stand and ecosystem dynamics. Fomitopsis widdringtoniae Masuka \& Ryv. is commonly found on Widdringtonia nodiflora in Malawi (Masuka and Ryvarden, 1993a), and is associated with the widespread decline of the host species. Armillaria, Amauroderma and Ganoderma species are the most common 
fungal causes of mortality in indigenous tree species. Heart-rot in indigenous merchantable timber species (e.g. B. plurijuga and $P$. angolensis) may reach an incidence of up to $19 \%$ in logs (Masuka and Ryvarden 1993b). This has prompted some recent studies (including those on the taxonomy and ecology of causal fungi) on the subject in Zimbabwe.

Table 1. Macrofungi associated with living trees in South Central Africa.

\begin{tabular}{|c|c|c|}
\hline Species & Paras & $\mathrm{d} \mathrm{part}^{2}$ (rot) \\
\hline Abortiporus biennis (Bull.: Fr.)Sing & $\mathrm{Bu}$ & root, butt \\
\hline Amauroderma argenteofulvum (Van der Byl)Doidge & $\mathrm{Bs}, \mathrm{Jg}$ & root \\
\hline A. fuscoporia Wakef. & $\mathrm{Bs}, \mathrm{Jg}$ & root \\
\hline A. preusii (Henn.) Steyaert & $\mathrm{Bs}, \mathrm{Jg}$ & root \\
\hline Armillaria (Fr.) Kummer spp. & $\mathrm{Bs}, \mathrm{Jg}, \mathrm{Pc}$ & root \\
\hline Aurificaria indica (Mass.) Reid & Csp & heart \\
\hline Fomitopsis widdringtoniae Masuka \& Ryv. & W & butt \\
\hline Ganoderma australe (Fr.) Pat. & $\mathrm{Kn}, \mathrm{Bg}$ & heart, butt \\
\hline G. colossum (Fr.) Baker & $\mathrm{Cm}$ & root, butt \\
\hline G. eminii (Henn.) Ryv. & Bs, Jg & root, butt \\
\hline G. lucidum (Fr.) Karst. & $\mathrm{Bs}, \mathrm{Jg}, \mathrm{Pc}, \mathrm{F}$ & root, butt \\
\hline G. sculptrutum Lloyd & $\mathrm{Bs}, \mathrm{Jg}(\mathrm{Eg})^{*}$ & root, butt \\
\hline Inonotus afromontanus Ryv. and Gilbn. & Psp & butt \\
\hline Perenniporia martius (Berk.) Ryv. & $\mathrm{Kn}$ & heart \\
\hline Laetiporus baudonii (Pat.) Ryv. & Bs, Jg & root \\
\hline L. percisinus (Berk. \& Curt.) Gilbn. & $\mathrm{Bu}$ & heart \\
\hline Phellinus allardii (Bres.) Ryv. & $\mathrm{Bsp}, \mathrm{Cm}$ & heart \\
\hline P. fastuosus (Lev.) Ryv. & $\mathrm{Cm}$ & heart \\
\hline P. lamaensis (Murr.) Ryv. & $\mathrm{Bu}, \mathrm{Kn}$ & heart \\
\hline P. merillii (Murr.) Ryv. & $\mathrm{Kn}$ & heart \\
\hline P. rimosus (Berk.) Pil. & Psp, $\mathrm{Pa}, \mathrm{Ba}, \mathrm{Bp}, \mathrm{Bs}$ & heart \\
\hline P. robustus (Karst.) Bourd. \& Galz. & $\mathrm{Bu}, \mathrm{Bs}, \mathrm{Cp}$ & heart \\
\hline P. senex (Nees. \& Mont.) Imaz. & $\mathrm{Bs}, \mathrm{Jg}$ & heart \\
\hline P. wahlbergii (Fr.) Reid & $\mathrm{Bu}, \mathrm{Bs}$ & heart \\
\hline
\end{tabular}

1 Bp, Baikiaea plurijuga; Bg, Brachystegia glauscesens; Bs, Brachystegia spiciformis; Ba, Burkea africana; Cm, Colophospermum mopane; Csp, Combretum sp.; F, Ficus spp.; Jg, Julbernardia globiflora; Kn, Khaya nyasica; Pa, Pterocarpus angolensis; Pc, Parinari curatelifolia; Psp, Phillipia sp., W, Widdringtonia nodiflora

.$^{2}$ But-rot defined as up to $1 \mathrm{~m}$ above ground; higher than that the term heart-rot applies.

* The fungus has recently adapted onto Eucalyptus grandis in Zimbabwe and causes up 4\% annual mortality in high risk areas whose previous indigenous vegetation is B. spiciformis or J. globiflora (Masuka and Nyoka, 1994).

\section{Mushrooms and mushroom poisoning}

Mushrooms are highly rated on the local gastronomic scale, have a unique contribution to the diets of many people, and are extensively collected during the rainy season (December-March). Country and regional guides to mushroom identification and mushroom poisoning have been produced. Morris's (1987) Mushrooms of Malawi and Storrs and Piearce's (1982) Do Not Eat These, Piearce (1981a) and more recently Ryvarden, Piearce, Masuka's (1994) An introduction to the Larger Fungi of South Central Africa are useful starting points for anyone studying mushrooms in this part of the world. Throughout the region fungi are normally equated with mushrooms (Khonga 1994, Masuka 1992a, Morris 1984, 
1987) and traditional classification systems - passed orally from one generation to the next - have existed to this day, though with urbanisation local, elderly people remain the only repository of such knowledge. The classification system is based principally on colour (there are 4-5 in the region), aroma, texture and taste. In Nigeria the shape, texture, taste and maturation period are the major taxonomic criteria employed by local people (Alabi 1994). A start has been made in Zimbabwe to prepare a checklist of vernacular names of fungi (in different languages and dialects) and to provide their English equivalents (where applicable) and scientific names. There is substantial folklore associated with edible and poisonous mushrooms though there are a few records of the use of macrofungi in traditional medicine, or for ritual purposes, and fungi are rarely used in artwork (Masuka 1992a, Piearce 1981a, Morris 1984, 1987). Perenniporia mundula (Wakef.) Ryv. is the only medicinal fungus used throughout the region, for treatment of pleurisy and impotence.

Termitomyces, Cantharellus, some species in the Russulales and Boletales are regarded as delicacies. A representative number of these, and other macrofungi, have been included in the book by Ryvarden et al (1994). Amanita zambiana Pegler \& Piearce, confined to South Central Africa and southern Zaire, is abundant and widely collected for consumption. The hard-textured species such as Lentinus cladopus Lev. and Polyporus tenuiculus (Beauv.) Fr. are edible but seldom collected; for consumption during the off-season.

There are about seven common Termitomyces species among which four are highly regarded. They have been studied in greater detail in Zambia (Piearce 1987). There are approximately 1800 termite species in Africa and South East Asia among which some 100 species in six genera grow, among them, about 20 species of Termitomyces (Bels and Pataragetvit 1982). The associated mounds can be invisible to about $10 \mathrm{~m}$ high. The relationship between Termitomyces and termites is an example of obligate symbiosis. The only other similar association of insects and fungi is that of the Attini, confined to Central and South America, which have been reported to grow a Leucocoprinus sp. (Holldobler and Wilson 1990). There is no evidence that the complementary habits of Termitomyces and Leucocoprinus sp. are mutually preemptive involving competitive exclusion, or accidental, reflecting the rarity of evolution along that path.

Amanita, Cantharellus, Lactarius and Russula, and also Termitomyces species cannot, as yet, be cultured. Their continued presence will depend on the protection of their natural environments. Mushroom collecting is regarded as a women's task. Mushroom productivity studies (counts by species and basidiocarps, basidiocarp diameters, determination of fresh and dry weights and nutritive values) in miombo forests in Zimbabwe have only been recently initiated. Aspects of mushroom collection, processing and consumption (and utilisation) patterns; importance in rural economy; and valuation, marketing and trade will also be considered. Such studies should result in a further understanding of their taxonomy and ecology.

Several cases of mushroom poisoning are reported each year, and children are most vulnerable. Most cases of poisoning reported generally fall within the class of gastrointestinal irritants (Ryvarden et al. 1994). Species (authentically) associated with poisoning are Chlorophyllum molybdites (Meyer: Fr.) Masse, and some Amanita species which are commonly mistaken for Macrolepiota and Agaricus species. Mushroom poisoning has attracted considerable attention from the print and electronic media in the region. Publications on the subject are by Flegg (1981), 
Gelfand and Harris, (1982), Robertson (1983) and Sharp (1983) for Zimbabwe; Storrs and Piearce (1982) for Zambia, Morris (1987) for Malawi, and (Ryvarden et. al. 1994) for the region.

Following introduction of exotic plantation species (Pinus spp.) poisonous species of Amanita [A. muscaria (L.:Fr.) Hooker], but also edible species such as Boletus edulis Bull.:Fr. and Suillus granulatus (L.:Fr.) O. Kuntze have become naturalised in those plantations. Most locals regard macrofungi under exotic plantations with very deep suspicion.

\section{Fungi on timber in service}

Recent studies in South Central Africa have also focussed on the identification of decay fungi on timber in service, ascertaining their temperature and moisture characteristics in culture, determining frequency of occurrence and extent of damage they cause, and on aspects of their control (Masuka and Ryvarden 1992). There is even greater scope for such studies as the construction of timber houses is now permitted in Zimbabwe (Standards Association of Zimbabwe, 1990). Furthermore, fungi responsible for deterioration of paints and other products may also be studied. Some survey work on the fungi causing decay of mine timber has also been conducted. The studies have revealed the presence of Gyrodontium boveanum (Montagne) Maas G. as an important rotter of mine timber, Coniophora puteana (Fr.) Karst., Antrodia vaillantii (Fr.) Ryv. and Gloeophyllum trabeum (Fr.) Murr. in pine timber, and Coriolopsis polyzona (Pers.) Ryv. and Trametes vesrsicolor (Fr.) Pil. in hardwood timber (Masuka 1992a).

\section{Saprotrophic and mycorrhizal macrofungi}

Most fungi in the region are saprotrophs on dead wood and on leaf litter, being inconspicuous to the unaided eye to large and sometimes brightly coloured species. The only well studied group is the Aphyllophorales (Masuka 1992b, Masuka and Ryvarden 1992b, 1993c, Mswaka 1992). This is so because the fungi are easy to collect and store. Collections in the region have yielded much information, and currently the region is better explored mycologically than most parts of Africa. Studies on the agarics and boletes have recently been resuscitated following collaborative collection trips with institutions and individuals in the United States of America.

The taxonomy of tropical fungi is in a continuing state of flux including that of relatively well studied groups such as the polypores (Ryvarden, 1994). New species continue to be discovered following thorough collecting (Masuka and Ryvarden 1993a) and many more polypores will be described soon from our region (Mswaka, Masuka and Ryvarden, unpublished). Such new discoveries have added to the instability of generic and species delimitations, whose descriptions are often based, for historical reasons, on temperate species. Current work on novel Dichomitus species from the region have repercussions on the generic delimitations of Grammothele, Grammothelopsis and Megasporoporia.

Collections of Aphyllophorales have yielded very valuable information (Table 2) and recent comparisons indicate that there are only $2-5 \%$ brown-rot fungi in the Polyporaceae in the tropics compared to $28-30 \%$ in temperate countries (Ryvarden, 1994). 
Table 2. Ecological roles of Aphyllophorales in Zimbabwe.

\begin{tabular}{lccr}
\hline Family & Mycorrhizal & Parasitic & Saprotrophic \\
\hline & 0 & 0 & 7 \\
Corticiaceae & 0 & 11 & 28 \\
Hymenochaetaceae & 0 & 0 & 6 \\
Lachnocladiaceae & 0 & 0 & 8 \\
Coniophoraceae & 0 & 8 & 11 \\
Ganodermataceae & 0 & 0 & 11 \\
Hericiaceae & 1 & 11 & 106 \\
Polyporaceae & 0 & 0 & 1 \\
Punctulariaceae & 0 & 0 & 2 \\
Schizophylaceae & 0 & 0 & 2 \\
Steccherinaceae & & 0 & 2 \\
Stereaceae & 1 & 0 & 0 \\
Thelepohoraceae & & & 4 \\
\hline
\end{tabular}

source: Masuka and Ryvarden (1993c)

The majority of the recorded species found in a study by Masuka and Ryvarden (1993c) had di- or tri-mitic systems of hyphae (Table 3), and there were significantly $\left(x^{2}=23.24, \mathrm{P}<0.01\right)$ more monomitic species in higher rainfall areas (Afromontane and moist ever-green forests) than in the drier areas (Baikiaea and Mopane forests).

Within the various forest types the species occurring there tend to be predictable. Thus while Phellinus rimosus is a common species in mopane woodlands Amauroderma and Ganoderma species do not occur there but predominate in miombo woodlands.

Table 3. Hyphal system and vegetation type.

\begin{tabular}{lrrr}
\hline Forest Type & \multicolumn{3}{c}{ Number of Species } \\
\hline & Monomitic & Di-/Tri-mitic \\
\hline Afromontane & 3 & $(38)$ & 5 \\
Baikiaea & 3 & $(9)$ & 29 \\
Deciduous tree savanna & 3 & $(10)$ & 28 \\
Eucalypts & 7 & $(22)$ & 25 \\
Miombo & 12 & $(15)$ & 67 \\
Moist evergreen & 24 & $(26)$ & 72 \\
Mopane & 3 & $(10)$ & 26 \\
Pine & 22 & $(41)$ & 32 \\
\hline
\end{tabular}

The figures in parenthesis indicate the percentage of species with a monomitic hyphal system.

An analysis of the species of the Coniophoraceae (Table 4) shows that only a few have been found in Africa. The data was compiled from various sources including Ginns (1976, 1978, 1982), Gilbertson and Ryvarden (1987) and Masuka and Ryvarden (1991). These observations are based on the morphological species concept. It is not known whether some of the species found in temperate 
environments are present in the tropics in a vegetative state but fail to produce basidiocarps because of adverse climatic and other conditions. One inherent flaw in such comparative mycogeographical studies is the dilemma of distinguishing between non collection and non occurrence of a species in an area.

Table 4. Representation of genera of the Coniophoraceae in Africa.

\begin{tabular}{lcc}
\hline Genus & \multicolumn{2}{c}{ Number of species in } \\
\hline & Genus & Africa \\
\hline Coniophora & 15 & 8 \\
Corneromyces & 1 & 1 \\
Gyrodontium & 3 & 2 \\
Jaapia & 2 & 0 \\
Leucogyrophana & 9 & 1 \\
Meruliporia & 1 & 0 \\
Pseudomerulius & 1 & 0 \\
Serpula & 3 & 1 \\
\hline
\end{tabular}

The Myxomycetes are extremely common but have not been collected for serious study in the region. Species of Lycogala are quite common in sheltered moist places. Among the common Ascomycetes known here are Cookeinia (Sarcosomataceae) though the whole group has been poorly studied. Daldinia, Hypoxylon and Xylaria species (Xylariaceae) are also quite common but the species have not been identified with certainty. Among the Heterobasidiomyectes only the common species of the Auriculariales, in Auricularia, and the Dacrymycetales, such as the conspicuous Dacryopinax, are known and often collected. The other order, the Tremellales, has not been studied in this part of the world.

Studies on the Agaricales of the region have been conducted by Pegler (1982), Pegler and Piearce (1980), Piearce (1981a, 1987), Morris (1987) and Ryvarden et al (1994). Pegler's (1977, 1986), A Preliminary Agaric Flora of East Africa and Agaric Flora of Sri Lanka have many applicable keys to agarics of South Central Africa and have formed a basis on which to build local knowledge. Studies on agarics have been most intensive in Zambia. However, a thorough and systematic inventory of the whole group has not been undertaken. Among the notable species are the poisonous Chlorophyllum molybdites, which can be mistaken for some Agaricus or Macrolepiota species. Collybia aurea (Beeli) Pegler (Tricholonmataceae) is a tropical African species whose dense and clustered habit makes it easily recognisable. The Russullales have also not been studied in any great detail. A few notable species in the genera Lactarius and Russula are however, confined to this region. Lactarius kabansus Pegler \& Piearce is only known as yet from Zimbabwe and Zambia, in miombo woodlands. Perhaps the least studied group of macrofungi is the boletes which occur scattered throughout miombo woodlands. Among the poorly studied Aphyllophorales are resupinate species - the 'corticiums' including species in Hymenochaete (Hymenochaetaceae). The gasteromycetes too have been poorly documented, though some preliminary work was conducted in Zambia (Piearce 1981b). A notable species in the Lycoperdales (Broomeiaceae) is Broomeia congregata Berk. only known in Africa south of the Sahara, often close to Acacia tree species. Another distinctive fungus is Podaxis pistillaris (Linn.:Pers.) Fr. 
(Podaxales) a tropical and sub-tropical species, in Southern Africa found mostly on termite mounds.

\section{Taxonomic approach}

The biological species concept is the basis of taxonomy in fungi, though there has been much debate on this subject (Clemencon 1977, Parmasto 1985, Boidin 1986, Hallenberg 1988). Morphological and anatomical characters of the basidiocarp, type of rot, reaction with selected chemicals, host range and specialisation and distribution (mycogeography) are routinely used in taxonomy. The approach is simple, inexpensive and pragmatic under our conditions were a greater number of species are unknown. As the taxonomic species concept emphasises the importance of developmental criteria, this has necessitated the analysis of the basic characteristics of the individual. Cultural, biochemical, incompatibility and genomic studies, and phenetic and cladistic analyses have not been used for fungi of the region to any great extent. Such studies could solve some delimitation problems, and are especially useful in economically important species and genera (e.g. Armillaria). A broader consideration of taxonomic characters is often done in mycologically better known regions, where molecular studies have gained prominence; and phenetic and cladistic analyses yield most valuable information there. However, there is hardly need for competing taxonomic approaches - the classical and culture-based methods - rather they should be regarded as being complementary. Indeed, in several cases the modern methods have corroborated delimitations arrived at using the classical or "conventional" taxonomic methods.

Morphological characters are polymorphic and it is impossible to reveal convergence phenomena or parallel evolution from their analysis. Funalia leonina $\mathrm{Kl}$. is primarily separated from Trametes by the presence of a thick mat of hairs on the upper surface of the basidiocarp. The presence of this feature in the species might just be an adaptation to predation by insects. Similarly, Coriolopsis species are separated from Trametes species by the coloured vegetative hyphae, and Pycnoporus is recognised as a distinct genus because of the presence of cinnabarin. Flavodon flavus (K1.) Ryv. is separated from Irpex by its yellow colour, but is micromorphologically similar - also having simple-septate hyphae and encrusted cystidia. An in depth analysis of the species' cultural and biochemical characters might reveal their true affinities. Furthermore, the yellow colour often fades with age.

Only a few species are known in culture (11 500 strains out of an estimated 254 000 strains in herbaria around the world or $17 \%$ of known - and only $0.8 \%$ of estimated number of - species present in the world, see Hawksworth 1994a,b). The rapid disappearance of species due to deforestation and other causes has given priority to less tedious, but workable approaches to taxonomy, and to collection for ex-situ conservation purposes in general.

In recent collaborative studies on the genus Armillaria the limitations of the morphological species approach have been poignantly manifold. Various features (and methods) are now being used to differentiate the Armillaria species in culture, to complement classical methods (Table 5). 
Table 5. Methods used to group Armillaria species from tropical Africa.

1.Fruiting in culture

2.Observation of morphology of basidiocarps, conditions conducive to fruiting, frequency of fruiting, morphology of single-spore isolates

3. Pairing tests

4. Comparison of growth in culture at different temperatures

5. Comparison of subterranean rhizomorphs obtained in a mist case

6. Biochemical and molecular analysis of proteins: esterase, whole cell DNA by hybridisation of restriction digests with mtDNA and random amplified polymorphic DNA analysis

Source: Mohammed and Guillaumin (1994).

Basidiocarps of the fungus are rarely produced under our conditions (Masuka, 1994). Studies by the "Zimbabwe Armillaria Group", under the EEC Project on African

Armillaria coordinated by the Oxford Forestry Institute, focussed on growth features and biochemical characteristics of the fungus in culture (Mwenje and Ride 1994) and on species screening for resistance and nursery pathogenicity experiments.

\section{CONSTRAINTS}

\section{Priorities and funding}

There is a lack of adequate, assured and continuous funding, as taxonomic studies have been relegated from the priority list; and applied pathology/mycology, including biotechnology, have gained prominence. It is however, important to note that knowledge of biodiversity, the .raw material for biotechnologies for agricultural development' (Boussingeut 1991), (which is heavily dependent on taxonomic studies) is (still) poor and fragmentary. Among the reasons for the lack of taxonomic studies is perhaps the opinion that taxonomic studies have no commercial value or a place in applied research. The importance of these studies has been underplayed leading to a marginalisation of the subject, hence taxonomy has become peripheral to the fields of agricultural or forest pathology. There is competition with other fields for funding, in addition to status and credibility. Only three out of 18 papers presented at the First African Regional Mycology Conference in Mauritius in 1990 dealt with fungal taxonomy (Hennebert 1994).

Countries in the region are pursuing structural adjustment programmes. These have generally, led to a reduction in public expenditure. In any budget cuts research operations, by their nature, usually suffer first and most. The salaries budget for some research institutions already account for $70 \%$ of the operational budget, further reducing financial resources allocated to research work.

\section{Personnel}

There is a lack of qualified staff. Those that have training in taxonomy often conduct taxonomic studies on a part-time basis, as there is no full-time taxonomist in the region. There is also a lack of status, recognition and generally, inadequate career paths for researchers in developing countries, and taxonomists are no exception. At this symposium whose theme is "Tropical Mycology: quo vadis?" only two out of the seven speakers are from the tropics. The respective number of permanent scientists listed in the directory of African mycologists for Malawi, Zambia and 
Zimbabwe are only 1, 3 and 7 (Buyck and Hennebert 1994). Only Zimbabwe has the personnel capable of conducting systematic inventories of macrofungi.

\section{Literature}

There are no monographs for the region. One has to depend on literature from elsewhere. The shortage of relevant information on tropical species has made any pioneering study a frustrating experience. With regards to the polypores Ryvarden and Johannson's (1980) Preliminary Polypore Flora of East Africa provides a valuable starting point, so do Pegler's $(1978,1986)$ monographs of agarics.

\section{Facilities}

The facilities available do not allow for advanced methods to be employed, so studies employing molecular approaches to taxonomy (enzyme, DNA analysis) can only be conducted at Universities (there is a shortage of equipment there as well), and will probably remain being of an academic interest only for the foreseeable future.

In all three countries the macrofungi herbaria fall under different institutions: the Zambian collection is at the Forestry Research Headquarters (ca. 2000 specimens), in Malawi the collections (ca. 500 specimens) are at the National Herbarium and Botanic Garden, while those in Zimbabwe are at the University of Zimbabwe (on temporary transfer to the Forestry Commission, Forest Research Centre since 1989, ca. 3000 specimens).

\section{Methodology}

Our priority in the region is to collect and document species before they disappear. This calls for the morphological and anatomical species approach to taxonomy. In well studied regions, studies have now concentrated on elucidating relationships between taxa and solving delimitation problems. Sometimes our approach (morphological) to species delimitation appears parallel to the biological species concept, and problems have been encountered in conclusively identifying species described on the basis of the latter concept. The shortage of equipment to conduct the required further analyses compounds the problem.

\section{Communication and networking}

Because of the constraints imposed by a shortage of funding scientists in the region do not travel often enough to meet and share ideas with peers. This has often led to unnecessary duplication, thus retarding the pace of progress in some studies. Joint project proposals are rarely made with institutions in the region. Opportunities for networking have often come from exogenous factors, being spear-headed from institutions outside the region.

\section{WINDOWS OF OPPORTUNITY}

The rate of deforestation on a global scale is estimated at 15 million hectares a year (UNEP, 1987) with greater losses taking place in developing countries and at present rates of deforestation about $40 \%$ of remaining forest cover might be lost by the year 2000 (Organisation for Economic Cooperation and Development, 1985). The estimated rate of deforestation is over 250000 hectares annually in the region (Table 6). The causes are shifting cultivation, expansion of agriculture, cattle 
ranching, the growth of industrial mining, and timber and wood exploitation and rapid urbanisation. These are exacerbated by high population growth rates of up to $3 \%$ per annum.

During the past ten years the need to preserve (plant) diversity has been popularised culminating in the conniving of the Earth Summit (and Agenda 21) in Brazil in 1992. Funding for conservation efforts has been mobilised and much of this has targeted developing countries. However, the need for a holistic ecosystem management approach has also illuminated the need to incorporate other components.

Table 6. Deforestation rates in the region.

\begin{tabular}{lcc}
\hline Country & $\begin{array}{r}\text { Total Area } \\
\left(\mathrm{x} 1000 \mathrm{~km}^{2}\right)\end{array}$ & $\begin{array}{c}\text { Rate of Deforestation } \\
(\% \text { per annum })\end{array}$ \\
\hline Malawi & 9408 & 0.93 \\
Zambia & 74072 & 0.53 \\
Zimbabwe & 38667 & 0.45 \\
\hline
\end{tabular}

Source: Singh (1991)

Fungi are important components of forest ecosystems, recycling immobilised nutrients and, through symbiotic associations, directly contributing to the well-being of forests. However, at present rates of deforestation some species might even be lost before they are known. A window of opportunity has been opened for conserving and inventorying fungi, and to further study their ecology and biology. Indeed, more than ever before, there is now scope for assessing absolute, generic, ecological and mycogeographical diversity of fungi.

There is still a receptive audience (in both public and private institutions) willing to cooperate, collaborate and fund joint studies, and to assist in training developingcountry scientists. The opportunity must not be missed.

There are vast opportunities for studying the mycorrhizal species in miombo woodlands, for their contribution to plant growth, and for opportunities to create manufacturing and processing industries to satisfy local and international demand for mushrooms and mushroom products. These studies will gain prominence due to the current wave of interest in non-timber forest products arising from a new realisation that woodland management is an integral component of social forestry programmes in the region.

A variety of national and regional literature has been published on mushroom poisoning because the only sure way to know whether a mushroom is safe to eat is to be able to identify it in advance accurately and with certainty (from own and others experience and books). Mushroom poisoning will continue to be an important public health concern. This should result in more taxonomic and ecological studies on mushrooms and related fungi in the region.

The biotechnological applications of fungi will soon feature prominently in the region, in concert with developments in other parts of the world. There is a search for emtomopathogenic fungi for use against scale insects (Aspidoproctus sp.) which have decimated extensive areas of Brachystegia-dominated forests in Zimbabwe. Such studies will, undoubtedly, have taxonomic and ecological aspects - which should contribute to a better inventory of microfungi of the region. Some work might 
focus on elucidating the biochemistry of the curative properties of fungi such as Perenniporia mundula, for the academic and practical challenges it presents. The increasing use of genetically uniform crops and trees in monocultures could give rise to more disease problems. That should keep mycofloristic studies of this important group of fungi alive. In addition, biological control options in agriculture and forestry will gain prominence in line with the need to employ environmentally benign methods of pests and disease control. Finally, the search for secondary metabolites for use in pharmaceutical and other industries should spur mycofloristic studies in the region, as hitherto unknown or little explored habitats are thoroughly inventoried. Indeed, the diversity of tropical fungi has been recognised together with their potential use in biotechnology (Subramanian 1992), so an increase in their inventorying and methodic study is envisaged.

Herbaria must be regarded as assets, and studies in these could provide a basis for checklists, and indicate priority areas for further field collecting and documentation studies.

\section{STRATEGIES}

\section{Training}

There is need to build capacity for fungal inventorying, taxonomic and other studies. This can be accomplished by organising fungal identification training courses. The International Mycological Association (IMA), International Mycological Institute (IMI) and the Committee for the Development of Mycology in Africa (CODMA) are planning to organise a Regional Fungal Identification Training Course for Africans actively engaged in research in the fields of pathology and mycology during the early part of 1995 . Short courses and exchange visits foster the sharing of information sharing, so should be encouraged.

\section{Fungal inventorying}

Intensified collection and documentation studies should be accorded the highest priority, otherwise many species will disappear before they are collected. Little explored habitats must also be inventoried. A series of ecological studies are either in progress or shall be initiated soon.

\section{Literature}

Access to literature should be improved through increased subscription to journals. Severe foreign exchange problems and the high subscription rates militates against these efforts -ultimately only a handful of journals might be subscribed to and, even fewer may be regularly available. Electronic literature databases such as CD-ROM, Internet etc. should be provided to allow for rapid and accurate literature searches. However, to enable this funding will need to be sought.

\section{Mobilise, organise and educate}

There is need to sensitise and organise schools into amateur clubs; organise poster competitions in schools; create awareness and fungal conservation societies; involve the media (regarding mushrooms); and publicise fungi through stamp and other anniversary commemorations. The importance of fungi in the maintenance of ecosystem diversity through symbiosis and saprotrophic activities, in biotechnology, as food and medicines; and for aesthetic purposes, should be continually highlighted. 
Environmental impact assessment studies should have a fungal component. Mycologists should mobilise and organise themselves and others to lobby governments, public and private organisations for this component to be included wherever such studies are being conducted. The importance of fungi as bio-indicators of change (habitat disturbance, pollution etc.), if clearly articulated, should result in an increase in fungus-related environmental studies.

\section{Herbaria}

Herbaria should be publicised for their scientific, educational, conservation and other purposes. This might lead to more collections being undertaken by individuals and local societies. Presently herbaria are grossly underutilised. Herbaria are a repository of myco-diversity - analogous to seed centres and gene banks - and must be regarded and treated as assets.

\section{Documentation}

Mycologists in the region will need to devote a considerable proportion of their time to documentation studies. A checklist of macrofungi recorded in Zimbabwe is now being prepared. There are no pathology or mycology newsletters, nor journals in the region. The launching of the African Mycology and Biotechnology Journal in Cairo, Egypt, in 1992 should provide an outlet for relevant publications (and African perspectives). Exploratory work on starting a regional newsletter has just commenced.

\section{Cooperation, collaboration and networkin}

Cooperation, collaboration and networking (with individuals and institutions within and outside the region) are prerequisites for any meaningful progress to be achieved on mycofloristic studies of the region. Attendance at conferences, symposia, workshops and seminars should further provide important fora for the exchange of ideas and information. Meagre financial, human and physical resources can then be appropriately deployed resulting in a sharpening of research focus and rapid progress. Faster methods of communication are needed. International collaborative/cooperative fungal inventorying efforts should be initiated, but need to be clearly defined and well planned (in the wake of property rights) and, of necessity, should encompass and build up and equip national centres in the respective countries.

\section{CONCLUSION}

The success of strategies outlined above depends on the availability of funds; local, regional and international support; and perhaps most importantly, the whims and aspirations of scientists in the region. We must initiate intensive studies soon, and even if we do not achieve our goals, in the words of Robert Louis Stevenson, '...to travel hopefully is a better thing than to arrive, and the true success is to labour.'

\section{REFERENCES}

Alabi R.O. 1994. Mycology and Nigerian culture: past, present and future. In Aspects of African Mycology. Hennebert, G.L. ed., The First Regional Conference on African Mycology Mauritius, 1315 June, 1990 Proceedings, pp. 43-56.

Angus A. 1962-66. Annotated list of plant pests, diseases and fungi in Northern Rhodesia/Zambia. Parts 
1-7 and Supplement. Mount Makulu Research Station. Zambia.

Bels P.J. and Pataragetvit S. 1982. Edible mushrooms in Thailand cultivated by termites. In Tropical Mushrooms, Biological Nature and Cultivation Methods. Chang, S.T. and Quimio, T.H. eds. Chinese University Press, Hong Kong, pp. 445-461.

Boidin J. 1986. Incompatibility and the species concept in the saprobic Basidiomycotina. Mycotaxon 26: 309-336.

Boussingeut J. 1991. Problems of assessment of biodiversity. In The Biodiversity of Microorganisms and Invertebrates: Its role in sustainable agriculture. Hawksworth, D.L. ed., CASAFA Report Series 4. pp. 31-35.

Buyck B. and Hennebert, G.L. 1994. Directory of African Mycology. Centre Technique de Cooperation Agricole et Rurale (CTA) and Mycotheque de I'Universite Catholique de Louvain (MUCL).

Clemencon H. The species concept in Hymenomycetes. Bibliotheca Mycologica 61: 1-444.

Doidge E.M. 1950. The South African Fungi and Lichens to the end of 1945. Bothalia 5:1-1094.

Flegg P.J. 1981. Mushroom poisoning. The Central Africa Journal of Medicine 27: 125-129.

Gelfand M. and Harris C. 1983. Poisoning by Amanita pantherina - a description of two cases. The Central Africa Journal of Medicine 28: 159-163.

Ginns J. 1976. Corneromyces Gen. Nov., Spec. Nov. (Aphyllophorales, Coniophoraceae). Mycologia 58: 970-975.

Ginns J. 1978. Leucogyrophana (Aphyllophorales): Identification of species. Canadian Journal of Botany 56(16): 1953-1973.

Ginn, J. 1982. A monograph of the genus Coniophora. Opera Botanica 61: 1-61.

Gilbertson R.L. and Ryvarden L. 1987. North American Polypores. Megasporoporia-Wrightoporia. Fungiflora. Oslo.

Hallenberg N. 1988. Species delimitation in the Corticiaceae (Basidiomycetes). Mycotaxon 18: 303 - 313.

Hawksworth D.L. 1994a. The tropical fungal biota: census, pertinence, prophylaxis, and prognosis. In Aspects of Tropical Mycology. Isaac S., Frankland J.C., Watling R. and Walley A. eds., Cambridge University Press, pp. 266-293.

Hawksworth D.L. 1994b. Fungal biodiversity: a largely untapped resource. In Aspects of African Mycology. Hennebert G.L. ed., The First Regional Conference on African Mycology. Mauritius, 1315 June, 1990, Proceedings, 185 pp.

Hawksworth D.L. (1993) The relevance of mycology to development. The African Mycology and Biotechnology Journal 1: 1-11

Hennebert G.L. 1994. ed. Aspects of African Mycology. The First Regional Conference on African Mycology. Mauritius, 13-15 June, 1990, Proceedings

Holldoble B. and Wilson, E.O. 1990. The Ants. Cambridge, Massachusetts: Harvard University Press.

Khonga E.B. 1994. The status of Mycology in Malawi. In Aspects of African Mycology. Hennebert G.L. ed., The First Regional Conference on African Mycology. Mauritius, 13-15 June, 1990, Proceedings, pp. 35-42.

Masuka A.J. 1992a. Tropical wood-inhabiting macrofungi. In Methodological Problems in the Study of Fungus Flora, Course of Lammi Biological Research Station, University of Helsinki, Finland, 22-27 march, 1992. Mimeo 29pp.

Masuka A. J. 1992b. Aspects of the ecology of Aphyllophorales on pines and eucalypts in Zimbabwe. Kirkia 14(1): 125-144.

Masuka A.J. (1994) Armillaria occurrence and management in Zimbabwe. In

Johannsson M. and Stenlid, J. eds. Proceedings of the Eighth International Conference on Root and Butt Diseases, Sweden and Finland, 9-16 August, 1993. International Union of Forestry Research Organisations Working party S2.06.01, pp.388-392.

Masuka A.J. and Ryvarden, L. 1991. The Coniophoraceae in Pinus and Eucalyptus plantations in Zimbabwe. XIII AETFAT Congress, Zomba, Malawi.2-11 April, 1991. Typescript 7pp.

Masuka, A.J. and Ryvarden, L. 1992a. Aphyllophorales on Pinus and Eucalyptus in Zimbabwe. Mycotaxon 44(1): 243-250.

Masuka A.J. and Ryvarden L. 1992b. Importance of Aphyllophorales in pine and eucalypt plantations and anticipated problems on timber in use. In Forestry Research in Zimbabwe. Piearce G. D. and Shaw P. eds. Proceedings of the Anniversary Seminar, "Forestry Research Advances in Zimbabwe", Mutare 27-31 August, 1990, pp. 109-113.

Masuka A.J. and Ryvarden, L. 1993a. Two new polypores from Malawi. Mycologia Helvetica 5: 143-148.

Masuka, A.J. and Ryvarden L. 1993b. Parasitic macrofungi on indigenous trees in Zimbabwe. In The Ecology and management of Indigenous Forests in Zimbabwe. Piearce G.D. and Gumbo D. eds., Proceedings of an International Symposium, Victoria Falls, Zimbabwe, 27-29 July, 1992, pp. 375382.

Masuka A.J. and Ryvarden L. 1993c. Aphyllophorales (Basidiomycetes) of Zimbabwe. The African 
Mycology and Biotechnology Journal 1(1): 24-32.

Masuka A.J. and Nyoka B.I. 1994. Susceptibility of Eucalyptus grandis provenances to Ganoderma sculptrutum. European Journal of Forest Pathology.25: 65-72

Mohammed C. and Guillaumin J.J. 1994. Armillaria in tropical Africa. In Aspects of Tropical Mycology. Isaac S., Frankland J.C., Watling R. and Walley A. eds., Cambridge University Press, pp. 207-217.

Morris B. 1984. Macrofungi of Malawi: Some ethnobotanical notes. Bulletin of the British Mycological Society, 18: 48-57.

Morris B. 1987. Common Mushrooms of Malawi. Oslo. Fungiflora.

Mswaka A.Y. 1992. A mycofloristic survey of the Polyporaceae s.l. of the indigenous forests of Zimbabwe. M.Ph. Thesis. University of Zimbabwe.

Mwenje E. and Ride J.P. 1994. Characterisation of Zimbabwean Armillaria isolates by morphological features and isozymes analysis of cell wall degrading enzymes. In Proceedings of the Eighth International Conference on Root and Butt Diseases. Johannsson M. and Stenlid, J. eds., Sweden and Finland, 9-16 August, 1993. International Union of Forestry Research Organisations Working party S2.06.01, pp. 393-401.

Organisation for Economic Cooperation and Development. 1985. The state of the environment 1985. Organisation for Economic Cooperation and Development, Paris.

Parmasto E. 1985. The species concept in Hymenochaetaceae (Fungi, Hymenomycetes). Plant Science, 94: $369-380$

Pegler D.N. 1977. A preliminary agaric flora of East Africa. Kew Bulletin Additional Series. VI. HMSO, London.

Pegler D.N. 1982. Agaricoid and boletoid fungi (Basidiomycotina) from Malawi and Zambia. Kew Bulletin, 37: 255-271.

Pegler D.N. 1986. Agaric Flora of Sri Lanka. Kew Bulletin Additional Series. XII. HMSO, London

Pegler D.N. and Piearce G. D. 1980. The edible mushrooms of Zambia. Kew Bulletin, 35: 475-491.

Piearce G.D. 1981. Introduction to Zambia Wild Mushrooms. Forest Department Production. Zambia.

Piearce G.D. 1981b. Records and collections of gasteromycetes in Zambia. Zambia Museums Journal, 6: 100-111.

Piearce G.D. 1987. The genus Termitomyces in Zambia. The Mycologist 1: 111-116.

Robertson V.J. 1983. Mushroom toxins. Zimbabwe Science News.17: 161-163.

Rothwell A. 1982. A revised list of plant diseases occurring in Zimbabwe. Kirkia, 12: 233-348.

Ryvarden L. 1994. Tropical polypores. In Aspects of Tropical Mycology. Isaac S., Frankland J.C., Watling R. and Walley A. eds., Cambridge University Press, pp.150-170.

Ryvarden L. and Johannson I. 1980. A Preliminary Polypore Flora of East Africa. Fungiflora. Oslo.

Ryvarden L., Piearce G. D. and Masuka A. J. 1994. An Introduction to the Larger Fungi of South Central Africa. Baobab Books. Harare.

Sharp C. 1983. Edible and poisonous fungi in Zimbabwe. Zimbabwe Science News 17: 134-138.

Singh K.D. 1991. An overview of deforestation in tropical Africa, the present state of knowledge and knowledge gaps. Regional Workshop for the Deforestation and forest degradation assessment. Nairobi, Kenya, 25 November - 13 December, 1991. Typescript. 7 pp.

Standards Association of Zimbabwe 1990. Code of Practice for Timber Buildings. Standards Association of Zimbabwe 173. Harare.

Storrs A.E.G. and Piearce G.D. 1982. Don't Eat These. A guide to some local poisonous plants (Storrs) and poisonous mushrooms (Piearce). Forest Department Production, Zambia.

Subramanian C.V. 1992. Tropical mycology and biotechnology. Current Science, 63: 167-172.

United Nations Environment Programme 1987. The state of the world environment. United Nations Environment Programme Report No. L14/16. Nairobi.

Wild H. and Barbosa A. 1967. Vegetation map of the Flora Zambesiaca Area.Supplement to Flora Zambesiaca. Collins, Salisbury. 


\title{
STRATEGIES FOR THE DEVELOPMENT OF TROPICAL MYCOLOGY
}

\author{
A PEERALLY \\ Faculty of Science, University of Mauritius, Reduit, Mauritius
}

\section{Introduction}

The need to develop biosystematics generally and mycology specifically has been the subject of some pertinent interventions during the last five to six years (Hawksworth and Bisby 1988, Hawksworth 1991, Hawksworth 1993, Peerally 1994, Haskell 1994, Rossman 1994, Zedan 1994, Bennett 1994, Jones 1994). Earlier Subramanian $(1982,1985)$ had highlighted various manners by which mycology could promote development in the tropics. Subramanian (1986) observed that there was no organisation in South or South-East Asia which could help anyone in the identification of fungi important in agriculture, forestry, medicine, public health and industry. We could very much make a similar observation today as we approach 2000A.D. It must be said that the development of mycology is of relevance not only to the tropical countries but to all industrialized countries as well. Any strategies for mycological development inescapably have to take into account the synergistic interaction between developing and developed countries.

This paper will attempt to review briefly the relevance of mycology to development, the various efforts in the tropics and in the North aimed at promoting biosystematics and/or mycology. These interventions and efforts already incorporate recommendations for the development of mycology world-wide and what is now needed really is to seek funds to support mycology in a more integrated and organised manner.

\section{Relevance of Mycology to Development}

Hawksworth (1993) detailed out, prioritized and categorized the relevance of mycology to economic development with particular reference to the tropics and a summary of his ideas is as follows:

(a) Food Security

Diseases in the fields

Post-harvest losses

Disease diagnosis

Biocontrol

Quarantine

Chemical control

Plant breeding for disease resistance

Mycorrhizas

Edible fungi

Mycoprotein

Mycotoxicology

Fermented foods and beverages 
(b) Waste Utilization

Upgrading of wastes into animal feeds

Composting

Biodegradation for paper making

Recycling of organic wastes

Bioremediation

Limitation of organic pollutants

(c) Public Health

Food safety

Mycotoxins

Mycoses

Fungi in AIDS

Fungi in allergy

(d) Biotechnology

Fungi as an unevaluated resource

Pharmaceuticals

Industrial enzymes

Pesticidal properties

Fermentation technologies

Flavours, fragrances

Delignification

(e) Maintenance of Biodiversity

Fungi play an important role in the maintenance of biodiversity through the maintenance of ecosystem function. Thus fungi are useful to higher plants in mycorrhizas, fungi and fungal enzymes play a key role in the digestive tracts of many feeding insects and even in the stomach of ruminant mammals. Fungi are important in nutrient cycling and natural biodegradation. Other fungi and various lichens have roles as bioindicators of ecological continuity.

\section{Status of Mycology in Tropical Countries}

While a few tropical countries like Australia and South-Africa have attained at least the critical mass in terms of applying mycology to development, most other tropical countries significantly lag behind. Hawksworth in his keynote address at the Second African Regional Mycology Conference (Hawksworth 1993) said: "Mycology has an immense amount to contribute to key aspects of development, but the subject is rarely accorded the attention it merits in national programmes aimed at increased sustainability. In the Tropics, and less developed regions generally, this is particularly true. Yet it is in those very areas that mycologists are generally few in number, and have scant resources?"

All aspects of mycology need strengthening in tropical countries, especially in view of the various continua such as mycology-biotechnology, mycologyagriculture, mycology-human health, mycology-industry. Areas which need urgent attention are crop diseases, post-harvest losses, new biological control possibilities, mycorrhizas, food storage and mycotoxins, edible fungi, the microbiology of fermented foods and beverages, waste utilization, environmental bioremediation, 
fungi causing human diseases and the isolation of novel and desired strains. (Hawkswort 1993).

In terms of constraints the major problems are the scant human resources, information and reference collection resources and funding for research, training and development work in mycology, as well as the inadequacy of appropriate career structures in Universities and research institutions, government bodies and in the private sector.

The International Mycological Directory (Hall and Hawksworth, 1990) lists 96 mycological organisations and institutions in the North, compared to 23 in the South of which 11 are in Australia alone. Only Argentina, Australia, India, Mexico, and the Republic of China have mycological societies. No African country has its own mycological society. Most lack even basic check lists, only nine of 53 African countries have any mycological collections included in the latest edition of Index Herbariorum (Holmgren et al. 1990). Only five African countries have culture collections listed in the current World Directory of Culture Collections (Takishima et al. 1989). Checklists are virtually non-existent.

Hawksworth (1993) went on to say that mycologists in Africa are isolated, and spatially remote from other mycologists, the major work in African mycology continues to be authored by Europeans.

Although a few mycologists in Asia, Latin America and Africa have lived up to international standards in their contribution, the magnitude of mycological problems in the tropics remains totally out of proportion with the available resources. The most poorly equipped are Africa, Central America and the Caribbean.

The IMA Regional Committees for Africa, Asia and Latin America are attempting to popularize mycology in their respective regions, but the meagre financial resources at their disposal barely allow meaningful activities to be organised, except for the occasional regional conferences. Plans of action which have been worked out as part of the deliberations of regional meetings have not taken off in most cases due to problems of networking resulting from financial constraints.

\section{International and National Organisations/Institutions Promoting Mycology}

\section{Associations and Societies}

In spite of the strong linkage in the continuum mycology-biotechnology, mycology is a discipline which cannot muster the media coverage not the public or private funding as biotechnology. There are good signs however that mycology, which has a brighter future now than was felt say a decade ago, is becoming more organised and coordinated. The International Mycological Association and D. L. Hawksworth have contributed significantly to the popularisation of mycology. The International Mycological Congress, formerly held every 6 years or so, is now organised every four years. IMA Regional Committees in Europe, Asia, Latin America and Africa are actively having their own regional conferences and some other activities. Other subcommittees of the IMA have contributed significantly in rationalizing nomenclatural problems.

The International Society for Human and Animal Mycology (ISHAM) formed in 1954 encourages the practice and study of all aspects of medical and veterinary mycology, and had 842 members from 68 countries in 1990. It publishes the Journal of Medical and Veterinary Mycology. It holds regular international meetings and 
occasionally ad hoc meetings and workshops.

The International Society of Mushroom Science (ISMS) focuses on fungal biotechnology, edible fungi, macromycetes and holds regular international congresses, and symposia on specific topics.

Other international mycology organisations are the Mycology Division, International Union of Microbiological Societies with interests in biotechnology, medical and veterinary fungi, micromycetes, mycotoxins and yeasts; the International Association of Lichenology and the International Pythium Group. The recently established World Society of Mushroom Biology and Mushroom Products (WSMBMP) is interested in all aspects of edible and non-edible, poisonous macrocycetes including those having medicinal attributes.

\section{Institutes}

International Mycological Institute (IMI)

A major world centre of excellence for the systematics of filamentous fungi, it provides specialist support and associated services in all aspects of applied mycology, including information and training.

\section{Centraalbureau voor Schimmelcultures (CBS)}

An institute of the Netherlands Academy of Sciences, it deals with all aspects of mycology, provides information and identification services and organises training courses regularly.

\section{Mycotheque Université Catholique de Louvain (MUCL)}

MUCL is part of the Belgian Coordinated Collection of Microorganisms (BCCM) financed and coordinated by the Science Policy Office of Belgium. Besides being a reputable fungal reference centre, MUCL encompasses systematic and applied mycology. It also offers mycological services and customized training facilities.

\section{American Type Culture Collection (ATCC)}

Its primary aim is to acquire ex-type cultures of all named taxa and strains of scientific and commercial interests. It provides information and identification services. It organises training courses and workshops regularly.

\section{US National Fungus Collection (USDA)}

It is dedicated to the systematic study of plant pathogenic fungi, those useful in biological control and of plant quarantine significance. It has developed an important computerized information system for the identification of pathogenic fungi.

\section{Microbiological Resources Centres (MIRCEN)}

The Microbiological Resources Centres consist of a network of centres of excellence in both tropical and developed countries dedicated to the application of microbiology to development, and emphasize bacteria as well as fungi. It is one of the organisations dealing with mycology that are mainly funded through the UN system. MIRCEN has also contributed significantly to training of personnel in applied microbiology. 


\section{The Biodiversity Debate}

The realization that biological sciences, molecular biology and biotechnology in particular, might hold the key to economic explosion and sustainable development in the decades to come is, as we know, significantly behind the global debate about biodiversity. The risk of environmental degradation with consequential loss of this biodiversity has also been an important element.

According to Hawksworth (1993) as little as 5\% of the world's fungi have yet been described out of a mycobiota of some 1.5 million, of which the major part is found in the tropics. This mycobiota is a largely untapped resource in terms of biotechnology. Their exploitation for commercial applications has naturally raised the important question of intellectual property and patent rights. The 1992 Biodiversity Convention of Rio, signed by 153 countries, clearly establishes:

(a) the sovereignty of Governments over their biodiversity,

(b) the principle of poor countries having a claim on the international community to help meet some of the costs of conserving biodiversity for global benefit through such mechanism as the Global Environment Facility (GEF), and

(c) the need for mutually sharing the benefits accruing from intellectual property and genetic resources.

Hawksworth (1993) pointed out that the benefits from fungi and other biotic materials exploited in the Northern Hemisphere largely accrue to the companies found in the North. There is therefore a need for most tropical countries to themselves become involved in the isolation of novel and desired strains, and to regulate their supply to overseas companies under mutually agreed terms of payment and royalty (Hawksworth 1993)

\section{Some Recent Initiatives for the Promotion of Systematics and/or Mycology}

(1) The setting up of Regional Committees for the Development of Mycology by the International Mycological Association provides new opportunities to create greater awareness at the level of policy makers, industrialists, the scientific community and even in the public at large.

(2) The meeting organized in 1987 at the Royal Society by the Systematics Association on the occasion of its Golden Jubilee spearheaded a new vision about systematics. The opening paper (Hawksworth and Bisby 1988) entitled "Systematics: The Keystone of Biology" provided substantial proof of the indispensability of systematics to biology.

(3) The Convention of Biological Diversity and the emergence of the concept of sustainable development have had an important impact through the creation of a world realization on the need to study and conserve biodiversity.

(4) A study conducted by CABI at the request of ODA on the priorities of Biosystematics Research in Support of Biodiversity in Developing Countries with particular emphasis on microorganisms and invertebrates was completed in 1993 (Hawksworth and Ritchie 1993). The report made some very useful recommendations for the development of tropical systematics.

(5) The WEFSA (Workshop on the Ecological Foundation of Sustainable Agriculture) has been another critical thrust. WEFSA I (Hawksworth 1991) drew attention to the inadequate current capabilities in matters of systematics of 
microorganisms and WEFSA III (Hawksworth 1994) examined this issue in depth and suggested ways to alleviate them.

(6) The Darwin Initiative for the Survival of Species, announced by John Major in Rio, focuses attention on strategies for the monitoring of biodiversity and on conservation and sustainable development. The Darwin Fellowship Scheme which includes awards mycology, is already operational.

(7) BIONET - International established by CABI at its 1993 Biennial Review Conference is a network with a global dimension and currently deals with arthropods, nematodes and microorganisms. BIONET is composed of subregional networks or LOOPS (Jones, 1994). The LOOPS will pool together resources to facilitate the pursuit of biosystematics endeavour using local resources. The LOOPS will coordinate and obtain support from the Bionet Technical Secretariat and from the world's major consortium of biosystematics institutions (BIOCON).

UNDP has already agreed to provide catalytic funding to BIONET International. The first BIONET LOOP was established in December 1993 for the Caribbean subregion (CARINET) involving 12 countries of the region. Similar LOOPS are in the pipeline for India, Eastern and Southern Africa. BIONET has an International Consultative Group with Dr. M. S. Swaminathan as its Chairman. CABI was asked by its 33 member countries at the Review Conference (14-17 June 1993) to do its utmost to assist the implementation of BIONET International.

(8) The U.S. National fungus collection has compiled electronic data from about 500,000 fungal specimens including rusts, smuts, polypores, asexual fungi and now ascomycetes as well. This database is important in providing identification of agriculturally important fungi and is not only useful for primary identification services, but also for substantiating new records (Rossman 1994).

(9) In terms of biochemical and molecular biosystematics, molecular studies are beginning to reveal hidden taxonomic criteria, especially where confusion exists with complexes of sibling species where molecular analysis may be usefully applied as with the former Armillaria mellea complex. (Rossman 1994).

\section{Reckoning with Some Constraints}

In the current attempt to organize, rationalize and consolidate ourselves as a world mycological community with common practices and objectives, we have to engage in some kind of corporate planning and in so doing we cannot escape analysing both strengths and weaknesses.

The following quotations speak for themselves:

1. "The practices of taxonomy and identification services are perceived as wrapped in white coats, obscured by Latin words, clouded in mists of camphor and formaldehyde, or even pickled in alcohol. They are seen as the preserve of an elite, to some extent parasitic, epiphytic, or at best, saprophytic upon the resources of other activities. Can this image be changed?" (Bennett 1994)

2. "Taxonomy must become an extrovert activity and escape the isolation of a Petri dish of introversion. The full value of taxonomic studies can only be demonstrated as a vital link in a process that creates income or achieves wider objectives". (Bennett, 1994)

3. "As a world community we have not come close to satisfy the most basic needs for identification services and resources to agriculture". (Rossman 1994) 
4. "The identification and characterisation of important organisms depends directly on the state of systematic knowledge of those organisms. At present, for many groups of pest organisms, knowledge on which to base accurate identification and characterisation and an understanding of the relationships of target organisms to related species is lacking". (Rossman 1994)

5. "For both fungi and insects any attempt to identify agriculturally important organisms can be unsatisfying in that an accurate identification is difficult. Between $25 \%$ and $50 \%$ of the specimens submitted to the USDA systematic laboratories cannot be accurately identified to the species level". (Rossman 1994)

6. "Many of the organisms belong to large genera which have never been monographed so that identification to the species level is impossible". (Rossman 1994)

\section{The Strategies}

Hawksworth (1993) has clearly emphasized that prospects for developing mycology in the tropics are good provided the prerequisite organisation and planning can be effected by 1995 . These prospects arise mainly from the new opportunities available through current awareness and interest in biodiversity and sustainable development, and through the Biodiversity Action Plans as proposed under the aegis of the Global Environment Facility.

It is evident from the points raised so far that the mycological scene globally is fairly buoyant with prospects of a better future in the years to come. It has been repeatedly emphasized how mycology hooks up with sustainable development, agriculture, industry, biotechnology, human health, food security, maintenance of biodiversity. Mycology is not and should not be sold as an applied science only. Fundamental mycology is an important as applied mycology. Mycology has both its Petri dish introversion and its biotechnological extroversion.

Another point should be emphasized. In the international, global outlook of mycology, in contrast to areas like the materials and the electronic technologies and others which are mainly the affairs of industrialized nations, an industrially useful fungal strain, for example, can be found anywhere but we need to know its identity and unravel its practical use.

The consolidation of mycology is naturally a priority for the tropical regions, but strategies for the development of mycology in the tropics should not ignore the fact that expanding facilities, research, infrastructure and services in the North will be of enormous benefits to the tropics.

The main actors in strategies for mycological development are:

(1) The international societies and associations like the IMA, ISMS, IUBS, WSMBMP and their regional committees, if any.

(2) The centres of excellence like IMI, ATCC, CBS, MUCL, USDA.

(3) Networks like BIONET, MIRCEN.

(4) Mycological laboratories in the North and South.

(5) National, regional and international funding agencies.

Strategies for promoting tropical mycology will have as main ingredients:

(a) Improving expertise of existing human resources through appropriate training opportunities.

(b) Devising a Tropical Post Graduate Training Programme in Pure and Applied Mycology. 
(c) Creating critical masses through national and regional pooling together of resources in priority areas.

(d) Creating national and regional mycological societies.

(e) Funding existing international mycological associations to enable them and their regional committees to operate efficiently.

(f) Encouraging the development of tropical centres of fungal identification through North-South joint ventures. Hawksworth (1992) emphasized that both national and regional capabilities in identification infrastructure are essential to meet the needs of agricultural and environmental services.

(g) Establishing an international task force (call it Project Neo-Saccardo) for the urgent preparation of monographs on important groups of fungi and available in electronic form. This would be of very significant help to taxonomists and nontaxonomists world-wide. In fact Rossman (1994) made the extremely pertinent observation that, in order to ease the identification requirements of both small and large scale countries, we need: (a) a world-wide network of primary contacts well trained and with relevant information tools at hand, (b) a cadre of back-up experts who provide identification of unusual species, and conduct research in systematic and (c) a mechanism to upgrade, revise and modernize the identification tools provided to primary contacts.

(h) Assisting developing countries to set up culture collections at least on a regional basis.

(i) Encouraging the development of centres of excellence in mycology in the tropics.

(j) Harnessing national and international support for established networks like BIONET and MIRCEN.

(k) For the purpose of attracting better funding possibilities it might be desirable to organize workshops/training seminars on topics which extrovert mycology: e.g. Mycology and Human Health (WHO might fund), Mycology and Pollutant Bioremediation (UNEP would be interested), Mycology and Industrial Development (UNIDO might certainly be interested), Mycology and Biotechnology, Mycology and the Maintenance of Biodiversity, Mycology and AIDS, Mycology and Allergy, Mycology and Protein Production, Mycology and Animal Feeds, Mycology and Fermented Foods, and many others.

The collective wisdom of the world's leading mycologists has been prominently seen at work during the past ten years or so, and it has started to yield positive results in terms of reasonable success in mobilising the scientific community, policy makers, and international agencies. The MUCL Centenary Celebration which focuses so much attention on tropical mycology is ample proof of the determination of the world's mycology community to function for the common good of humanity.

\section{References}

Ainsworth A.M. and Hawksworth D.L. eds 1992. Biodiversity in the Caribbean. CAB International, Wallingford, U.K

Bennett A. 1994. A review of existing identification services and future needs. In The identification and Characterization of Pest Organisms. Hawksworth D. L. ed., CAB International, Wallingford, U.K. pp. $57-68$

Hall G.S. and Hawksworth D.L. eds 1990. International Mycological Directory. 2nd edition. CAB International, Wallingford, U.K. 
Haskell P.T. 1994. Are biosystematic services necessary, desirable and possible? In: The Identification and Characterization of Pest Organisms. Hawksworth D.L. ed.,CAB International, Wallingford, U.K. pp. 3-16.

Hawksworth D.L. ed. (1991). The biodiversity of microorganisms and invertebrates: Its role in sustainable agriculture. In The Identification and Characterization of Pest Organisms. CAB International, Wallingford, U.K.

Hawksworth D.L. 1993. The relevance of Mycology to development. The African Journal of Mycology and Biotechnology 1(1): 1-11.

Hawksworth D.L. ed. 1994. The Identification and Characterization of Pest Organisms. CAB International, Wallingford, U.K

Hawksworth D.L. and Bisby F. A., (1988). Systematics: The keystone of Biology. In Prospects in Systematics. Hawksworth D. L. ed.,Clarendon Press, Oxford.

Hawksworth D. L. and Ritchie J. M., eds. 1993. Biodiversity and biosystematic priorities. Microorganisms and invertebrates. CAB International, Wallington, U.K.

Holmgren P.K., Holmgren N.H. and Barnett L.C. 1990. Index Herbariorum. Part 1: The Herbaria of the World, 8th Edition. Regnum Vegatabile 120. New York: New York Botanical Garden.

Jones T. (1994). BIONET - International: A global network for biosystematics of arthropods, nematodes and microorganisms. In The Identification and Characterization of Pest Organisms. Hawskworth D.L. ed. CAB International, Wallingford, U.K. pp. 81-92.

Peerally A. 1994. Biodiversity: adding weight to the debate: Proceedings Seminar (1993) on Plant Genetic Resources: Towards a National Strategy for Mauritius. IBPGR, University of Mauritius, Ministry of Agriculture.

Rossman A.Y. 1994. The need for identification services in agriculture. In The Identification and Characterization of Pest Organisms. Hawskworth D.L. ed. CAB International, Wallingford, U.K. pp. 35-46.

Subramanian C.V. 1982. Tropical mycology: future needs and development. Current Science 51: 321-325.

Subramanian C.V. 1986. Foreward UNESCO Workshop on Progress on Applied Mycological Research in the Tropics, University of Singapore, May 1985. Proceedings of the Indian Academy of Sciences, Plant Science 96: 333-34.

Takishima Y., Shimura J., Udagawa Y. and Sugawara H. 1989. Guide to World Data Center on Microorganisms with a list of Culture Collections in the World. Saitama: World Data Center on Microorganisms.

Zedan H. 1994. Pest organisms: number, ecosystem impact and developing country needs. In The Identification and Characterization of Pest Organisms. Hawskworth D.L. ed. CAB International, Wallingford, U.K. pp. 17-34. 


\title{
THE ROLE OF THE MICROBIOLOGICAL RESOURCES CENTRES (MIRCENS) IN THE DEVELOPMENT OF MYCOLOGY IN THE TROPICS ${ }^{2}$
}

\author{
E.J. DA SILVA
}

\section{Introduction}

It is a pleasure to have the opportunity, albeit even in abstentia, to be associated with the Centenary Celebration of the MycothPque de l'UniversitÚ Catholique de Louvain. In this regards, UNESCO notes with satisfaction the herculean work carried out over several decades under the inspiring leadership of Professor G; Hennebert in building up this culture collection of fungi. While in the 1950's the collection maintained a few hundred cultures of fungi, reference to the classical World Directory of Collections of Microorganisms, produced in 1972 by John Wiley \& Sons, Inc., with the financial support of UNESCO, shows that MUCL then held 1183 species of fungi. Those were represented at the time by 14,500 cultures of fungi and 500 cultures of yeasts. Moreover, the interests of the collection were distinctly confined to agricultural and industrial mycology. Charging no fee for distributing and exchange of cultures, providing research qnd teaching services, MUCL had no published catelogue.

Twenty-two years later, the picture is different. From a one-time staff component of 1 , the total strength has grown to 9. Moreover, a fee is levied for the distribution and exchange of 24,000 cultures of fungi, including 2000 cultures of yeasts. Storage, identification, training and consultation services are provided in the management of culture collection, propagation, culture and preservation methods, and in quaratine and shipment regulations. Still again, the interests of the culture collection have grown. These range from agriculture, applied and industrial microbiology, fermentation, freeze drying, food science, forest microbiology, and biodeterioiation, to systematics and taxonomy.Finally, in addition to sponsorship by the University, there has been support from the Belgian Science Policy Office, and the then Commission of the European Communities (CEC).

This admirable yet painstaking evolution of MUCL, in just two decades, from its early humble existence into its present day acknowledged national and international stature and status, is but a powerful reminder of the efforts and time required for the development of such culture collections in the developing word. Furthermore, the obstacles and challenges, encountered by mycologists in different regions of the developing world, are virtually the same as those encountered by virologists, protozoologists, phycologists and even bacteriologists.

Although well established fungal culture collections do exist, e.g. the Centraalbureau voor Schimmelcultures in The Netherlands, the CAB International Mycological Institute in the U.K., and the Mycological Culture Collection in the USA, proposals for funding and setting up such collections have often received

\footnotetext{
2 .Presented by Jean Mouchacca, Laboratoire de Cryptogamie, Muséum National d'Histoire Naturalle, Rue Buffon 12, 75005, Paris, France. ${ }^{3}$ I agree with Barr (1983) that we are in need of a suitable term referring to fungi, in parallel to the terms "flora" and "fauna". However, from the two terms proposed by her, I consider "funga" as more suitable than "mycota" (preferred by Barr 1983: 8), because the latter term is also used as a suffix for naming taxa at the Division level.
} 
token support. The stark reality of the scarcity of such resources in times of economic repression and emerging competitive priorities such as environmental management or greening of the deserts is to be found every time a current culture collection changes status to that of being an endangered culture collection -- be it mycological, viral, protozoal or bacterial in content.

This is evident from the excellent initiative of the World Federation of Culture Collections (WFCC) in releasing "Living Resources for Biotechnology" which is a series of practical books that provide primary data and guides to sources for information on matters relating to the location and use of animal cells, bacteria, filamentous fungi and yeasts. It is worth noting that each volune contains specialized information together with material on general matters (information centres, patents, consumer services, and international coordination of culture collection activities) that is common to each group treated, i.e. animal cells, filamentous fungi, bacteria and yeasts. Moreover, each volume also provides valuable information on resource centres located world-wide.

Of late much concern has been voiced about the lack of attention given to these research centres, and by consequence to specialized areas such as mycology, protozoology, entomology, and the like. This concern has been eloquently articulated in the run-up to and the follow-up of the 1992 U.N. Conference on Environment and Development in the Rio de Janerio, Brazil (Hawskwoth and Ritchie, 1993).

In order to address the topic of the assignment, a brief review of the MIRCEN network appears to be useful.

The international support of culture collections -- the treasure houses of the planet's microbial (and, of course, mycological) genetic heritage, can be traced back to the early catalytic support provided by UNESCO, in 1946. Several of today's well-known culture collections have been beneficiaries of such support (Da Silva et al. 1977). Moreover, support was extended also to relevant publication such as the Internatinal Bulletin of Bacteriological Nomenclature and Taxonomy.

In 1962, UNESCO's Twelfth General Conference adopted a resolution by the Government of Japan to initiate and intensify research and training activities in microbial biotechnology in view of the growing domestication and use, at that time, of microbial resources in the sector of food, energy, industry, medicine, and agriculture. As a consequence, UNESCO, in 1963, initiated a series of GIAM conferences and in subsequent years, undertook joint collaborative activities with the Panel on Microbiology of the International Cell research Organizations (ICRO), the International Association for Microbiological Societies (now known as IUMS), the International Organisation for Biotechnoloy and Bioengeneering (IOBB), and the World Federation of Culture Collections (WFCC).

The 16th. Session of UNESCO's deliberations in 1970 were enriched by a resolution from the Governments of Denmark, Finland, Norway and Iceland calling for the establishment of specialized microbial research centres in developing countries. This foresight by these Governments, in its historic perspective, was the essence of what today is known as the MIRCEN network.

The origins of the MIRCEN network can be traced back to the early days of the UNESCO/IRCO Panel on Mircobiology. Established in 1965, the Panel was principally concerned with:

(i) the establishment of an international network for the preservation and exchange of cultures; 
(ii) the promotion of the use of micro-organisms as a natural resource; and,

(iii) the world-wide training of microbiologists (Porter 1974, Bull and Da Silva 1983).

Following the United Nations Conference on the Human Environment at Stocklohm, 1972, experts from UNEP, UNESCO, and the international scientific community, represented in ICRO, met in 1974 and 1975 at the UNEP Secretariat at Nairobi, to formulate jointly a world-wide programme aimed at the presevation of microbial gene pools and to make them accessible to developing countries through the establishment of a network of microbiological resources centres (MIRCENs). Subsequently, a UNEP/UNESCO/ICRO project titled "Development of an Integrated Programme in the Use and Preservation of Microbial Strains for Deployment in Environmental Management" (July 1975 - October 1984) was formulated and submitted by UNESCO/ICRO, and approved by UNEP for implementation. The objectives of the MIRCEN network are:

(i) to provide a global intrastructure which would incorporate national, regional, and inter-regional co-operating laboratories geared to the management, distribution, and utilization of the microbial gene pools;

(ii) to reinforce the conversation of micro-organisms, with emphasis on Rhizobium gene pools in developing countries with an agrarian base;

(iii) to foster the development of novel technologies native to specific regions;

(iv) to promote the economic and environmental applications of microbiology;

and,

(v) to serve as focal centres in the network for the training of manpoer and diffusion of microbiological knowledge.

The first development in the World Network of Microbiological Resource Centres was the development of the World Data Centre for Microorganisms (WDCM), in 1975, at the University of Queensland, Brisbane, Australia. The WDCM houses a master copy of the World Directory of Collections of Culture of Microorganisms and serves as a pivotal point for fostering development of culture collections in developing countries in an international cooperation (Da Silva 1986).

To help provide permanency and continuity to the work of the WDCM, the World Data Centre, has been relocated, from July, 1986 to the Life Sciences Research Information Section, RIKEN, Tokyo, Japan.

Today, the World Network of Microbial Resources Centres (MIRCENs) is comprised of the BNF and Biotechnology MIRCENs. Culture collections continue to occupy an integral role in the network and the emphasis is more on strengthening current assets rather than funding new ventures.

To return to the theme of mycology, it is evident that there is a distinct need for basic training especially at research sites and institutions in the developing world. Emphasis to date has been on specialized training at established institutions in the developed world. Important and valuable as training is, there needs to be a concerted effort that focuses on the awareness and beneficial inputs of the mycological sciences in improving the quality of life and by consequent the environment. Emphasis should be on responding to the needs for food, health, and energy coupled to the adaptation rather than the adoption of new skills and techniques for use by researchers in the developing word.

Fungal collection such as that of the Mycothèque de l'Université Catholique de Louvain and the IMI MIRCEN have important roles to play in taking the message into developing countries by organizing training courses and participating as 
"Professors" in these countries. Such pioneering roles help to create awareness in university administrators, help update curricula and syllabi, and help attract industrial support and participation. An example is the excellent contribution of Mycothèque de l'Université Catholique de Louvain in the development of a basic unit in the University of Burundi, Bujumbura, Burundi. The grass-roots approach has never failed. One of the best examples is the case of the study of mushrooms that has emerged on the international scientific scene as an important theme for international co-operation. In fact, these "mushroomologists" have their own society, their own computer network, their own newsletter, and their own programme of scientific activities.

In wishing the Organizers of this meeting every success, UNESCO, through its decentralized programme activities, and the Participation Programme for Member States, in which national culture collections in Bangladesh, Iran, and Paskistan have been strengthened, is ready to assist in the delivery of this message and would like, publically to thank all those scientists who have strengthen the bonds of international scientific co-operation.

\section{References}

Bull A. and Da Silva E.J. 1983. World networks for microbial technology. Society for General. Microbiology Quarterly. 10, 6-7.

Da Silva E.J. 1986. Culture Collections, a field for international cooperation. Symbiosis 2(1): 249-263.

Da Silva E.J., Burgers A.C.J. and Olembo R.J. 1977. UNEP-UNESCO and the international community if culture collections. Proc. Third Int. Conf. Culture Collections, Bombay, 1977, pp. 107-120.

Hawksworth D.L. and Ritchie J.M. 1993. Biodiversity and Biosystematic priorities: Microorganisms and invertebrates. CAB International, Wallingford. $70 \mathrm{pp}$.

Porter J.R. 1974) UNESCO/ICRO Panel on Microbiology. A.J.M. News 40:259-265. 


\section{DISCUSSION}

\section{FUNGAL TAXONOMY AND TROPICAL MYCOLOGY: QUO VADIS?}

Hennebert. It is now the time for questions and discussion. We heard this afternoon different options in approaching fungal taxonomy. In regard of the need developing tropical mycology, we may question what kind of approach is now suitable.

Dr Kurtzman and Dr Blackwell showed that molecular analysis can provide reasonable answers to problems left by the so-called classic - meaning morphologybased - taxonomy. But they made clear that such answers, if sometimes leading to definite nomenclatural changes in yeast taxonomy, are most often indicating new relationships between taxa that need further investigation before definite nomenclatural changes are made like in the Ascomycetes.

In another hand, Dr Rammeloo and Dr Roquebert pointed out the fact that the two century-old taxonomic framework of classification of the fungi is morphologybased, and that is more practical to build on it or revise it that to construct another one de novo. Dr Rammeloo also indicated the particular need for more precision, consistency and coherence in morphological criteria and their expression. Dr Roquebert showed promising perspectives in that direction with new types of microscopy that may diminish subjective interpretations.

For mycologists wanting to cover as soon as possible the largest part of the still unexplored and disappearing fungal diversity in mainly tropical areas, the question of methodology is surely justified. Mycologists in those areas have not so many facilities, as we heard from Dr Masuka and Dr Peerally. They are two taxonomic approaches to fungal diversity. Which of these two taxonomic approaches should they adopt? What option should we propose? Your opinions are welcome.

Webster. Let me ask Dr Kurtzman and Dr Blackwell if they feel that we are here approaching a finality in ascomycete classification whether we are moving to a stable state or we are moving to a phase that would become to develop much further.

Kurtzman. That is a very good question actually. I would like to think, on the basis of ribosomal RNA and rDNA sequence comparisons, that we are placing our systematics in a framework that would not change too much. And we have seen from comparing these molecules with other molecular sequences that they are giving pretty much the same outline. That is what we see now, and what we will see for some time.

Blackwell. I agree with Dr Kurtzman. Some people have expressed that they believe that some of these advances have been so fast that they have not been considered using ribosome genes, and it does not seem that other genes could do it. This may be pessimistic, but we have not studied enough taxa to know this. I think we are coming very soon to something quite stable for most of the Ascomycetes. Also I tend to be taxonomically very conservative, and I don't like to make taxonomic changes at this point to confuse the issue for a time.

Hennebert. At what level do you see this?

Blackwell. At the order level, for now, but later, at other levels. 
Hennebert. It seems that also morphologists are waiting to define orders within the Ascomycetes.

Blackwell. The things that we have been doing and in what we believe, is that in the Ophiostomales there has always been the same ravel. The genes we have done so far gave the same story. And if you sequence a gene that is evolving very quickly, it might be you will not get the same story. But, you will get a lot of noise that you may not be able to distinguish it. We are making gene trees. By doing that, we are making a lot of headeway from what has been made.

Hennebert. But doing such an approach is the classical taxonomy keeping some reference?

Blackwell. In my work certainly. I am a classical taxonomist, you know for thirty years. I am using morphology but it sometimes come to a certain point that I cannot decide what is right or wrong. Then I look for gene analysis possibly to provide an answer.

Prillinger. I think it is absolutely right that there is a necessary combination of classical taxonomy and systematics with molecular analysis, especially taking into account that there is in molecular biology a dependence on applied computer programs. Indeed it is possible that depending on the program we use we get different trees, so we have trees. Another aspect is that we need other characters, other methods to prove our results. It is therefore absolutely necessary for all people working on systematics not to use one method only either molecular analysis, or morphology, or anatomy, but to combine theses methods.

Hennebert. There are indeed many different programs giving different trees and neither the molecular biologists nor the fungal taxonomists know really how those programs are constructed, on the basis of what algorithms, and what is the significance and real value of these algorithms for our taxonomic purposes.

Perhaps, and that is not a joke, mycologists should be good computer scientists, and not only good biologists, and, I should add, good molecular biologists. Therefore, if things are such, the required training to become a mycologist should change and be much broader.

Whalley. I have a question for Meredith Blackwell. Meredith, do you remember the paper by Gowan and Vilgalys (1991, Am. J. Bot. 78:1603-1607). They looked at Xylaria magnoliae and they looked at the ribosomal length polymorphisms from different stromata taken from the same fruit and from the different fruits in the same region and then from different localities. And they got quite a lot of variations in their data. In fact they were getting more variations, I think, on the DNA material that they would on the classical morphological characters. The question I ask is how much molecular data do you need to be confident in the results?

Blackwell. It all depends. I do know the opposite problem too, with little rDNA variation within large specimen variability. If I consider the biology of the organism, questions are how is its dispersal, how it is getting here or there. If you count its 
population, is it really a population? And what is an effective population size? All these things ought to be known before being able to interpret the molecular data. Does it help?

Whalley. Thank you, Meredith. It does indicate that interpretation of molecular data must be done with great care.

Prillinger. I feel there is also different information from ribosomal DNA. If we look to a recent paper of John Taylor and Berbee, and to your paper of today, Mrs Blackwell, and to the paper of Dr Kurtzman, we have different interpretations of the Endomycetes. For John it is a side group of the Ascomycetes near the filamentous ones. In the Kurtzman paper it is a primitive group of fungi. What do we have to believe?

Blackwell. What I think of the Endomycetes? I think they are sister taxa to the filamentous Ascomycetes, do'nt you? It is somewhat deceptive when you have different arrangements from the tree made from different numbers of taxa. But I think now, looking with clean eyes, that, with the increasing number of species included in the trees, we see that the Endomycetales and the filamentous Ascomycetes are sister taxa.

Kurtzman. These are indeed not far away.

Blackwell. Dr Sugiyama what do you think?

Sugiyama. I agree with Dr Blackwell. From phylogenetic analysis of the nuclear small subunit rRNA (18S rRNA) sequence data, we found three major lineages within the Ascomycetes. their distinction supported by very strong bootstrap confidence. These are the Archiascomycetes (early Ascomycetes), the Hemiascomycetes (yeast Ascomycetes) and the Euascomycetes (filamentous Ascomycetes). The Archiascomycetes comprise Taphrina, Protomyces, Saitoella, Schizosaccharomyces and Pneumocystis. The Archiascomycete lineage diverged first among the ascomycetes, whereas the Hemiascomycetes and Euascomycetes as sister taxa evolved more recently.

Korf. I like to shift a little bit from phylogeny and relationships of organisms, which is indeed an example of the most exciting things that come out molecular work, to look, in fact, at "fungal taxonomy and tropical mycology: quo vadis?", as our topic today, specifically at biodiversity and particularly at biodiversity in the tropics.

The question I want to rise is how to go about training fungal taxonomists. That is mostly only done in temperate areas by people that have been trained in temperate areas also. My own experience with both temperate and tropical floras had led me to realize that we have that tremendous history of a development of morpho-anatomical systems primarily based upon temperate floras.

When I went to the tropics, I discovered something that made me change all my viewpoints upon how one has to train a mycologist and I always insist on, and force all my students to collect in the tropics. It is not only that the tropics are loaded with a lot of species, and we know from the biodiversity information that the number of species in the tropics may be usually in excess of that in the temperate areas, and 
that they have been vastly understudied, as has already been said several times today. What really strickes me as most amazing was that my generic concepts were immediately challenged the moment I moved to the tropics. I kept finding things that were neither this nor that, and when I hear of my colleagues here saying, we want to identify at least to genus, I do not know how you can, given the way current taxonomy is arranged. There are temperate genera. If you are going to work in the tropics you will have to find that you are going to have all new systems, you are going to have to develop them. And you are going to find lots of species that you cannot put names on.

Now, I spent all my life travelling all over the world convincing the dean of my College that I could not possibly study the fungi in Ithaca, New York, without going to the Canary Islands, to Africa, to Asia, Not true? I could spend all my life studying the fungi outside my door and I would still not have had all the answers because I don't know two-thirds of the fungi outside my door. But the tropics really change your perspective. And I am sorry for the fact that there are not many places in the tropics where taxonomy is being per persued today, and if it is going to be persued it has to be persued with the idea that the tropics have lots of unanswered questions, lots of things to be described, and that we indeed must go there and collect these things. Rammeloo, Masuka and Peerally have clearly shown that the traditional morpho-anatomic methods are what is going to have to be done if we want to document what is there, and to go and collect them in order to explore that tropical flora.

Where we are going to find the mycologists being trained to monograph large genera, or to find financial support for people producing large monographs? That is a big and unsolved problem. And I want to sound an alarm. An alarm that does not seem to have been voiced today. We must stress, no matter how exciting molecular techniques are, and they are exciting - and if I was a young student again, I am sure I would be a molecular taxonomist - they will not, and cannot, address the problem of biodiversity or cataloguing of our tropical floras.

In addition, classical mycologists are losing out in these areas. When I look about me, I see mycological taxonomic positions in numerous universities all over the world, in museums, being replaced by either nobody or by molecular taxonomists whose interests are not in producing monographs, or in cataloguing the tropical floras that we know so poorly.

I just wanted to point out my worries for the future of our understanding of biodiversity in the tropics.

(Applause.)

Webster. I truly share what Dr Korf has said.

Hughes. Grégoire, it was a surprise and a pleasure to be invited here. Yes, in order to survive the Ottawa winters, I have been a long time in the tropics. When I think of the tropics, I think of the tremendous leaf surface of the living leaves, and probably in these land areas where the leaves and the nutrient within are separated from the fungi outside by a thin cuticle only.

I stated as a novice at IMI in 1945. In 1946, Hansford's wonderful work on 'Foliicolous Ascomycetes and their parasites' appeared. The availability of this work and the riches in herbarium IMI, followed by a 3 month foray in Ghana in 1949 
further impressed upon me the enormous microfungus diversity even in the small area that I covered.

Flat leaf surfaces in the tropics support a vas array of Ascomycetes. For the most part theses have been assigned to well over 30 families in various orders. Some of the fungi are entirely superficial and comprise several families of the pleomorphic sooty moulds. Others are leaf parasites and, as Hansford has stressed, various devices have evolved to tap the nutrient resources of the leaf. These fungi can cause severe damage or may be benign parasites which cause minimal damge to the leaves, such as the Meliolaceae, Asterinaceae and several other families. A third group of common Ascomycetes comprises the mycoparasites of other foliicolous fungi.

But the numerous families of foliicolous Ascomycetes are by no means well defined. There is a great need of studies on the hyphal behaviour on leaf surface, perithecium ontogeny, development morphology of the type species as well as the identification of thei anamorphs. Such studies can be carried out adequately with ordinary microscope using current simple and straightforward techniques. We have much to learn about, and from these beautiful Ascomycetes.

Subramanian. I am entirely agreeing with much of what has been said here. I feel really happy that we are going to make great progress in the field of molecular taxonomy. But I think, for the developing countries, which don't have a complete inventory of their fungal resources, they have to rely on the ordinary light microscope and the simple tools which are at their command.

And, to begin up, the collections and the herbaria from those regions, which remain largely unexplored, have to be done by the people in these regions. Such a task needs, of course, cooperation and help which they can get from mycologists in the advanced countries.

Just to make an example. how pertinent observations can be made by ordinary light microscope, my good friend Stan Hughes here, his work in his 1953 paper on conidiogenesis and the classification of the Hyphomycetes was based entirely on observations under the ordinary light microscope. And so was it earlier with Vuillemin, Mason and other people. And we do know now how much of it has been confirmed by studies with better tools, like the transmission electron microscope, the scanning electron microscope and, maybe, studies on the biosynthesis of certain metabolites may also confirm some of these things.

The important thing to do, is to use the ordinary light microcope, the phase contrast microscope, in the best way, to study the fungi. They are so beautiful. They are everywhere. Professor Korf said there are so many new things in the tropics. Almost everything is new! But of course it is also interesting to see the old fellows when you collect. Some beautiful things also occur in the tropics that are in the temperate regions.

I think we ourselves must define our priorities in developing countries. And the first thing to do is the inventory of the fungi using simple tools which may be easy to get, the ordinary light microscope, and the literature. Getting past literature is more difficult. But there again, things are better now. Things were not the same when Saccardo compiled his Sylloge fungorum, we have better things today to come up with lists of fungi. So I thing the first priority is the inventory.

The second priority is to have monographs. This has been expressed already. 
I believe it is up to us, people of the developing countries, to do this job. And I am sure if we seek for cooperation of the mycologists in other countries, we will get it, as I got it when I started my work in mycology.

(Applause)

Parmasto. I wish to comment about the importance of collecting and use of tropical specimens.....

First, you make a data matrix, trying to make a system of the species you have classified using phenetic or cladistic methods. Then you are adding data and many other species and your cladogram is almost totally changing. That means that we not only have need to know more species of the tropics, but also more various collections of each species.

Secondly, if nowadays there are not many possibilities to use molecular methods in tropical countries, it is possible to collect specimens in such a way that they will be usable in future for such studies. That means that specimens must be collected and preserved in such a way that their DNA are not damaged, f. i. by chemicals, high temperature, or simply contaminations...As far as I know no one has been using well preserved spore print on cellophane for molecular studies. One spore is enough for sequencing. I hope that somebody shall do it and tell how it does work.

Third, it concerns molecular data as well as morphological ones. The molecular data are mainly based on morphological classification of the species. They are meant to check each other, inducing revision of the morphological classification, but also revisiting the exact value of the numerical treatment of sequences. Only after that process, they are to unite. It seems that it is not so important how to make the sequencing. Half of the work comes after sequencing, how to use the data. An seen on the posters, they are nice cladograms, made using neigbour-joining method. When using cladistic methods, instead of one cladogram, one shall get 3466 cladograms of equal length. That implies that training young taxonomists must include such courses as philosophy of sciences and methodology of sciences. We are a little bit too old, including me, to understand what to do with all the data we have, but let them have a little more "soil under their feet", a better background to understand what they do.

The last not the least, seventy to eighty percent of new fungus species are based on one collection. The percentage of one-specimen species has been very stable for the last forty years. As a result, description of such new species does not give any information about the variability of the species. Half of these new species may never been found later again. Because they are specimens but not real species, they are producing noise in data treatment. We should avoid that situation.

(Applause)

Masuka. Yes, it was good to get some corroboration on the most important things to do, that is to go out, to collect, to document and to make inventoies. That is priority for us. We accept, of course, what temperate scientists are saying on molecular taxonomy.

But wa are convinced that the priority is finally to compile check lists of the fungi. We know that check lists form the base for our studies. Then, of course we must do monographs.

An important thing that was hightlighted is that, as Professor Perally said, in our work agenda in developing countries we must seek for collaboration and cooperation 
with laboratories in the developed world. That has been so in the past and that must continue.

Arnold. I would like to indicate that only one existing system of the fungi is a complete one, the one of Saccardo, contructed on the base of all fungi known at his time. All the following systems are incomplete, as constructed on only a part of the fungi: Ascomycetes or Basidiomycetes or Fungi Imperfecti. If one wants to make a system on the basis of molecular data, one must study about 75000 existing species. You can imagine how many years such a work needs..

We have to combine all data on morphology, anatomy, biochemistry of these species with the data of molecular biology. This would make a system, may be almost natural.

Hawksworth. I feel the time has come to startt thinking in terms of different practices. This is an issue that has been brought up many times over the years, and initially by the mycologist J.H. Corner in 1946 who, having to cope with a tropical flora of not only the fungi but the trees that are unknown, decided to monograph the trees first, viz. the two volume 'Trees of Malaya'. Then he was able to continue work on the fungi, something he is still doing. Corner's way of operating, drawing carefully all that he saw, led him see things that others had not seen before, often because they were looking with temperate trained eyes and with preconceived ideas. A fresh look is very valuable, and a great deal of the work can be done locally.

We also have to consider making data available. Dr Huhtinen, at the First Intensive Workshop on Ascomycetes Systematics, in Paris last year, pointed out that also we have to think more about data capture, as well as trying to get fungi into culture close to the site of collection while the specimens are still fresh. With the resources we get, we can carry out the other work, make comprehensive drawings, look at all possible characters and determine ways to exchange these data electronically. We have been thinking about this at the IMI. This is also true in the insects. We can not think just about conventional publications, as the volume of data will be so great. In the case of insects, it has been estimated that something like 1-6 $\mathrm{km}$ of shelves would be needed to accommodate the literature on the Earth's insects at the rate of two pages per species. The task is absolutely enormous, so we have to develop new ways of working.

In the tropics, this means really developing a taxonomy as we go along. Dr Subramanian, Dr Korf, and others have mentioned today that a vast number of fungi are unknown. So it is not a matter of working with a key that exists, because we will find very quickly a huge number of genera, even of families, not documented in the keys. The same phenomenon arises in specialized habitats even in Europe, as some of you will know.

What I have been very pleased to see florishing in the IMA, is the regional committees. This is has been a noticeable development, especially in the African Committee, that we have been hearing about today: two major conferences have been held, a third one is being planned next year, together with a training course, a new journal has been launched, and an African Center of Biotechnology and Ecology in Cairo has recently managed to secure almost two millions dollars to actually set it up.

This move should be linked with other programmes that have been mentioned several times today. There are entomologists meeting at this very moment $20 \mathrm{~km}$ 
from here in Belgium looking at the development of BioNET INTERNATIONAL in Europe. BioNET is a concept that CAB INTERNATIONAl, as a peculiar intergovernmental organization mainly owned by developing countries, has developed in agreement with major institutions around the world, particularly in North America and in Europe, to establish a number of regional biosystematics loops which bring together the expertise in different regions. It is amazing how many specialists there are in now developing countries, but who don't necessarily know each other at all, being in different departments of a university or in different institutions.

This networking can be linked with a building up of collection resources locally, and there is money for this type of activity to be sought. I mentioned the year 1995 as a critical date for planning in mycology, in a paper I gave in 1992. That date still stands, as it will be the watershed as to how the funding of the Global Environment Facility will be used. The fund has a large amount of money available for biodiversity and related actions, with the 2.1 billion dollars so far pledged to this fund. The fund is being re-structured in November 1994. Mycologists need to think how we actually tackle that funding source. And because the fund scale is so huge, you cannot tackle it just as mycologists. We have to present ourselves as systematists, linking with other groups.

The major problem is to increase recognition that, in order to support the development and strengthen country capabilities, the training of people in the developing countries is visible. Yet it is up to them to work on their priorities and make their submissions for support. IMI has been fortunate in securing biodiversity related money under the Danish Initiative scheme, not just for mycology but including entomology and parasitology. Through this initiative, 22 fellowships for systematists with some training are already established in positions in developing countries and founded. Money is around if the right sort of proposalsis is made and if we think much more as biologists and systematists, rather than only as mycologists.

Demoulin. Just a small point, I wish would not be forgotten concerning tropical mycology. Is it true that we need to put inventory as a priority? There is also a thing, perhaps more experimental, but within reach of many laboratories, including those in developing countries, which is quite interesting. It is ecophysiology and autoecology of the species and of the strains and the study of the differences between the tropics and the temperate zone. Just to give an example, with a case that is studied in Papua New Guinea by several Belgian laboratories. We found the North-temperate Polyporus squamosus in Papua New Guinea. The species has a well defined North-temperate ecology concerning the optimum temperature. Although it is definitely not well adapted in that tropical area, it is still fruiting there. There are many similar cases that can be studied in an ordinary laboratory, with not much equipment.

Hennebert. I shall try to conclude in few words. It comes out the discussion particularly, that there is a priority on a classical approach of the large unexplored diversity of fungi in tropical countries. A priority and urgent duty is to describe and make the inventory of this mycoflora, using microscope and culture in situ, for which well trained young mycologists are needed. And on that basis, elaboration of monographs should provide the necessary tools to further investigations. 
This does not at all exclude research in molecular taxonomy, to solve taxonomic problems, afterwards. There are lots of problems arising every day in fungal taxonomy in classifying tropical fungi. As Dr Korf and Dr Hawksworth said, when collecting in tropical countries, we need almost to forget our temperate system of fungi and be open to construct new concepts. Taxonomy is an evolving field where revision is current.

Moleclar taxonomy does not supercede morpho-anatomical taxonomy. On the contrary, they are complementary to each other. Good phylogeny based on molecular data may demonstrate relationships that might lead one to amend the system of the fungi at the highest ranks. But the molecular approach has not opposed the species concept as defined by classical taxonomists as wrong.

Another issue from this discussion is the important need of a new training profile with collaboration from both tropical and temperate regions. That training should cover different disciplines, including computer science, cladistics, molecular biology, ecophysiology, without neglecting at all the traditional but unavoidable and basic methodologies of fungal taxonomy: collecting, microscopying, drawing, isolating fungi into pure culture, studying type materail, interpreting past literature, writing descriptions, constructing keys, writing monographs, building expert systems of identification and managing herbaria and culture collections. These are the first acts for a mycologist in tropical and unexplored areas of the world.

Thanks to all of you for your contribution. 


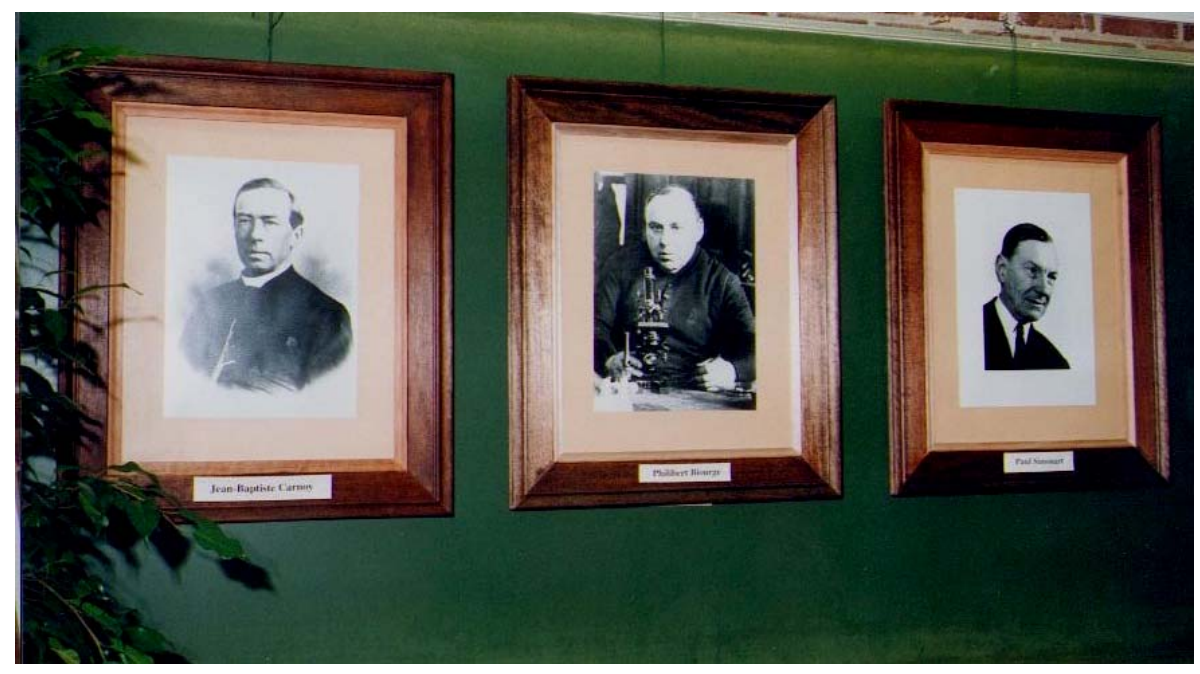

The founders of the Mycothèque MUCL:

Pr. Jean-Baptiste Carnoy (1894), Pr. Philibert Biourge (1894-1934), Pr. Paul Simonart (1934-1964).

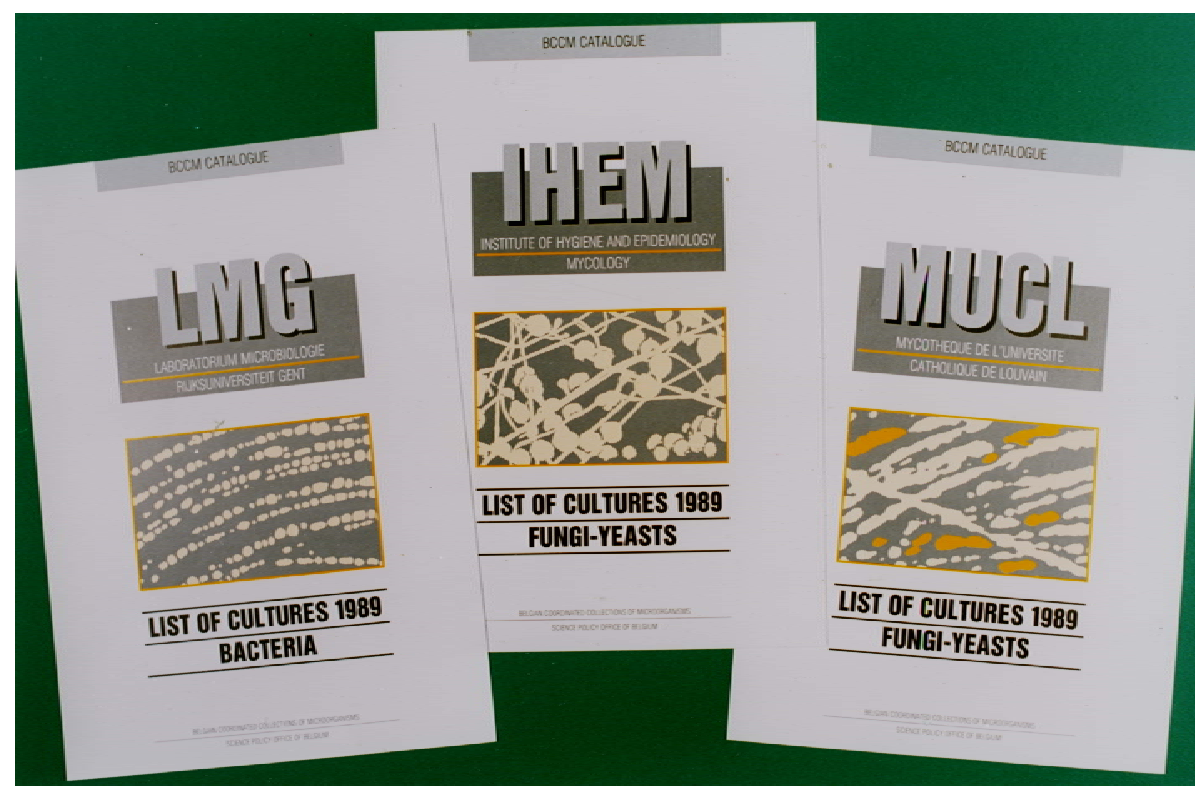

The first public catalogues of the three Belgian Collections of Microoganisms coordinated in the BCCM (1987) 


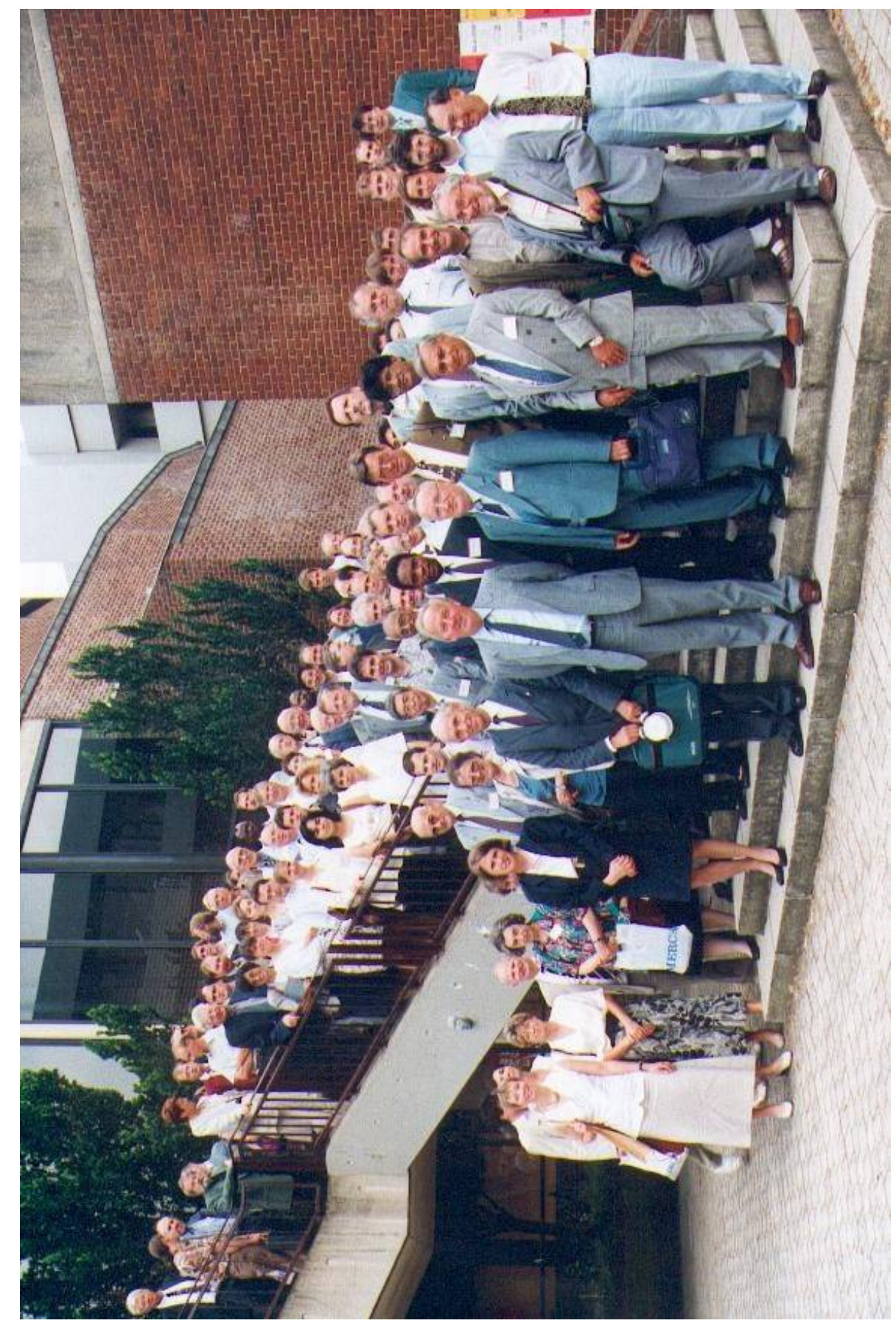



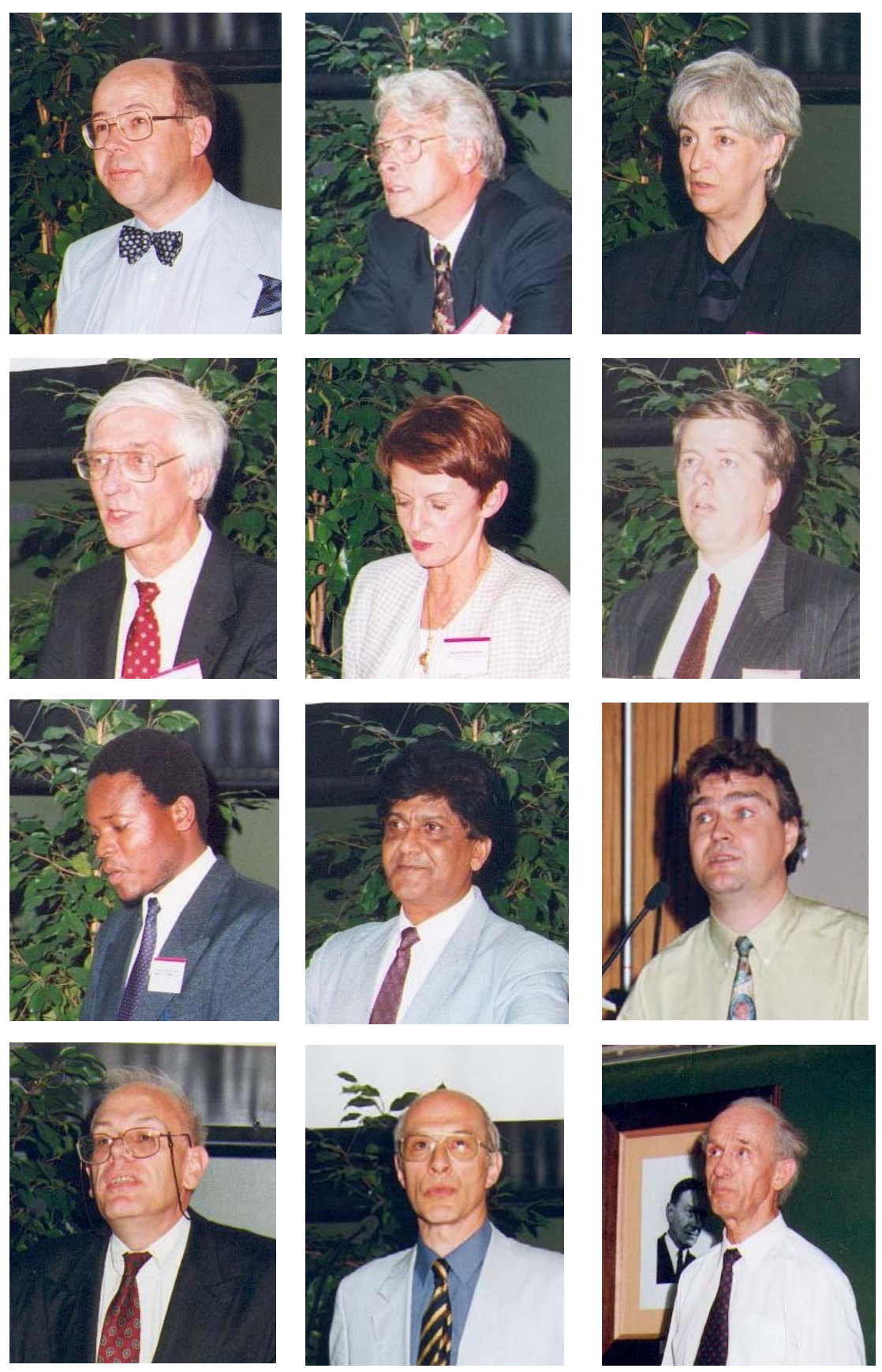

Pr. Drs D.L. Hawksworth (IMI, UK), D. van der Mei (CBS, NL), M. Blackwell (MSA, USA), C.P. Kurtzman (NRRL, USA), M.-F. Roquebert (CP, FR), J. Rammeloo (BR, BE), A.J. Masuka (FRC, Zimbabwe ), A. Peerally (MU, Mauritius), J. De Brabandere (SPPS, BE), P. Rouxhet (Prorecteur UCL), H. Naveau (Dept. Head, UCL) \& G.L. Hennebert (UCL) 

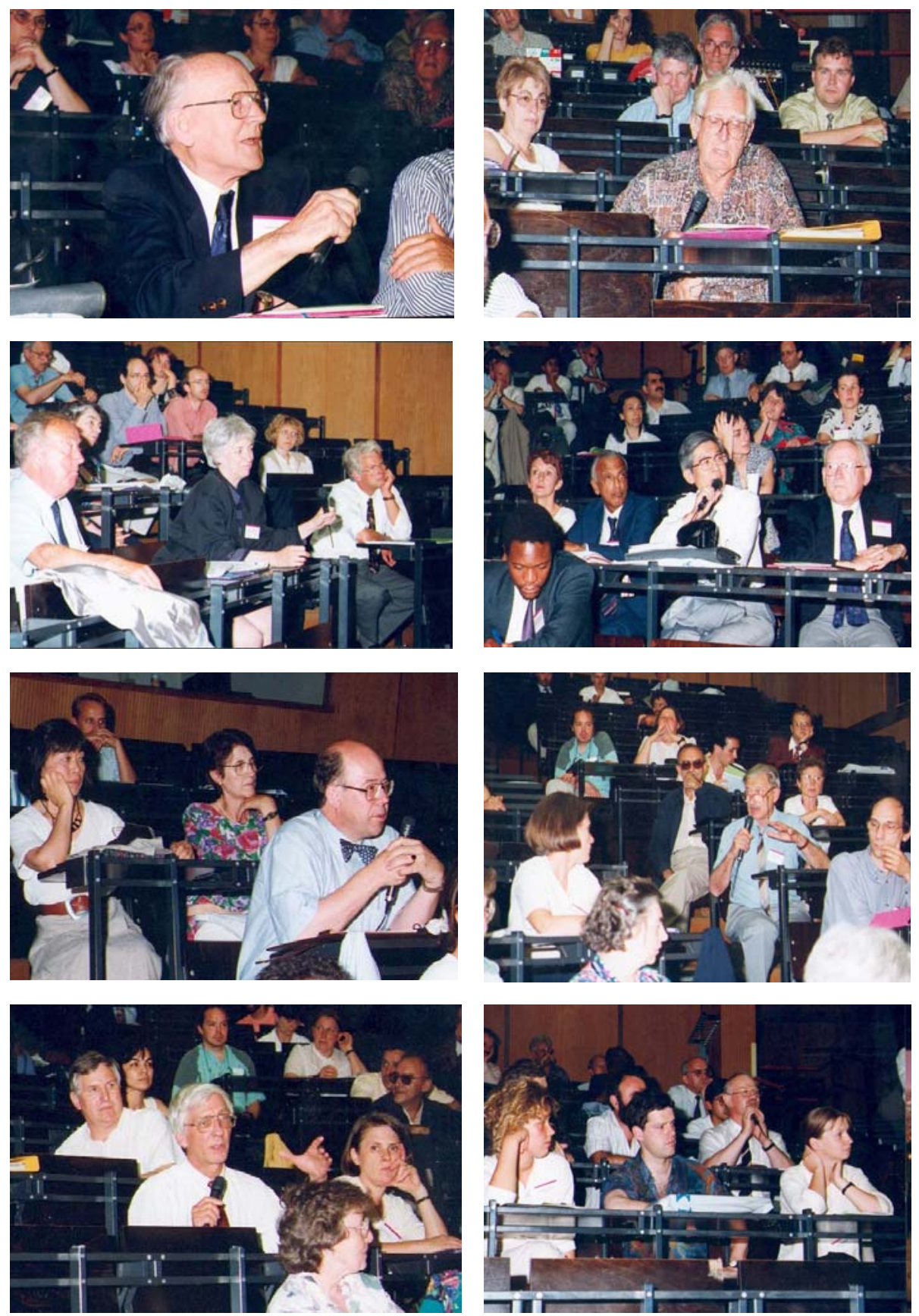

Drs (1) Hughes, (2) Cerisier, Kersters, Korf, Gams, De Brabandere, (3) Webster, Blackwell, van der Mei, (4) Masuka, Roquebert, Subramanian, Sugyama, Spencer

(5) Mrs Korf, Mrs Hennebert, Hawksworth, (6) Martini-Vaughan, Cimerman, Uruburu, Parmasto, Fraiture, (7) Maraîte, Kurtzman, Marson, Martini-Vaughan, Uruburu, (8) Verbeken, Walley. 

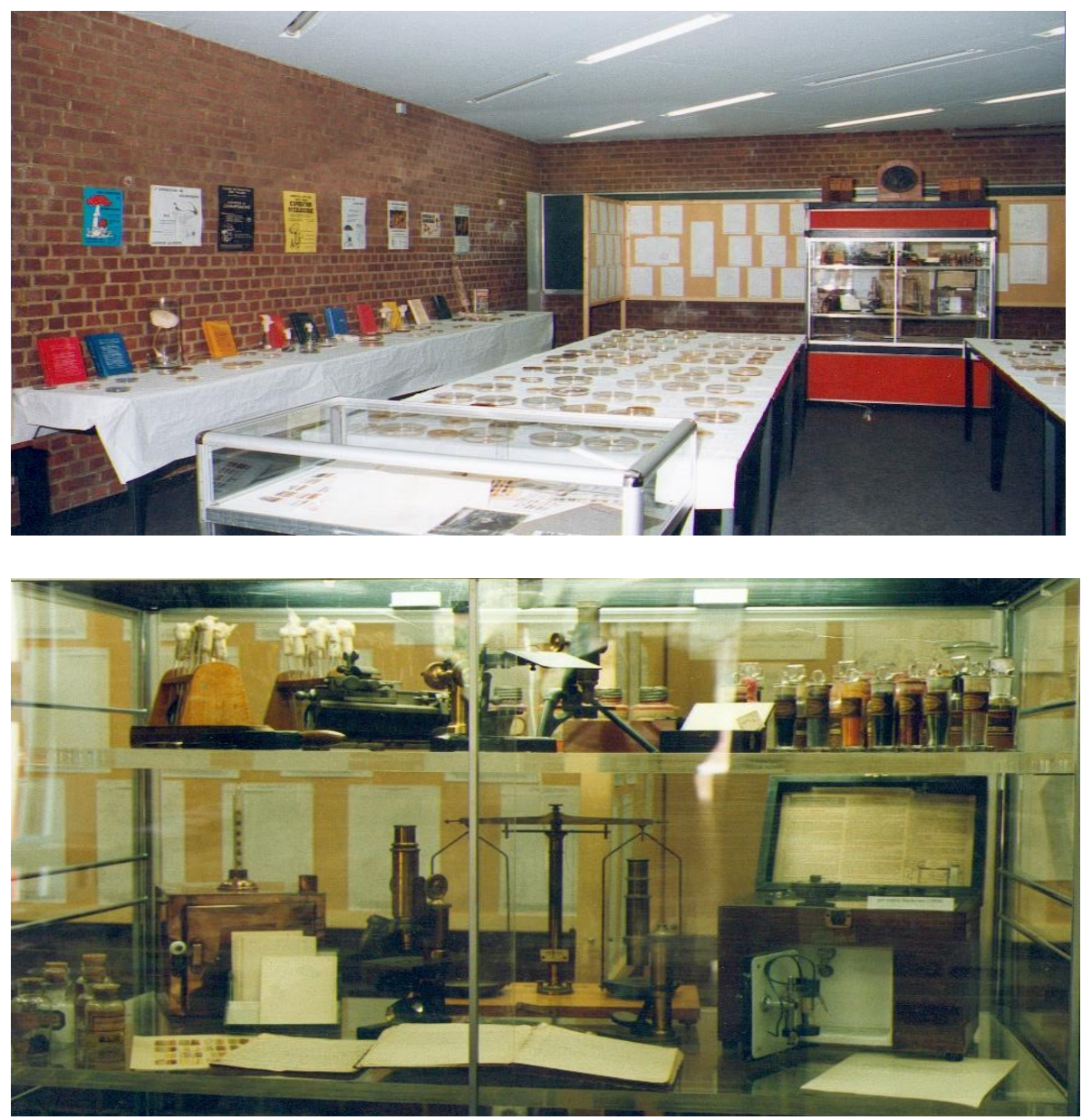

Fig. 1. View on the exhibition of cultures from three classes of fungi, original drawings of Hyphomycetes, catalogues of culture collections and publications.

Fig. 2. Ancient equipments and manuscripts from Pr. $\mathrm{Dr} \mathrm{Ph}$. Biourge.

Fig. 3. Evidence of the MUCL activity in applied mycology.

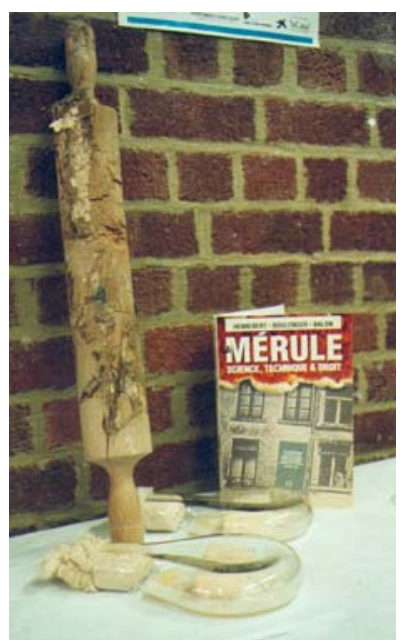



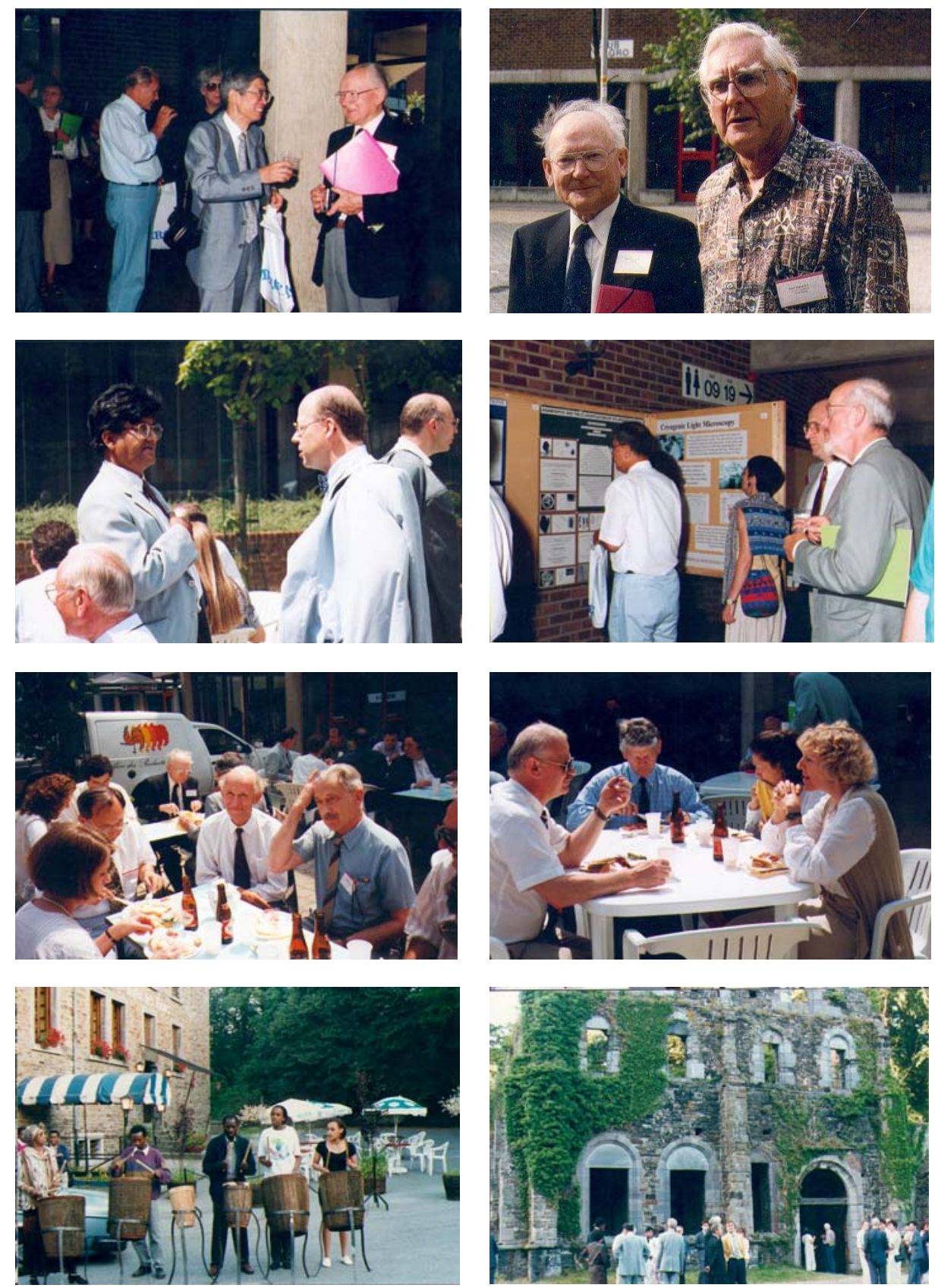

Openair lunch with breads, cheeses and beers from Wallony, at the Faculty in Louvain-la-Neuve: Figs. 1, Dr Sughyama, Dr.Hughes; 2, Dr Hughes, Dr Korf; 3, Dr Peeraly, Dr Hawksworth; 4, Dr. Vanderveken; 5, Dr Nguyen, Hennebert, Dr Parmasto; 6, Dr Walley, Dr. Kersters, Dr.Spencer.

7-8. African concert near the ruins of the Abbey of Villers-la-Ville 


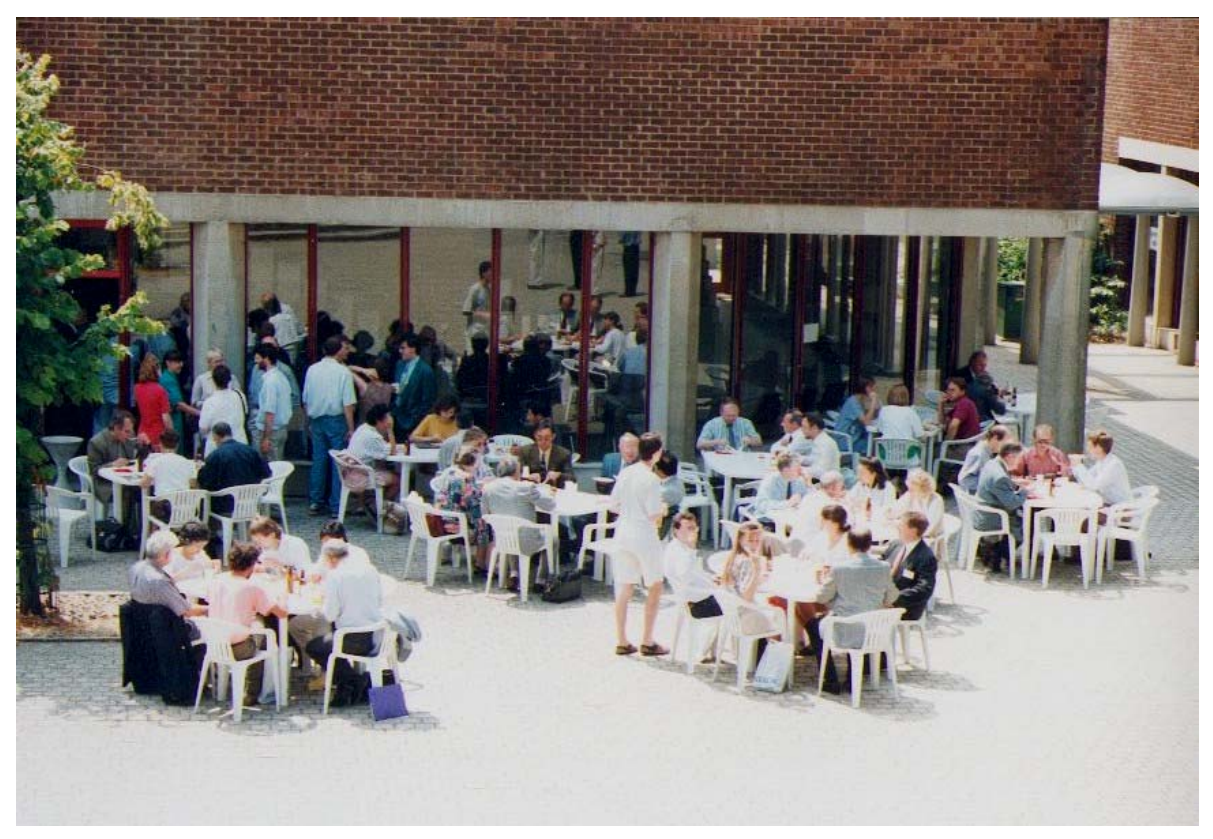

The lunch, at the Faculty in Louvain-la-Neuve

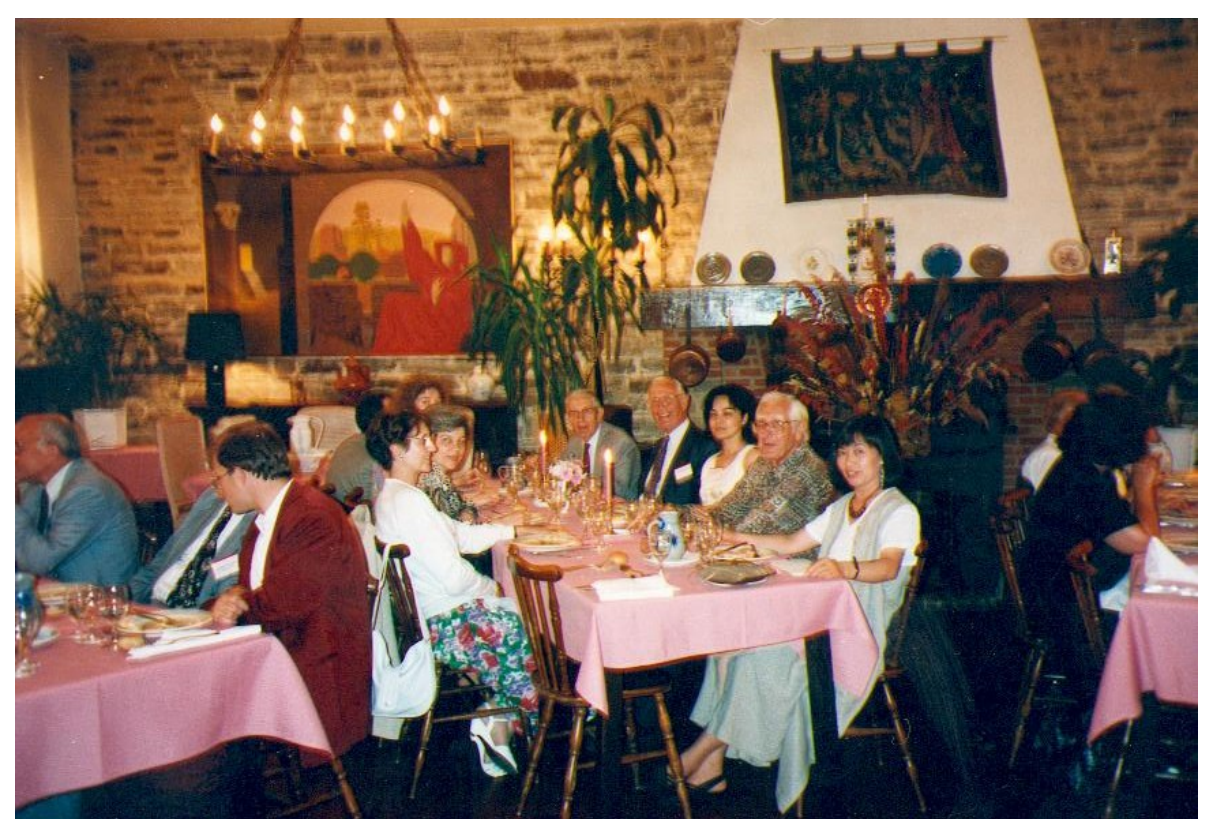

The diner, in the Abbey hôtel of Villers-la-Ville 


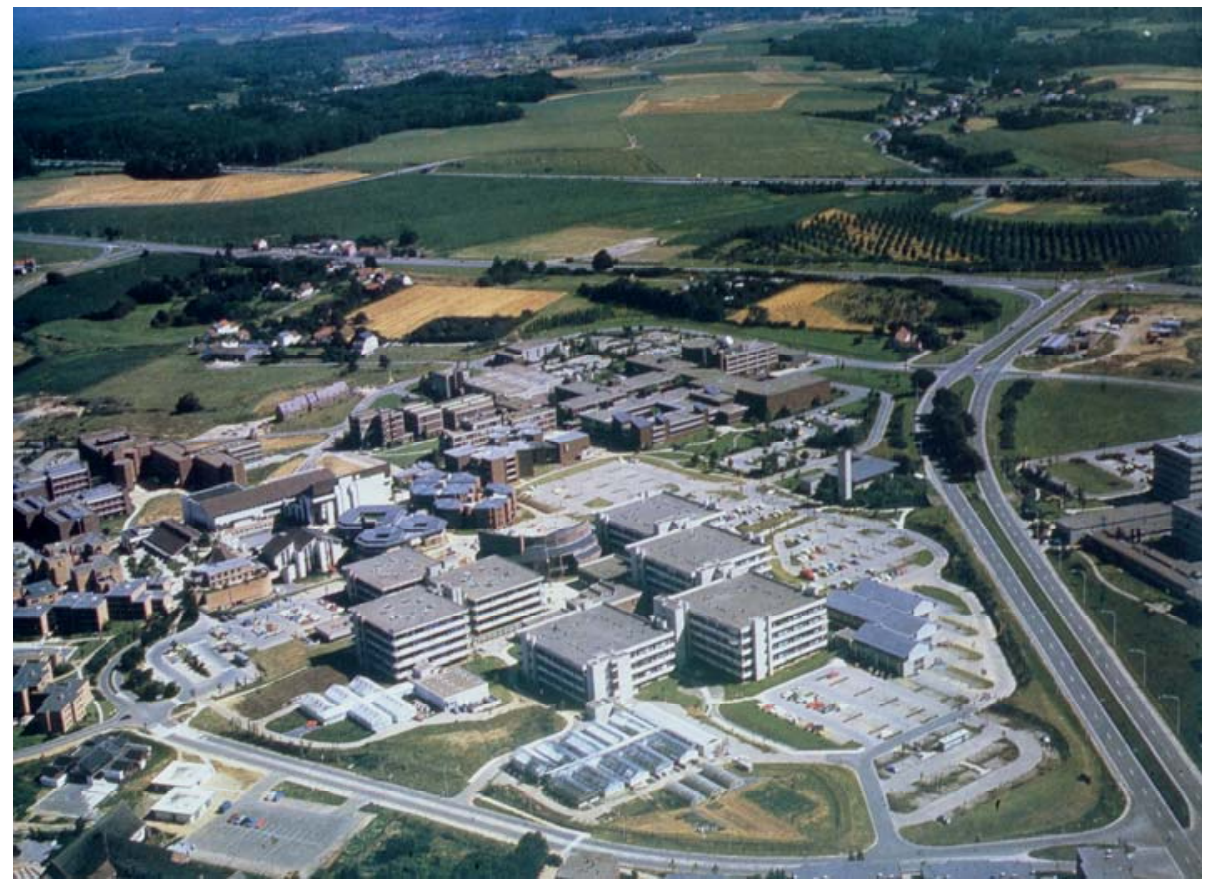

The Faculties of Agronomy and of Biological Sciences, Louvain-la-Neuve

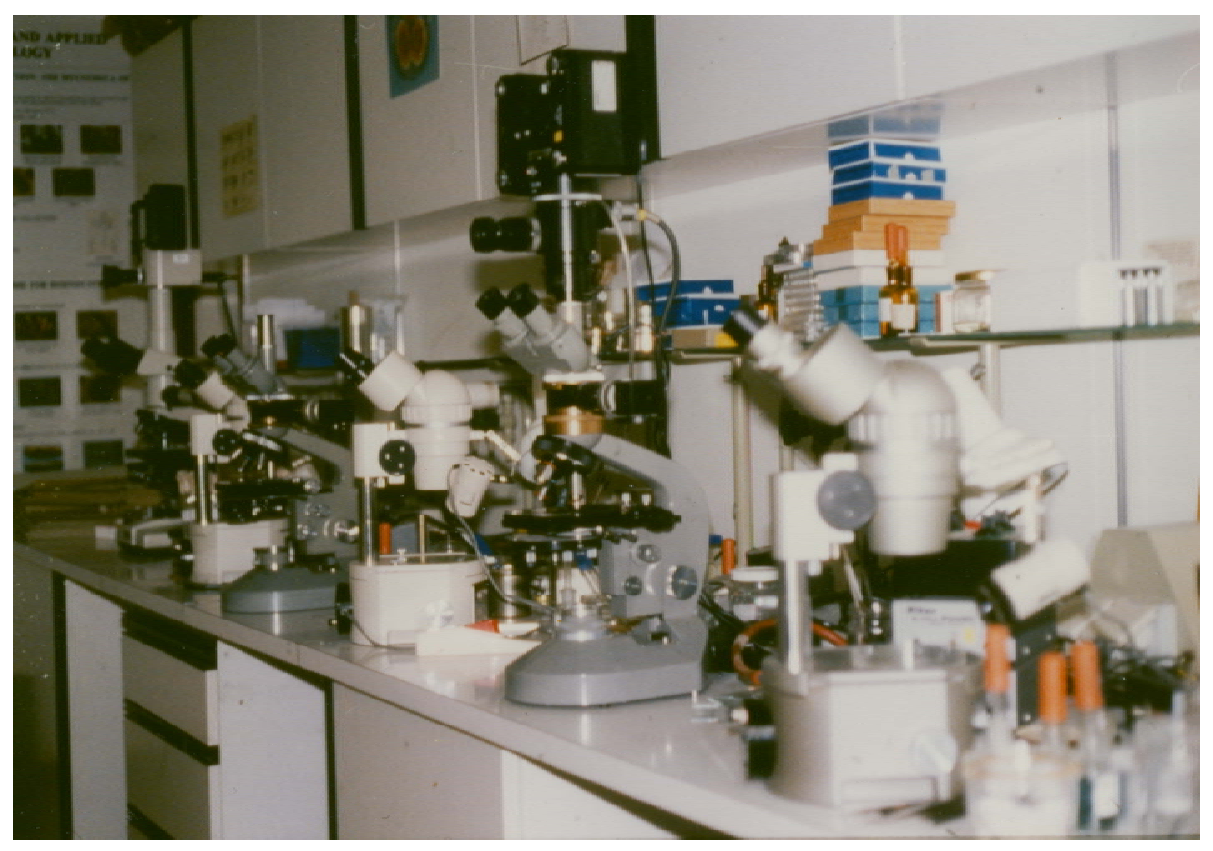

The Laboratory of Systematic and Applied Mycology (MUCL) 


\section{POSTERS}




\title{
FUNGUS COLLECTIONS AND SERVICES
}

\author{
THE MYCOTHEQUE OF THE UNIVERSITE CATHOLIQUE DE LOUVAIN \\ (MUCL) \\ 100 years 1894-1994 \\ GREGOIRE L. HENNEBERT
}

MUCL, 3, Place Croix du Sud, 1348 Louvain-la-Neuve, Belgium

The genealogy of the MUCL culture collection is presented. Under the pulse of Carnoy, Philibert Biourge inaugurated his young collection of yeasts, molds and bacteria in 1894. He left the hand to his young colleague Paul Simonart in 1938 Simonart named the collection "Mycothèque Philibert Biourge" in 1939. He maintained the collection untill 1969, when he passed it to the author.

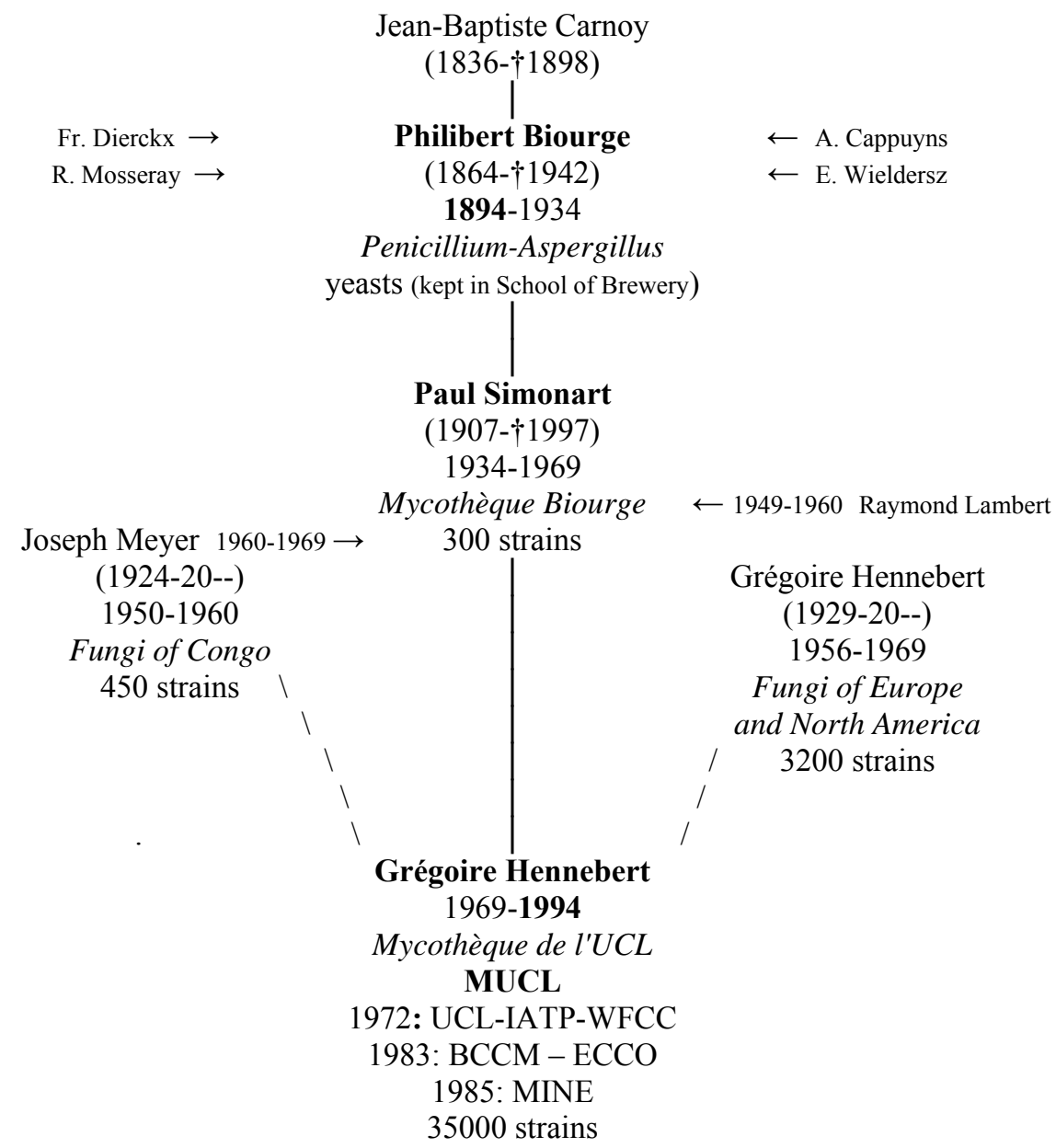


Hennebert integrated the Biourge's Collection with his own one into the new "Mycothèque de l'Université Catholique the Louvain" internationally recognized by the IAPT in 1969 ant the WFCC in 1972. MUCL, in 1983, became one of the two first collections of the Belgian Coordinated Collections of Microorganisms BCCM and a member of the European Culture Collection Organization ECCO. In 1985, it is a member of the Microbial information network, and in 1992, an International Depository Authority IDA under the Budapest Treaty.

The Collection made important contributions to fungal taxonomy (François Dierckx in Penicillium; Philibert Biourge in Penicillium; René Vandendries in Basidiomycetes; Raoul Mosseray in tropical Aspergillus, Paul Henrard in tropical Xylaria, Pr. Joseph Meyer in African soil Hyphomycetes, .... Others developed applied mycology and biotechnology: Ernest Wieldiers, the Bios production by yeast; Alphonse Cappuyns, the citric acid production by Aspergillus niger; Pr. Paul. Simonart: the discovery of the griseofulvin among metabolites of Penicillium griseofulvum; Pr. P. Simonart and Pr. R. Lambert, the quality control of dairy products and the invention of the milk aseptisation by ultracentrifugation. From its start, the collection has been oriented both to fungal taxonomy and to applied mycology.
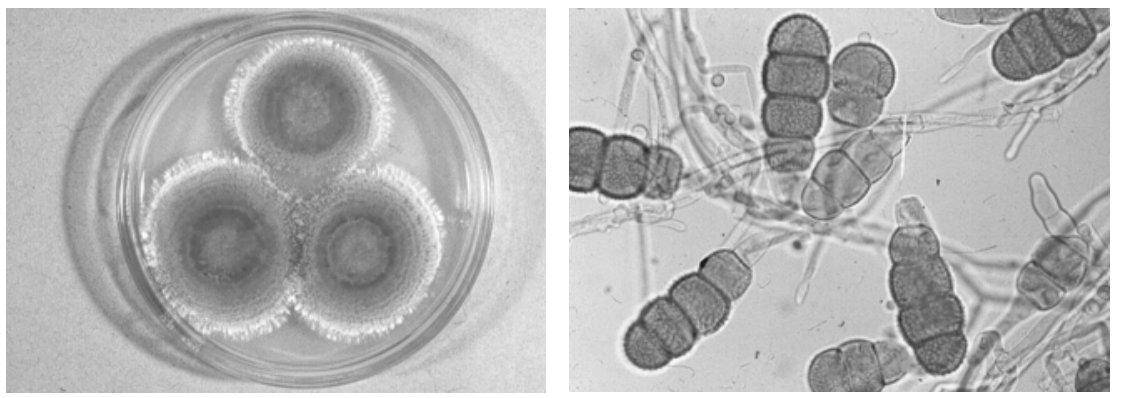

\title{
MUCL, a Public Service Collection
}

\author{
C. Decock, Ch. Moulliard, L. Nelissen, P. Massart, P. Evrard, N. Jamin, P. Charue and G.L. \\ HENNEBERT \\ Mycothèque de l'Université Catholique de Louvain, Unité de Microbiologie, Faculté des Sciences \\ Agronomiques, Place Croix du Sud 3, B-1348 Louvain-la-Neuve, Belgium
}

The MUCL culture collection is a partner of the Belgian Coordinated Collections of Microorganisms (BCCM), the four complementary collections of fungi, yeast, bacteria and plasmids in Belgium. Besides the mycological research, MUCL has developed many services for the scientific and industrial community. The Mycothèque MUCL is indeed recognized as a public service collection. Its main characteristics are the field collection, identification, isolation and preservation of a constantly increasing patrimony of fungal strains (yeast and fungi), of taxonomic, environmental, (agro)-industrial and biotechnological interest. 


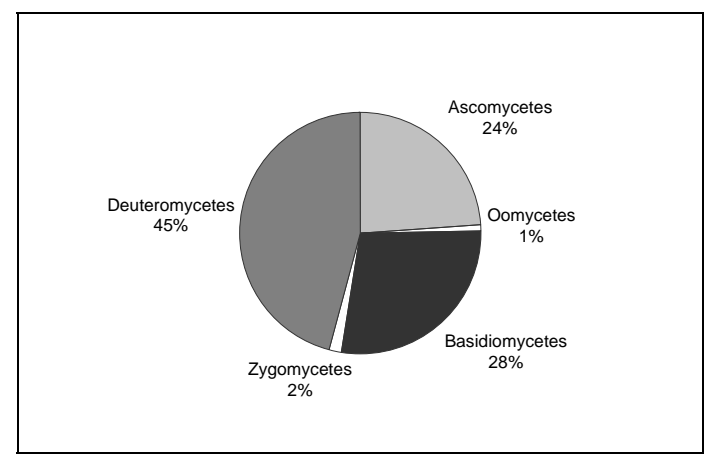

As public collectiopn, MUCL is aimed to offer many confidential services (Anomymous 1973, 1994) such as:

1) Identification of yeast and fungi by means of morphological, physiological, biochemical and gene-sequencing analysis. Isolation and identification of fungi from submitted products.

2) Material deterioration and material testing for resistance to fungicides and bioassays of fungicides according international standards.

Survey and expertise of fungal decay and mycoflora in buildings and industries, with recommendations of contrôl.

3) Fungal analysis of agricultural and industrial food products.

4) Screening and characterization of strains with particular properties, and creation of strains with specific enhanced property.

4) Distribution of strains. Biomass production;

5) Patent deposit under the Budapest Treaty. Confidential safe deposit of industrial strains. Deposit of strains for the MUCL public collection.

6) Training in general, taxonomic and applied mycology with special attention to undergraduate and gradute researchers from abroad.

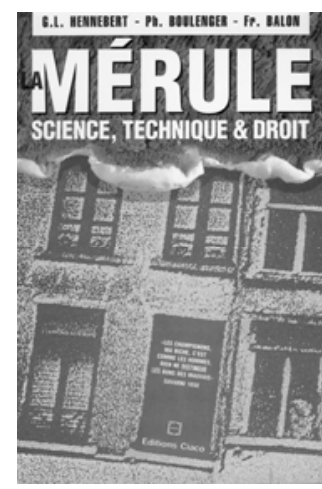

The MUCL collection currently holds thirty thousands of strains of filamentous and yeast fungi of all groups especially Zygomycetes, Ascomycetes, Hyphomycetes and Basidiomycetes, and includes taxonomically and biotechnologically important reference strains. Since 1992, the 2d edition of the MUCL catalogue containing 7300 strains of filamentous and yeast fungi is available on request at MUCL. A particular attention has been put on the incorporation of reference strains for industry, for identification and for bioassays and material testing.

Anonymous 1973. Laboratoire de Mycologie systémùatique et appliquée. Revue des Fermentations et Industries alimentaires 28(2): 80-81.

Anonymous 1994. La Mycothèque de l'UCL a cent ans. Champignons: un siècle de passion. UCL Quinzaine universitaire 36: 1.

P.S. In 1998, a third edition of the MUCL List of Cultures was issued with 8300 selected strains of fungi. 


\title{
MUCL, a Fungus Culture Collection, a service to African fundamental and applied mycology.
}

\author{
GREGOIRE L. HENNEBERT
}

MUCL, 3, Place Croix du Sud, 1348 Louvain-la-Neuve, Belgium

It is the aim of the MUCL fungus culture collection to make its expertise in fundamental taxonomy of fungi and in aspects of applied mycology available to serve African countries. Such a contribution could be offered by means of eveloped ence, exchange of materials, training and research assistance in situ in Africa, training and research periods at MUCL. Training and research grants are available from the Lomé agreements, by the GD VIII and GD XII of the ECC, by the National Agencies for Cooperation and Development of Switzerland and Canada, and by international arrangements for higher education and development such as Association des Universités Partiellement ou Entièrement de Langue Française AUPELF, Association des Universités Africaines, International Foundation of Science, International Development Research Center, United Nations University, World Bank, FAO, UNESCO and MIRCEM, and from Belgium by the General Agency for Cooperation and Development and by the Commission for International Cooperation of the Catholic University of Louvain.

MUCL has been collaborating with the University of Burundi, Faculty of Agricultural Sciences, Bujumbura, in establishing a Laboratory of Microbiology mainly oriented toward food microbiology.

During the last decade, the Laboratory of Microbiology LMUB (see photos) has carried out, owing national and international financial support, several projects for food sanitation and quality.
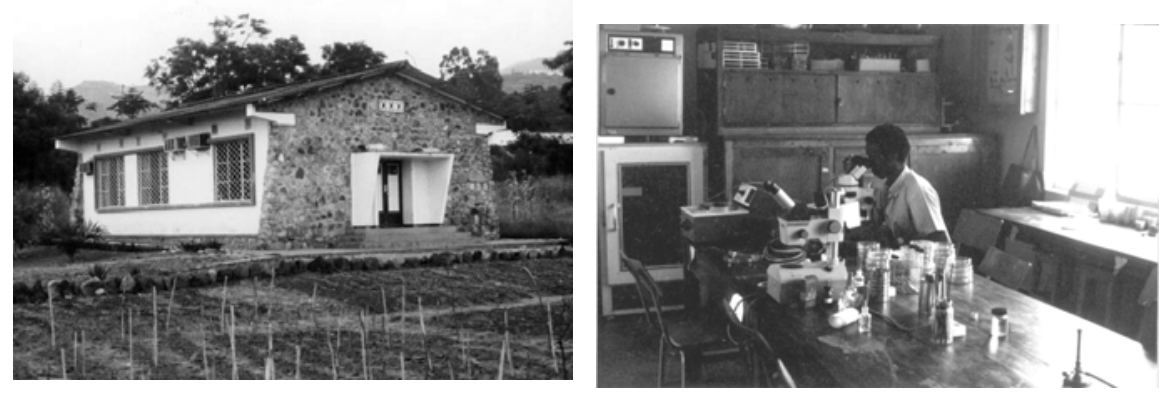

- Fungal deterioration of cereals and legumes in storage in Burundi (CEC)

- Improvement of storage conditions for cereals and legumes in Burundi (AGCD).

- Microbiological quality control of dried fish ndagala from Lake Tanganika (FED)

- Selection of an appropriate drying technology for optimal microbiological and sanitary quality of dried fish (UB)

- Quality control of indigenous and imported food products as a current service of the Laboratory LMUB since 1985.

MUCL is also eveloped research project in applied mycology for the Tropics, particularly on food production with different African countries. With Brazzaville and Matsoumba, Congo, MUCL investigated the solid fermentation of cassava by 
Rhizopus oryzae for its protein enrichment. With Guinea, Zaïre and Cameroon, diverse levels of collaboration, guidance, local experiments and $\mathrm{PhD}$ research, concerned the domestication of native species of edible mushrooms, the evaluation of local wastes as cultivation substrate and the improvement of yield and protein content by appropriate substrate composition (Tshinyangu 1994).

The production of mycorrhizae is another field of interest for the Tropics. Original mycorrhizogenic fungi, like Suillus cothurnatus from Perou, Pisolithus arhizus from different continents, are cultured and used in the mycorrhization of Pinus spp, both in laboratory experiments and in the field for reforestation.

Also MUCL provides skill and training in many aspects of fundamental and applied mycology. In fungal taxonomy, it offers training in isolating, identifying, describing and publishing local fungi. MUCL has contributed to the development of an African Culture Collection of fungi and yeasts at the Laboratory of Microbiology, University of Burundi, at Bujumbura. The Collection LMUB contains more than 800 strains. Duplicates are deposited in MUCL.

Robert et al. (1994) have set up an expert programme for the identification of yeasts, which has been elaborated and tested on a large collection of yeasts isolated by the first author from Burundi.

MUCL, for the I.M.A. International Committee for the Development of Mycology in Africa, and with the financial support of the Centre Technique de Coopération Agricole et Rurale ACP-CEE Convention de Lomé, has published the first Directory of African Mycology, a tool that should enhance mutual contacts and collaboration between African mycologists themselves and with non-African mycologists.

Ndenzako C. 1986. Les altérations fongiques du Sorgho en cours de stockage. Cas de la provinde de Kirundo, Burundi. Université du Burundi, Bujumbura, 143p.

Ngabonziza P. 1987. La croissance des moisissures de stockage et l'activité de l'eau. Le genre Aspergillus. Université du Burundi, Bujumbura, 59p.

Simbizi J. 1987. La contamination fongique du poisson séché au Burundi. Université du Burundi, Bujumbura, 68p.

Nyandwi A. 1987. Contamination fongique du Haricot avant et après la récolte: recherche sur la flore du stockage. Université du Burundi, Bujumbura, 110p.

Baert W. 1988. Valorisation de la Bagasse de Canne à sucre par la production de Pleurotes en Côte d'Ivoire. Fac. Sc. Agronomiques, UCL, 52p.

Tshinyangu K. 1994. Production et valeur alimentaire du Pleurotes en fonction du substrat. PhD thesis UCL, 255p.

Robert V.,De Bien J., Buyck B. and Hennebert G.L. 1994. ALLEV, a new program for computer-assisted identification of yeasts. Taxon, 43: 433-439.

Buyck B. and Hennebert G.L. 1991. Directory of African Mycology.MUCL-IMA-CTA publ. Louvain-laNeuve, 189p. 


\title{
THE ACTIVITIES AND MYCOLOGICAL COLLECTIONS OF THE BELGIAN NATIONAL BOTANiCAL GARDEN (BR)
}

\author{
ANDRÉ FRAITURE \\ Belgian National Botanical Garden (BR), Brussels
}

\section{The study of African Fungi}

The first works of African mycology published by the Botanical Garden were edited by É. De Wildeman and T. Durand, who were both directors of the National Botanical Garden in Brusseles (Bresadola and Saccardo, 1899; De Wildeman and Durand, 1901; De Wildeman 1905-1907; De Wildeman 1903-1912). The study is about collections which were carried out by various collectors (principally A. Dewèvre and H. Vanderyst) and identified by J. Bresadola, P. Hennings, P.-A. Saccardo, and H. and P. Sydow. This materiel consists of more than 450 taxons of which close to 400 are new to mycology.

The first mycologist from the Botanical Garden to have worked systematically on the collections of African fungi was Maurice Beeli (1879-1957), who worked there as a scientific collaborator for more than 30 years (Heinemann 1959). Although never having stayed in Africa, he worked extensively to develop the study of the mycoflora of this continent. Following on his advice, Mme Goossens-Fontana started collecteing from 1919 numerous specimens for the Botanical Garden, while also adding notes and watercolours (more than 900). This collection constitutes a remarkable source of knowledge about African fungi. Between 1920 and 1940, M. Beeli published close to 30 studies on the African fungi preserved in the Botanical Garden, 11 of which are recognized in the "Fungi Goossensiani".

The efforts of M. Beeli to highlight these collections succeeded in the publication of the "Iconographical Flora of Fungi from the Congo", the 17 volumes of which were published between 1935 and 1970. This flora was enlarged by the "Illustrated Flora of Central African Fungi", the 16th. volume of which is in the course of publication. It presents descriptions and identifcation keys, accompaigned by line drawings and colours prints, for differents groups of Basidiomycetes, Ascomycetes, and Myxomycetes. Several Belgian (P. Heinemann, M. Beeli, B. Buyck, J. Rammeloo, D. Thoen) and foreign mycologists (J. Boidin, E.J.H. Corner, R.W.G. Dennis, H. Dissing, R. Heim, E. Horak, M. Lange, M. Le Gal, R.A. Maas Geesteranus, D.N. Pegler, H. Romagnesi, R. Singer, R. Watling) collaborated in this work.

The presence of R. L. Steyaert (1905-1978) (Bienfait, 1979) at the Botanical Garden also needs mentioning. He conducted the greatest part of his career in Africa, then at the Commission for the Study of the Flora of the Belgian Congo and Ruanda-Urundi. He was interested in African phytopathology and studied primarily the genera Pestalotia, Monochaetia and Ganoderma. He left an abundance of material at the Botanical Garden.

\section{The Study of European Fungi}

From the second half of the last century, collaborators with and personnel of the Botanical Gardien became interested in the mycoflora of Belgium. É. Bommer (1832-1910) (Rousseau, 1910) and E. Rousseau ( \pm 1845-1926) gathered a great deal of materiel for the herbarium and published several floristic catalogues. Paul Nypels 
(1865-1909) was interested in species of harmful plants. Émile De Wildeman (18661947) (Robyns, 1948) carried out the cryptogamic part of the "Prodrome of the flora from Belgium" (De Wildeman 1898, 1899).

During the first half of this century, Beeli published several works and numerous notes concerning the mycoflora of Belgium. In the 1960's, Steyaert published several articles on the Ganoderma of the European flora.

The series "Icones Mycologicae" debuted in 1982. It presents high quality illustrations and detailed descriptions of various species of fungi. In this same year, 130 species, mostly European, were described in this way.

The distribution of various species of fungi was studied as well. The "Distributiones fungorum Belgii et Luxemburgi", the second volume which should appear in 1994, presents annotated distribution maps. Today, a cartography programme developed by A. Empain lets one produce distribution charts by computer.

Finally, the Laboratory conducts public service activities as well. This service consists mainly of identifying fungi and providing expertise about fungi which grow in homes. The Laboratory is also consulted by the Centre of Anti-Poisons when there are possible cases of mushroom poisoning.

\section{The Fungus Herbarium}

The Mycology Herbarium of the National Botanical Garden Botanique National consists of about 126,000 specimens. It is divided into three parts, according to the geographical source of the collection:

- the European Herbarium gathers together specimens collected in Europe and in the countries of the mediterranean bassin; it contains some 88,000 specimens;

- the African Herbarium is constituted of specimens collected in Afrique, south of the Sahara; and accounts for some 25,000 specimens;

- the General Herbarium unites the exsiccata coming from the other regions of the world, and contains about 13,000 specimens.

Among these specimens figure close to 2,500 types (1,100 for the African Herbarium, 900 for the European Herbarium and 500 for the General Herbarium). The Herbarium consists of type species created notably by the following mycologist: M. Beeli, J. Boidin, E. Bommer and E. Rousseau, H.F. Bonorden, G. Bresadola, B. Buyck, E.J.H. Corner, G. De Notaris, R.W.G. Dennis, H. Dissing, L. Fuckel, C.G. Hansford, R. Heim, P. Heinemann, P. Hennings, E. Horak, K. Kalchbrenner, P. Karsten, J. Komarov, M. Lange, M. Le Gal, M.A. Libert, R.A. Maas Geesteranus, Ém. Marchal, J. Moureau, V. Mouton, G. Passerini, C.H. Peck, D.N. Pegler, F. Petrak, J. Rammeloo, H. Romagnesi, H. Rehm, H. Riess, C. Roumeguère, L. Ryvarden, P.A. Saccardo, R. Singer, R.L. Steyaert, P. Sydow, C. Torrend, F. Von Thümen, R. Watling, G. Winter.

As well, the mycological herbarium contains several collections of watercolours representing fungi. These watercolours were produced by several artist-mycologists; the most important collections are those from Marthe Goosens-Fontana (African mycoflora) and Omer Van de Kerckhove (European mycoflora), but also those from M. Beeli, J. Bruylants, P. Heinemann, L. Imler, E. Klopfenstein, G. Malençon.

Also, the Laboratory possesses a collection of \pm 6.000 slides, in addition to numerous dossiers containing notes, descriptions, and optic- and electronmicroscope photographs, etc... 


\section{The Mycological Library}

The Library of the Belgian National Botanical Garden contains close to 2000 books on mycology, among those which happened to be originals from Ch. D. Badham, J.B. Barla, M.J. Berkeley, A.N. Berlese, É. Boudier, J. Bresadola, J.B.F. Bulliard, C. Clusius, M.C. Cooke, A.C.J. Corda, L. Forquignon, E.M. Fries, L. Fuckel, C.C. Gillet, R. Hesse, H. Hoffman, D.G.F. Hoffmann, K. Kalchbrenner, P.A. Karsten, J. Kickx, P. Kummer, E. Lambotte, C. Linnaeus, N. Patouillard, M. Paulet, C.H. Persoon, L. Quélet, P.A. Saccardo, J.C. Schaeffer, J. Schroeter, L. Secretan, C. Spegazzini, L.-R. \& C. Tulasne, G. Van Sterbeeck, D. Viviani, G.D. Westendorp, O. Wunsche. The library also contains a collection of 100 periodicals that are strictly mycological, al though not all sets are complete, as well as numerous other public periodicals that sporadically contain articles on mycology. Finally, there is also a collection off-prints.

\section{References}

Bienfait A. 1979. René Léopold Steyaert (1905-1978). Bulletin of theNational Botanical Garden of Belgium 49(1/2): 3-9.

Bresadola J. and Saccardo P.-A. 1899. Fungi congoenses. Bulletin of the Royal Botanical Society of Belgium 38(2):152-168 + 5 pl. [Collection of Alfr. Dewèvre, 87 taxons, 17 which are new; De Wildeman and Durand 1901 made numerous references to this publication calling it, "Durand and De Wildeman, Materials for the Flora from the Congo 5: 33-48 (1899)"].

De Wildeman É. 1898. Prodrome of Belgian Flora. Pt.I, vol. 1-3, and Pt.II, vol. 4. Castaigne, Brussels. $543+160 \mathrm{pp}$

De Wildman, É. 1899. Prodrome of Belgian Flora. Pt.II, vol. 5 and 6. Castaigne, Brussels. pp. 161-480.

De Wildeman É. and Durand T.. 1901. Reliquiae Dewevreanae. Annals of the Museum of the Congo. Botanique, Series 3, 2: 269-287. [It consists of a reprise, assorted commentaries, and identifications published by Bresadola and Saccardo (1899) about the collections of Alfr. Dewèvre]

De Wildeman É. 1905-1907. Mission Émile Laurent 1903-1904. 3d ed., 2 vols. Brussels. CCXXV, 617 p. +1 map and CLXXXV prints.

De Wildeman É. 1903-1912. Studies of systematic and botanical geography on the flora of the Low-and Middle-Congo. Annales of the Museum of the Congo, Botanique, Series 5, volumes 1-3.

Heinemann P. 1959 Maurice Beeli (1879-1957). Bulletin of the National Botanical Garden in Brussels 39(1): $1-6+1$ portrait.

Robyns W. 1948. Émile De Wildeman (1866-1947). Bulletin of the National Botanical Garden in Brussels 19: 1-35 + 1 portrait.

Rousseau E. 1910. Madame J.E. Bommer, née Elisa Destrée. Bulletin of the Royal Botanical Society of Belgium 47(2):256-261 + 1 portrait. 


\section{CLASSICAL TAXONOMY OF THE FUNGI}

ANAMORPH-TELEOMORPH CONNECTIONS IN THE XYLARIACEAE

M.A. Whalley, G.P. SharPles AND A.J.S. WHALLEY

School of Biomolecular Sciences, Liverpool John Moores University, Byrom Street, Liverpool L3 3AF, UK.

The Xylariaceae is a large family belonging to the ascomycotina and encompassing perhaps as many as 40 genera (Eriksson and Hawksworth 1993). The family is worldwide in distribution but exhibits its greatest diversity in the tropics and subtropics (Rogers 1993, Whalley 1993). The majority of species occur on angiospermous wood but there is also good representation on leaves, fruits, animal dung, and even on abandoned insect nests (Whalley 1985). To date most species investigated produce both teleomorphic and anamorphic states.

Traditionally, but also for convenience and sometimes through necessity, the teleomorph has assumed the central role in taxonomic decision making and has been the source of data for most identification keys. There are, however, situations where a teleomorphic form is absent and under these conditions the anamorph inherits the leading role for identification purposes.

The ability to relate anamorphs with known teleomorphs is dependent on the taxon involved and the ecological situation in which it occurs. Thus in many of the Xylariaceae anamorphs occur on the immature teleomorph and recognition of the connection is readily made. In other taxa they can occur separated both in time or space and connecting the two states now becomes more difficult and uncertain. A further challenge is provided by the apparent widespread occurrence of these fungi as endophytes of a wide range of plants where in most cases identification of the fungus is limited to features of the anamorphic state (Petrini and Petrini 1985). To what extent confident assignment to a known xylariaceous taxon can be based on the anamorph alone is open to discussion although several authors have reported species distinctive characteristics which enable identification in the absence of a teleomorph (e.g. Greenhalgh and Chesters 1968, Jong and Rogers 1972, Petrini and Muller 1985, Petrini 1992).

In the case of these endophytic isolates many undoubtedly have xylariaceous affinities but Rogers (1993) urged caution in making assignations without a clear connection to a teleomorph stating "in my laboratory anamorph and teleomorph connections are considered as definite only after ascospore cultures have been established or after cultures from natural substrata have been equated with known culture types that originated from ascospores". To what extent molecular methods can be used to make anamorph-teleomorph connections in the Xylariaceae in the absence of a teleomorph is unknown. Recent examination of ribosomal DNA restriction patterns in single conidial cultures of $X$. magnoliae J.D. Rogers derived from stromata from single host fruits, from different host fruits and from different host tree localities indicate considerable variability in ribosomal DNA phenotypes within localities and from single fruits (Gowan and Vilgalys 1991). Therefore the amount of molecular data required before unequivocal identification of a particular xylariaceous taxon can be achieved under a range of different situations is an unknown quantity but is likely to be more extensive than originally expected. 


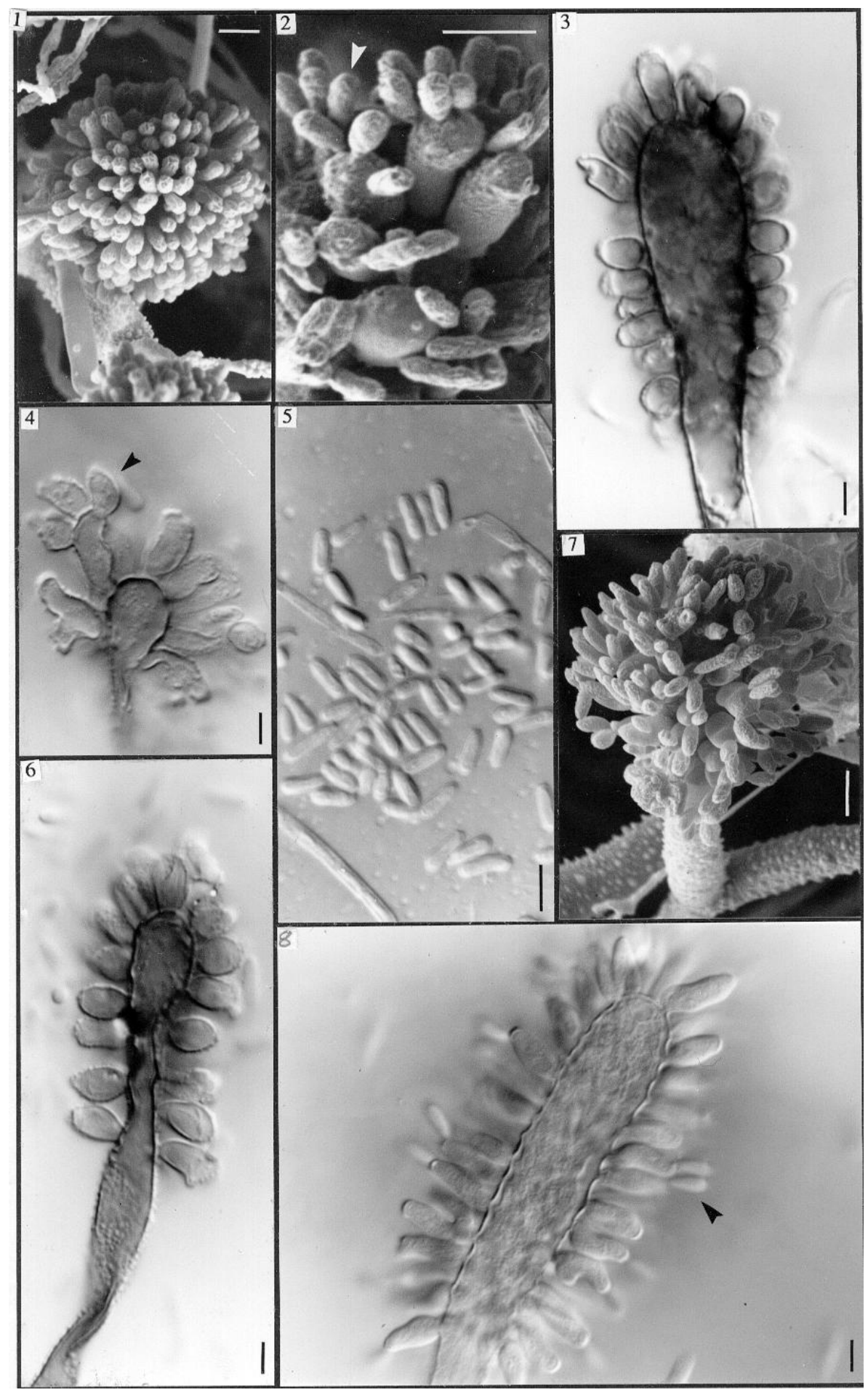

122 
Figs 1-8. Xylocladium anamorph of Camillea leprieurii (Mont.) Mont. and C. tinctor (Berk.) Laessoe, Rogers \& Whalley. Scale bar $=5$ um. Figs. 1-3. C. leprieurii (applanate form). Figs. 1-2. Ampullae, conidiogenous cells and conidia (arrowed). Fig. 3. Ampullae with conidiogenous cells. Figs. 4-6. C. leprieurii (erect form). Fig. 4. Formation of secondary conidiogenous cells (arrowed). Fig. 5. Conidia. Fig. 6. Ampullae bearing conidiogenous cells with scars from conidial production. Figs 7-8. C. tinctor. Fig. 7. Ampullae with numerous conidia. Fig. 8. Ampullae with conidiogenous cells and conidia (arrowed). Figs 1, 2, 7. Cryo SEM micrographs. Figs 3-6, 8. Differential interference contrast microscopy.

Regardless of the form genera to which Xylariaceous anamorphs are assigned conidiogenesis has been described as holoblastic coupled with a more or less sympodial proliferation of the conidiogenous cell (eg. Greenhalgh and Chesters 1968, Jong and Rogers 1972, Petrini and Muller 1986). The conidia which are produced singly characteristically possess a flattened base relating to their former point of attachment to the conidiogenous cell. Corresponding succession scars on the conidiogenous cell mark the production of single conidia. Since the taxonomic value of the anamorphic state in the Xylariaceae was first recognised by Chesters and Greenhalgh (1964) many xylariaceous taxa have been cultured from ascospores and their anamorphs described and assigned to particular form genera (e.g. Martin 1967, Jong and Rogers 1972, Petrini and Muller 1985). Initial attempts to relate anamorph type with taxonomic groupings were apparently not considered particularly successful at that time (Greenhalgh and Chesters 1968) but the subsequent accumulation of additional data and re-organization of some of these taxonomic groupings has resulted in the recognition of stable anamorph-teleomorph patterns in many genera (Rogers 1985, 1993, Petrini and Muller 1986).

The allocation of particular anamorphs to form genera can in many cases be open to personal interpretation but there is overall agreement that certain genera have clear anamorph connections. Thus Camillea Fr. possesses a Xylocladium Sydow (=Basidiobotrys Hohnel) anamorph (Figs 1-8) (Laessoe, Rogers and Whalley 1991) whilst the dung inhabiting genera Poronia Willd. and Podosordaria Ellis. \& Holw. produce a Lindquistia Subram. \& Chandrashekara state (Fig. 18) (Stiers, Rogers and Russell 1973, Subramanianand Chandrashekara 1977, Rogers and Laessoe 1992). In these three xylariaceous genera the assigned form genera are currently known to exhibit an exclusive association.

However other anamorphic forms, although distinctively xylariaceous, are more widely occurring and can be associated with a range of, all be it, in the main closely related teleomorphic genera. Nodulisporium Preuss (Figs 9-14) is the typical anamorphic form in Hypoxylon Bull. s. str., Daldinia Ces. \& De Not., Biscogniauxia O. Kuntze, Rhopalostroma Hawksw. and several other genera (Martin 1967, Greenhalgh and Chesters 1968, Jong and Rogers 1972, Hawksworth and Whalley 1985). Geniculosporium Chesters \& Greenhalgh (Figs 15-16) is associated with Leprieuria Laessoe, Rogers \& Whalley (Samuels and Muller 1980) Nemania S.F. Gray, many species of Rosellinia Ces. \& De Not. (Greenhalgh \& Chesters 1968, Martin 1967, Jong and Rogers 1972, Petrini and Muller 1986, Petrini 1992) Phylacia Lev. (Rodrigues and Samuels 1989), Anthostomella Sacc. (Francis, Minter and Caine 1980) and Euepixylon Fuisting (Whalley 1976, Laessoe and Spooner 1994). 


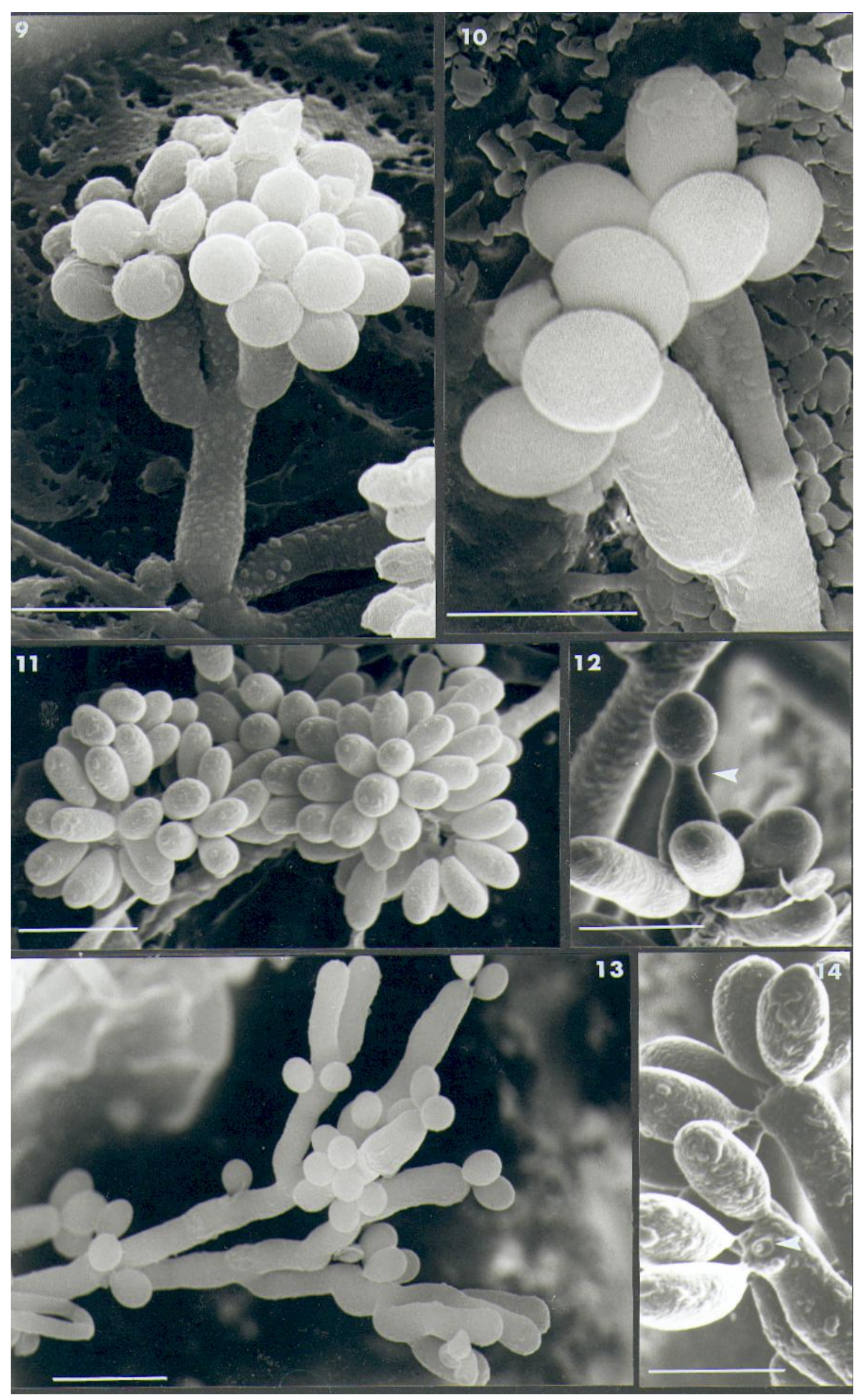

124 
Figs 9-14. Nodulisporium anamorph of Biscogniauxia nummularia (Bull.:Fr.) O. Kuntze and Daldinia eschscholtzii (Ehrenb.) Rehm.

Scale bar $=10$ um. Figs 9, 10, 13. B. nummularia conidiogenous cells and conidia. Figs. 11, 12, 14. D. eschscholtzii. Fig. 12. Young conidium developing (arrowed). Fig. 14. Succession scar on conidiogenous cell marking the former point of attachment of a conidium (arrowed). Figs. 9-14. Cryo SEM micrographs.

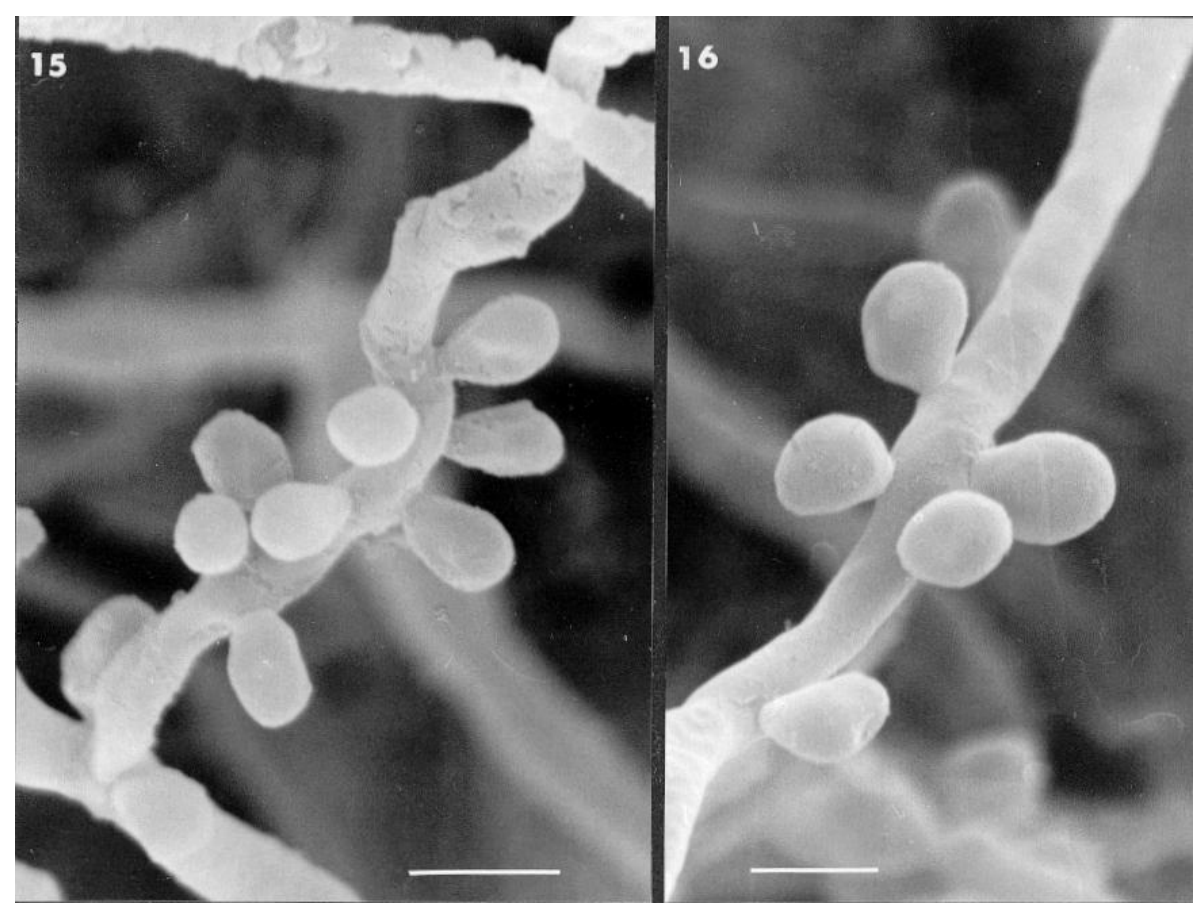

Figs 15-16. Geniculosporium anamorph of Nemania serpens (Pers.:Fr.) Gray. Scale bar $=10$ um. Cryo SEM micrographs.

Geniculosporium Chesters \& Greenhalgh (Figs 15-16) is associated with Leprieuria Laessoe, Rogers \& Whalley (Samuels and Muller 1980) Nemania S.F. Gray, many species of Rosellinia Ces. \& De Not. (Greenhalgh and Chesters 1968, Martin 1967, Jong and Rogers 1972, Petrini and Muller 1986, Petrini 1992) Phylacia Lev. (Rodrigues and Samuels 1989), Anthostomella Sacc. (Francis, Minterand Caine 1980) and Euepixylon Fuisting (Whalley 1976, Laessoe and Spooner 1994). If Nodulisporium can be seen as the main anamorphic type in the family it should be noted that there are variations on the theme and various authors have selected Periconiella Sacc., Virgariella S.J. Hughes or Rhinocladiella Nannf. as more appropriate depending on subtlety of branching and degree of complexity of the conidiophores. Thus Petrini and Muller (1986) elected to place anamorphic states of Biscogniauxia in Periconiella but Gonzalez and Rogers (1992) preferred Nodulisporium. Jong and Davies (1974) selected the similar Sporothrix Hektoen \& C.F. Perkins as the most appropriate form genus to accommodate Phaeosporis melasperma (Nyl.) Clem. (=Areolospora terrophila S.C. Jong \& E.E. Davies. 
An interesting and unusual variation is shown by $D$. occidentalis Child where the anamorph, although essentially a Nodulisporium, produces its conidia holoblastically where each scar apparently represents the former production site of a single conidium. Thus the conidiogenous cells are annellated and do not exhibit the usual sympodial proliferation typical for a Nodulisporium (Petrini and Muller, 1996). A similar observation has been made for $X$. longipes Nits. (Rogers 1983). To what extent these variations are significant in relation to systematic arrangements in the Xylariaceae is unknown but it should be noted that even the distinction between Nodulisporium and Geniculosporium has been questioned (Cole 1971, Kendrick and Carmichael 1973). However the clear association of Geniculosporium with Nemania, Rosellinia and a number of other closely related genera provides powerful reason for maintaining separate status for these two genera. The Dematophora Hart. state associated with Rosellinia necatrix Prill. can be considered as a synematious form of Geniculosporium and therefore still in harmony with the anamorphs produced by most other species of Rosellinia (Teixeira de Sousa and Whalley 1991).

However a fundamental question raised by Cole (1973) which still requires attention concerns his comment that "it is often difficult to be sure whether a conidiophore or conidiogenous cell has proliferated sympodially when only the mature state is examined". According to Cole " in Nodulisporium hinnuleum (Preuss) G. Smith conidia are produced in a basipetal succession, clustering about the apex of the conidiogenous cell, which does not itself change in length during the process of conidium formation". This is in conflict with the usual interpretation of Nodulisporium anamorphs in the Xylariaceae where the conidia are produced by continuous acropetal succession with the youngest conidium produced at the apices of a succession of growing points produced by a sympodially proliferating conidiogenous cell (Greenhalgh and Chesters 1968).

Although the overall pattern of anamorphic type in the Xylariaceae can be viewed as one of consistency there are some notable exceptions. As Ju, Gonzalez and Rogers (1993) stated "one of the hallmarks of taxa of family Xylariaceae is the type of hyphomycetous anamorph that features dry amerosporous conidia produced holoblastically from sympodially proliferating conidiogenous cells". Their discovery of three Xylariaceous taxa [Lopadostoma turgidum (Pers.:Fr.) Traverso; Creosphaeria sassafras (Schwein.:Fr.) Ju, Gonzalez \& J.D. Rogers and a fungus close to Nummularia viridis Theiss.] that, "in culture, produce more or less slimy scolecosporous conidia holoblastically from sympodially proliferating or sympodially and percurrently proliferating conidiogenous cells" (Ju et al. 1993) is an interesting development. It leads them to conclude "that these fungi represent a heretofore unrecognized evolutionary line in the Xylariaceae" ... and... "the relationships among these three taxa are presently unknown" (Ju et al. 1993). Although these undoubtedly represent a major departure from the 'norm' for the family there are other curiosities. Padixonia C.V. Subraman. (Fig. 20) is unique to Xylaria furcata Fr., one of several species associated with termite nests (Subramanian 1972) and Acanthodochium Samuels, Rogers and Nagasawa (Fig. 19) has to date been reserved for anamorphs of Collodiscula japonica Hino \& Katumoto and Astrocystis Berk. \& Broome (Samuels, Rogers and Nagasawa 1987, Ju and Rogers 1990). 


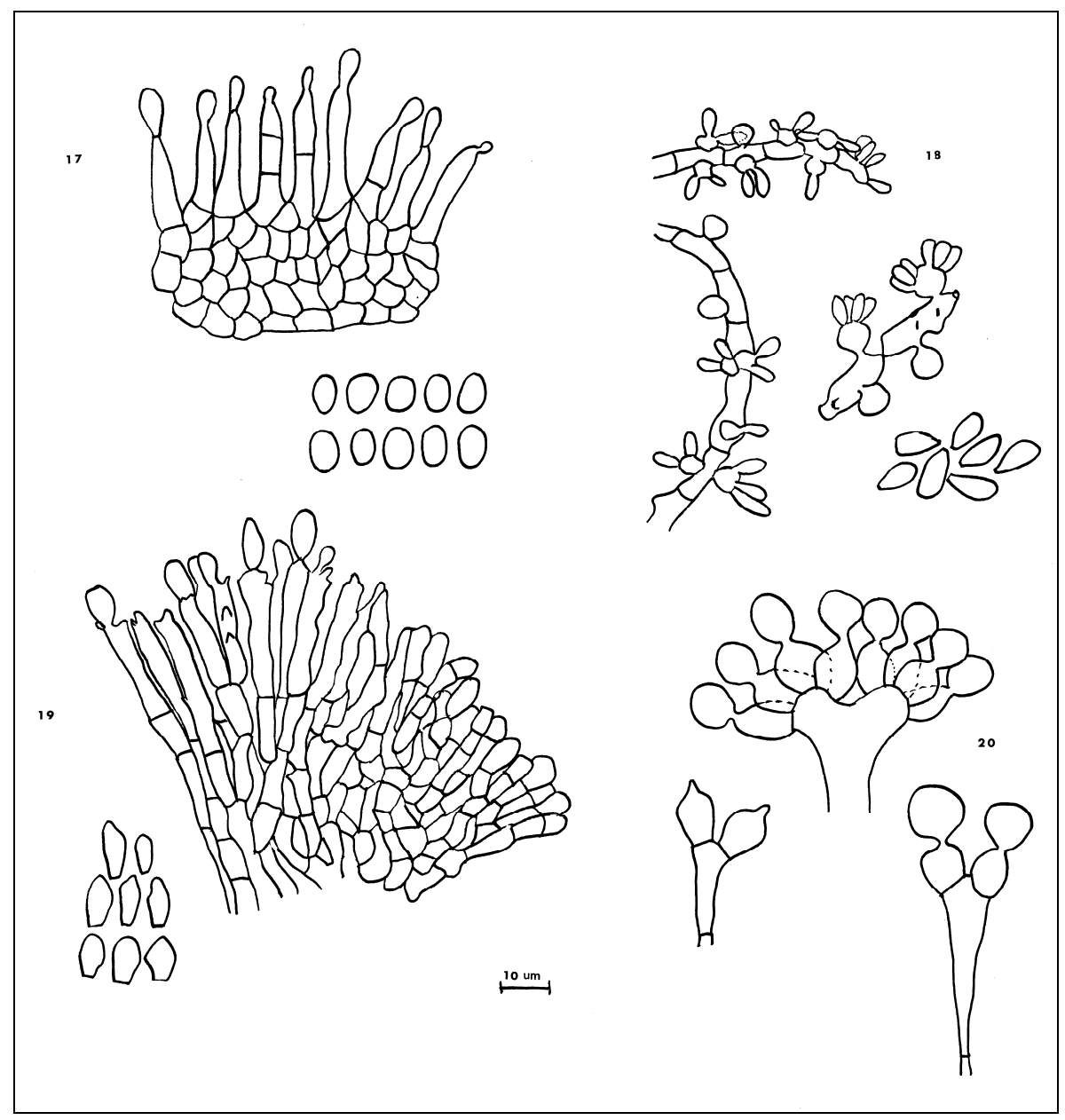

Figs 17-20. Conidiogenous cells and conidia of Fig.17. Hadrotrichum (after Petrini and Candoussau), Fig. 18. Lindquistia (after Subramanian and Chandrashekara), Fig. 19. Acanthodochium (after Samuels et al.), Fig. 20. Padixonia (after Subramanian).

Other isolated anamorphic forms which deserve consideration include Hadrotrichum Fuckel which was the chosen form genus for the anamorph of Hypoxylon deustum (Hoffm.:Fr.) Grev. (= Kretzschmaria deusta (Hoffm.:Fr.) P.M.D. Martin (Petrini \& Muller, 1986) and the Xylocoremium flabelliforme (Schwein.:Fr.) J.D. Rogers anamorph of Xylaria cubensis (Mont.) Fr., X. allantoidea (Berk.) Fr. and allies (Callan and Rogers 1990). We draw attention to Hadrotrichum pyrenaicum Petrini \& Candoussau and the statement that "Hadrotrichum pyrenaicum has probably been overlooked, or confused, for a long time with immature forms of Hypoxylon spp., although a microscopical examination leaves no doubt as to its identity. No connection with any known species of Hypoxylon could be established even though the presence of Nodulisporium - like conidiophores and conidia in pure culture indicate a possible link" (Petrini and Candoussau 1983). Later Petrini and Muller (1986) connected H. pyrenaicum with Hypoxylon. 
moravicum Pouzar. It is interesting to note that Hypoxylon fraxinophillum Pouzar (=H. argillaceum Berk.) is also restricted to Fraxinus (Pouzar 1972) and it develops conidiophores, which as illustrated by Greenhalgh \& Chesters (1968), are remarkably similar to those of $H$. pyrenaicum. It certainly would not be unexpected to find similar anamorphs in these closely related taxa but the presence of a Hadrotrichum anamorph in Hypoxylon deustum as recorded by Petrini and Muller (1986) is much more difficult to explain in terms of teleomorph relationships. It is generally agreed that Hypoxylon deustum has little in common with Hypoxylon s. str. and it is usually referred to Ustulina Tul. \& C. Tul. or to Kretzschmaria Fr. if the proposal to synonomize these genera is accepted (Laessoe, 1994).

Regardless of argument over the finer details of assignment to hyphomycetous genera the value of the anamorph in developing systematic arrangement within the Xylariaceae must not be undervalued. As stated by Rodrigues and Samuels (1989) in reference to Phylacia "Anamorphs can be useful in determining relationships of fungi" and "the anamorphs that we observed are species of Geniculosporium (sensu Petrini and Petrini 1985) and are wholly consistent with anamorphs that have previously been described for the family Xylariaceae". In a similar vein Callan \& Rogers (1990) stated that "Xylaria Hill ex Schrank is increasingly recognized as a diverse and complicated assemblage of taxa. It has become apparent that the biology and systematics of Xylaria and its allies can be understood only after consideration of the holomorphs".

Table 1. Teleomorph-anamorph connections in the Xylariaceae

\begin{tabular}{|ll|}
\hline \multicolumn{1}{|c|}{ TELEOMORPH } & \multicolumn{1}{c|}{ ANAMORPHS } \\
\hline Anthostomella Sacc. & Nodulisporium \& Virgariella \\
Astrocystis Berk. \& Broome & Acanthodochium \\
Biscogniauxia Kuntze & Geniculosporium, Periconiella \& \\
& Nodulisporium \\
Calceomyces Udagawa \& Ueda & Nodulisporium \\
Camillea Fr. & Xylocladium \\
Collodiscula * I.Hino \& Katum. & Acanthodochium \\
Daldinia Ces. \& De Not. & Nodulisporium \\
Entonaema A.Moller & Nodulisporium \\
Euepixylon * Fuisting & Geniculosporium \\
Hypoxylon Bull. & Nodulisporium, Virgariella \\
& \&Rhinocladiella \\
Induratia Samuels, E.Mull. \& O.Petrini & Nodulisporium \\
Leprieuria Laessoe, J.D.Rogers \& Whalley. & Geniculosporium \\
Nemania * S.F.Gray & Geniculosporium \\
Obolarina * Pouzar & ? Rhinocladiella \\
Phaeosporis Clem. & Sporothrix \\
Phylacia Lev. & Geniculosporium \\
Podosordaria Ellis \& Holw. & Lindquistia \\
Poronia Willd. & Lindquistia \\
Rhopalostroma D.Hawks. & Nodulisporium \\
Rosellinia De Not. & Geniculosporium, Dematophora \\
Thamnomyces Ehrenb. & \& Nodulisporium \\
Theumenella Penz. \& Sacc. & Nodulisporium \\
Xylaria Hill ex Schrank & Nodulisporium \\
* not recognised by Eriksson \& Hawksworth ( 1993 ) & Xylocoremium \\
\hline
\end{tabular}

The value of cultural studies in the Xylariaceae is clearly proven: at their least the anamorphs provide a range of new characters for taxonomic purposes but their 
outstanding contribution lies in the confirmation of connections with known teleomorphs and the subsequent development of more natural groupings. Currently accepted anamorph teleomorph connections in the Xylariaceae are listed in Table 1; others will undoubtedly follow.

\section{Acknowlegments}

We wish to thank the British Mycological Society for permission to reproduce Figures 1-8 from Mycological Research.

\section{References}

Callan B. and Rogers J.D. 1990. Teleomorph-anamorph connections and correlations in some Xylaria species. Mycotaxon 36, 343-369.

Chesters C.G.C. and Greenhalgh G.N. 1964. Geniculosporium serpens gen. et sp. nov., the imperfect state of Hypoxylon serpens. Transactions of the British Mycological Society 47, 393-401.

Cole G.T. 1971. The Sympodula and the Sympodioconidium. In Taxonomy of Fungi Imperfecti Kendrick W.B. ed., University of Toronto Press, Toronto. Canada, pp. 141-159.

Eriksson O. and Hawksworth D.L. 1993. Outline of the ascomycetes - 1993. Systema Ascomycetum 12, 51-257.

Francis S.M., Minter D.W. and Caine T.S. 1980. Three newspecies of Anthostomella. Transactions of the British Mycological Society 75, 201-206.

Gonzalez F.S.M. and Rogers J.D. 1993. Biscogniauxia and Camillea in Mexico. Mycotaxon 47, 229-258.

Gowan S.P. and Vilgalys R. 1991. Ribosomal length polymorphisms within populations of Xylaria magnoliae (Ascomycotina). American Journal of Botany 78, 160-1607.

Greenhalgh G.N. and Chesters C.G.C. 1968. Conidiophore morphology in some British members of the Xylariaceae. Transactions of the British Mycological Society 51, 57-82.

Hawksworth D.L. and Whalley A.J.S. 1985. A new species of Rhopalostroma with a Nodulisporium anamorph from Thailand. Transactions of the British Mycological Society 84, 560-562.

Jong S.C. and Davies E.E. (1974). Areolospora, a new humicolous genus in the Xylariaceae. Norwegian Journal of Botany 21, 23-30

Jong S.C. and Rogers J.D. 1972. Illustrations and descriptions of conidial states of some Hypoxylon species. Washington Agricultural Experiment Station Technical Bulletin 71, 1-51.

Ju Y.-M. and Rogers J.D. 1990. Astrocystis reconsidered. Mycologia 82, 42-349.

Ju Y.-M., Gonzalez F.S.M. and Rogers J.D. 1993. Three xylariaceous fungi with scolecosporous conidia. Mycotaxon 47, 219-228.

Kendrick W.B. and Carmichael J.W. 1973. Hypomycetes. In The Fungi: an Advanced Treatise., Volume 4A, A taxonomic Review with Keys: Ascomycetes and Fungi Imperfecti. Ainsworth G.C., Sparrow F.K. and Sussman A.S.eds., Academic Press, New York, pp. 323-509.

Laessoe T. 1994. Index Ascomycetum. 1. Xylariaceae. Systema Ascomycetum (in press).

Laessoe, T., Rogers, J.D. and Whalley, A.J.S. (1989). Camillea, Jongiella and light-spored species of Hypoxylon. Mycological Research 93, 121-155.

Laessoe T. and Spooner B.M. 1994. Rosellinia \& Astrocystis (Xylariaceae): new species and generic concepts. Kew Bulletin 49, 1-70.

Martin P. 1967. Studies in the Xylariaceae: 1. New and old concepts. Journal of South African Botany 33, 205-240.

Petrini L.E. 1992. Rosellinia species of the temperate zones. Sydowia 44, 169-281.

Petrini L.E. and Muller E. 1986. Untersuchungen uber die Gattung Hypoxylon (Ascomycetes, Xylariaceae) und verwandte Pilze. Mycologia Helvetica 1, 501-639.

Petrini L.E. and Petrini O. 1985. Xylariaceous fungi as endophytes. Sydowia 38, 216-234.

Petrini O. and Candoussau F. 1983. Hadrotrichium pyrenaicum nov. sp., a new deuteromycete from the Pyrenees (France). Mycotaxon 18, 91-95.

Pouzar Z. 1972. Hypoxylon fraxinophilum spec. nov. and H. moravicum spec. nov., two interesting species found onFraxinus angustifolia. Ceska Mykologie 26, 129-17.

Rodrigues K.F. and Samuels G.J. 1989. Studies in the genusPhylacia (Xylariaceae). Memoirs of the New York Botanical Garden 49, 290-297.

Rogers J.D. 1983. Xylaria bulbosa, Xylaria curta and Xylaria longipes in Continental United States. Mycologia 75, 457-467.

Rogers, J.D. 1985. Anamorphs of Xylaria: Taxonomic considerations. Sydowia 38, 255-262.

Rogers J.D. 1993. Teleomorph, Anamorph, and Holomorph considerations in the Xylariaceae. In The Fungal Holomorph: Mitotic, Meiotic and Pleomorphic Speciation in Fungal Systematics. Reynolds 
D.R. and Taylor J.W. eds., CAB International: Wallingford, UK, pp. 179-182.

Rogers, J.D. and Laessoe T. 1992. Podosordaria ingii sp. nov. and its Lindquistia anamorph. Mycotaxon 44, 435-443.

Samuels G.J. and Muller E. 1980. Life history studies of Brazilian Ascomycetes 8. Thamnomyces chordalis (anam.: Nodulisporium) and Camillea bacillum (anam.: Geniculosporium) with notes on taxonomy of the Xylariaceae. Sydowia 33, 274-281.

Samuels G.J., Rogers J.D. and Nagasawa E. 1987. Studies in the Amphisphaeriaceae (sensu lato) 1. Collodiscula japonica and its anamorph, Acanthodochium collodisculae. Mycotaxon 28, 453-459.

Stiers D.L., Rogers J.D. and Russell D.W. 1973. Conidial state of Poronia punctata. Canadian Journal of Botany 51, 481-484.

Subramanian C.V. (1972). Padixonia, a new genus of hyphomycetes. Current Science 41, 282-283.

Subramanian C.V. and Chandrashekara, K.V. 1977. Lindquistia, a new hyphomycete genus. Boletin de la Sociedad Argentina de Botanica 18, 145-151.

Teixeira de Sousa A.J. and Whalley A.J.S. 1991. Induction of mature stromata in Rosellinia necatrix and its taxonomic implications. Sydowia 43, 281-290.

Whalley A.J.S. 1976. Notes on the conidial state of Hypoxylon udum. Transactions of the British Mycological Society 67, 515-517.

Whalley A.J.S. 1985. The Xylariaceae: some ecological considerations. Sydowia 38, 369-382.

Whalley A.J.S. 1993. Tropical Xylariaceae: their distribution and ecological characteristics. In Aspects of Tropical Mycology. Isaac S., Frankland J.C., Watling R. \& Whalley A.J.S. eds., Cambridge University Press: Cambridge, UK, pp. 103-119. 


\title{
ANAMORPHS AND THE CLASSIFICATION OF XYLARIACEOUS FUNGI
}

\author{
KATLEEN VAN DER GUCHT
}

Laboratorium Plantkunde, Vakgroep Morfologie, Systematiek \& Ecologie, Universiteit Gent, K.L. Ledeganckstraat 35, 9000 Gent, België.

\section{Introduction}

As Rogers (1985) pointed out Xylaria Hill ex Schrank is a complex and difficult genus. Several mycologists (Fries 1851, Miller 1942, Dennis 1961, 1970, 1974, Joly 1968, Martin 1970, Bertault 1984) have tried to organize the genus into well-defined groups, based upon stromatal characteristics. Though these efforts have some merit, most of the proposed schemes are difficult to use consistently and the groupings tend to be artificial due to variability and often ambiguous nature of some of the major characters employed.

\section{Classifiction}

Rogers (1985) was the first to propose a subdivision of the genus Xylaria based upon anamorphic characteristics. The division is primarily based on two aspects of Xylaria anamorphs: the place of the anamorph in the life cycle, and the morphology of the conidiogenous structure. He recognizes 8 groups divided upon 4 sections (see table 1).

Table 1. The subdivision of Xylaria into four sections based on anamorph characteristics (Rogers 1985).

\begin{tabular}{|l|l|}
\hline sections & characteristic features \\
\hline Section 1 & $\begin{array}{l}\text { Conidia produced from palisade of conidiogenous cells over entire young } \\
\text { telemorphic stromata or at least not limited to specialized appendages. } \\
\text { Conidiogenesis holoblastic. Conidia produced in more or less sympodial } \\
\text { sequence, seceding individually and passively. } \\
\text { This section contains 5 groups: Xylaria polymorpha group, } X \text {. hypoxylon group, } X . \\
\text { multiplex group, } X \text {. pyramidata group and the } X \text {. pedunculata group. }\end{array}$ \\
\hline Section 2 & $\begin{array}{l}\text { Conidia produced on special and localized peg- or hair-shaped appendages on } \\
\text { young teleomorphic stromata. Conidiogenesis apparently holoblastic. Conidia } \\
\text { apparently seceding individually and passively. } \\
\text { This section is represented by the } X \text {. comosa group. }\end{array}$ \\
\hline Section 3 & $\begin{array}{l}\text { Conidia produced on special anamorphic stromata or conidiomata which usually } \\
\text { are produced earlier in the year than teleomorphic stromata. Teleomorphic } \\
\text { stromata never bear conidia. Conidiogenesis holoblastic. Conidia produced in } \\
\text { more or less sympodial sequence, seceding individually and passively. } \\
\text { This section is represented by the } X \text {. cubensis group. }\end{array}$ \\
\hline Section 4 & $\begin{array}{l}\text { Conidia produced on young teleomorphic stromata. Conidiogenesis apparently } \\
\text { holoblastic. Conidia produced in tandem, seceding forcibly. } \\
\text { This section is represented by the } X \text {. furcata group. }\end{array}$ \\
\hline
\end{tabular}

The division proposed by Rogers (1985) has special merit, since the recognized sections may represent more natural assemblages of Xylaria species. However, as the author pointed out many more anamorphic data must be obtained and correlated with teleomorphic data to fill in gaps and thereby ameliorate and/or alter the proposed system.

The subdivision proposed here, which became apparent through my study of anamorphic and teleomorphic features of Papua New Guinean material, is inspired 
on the work of Rogers (1985), but differs in the grouping of some of the species. These differences can largely be explained by the fact that I have been able to study the anamorphic state (including the anamorph as found in nature) of a number of species for which there were no previous data.

Based on the Papua New Guinean material 5 groups could be recognized.

\section{Xylaria anisopleura group}

Members of this group are $X$. anisopleura (Mont.) Fr., X. schweinitzii Berk. \& M.A. Curtis, and, judging from the teleomorphic features, $X$. scruposa (Fr.) Fr.

- Teleomorphic features: This group is well characterised by the (usually) large teleomorphic stromata with a roughened stromatal surface due to wrinkles, warts and tomentum, with greyish to brownish scales when young, becoming blackish to black at maturity; ostioles umbilicate, appearing as small hemispherical black discs; apical apparatus rectangular, constricted sub-apically; ascospores fairly large, over $15 \mu \mathrm{m}$ long, usually with a short, straight to curving germ slit, obliquely oriented.

- Anamorph in nature: The anamorph as found in nature is formed on stromata growing side by side with mature teleomorphic stromata. These stromata are, except for being smaller, similar to the teleomorphic stromata (e.g. clavate to spathulate for $X$. anisopleura (Mont.) Fr., and cylindric-fusoid for X. schweinitzii Berk. \& M.A. Curtis).

Most probably, the anamorph in this group develops on young immature teleomorphic stromata, iIn contrast to the taxa of the $X$. cubensis group (see further) where the anamorph develops on stromata distinct from the teleomorphic stromata.

- Cultural characteristics: The colony colours are white, grey and black, and the mycelium is characterised by the presence of coiled hyphae. The conidia are produced upon cylindrical stromata and are ellipsoid. In most instances, the cultures remained sterile, or the material did not germinate at all.

This group represents part of the $X$. polymorpha group as recognized by Rogers (1985) (see further).

\section{Xylaria feejeensis group}

Members of this group are X. feejeensis (Berk.) Fr. and X. luteostromata Lloyd.

- Teleomorphic features: Characteristic for this group are the slender stromata, usually less than $5 \mathrm{~mm}$ diam., with a surface finely and reticulately cracked into small, angular closely spaced scales so as to outline the individual perithecia; ostioles finely papillate in the centre of a dome-shaped to hemispherical black disc; apical apparatus more or less quadrate; ascospores rather small (up to $11 \mu \mathrm{m}$ long) with a straight germ slit running almost full spore length.

- Anamorph in nature: unknown.

- Cultural characteristics: Colonies appressed, white to grey (usually with a reddish to orange shine). The mycelium is characterised by the presence of highly coiled hyphae. Conidia are produced upon cylindrical stromata or on small rudimentary stromata. Conidia subglobose, obovoid to cylindrical.

Rogers (1985) placed X. feejeensis (Berk.) Fr. together with X. anisopleura (Mont.) Fr. and $X$. schweinitzii Berk. \& M.A. Curtis in the X. polymorpha group. Though cultural characteristics indicate that the $X$. feejeensis group and the $X$. anisopleura group may be closely related, it is my opinion that based on the above mentioned differences in teleomorphic and anamorphic features warrent their classification in two distinct groups. 


\section{Xylaria apiculata group}

Members of this group are $X$ apiculata Cooke, X. palmicola G. Winter and $X$. mellisii (Berk.) Cooke.

- Teleomorphic features: Stromata with cylindric-conical fertile parts, on narrow hirsute stipes, and attenuated acute sterile apices; surface with peeling or flaking greyish to brownish outer layer, the remnants persisting as conspicuous plates or shreds in some species or not noticeably persisting in other species; ostioles umbilicate, usually obscure, or slightly raised; ascospores over $10 \mu \mathrm{m}$ long with a straight or slightly spiralling germ slit, from less than to almost full spore length.

- Anamorph in nature: formed on filiform apiculi or branches, white to grey, of immature teleomorphic stromata. Conidia obovoid, small, 3.5-5.5x1-2.5 $\mu \mathrm{m}$.

- Cultural characteristics (after Rogers and Samuels 1986, Rogers et al. 1988).

Colonies velvety, white, becoming grey to black (with a greenish shine). Slender cylindrical stromata are usually formed, less than $2 \mathrm{~mm}$ diam., covered with dark hyphae and with an acute white tip. Conidia rarely forming in culture, arising from loosely dispersed, aerial hyphae along the white upper part of the stroma.

Rogers (1985) recognized a $X$. multiplex group with the following members: $X$. apiculata Cooke, $X$. arbuscula Sacc. $(=X$. mellisii (Berk.) Cooke), X. multiplex (Kunze) Fr. and $X$. schreuderiana van der Bijl. I agree on the grouping of $X$. apiculata Cooke and $X$. arbuscula Sacc. but according to my observations, $X$. multiplex (Kunze) Fr. belongs to a group clearly distinct from the here proposed $X$. apiculata group (see further).

\section{Xylaria grammica group}

Members of this group are $X$. grammica (Mont.) Fr., X. papulis Lloyd, $X$. multiplex (Kunze) Fr. and $X$. cf. pallida Berk. \& Cooke.

- Teleomorphic features: Immature stromata brightly coloured, white or yellow. The brightly coloured surface layer is worn off in mature stromata. Surface of mature stromata smooth, with remnants of the bright-coloured surface layer, or characterized by an outer layer which splits longitudinally around the ostioles, exposing the underlying black surface. Ostioles punctiform and located in the black stripes, or finely papillate, often surrounded by an annular disc; ascospores inaequilaterally ellipsoid with a straight germ slit running almost full spore length.

- Anamorph in nature: formed on wart-like structures developed upon immature bright coloured teleomorphic stromata.

- Cultural characteristics: Colonies at first brightly coloured (e.g. light yellow), later darkening from centre outwards, finally becoming black with a greenish shine. Mycelium characterized by the presence of thick walled, dark brown hyphae, with short protuberances, breaking down into segments. Cylindrical stromata covered with villose black hyphae are formed, but they usually remain sterile, or the conidiogenous structures are produced on wart-like structures upon the cylindrical stromata. Anamorph usually formed on small tufts or subglobose stromata in the centre of the colony. Conidia elongated ellipsoid.

This is a conspicuous group due to the bright colouration of the colonies as well as of the immature stromata, the production of conidia on wart-like structures, and the shape of the conidia. This group was not previously recognized. 


\section{Xylaria cubensis group}

Members of this group are $X$. cubensis (Mont.) Fr., X. allantoidea (Berk.) Fr. and $X$. poitei (Lév.) Fr. This group corresponds to the $X$. cubensis group as described by Rogers (1985).

- Teleomorphic features: Stromata brown, bronze or copper-coloured, becoming blackened with age; surface smooth and plane; ostioles papillate; ascospores inaequilaterally ellipsoid, germ slit inconspicuous or conspicuous, straight, running almost full spore length.

- Anamorph in nature: produced on special anamorphic stromata which develop earlier in the year than the teleomorphic stromata.

- Cultural features: Colonies orange-white. Anamorph formed on cylindrical to flabelliform stromata. Conidiogenous cells long cylindrical. Conidia obovoid.

\section{Conclusion}

It was not possible to place all the Xylaria taxa, found in Papua New Guinea, in one of the above circumscribed groups, especially due to the lack of observations on the anamorphic state.

It is obvious that much more study needs to be done before one can come to a more natural subdivision within the genus Xylaria. In my opinion, however, the subdivision as proposed by Rogers (1985) together with the scheme presented above, though both far for complete and in need of further corroboration, provide an indication that the combination of anamorphic and teleomorphic features can lead to a less artificial grouping, and may thus provide insight into the evolution of the large and complex genus Xylaria.

It is also admitted that a subdivision of a complex genus like Xylaria should ideally be carried out in the frame-work of a worldwide revision rather than being based on a study of the funga ${ }^{3}$ of one region. However, I have judged it worthwhile to propose the scheme obtained, such that it can be verified and complemented through studies on taxa from other regions.

\section{References}

Barr M.E. 1983. The ascomycete connection. Mycologia 75: 1-13.

Bertault R. 1984. Xylaires d'Europe et d'Afrique du Nord. Bull. Soc. Mycol. France 100: 139-175.

Dennis R.W.G. 1961. Xylarioideae and Thamnomycetoideae of Congo. Bull. Jard. Bot. État 31: 109-154.

Dennis R.W.G. 1970. Fungus flora of Venezuela and adjacent countries. Kew Bull., Addit. Ser. 3: 1-531.

Dennis R.W.G. 1974. Xylariaceae from Papua and New Guinea. Bull. Mens Soc. Linn. Lyon, num. spéc. 43: $127-138$.

Fries N. 1851. Novae symbolae mycologicae. Nova Acta Regiae Soc. Sci. Upsal. ser. 3 1: 17-136.

Joly P. 1968. Eléments de la flore mycologique du Viet-Nam, Troisième contribution: A propos de quelques Xylarias. Rev. Mycol. Paris 33: 155-207.

Martin P. 1970. Studies in the Xylariaceae: VIII. Xylaria and its allies. J. S. African Bot. 36: 73-138.

Miller J.H. 1942. South African Xylariaceae. Bothalia 4: 251-272.

Rogers, J.D. 1985. Anamorphs of Xylaria: Taxonomic considerations. Sydowia 38: 255-262.

Rogers J.D., Callan, B.E., Rossman, A.Y.and Samuels, G.J. 1988. Xylaria (Sphaeriales, Xylariaceae) from Cerro de le Neblina, Venezuela. Mycotaxon 31: 103-153.

Rogers, J.D. and Samuels, G.J. 1986. Ascomycetes of New Zealand. 8. Xylaria. New Zealand J. Bot. 24: 615-650. 


\title{
First occurrence in nature of the teleomorph of Cochliobolus
}

\author{
R. RAEMAEKERS* AND J. COOSEMANS** \\ *Belgian Administration for Development Cooperation (BADC), \\ **Faculty of Agricultural and Applied Biological Sciences, K.U. Leuven
}

\section{Introduction}

The genus Cochliobolus was erected by Drechsler (1934) to include those fungi with teleomorphs producing asci with helically coiled filiform ascospores. Teleomorphs of Cochliobolus are rare in nature (Sivanesan 1987). The teleomorph Cochliobolus sativus (Ito \& Kurib.) Drechsler ex Dastur (anamorph Bipolaris sorokiniana (Sacc.) Shoem., syn. Helminthosporium sativum Pammel, King \& Bakke) syn. Drechslera sorokiniana (Sacc.) Subram. \& Jain, has not been recorded in the field (Sivanesan 1987, personal comm. 1990, Tinline and Dickson 1958).

Sivanesan (1987) reports that several Cochliobolus spp. were produced since 1927 by pairing opposite mating strains of single spore isolates at temperatures above $20{ }^{\circ} \mathrm{C}$ on agar media prepared with plant material. Cochliobolus sativus was produced in the laboratory and described by Ito and Kuribayashi, and by Tinline (1951) and Tinlin and Dickson (1958).

This paper describes the occurrence in nature of C. sativus in Zambia following the introduction of rainfed wheat, on which annually epidemics of Bipolaris sorokiniana diseases occur (Raemaekers 1991, Raemaekers et al. 1991). The naturally occurring teleomorph is compared with the teleomorph produced by Ito \& Kuribayashi and by Tinline (1951) in the laboratory.

\section{Materials and methods}

Rainfed wheat or summer wheat research was re-introduced by the Department of Agriculture (Zambia) in the late 1970s. The research described was carried out at Mount Makulu Research Station (MMRS) near Lusaka, where wheat plots were seeded early January and harvested in May. At this and at other locations in the country, B. sorokiniana diseases (spot blotch, head blight, stem break and black point) were destructive (Raemaekers 1991).

The weather conditions during the period from harvest to seeding of the new crop (autumn to summer) when the teleomorph develops are summarised (Table 1).

To examine the above-ground crop residues from autumn till summer, wheat culms with and without ears were collected after plot-combining and taken to an area (garden) protected from bushfires. There, the straw was spread on a net of chickenwire $(1 \mathrm{x} 8 \mathrm{~m})$ suspended $\pm 20 \mathrm{~cm}$ above soil level in an area without shade. It was exposed to normal weather conditions, similar to those occurring in the adjacent fields. This procedure started in 1985 but due to circumstances it had to be repeated annually till 1989-90. Straw from the Mbala area, 1,000km north of Lusaka, was collected after harvest and exposed to the weather in the same way. Partially decomposed straw, used as mulch under coffee trees in the same area, was also collected.

Selected parts were examined at regular intervals by stereoscope and fruiting bodies were carefully removed, often from in between a dense mat of conidia and conidiophores. They were cleaned following a method described by Tinline (1951). A cleaned fruiting body would then be mounted in a drop of sterile distilled water and squashed for microscopic examination. 
Table 1. Weather conditions from autumn to summer at MMRS ${ }^{1}$

\begin{tabular}{|l|l|l|l|l|l|l|}
\hline month & $\begin{array}{l}\text { mean } \\
\text { temp. } \\
{ }^{\circ} \mathrm{C}\end{array}$ & $\begin{array}{l}\text { mean } \\
\max \text { temp. } \\
{ }^{\circ} \mathrm{C}\end{array}$ & $\begin{array}{l}\text { mean } \\
\text { min temp. } \\
{ }^{\circ} \mathrm{C}\end{array}$ & $\begin{array}{l}\% \\
\text { R.H. }\end{array}$ & Sunshine hrs/day & $\begin{array}{l}\text { rainfall } \\
\text { mm }\end{array}$ \\
\hline May & 17.3 & 24.5 & 11.3 & 57 & 9.1 & 5 \\
June & 16.1 & 23.3 & 9.8 & 55 & 8.5 & 0 \\
July & 16.0 & 23.2 & 9.2 & 51 & 9.0 & 0 \\
August & 18.3 & 25.7 & 11.9 & 44 & 9.1 & 0 \\
September & 22.0 & 29.4 & 16.4 & 37 & 9.5 & 1 \\
October & 24.8 & 31.8 & 18.4 & 40 & 8.8 & 9.6 \\
November & 22.7 & 29.0 & 17.9 & 59 & 5.7 & 222 \\
December & 21.1 & 26.3 & 17.2 & 74 & 4.9 & 166 \\
January & 21.0 & 26.3 & 17.4 & 75 & . & \\
\hline
\end{tabular}

${ }^{1}$ MMRS: Mount Makulu Central Agricultural Research Station, Chilanga, Zambia. (Meteorological Department, Lusaka, 1975).

Asci and single spores were placed on PDA with bactericide. Single spores were separated under the stereoscope into a small drop of sterile distilled water and transferred to the agar. Petri dishes were sealed and kept at room temperature in the laboratory where they were exposed to daylight.

Plants of the susceptible wheat variety 'Loerie' were grown in pots in the greenhouse for pathogenicity tests. At heading, the bottom leaves were removed and the two top leaves were kept for inoculation. In the laboratory, these plants were atomised with distilled water. A drop of sterile distilled water was placed a few $\mathrm{cm}$ away from the leaf base either on the flag leaf or the penultimate leaf. The following treatments were compared with a control: single B. sorokiniana conidium from wheat straw, single conidium from monoascosporic isolate, single ascospore, groups of ascospores, ascus. Spores were collected with fine pointed tweezers under the stereoscope and transferred to the drop of water on the leaf. The plants were covered with plastic bags after being atomised with distilled water. The pots were placed on a bench in a tray with water. The bags were removed 24 hours later. The maximum and minimum temperatures in the laboratory were 29 and $22^{\circ} \mathrm{C}$ respectively during the period 15 to 30 March 1990.

\section{Results}

Ascocarps developed in abundance towards the end of the year on the straw; this occurred annually during the years of observations. Most ascocarps were observed on dark brown discoloured straw and ears, the discolouration being the result of severe infection during crop life. Conidia and conidiophores were also present in abundance on this dry material. The ascigerous stage was present predominantly on the upper part of the plant including glumes, rachis, peduncle, top internode and nodes, but not on the lower internodes. It was not observed on the infected grains in the glumes. Most of the leaves were disintegrated by the time the ascocarps started to develop; fruiting bodies did develop in and underneath leaf sheaths.

Mature fruiting bodies started to develop in December. By March-April, most ostioles were open and such ascocarps were found to be empty upon examination. A few immature (nonseptate) spores were observed by mid-December. By late December the first septate ascospores were noticed. The first observation of such a spore was on 24.12.85. By mid-January, pseudothecia with immature and mature asci were present. One hundred and four fruiting bodies were examined mid- 
January 1986. Only $15 \%$ of those contained asci (with mature and immature ascospores). In February and March 1990, only one third of all asci examined in many fruiting bodies contained ascospores. The other asci were either empty or filled to various degrees with granular protoplasma. Pseudothecia always contained a mixture of asci with and without ascospores. Ascocarps developed on the Mbala straw, but less frequent than on the more infected MMRS straw. A few ascocarps with mature ascospores were found on the partially decomposed straw (mulch).

Globose superficial pseudothecia were smooth and black, occasionally with setae. Most of them developed amidst concentrations of conidia and conidiophores from the infection during crop growth. Often they were held to the straw by strips of disintegrating epidermis through which they had grown. They were usually scattered on the wheat tissue. Occasionally they developed closely together in groups of 4 to 6. Most ascocarps had a short subcylindrical ostiolar beak. Several pseudothecia were observed without beak; one fruiting body had two beaks. Upon contact with water on a glass slide, or after being crushed by a needle if not opening spontaneously, the mature fruiting bodies released their content of asci and paraphyses together. Asci in all stages of development appeared.

The number of asci per ascocarp varied between 1 and 39 with a mean of 16 . One ascocarp with 50 asci was observed. The number of asci with spores varied between 1 and 9 per ascocarp. The number of ascospores per ascus varied from 1 to 8, with a mean of 5. Similar to descriptions by Drechsler (1934) and Tinline (1951), the shape of the asci was subcylindrical to clavate, slightly curved and shortstipitate.

When the ascus was filled with spores, they were always coiled in a tight helix, the typical characteristic of the genus Cochliobolus. Coiled spores discharged simultaneously through the ascus apex which ruptured circumscissally. The ascospores uncoiled gradually in water. Asci contained any number of spores from one to eight. When an ascus contained one ascospore only, this one was bent or positioned irregularly within the ascus. Nonseptate spores were hyaline; mature spores somewhat olivaceous. They were long, filiform and with many septa. Constrictions at the septa were visible, especially at or prior to germination. Mature ascospores broke up easily into segments which germinated individually.

Single septated ascospores quickly germinated on PDA and after 2 to 3 hours the developing germtube was visible. Nonseptate ascospores did not germinate. Spores not liberated from within the ascus germinated in situ through the ascus wall on PDA.

Table 2. Comparison of measurements of C. sativus naturally occurring versus laboratory produced teleomorph (units in $\mu \mathrm{m}$ )

\begin{tabular}{|l|r|r|r|r|}
\hline & \multicolumn{2}{|c|}{ C. sativus in nature } & \multicolumn{2}{c|}{ C. sativus in laboratory ${ }^{1}$} \\
& \multicolumn{2}{|c|}{ mean } & \multicolumn{1}{c|}{ Tinline 1951} \\
\hline ascocarp width & $240-660$ & $(440)^{2}$ & $310-460$ & $370-530$ \\
ascocarp height & $180-576$ & $(383)$ & $270-420$ & $340-470$ \\
beak width & $50-144$ & $(85)$ & $90-140$ & $80-110$ \\
beak length & $40-90$ & $(69)$ & $66-190$ & $90-150$ \\
ascus length & $43-259$ & $(162)$ & $160-255$ & $110-220$ \\
ascus width & $10-39$ & $(27)$ & $27-42$ & $32-45$ \\
ascospore length & $113-420$ & $(220)$ & $182-343$ & $160-360$ \\
ascospore width & $7-15$ & $(9)$ & $5-10$ & $6-9$ \\
number of septa & $3-11$ & $(7)$ & $6-14$ & $6-13$ \\
\hline
\end{tabular}


Table 2 compares the measurements of ascocarps, asci and ascospores of $C$. sativus occurring in nature with those recorded by Ito and Kurib (Tinline 1951) of artificially produced teleomorphs made by mating compatible fertile strains under controlled laboratory conditions.

In general, the observations show the species to have a wider range of measurements than previously recorded. Some ascocarps found in nature were smaller while others were larger than those produced in the laboratory. Longer beaks were recorded on the laboratory produced teleomorphs. The ascus measurements include both mature and immature asci. Immature asci varied in length between 50 and $250 \mu \mathrm{m}$ and in width between 10 and $38 \mu \mathrm{m}$. The length of mature asci fluctuated between 43 and $246 \mu \mathrm{m}$. In the range of 50 to $110 \mu \mathrm{m}$ only immature asci were observed. Most ascus lengths fell within the range covered by Tinline's and Ito's, but the asci were somewhat narrower than theirs. Most measurements of ascospore length and width were within the range observed by both authors. Some were wider and shorter; longer ascospores were observed as well. Nonseptate ascospores measured $134-276 \mu \mathrm{m}$ by 5-10 $\mu \mathrm{m}$. The maxima of 14 and 13 septa as observed by Tinline and Ito \& Kuribayashi respectively were not observed.

Monoascosporic cultures yielded Bipolaris sorokiniana with typical brown conidia and dark mycelium on PDA, identical to cultures from single conidia directly transferred from straw to the agar medium.

In the pathogenicity tests, all inoculations produced tiny brown specks within 48 hours. Within 4 days such a speck developed into a light brown elliptical lesion with a dark centre and a yellow halo. Six days after inoculations, small parts of the lesions were plated on PDA and B. sorokiniana was recovered.

\section{Discussion}

This is the first report of abundant formation in nature and annual re-occurrence in spring of the teleomorph of Cochliobolus sativus. A reference sample was deposited in 1990 at the International Mycological Institute under IMI-number 34112 and the fungus was confirmed as C. sativus (Sivanesan, personal communication). Moist chamber tests and plating of infected plant parts on agar media never yielded the teleomorph during the period 1974-90.

Tinline (1951) considered the slight differences between his measurements and those by Ito and Kurib. to be due to variant strains of the fungus, and environmental and cultural differences. The differences observed here between the laboratory produced and naturally occurring $C$. sativus may be attributable to different conditions during development in 'controlled environment' in Canada and Japan and 'normal weather conditions' in Zambia.

The occurrence of asci with fewer than 8 ascospores, also observed by others (Drechsler 1934, Nelson 1964, Tinline 1951), is attributed to the chromosomal behaviour in the developing ascus (Hrushovetz 1956). This may also explain why many asci were either empty or partially filled with granular protoplasma, at a time when most asci were expected to contain mature ascospores.

The presence of the perfect stage in central and northern Zambia at locations $1,000 \mathrm{~km}$ apart and the severity of $B$. sorokiniana infections in rainfed wheat experiments in central Zimbabwe (Raemaekers 1987) are an indication that the teleomorph could develop abundantly in a large region of southern Africa, if a susceptible crop such as summer wheat is introduced.

Tinline and Dickson (1958) reported that the formation and maturation of $C$. 
sativus was greatly affected by temperature, requiring more precise environmental conditions than the vegetative stage. Sexually the fungus developed only on natural media between 16 and $24^{\circ} \mathrm{C}$ with the optimum at $20^{\circ} \mathrm{C}$, whereas asexually it grew on natural and synthetic media between 4 and $36^{\circ} \mathrm{C}$ with optimum growth at $28^{\circ} \mathrm{C}$. Shoemaker (1955) investigated the temperature range and observed that the formation of male and female reproductive organs in C. sativus took place at 24 and $20^{\circ} \mathrm{C}$ respectively. Beyond these temperatures no fertilisation took place and only protothecia developed. Similar temperatures as those at which the teleomorph was produced in the laboratory occur in Zambia during spring and early summer.

C. sativus and other Cochliobolus species are heterothallic (Nelson 1957, Tinline 1951). Opposite mating strains are randomly distributed in nature, and may even occur on the same lesion (Nelson 1957). The dense concentration of Bipolaris sorokiniana on wheat straw in Zambia therefore is thought to contain both mating strains.

What the implications are of finding the complete life cycle of Cochliobolus sativus in nature in Zambia is not yet clear. It was demonstrated that it can serve as primary inoculum along with conidia from debris. Its role will be better understood when rainfed wheat production expands and wheat-wheat rotations are introduced. Production of more virulent $C$. sativus strains is not excluded.

\section{References}

Drechsler C. 1934. Phytopathological and taxonomic aspects of Ophiobolus, Pyrenophora, Helminthosporium, and a new genus, Cochliobolus. Phytopathology 24: 953-983.

Hrushovetz S.B. 1956. Cytological studies of ascus development in Cochliobolus sativus. Can. J. Bot. 34: 641-651.

Meteorological Department. 1975. Climatological summaries for Zambia. Periods ending December 1970. Meteorological Department Lusaka. Government Printer, Lusaka, Zambia. 59 pp.

Nelson R.R. 1957. Heterothallism in Helminthosporium maydis. Phytopathology 47: 191-192.

Nelson R.R. 1964. The perfect stage of Helminthosporium cynodontis. Mycologia 56: 64-69.

Raemaekers R. 1987. Annual report 1986. Rainfed and irrigated wheat pathology-breeding. Belgian Development Cooperation. Mount Makulu Research Station, Zambia. 138 pp.

Raemaekers R. 1991. Contribution to the epidemiology of Bipolaris sorokiniana diseases and the development of rainfed wheat, a new crop in Zambia. Ph.D. thesis nr. 205. Dissertationes de Agricultura. Faculteit der Landbouwwetenschappen, Katholieke Universiteit Leuven. 136 pp.

Raemaekers R.H., Nawa I.N., Chipili J. and Sakala A. 1991. Revised checklist of plant diseases in Zambia. Agricultural Editions nr.18, General Administration for Development Cooperation, Brussels. $119 \mathrm{pp}$.

Shoemaker R.A. 1955. Biology, Cytology, and Taxonomy of Cochliobolus sativus. Can. J. Bot. 33: $562-$ 576.

Sivanesan A. 1987. Graminicolous species of Bipolaris, Curvularia, Drechslera, Exserohilum and their teleomorphs. C.A.B. International Mycological Institute (CMI). 261 pp.

Tinline R.D. 1951. Studies on the perfect stage of Helminthosporium sativum. Can. J. Bot. 29: 467-478.

Tinline R.D. and Dickson J.G. (1958). Cochliobolus sativus. I. Perithecial development and the inheritance of spore color and mating type. Mycologia 50(5): 697-706. 


\title{
PYXIDIOPHORA, A POSSIBLE TELEOMORPH OF PLEUROCATERA ACICULARIS
}

\author{
GÜNTER R.W. ARNOLD
}

Friedrich-Schiller Universität Jena, Pilzkulturensammlung, Freiherr-Vom-Stein-Allee 2, D-9925, Germany

Abstract. In old cultures of the recently re-discovered hyphomycete Pleurocatena acicularis mature perithecia were found which doubtless are the teleomorph of this fungus and belongs to the genus Pyxidiophora. Until now a connection between Pyxidiophora and Pleurocatena was unknown.

\section{Introduction}

Establishing connections between anamorphs and teleomorphs, or to find a teleomorph of an existing anamorph is one of the tasks even of the modern mycology. There are various methods and techniques to gain positive results, as was pointed out by Kendrick (1979). The factor of a lucky chance should not be forgotten. It was indeed one lucky chance which led us to a surprising possible connection. This "possible" should be underlined, because the result until now was impossible to repeat. The fact is that in our laboratory the teleomorph of Pleurocatena acicularis was discovered.

\section{Material and Methods}

A pure culture of the hyphomycete Pleurocatena acicularis was obtained from conidia from the natural host and subcultured.

The fungus was isolated from a minute colony on Hymenochaete tabacina, covered partially with Hypocreopsis lichenoides, collected by Björn Brandt on Salix aurita, Germany, Mecklenburg-Vorpommern, between Bad Sülze and Triebsees, 15.03.1992, and identified by G.R.W. Arnold (A 92/39.2 = MW i 3147). The fungus, surely, is mycophilic.

The growth in liquid Czapek medium is almost zero. Subcultures were obtained on malt-agar (MA), oat-meal-agar (OMA) and synthetic nutrient agar (SNA) after Nirenberg, incubated in the incubator-room at $21 \pm 1^{\circ} \mathrm{C}$ and a relative humidity of $76 \%$. The growth of the fungus was checked at great intervals (4-6 weeks).

\section{Results}

$P$. acicularis proved to be a highly difficult fungus. The mycelial growth was, in general on all three employed media, slow and scanty; the best growth was observed on MA, the poorest on SNA. A thin, whitish superficial mycelium was developed. Conidiation, characteristic for P. acicularis (Fig. 1), was satisfaying on MA, not so good on OMA, and rare on SNA. But after a period of four months small darkish points were detected with naked eye, which under the dissecting microscope were identified as ascomata! A contamination of Pleurocatena with an ascomycete was to be excluded. Therefore, the observed ascomycete had to be connected with Pleurocatena acicularis. The perithecia were scattered over the elder parts of the colony which at this time covered almost completely the surface of the agar slant in a $10 \mathrm{~cm}$ Petri dish; they were superficial, comparatively simple, slender and elongated piriforme, almost translucent, with a fimbriate neck (Fig. 2). Asci were not seen. The ascospores were very peculiar and characteristic: long guttuliforme, with a broader 
apex and an attenuated basal part, thinwalled, hyaline, two-to multicelled. In some cases a dark eye-spot could be observed in the upper part of the ascospore (Fig. 3).

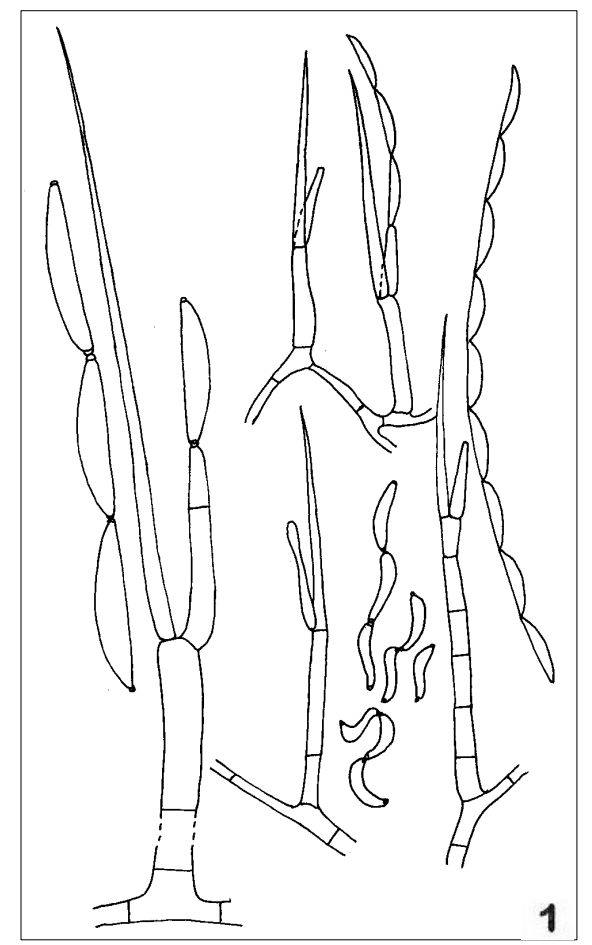

Fig.1. Pleurocatena acicularis: conidiophores and conidia

Until now, the existence of a teleomorph-connection of Pleurocatena acicularis was unknown. On the basis of tho described characters, it is proposed to place this ascomycetous fungus in the genus Pyxidiophora, tentatively. It certainly differs from the described species of Pyxidiophora which have anamorphs belonging to the genera Chalara and Thaxteriola (Lunquist 1980, Blackwell et al.1986).

\section{Conclusions}

The formation of a teleomorph of the recently rediscovered and cultivated hyphomycete Pleurocatena acicularis (Arnold 1995) was an unexpected surprise. Attempts have to be made to improve the conditions for growth of the fungus, to repeat the production of perithecia based on mono-conidial and mono-ascosporic cultures, to study the physiology of nutrition, biochemistry, a possible antibiotic activity of the fungus, and its ontogeny and cytology. 

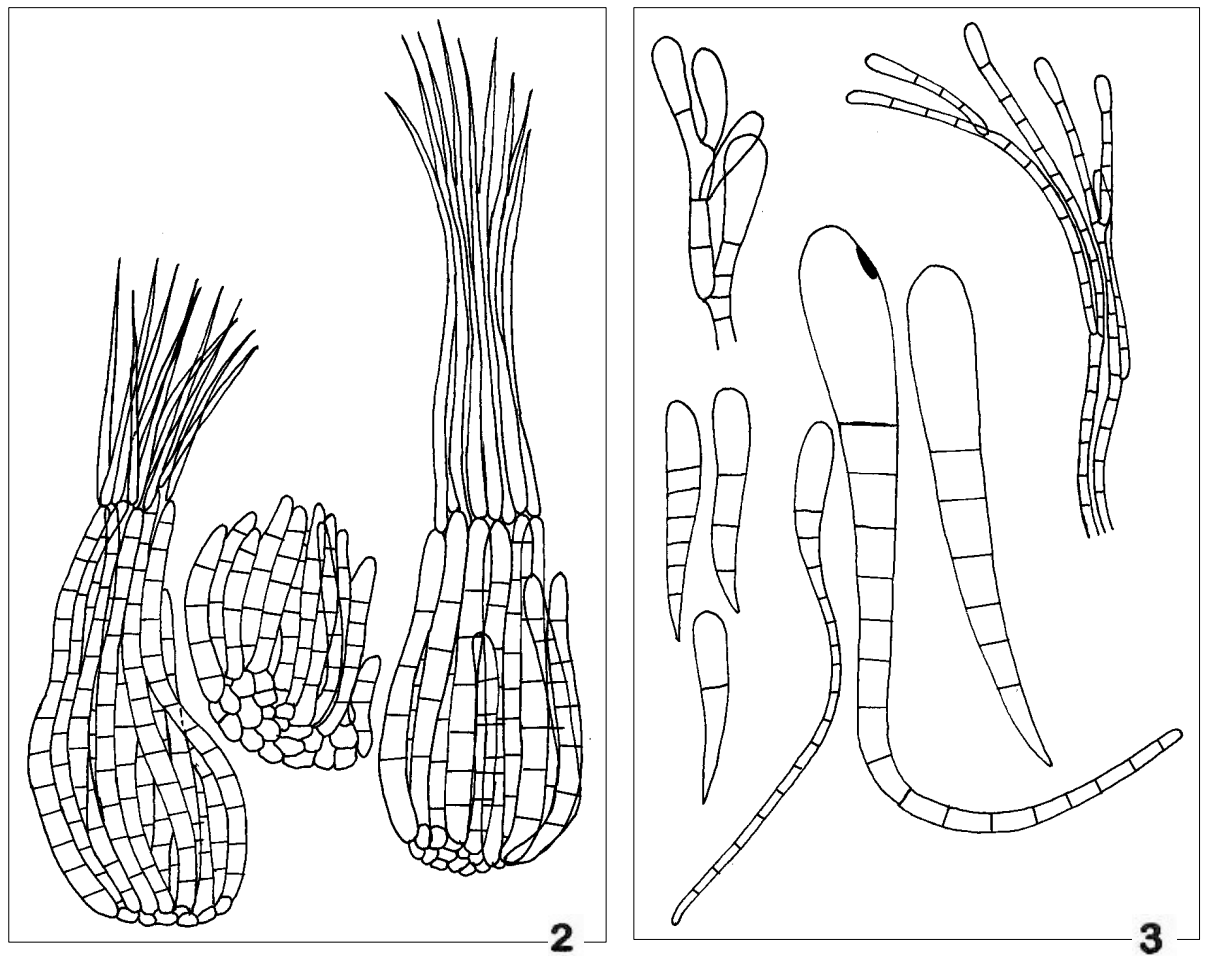

Fig.2. Pyxidiophora teleomorph of Pleurocatena acicularis: three stages of perithecia

Fig.3. Pyxidiophora teleomorph of Pleurocatena acicularis: ascospores of different kinds, and"Hullhyphen" of the earlier stages of perithecial development

\section{References}

Arnold G.R.W. 1995. Pleurocatena acicularis, ein seltener, wiederentdeckter Hyphomyzet. Feddes Repertorium.

Blackwell M. Perry T.J., Bridges J.R. and Moser J.C. 1986. A new species of Pyxidiophora and its Thaxteriola anamorph. Mycologia 78: 605-612,.

Kendrick W.B, Samuels G.J., Webster J. and Luttresll E.S. 1979. Techniques for establishment connections between Anamorph and Teleomorph. In The Whole Fungus, Kendrick, B. ed., National Museum of Natural Sciences, National Museums of Canada, Ottawa, and The Kananaskis Foundation 2: $635-651$.

Lundquist N. 1980. On the genus Pyxidiophora sensu lato (Pyrenomycetes). Botaniska Notiser 133: 121144. 


\title{
ILLUSTRATIONS OF THE CULTURAL FEATURES OF GYRODONTIUM BOVEANUM (Montagne) MaAs Geesteranus
}

\author{
CONY DECOCK* AND ANXIOUS J. MASUKA** \\ * Mycothèque de l'Université catholique de Louvain. Louvain-la-Neuve, Belgium \\ ** Forest Research Center,P.O. Box HG 595,Highlands, Harare, Zimbabwe
}

Abstract. Main cultural features of Gyrodontium boveanum (Montagne) Maas Geesteranus are illustrated on the basis of two cultures which recently became available to the authors. One was obtained from a specimen of Gyrodontium boveanum (Montagne) Maas Geesteranus collected by the authors in the small wet forest surrounding the Victoria Falls (Zimbabwe), inside an empty dead trunk. The second was kindly provided by the IFO, Osaka, Japan, under the name Gyrodontium versicolor (Berkeley \& Broome) Maas Geesteranus.
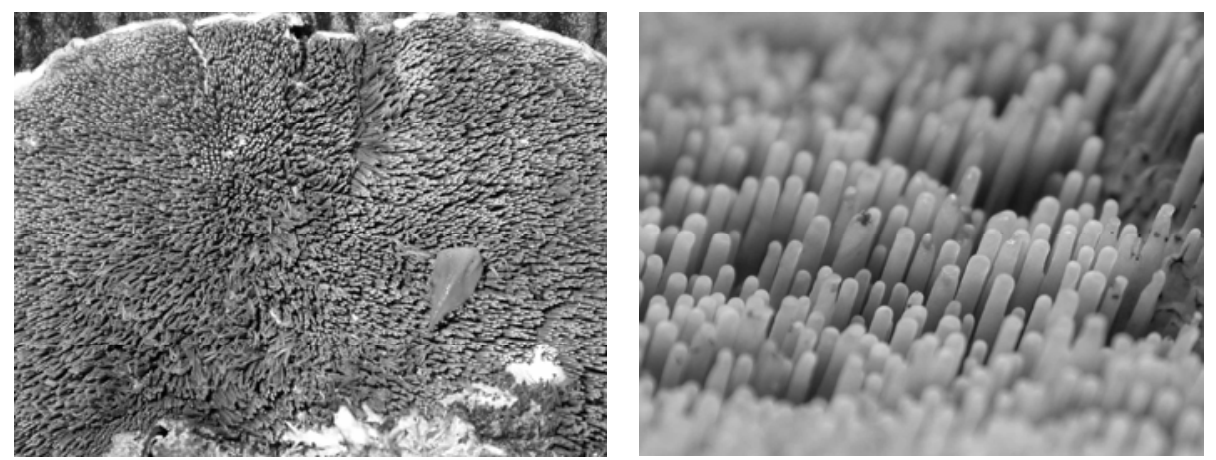

In culture on Malt Agar $2 \%$ or on Cherry decoction agar at $25^{\circ} \mathrm{C}$, both strains produce arthroconidia. Those arthroconidia rapidly become thick walled, turn yellow brown and round off, soon disjoining and forming a brown powdery layer at the colony surface. Fresh cultures obtained from teeth produced fruitbodies in Petri dishes but, after several subculturing, new fruitbody formation were no more observed. Thin strands are also formed.
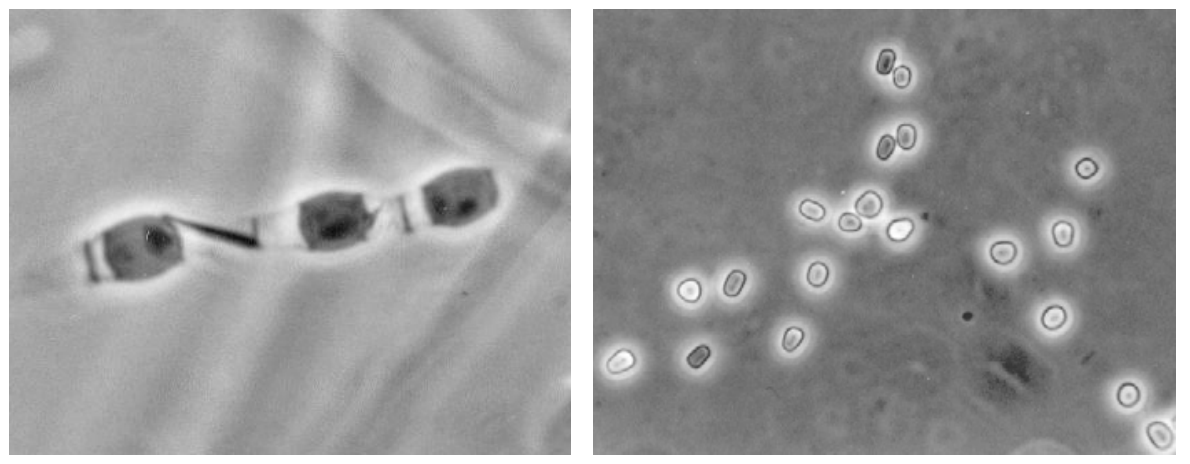
Several authors have reported the occurrence of yellow brown thick walled spores in the flesh of Gyrodontium basidiocarp, sometimes forming a dense brown powdery layer (Reid 1963, Maas Geesteranus 1964). Although they interpreted such spores either as chlamydospores or as basidiospores, owing to their close resemblance to true basidiospores, both authors were doubtful about their origin. Study of pure culture allowed to determinate the nature of these spores.

\title{
References
}

Reid D.A. 1963. New or interesting records of Australasian Basidiomycetes: V, Kew Bull. 17: 267-308. Maas Geesteranus R.A. 1964. Notes on Hydnums II, Persoonia 3: 155-192.

\section{THE LACTARIUS GYMNOCARPUS COMPLEX IN TROPICAL AFRICA}

\author{
A. VERBEKEN
}

University of Ghent, K.L. Ledeganckstraat 35, B-9000 Gent, Belgium

Abstract. The Lactarius gymnocarpus group has been studied as part of a revision of the genus Lactarius in tropical Africa.

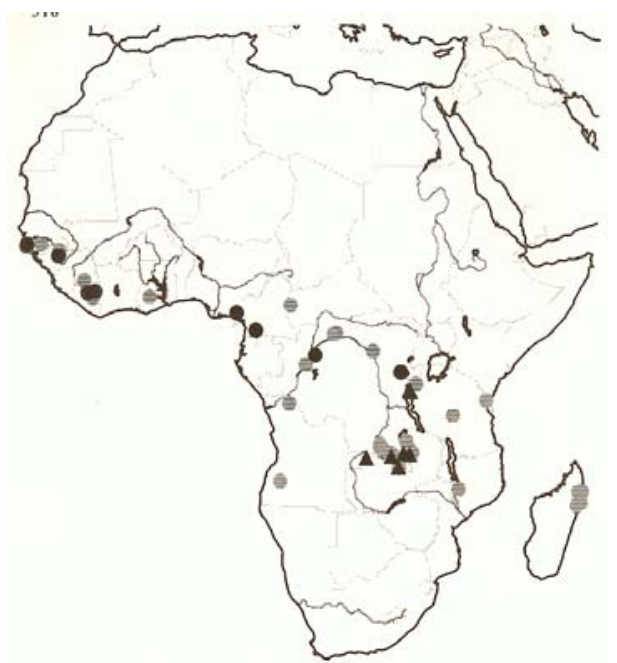

Fig. Collecting sites of Lactarius gymnocarpus $(\bullet)$ and L. longisporus $(\mathbf{\Delta})$ and other Lactarius species (०).

Detailed study of morphological and anatomical characters combined with ecological data of 99 collections of so called or close to Lactarius gymnocarpus Heim maily from African miombo woodland lead us to recognize 6 new species to Lactarius gymnocarpus. They are L. pseudogymnocarpus, L. gymnosporus, L. gymnocarpoides, L. luteopus, L. medusae and L. flammans. The diagnostic differences are illustrated by drawings, photographs and distributions maps. 


\title{
SCORIAS SPONGIOSA (SCHW.)FRIES FROM KENYA
}

\author{
R. MIBEY
}

University of Nairobi, Department of Botany, P.O. Box 30197 Nairobi, Kenya

Abstract. This paper describes the "sooty mould" fungus, Scorias spongiosa (Schw.) Fries from Kenya usually found associated with scale insects of the genus Coccus. The fungus is found mainly during dry weather conditions.

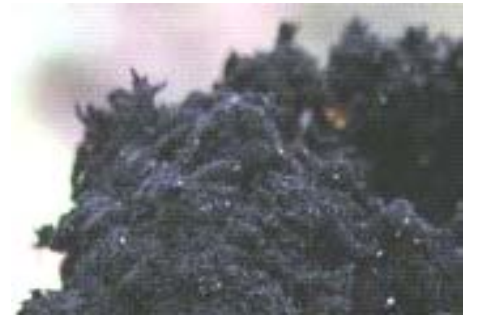

\section{AN INTERESTING SPECIES OF PUCCINIA ON GENTIANA}

EVANGELIA KAPSANAKI-GOTSI AND I. VOVOU

University of Athens, Department of Biology, Section of Ecology and Systematics, Panepistimiopolis, 157 84 Athens, Greece

Abstract. An interesting specimen of Puccinia living on Gentiana cruciata L. has been collected near Vikos gorge, at Ipiros, Greece. A detailed study of its morphological characteristics, revealed some remarkable differences from the known species of Puccinia on Gentiana. It seems to be closely related to Puccinia gentianae (F. Strauss) Rohl., which is widespread in the northern hemisphere, but unknown from Greece. A comparative study of our specimen with authentic material of $P$. gentianae is necessary, for the determination of its exact taxonomic position.

It is the first time that the genus Gentiana is reported from Greece, as a host of a rust fungus.

\section{CULTURAL CHARACTERISTICS AND THEIR TAXONOMIC POTENTIAL IN LENTINELLUS SPECIES}

ZAPI GONOU-ZAGOU AND MARIA PANTIDOU

University of Athens, Department of Biology, Section of Ecology and Systematics, Panepistimiopolis, 157 84 Athens, Greece

Abstract. Mycelial cultures of three species of the genus Lentinellus Karsten have been studied. The cultural, macroscopical and microscopical, as well as cytological characters of the species and their ability to produce polyphenol oxidases are presented. The aim of this study is not only to give the culture features of the three species but also to get additional viewpoints for a better understanding of the 
taxonomic relationships within the genus Lentinellus. Thus there is a taxonomic approach in which the study of cultures is intended to contribute to the evaluation of fungal relationships.

\title{
RESPONSES TO THE THERMAL STRESS IN THE INTERSTERILITY GROUPS OF GANODERMA AUSTRALE IN TAIWAN
}

\author{
ZUEI-CHING CHEN \\ Department of Botany, National Taiwan University, Taipei, Taiwan 10764, R.O.C.
}

Abstract. The diversity of responses to thermal stress of two "intersterility groups" of Ganoderma australe (Fr.) Pat. in Taiwan was studied and its impact on the migration of tropical fungi to the temperate region in Asia was discussed. The intersterility Group 1 (isolate TAI-01) is a tropical strain distributed in the lowland area of Taiwan and has a higher optimum growth temperature range i.e., $28^{\circ}-32^{\circ} \mathrm{C}$ than that of Group 2. The tropical strain responded to $40^{\circ} \mathrm{C}$ heat shock treatment by synthesizing seven heat shock proteins with mol. wt. of $13.5-98 \mathrm{kDa}$. The intersterility Group 2 (isolate TAI-05) is distributed in the high land area of $1.650 \mathrm{~m}$ altitude of Taiwan and has an optimum growth temperature range of $24^{\circ}-28^{\circ} \mathrm{C} \mathrm{but}$ showed no response to heat shock. Instead, it responded to cold shock by synthesizing a cold shock protein of $41 \mathrm{kDa}$. Evidently the Group 2 of G. australe in Taiwan has been reproductively isolated from Group 1 and has adapted to the habitat of the temperate climate and is a temperate strain. The migration of tropical fungi into the temperate zone requires a speciation process and an induction of coldshock proteins. The subtropical island Taiwan can provide an excellent bridge for the floristic migration between the Torrid and the Temperate species of fungi in Asia. 
MOLECULAR TAXONOMY

\title{
18S RRNA AND THE FUNGAL TREE: EVOLUTIONARY RELATIONSHIPS AMONG ASCOMYCETES, BASIDIOMYCETES, ZYGOMYCETES, AND CHYTRIDIOMYCETES.
}

\author{
Yves VAN DE PEER ${ }^{1}$, Grégoire L. HENNEBERT ${ }^{2}$, Rupert DE WACHTER ${ }^{1}$ \\ ${ }^{1}$ Departement Biochemie, Universiteit Antwerpen (UIA), Universiteitsplein 1, B-2610 Antwerpen, \\ ${ }^{2}$ Mycothèque de l'Université Catholique de Louvain, B-1348 Louvain-la-Neuve, Belgium
}

\section{Introduction}

A valuable and reliable classification of organisms should ideally be based on their natural evolutionary relationships. Although morphology is an inevitable tool for the description of a species, drawing conclusions about its genealogy from morphological or biochemical characteristics is far more difficult. This is especially true for prokaryotes, but also for eukaryotes with simple morphology, like yeasts. But even for organisms with a more pronounced morphology, there can be misinterpretation of the data due to convergent evolution and disagreement about phenotypic characteristics being ancestral or derived.

However, the evolutionary history of an organism is recorded in its genes and the development of sequence analysis techniques has allowed reconstruct biological evolution by comparing the structure of genes or gene-products in different species. One of the molecules exceedingly suited for this purpose is the small ribosomal subunit RNA (further abbreviated as SSU rRNA) or 18S rRNA (in eukaryotes). SSU rRNA, present in all living organisms, as well as in organelles like plastids and mitochondria, has a conserved structure and an average chain length of about 1800 nucleotides in eukaryotes. As a consequence of its popularity in the construction of evolutionary trees, over 3000 complete (or nearly complete) sequences have been determined. They are recorded in our SSU rRNA database, which is made available to the scientific community (Neefs et al. 1993). About 700 of these sequences are from eukaryotes and about 165 of these are from "true" fungi, covering a wide range of different genera and families.

\section{Results}

Figure 1 shows an evolutionary tree based on all SSU rRNA sequences known to date of ascomycetes, basidiomycetes, zygomycetes, and chytridiomycetes. Only one rRNA sequence is included if several are determined for the same species, usually by different authors. Dissimilarity, being the fraction of substitutions between two sequences, was converted into evolutionary distance by using the equation of Jukes and Cantor (1969), which corrects for multiple mutations per site. The tree topology was inferred by the neighbour-joining method of Saitou and Nei (1987). The complete alignment was used for tree construction. Confidence values for individual branches were determined by a bootstrap analysis in which 100 bootstrap trees were generated from resampled data (Felsenstein 1985). Distance calculation, tree construction and bootstrap analysis were performed with the software package TREECON (Van de Peer and De Wachter 1993). 


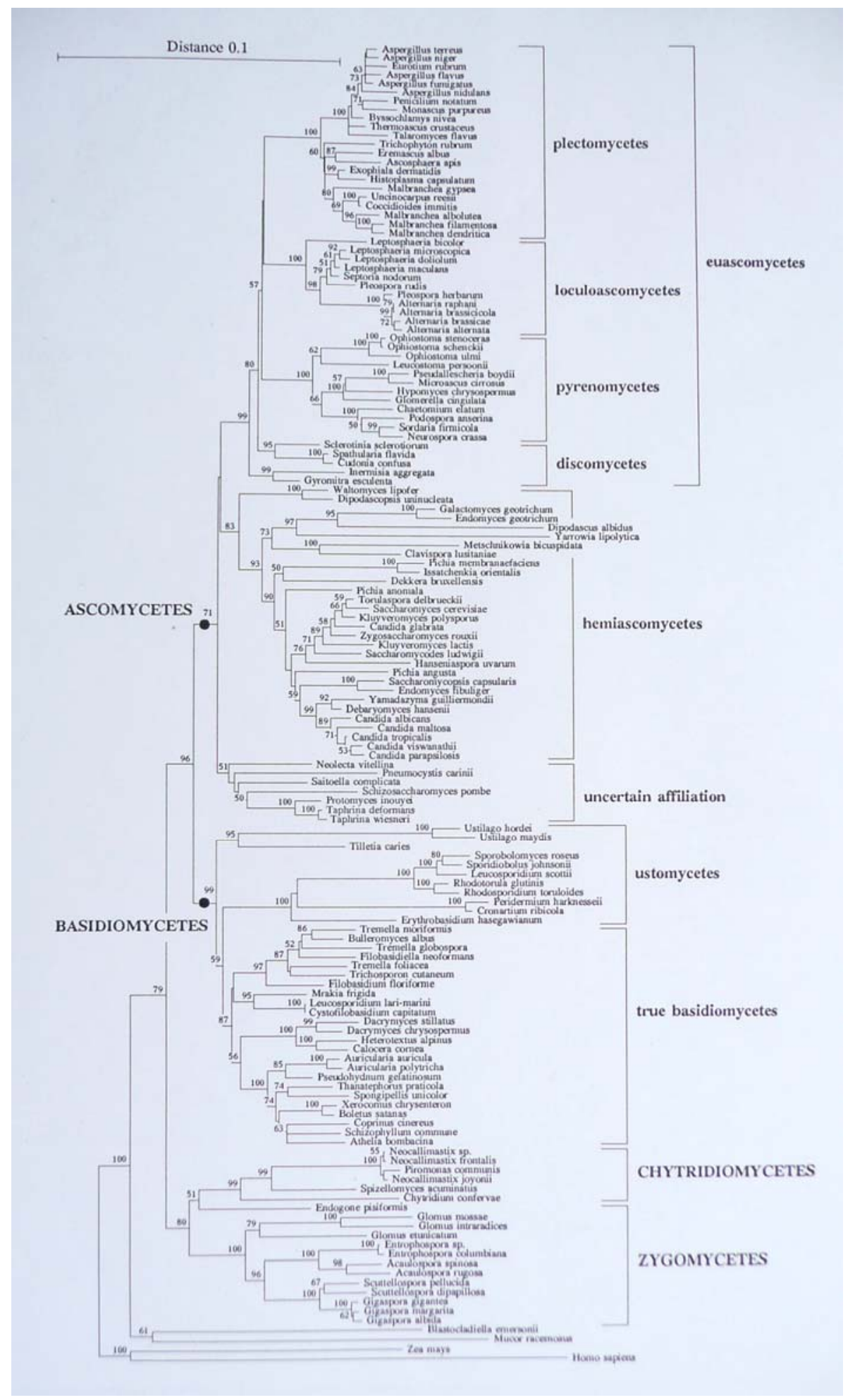

148 
Fig.1. Evolutionary tree based on 145 SSU rRNA sequences of chytridiomycetes, zygomycetes, ascomycetes, and basidiomycetes. Zea mays and Homo sapiens were included as outgroup organisms. The distance between two organisms or groups of organisms, measured in substitutions per nucleotide, is obtained by summing the lengths of the connecting branches along the horizontal axis, using the scale on top. Bootstrap percentages higher than $50 \%$ are placed alongside the node considered.

\section{Phylogeny of Chytridiomycetes and Zygomycetes}

In the tree of Fig. 1, the chytridiomycete Blastocladiella emersonii and the zygomycete Mucor racemosus form the first lines of divergence within the fungi. Next there is a splitting of, on the one hand, Ascomycetes and Basidiomycetes, and on the other hand, the remaining Zygomycetes and Chytridiomycetes. Thus, based on this result, the Chytridiomycetes as well as the Zygomycetes seem polyphyletic. However, since both Blastocladiella and Mucor form long branches because of a higher evolutionary rate, their divergence should be interpreted with caution. It is a known phenomenon that long branches can be pulled to some extent towards the root of the tree (Olsen 1988), because of an underestimation in the Jukes-Cantor conversion of dissimilarity into evolutionary distance (Golding 1983). Furthermore, until now, only one representative is sequenced for the Blastocladiales and for the Mucorales and it is possible that addition of more representatives of these orders may change and stabilize their respective position in the tree. Blastocladiella and Mucor not considered, Zygomycetes and Chytridiomycetes are clustered together and seem to form a monophyletic lineage, supported at a fairly high bootstrap level (80\%). In this tree, the zygomycete Endogone pisiformis is clustered with the Chytridiomycetes, as also noticed by Bruns et al. (1992). However, its position is unstable (only supported in $51 \%$ of the bootstrap samples) and depends on the composition of the dataset.

\section{Phylogeny of Ascomycetes}

Basidiomycetes and Ascomycetes clearly form two separate monophyletic groups based on SSU rRNA data (Bruns et al. 1992, Van de Peer et al. 1992, Wilmotte et al. 1993, this study). Furthermore they are sister groups supported at a high bootstrap level (96\%). The Ascomycetes are subdivided into two major lineages: the Euascomycetes and the Hemiascomycetes. Within the Euascomycetes, three clearly separated groups can be distinguished, viz. Plectomycetes, Loculoascomycetes, and Pyrenomycetes, while the Discomycetes are divided over two different lineages. Overall, this subdivision of the Euascomycetes agrees well with classical taxonomic views.

The Hemiascomycete cluster consists of yeasts and yeast-like fungi, most of which were formerly classified as Deuteromycetes. Based on SSU rRNA it is clear that some of these yeast genera like Candida and Pichia, are heterogenous and it would be meaningful to reconsider their taxonomy. A detailed discussion of many of the species included in both the Euascomycete and the Hemiascomycete cluster is given in Wilmotte et al. (1993).

Within the Ascomycetes, a third cluster, although not supported by bootstrap analysis, is formed by the genera Neolecta, Pneumocystis, Taphrina, Schizosaccharomyces, Protomyces, and Saitoella. The exact taxonomic position of these species is not clear and deserves further attention.

\section{Phylogeny of Basidiomycetes}

Within the Basidiomycetes, three main lineages can be discerned, two leading to 
the Ustomycetes and one leading to the true Basidiomycetes, two taxa created by Moore (1972) on the basis of differences in septal ultrastructure and life cycle. According to this tree and some previously published trees based on SSU rRNA (e.g. Wilmotte et al. 1993), the Ustomycetes seem to be paraphyletic, although this finding is not supported at a high level by bootstrap analysis.

\section{Acknowledgements}

This work was supported by the Federal Office for Scientific, Cultural and Technical Matters of the Belgian State, by the Programme on Interuniversity Poles of Attraction (contract 23) of the Belgian State, and by the F.K.F.O.

\section{References}

Bruns T.D., Vilgalys R., Barns S.M., Gonzales D., Hibbett D.S., Lane D.J., Simon L., Stickel S., Szaro T.M., Weisburg W.G. and Sogin M.L. 1992. Evolutionary relationships within the fungi: analysis of nuclear small subunit rRNA sequences. Mol. Phyl. Evol. 1:231-241.

Felsenstein J. 1985 Confidence limits on phylogenies: an a pproach using the bootstrap. Evolution 39: 783-791.

Golding G.B. 1983. Estimates of DNA and protein sequence divergence: an examination of some assumptions. Mol. Biol. Evol. 1: 125-142.

Jukes T.H. and Cantor C.R. 1969. Evolution of protein molecules. In: Mammalian Protein Metabolism, Munro H.N. ed., Academic Press, New York pp 21-132.

Moore R.T. 1972. Ustomycota, a new division of higher fungi. Antonie v. Leeuwenhoek 38: 567-584.

Neefs J.-M., Van de Peer Y., De Rijk P., Chapelle S. and De Wachter R. 1993 Compilation of small ribosomal subunit RNA structures. Nucl. Acids Res. 21: 3025-3049.

Olsen G.J. 1988. Phylogenetic analysis using ribosomal RNA. Methods in Enzymology 164: 793-812.

Saitou N. and Nei M. 1987. The neighbor-joining method: a new method for reconstructing phylogenetic trees. Mol. Biol. Evol. 4: 406-425.

Van de Peer Y., Hendriks L., Goris A., Neefs J.-M., Vancanneyt M., Kersters K., Berny J.-F., Hennebert G.L. and De Wachter, R. 1992 Evolution of basidiomycetous yeasts as deduced from small ribosomal RNA sequences. System. Appl. Microbiol. 15: 250-258.

Van de Peer Y.and De Wachter R. 1993. TREECON: a software package for the construction and drawing of evolutionary trees. Comput. Applic. Biosci. 9: 177-182.

Wilmotte A., Van de Peer Y., Goris A., Chapelle S., De Baere R., Nelissen B., Neefs J.-M., Hennebert G.L. and De Wachter R. 1993. Evolutionary relationships among higher fungi inferred from small ribosomal subunit RNA sequence analysis. System. Appl. Microbiol. 16: 436-444. 


\title{
A RAPID MOLECULAR TECHNIQUE TO DISTINGUISH CYLINDROCLADIUM SPECIES
}

\author{
P.W. CROUS ${ }^{1}$, A. KORF ${ }^{2}$ AND W.H. VAN ZYL ${ }^{2}$ \\ Departments of ${ }^{1}$ Plant Pathology and ${ }^{2}$ Microbiology, University of Stellenbosch, South Africa
}

\section{Introduction}

In a recent monograph of Cylindrocladium Morgan, Crous and Wingfield (1994) recognized 22 species and 2 varieties. Four of these species, namely C. clavatum Hodges \& May, C. pteridis Wolf, C. gracile (Bugn.) Boesewinkel and C. hawksworthii Peerally are known to have 1-septate conidia and thin-walled stipe extensions terminating in a clavate vesicle. C. hawksworthii can easily be distinguished in having curved conidia, whereas those of C. clavatum, C. gracile and C. pteridis are straight. The latter three species have similar temperature requirements for growth, and are primarily distinguished on cultural characteristics and dimensions of conidia and stipe extensions. These species represent a range in size of conidia and stipe extensions from the smaller C. clavatum and C. gracile to the larger C. pteridis (Crous and Wingfield 1994). Calonectria teleomorphs have been described for C. pteridis, C. gracile and recently also for C. clavatum (Crous et al. 1993b, El-Gholl et al. 1993). However, an examination of the type strains of Cylindrocladium clavatum and Calonectria clavata Alfieri et al. found them to be morphologically distinct.

Complementing alpha and beta taxonomy, several molecular techniques have recently been employed in Cylindrocladium, ranging from total protein and isozyme banding patterns to DNA restriction fragment length polymorphisms (RFLPs) (Crous et al. 1993a, 1993c). These techniques proved to be time consuming, and in the case of proteins, often influenced by host and geographical variation. It has been shown, however, that DNA RFLPs can clearly distinguish variation between and among species, and therefore substantially enhance attempts to allocate isolates in Cylindrocladium (Crous et al. 1993a). In the Hypocreales, ribosomal DNA (rDNA) restriction fragment length polymorphisms (RFLPs) have been successfully used as a rapid technique to demonstrate species-specific differences in Fusarium Link (Lodolo et al. 1992). The aim of the present study, therefore, was to use this technique to establish variation among the four Cylindrocladium species discussed above, and to determine the correct taxonomic position of the type strain of Calonectria clavata.

\section{Morphological characterization}

Material and Methods

The following type and verified strains of Cylindrocladium and Calonectria spp. were studied: Cylindrocladium clavatum (PPRI $3994=$ CPC 328), (ATCC 22833, ex type strain); Calonectria clavata $(078-1261=$ ATCC 66388 and 078-1543 = ATCC 66389, ex type strains); Cylindrocladium gracile (PC 551197, ex type strain); Calonectria gracilis Crous, Wingfieldand Alfenas (PPRI $4176=$ AR 2677, ex type strain), Cylindrocladium hawksworthii (MUCL 30866, ex type strain); Cylindrocladium pteridis (PPRI $4157=\mathrm{UFV} 43$ ). 
Single-conidial isolates were cultured on $2 \%$ malt extract agar (MEA) (Oxoid), plated onto carnation-leaf agar (CLA), incubated at $25{ }^{\circ} \mathrm{C}$ under near-ultraviolet light, and examined after $7 \mathrm{~d}$. Only material occurring on carnation leaves was examined. Mounts were prepared in lactophenol cotton blue, and measurements made at $1000 \times$ magnification.

\section{Chromosomal DNA isolation}

Single-conidial isolates were grown on MEA, and plugs of seven-day-old cultures transferred into $500 \mathrm{ml}$ Erlenmeyer flasks containing $100 \mathrm{ml}$ glucose-yeast extract broth (Biolab). Cultures were incubated for 7-14 d in the dark at $25{ }^{\circ} \mathrm{C}$ until sufficient growth occurred. Mycelia were harvested by filtration (Whatman No. 1 filter paper), the mycelial mat immersed into liquid nitrogen and chromosomal DNA isolated. Chromosomal DNA was subsequently redissolved in $200 \mu \mathrm{TE}$ buffer ( $\mathrm{pH}$ 8.0).

\section{Restriction enzyme analysis and Southern hybridization}

Chromosomal DNA (ca. $5 \mu \mathrm{g}$ ) of each isolate was subjected to restriction digestion with EcoRI, HindIII and XhoI for $3 \mathrm{~h}$ respectively, according to the recommendation of the suppliers (Boehringer Mannheim). The DNA was separated on horisontal $0.8 \%$ agarose gels and transferred to Hybond-N nylon membranes (Amersham) according to standard procedures. The Neurospora crassa rDNA was purified from plasmid pMF2 (Russell et al. 1984) as a $6.3 \mathrm{~kb}$ PstI fragment and labelled with [ $\$-32 \mathrm{P}] \mathrm{dATP}$ (Amersham). The Southern hybridizations and stringency washes were performed according to the method of Sambrook et al. (1989).

\section{Morphological characterization}

\section{Results}

All the species examined in the present study produced stipe extensions terminating in clavate vesicles. Based on conidium morphology on CLA, two groups could be distinguished. Conidia of C. clavatum, C. gracile and C. pteridis were straight, whereas those of $C$. hawksworthii and Calonectria clavata were curved. Conidia of Cylindrocladium clavatum were 38-52 x 4-6 $\mu \mathrm{m}$, overlapping with the slightly larger conidia of Cylindrocladium gracile (PC 551197), which were 40-56 x 3.5-5 $\mu \mathrm{m}$. Conidia of Calonectria gracilis were 40-65 x 4-5 $\mu \mathrm{m}$, overlapping to some degree with the lower range of Calonectria pteridis, which were 62-121 x 5-6 $\mu \mathrm{m}$. The same trend was also observed for their ascospores, those of C. gracilis being 1 - septate, (27-)36.5(-50) x (4-)5(-6) $\mu \mathrm{m}$, and those of C. pteridis 1(-3)septate, (30)-51.5(-75) $\times(4.5) 5.5(-7) \mu \mathrm{m}$. Of the two species with curved conidia, those of C. hawksworthii (42-76 x 4-4.5 $\mu \mathrm{m})$ were similar in length, but slightly narrower than those of Calonectria clavata $(50-80 \times 5-6 \mu \mathrm{m})$.

\section{Restriction enzyme analysis and Southern hybridization}

Using the Neurospora crassa rDNA probe five distinct EcoRI, XhoI and HindIII restriction patterns were obtained for the different strains (Figs. 1-3). The South African collection of Cylindrocladium clavatum (CPC 328) was similar to that of the type culture of C. clavatum (ATCC 22833). However, the type culture of C. gracile (PC 551197) could not be distinguished from that of C. clavatum. The two heterothallic isolates of Calonectria clavata were similar to each other, but distinct from that of Cylindrocladium clavatum, and all other species studied. The profile of 
Calonectria gracile (AR 2677) was distinct from Cylindrocladium gracile (PC 551197), and closer to that of Cylindrocladium pteridis.

\section{Discussion}

Type and verified strains of four Cylindrocladium species were investigated in this study. Three of these have been associated with Calonectria teleomorphs, and type strains of the latter were also included. Comparisons were done on the basis of morphology, culture characteristics and nuclear DNA polymorphisms.

\section{Cylindrocladium hawksworthii}

The species is known from two collections made in Mauritius (Peerally 1991). It is distinguished from other species in Cylindrocladium in having primarily clavate vesicles, and curved 1-septate conidia. The phenomenon of curved macroconidia in Cylindrocladium is known from C. curvatum Boedijn and Reitsma (1950) with sphaeropedunculate vesicles, and C. variabile Crous et al. (1993a), with sphaeropedunculate to ellipsoidal or clavate vesicles and (1-)3(-4)-septate conidia. The present study also found the two heterothallic strains of Calonectria clavata to have prominently curved macroconidia. The latter strains could, however, be distinguished from Cylindrocladium hawksworthii by their larger conidium dimensions and septation, as well as distinct rDNA restriction patterns.

\section{Cylindroladium clavatum and Cylindrocladium gracile}

C. clavatum was described by Hodges and May (1972) with conidia being 37.5$48 \times 3.5-5.5 \mu \mathrm{m}$. Bugnicourt (1939) described Cylindrocarpon gracile with conidial dimensions as being 24-48 x 2.5-4 $\mu \mathrm{m}$. Boesewinkel (1982) transferred Cylindrocarpon gracile to Cylindrocladium, and retained it as a separate species because of its longer stipe extensions and narrowly clavate vesicles.

Results obtained with rDNA restriction patterns for EcoRI, XhoI and HindIII in the present study clearly indicate, however, that the type culture of Cylindrocladium gracile (PC 551197) is indistinguishable from that of C. clavatum (ATCC 22833). The similarity in morphology and rDNA restriction pattern suggests therefore that the two species are synonymous. Because gracile is the older epithet, C. gracile Bugnicourt is to be accepted as the earlier name for this species.

\section{Calonectria pteridis (anam. Cylindrocladium pteridis) and Calonectria gracilis}

Of the 1-septate species with clavate vesicles, C. pteridis has the largest macroconidia, and frequently also forms curved microconidia in culture. In the present study curved microconidia have also been observed for Calonectria clavata. Similar to C. clavata, C. pteridis has also been found to be heterothallic, with successful matings producing the teleomorph in culture (Crous et al. 1993b).

A homothallic strain with conidial dimensions of 40-65 x 4-5 $\mu \mathrm{m}$ was recently obtained from Brazil. Because these dimensions overlapped considerably more with that of C. gracile than C. pteridis, and C. pteridis was known to be heterothallic with larger, 1(-3)-septate ascospores, the isolate was described as the Calonectria teleomorph of Cylindrocladium gracile (Crous et al. 1993b). As stated above, the present study found Cylindrocladium gracile to be distinct from Calonectria gracile, and synonymous with Cylindrocladium clavatum.

The rDNA restriction patterns obtained with EcoRI and HindIII showed only minor differences between Calonectria gracile and Calonectria pteridis. These two 
isolates could, however, easily be distinguished with the restriction enzyme XhoI. Based on the differences in the banding patterns, as well as conidium and ascospore dimensions, it would appear that Calonectria gracilis represents a distinct species between Cylindrocladium clavatum and Cylindrocladium pteridis. We are, however, of the opinion that additional isolates of Calonectria pteridis will have to be studied to suitably resolve the validity of Calonectria gracilis.
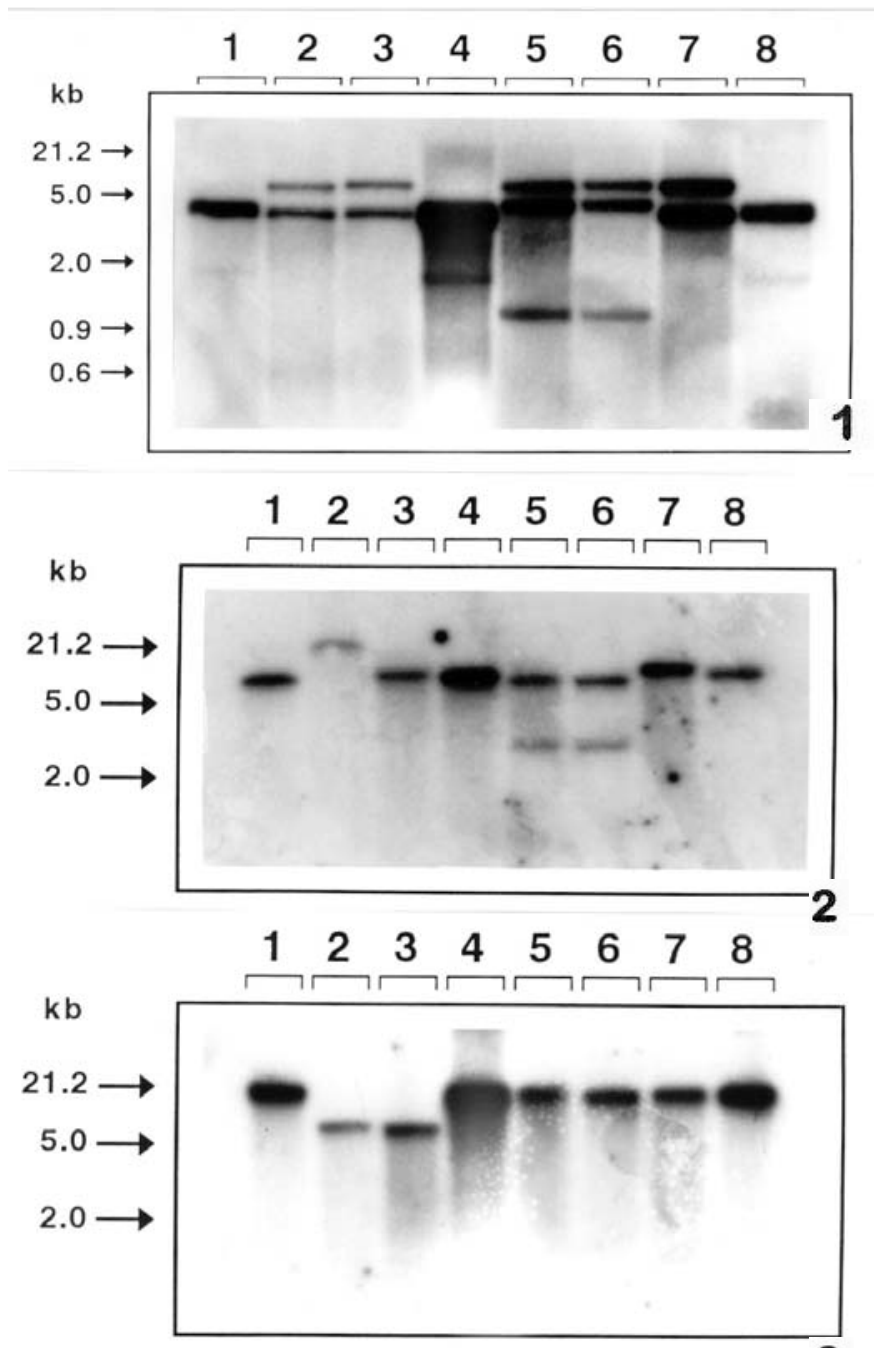

Fig.1. rDNA hybridization patterns for EcoRI-digested nDNA of strains of Cylin_..._ladium and Calonectria species. Lane 1: Cylindrocladium clavatum PPRI 3994. Lane 2: Calonectria gracilis PPRI 4176. Lane 3: C. pteridis PPRI 4157. Lane 4: Cylindrocladium gracile PC 551197. Lanes 5 and 6: Calonectria clavata ATCC 66388 and 66289. Lane 7: Cylindrocladium hawksworthii MUCL 30866. Lane 8: C. clavatum ATCC 22833. Size markers are lambda DNA digested with EcoRI and HindIII.

Fig.2. rDNA hybridization patterns for Xhol-digested nDNA of strains of Cylindrocladium and Calonectria species. Details as in Fig. 1.

Fig.3. rDNA hybridization patterns for HindIII-digested nDNA of strains of Cylindrocladium and Calonectria species. Details as in Fig. 1 


\section{Calonectria clavata}

The two heterothallic strains of Calonectria clavata are morphologically distinct from all presently described species of Cylindrocladium, C. clavatum included. Furthermore, their rDNA restriction patterns (Figs. 1-3) show these two isolates to be similar, but distinct from all other species investigated. These results therefore suggest that these isolates represent a presently undescribed species of Cylindrocladium.

\section{Conclusion}

The present study has found rDNA RFLPs to be effective in distinguishing among morphologically similar species of Cylindrocladium. Our results further suggest that this is also an excellent technique for validating anamorph teleomorph relationships where the two states have been described from separate collections. With the recently completed monograph of Cylindrocladium, results obtained using this technique can now be integrated with alpha and beta taxonomic criteria. This approach would help to determine the morphological and genetic range of species occurring in morphologically similar complexes.

\section{Acknowledgments}

We thank Dr. N.E. El-Gholl (Division of Plant Industry, Florida, USA), Dr. J. Mouchacca (Laboratoire de Cryptogamie, Paris, France), Pr A.C. Alfenas (Department of Plant Pathology, Viáosa, MG, Brazil) and the curators of MUCL and ATCC for providing cultures examined in this study. Financial support for this study was provided in the form of a rolling grant from the Foundation for Research Development to the first and junior author.

\section{References}

Boedijn K.B.and Reitsma J. 1950. Notes on the genus Cylindrocladium. Reinwardtia 1: 51-60

Boesewinkel H.J. 1982. Heterogeneity within Cylindrocladium and its teleomorphs. Trans. Br. mycol. Soc. 78: $553-556$

Bugnicourt F. 1939 Les Fusarium et Cylindrocarpon de l'Indochine. Encycl. Mycol. 11: 1-206

Crous P.W., Wingfield, M. J. 1994. A monograph of Cylindrocladium, including anamorphs of Calonectria. Mycotaxon 51: 341-435

Crous P.W., Janse B.J.H., Victor D., Marais G.F.and Alfenas A.C. 1993a. Molecular characterization of Cylindrocladium spp. with three-septate conidia and ovoid-like vesicles. System. Appl. Microbiol. 16: 266- 273.

Crous P.W., Wingfield M.J.and Alfenas A.C. 1993b. Additions to Calonectria. Mycotaxon 46: 217-234.

Crous P.W., Wingfield M.J.and Alfenas A.C. 1993c. Cylindrocladium parasiticum sp. nov., a new name for C. crotalariae. Mycol. Res. 97: 889-896.

El-Gholl N.E., Alfieri S.A.and Barnard E.L. 1993. Description and pathogenicity of Calonectria clavata sp. nov. Mycotaxon 48: 201-216.

Hodges C.S.and May L.C. 1972. A root disease of pine, Araucaria, and Eucalyptus in Brazil caused by a new species of Cylindrocladium. Phytopathology 62: 898-901.

Lodolo E.J., Van Zyl W.H.and Rabie C.J. 1992. A rapid molecular technique to distinguish Fusarium species. Mycol. Res. 97: 345-346.

Peerally A. 1991. Cylindrocladium hawksworthii sp. nov. pathogenic to water-lilies in Mauritius Mycotaxon 40: 367-376.

Russell P.J., Wagner S., Rodland K.D., Feinbaum R.L., Russel J.P., Bret-Harte M.S., Free S.J.and Metzenberg R.L. 1984. Organization of the ribosomal ribonucleic acid genes in various wild-type strains and wild-collected strains of Neurospora. Mol. Gen. Genet.196: 275-282.

Sambrook J., Fritsch E.F.and Maniatis T. 1989. Molecular cloning: a laboratory manual. 2nd ed. Cold Spring Harbor/NY, Cold Spring Harbor Laboratory Press.

(The present study forms part of a paper submitted to System. Appl. Microbiol.) 


\title{
IDENTIFICATION OF BASIDIOMYCETOUS YEASTS USING WHOLE- CELL PROTEIN ELECTROPHORESIS
}

\author{
M. VANCANNEYT ${ }^{1}$, G. L. HENNEBERT ${ }^{2}$ AND K. KERSTERS ${ }^{1}$ \\ ${ }^{1}$ Laboratorium voor Microbiologie, Universiteit Gent, K. L. Ledeganckstraat 35, B-9000 Gent, Belgium; \\ ${ }^{2}$ Mycothèque de l'Université Catholique de Louvain, Place Croix du Sud 3, B-1348 Louvain-la-Neuve, \\ Belgium
}

\section{Introduction}

There is a continuing need for simple and reliable laboratory procedures to differentiate yeast species. With an increasing number of species it becomes obvious that the conventional diagnostic tests frequently provide insufficient phenotypic characterization to permit unambiguous assignment at the species level. In this study the use of one-dimensional sodium dodecyl sulphate-polyacrylamide gel electrophoresis of whole-cell proteins (SDS-PAGE) as a possible method for classification and identification of basidiomycetous yeasts is investigated.

\section{Materiel and methods}

Approximately 240 different strains representing at least 70 different species in the basidiomycetous genera Cystofilobasidium, Cryptococcus, Filobasidium, Filobasidiella, Kondoa, Leucosporidium, Mrakia, Rhodosporidium, Rhodotorula and Tremella were studied. Cells were cultivated in liquid cultures and harvested in the stable growth phase. The methods used for preparation and electrophoresis of the SDS-protein extracts were those described previously (Vancanneyt et al. 1992b). Protein patterns were scanned and digitized. Numerical analysis was performed using the Pearson product moment correlation coefficient and UPGMA clustering.

\section{Results}

Except for the genus Mrakia, all species of the teleomorphic genera Cystofilobasidium, Filobasidium, Filobasidiella, Kondoa, Leucosporidium, Mrakia and Rhodosporidium constituted separate protein electrophoretic clusters (Vancanneyt et al. 1992a). The species of the genus Mrakia (M. frigida, M. gelida, $M$. nivalis and $M$. stokesii) showed highly similar protein patterns, suggesting that these four species may be synonymous. Strains of two varieties of Filobasidiella neoformans, F. neoformans var. neoformans and F. neoformans var. bacillispora could not be differentiated, neither by protein electrophoresis, visually, or by numerical analysis.

A study of 107 Rhodosporidium and Rhodotorula strains revealed after numerical analysis of protein electrophoregrams eighteen clusters (Vancanneyt et al. 1992b). Only the type strain was included for the following ten species: Rhodotorula armeniaca, Rt. auriculariae, Rt. bacarum, Rt. bogoriensis, Rt. diffluens, Rt. hordea, Rt. hylophila, Rt. ingeniosa, Rt. muscorum and Rt. philyla. All these type strains demonstrated a qualitatively well-characterized protein profile, which might indicate that each of these species occupies a unique and separate taxonomic position within the genus Rhodotorula. Homogeneous and separate protein clusters were found for strains of the species Rt. fragaria, Rt. javanica, Rt. lactosa, Rt. pustula and Rt. sonckii. Intraspecific heterogeneity was demonstrated 
within the following seven Rhodotorula species: Rt. acheniorum, Rt. araucariae, Rt. aurantiaca, Rt. foliorum, Rt. glutinis, Rt. graminis and Rt. minuta. One or more strains of the anamorphic species Rt. glutinis grouped with strains of Rs. sphaerocarpum, Rs. toruloides, Rs. diobovatum strains, Rs. kratochvilovae and Rt. mucilaginosa strains. For Rt. graminis, the type strain grouped with Rs. diobovatum strains and most of the strains investigated grouped with Rs. paludigenum strains.

Table 1: DNA base composition and type of coenzyme Q within protein electrophoretic groups of Rhodotorula and Rhodosporidium strains.

\begin{tabular}{|c|c|c|c|c|}
\hline $\begin{array}{l}\text { Protein } \\
\text { group }\end{array}$ & \multicolumn{2}{|c|}{ Number of strains } & mol \%(G+C) & Coenzyme Q \\
\hline \multirow[t]{2}{*}{1} & Rt. glutinis & 2 & $59.6-60.7$ & Q10 \\
\hline & Rt. mucilaginosa & 15 & & \\
\hline \multirow[t]{2}{*}{2} & Rt. glutinis & 1 & $62.0-63.0$ & Q10 \\
\hline & Rs. sphaerocarpum & 4 & & \\
\hline \multirow[t]{3}{*}{3} & Rt. glutinis & 1 & $59.9-60.0$ & Q9 \\
\hline & Rt. sp. & 1 & & \\
\hline & Rs. toruloides & 6 & & \\
\hline 4 & Rt javanica & 2 & ND & Q9 \\
\hline \multirow[t]{2}{*}{5} & Rt. graminis & 4 & $62.5-63.0$ & Q10 \\
\hline & Rs. paludigenum & 4 & & \\
\hline 6 & Rt. pustula & 2 & ND & ND \\
\hline \multirow[t]{2}{*}{7} & Rt. aurantiaca & 1 & 61.3 & Q10 \\
\hline & Rt. graminis & 1 & & \\
\hline \multirow[t]{3}{*}{8} & Rt. glutinis & 7 & $66.4-67.2$ & Q10 \\
\hline & Rt. graminis & 1 & & \\
\hline & Rs. diobovatum & 5 & & \\
\hline \multirow[t]{2}{*}{9} & Rt. glutinis & 2 & $64.2-65.0$ & Q10 \\
\hline & Rs. kratochvilovae & 1 & & \\
\hline 10 & Rt. fragariae & 2 & ND & Q10 \\
\hline 11 & Rt. acheniorum & 2 & 52.8 & Q10 \\
\hline 12 & Rs. dacryoidum & 3 & ND & ND \\
\hline 13 & Rt. aurantiaca & 2 & $58.4-59.3$ & Q10 \\
\hline 14 & Rt. aurantiaca & 2 & 55.1 & Q10 \\
\hline 15 & Rt. minuta & 10 & $48.6-51.0$ & Q10 \\
\hline 16 & Rt. lactosa & 2 & 56.6 & Q9 \\
\hline 17 & Rt. sonckii & 2 & ND & Q10 \\
\hline 18 & Rt. aurantiaca & 2 & $57.6-57.9$ & Q9 \\
\hline
\end{tabular}

ND: not determined

A study of 78 Cryptococcus strains, 3 Filobasidium strains and 10 Tremella reference strains revealed after numerical comparison eighteen protein electrophoretic clusters (Vancanneyt et al. 1994). Only the type strain was included for the following Cryptococcus species: Cr. curiosus, Cr. dimennae, Cr. flavus, Cr. heveanensis, Cr. magnus, Cr. marinus, Cr. skinneri and Cr. tsukubaensis. These type strains showed a well-characterized protein fingerprint. Homogeneous and separate protein clusters were found for strains of the species Cr. amylolentus, Cr. curvatus, Cr. gastricus and Cr. macerans. A significant protein electrophoretic heterogeneity was observed within the species Cr. albidus, Cr. humicola, Cr. laurentii and $\mathrm{Cr}$. luteolus. Highly similar protein patterns were observed for some strains of Cryptococcus albidus and the investigated strains of Cr. kuetzingii, for the 
type strain of Cr. elinovii and the studied strains of Cr. terreus and for a strain of Cr. luteolus and two strains of Cr. laurentii. Concerning the reference strains of the genus Tremella, T. aurantia, T. brasiliensis and T. globospora showed a unique protein pattern. A highly similar protein fingerprint was found between one strain of Cr. Laurentii and T. foliacea. A qualitatively and quantitatively highly similar protein pattern was found for respectively T64. coalescens and T. mesenterica, T. fuciformis and T. samoensis and T. encephala and T. subanomala.

The taxonomic validity of the obtained groupings was evaluated using other chemotaxonomical criteria such as the determination of the DNA base composition and the ubiquinone type (Table 1 and 2; Vancanneyt et al. 1992a, b, 1994). These data confirmed the protein electrophoretic heterogeneity within several anamorphic Rhodotorula and Cryptococcus species and indicated a possible close relationship between strains of different species.

Table 2: DNA base composition and type of coenzyme Q within protein electrophoretic groups of Cryptococcus, Filobasidium and Tremella strains.

\begin{tabular}{|c|c|c|c|c|}
\hline $\begin{array}{l}\text { Protein } \\
\text { group }\end{array}$ & Species & Number of strains & $\mathrm{mol} \%(G+C)$ & Coenzyme Q \\
\hline 1 & Cr. curvatus & 6 & $56.7-56.9$ & Q10 \\
\hline 2 & Cr. amylolentus & 2 & ND & Q9 \\
\hline \multirow[t]{2}{*}{3} & Cr. laurentii & 2 & $54.7-55.2$ & Q10 \\
\hline & Cr. luteolus & 1 & & \\
\hline 4 & Cr. laurentii & 3 & $57.2-58.1$ & ND \\
\hline \multirow[t]{2}{*}{5} & Cr. elinovii & 1 & $54.7-55.0$ & Q10 \\
\hline & Cr. terreus & 3 & & \\
\hline \multirow[t]{2}{*}{6} & Cr. albidus var. aerius & 1 & $53.7-54.2$ & Q10 \\
\hline & Cr. albidus var. albidus & 1 & & \\
\hline 7 & Cr. albidus var aerius & 2 & $49.8-50.1$ & ND \\
\hline \multirow[t]{2}{*}{8} & Cr. albidus var. albidus & 7 & $51.3-53.0$ & Q10 \\
\hline & Cr. kuetzingii & 4 & & \\
\hline 9 & Cr. albidus var. albidus & 4 & $49.1-49.6$ & Q10 \\
\hline \multirow[t]{2}{*}{10} & T. encephala & 1 & $47.6-47.7$ & Q10 \\
\hline & T. subanomala & 1 & & \\
\hline \multirow[t]{2}{*}{11} & T. fuciformis & 1 & $55.9-56.1$ & Q10 \\
\hline & T. samoensis & 1 & & \\
\hline 12 & Cr. albidus var. albidus & 3 & $52.8-53.5$ & Q10 \\
\hline \multirow[t]{2}{*}{13} & Cr. ater & 2 & $51.3-52.0$ & Q10 \\
\hline & F. foliforme & 3 & & \\
\hline 14 & Cr. humicolus & 2 & 57.4 & ND \\
\hline 15 & Cr. gastricus & 2 & ND & ND \\
\hline 16 & Cr. macerans & 4 & ND & ND \\
\hline \multirow[t]{2}{*}{17} & T. coalescens & 1 & $46.4-47.2$ & ND \\
\hline & T. mesenterica & 1 & & \\
\hline 18 & Cr. humicolus & 8 & $61.1-62.3$ & ND \\
\hline
\end{tabular}




\section{Conclusion}

We conclude that SDS-PAGE of cellular proteins allows a rapid and reliable grouping of a large number of yeast strains. The majority of the species are delineated as separate clusters. The technique is successful in delineating anamorph/teleomorph relations, in revealing synonymy between species and in demonstrating taxonomic heterogeneity within several species. Moreover, the possibility of computerized processing of the patterns suggests a method useful for analytical characterization of yeasts. Identification of new isolates becomes an easy routine procedure once a database of reference protein electrophoregrams is constructed.

\section{References:}

Vancanneyt M., Coopman R., Tytgat R., Berny J.-F., Hennebert G.L. and Kersters K. 1992a. A taxonomic study of the basidiomycetous yeast genera Rhodosporidium and Rhodotorula based on whole-cell protein patterns, DNA base compositions and coenzyme Q types. J. Gen Appl. Microbiol. 38: $363-372$.

Vancanneyt M., Van Lerberge, E., Berny J.-F., Hennebert G.L. and Kersters K. 1992b. The application of whole-cell protein electrophoresis for the classification and identification of basidiomycetous yeast species. Antonie van Leeuwenhoek 61: 69-78.

Vancanneyt M., Coopman R., Tytgat R., Hennebert G.L. and Kersters K. 1994. Whole-cell protein patterns, DNA base composition and coenzyme Q types in the yeast genus Cryptococcus Kützing and related taxa. Syst. Appl. Microbiol. 17: 65-75. 


\title{
PHYTOPATHOGENIC FILAMENTOUS (ASHBYA, EREMOTHECIUM) \\ AND DIMORPHIC FUNGI (NEMATOSPORA) WITH FALCATE ASCOSPORES AS NEW MEMBERS WITHIN THE SACCHAROMYCETACEAE.
}

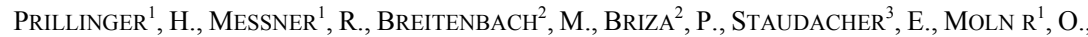 \\ WEIGANG $^{4}$, F., LOPANDIC ${ }^{1}$, K., IBL ${ }^{5}$, M., HIMMLER ${ }^{1}$, G. \\ ${ }^{1}$ Univ. Bodenkultur, Inst. f. Angew. Mikrobiol.; Nußdorfer Lände 11, A-1190 Wien; ${ }^{2}$ Univ. Salzburg, \\ Inst. f. Genetik u. Allgemeine Biologie; Hellbrunnerstr. 34, A-5020 Salzburg; ${ }^{3}$ Univ. Bodenkultur, Inst. f. \\ Chemie; Gregor Mendel Str. 33, A-1180 Wien; ${ }^{4}$ Hewlett-Packard Ges. m. b. H.; Lieblgasse 1, A-1222 \\ Wien; ${ }^{5}$ Codon Genetic Systems Ges. m.b.H.; Colloredogasse 29/13 A-1180 Wien.
}

\section{Introduction}

Although geneticists and molecular biologists commonly separate yeasts and filamentous fungi, Meyen, 1838 has already acknowledged in the name Saccharomyces the fact that yeasts are fungi (van der Walt 1987). Wickerham $(1951,1952)$ concluded that the classification of yeasts will be incorrect as long as the related filamentous forms had been studied thoroughly by persons who knew these fungi as well as yeasts.

In the present work we investigated the phylogenetic relationship between the saprophytic and predominantly unicellular yeast genera Saccharomyces and Kluyveromyces and the plant pathogenic, filamentous fungi Ashbya gossypii and Eremothecium ashbyi. Dimorphic yeast, Nematospora coryli, parasitic on hazelnuts and soybeans was included in our investigations. To characterize yeasts and filamentous fungi from these genera on the molecular level we have chosen the cell wall monosaccharide composition, the presence of DL-dityrosine-containing macromolecules from ascospore walls (Briza et al. 1990), the ubiquinone spectra, and the DNA sequence information from the small and large ribosomal subunit genes as well as from the rapidly evolving ITS1 and ITS2 regions.

\section{Material and Methods}

Cell wall sugars/Ubiquinone-system. The qualitative and quantitative monosaccharide pattern of purified yeast and hyphal cell walls and ubiquinone spectra were determined as described in Prillinger et al. (1993) and Messner et al. (1994a). Dityrosine analysis was performed according to Briza et al. (1994). Analysis of ribosomal DNA-sequences: Sequences were obtained by direct, automatic sequencing of PCR products (Codon Genetic Systems, Vienna; Messner et al. 1994b).

\section{Results and Discussion}

Ascospore morphology and the presence of hyphal growth were considered to separate the genera Ashbya, Eremothecium, and Nematospora at least on the family level (Metschnikowiaceae: von Arx and van der Walt 1987) from other yeast genera belonging to the Endomycetales. The ascospores of Saccharomyces species are smooth and spherical, in Kluyveromyces they are reniform. Falcate ascospores, often whiplike at one end and occasionally septate are known in Nematospora coryli, Ashbya gossypii and Eremothecium ashbyi (von Arx and van der Walt 1987).

Kurtzman (1993), Kurtzman and Robnett (1994), and Prillinger et al. (1990, 1993) presented molecular evidence that the Endomycetales except the Schizosaccharomycetaceae are homogenous. The Schizosaccharomycetaceae were 
included in the new order Schizosaccharomycetales. According to Kurtzman \& Robnett (1994) and Prillinger et al. (1993) representatives of the Endomycetales like Ashbya, Eremothecium, Kluyveromyces, Nematospora, and Saccharomyces investigated in this study are primitive fungi ancestral to filamentous Asco- and Basidiomycetes.

To clarify the phylogenetic relationship between the saprophytic unicellular species of Kluyveromyces and Saccharomyces on the one hand and the phytopathogenic dimorphic (Nematospora) or filamentous Ashbya or Eremothecium species on the other hand we used a polyphasic molecular approach. In the present paper we have investigated:

1. The qualitative and quantitative monosaccharide composition of purified yeast and fungal cell walls after hydrolysis with trifluoroacetic acid.

2. The presence of dityrosine in the cell walls of ascospores.

3. The major component of the ubiquinone system.

4. The DNA sequence of six regions of the ribosomal RNA genes.

The cell wall carbohydrate pattern of the phytopathogenic filamentous fungi $A$. gossypii and E. ashbyi comes close to the characteristic mannose-glucose pattern of several Saccharomyces and Kluyveromyces species (Prillinger et al. 1990). Whereas the proportion of mannose appeared to be commonly higher in the Kluyveromyces and Saccharomyces yeast strains as well as in N. coryli, glucose dominates in the filamentous strains of A. gossypii and E. ashbyi.

As far as ascospores could be observed on different sporulation media (Kregervan Rij 1984), dityrosine was detected in all species of Saccharomyces, Kluyveromyces, and the two filamentous fungi A. gossypii and E. ashbyi. Dityrosine was absent in sporulating cultures from Nadsonia fulvescens (Ubiquinone Q-6), Debaryomyces hansenii (Q-9), Pichia farinosa (Q-9), and Schizosaccharomyces pombe (Q-10).

As already shown by Yamada et al. (1977, 1987), the ubiquinone Q-6 was present in all species of the genera Kluyveromyces and Saccharomyces as well as in A. gossypii and E. ashbyi. Ubiquinone Q-5 was found in the type strain of Nematospora coryli. According to Yaada et al. (1987) there is an additional strain of $N$. coryli where ubiquinone Q-6 was found. Conspecificity was established for both strains based on partial base sequences of $18 \mathrm{~S}$ and 26S ribosomal DNA (Yamada and Nagahama 1991).

For molecular phylogeny based on DNA sequences three different targets were chosen. Regions of 800 to 900 bases length of the genes for the small and large ribosomal subunit as well as both the complete internal transcribed spacers ITS1 and ITS2 had been sequenced (Messner et al. 1994b).

Separate phylogenetical computation of these three types of ribosomal sequences results in the clustering of the Saccharomyces species, the Kluyveromyces species, E. ashbyi, N. coryli, and A. gossypii for all regions investigated. As illustrated by Fig. 1 deriving from ITS1 and ITS2 data, the genera Saccharomyces and Kluyveromyces are well defined by 82 and $98 \%$ bootstrap confidence, respectively. E. ashbyi, $N$. coryli and A. gossypii are clustered here at the species level, and the separation of these three subclades from Candida by tree topology and a bootstrap value of $65 \%$ is quite good for sequences of the rapidly evolving ITS regions. 


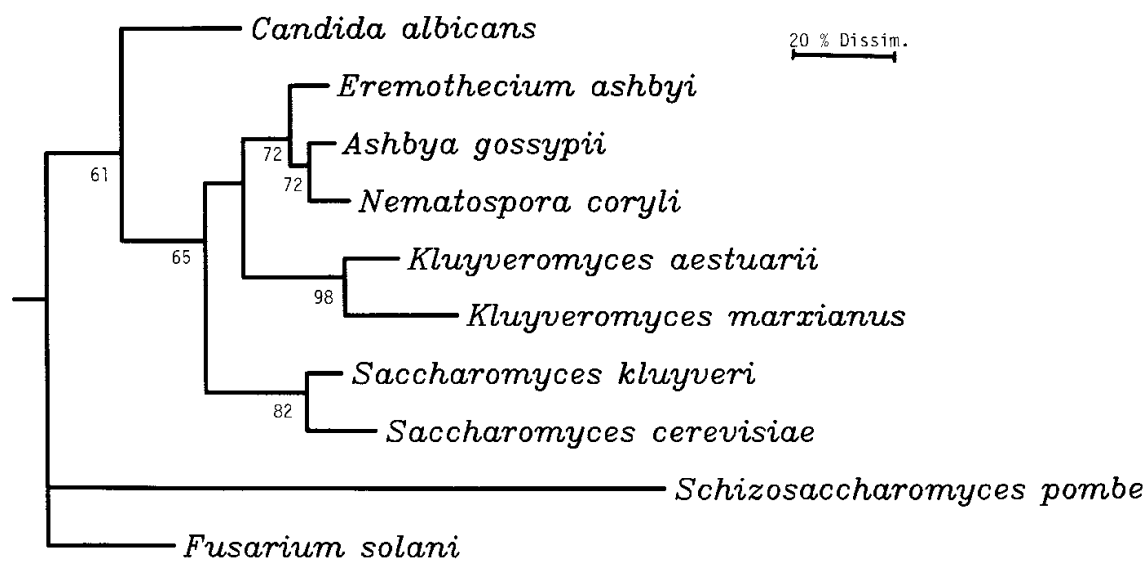

Fig. 1. Cladogram based on both internal transcribed spacer regions ITS1 and ITS2. An alignment of 655 bases in length was computed under maximum likelihood hypothesis. Bootstrap confidence values derived from 600 repeats.

Our data corroborate the work of Kurtzman and Robnett (1994), who found by ribosomal sequences a close relationship between Ashbya, Eremothecium, and Nematospora together with Holleya tight enough to be congeneric. The genus Metschnikowia, however, appears to be only distantly related according Kurzmann and Robnett (1994).

\section{Conclusions}

1. Phenotypic criteria like ascospore shape and ornamentation or presence or absence of hyphae are in most cases unreliable for definition of families in the Endomycetales.

2. Based on a similar cell wall carbohydrate composition, the presence of dityrosine in endospores, which are formed by free cell formation, and a high degree of ribosomal DNA sequence similarity especially with respect to the ITS1 and ITS2 regions we redefine the family Saccharomycetaceae Winter to include unicellular saprophytic fungi like the genera Kluyveromyces and Saccharomyces, as well as dimorphic or filamentous parasitic fungi like species of the genera Ashbya, Eremothecium, Holleya, and Nematospora.

3 . Our data suggest that unicellular ascomycetous yeasts like Kluyveromyces and Saccharomyces have evolved from filamentous plant pathogens with ontogenetic saprophytic yeast stages. The lack of the respective parasitic filamentous forms can be explained by an extinction of the specific hosts.

4. Molecular characteristics like cell wall sugars or ribosomal DNA sequence information are reliable tools to trace ascomycetous yeasts back to a polykaryotic coenocytic ("siphonal") ancestor (Prillinger 1987).

\section{Acknowledgements}

For kindly providing type strains we are indebted to Dr. C. P. Kurtzman (Peoria, U.S.A.), Prof. U. Stah and K. Scheide (T.U. Berlin). Supported by grants from the "Jubiläumsfonds der Österreichischen Nationalbank" and FWF (project: PO9255-Bio). 


\section{References}

Arx J.A. von, and van der Walt J.P. 1987. Ophiostomales and Endomycetales. Stud. Mycol. 30: 167-176.

Briza P., Ellinger A., Winkler G. and Breitenbach M. 1990. Characterization of a DL-dityrosine containing macromolecule from yeast ascospore walls. J. Biol. Chem. 265: 15118-15123.

Briza P., Eckerstorfer M. and Breitenbach M. 1994. The sporulation-specific enzymes encoded by DIT1 and DIT2 genes catalyze a two-step reaction leading to a soluble LL-dityrosine-containing precursor of the yeast spore wall. Proc. Natl. Acad. Sci. USA 91: 4524-4528.

Kreger-van Rij N.J.W. 1984. The yeasts - a taxonomic study. Elsevier Sci. Publ., Amsterdam, 1082 p.

Kurtzman C.P. 1993. Systematics of the ascomycetous yeasts assessed from ribosomal RNA sequence divergence. Antonie van Leeuwenhoek 63: 165-174.

Kurtzman C. P. and Robnett C. J. 1994a. Orders and families of ascosporogenous yeasts and yeast-like taxa compared from ribosomal RNA sequence similarities. In Ascomycete Systematics: Problems and Perspectives in the Nineties. Hawksworth, D. L. ed., Plenum Press, New York, USA pp 249-260.

Messner R., Prillinger H., Altmann F., Lopandic K., Wimmer K., Molner O. and Weigang F. 1994a. Molecular characterization and application of random amplified polymorphic DNA analysis of Mrakia and Sterigmatomyces species.Int. J. Syst. Bacteriol. 44: 694-703.

Messmer R., Prillinger H., Ibl L. and Himmler G. 1995 Sequences of ribosomal genes and internal transcribed spacers. GenBank acc. N UO9327. Mol. Evol. Biol. 4:406-425.

Messner R., Prillinger H., Ibl M. and Himmler G. 1995. Sequences of ribosomal genes and internal transcribed spacers move three plant parasitic fungi, Eremothecium ashbyi, Ashbya gossypii, and Nematospora coryli, towards Saccharomyces cerevisiae. J. Gen. Appl. Microbiol. 41: 31-42.

Meyen J. 1838. Wiegmann Arch. Naturgesch. 4: 1-186.

Prillinger H., 1987. Yeasts and anastomoses: their occurrence and implications for the phylogeny of Eumycota. In Evolutionary biology of the fungi. Rayner A.D.M. et al. eds., Cambridge Uiv. Press pp. 355-377

Prillinger H., Dörfler C., Laaser G., Eckerlein B. and Lehle L. 1990. Ein Beitrag zur Systematik und Entwicklungsbiologie höherer Pilze: Hefe-Typen der Basidiomyceten. Teil I. Schizosaccharomycetales, Protomyces-Typ. Z. Mykol. 56: 219-250.

PrillingerH., Oberwinkler F., Umile C., Tlachac K., Bauer R., Dörfler C. and Taufratzhofer E. 1993. Analysis of cell wall carbohydrates (neutral sugars) from asco- and basidiomycetous yeasts with and without derivatization. J. Gen. Appl. Microbiol. 39: 1-34.

van der Walt J.P. 1987. The yeasts: a conspectus. In The expanding realm of yeast-like fungi. De Hoog G.S., Smith M.T. and Weijman A.C.M. eds. Stud. Mycol. 30: 19-31.

Wickerham L.J. 1951. Taxonomy of yeasts. 1. Techniques of classification. 2. A classification of the genus Hansenula. Tech. Bull. 102: 56 p., U.S. Dept. Agric. Washington D.C.

Wickerham L.J. 1952. Recent advances in the taxonomy of yeasts. Annu. Rev. Microbiol. 6: 317-332.

Yamada Y.and Nagahama T. 1991. The molecular phylogenyof the ascomycete yeast genus Holleya Yamada based on the partial sequence of $18 \mathrm{~S}$ and 26S ribosomal RNAs. J. Gen. Appl. Microbiol. 37: 199-206.

Yamada Y., Nojiri M., Matsuyama M. and Kondo K., 1977. Coenzyme Q system in the classification of the ascosporogenous yeast genera Debaryomyces, Saccharomyces, Kluyveromyces and Endomycopces. J. Gen. Appl. Microbiol. 22: 325-337.

Yamada Y., Banno I., Arx J.A. von, and van der Walt J.P. 1987. Taxonomic significance of the coenzyme Q system in yeasts and yeast-like fungi. In The expanding realm of yeast-like fungi. De Hoog G.S., Smith M.T. and Weijman A.C.M. eds. Stud. Mycol. 30: 299-308. 


\section{GENETIC ORGANIZATION OF THE YEAST YARROWIA LIPOLYTICA}

Serge Casaregola, Huu Vang NGuyen, Chantal Feynerol, Monique Diez \& Claude Gaillardin

Collection de Levures d'Intérêt Biotechnologique (CLIB)

Laboratoire de Génétique Moléculaire et Cellulaire, INRA-CNRS,

Centre de Biotechnologies Agro-Industrielles, Institut National Agronomique,

78450 Thiverval-Grignon, France

\section{Introduction}

Yarrowia lipolytica (otherwise known as Candida lipolytica and Saccharomycopsis lipolytica) is a dimorphic yeast which forms both yeast-like cells and true mycelium. It can grow on hydocarbons and has been used for the production of single-cell protein and citric acid as well as various other various metabolites. In addition, $Y$. lipolytica is able to secrete naturally a number of enzymes in large amount. These characteristics make this yeast an organism with high biotechnogical potential.

Although sexuality in this yeast was discovered more than 20 years ago, genetic studies were hampered by unusual features including low mating frequencies, low fertility of hybrids, irregular meiotic segregation and mitotic haploidization. Despite the development of programs to generate numerous mutants and to improve mating procedures and tetrad analysis (Gaillardin et al. 1973, Ogrydziak et al. 1978), the construction of a genetic map did not allow the detection of any centromere-linked genes and the number of chromosomes in this yeast.

Recent studies in our laboratory (Naumova et al. 1993) have defined, using Pulse Field Gel Electrophoresis (PFGE), the existence of three groups of electrophoretic karyotypes amongst 27 natural isolates. Moreover, within these groups an important variability of the number of chromosomes (from 3 to 6 varying in size from 2 to 6 $\mathrm{Mb}$ ) was observed as well as an important chromosomal-length polymorphism. Hybridization studies with few cloned genes to these karyotypes revealed that these markers were not linked to specific chromosomes when strains were compared. This result is in agreement with the absence of centromere linkage observed with tetrad analysis during the establishment of the genetic map (Ogrydziak et al. 1982). Taken together, these results could account for the genetic anomalies displayed by this species.

In a different approach based on the separation of chromosomes by PFGE and the assignement of cloned genes to these chromosomes, we set up the construction of a physical map of the genome of $Y$. lipolytica, in order to facilitate genetic studies and to gain better insights on the unusual genetic characteristics of this yeast.

\section{Material and Methods}

Strains used in this study

\begin{tabular}{|l|l|l|}
\hline E150 & lab. strain, from the crossing W29 x CBS6124-2 & CLIB 122 \\
\hline E129 & lab. strain, from the crossing W29 x CBS 6124-2 & CLIB 121 \\
\hline H222 & lab. strain from H. Weber (Germany) & CLIB 80 \\
\hline CX161-1B & lab. strain from D.M. Ogrydziak (U.S.A.) & CLIB 153 \\
\hline W29 & wild type, ATCC 20460 & CLIB 89 \\
\hline CBS 6124-1 & monosporous segregant from the wild type CBS 6124 & CLIB 77 \\
\hline CBS 6124-2 & monosporous segregant from the wild type CBS 6124 & CLIB 78 \\
\hline B204-12D & lab. strain from G. Barth (Swizerland) & \\
\hline
\end{tabular}


Preparation of chromosomal DNA:

This was essentially performed as described by Naumova et al. (1993).

\section{Chromosome separation}

Chromosomes were separated in $0.8 \%$ agarose gels run in $0.5 x$ TAE buffer at $12^{\circ} \mathrm{C}$ in a Bio-Rad CHEF-DRII. Electrophoresis was carried out at $50 \mathrm{~V}$ for $48 \mathrm{hrs}$ with a switching time of $2400 \mathrm{~s}, 43 \mathrm{~V}$ for $70 \mathrm{hrs}$ with a switching time of $3000 \mathrm{~s}$ and $40 \mathrm{~V}$ for $47.7 \mathrm{hrs}$ with a switching time of $3300 \mathrm{~s}$.

\section{Southern blotting and DNA/DNA hybridization}

DNA from agarose was depurinated, denaturated and alkali-transferred overnight to GeneSceen membranes. The membranes were then neutralized.

DNA used as probe was directly ${ }^{32} \mathrm{P}-\mathrm{dCTP}$ labelled with the random priming method (MegaPrime, Amersham) in low-melting agarose or after purification from agarose gel with the GeneClean kit. Hybridizations were performed at $65{ }^{\circ} \mathrm{C}$ in $3 \mathrm{xSSPE}$ with Denhardt and dextransulfate. The final wash was in $40 \mathrm{mM}$ Na-phosphate/1\% SDS at $65{ }^{\circ} \mathrm{C}$.

\section{Results and Discussion}

Important efforts were devoted to the improvement of $Y$. lipolytica chromosomes separation by PFGE and to the transfer of DNA to membranes in Southern blotting. For this purpose, a laboratory strain, E150, was chosen and we were able to show that this strain possesses at least 5 chromosomes whose sizes vary from 2.6 to 4.9 $\mathrm{Mb}$ using the chromosomes of Schizosaccharomyces pombe as size markers. The size of the Y. lipolytica genome is thus estimated at $18.1 \mathrm{Mb}$.

36 genes, mostly cloned in the Laboratoire de Génétique Moléculaire et Cellulaire, others kindly provided by foreign laboratories, were used as probes in hybridization experiments to the PFGE separations. This allowed the obtention of 5 linkage groups comprising:

- 2 markers on Chrom. I

- 6 markers on Chrom. II

- 12 markers on Chrom. III

- 7 markers on Chrom. IV

- 11 markers on Chrom. V.

It must be pointed at that:

(1) 3 centromeres were used as probes defining the chromosomes I, III and IV. This allows for the first time, a certain number of markers to be linked to centromeres.

(2) rDNA clusters were shown, unlike other yeasts, to be present on 4 chromosomes out of 5. It is remarkable that, unlike other yeasts too, $Y$. lipolytica possesses at least 2 classes of rDNA units (Fournier et al. 1986). The rDNA clusters being highly variable in size, their presence on most of the chromosomes of the strain tested could explain the chromosome-length polymorphism observed in different $Y$. lipolytica strains.

In order to validate the construction of a physical map of the genome, we tested the distribution of the already assigned markers in the E150 strain to a number of widely used wild type and laboratory strains.

First, electrophoretic karyotypes of 7 different isolates were obtained. This allowed us to confirm the results obtained by Naumova et al. (1993) on the 
important variability of the genome structure of realy related $Y$. lipolytica isolates. Then, a pool of genes (Bio6, His1, Pho2, PGK) known to hybridize to the E150 chromosome III was used as probes to hybridize to the chromosome separations of the 7 isolates. Preliminary results indicate that the linkage previously obtained with the 4 genes in E150 was not conserved in these strains, including in the parental strains of E150. Indeed, whereas only one signal was observed on the chromosome III of E150, hybridization signals were scattered over the separations of the other strains.

Surprisingly, in the same test performed with a marker of the E150 chromosome I, the Ryl2 gene, a single signal on the smallest chromosome of each separation was observed, indicating that this chromosome did not display important length polymorphism or gross rearrangement from strain to strain. This is to be linked to the fact that the chromosome $\mathrm{I}$ is not carrying rDNA clusters unlike the other chromosomes.

These observations lead us to incriminate homologous recombination between the rDNA clusters on most of the chromosomes to explain the chromosomal rearrangements and the specific genetic features of $Y$. lipolytica. This hypothesis is being tested through the study of the conservation of linkage groups at meiosis and a better characterization of the location of the rDNA clusters in respect with the assigned markers.

\section{Acknowledgements}

We are indebted to Dr. Ph. Fournier who initiated this work, set up the PFGE conditions and showed constant interest and support. We would like to thank all our colleagues for kindly providing us with the cloned genes. This work was supported by the Insititut National de la Recherche Agronomique and by an EEC grant (BIOT-CT91-0267 DSCN).

\section{References}

Gaillardin C.M., Charoy V. and Heslot H.. 1973. A study of copulation, sporulation and meiotic separation in Candida lipolytica. Arch. Microbiol. 92: 69

Fournier P., Gaillardin C.M., Persuy M.A., Klootwijk J. and van Heerikhuizen H. 1986. Heterogeneity in the ribosomal family of the yeast Yarrowia lipolytica: genomic organization and separation studies. Gene 42: 273

Naumova E., Naumova G., Fournier P., Nguyen H.V. and Gaillardin C.M. 1993. Chromosomal polymorphism of the yeast Yarrowia lipolytica and related species: electrophoretic karyotyping and hybridization with cloned genes. Current Genet. 23(5-6): 450-454.

Ogrydziak K.D., Bassel D., Contopoulou J.R. and Mortimer R.K. 1978. Development. of. genetic techniques and the genetic map of the yeast. Saccharomycopsis lipolytica. Molec. Gen. Genet. 163: 229-239.

Ogrydziak K.D., Basel J. and Mortimer R.K. 1982 Development of the genetic map of the yeast Saccharomycopsis lipolytica. Molec. Gen. Genet. 188: 179 


\title{
TAXONOMIC REVISION OF SACCHAROMYCES SENSU STRICTO STRAINS OF NCAIM
}

\author{
J. TORNAI-LehoczKI., D. Dlauchy., G. Peter
}

National Collection of Agricultural and Industrial Microorganisms, Budapest, H-1118, Somloi ut 14-16, Hungary

\section{Introduction}

In the last decades, classification of species in the Saccharomyces sensu stricto group have been a hot issue (Lodder 1970, Kreger-van Rij 1984, Barnett 1992). Using conventional identification tests for the determination of phenotypic characters delimitation of species within the group cannot be done satisfactorily. On the basis of nDNA/nDNA reassociation studies, recently four species have been reestablished, namely Saccharromyces cerevisiae, S. paradoxus, S. bayanus, and S. pastorianus (Vaughan-Martini and Martini 1987, 1989, 1993). In 1990, Rodrigues de Sousa et al. investigated the fructose proton symport activity in species of Saccharomyces sensu stricto group. They reported that four strains of $S$. pastorianus and five strains of $S$. bayanus tested showed fructose proton symport activity whereas five strains of $S$. cerevisiae and three strains of $S$. paradoxus did not. They suggested that active fructose transport is a reliable phenotypic character that correlates with species delimitation based on the genotype.

The aim of our study was to check the reliability of the active fructose proton symport in differentiating 72 species of Saccharomyces sensu stricto, using yeast strains isolated from Hungarian wine and beer.

\section{Yeast strains}

\section{Material and Methods}

The type strains of Saccharomyces sensu stricto species and some of their synonyms and 72 wine and brewer yeast strains identified earlier with species belonging to Saccharomyces sensu stricto were studied. Thirteen type strains were obtained from the Industrial Yeast Collection of the Dipartimento di Biologia Vegetale, Universita di Perugia, Italy (DBVPG) and the Culture Collection Unit, Fermentation Section, Northern Utilization Research Branch, U.S. Department of Agriculture, Peoria, Illinois, USA (NRRL). The other strains were obtained from the National Collection of Agricultural and Industrial Microorganism, Budapest, Hungary (NCAIM).

Yeast strains were characterized and identified according to methods and keys of Kreger-van Rij (1984) and Barnett et al. (1990).

\section{Measurement of fructose transport}

Detection of active fructose transport system was performed according to the method of Rodrigues de Sousa et al. (1990). Yeast strains were propagated in a liquid mineral medium (van Uden 1967) with $0.5 \%$ (w/v) fructose (Merck). Proton symport activity was tested by recording the alkalinization of an aqueous cell suspension after addition of fructose to a final concentration of $10 \mathrm{mmol} \mathrm{1-1} \mathrm{using} \mathrm{a}$ RADELKIS OP-211/1, (Hungary) pH meter (precision $\pm 0.05 \mathrm{pH}$ ) with OP-0808P $\mathrm{pH}$ sensitive combination glass electrode, and registered with a Potentiometric $\mathrm{OH}$ 814/1 type recorder (RADELKIS, Hungary). 
Table 1. Active fructose transport of yeast strains belonging to Saccharomyces sensu stricto group

\begin{tabular}{|c|c|c|c|}
\hline $\begin{array}{l}\text { Species } \\
\text { original epithet }\end{array}$ & $\begin{array}{l}\text { Collection } \\
\text { numbers }\end{array}$ & $\begin{array}{l}\text { Isolation } \\
\text { source }\end{array}$ & $\begin{array}{l}\text { Fructose } \\
\mathrm{H}+\text { symport }\end{array}$ \\
\hline \multicolumn{4}{|c|}{ Saccharomyces cerevisiae } \\
\hline Sacch. cerevisiaeT & DBVPG 6173 & beer & - \\
\hline Sacch. cerevisiae & NCAIM Y.00201 & wine & - \\
\hline Sacch. cerevisiae & NCAIM Y.00204 & wine & - \\
\hline Sacch. cerevisiae & NCAIM Y.00205 & wine & - \\
\hline Sacch. cerevisiae & NCAIM Y.00206 & wine & - \\
\hline Sacch. cerevisiae & NCAIM Y.00222 & wine & - \\
\hline Sacch. cerevisiae & NCAIM Y.00223 & wine & - \\
\hline Sacch. cerevisiae & NCAIM Y.00225 & wine & - \\
\hline Sacch. cerevisiae & NCAIM Y.00227 & wine & - \\
\hline Sacch. cerevisiae & NCAIM Y.00228 & wine & - \\
\hline Sacch. cerevisiae & NCAIM Y.00235 & wine & - \\
\hline Sacch. cerevisiae & NCAIM Y.00237 & wine & - \\
\hline Sacch. cerevisiae & NCAIM Y.00238 & wine & - \\
\hline Sacch. cerevisiae & NCAIM Y.00248 & wine & - \\
\hline Sacch. cerevisiae & NCAIM Y.00249 & wine & - \\
\hline Sacch. cerevisiae & NCAIM Y.00252 & wine & - \\
\hline Sacch. cerevisiae & NCAIM Y.00254 & wine & - \\
\hline Sacch. cerevisiae & NCAIM Y.00255 & wine & - \\
\hline Sacch. cerevisiae & NCAIM Y.00256 & wine & - \\
\hline Sacch. cerevisiae & NCAIM Y.00259 & wine & - \\
\hline Sacch. cerevisiae & NCAIM Y.00261 & wine & - \\
\hline Sacch. cerevisiae & NCAIM Y.00294 & wine & - \\
\hline Sacch. cerevisiae & NCAIM Y.00295 & wine & - \\
\hline Sacch. cerevisiae & NCAIM Y.00297 & wine & - \\
\hline Sacch. cerevisiae & NCAIM Y.00299 & wine & - \\
\hline Sacch. cerevisiae & NCAIM Y.00301 & wine & - \\
\hline Sacch. cerevisiae & NCAIM Y.00303 & wine & - \\
\hline Sacch. cerevisiae & NCAIM Y.00304 & wine & - \\
\hline Sacch. cerevisiae & NCAIM Y.00305 & wine & - \\
\hline Sacch. cerevisiae & NCAIM Y.00307 & wine & - \\
\hline Sacch. cerevisiae & NCAIM Y.00309 & wine & - \\
\hline Sacch. cerevisiae & NCAIM Y.00312 & wine & - \\
\hline Sacch. cerevisiae & NCAIM Y.00368 & wine & - \\
\hline Sacch. cerevisiae & NCAIM Y.00370 & wine & - \\
\hline Sacch. cerevisiae & NCAIM Y.00378 & wine & - \\
\hline Sacch. cerevisiae & NCAIM Y.00417 & wine & - \\
\hline Sacch. cerevisiae & NCAIM Y.00418 & wine & - \\
\hline Sacch. cerevisiae & NCAIM Y.00419 & wine & - \\
\hline Sacch. cerevisiae & NCAIM Y.00420 & wine & - \\
\hline Sacch. cerevisiae & NCAIM Y.00424 & wine & - \\
\hline Sacch. cerevisiae & NCAIM Y.00425 & wine & - \\
\hline Sacch. cerevisiae & NCAIM Y.00426 & wine & - \\
\hline Sacch. cerevisiae & NCAIM Y.00427 & wine & - \\
\hline Sacch. cerevisiae & NCAIM Y.00429 & wine & - \\
\hline Sacch. cerevisiae & NCAIM Y.00431 & wine & - \\
\hline Sacch. cerevisiae & NCAIM Y.00442 & wine & - \\
\hline Sacch. cerevisiae & NCAIM Y.00445 & wine & - \\
\hline Sacch. cerevisiae & NCAIM Y.00768 & wine & $+*$ \\
\hline Sacch. cerevisiae & NCAIM Y.00769 & wine & $+*$ \\
\hline Sacch. cheresiensis & NCAIM Y.00817 & wine & - \\
\hline Sacch. prostoserdovii & NCAIM Y.00818 & wine & - \\
\hline Sacch. beticus & NCAIM Y.00819 & wine & - \\
\hline
\end{tabular}


Saccharomyces bayanus

Sacch. bayanusT

Sacch. globosusT

Sacch. heterogenicusT

DBVPG 6171

NRRL-Y 12645

NRRL-Y 1354

Sacch. inusitatusT

Sacch. abuliensisT

NRRL-Y 12648

NRRL-Y 11845

Sacch. bayanus

Sacch. bayanus

Sacch. bayanus

NCAIM Y.00500

NCAIM Y.00826

NCAIM Y.00827

Sacch. bayanus

Sacch. bayanus

NCAIM Y.00828

NCAIM Y.00829

NCAIM Y.00830

Sacch. bayanus

NCAIM Y.00831

beer

pear juice

apple juice

beer

Mesophylax adopersus

wine

wine

wine

wine

wine

wine

wine

NT DBVPG 6047

NCAIM Y.00820

NCAIM Y.00821

NCAIM Y 00822

NCAIM Y.00832

NCAIM Y.00833

NCAIM Y.00834

NCAIM Y.00770

NCAIM Y.00835

NCAIM Y.00823

NCAIM Y.00824

$\begin{array}{ll}\text { beer } & + \\ \text { beer } & + \\ \text { beer } & + \\ \text { beer } & + \\ \text { beer } & + \\ \text { beer } & + \\ \text { beer } & + \\ \text { beer } & -* \\ \text { beer } & -* \\ \text { beer } & -* \\ \text { beer } & -*\end{array}$

Saccharomyces paradoxus

Sacch. paradoxusNT

DBVPG 6411

Sacch. paradoxus

NCAIM Y.00223

NCAIM Y.00399

Sacch. paradoxus

NCAIM Y.00825

$\mathrm{T}=$ Type strain; $\mathrm{NT}=$ Neotype strain; $*=$ Differing results. Strains have been examined for nDNA/nDNA homology, see Table 2; NCAIM = National Collection of Agricultural and Industrial Microorganisms; DBVPG = Industrial yeast collection of the Dipartimento di Biologia Vegetale,

Universitä di Perugia, Italy; NRRL = ARS Culture Collection, Northern Regional Research Laboratory,

U.S. Department of Agriculture, Peoria, Illionis, USA

Table 2. Results of nDNA/nDNA homology \%

\begin{tabular}{lccc}
\hline & $\begin{array}{l}\text { Sacch. } \\
\text { cerevisiaeT } \\
\text { DBVPG 6173 }\end{array}$ & $\begin{array}{l}\text { Sacch. } \\
\text { pastorianusT } \\
\text { DBVPG 6047 }\end{array}$ & $\begin{array}{l}\text { Sacch. } \\
\text { bayanusT } \\
\text { DBVPG 6171 }\end{array}$ \\
\hline Sacch.cerevisiae NCAIM Y.00768 & 52 & 98 & - \\
Sacch.cerevisiae NCAIM Y.00769 & 50 & 97 & - \\
Sacch.carlsbergensis NCAIM Y.00770 & 96 & 50 & - \\
Sacch.carlsbergensis NCAIM Y.00835 & 98 & 48 & - \\
Sacch. uvarum NCAIM Y.00823 & 89 & 45 & - \\
Sacch. pastorianus NCAIM Y.00824 & 94 & 42 & - \\
Sacch. bayanus NCAIM Y.00826 & 96 & - & 18 \\
Sacch. bayanus NCAIM Y.00827 & 94 & - & 19 \\
Sacch. bayanus NCAIM Y.00828 & 90 & - & 22 \\
Sacch. bayanus NCAIM Y.00829 & 88 & - & 20 \\
Sacch. bayanus NCAIM Y.00830 & 89 & - & 12 \\
Sacch. heterogenicus NCAIM Y.00831 & 90 & & -
\end{tabular}

$\mathrm{T}=$ Type strain; NCAIM = National Collection of Agricultural and Industrial Microorganisms

DBVPG = Industrial yeast collection, Dipartimento di Biologia Vegetale, Universita di Perugia,Italy 


\section{nDNA/nDNA reassociation studies}

Extraction and purification of nDNA was accomplished by a combination of procedures by Marmur (1961) and Price et al. (1978). nDNA/nDNA reassociation was determined spectrophotometrically using a Gilford Response 2 instrument with a thermoprogrammer, according to Kurtzman et al. (1980). nDNA/nDNA homology $\%$ was calculated from the Cot curves (Britten and Kohne 1968) using the formula of Seidler and Mandel (1971).

\section{Results and Discussion}

Presence or absence of active fructose transport in 72 yeast strains, which were identified earlier as belonging to the Saccharomyces sensu stricto group and in eight Saccharomyces type strains are summarized in Table 1. Results obtained with type strains corresponded in all cases with those of Rodrigues de Sousa et al. (1990). Thirteen out of 72 wine and brewer strains differed in active fructose transport ability from that expected for the species identified earlier. These strains were examined for deoxyribonucleic acid relatedness. Results of nDNA/nDNA reassociation studies are summarized in Table 2.

Results of active fructose transport investigation in accordance with results of nDNA/nDNA homology confirmed that species of Saccharomyces sensu stricto can be divided into two groups: active fructose transport is present in $S$. bayanus and $S$. pastorianus and active fructose transport is absent in S. cerevisiae and S. paradoxus in agreement with finding of Rodrigues de Sousa et al. (1990). Vaughan-Martini and Martini (1993) by testing 12 strains of the four species also confirmed that they can be discriminated on the basis of this property. Molecular taxonomy has reformed yeast classification and has become of prime importance in the differentiation of species (Kurtzman 1987). While nDNA/nDNA reassociation method is an excellent research tool, it is too expensive and sophisticated for routine yeast identification. Active fructose transport can be determined easily and this phenotypic character correlates with the molecular classification of species in the group of Saccharomyces sensu stricto. Hence it is a useful method for the identification of yeast strains belonging to Saccharomyces sensu stricto. Investigations are in progress to find appropriate and reliable conventional physiological tests as suggested by Vaughan-Martini and Martini (1993) to differentiate between pairs of Saccharomyces species differentiated on the basis of the presence or absence of active fructose transport.

\section{References}

Barnett J.A. 1992. The taxonomy of the yeast Saccharomyces Meyen ex Rees: a short review for nontaxonomists. Yeast 8: 1-23.

Barnett J.A., Payne R.W. and Yarrow D. 1990. Yeasts: characteristics and identification. 2nd ed., Cambridge Univ. Press, Cambridge, pp. 594-602.

Britten R.J. and Kohne D.E. 1968. Repeated sequences in DNA. Science 161: 529-540.

Kreger-van Rij N.J.W. ed. 1984.The Yeasts. A Taxonomic Study. 3rd ed. Elsevier Science Publishers, Amsterdam, pp.379-395.

Kurtzman C.P., Smiley M.J. and Johnson C.J. 1980. Emendation of the genus Issatchenkia Kudriavzev and comparison of species by deoxyribonucleic acid reassociation, mating reaction and ascospore ultrastructure. International Journal of Systematic Bacteriology 30: 503-513.

Kurtzman C.P. 1989. Molecular taxonomy. In The Yeasts. Rose A. H. and Harrison J. S. eds., 2nd ed., Academic Press, London, pp. 63-89.

Lodder J. ed. 1970. The Yeasts: A Taxonomic Study. North-Holland Publ. Co., Amsterdam, pp. 555-718.

Marmur J. 1961. A procedure for the isolation of DNA from microorganisms. Journal of Molecular Biology 3: 208-218. 
Price C.W., Fuson G.B. and Phaff H.J. 1978. Genome comparison in yeast systematics: delimitation of species within the genera Schwanniomyces, Saccharomyces, Debaromyces, and Pichia. Microbiological Reviews 42: 161-193.

Rodrigues de Sousa H., Spencer-Martins I. and van Uden N. 1990. Active fructose transport in Saccharomyces sensu stricto. Taxonomic implications. Acta Varia 5:127-134.

Seidler R. J. and Mandel M. 1971. Quantitative aspects of deoxyribonucleic acid renaturation: Base composition, state of chromosome replication, and polynucleotide homologies. Journal of Bacteriology 106: 608-614.

van Uden N. 1967. Transport-limited fermentation and growth of Saccharomyces cerevisiae and its competitive inhibition. Archives of Microbiology 58: 155-168.

Vaughan Martini A. and Martini A. 1989. Saccharomyces paradoxus comb. nov., a newly separated species of the Saccharomyces sensu stricto complex based upon nDNA/nDNA homologies. Systematic and Applied Microbiology 12: 119-122.

Vaughan Martini A. and Martini A. 1987. Three newly delimited species of Saccharomyces sensu stricto. Antonie van Leeuwenhoek 53: 77-84.

Vaughan Martini A. and Martini A. 1993. A taxonomic key for the genus Saccharomyces. Systematic and Applied Microbiology 16: 113-119. 


\title{
PHYLOGENIC RELATIONSHIPS WITHIN THE SACCHAROMYCES CEREVISIAE COMPLEX SPECIES.
}

\author{
Dilnora E. GOUliamova ${ }^{1}$ GRÉGOIRE L. HENNEBERT $^{2}$ \\ ${ }^{1}$ Institute of Microbiology, Acad. G. Bonchev str. 26, 1113 Sofia, Bulgaria \\ ${ }^{2}$ Mycothèque de l'Université Catholique de Louvain, Place Croix du Sud 3, B-1348 Louvain-la-Neuve, \\ Belgium
}

\section{Introduction}

Saccharomyces cerevisiae, as conceived by Kreger-van Rij (1984), is a large species covering 75 differently named taxa (S. cerevisiae and 75 synonyms). That is the taxon she called Saccharomyces cerevisiae sensu lato.

Vaughan-Martini and Martini (1987) demonstrated by DNA/DNA reassosiation the weak relatedness between the type or neotype strains of three of the synonymyzed taxa (S. pastorianus, S. bayanus, S. paradoxus) and the neotype strain of $S$. cerevisiae, while relatedness between two of the synonimized species themselves is also weak, as shown here.

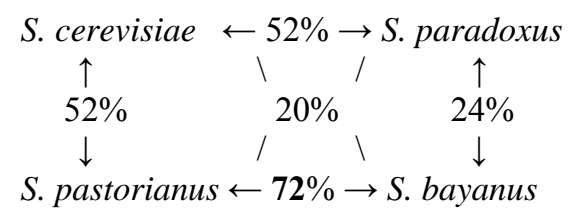

That means that Saccharomyces cerevisiae as a large species cannot stand. It is a group of related but distinct species: three species are shown distinct from Saccharomyces cerevisiae sensu stricto.

To be convincing those results needed confirmation. Kurtzman and Robnett (1991) proved the non-similarity between the three synonymized species and S. cerevisiae, using partial sequencing (300 bases) in two regions of $28 \mathrm{~S}$ rRNA and o,e region of $18 \mathrm{~S}$ rRNA. But, they used to charactere $S$. pastorianus the type strain of $S$. carbergensis and therefore were characterizing the later species instead.

\section{Material and methods}

The purpose of the present work is to confirm the Vaughan-Martini and Martini's statement (1987) by using the proper type strains of the same four species names: MUCL 31497, NT of S. cerevisiae, MUCL 31496, NT of S. pastorianus, MUCL 31495, T of S. baynus, MUCL 3149, NT of S. paradoxus.

\section{rRNA extraction}

Cells were grown at $25^{\circ} \mathrm{C}$ in $100 \mathrm{ml}$ of DYP liquid medium (dextrose, yeast extract, peptone) on rotary shaker at 200rpm during 16 hours. Celles were harvested by centrifugation, washed with BPS buffer and freeze dried. Undergraded rRNA was isolated according two procedures, one by Guadet et al. (1989) and the other by guanidinium thiocyanate (Promega). Purity of rRNA samples was estimated from spectrophotometric absorbance ratios $260 / 280=1.70-2.00$ and their integrity by denaturating agarose gel electrophoresis (Fig. 1). 

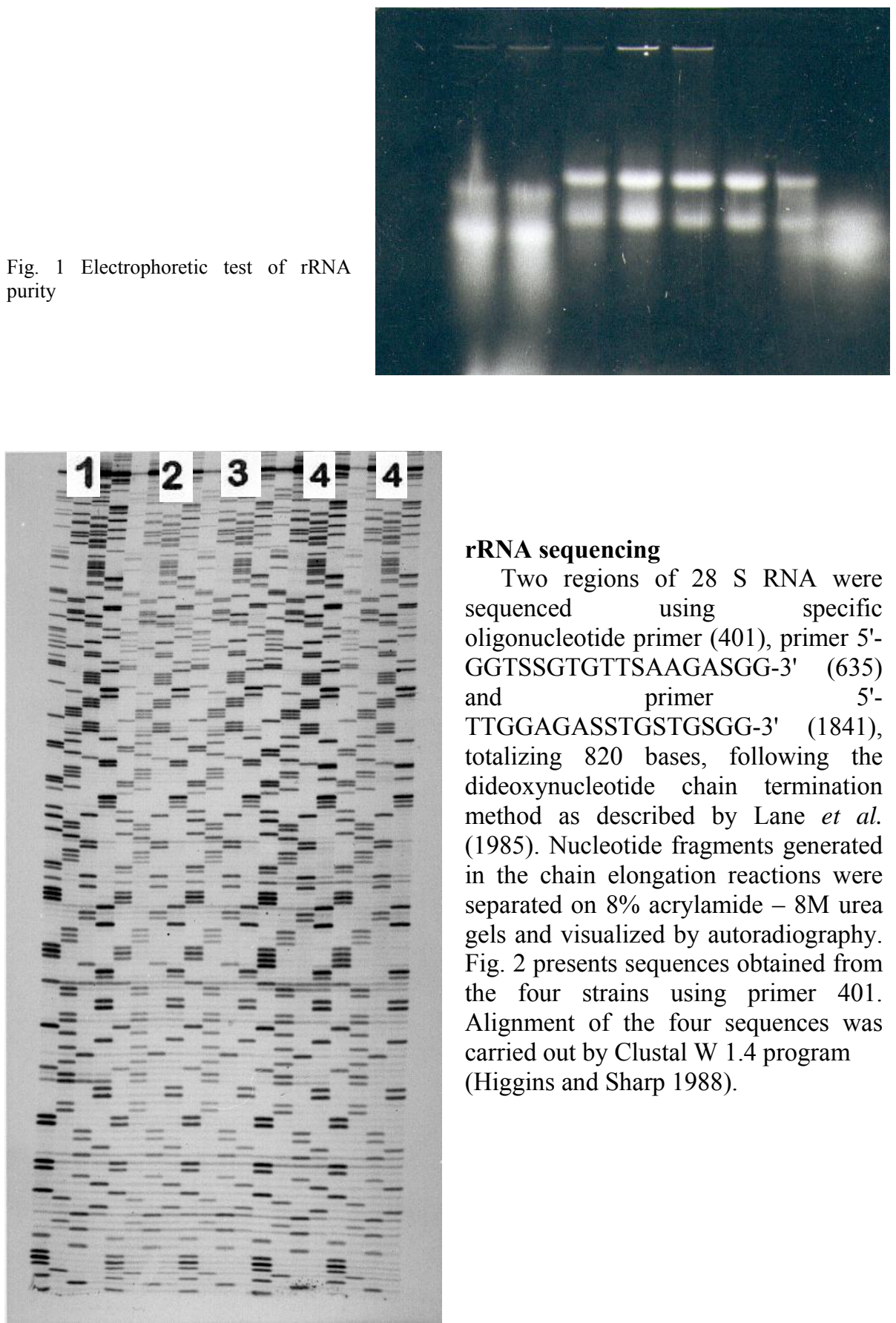

\section{rRNA sequencing}

Two regions of $28 \mathrm{~S}$ RNA were sequenced using specific oligonucleotide primer (401), primer 5'GGTSSGTGTTSAAGASGG-3' (635) and primer TTGGAGASSTGSTGSGG-3' (1841), totalizing 820 bases, following the dideoxynucleotide chain termination method as described by Lane et al. (1985). Nucleotide fragments generated in the chain elongation reactions were separated on $8 \%$ acrylamide $-8 \mathrm{M}$ urea gels and visualized by autoradiography. Fig. 2 presents sequences obtained from the four strains using primer 401. Alignment of the four sequences was carried out by Clustal W 1.4 program (Higgins and Sharp 1988).

Fig. 2 Sequences of $28 \mathrm{~S}$ rRNA portion from 1, S. pastorianus, 2, S. bayanus, 3, S. cerevisiae, 4, S. paradoxus 


\section{Phylogenetic tree}

A phylogenetic tree depincting the four sequenced species has been produced using the YVDP program (Van de Peer and De Wachter 1993) and including the sequences of another strain of $S$. cerevisiae and of five other fungi available from $\mathrm{Pr}$ De Wachter's data base, University of Antwerp. Evaluation of the branching reliability has been obtained by bootstrapping.

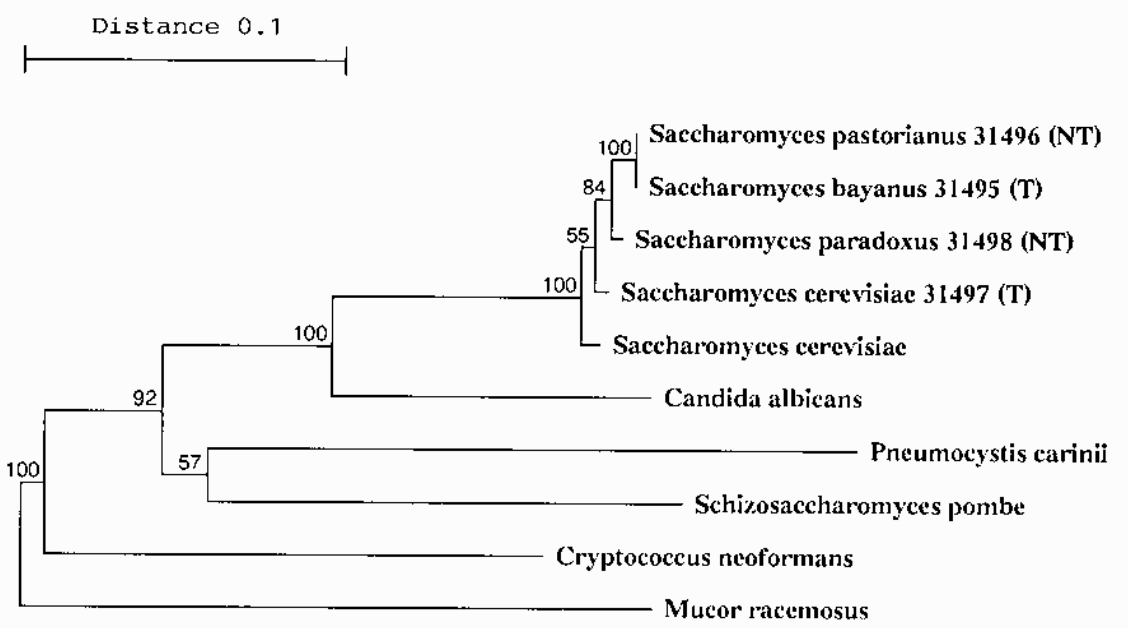

Fig. 3.Phylogenetic tree constructed using YVDP software.

\section{Results}

The obtained tree (Fig. 3) does not differ from the one obtained by Kurtzman and Robnett (1991) exepted by the fact that Saccharomyces cerevisiae in our tree seems to be phylogenetically older than the derivated species. This might result from sequencing a larger portion of rRNA but might also be due to the fact that the medial branching of the cluster is poorly reliable with $55 \%$ bootstrap.

In both trees the four tested Saccharomyces species cluster together separately from other fungal species. Saccharomyces pastorianus and S. bayanus cluster closely together with high bootstrap valuie (100\%) and confirm the DNA relatedness of $72 \%$ as shown by Vaughan-Martini and Martini (1987). It also confirms the weak DNA relatedness of $20 \%$ between S. cerevisiae and S. bayanus and between $S$. paradoxus and S. pastorianus.

S. carlsbergensis sequenced by Kurtzman and Robnett (1985) falls into the same position than S. pastorianus. But their synonymy needs demonstration.

It is noteworthy to observe the close relatedness between Candida albicans and Saccharomyces cerevisiae, both hemiascomycetous species, and the segregation of Pneumosystis carinii and Schizosaccharomyces pombe from the Hemiascomycetes, as it has been shown by $18 \mathrm{~S}$ rRNA sequencing. But the two latter taxa are certainly not mutually related as shown by $57 \%$ bootstrap value.

The other yeast Cryptococcus neoformans, is here also confirmed to be very distinct, in the same position as found in $18 \mathrm{~S}$ rRNA trees where it culsters together with Basidiomycetes. 


\title{
Conclusions
}

Our results based on the sequence of two portions of $28 \mathrm{~S}$ rDNA, confirms the distinction of Saccharomyces cerevisiae from the three first thought synonyms $S$. paradoxus, S. pastorianus and $S$. bayanus and the close relatedness between $S$. pastorianus and S. bayanus.

\section{References}

Guadet J., Julien J., Lafey JF. and Brygoo Y. A989. Phylogeny of some fusarium species, as determined by large subunit rRNA sequence comparison. Mol. Biol. Evol. 6: 227-242.

Higgins D.J. and Sharp P.M. 1988. Clustal: a package for performing multiple sequence alignment on a microcomputer. Gene 73: 237-244.

Kreger-van Rij N.J.W. ed. 1984 The Yeasts, a taxonomic study. $3^{\text {rd }}$ edn. Elsevier Science Publ. Amsterdam.

Kurtzman C. and Robnett C. 1991. Phylogenetic relationships among Saccharomyces, Schizosaccharomyces, Debaryomyces and Schwanniomyces determined from partial ribosomal RNA sequences. Yeast 7: 61-72.

Martini-Vaughan A. and Martini A. 1987. Three newly delimited species of Saccharomyces sensu stricto. Antonie van Leewenhoek 53: 77-84.

Van de Peer Y. and De Wachter R. 1993. TREECON, a software package for the construction ans drawing of evolutionary trees. Comput. Appl. Biosc. 9: 177-182.

\section{WHAT ABOUT THE "SPECIES GROUP" CONCEPT IN ASPERGILLUS FLAVUS ?}

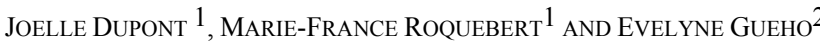 \\ $1_{\text {Laboratoire de Cryptogamie, M.N.H.N., } 12 \text { Rue Buffon, } 75005 \text { Paris }}$ \\ ${ }^{2}$ Unité de Mycologie, Institut Pasteur, 25 rue du Dr. Roux, 75015 Paris
}

Abstract. Whemer (1901) was the first to introduce the idea of "species groups" to accommodate the great diversity and variability of Aspergilli.

This concept is the foundation of the actual taxonomy of the genus.

The flavus group contains 16 species (Christensen 1985) from yellow green to brown in color. Some of them are morphologically well defined but others of industrial importance, referred as the Koji molds, A. oryzae, A. sojae, A. tamarii are difficult to differentiate from the toxinogenic and cosmopolitan species $A$. flavus and A. parasiticus.

Numerous technics have been applied to find new characters: electrophoretic mobility patterns of proteins and specific enzymes, pyrolysis profiles, DNA homology, GC contents of DNA. We propose a view of the flavus group through the comparison of sequences of the $5^{\prime}$ end of $28 \mathrm{~S}$ rRNA. Two points are clear and make the group heterogeneous:

- the complete similarity of 10 "species", including the Koji molds and the toxinogenic species,

- the split of A. zonatus and A. clavatoflavus together with the teleomorphic Penicilliopsis. 


\title{
A COMPARATIVE STUDY OF ELECTROPHORETIC KARYOTYPES IN THE YEAST GENERA TORULASPORA LINDNER AND ZYGOSACCHAROMYCES BARKER
}

\author{
GUIDO CAPRIOTTI AND ANN VAUGHAN-MARTINI
}

Dipartimento di Biologia Vegetale, Sez. Microbiologia Applicata, Universita degli Studi di Perugia, Italy

Abstract. In the most recent edition of the monograph The Yeast, a taxonomic study (Yarrow 1984) the genera Torulaspora and Zygosaccharomyces were formally separated from Saccharomyces. These genera differ from Saccharomyces in having a predominately haploid vegetative phase and in the mechanism of ascus formation: conjugation of independent cells that can be preceded by the formation of specific protuberances or by the union of a cell with its bud.

While the distinction between Torulaspora and Zygosaccharomyces is usually straightforward, some problems have been encountered in the separation of species within Zygosaccharomyces since several taxa are characterized by limited assimilative and fermentative profiles. Since these genera has been clarified by DNA/DNA reassociation experiments (Price et al. 1978, Kurtzman 1991), the definition of electrophoretic karyotypes of all type strains of the two genera can be useful as an aid for the identification of unknown strains.

\section{References}

Kurtzman C.P. 1991. DNA relatedness among species of the genus Zygosaccharomyces. Yeast 6: $213-$ 219.

Price C.W., Fuson G.B. and Phaff H.J. 1978. Genome comparison in yeast systematics. Delimitation of the species within the genera Schwanniomyces, Saccharomyces, Debaryomyces and Pichia. Microbiol. Rev. 42: 161-193.

Yarrow D. 1984. Saccharomyces Meyen ex Reess. In The yeasts, a taxonomic study. Kreger-van-Rij N.J.W. ed. Amsterdam, Elsevier Science Publishers. pp.379-395. 


\title{
FUNGAL BIODIVERITY
}

\section{BIODIVERSITY OF CYLINDROCLADIUM ON WATER-LILIES AT SSR PAMPLEMOUSSES BOTANIC GARDEN IN MAURITIUS}

\author{
A. PeERAlly
}

Abstract. An interesting case of fungal biodiversity within the same genus is described. Six species of Cylindrocladium are reported to occur on water-lilies in three contiguous ponds in Mauritius. The species are C. hawksworthii, recently descibed as a new species, C. quinqueseptatum, C. colhounii, C. floridanum, C. camelliae and C. infestans, all of which are pathogenic. Of these species with the exception of $C$. hawksworthii, the five others were previously observed to occur on tea in Mauritius.

\section{Introduction}

The taxonomy and pathology of the genus Cylindrocladium has fairly recently been comprehensively reviewed (Peerally 1991a). In the present paper the occurrence of six species of Cylindrocladium is reported on water-lilies at Pamplemousses Botanic Garden in Mauritius.

Three species of water-lilies, Nelumbo nucifera Gaertn., Nymphaea lotus L. and Victoria amazonica L. have, for many years, been known to be affected by a foliage disease (Fig. 1a-c). These water-lilies grow on three contiguous ponds, two of which contain $N$. nucifera and $N$. lotus, while the third larges pond bears $N$. lotus and the spectacular $V$. amazonica, of significant attraction to visitors. The occurrence of Cylindrocarpon quinqueseptatum Boedijn \& Reitsma and of C. hawksworthii on $N$. nucifera and $N$. lotus has already been described (Peerally 1991b), since the latter pathogenen was reported as a new species.

The associatopn of Cylindrocarpon as a pathogen of tea had been the subject of previous papers (Peerally 1973, 1974a-C, 1976a, b, 1991a), and the species reported are:

C. colhounii Peerally

C. floridanum Sob. \& Seymour

C. clavatum Hodges \& May

C. camelliae Venkataaara \& Venkata Ram

C. quiqueseptatum Boedijn \& Reitsma

C. infestans (Boersew.) Peerally.

The occurrence of two or more species of Cylindrocladium on the same hosts has been frequently reported in the literature. Thus significant mortality rates of seedlings of Eucalytus grandis and E. terreticornis were due to a disease complex which included C. quinqueseptatum, C. clavatum and C. ilicicola (Anon. 1982), Mohanan and Sharma (1986) found several species of Cylindrocladium associated with Eucalyptus spp. causing a complex damping off, stem canker and leaf and shoot blight in Kerala, India.

Several papers in the literature have emphasized the disease enhancing effects of the high moisture levels with Cylindrocladium diseases. Thus Barnard (1984) reported extensive damage of E. grandis and E. robusta seedlings under very humid conditions caused by overhead irrigation. Various workers (Petch 1921, Loos 1951, Wabster 1954) reported that the disease incidence by $C$. theae on tea to be enhanced by wet conditions and was checked with the cessation of rain. 


\section{Material and method}

The Cynlindrocladium species reported in this paper were all isoltad from diseased leaves of three species of water-lilies. All six species were examined from incubated diseased material and from colonies on PDA isolated from diseased leaves after surface sterilistation. Artificially and naturally infected leaves (Fig. 2a) of water-lilies were maintained in boxes with wet cotton to induce disease and sporulation which occurred very easily after a few days at $22-27^{\circ} \mathrm{C}$.

\section{Results}

The occurrence of Cylindrocladium on the three species of water-lilies wa as follows:

Host species

Nelundo nucifera

Nymphaea lotus

\section{Victoria amazonica}

\section{Cylindrocladium species}

C. quinqueseptatum, C. hawksworthi

C. quinqueseptatum, C. hawksworthii,

C. colhounii

C. colhounii, C. floridanum,

C. camelliae, C. infestans

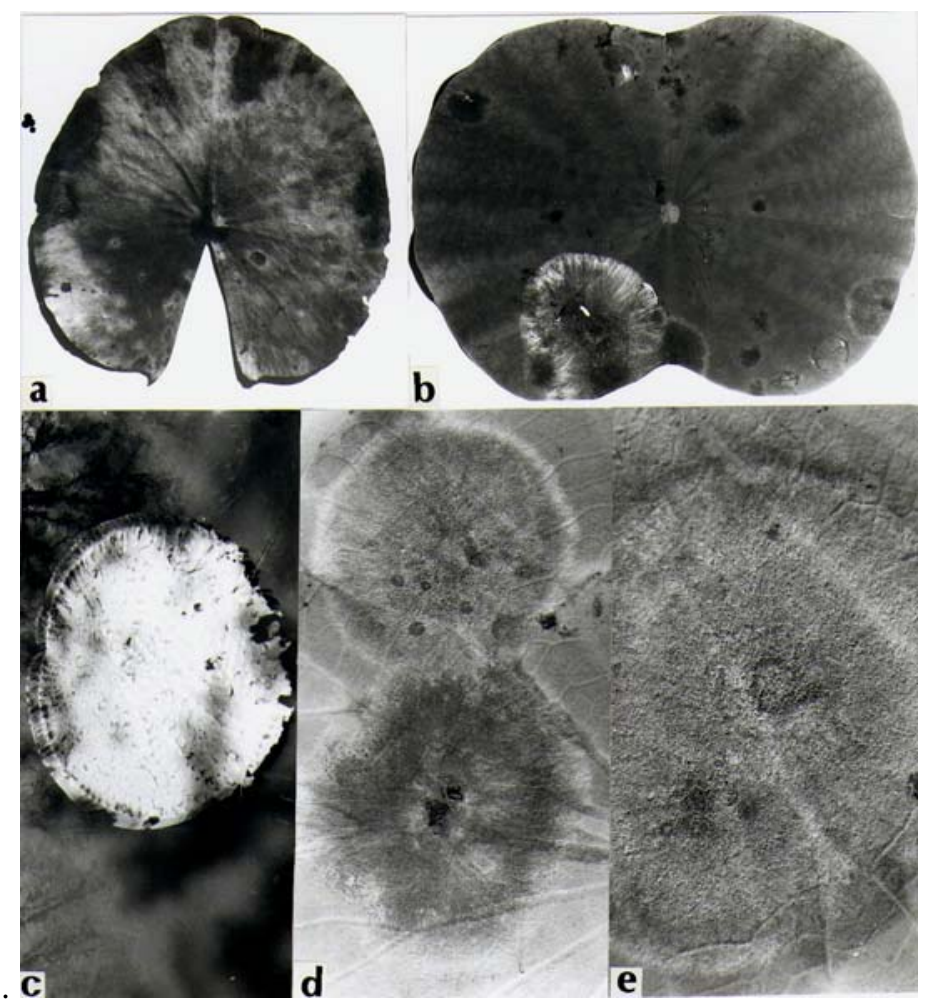

Fig. 1. a. Necrotic spots on Nymphaea lotus leave. b. Spot on Nelumbo nucifera leaf. c. Spot on Victoria amazonica leaf. d. Cylindrocladium hawksworthii (upper colony) and C. quinqueseptatum (lower colony) on $N$. nucifera. e. C. hawksworthii conidial masses on $N$. nucifera. 
Spots on Nelundo nucifera are grey becoming brown to black and coalesce to give large circular necrotic areas. The masses of conidiophores and conidia of $C$. quinqueseptatum are generally less dense (Fig. 1d) than those of C. hawksworthii (Fig. 1d, e) which look decidedly denser, more powdery and white.

Spots on Nymphaea lotus are initially water-soaked areas turning brown and later black.

On Victoria amazonica (Fig. 1c) there are conspicuous water-soaked areas which soften to produce a soft rot. It can be easily observed under a microscope that in infected areas the host cells separate easily due to the dissolution of the middle lamella. Areas of infected leaves of $V$. amazonica producing condidiophoes of $C$. infestans and C. camelliae are clearly discernible.

\section{Species description}

\section{Cylindrocladium colhounii Peerally (Fig. 2b)}

Coniophores 308-413 $\mu \mathrm{m}$ long, with strile appendage, much shorter without appendage. Stipe narrower towards an apical slender vesicle 7.8-82.0 x 2.0-5.2 $\mu \mathrm{m}$. Primary conidiophore branches aseptate, rarely 1-septate, 13-26 $\mu \mathrm{m}$ long; secondary branches aseptate, 7.8-18.2 $\mu \mathrm{m}$ long; tertiary branches aseptate 7.8-10.4 $\mu \mathrm{m}$. Phialides in groups of 2-4, occasionally arising directly from the stipe, 7.6-13.6 $\mu \mathrm{m}$ long. Conidia hyaline, cylindrical, straight, 3-septate, rarely 1- or 2-septate, never 4or 5-septate, 38.3-84.2 x 3.4-5.7 $\mu \mathrm{m}$, in average $66.0-5.0 \mu \mathrm{m}$.

\section{Cylindrocladium quinqueseptatum Boedijn \& Reitsma (Fig.2c, d)}

Conidiophores branches arising laterally from a stipe; primary branches aseptate or 1-septate, 8.4-26.6 $\mu \mathrm{m}$ long; secondary and tertiary branches aseptate, 11.5-15.6 $\mu \mathrm{m}$. Phialides hyaline, 13.0-15.6 $\mu \mathrm{m}$ long. Conidia hyaline, cylindrical, straight, $1-$ to 6-septate, usually 5-septate, old conidia in culture developing more than 6 septa, $59.8-104.6$ x 5.2-7.0 $\mu \mathrm{m}$ in vivo, 72.8-119.6 x 5.2-7.8 $\mu \mathrm{m}$ in vitro. Sterile filament terminating in a narrowly clavate vesicle $2.5-3.0 \mu \mathrm{m}$ wide.

\section{Cylindrocladium hawksworthii Peerally (fig. 3)}

Conidiophores erect, hyaline, septate, 250-500 $\mu \mathrm{m}$ long. Stipe conspicuously setate; primary conidiophore branches 1-septate or aseptate 12-80 $\mu \mathrm{m}$ long; secondary branches 1-septate or aseptate 12-45 $\mu \mathrm{m}$ long; tertiary branches usually aseptate 8-30 $\mu \mathrm{m}$ long; quaterny branches usually aseptate 7-35 $\mu \mathrm{m}$ long; sterile appendage 160-280 $\mu \mathrm{m}$; phialides solitary or in small groups, sometimes arising directly from the stipe, hyaline, aseptate, 7-13 $\mu \mathrm{m}$ long; conidia hyaline, cylindrical, curved, 1-septate, 36-62 x 3-5 $\mu \mathrm{m}$.

\section{Cylindrocladium floridanum Sob. \& Seymour (Fig. 4a-d)}

Conidiophores banches arising lateraly from a stipe; primary and secondary branches asteptate or 1-septate, 10.2-26 $\mu \mathrm{m}$ long; tertiary branches aseptate, 7.8-10.2 $\mu \mathrm{m}$. Phialides hyaline, 7.8-15.6 $\mu \mathrm{m}$. Conidia hyaline, cylindrical, 1-septate, 36.4$57.2 \times 2.6-4.6 \mu \mathrm{m}$. Sterile filmaments temenating in a globose vesicle, $8-18 \mu \mathrm{m}$ diam. Secondary sterile filaments present in most isolates. 

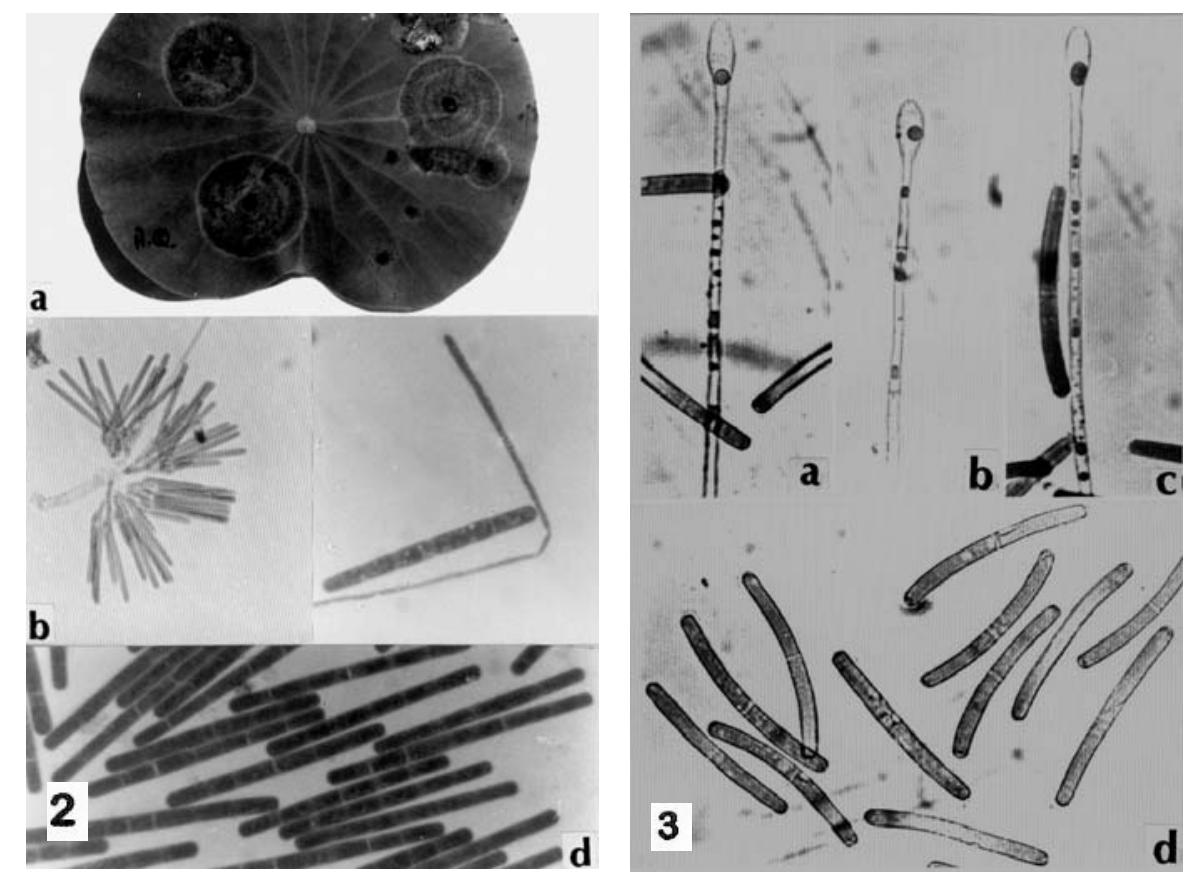

Fig.2. Cylindrocladium colhounii. b. Conidiophore and conidia. Cylindrocladium quinqueseptatum. a. spots on in vitro inoculated Nelundo nucifera leaf. c. Sterile appendate with vesicle. d. Conidia.

Fig. 3. Cylindrocladium hawksworthii. a-C. Sterile appendage with vesicle. d. Conidia.

\section{Cylindrocladium camelliae Venkatara \& Venkata Ram (Fig. 5c-g)}

Conidiophores of two types: penicillate or verticillate. In penicillate stipe, sterile filament usually present; primary branches aseptate, 12.0-28.2 $\mu \mathrm{m}$ long; secondary branches aseptate, 8.4-19.6 $\mu \mathrm{m}$; phialides navicular, hyaline, 9.2-18.6 $\mu \mathrm{m}$. In verticillate stipe, sterile filament absent, phialides 16.8-31.2 $\mu \mathrm{m}$ long. Conidia hyaline cylindrical, 1-septate, 9.6-15.6 x 1.8-2.2 $\mu \mathrm{m}$. Sterile filament elongate, aseptate, thick-walled, 60.0-110.6 $\mu \mathrm{m}$, apically vesiculate. Vesicle lanceolate or ellipsoidal, sometimes lanceolate curved, thin walled, 4.0-5.2 $\mu \mathrm{m}$ wide. Chlamydospores present. Microconidia unfrequent in culture.

\section{Cylindrocladium infestans (Boesew.) Peerally (Fig 5a, b)}

Conidiophores of two types: penicillate or most often subverticillate. Penicillate conidiophores 190-250 $\mu \mathrm{m}$ long; primery branches 13-22 $\mu \mathrm{m}$ long; secondary branches 8-16 $\mu \mathrm{m}$ long; phialides avicular 5-15 $\mu \mathrm{m}$. In verticillate conidiophores, significantly phialides longer, $14-32 \mu \mathrm{m}$ long. Sterile filament 70-130 $\mu \mathrm{m}$ long, ending a a cylindrical or narrowly lanceolate vesicle $20-70$ x $4-5 \mu \mathrm{m}$. Chlamydospores présent. Microconidia unfrequent in culture. 

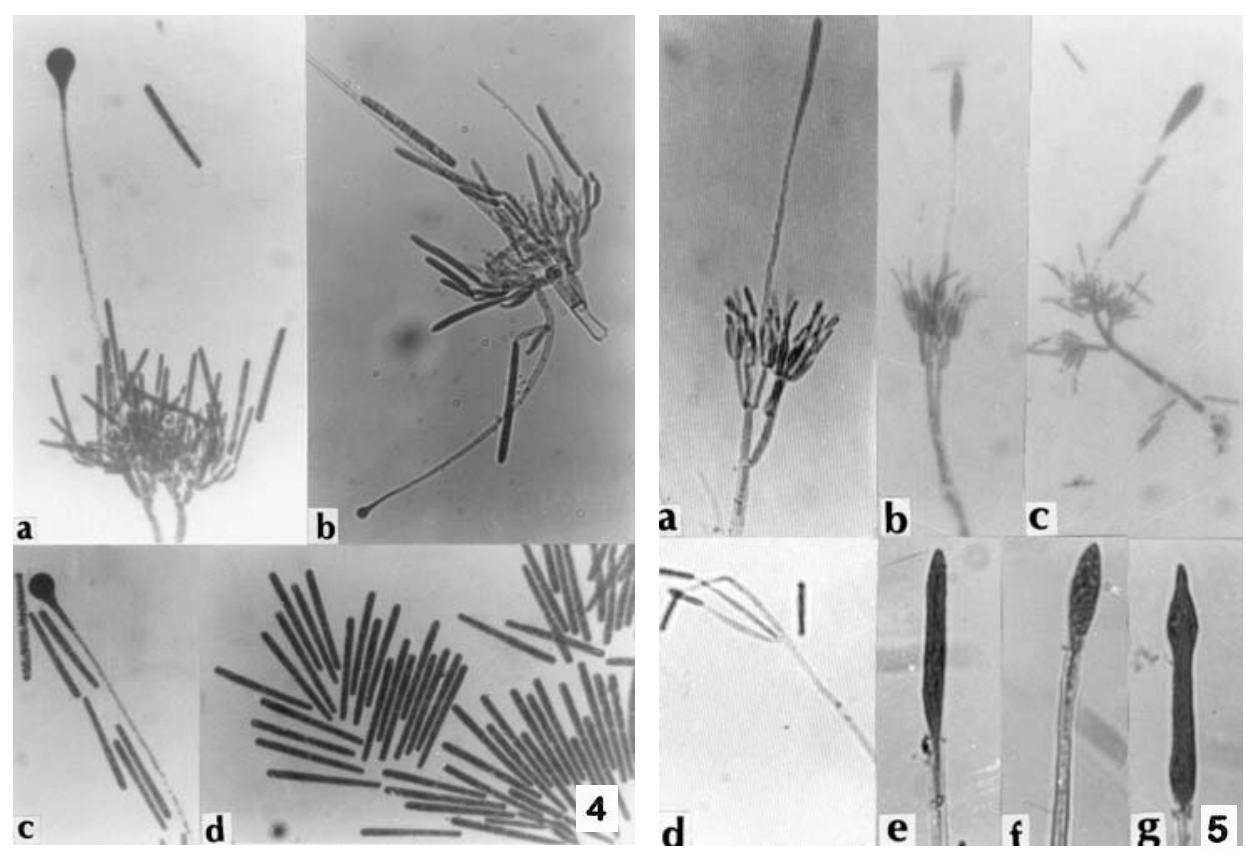

Fig. 4. Cylindrocladium floridanum. a, b. Conidiophores. c. Sterile appendage with vesicle. d. Conidia.

Fig. 5. Cylindrocladium infestans. a. Penicillate conidiophore. d. vertcillate conidiophore Cylindrocladium camelliae. b-c. Penicillate conidiophores. e-g. Vesicles .

\section{Discussion}

Six species of Cylindrocladium pathogenic on water-lilies in Mauritius are described from three contiguous ponds in Pamplemousses Botanic Garden. In addition to the pathology of this disease complex it is interesting to highlight the biodiversity and ecological aspects. Several workers like Peerally (1974b), Rattam and Dhanda (1985) and Mohanan and Sharma (1986), have reported mixed infections involving two or morespecies of Cylindrocladium. The foliage disease of water-lilies in Mauritius associated with six species of Cylindrocladium is a particularly stricking example of the biodiversity of a single fungus genus on three species of water-lilies growing within a very small area. The fact that this disease on water-lilies occurs in a particulaly humid habitat is quite characteristic of Cylindrocladium, which has been constantly reported to thrive best under very humid conditions in both artificial and natural growing conditions. Of the six species occuring on water-lilies five are pathogenic to tea in Maritius in the central plateau, about $300 \mathrm{~km}$ away from the Pamplemousses Gargen. The sixth species, C. hawksworthii, was described as a new species which has not so far been observed on tea or any other host.

\section{Acknowlegements}

My appreciation is due to $\mathrm{Mr} \mathrm{V}$. Aumeer for technical assistance.

\section{References}


Anonymous 1982. Nursery diseases of Eucalyptus in Kerala. Evergree 8: 2-4. Div. of Pathology, Kerala Forest Res. Inst., Peechi, India.

Barnard E.L. 1984. Occurrence, impact and fungicidal control of girdling stem cankers caused by Cylindrocladium scoparium on Eucalyptus seedlings. Plant Disease 68: 471-473.

Loos C.A. 1951. Pathological problems. Tea Quartely 22: 27-30.

Mohanan C. and Sharma J.K. 1986. Epidemiology of Cylindrocladium diseases of Eucalyptus . In Eucalyptis in India: Past, present and future. Proceedings of the National Seminar, Kerala Forest Inst. India.

Peerally A. 1972a. A new disease on tea. Revue agricole et Sucrière de l'Ile Maurice 51: 115-117.

P eerally A. 1972b. A decline of tea bushes associated woth a root rot. Revue Agricole et Sucrière de l'Ile Maurice 51:147-152.

Peerally A. 1973. Calonectria colhounii sp. nov., a common parasite of tea in Maritius. Trans. Brit. mycol. Soc. 61: 89-93.

Peerally A. 1974a. Cylindrocladium clavatum. CMI Descriptions of Pathogenic Fungi and Bacteria 422.

Peerally A. 1974b. Calonectria quinqueseptata (conidial state: Cylindrocladium quinqueseptatum). CMI Descriptions of Pathogenic Fungi and Bacteria 423.

Peerally A. 1974c. Cylindrocladium camelliae. CMI Descriptions of Pathogenic Fungi and Bacteria 428.

Peerally A. 1974d. An elucidation of certain diseases of tea caused by Calonectria spp. Revue Agricole et Sucrière de l'Ile Maurice 53: 57-66.

Peerally A. 1991a. The classification and phytopathology of Cylindrocladium species. Mycotasxon 40: 323-366.

Peerally A. 1991b. Cylindrocladium hawksworthii sp. nov. pathogenic in water-lilies in Mauritius. Mycotaxon 40: 367-376.

Petch T. 1921. Report of the Botanist/Mycologist. Tropical Agriculturist 57: 318-319.

Rattan G.S. and Dhanda R.S. 1985. Leaf blight and seedling diseases of Eucalyptus caused by Cylindrocladium spp. in Punjab. Annals of Biology (India) 1: 184-188.

Webster B.N. 1854. Notes on pathological matters. Tea Quarterly 25: 17-19. 


\title{
THE OCCURRENCE OF WOOD DECAYING FUNGI IN BELGIUM HOUSES
}

\author{
CONY DECOCK AND GREGOIRE L. HENNEBERT
}

Mycothèque de l'Université catholique de Louvain (MUCL) Laboratoire de Mycologie Systématique et Appliquée, Place Croix du Sud 3, 1348 Louvain-la-Neuve, Belgium

\begin{abstract}
From 1990 to 1993, 602 cases of timber decay from anish houses have been analysed at MUCL. 18 species of wood decaying fungi have been recorded. 11 species cause a brown rot and represent more than $72 \%$ of the timber decay while 7 species cause white rot. Serpula lacrymans occurs in $46 \%$ of the cases and is the more frequent species. After this, Donkioporia expansa, Coniophora marmorata and Asterostroma ochroleucum are observed at a respective frequency of $11 \%, 7.3 \%$ and $6.3 \%$. Noteworthy, Coniophora marmorata is here much more common than Coniophora puteana, reputed in other countries as most frequent. Another species, rarely documented as a wood decaying fungus in houses, is Asterostroma ochroleucum. The fungus is causing a white rot of wood and expands easily on and in plasterwork and masonry in very wet areas.

Keywords. Timber decay, house fungi, Serpula lacrymans, Donkioporia expansa, Coniophora marmorata, Asterostroma ochroleucum.
\end{abstract}

\section{Introduction}

Fungal decay in houses immediately suggests an attack by the true dry rot fungus, Serpula lacrymans. Indeed, Serpula lacrymans is the most frequently reported but also the most destructive fungi in all North Western Europe. Nevertheless, other lignicolous basidiomycetes occur in buildings beside Serpula lacrymans. For instance Coniophora puteana, the cellar fungus, is known as frequent. But few informations are available over the presence and frequency of others species than the true dry rot in buildings of central and western Europe. Koch (1985) gives an overview of the principal wood-rot fungi occurring in Danish buildings for years 1946-1983. More recently, Paajanen and Viitanen (1989) do the same for Finland for the years 1978 to 1988.

From 1990 to 1993, 683 cases of timber decaying fungi were analysed by MUCL. This paper presents a brief draft of the species encountered and their frequency in Belgian housing with some comments on noteworthy species.

\section{Situation in Belgium}

Between 1990 and 1993, 683 timber decaying fungi were analysed at MUCL. Most samples were sent or brought to the laboratory by private owners or repairing companies. $92.4 \%$ of the samples were identified to the species level, doubtful cases identified only to the genus level, representing 7.6\%. 18 species belonging to 8 families have been recorded. Table 1 gives the list of species encountered, their respective case number and frequency. Table 2 gives the families with their respective species number, incidence and kind of rot (classification according to Jülich 1981). 11 species cause a brown rot -or laccase negative rot- while 7 species cause a white rot -or laccase positive rot. Incidence of brown rotter is much more important than that of white rotter. Indeed, more than $72 \%$ of timber decay is by brown rot against only $28 \%$ by white rot. Almost two third $(62.7 \%)$ of the decay are assigned to members of the Coniophoraceae represented by 3 genera and 5 species. 


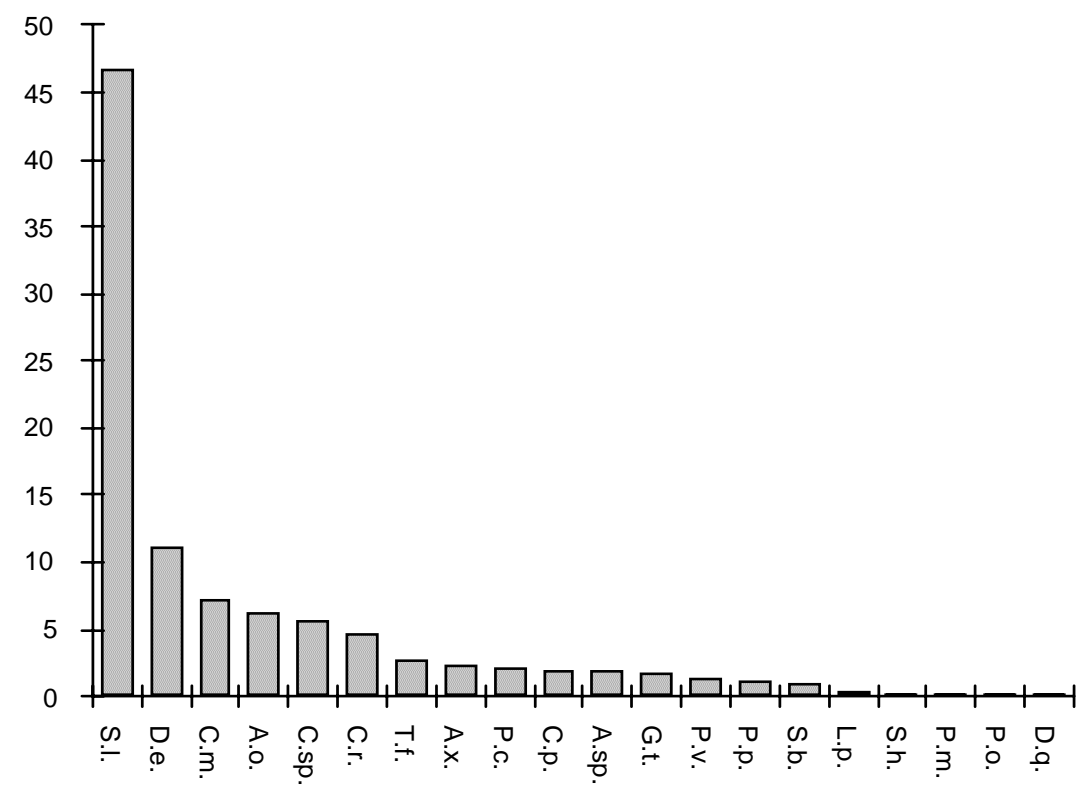

S.l.: Serpula lacrymans - D.e.: Donkioporia expansa - C.m.: Coniophora marmorata - A.o.: Asterostroma ochroleucum - C. sp.: Coniophora species - C.r.: Coprinus radians - T.f.: Trechispora farinacea - P.c.: Phellinus contiguus - A.x.: Antrodia xantha - C.p.: Coniophora puteana - A.sp.: Antrodia species - G.t.: Gloeophyllum trabeum - P.p.: Paxillus panuoides - P.v.: Poria vaillantii - S.b.: Sistotrema brinkmanii - L.p. Leucogyrophana pinastri - S.h.: Serpula himantioides - P.m.: Perenniporia medulla-panis - P.o.: Pleurotus ostreatus - D.q.: Daedelea quercina.

Table 1. List of species seen during the studies and their respective number, frequency and kind of rot.

\begin{tabular}{|l|c|c|c|}
\hline \multicolumn{1}{|c|}{ Species } & raw number & Percentage & Kind of rot \\
\hline Serpula lacrymans & 319 & 46.7 & Brown \\
\hline Donkioporia expansa & 76 & 11.12 & White \\
\hline Coniophora marmorata & 50 & 7.32 & Brown \\
\hline Asterostroma ochroleucum & 43 & 6.29 & White \\
\hline Coniophora sp. & 39 & 5.7 & Brown \\
\hline Coprinus radians & 32 & 4.68 & White \\
\hline Trechispora farinacea & 19 & 2.78 & White \\
\hline Antrodia xantha & 16 & 2.34 & Brown \\
\hline Phellinus contiguus & 15 & 2.19 & White \\
\hline Coniophora puteana & 14 & 2.05 & Brown \\
\hline Antrodia sp. & 13 & 1.90 & Brown \\
\hline Gloeophyllum trabeum & 12 & 1.75 & Brown \\
\hline Antrodia vaillantii & 10 & 1.46 & Brown \\
\hline Paxillus panuoides & 8 & 1.17 & Brown \\
\hline Sistotrema brinkmanii & 7 & 1.02 & Brown \\
\hline Leucogyrophana pinastri & 3 & 0.44 & Brown \\
\hline Serpula himantioides & 2 & 0.30 & Brown \\
\hline Perenniporia medulla-panis & 2 & 0.30 & White \\
\hline Pleurotus ostreatus & 2 & 0.30 & White \\
\hline Daedalea quercina & 1 & 0.15 & Brown \\
\hline \hline TOTAL & 683 & 100 & \\
\hline \hline
\end{tabular}


Table 2. List of families encountered with their respective frequency and kind of rot.

\begin{tabular}{|c|c|c|c|c|c|c|}
\hline \multirow{2}{*}{ Fungal family } & \multicolumn{2}{|c|}{ Brown rot } & \multicolumn{2}{c|}{ white rot } & \multicolumn{2}{c|}{ Total } \\
\cline { 2 - 7 } & species & $\%$ incidence & species & $\%$ incidence & species & $\%$ incidence \\
\hline Coniophoraceae & 5 & $62.5(427)$ & - & $0(0)$ & 5 & $62.5(427)$ \\
\hline Polyporaceae & 4 & $7.6(52)$ & 2 & $11.4(78)$ & 6 & $19(130)$ \\
\hline Lachnocladiaceae & - & $0(0)$ & 1 & $6.3(43)$ & 1 & $6.3(43)$ \\
\hline Coprinaceae & 0 & $0(0)$ & 1 & $4.7(32)$ & 1 & $4.7(32)$ \\
\hline Corticiaceae & 1 & $1.0(7)$ & 1 & $2.8(19)$ & 2 & $3.8(26)$ \\
\hline Hymenochaetaceae & - & $0(0)$ & 1 & $2.2(15)$ & 1 & $2.2(15)$ \\
\hline Paxillaceae & 1 & $1.2(8)$ & - & $0(0)$ & 1 & $1.2(8)$ \\
\hline Pleurotaceae & - & $0(0)$ & 1 & $0.3(1)$ & 1 & $0.3(1)$ \\
\hline $\begin{array}{c}\text { Total number of } \\
\text { species }\end{array}$ & 11 & $72.3(493)$ & 7 & $27.7(190)$ & 18 & 100 \\
\hline
\end{tabular}

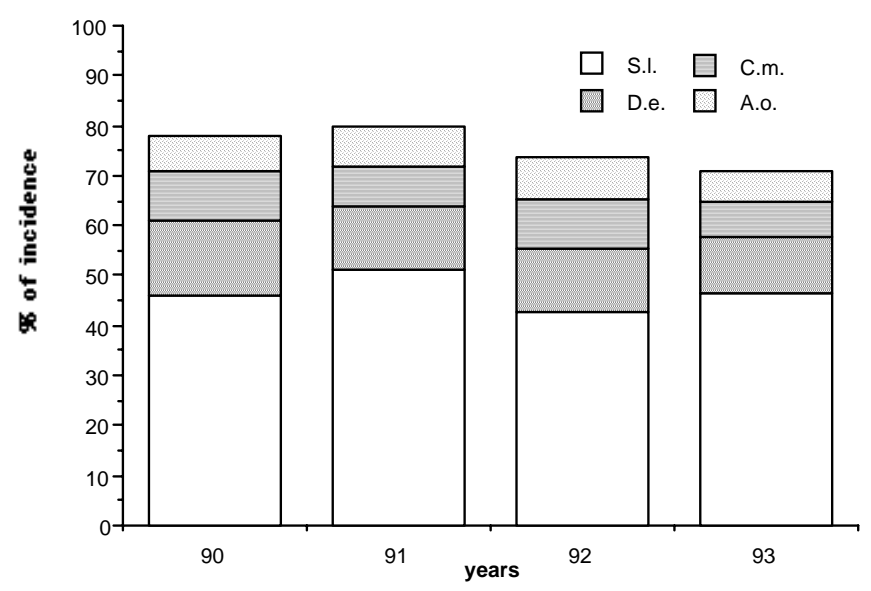

Fig. 2. Frequency of the 4 principal species year by year.

\section{Discussion}

Before discussing the present data, we must advice that relative occurrence given above are not necessarily representative of the real Belgian situation. In first hand, we do not have information from all around Belgium. Most of the specimens analysed originate from Brussels and Louvain-la-Neuve areas. In second hand, we have not collected all the samples ourselves in a randomly way. Most were sent to MUCL and we can not certify they were always collected randomly. It may occur that a selection has been applied before. This is particularly obvious for Serpula lacrymans. Indeed, information about the dry rot in house has been widely spread in the public the last years in Belgium by means of media, books and vulgarisation leaflets (Leclercq 1989, Rammeloo et al. 1989, 1992, Hennebert et al. 1990). Peoples dealing with timber decay (owners, repair companies, architects and insurance experts) may have acquired by this way a relatively good knowledge of the true dry rot symptoms allowing them to identify Serpula lacrymans. Samples sent to the laboratory might be only the most critical ones. This can alter the real species occurrence, leading to some under-estimation of Serpula lacrymans and consequently to some over-estimation of the others species. 

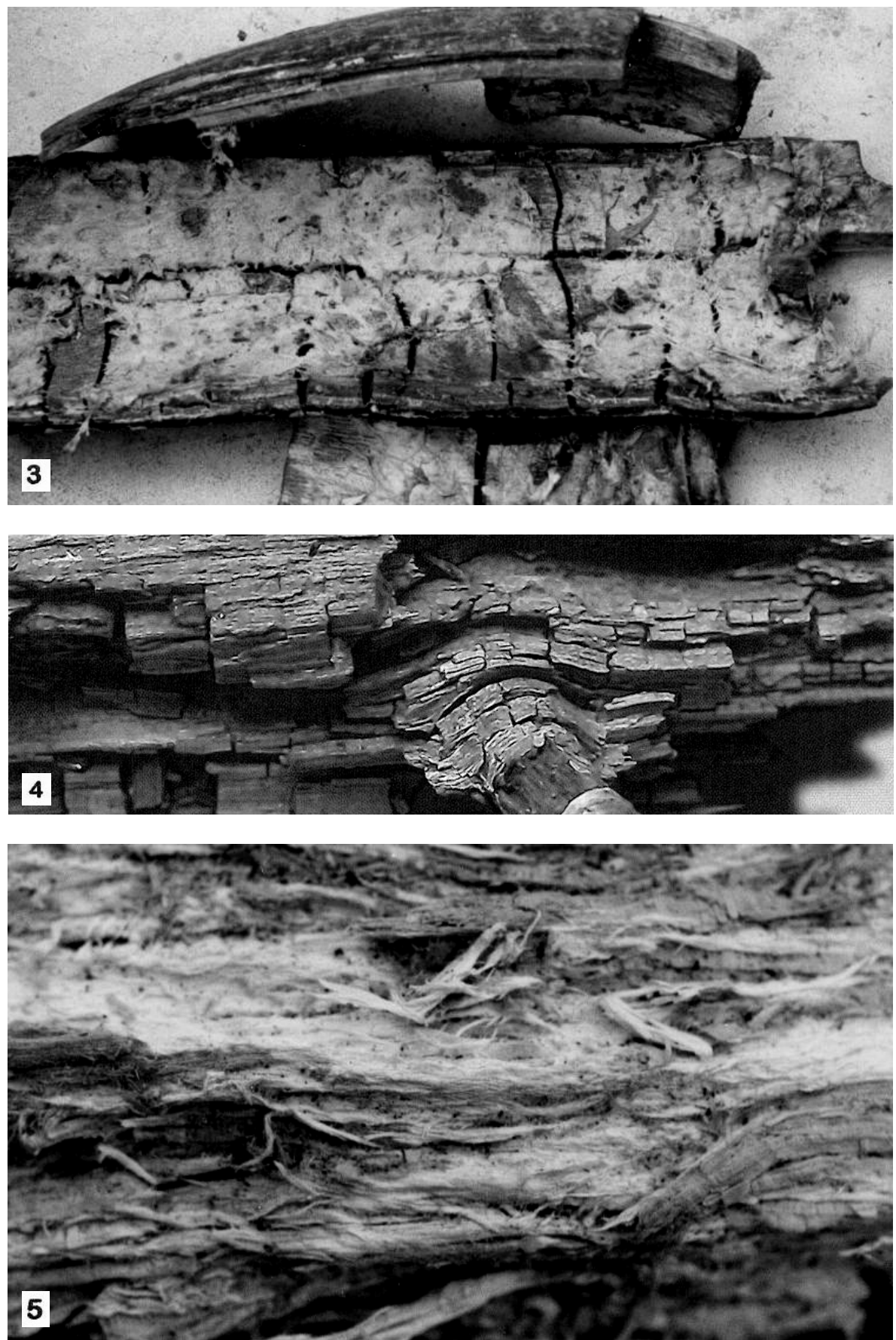

Fig.3. Cubic rot or Brown rot due to Serpula lacrymans.

Fig.4. Micro-cubic rot due to Coniophora puteana

Fig.5. Fibrous rot or White rot due to Donkioporia expansa. 
The occurrence given above must therefore be regarded more as a general overview rather than the real situation which could be slightly different. Nevertheless, for the other species than S. lacrymans, the number of cases per year represents a good draft of the situation. More than the real occurrence, the presence and the relative position of each species are important.

\section{Serpula lacrymans (Wulfen:Fries) Schroeter}

Serpula lacrymans, the true dry rot fungus, as in all north western European countries, is by far the most important species involved in timber decay. Almost $50 \%$ of the timber breakdown is attributed to that species. It is also the most destructive fungi for timber.

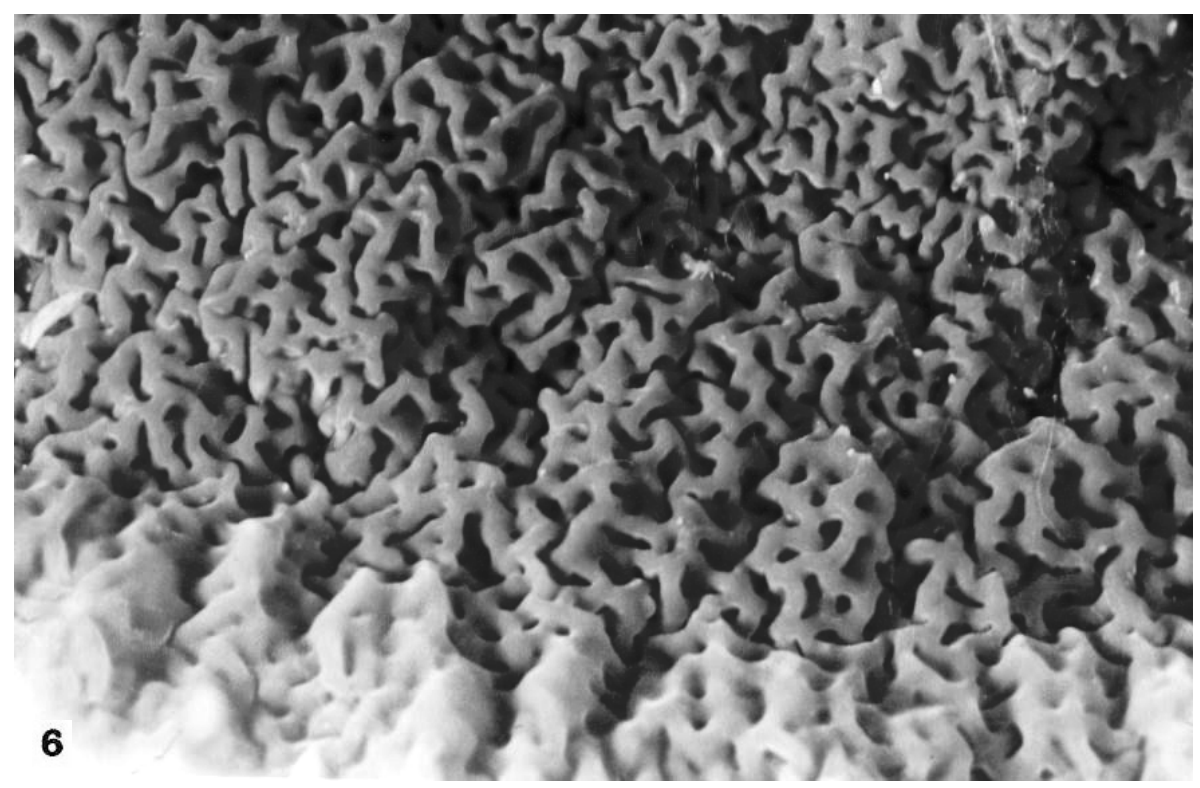

Fig. 6. Hymenium of the fruitbody of Serpula lacrymans.

\section{Donkioporia expansa (Desmazières) Kotlaba \& Pouzar}

Syn. Boletus expansus Desmazières

Poria expansa (Desmazières) H. Jahn

Phellinus cryptarum Karsten

Polyporus megaloporus Persoon

Phellinus megaloporus (Persoon) Heim

Poria megalopora (Persoon) Cooke

Donkioporia expansa (Desmazières) Kotlaba \& Pouzar comes in second position with an occurrence of $11 \%$ (76 cases in 4 years). This species causes a white rot mainly of deciduous wood but can also be found on coniferous (Cartwright and Findlay 1958, Findlay 1950, Buchwald 1986). 
According to Heineman (1979), the earliest Belgian record of this species dates on 1854. This author considered this species rare in Belgium and cited only 5 more records after the first one (Heineman 1979). Since then, in 1989, Guillitte (in Rammeloo et al. 1989) noted a very weak occurrence of only $2 \%$ (5 cases over 250 samples between 1985 and 1988). In 1992, the same author (in Rammeloo et al. 1992) mentioned 71 cases from 1985 to 1991 (for 749 wood decaying fungi analysed in 7 years), thus \pm 10 cases/year. In the present analysis one the 4 last years, 76 cases have been identified with an average of 19 cases/year

D. expansa was also considered as scarce in others western European countries. The species is known in Belgium since at least 1922 (as P. cryptarum) after the destruction of the Versailles Palace woodwork (Mangin and Patouillard 1922). There is no recent data available on the occurrence of the species in Belgium. In England, Findlay (1950) reports the species in «a dozen or more buildings». Jülich (1984) reports the same as rare in CS, D, DDR, GB and NL. Ritter (1983) draws attention on the species in a small report. As far as we know, Buchwald (1986) was the first to demonstrate that $D$. expansa occurs much more frequently in Germany than previously known. He reported an average of 15 to 20 cases/year while the species was stated as seldom.

Nor Koch (1985) nor Paajanen and Viitanen (1989) do mention the species in their survey of timber decaying fungi in North Europe. According to Ryvarden and Gilbertson (1993) D. expansa is a Central and Southern species.

Buchwald (1986) draws the attention on the fact that $D$. expansa is not strictly restricted to hardwood (oak and chestnut mainly) as stated previously but is also able to decay softwood. Well, in the past, most records were made on oak or chestnut wood (Cartwright and Findlay 1958, Findlay 1950), but it is well demonstrated now that th species occurs also on coniferous wood. Numerous collections are made on softwood now since oak wood is less used in modern buildings.

For more literature concerning this species, see Mangin and Patouillard 1922 (under P. cryptarum), Cartwright and Findlay 1958 (under P. cryptarum), Domanski and Orlicz 1967 (under Polyporus megaloporus Persoon), Dörfelt and Sommer 1973 (under Poria expansa), Heineman 1979 (under D. expansa), Ritter 1983 (under D. expansa), Buchwald 1986 (under D. expansa),

\section{Coniophora marmorata Desmazières.}

Coniophora marmorata Desmazières represent $7.9 \%$ of the specimens analysed (40 cases over 520) while Coniophora puteana represent only $2.4 \%$ (12 cases). Up to 1992, no mention of this species existed for Belgium. Ginns (1982), in its monograph of the genus Coniophora cited only 9 specimens. Few data on its occurrence in Europe are available. C. marmorata seems to be restricted to western, central and southern Europe. Indeed, nor Koch (1985) nor Paajanen and Viitanen (1989) mention the species in their survey. At the same, Hallenberg (1985) do not mention the species for northern Europe. However Beech-Andersen (1992) mentions the species in Denmark. 


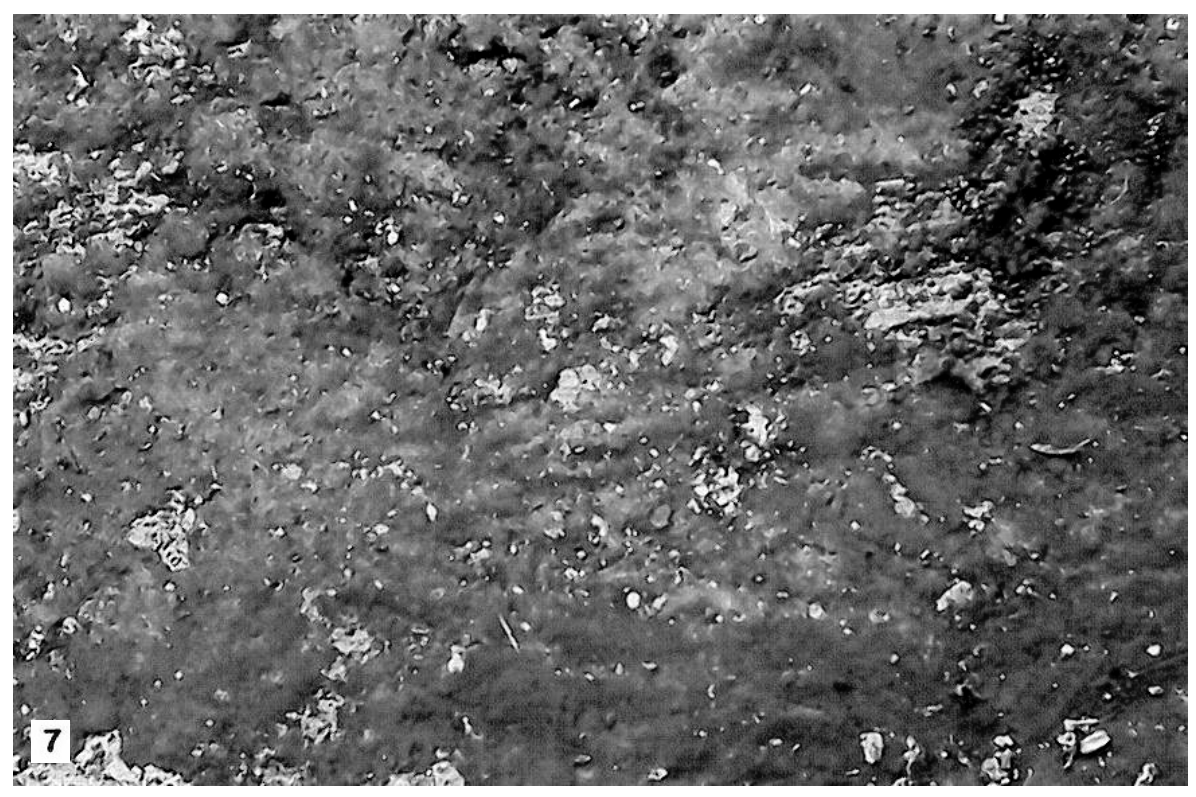

Fig. 7. fruitbody of Coniophora marmorata on wood.

Table 2. Main distinguishing features between C. marmorata and C. puteana(Ginns, 1982).

\begin{tabular}{|c|c|c|}
\hline Speciies & Spores & Mycelium \\
\hline C. marmorata & $\begin{array}{l}\text { subglobose } \\
\text { to ovoid } \\
\text { dextrinoid } \\
7-10 \mu \mathrm{m} \times 4.5-7(-8) \mu \mathrm{m}\end{array}$ & $\begin{array}{l}\text { dimitic with } \\
\text { tan to pale yellow } \\
\text { skeletal hyphae } \\
2-7 \mu \mathrm{m} \text { wide }\end{array}$ \\
\hline C. puteana & $\begin{array}{l}\text { broadly } \\
\text { ellipsoid } \\
\text { non dextrinoid } \\
9.5-14 \text { x 6-7.5 um }\end{array}$ & monomitic \\
\hline
\end{tabular}

\section{Asterostroma ochroleucum Bresadola.}

Asterostroma ochroleucum Bresadola represents $6.2 \%$ of samples analysed (43 cases over 683).

A. ochroleucum produces a white fibrous rot of both hardwood and coniferous wood. It expands easily on and in plasterwork and masonry in very wet areas forming whithish to ochraceous strands network which can be mistaken for $S$. lacrymans strands with the naked eyes. It is easily recognised under the microscope by its typical brown asterosetae. When fruitbodies is present, which occurs scarcely in Belgian buildings, spores are globose to subglobose and strongly tuberculate (fig. 9) with amyloid tubercule. 


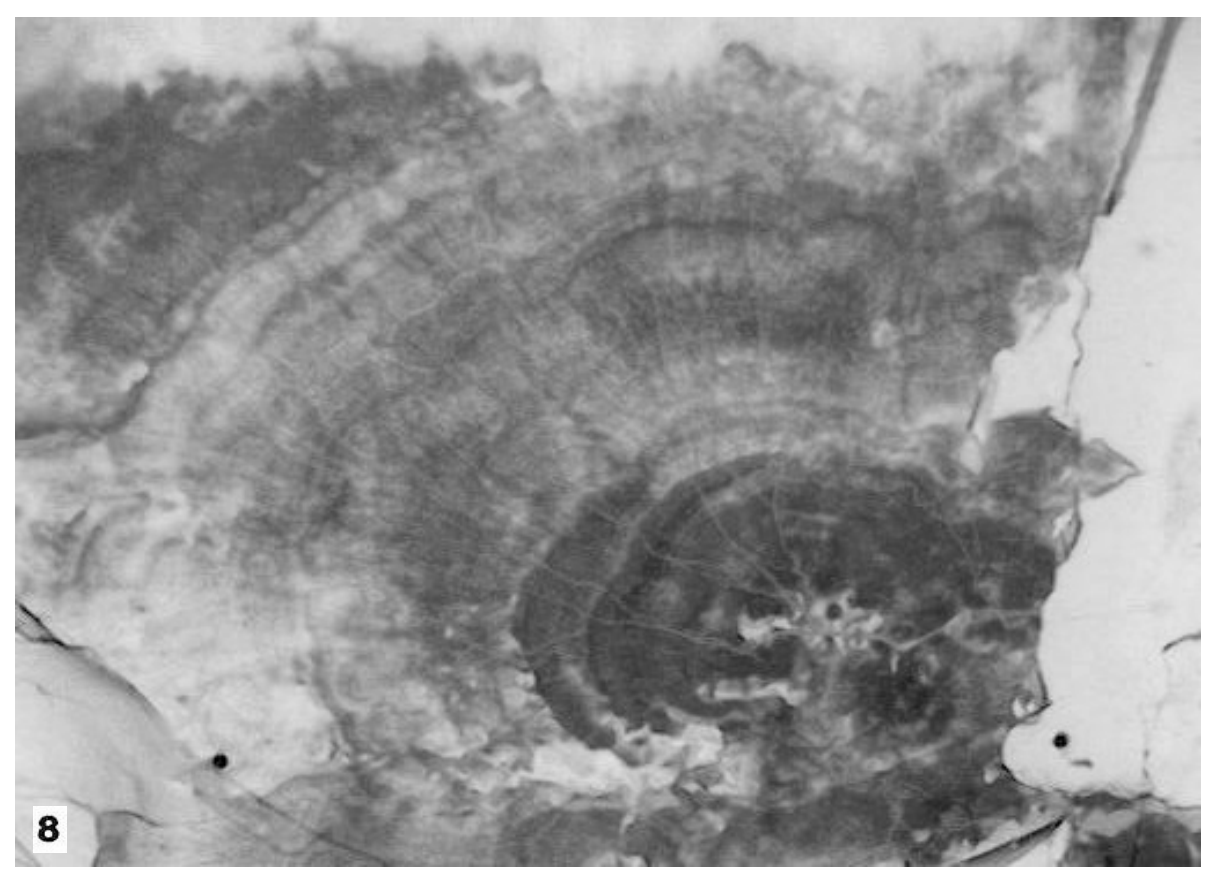

Fig.8. Mycelium et fruitbody (in centre) of Asterostroma ochroleuca on plasterwork.

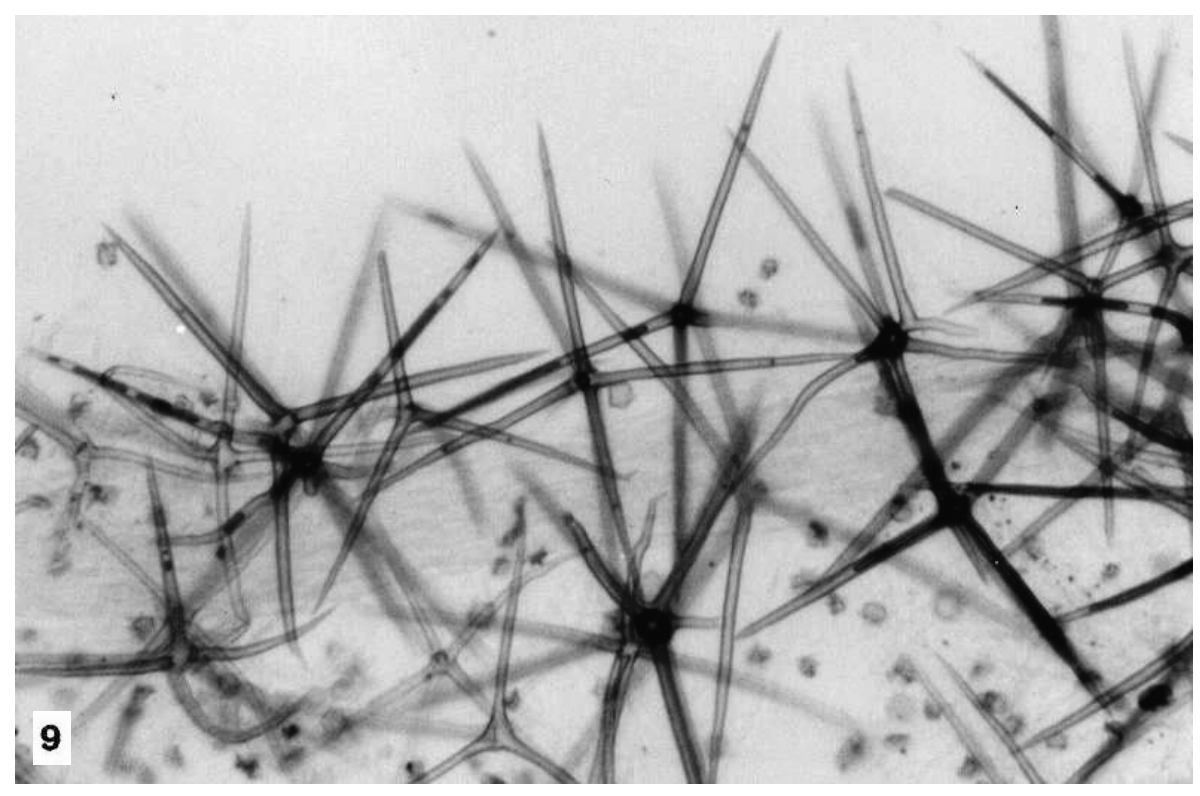

Fig.9. Asterosetae and tuberculate spores of Asterostroma ochroleucum.

Few data are available on the occurrence of the species in building, in Europe. Findlay (1950) found the species on several occasions in England while Koch, according to Hallenberg (1985), mentioned it as quite common in Denmark. 
However, she did not mention it in her survey of wood decay fungi in Danish houses (Koch 1985).

This species is more important than previously known.

\section{Conclusions}

Like in all North and Western European countries, Serpula lacrymans is the first cause of wood decay in Belgium. It is also the most destructive fungus. From the data analysed, the species is involved in almost $50 \%$ of the wood damage in Belgium. And its occurrence is most probably under-estimated.

Donkioporia expansa is much more important than previously established. It has to be looked for, not only on oak wood but also on coniferous wood.

Coniophora marmorata is also an important wood decayer and has been certainly mistaken for C. puteana in the past.

Asterostroma ochroleucum is not uncommon in Belgian buildings and was certainly overlooked till recently..

\section{References}

Beech-Andersen J. 1993. The dry rot fungus and other fungi in houses. Internat. Res. Group on Wood Pres. IRG/WP 2389: 1-46.

Buchwald G. 1986. On Donkioporia expansa (Desm.) Kotlaba et Pouzar. Internat. Res. Group on Wood Pres. IRG/WP1285: 1-9.

Cartwright K.S.G. and Findlay W.P.K. 1958. Decay of timber and its prevention. Her Majesty's Stationery Office, London $356 \mathrm{pp}$.

Domanski S. and A Orlicz. 1967. Polyporus megaloporus Pers. in the Family Polyporaceae s. str. Acta Mycologica 3: 51-62.

Dörfelt H. and B. Sommer 1973. Poria expansa (Desm.) Jahn in Botanischen Garten halle Gefunden. Mykologisches Mitt. Bl. 17: 44-47.

Findlay W.P.K. 1950. A note on the fungi of less common occurrence in houses. Trans. Brit. Mycol. Soc. 34: 35-37.

Ginns J., 1982. A monograph of the genus Coniophora (Aphyllophorales, Basidiomycetes). Opera Botanica 61: 1-61.

Hallenberg N. 1985. The Lachnocladiaceae and Coniophoraceae of North Europa. Ed. Fungiflora, Oslo. 96 pp.

Heineman P. 1979. Poria expansa, polypore peu connu ou méconnu en Belgique. Dumortiera 13: 1-2.

Hennebert G.L., Balon F. and Boulenger P. 1990. La mérule: science, technique et droit. Ed. Ciaco, Bruxelles, $250 \mathrm{pp}$.

Jülich W. 1981. Higher taxa of basidiomycetes. Bibliotheca Mycologica 85, ed. J. Cramer. 485 pp.

Jülich W. 1984. Kleine Kyptogamenflora Bd. II. Ed. Fischer Verlag, Stuttgart: XXX pp.

Koch A.P. 1985. Wood decay in danish buildings. Internat. Res. Group on Wood Pres. IRG/WP 1261: 1 9.

Mangin L. and Patouillard N. 1922. Sur la destruction des charpentes au Belgique de Versailles par le Phellinus cryptarum Karst. C.R. Acad.Sci.Paris 175: 389-394.

Paajanen L. and Viitanen H. 1989. Deacy fungi in Finnish houses on the basis of inspected samples from 1978 to 1988. Internat. Res. Group on Wood Pres. IRG/WP 1401: 1-5.

Rammeloo J., O. Guillitte, G. Draye, M. Van Leemput, F. Deroy and S. Roland, 1989. La mérule et autres champignons nuisibles dans les bâtiments: une approche multidisciplinaire. Jardin Botanique National de Belgique : 55 pp.

Rammeloo J., Guillitte O., Draye G., Van Leemput M., Deroy F. and Roland S., 1992. La mérule et autres champignons nuisibles dans les bâtiments: une approche multidisciplinaire, $2 \mathrm{~d}$ éd. Jardin Botanique National de Belgique $55 \mathrm{pp}$.

Ritter G. 1983. Neufund von Donkioporia expansa. Boletus 7(1): 3-4. 


\title{
SYMBIOTIC FUNGI IN THE GALLERIES OF THE STRIPED BARK BEETLE, TRYPODENDRON LINEATUM (COLEOPTERA, SCOLYTIDAE)
}

\author{
G. BABUDER AND F. POHLEVEN
}

Department of Wood Science and Technology, Biotechnical Faculty, University of Ljubljana, Slovenia

\begin{abstract}
Symbiotic fungi from the galleries of the striped bark beetle, Trypodendron lineatum (O1.) found in spruce logs lying at the timber storage yard of a woodprocessing industry in Ljubljana were studied. In laboratory conditions the following fungi were isolated and determined: Trichoderma harzianum Rifai, yeast Pichia anomala Hansen (Kurzman), blue-stain fungus Ceratocystis piceae (Münch) Bakshi (anam. Graphium) and a fungus closely similar to Ceratocystis araucariae (anamorph) (in the following text designed as Fs Ca). Isolated fungi stained the wood but did not cause any major weight loss of wood samples in laboratory conditions. The hyphae of symbiotic fungi primarily colonized the parenchyma tissues of the sapwood, and also tracheids. Enzyme activity of fungi was studied by using simple laboratory test methods. The presence of specific enzymes cooperating in wood decay was established.
\end{abstract}

\section{Introduction}

The striped bark beetle, Trypodendron lineatum (Ol.) is considered to be one of the most serious pest among ambrosia beetles in the northern hemisphere (Uusvaara and Loyttyniemi 1975). Ambrosia beetles are coleopteran insects (Scolytidae and Platypodidae) that indirectly utilize wood.

The damage is caused by the female beetle of T. lineatum tunneling in sapwood and introducing into it spores of mutualistic fungi which then grow in the gallery. Such fungi can spread and stain the wood near gallery or up to several centimeters from the gallery. They convert wood components into the fungal growth which serves as food for the beetle (Baker, 1963; Batra, 1963). T. lineatum attacks logs of autumn and winter felled coniferous trees lying unpeeled, and also weakened or dying trees (Anilla, 1975).

From the first reports of symbiosis between insects and fungi till the early 1960s, the concept of one ambrosial fungus per one ambrosia beetle was emphasized. But some subsequent studies confirmed the view that each ambrosia beetle is symbiotically associated with more than one fungus (Baker 1963, Norris 1965). Experimental results show that different species of bacteria, yeasts, yeastlike fungi and ambrosial fungi compose the symbiotic community of microorganisms with the ambrosia beetle. Norris (1979) used for the assemblage of symbiotic micro- and macroorganisms (including the beetle) the term a multi-species complex or a supraspecies. The activities of the microbial complex as a whole, not just those of the ambrosial fungus, allow such beetles to indirectly utilize nutrient-poor substrates as wood.

Nevertheless, many questions concerning the relationship and interaction between symbiotic fungi and ambrosia beetles are still open. The following problems are perhaps the most interesting for research in near future: isolation and determination of fungi species associated with the ambrosia beetles in different countries, antagonism and synergism among symbiotic fungi in vivo and in vitro, the influence of fungi on wood decay and utilization of wood components, succession of fungi in attacked wood etc. 


\section{Isolation of fungi}

\section{Materials and Methods}

Spruce logs containing galleries of active T. lineatum were identified by the presence of the whitish bore-dust at the entrance holes in bark crevices and by chiseling into galleries. $20 \mathrm{~cm}$-thick disks of the logs were removed in areas containing the highest number of the entrance holes. These disks were then brought to laboratory for dissection and isolation of associated fungi.

Isolations were made from the different parts of the galleries: entrance tunnels, lateral mother tunnels, axial larval cradles, wood near tunnels and abandoned tunnels. Samples were taken with sterile forceps and scalpel. Each sample was brought on the surface of potato-dextrose agar (PDA).

Adult females were aseptically removed from the tunnels and allowed to walk on agar surfaces, to transfer the spores of fungi to the medium.

\section{Determination of decay capacity}

Decay capacity of isolated fungi was determined by partly modified EN 113. In laboratory conditions the wood samples of spruce (P. abies (L.) Karst.) and red pine (P. sylvestris L.) were exposed to fungi activity on malt extract agar (MEA) in Kolle flasks for 16 weeks. The decay of wood samples was determined by measuring weight loss.

\section{Enzyme activity}

The presence of specific enzymes and their activity in the symbiotic fungi were demonstrated by simple test methods. Cellulase activity was determined according to Rautela and Cowling (1966), that of oxidase according to Bavendamm (1928) and Jorgensen and Vejlby (1953). Activity of laccase, which is important for the splitting of the phenol ring of the lignin complex, was determined according to Stalpers (1978).

\section{Results and discussion}

From the different parts of the galleries of $T$. lineatum, four species of symbiotic fungi were constantly isolated: Trichoderma harzianum Rifai, yeast Pichia anomala (Hansen) Kurzman, blue-stain fungus Ceratocystis piceae (Münch) Bakshi (anam. Graphium) and a fungus closely similar to Ceratocystis araucariae (anam. ' $F s C a$ ') (Table 1).

The ambrosial fungus of $T$. lineatum known as Ambrosiella ferruginea (Mathiesen-Käärik) Batra was not isolated. The four species of fungi were found during the pupa, callow adult and adult beetle stage. Some other microorganisms were isolated from either the beetles or their galleries, but not from both. They were found at only one, or a very few locations on beetles or in galleries. Therefore, they were not considered as symbionts and not determined.

Nevertheless, no report on the establishment of T. harzianum, $P$. anomale and FsCa from the galleries of T. lineatum were found in the literature. Consequently, this might be the first observation of the symbiosis of $T$. lineatum with these fungi. It is known for a long time that $C$. piceae is an important member of the fungal community of microbes associated with different ambrosia and bark beetles (Bakshi 
1950, Wilson 1959, Käärik 1971). Because of limited and extremely slow growth $P$ anomala was not used in further experiments.

Table 1: Symbiotic fungi isolated from the different parts of the galleries of Trypodendron lineatum (O1.).

\begin{tabular}{|l|r|r|r|r|r|}
\hline Symbiont & Entrance tunne & Lateral mother tu & Axial larval cr & Wood near & \multicolumn{2}{|c|}{ Abandoned tunnel } \\
\hline T. harzianum & + & + & + & + & + \\
\hline P. anomala & + & + & + & - & - \\
\hline C. piceae & + & + & + & + & + \\
\hline FSCa & + & + & + & + & + \\
\hline
\end{tabular}

The symbiotic fungi caused the stain of spruce and red pine wood after 16 weeks, but did not produce any major weight loss of wood samples. Weight loss was found to be the highest in samples exposed to $C$. piceae, and medium weight loss in samples exposed to FsCa which caused the most intensive wood staining. The lowest weight loss was observed in samples exposed to T. harzianum which discoloured the wood by forming masses of pigmented spores on the wood surface (Table 2). C. piceae caused $11.4 \%$ weight loss in American beech and 17.3 $\%$ in sugar maple after 3 months (Eslyn and Davidson, 1976). Fungus can act as typical soft-rot and attack the S2 layer of tracheid walls (Levy, 1967).

Table 2: Weight loss of wood samples (\%) produced by symbiotic fungi of Trypodendron lineatum (Ol.).

\begin{tabular}{|l|c|c|c|}
\hline Symbiont & cellulase & oxidase & laccase \\
\hline T. harzianum & + & - & \\
\hline C. piceae & ++ & ++ & +++ \\
\hline FsCa & +++ & - & +++ \\
\hline
\end{tabular}

a) mean values of weight loss of ten samples; b) standard deviation

Specific enzymes are needed for the fungi to penetrate the wood elements. Cellulase was proved in T. harzianum, C. piceae and FsCa. Laccase activity was observed in FsCa and C. piceae which produced also oxidase.T. harzianum did not synthesize any of these enzymes (Table 3).

Table 3: Enzyme activity of symbiotic fungi of Trypodendron lineatum (Ol.). - = no activity; + = slight

\begin{tabular}{|l|c|c|c|c|}
\hline \multirow{2}{*}{ Symbiont } & \multicolumn{2}{|c|}{ Red pine } & \multicolumn{2}{c|}{ Spruce } \\
\cline { 2 - 5 } & $\overline{\mathrm{X}}^{\mathrm{a}}$ & $\mathrm{SD}^{\mathrm{b}}$ & $\overline{\mathrm{X}}^{\mathrm{a}}$ & $\mathrm{SD}^{\mathrm{b}}$ \\
\hline T. harzianum & 0.05 & 0.002 & 0.07 & 0.002 \\
\hline C. piceae & 2.21 & 0.012 & 1.02 & 0.009 \\
\hline FsCa & 0.79 & 0.012 & 0.12 & 0.006 \\
\hline
\end{tabular}

activity: $++=$ medium activity; $+++=$ strong activity.

\section{References}

Annila E. 1975. Effect of felling date of trees on the attack density and flight activity of Trypodendron lineatum (Oliv.) (Col., Scolytidae). Comm. Inst. For. Fenn. 86: 6, 28 p.

Baker J.M. 1963. Ambrosia beetles and their fungi, with particular reference to Platypus cylindrus Fab. Sym. Soc. Gen. Microbiol. 13: 232-265. 
Bakshi B.K. 1950. Fungi associated with ambrosia beetles in Great Britain. Trans. Brit. Mycol. Soc. 33: 111-120.

Batra L. R. 1963. Ecology of ambrosia fungi and their dissemination by beetles. Trans. Kansas Acad. Sci. 66: $213-236$

Bavendamm W. 1928. Uber das Vorkommen und den Nachweis von Oxisasen bei Holzzerstorenden Pilzen. Zuschr. Pflanzenkrank. u. Pflanzenschutz 38: 257-276.

Eslyn W.E. and Davidson R.W. 1976. Some wood-staining fungi from pulpwood chips. Mem. N.Y. Bot. Garden 28(1): 50-57.

Jorgensen E. and Vejlby K. 1953. A new polyphenol oxidase test. Physiologia Plantarum 6: 533-537.

Käärik A. 1971. The relation beetwen blue-stain fungi and bark beetles. IRG/WP Bull.19, 6 p.

Levy J.F. 1967. Decay and degrade of wood by soft-rot fungi and other organisms. Inter. Pest Control (Nov./Dec.): 28-34.

Norris D.M. 1965. The complex of fungi essential to the growth and development of Xyleborus sharpi in wood. Mat. u.Organ. Beih. 1: 523-529.

Norris D.M. 1979. The mutualistic fungi of Xyleborini beetles. In Insect fungus symbiosis.Batra, L.R. ed., Allanheld, Osmun and Co, Montclair, pp.53-63.

Rautela G.S.and Cowling E.B. 1966. Simple cultural test for relative cellulolitic activity of fungi. App. Microb.: 892-898.

Stalpers, J.A. 1978. Identification of wood-inhabiting Aphyllophorales in pure cultures. CBS Studies in mycolog 16: 1-248.

Uusvaara O. and Loyttyniemi K. 1975. Effect of injury caused by the ambrosia beetle (Trypodendron lineatum Oliv., Coleoptera, Scolytidae) on saw timber quality and value. Folia Forestalia 231: 1-14.

Wilson C.L. 1959. The Columbian timber beetle and associated fungi in white oak. For. Sci. 5: 114-127. 


\title{
CERCOSPOROID FUNGI ASSOCIATED WITH THE EYESPOT DISEASE OF GRAMINICOLOUS HOSTS
}

\author{
G.F. CAMPBELL ${ }^{1}$, B. ROBBERTSE ${ }^{2}$, P.W. CROUS ${ }^{2}$, AND B.J.H. JANSE ${ }^{1}$ \\ Departments of ${ }^{1}$ Microbiology and ${ }^{2}$ Plant Pathology, University of Stellenbosch, South Africa
}

\section{Introduction}

The fungus commonly associated with eyespot disease symptoms of wheat was first described as Cercosporella herpotrichoides Fron (Sprague 1936). Based on the unthickened, inconspicuous conidial scars, several Cercosporella species were reallocated to the newly erected genus Pseudocercosporella Deighton (1973). Two varieties of $P$. herpotrichoides (Fron.) Deighton were distinguished, namely $P$. herpotrichoides var. herpotrichoides and $P$. herpotrichoides var. acuformis Nirenberg (Nirenberg 1981). In addition, P. anguioides Nirenberg and P. aestiva Nirenberg were also described from eyespot symptoms on cereals in Germany (Nirenberg 1981). In a study of the various Mycosphaerella Johanson species and their respective anamorphs (von Arx 1983), P. herpotrichoides was reallocated to the genus Ramulispora Miura as R. herpotrichoides (Fron) Arx . This decision was chiefly based on the fact that Ramulispora species had conidia forming lateral branches. The genus was furthermore restricted to all species occurring on Poaceae. Pseudocercosporella species were recognized as anamorphs of Mycosphaerella (Corlett 1991). The description of the teleomorph of Ramulispora herpotrichoides as Tapesia yallundae Wallwork \& Spooner (1988) [0culimacula yallundae (Wallroth \& Spooner) Crous \& W. Gams, 2003, ndlr], further supported its separation from Pseudocercosporella.

New combinations for the varieties of $R$. herpotrichoides were subsequently introduced as $R$. herpotrichoides var. herpotrichoides (Fig. 1) and var. acuformis (Nirenberg) Boerema, Pieters \& Hamers (1992). This variety was later reconsidered at species level as R. acuformis (Nirenberg) Crous. Braun (1993) furthermore reduced Pseudocercosporella. anguioides to an additional variety of $R$. herpotrichoides. No reasons were given, however, for not retaining it as a separate species in Ramumispora. Because of the uncertainty surrounding the generic placement of Pseudocercosporella aestiva (Fig. 2), the latter species was not introduced into Ramulispora.

Using isozyme and mitochondrial DNA (DNA) banding patterns, the three varieties of Ramulispora herpotrichoides could clearly be distinguished from $P$. aestiva (Julian \& Lucas 1990, Priestly et al. 1992; Nicholson et al. 1993), which appeared to be unrelated to $R$. herpotrichoides. The aim of the present study, therefore, was to use scanning electron microscopy (SEM) and random amplified polymorphic DNA (RAPDs) to investigate the generic and species status of the four taxa discussed above.

\section{Scanning electron microscopy}

\section{Material and Methods}

Verified isolates of $R$. herpotrichoides var. herpotrichoides (CBS 494.80), var. acuformis (CBS 495.80), var. anguioides (86.45.64C) and P. aestiva (CBS 497.80) were used to compare their conidial morphology using SEM. Sporulation of isolates were obtained after 2 wks on synthetic nutrient agar (SNA) (Nirenberg 1981) at 
$15^{\circ} \mathrm{C}$. Specimens for SEM were cut from colonized agar, fixed overnight in $3 \%$ glutaraldehyde, and rinsed in $0.05 \mathrm{M} \mathrm{Na}$ Cacodylate buffer the next day. The material was further fixed in $2 \%$ Osmium tetroxide in $0.05 \mathrm{M} \mathrm{Na}$ Cacodylate buffer, rinsed in the latter buffer, and dehydrated in a series of alcohol concentrations. Specimens were critical point dried, coated with gold and viewed with a Jeol JSM 6100 scanning electron microscope. Specimens were also viewed using kryofixation.

\section{DNA isolation}

Colonized agar plugs from 14-d-old colonies grown on PDA were inoculated into $200 \mathrm{ml} \mathrm{liquid}$ cultures $(8 \mathrm{~g} / 1$ yeast extract and $5 \mathrm{~g} / 1$ glucose $)$. Cultures were incubated at $25^{\circ} \mathrm{C}$ for $10 \mathrm{~d}$ on an orbital shaker and the mycelia harvested by filtration (Whatman no.1 filter paper). Freeze-dried mycelia mixed with river sand was ground to a fine powder with a mortar and pestle. Twenty $\mathrm{ml}$ lysis buffer $(3 \%$ [v/v] SDS; $1 \%$ [v/v] 2-mercaptoethanol; $50 \mathrm{mM}$ Tris [pH 7.2] and $50 \mathrm{mM}$ EDTA) was added and the mixture incubated at $65^{\circ} \mathrm{C}$ overnight. Cetyltrimethylammonium bromide $(\mathrm{CTAB})$ and $5 \mathrm{M} \mathrm{NaCl}$ were then added to a final concentration of $1 \%$ [v/v] and $0.7 \mathrm{M}$ respectively and incubated for $4 \mathrm{hrs}$ at $65^{\circ} \mathrm{C}$. The mixture was extracted with phenol/chloroform/isoamylalcohol (25:24:1) (PCI), and followed by two extractions with chloroform/isoamylalcohol. The resulting aqueous fraction was mixed with $7.5 \mathrm{M} \mathrm{NH4OAc}$ and 0.54 volumes isopropanol and incubated at $-20^{\circ} \mathrm{C}$ overnight to precipitate the nucleic acids. After centrifugation at $5000 \mathrm{rpm}$ for 5 min, the pellet was washed with $70 \%$ [v/v] ethanol, vacuum dried and dissolved in $300 \mu 1$ TE buffer (10 mM Tris [pH 8.0] and $1 \mathrm{mM}$ EDTA). The sample was treated with Rnase A $(10 \mathrm{mg} / \mathrm{ml})$ and incubated at $37^{\circ} \mathrm{C}$ for $90 \mathrm{~min}$ after which the DNA was PCI extracted, precipitated with isopropanol in the presence of NH4Oac, washed, vacuum dried and redissolved in $300 \mu \mathrm{l}$ TE buffer.

\section{RAPD analysis}

The 10-mers used as random primers were purchased from Operon Technologies (Kit E, Operon Technologies Inc. Alameda CA94501, USA). Taq Polymerase together with its $10 \mathrm{X}$ concentrated buffer $\left(100 \mathrm{mM}\right.$ Tris- $\mathrm{HCl}[\mathrm{pH} 8.3]\left[20^{\circ} \mathrm{C}\right] ; 15$ $\mathrm{mM} \mathrm{MgCl} 2 ; 500 \mathrm{mM} \mathrm{KCl}$ ) was supplied by Boehringer Mannheim (Boehringer Mannheim, Johannesburg, South Africa). PCR reaction mixtures (50 $\mu \mathrm{l}$ final volumes) contained $25 \mathrm{ng}$ genomic DNA, dATP, dCTP, dGTP and dTTP each at $200 \mu \mathrm{M}$ final concentration, $100 \mathrm{nM}$ oligonucleotide primer, $1 \mathrm{X}$ Taq polymerase buffer and 1 unit of Taq polymerase. One $\mathrm{mM} \mathrm{MgCl} 2$ was also added. Each reaction was overlaid with a $100 \mu \mathrm{l}$ of mineral oil to prevent evaporation. The random sequence primers used were OPE-14 5'TGCGGCTGAG and OPE-15 5'ACGCACAACC. Amplifications were conducted in a Hybaid Omnigene (Hybaid Ltd., Waldegrave Rd., Middlesex, UK) thermal cycler. Samples were subjected to 60 repeats of the following cycle: $1 \mathrm{~min}$ at $94^{\circ} \mathrm{C}, 1 \mathrm{~min}$ at $36^{\circ} \mathrm{C}$ and $2 \mathrm{~min}$ at $72^{\circ} \mathrm{C}$. After the last cycle, a final extension step of $72^{\circ} \mathrm{C}$ for $5 \mathrm{~min}$ was included, followed by cooling to $4^{\circ} \mathrm{C}$ until recovery of the samples. Electrophoresis of aliquots $(15 \mu \mathrm{l})$ of amplification products was through $1.4 \%$ agarose gels using $1 \mathrm{X}$ TAE buffer. Phage lamda DNA digested with EcoRI and HindIII was used as a standard. 


\section{Generic placement}

\section{Results and Discussion}

When von Arx (1983) separated the graminicolous Pseudocercosporella species into Ramulispora, it was chiefly based on the hosts falling in the Poaceae, and the conidia having the ability to form lateral branches.

All species of Pseudocercosporella studied by the present authors have been found to have conidia arranged in dry masses. However, conidia of R. sorghi (Ell. \& Ev.) Olive \& Lefebvre, the type species of Ramulispora, aggregate in slimy masses. The same phenomenon has also been observed for $R$. herpotrichoides.

With the description of the teleomorph of $R$. herpotrichoides var. herpotrichoides in Tapesia, additional support was given for the separation of these anamorphs from Pseudocercosporella. Furthermore, based on the research of King (1990), T. yallundae var. acuformis Boerema, R. Pieters \& Hamers (1992) has also recently been described as the teleomorph for $R$. herpotrichoides var. acuformis.and later named Tapesia acuformis [Oculimacula acuformis (Boerema, R. Pieters \& Hamers) Crous \& W. Gams, 2003; ndlr].

Other than Tapesia and Mycosphaerella teleomorphs, the formation of lateral conidium branches is the chief morphological character proposed by von Arx (1983) to separate Pseudocercosporella from Ramulispora. Von Arx (1983) clearly illustrated lateral branches in Ramulispora sorghi and $R$. herpotrichoides as is typically observed in other Cercosporoid genera such as Mycovellosiella Rangel (Crous \& Braun 1994). However, in a paper dealing with the morphology of $R$. sorghi, Olive et al. (1946) presented several figures indicating that the lateral branches in $R$. sorghi are in fact secondary conidia forming directly on the surface of primary conidia. Chang and Tyler (1964) were the first to report microcyclic conidiation in $R$. herpotrichoides var. herpotrichoides. Fernandez et al. (1991) recently defined this process of conidiation in Cercospora kikuchii (Matsumoto \& Tomoyasu) M.W. Gardner. Although Chang and Tyler (1964) reported short secondary conidiophores to be present, the latter was not observed in our study. Secondary conidia were, however, found to develop directly on the surface of primary conidia. Microcyclic conidiation, also known as iterative germination or precocious sporulation was defined as the recapitulation of conidiation following conidial germination without an intervening phase of mycelial growth.

The phenomenon observed for $R$. herpotrichoides and $P$. aestiva in the present study was identical to that illustrated by Olive et al. (1946) for R. sorghi, and distinct from microcyclic conidiation (sensu Fernandez et al. 1991), and lateral branches (sensu Crous \& Braun 1994). We conclude, therefore, that in Ramulispora the formation of secondary conidia and not lateral branching or microcyclic conidiation occurs. The process should therefore be seen as conidia forming directly on the surface of the primary conidia, without the presence of an intervening germ tube with conidiogenous locus.

From these data it is clearly shown that the three varieties discussed above are presently best retained in Ramulispora, as they cannot be accommodated in Pseudocercosporella. The generic placement of $P$. aestiva remains, however, uncertain. The present study suggests that the possible collection of its teleomorph and further molecular studies will prove necessary to resolve the generic position of P. aestiva. 


\section{Species delimitation}

\section{Ramulispora herpotrichoides var. herpotrichoides \& var. acuformis}

Several morphological criteria are used to distinguish var. herpotrichoides and var. acuformis. Conidia of var. herpotrichoides are curved, whereas those of var. acuformis are straight (Nirenberg 1981). Our SEM study showed both var. herpotrichoides and var. acuformis to have polyblastic conidiogenous cells (Fig. 3). When cultured on PDA, var. herpotrichoides has fast growing colonies with smooth margins, whereas $R$. herpotrichoides var. acuformis has slow growing colonies with feathery margins (Lange-de la Camp 1966, Scott et al. 1975). The two varieties also differ in their pathogenicity towards wheat and rye, with var. herpotrichoides being more pathogenic to wheat than to rye, and var. acuformis being equally pathogenic to wheat and rye (Lange-de la Camp 1966, Hollins et al. 1985). Furthermore, an ultrastuctural study showed that the infection process on a wheat coleoptile was also distinctly different for var. herpotrichoides and var. acuformis (Daniels et al. 1991). Distinct mating populations have also recently been isolated for var. acuformis and var. herpotrichoides in England (P. Dyer, personal communication). Molecular markers including isozymes, restriction fragment length polymorphisms (RFLPs) and RAPDs have successfully been used to distinguish var. herpotrichoides and var. acuformis (Julian and Lucas, 1990; Nicholson et al. 1991, 1993, Nicholson and Rezanoor 1994, Priestley et al. 1992, Thomas et al. 1992).

Using RAPDs, the present study supported the distinction made between var. herpotrichoides and var. acuformis based on general morphology, cultural characteristics, pathology, and molecular studies of other workers discussed above. The F-values obtained with two primers in the present study indicated var. herpotrichoides to have $50 \%$ similarity to var. acuformis. Using four primers, this figure decreased to $35 \%$ (Fig. 5, Table 1). This low percentage similarity, together with the other differences discussed above, suggests that these two varieties should be seen as two separate species.

\section{Ramulispora herpotrichoides var. anguioides}

Nirenberg (1981) described var. anguioides as having grey- pink, velvety colonies on PDA with even colony margins. Conidia were described as forming on simple, seldomly branched conidiophores. Successful protoplast fusion between var. herpotrichoides, var. acuformis and var. anguioides has recently been obtained by Hocart \& McNaughton (1994), indicating that these varieties are closely related.

Using isozyme electromorphs, var. anguioides has been shown to be distinct from var. herpotrichoides, var. acuformis and P. aestiva (Julian and Lucas 1990, Priestley et al. 1992). However, var. anguioides has been shown to have a certain percentage of similarity with var. acuformis. Using RFLPs, Nicholson et al. (1993) found that strong hybridization occurs between var. anguioides and var. herpotrichoides, indicating that they may be closely related. However, DNA electromorphs of the three varieties were distinct.

Using RAPDs, the present study found var. anguioides to be distinct from the other two varieties and $P$. aestiva. F-values showed var. anguioides to have $17 \%$, $9 \%$ and $8 \%$ similarity to var. herpotrichoides, var. acuformis and P. aestiva respectively (Fig. 5, Table 1). 


\section{Pseudocercosporella aestiva:}

Nirenberg (1981) described the conidiophores of var. anguioides as hyaline, thin, polyblastic, simple, and seldomly branched. Results obtained in the present study found all four taxa could have polyblastic conidiogenous cells. However, the conidiogenous cells of var. herpotrichoides were frequently swollen and ampulliform, whereas those of P. aestiva were mostly reduced (Fig. 4).

Isozyme and RFLPs have indicated little relationship between $P$. aestiva and the three varieties of $R$. herpotrichoides (Julian and Lucas 1990, Nicholson et al. 1993, Priestley et al. 1992).

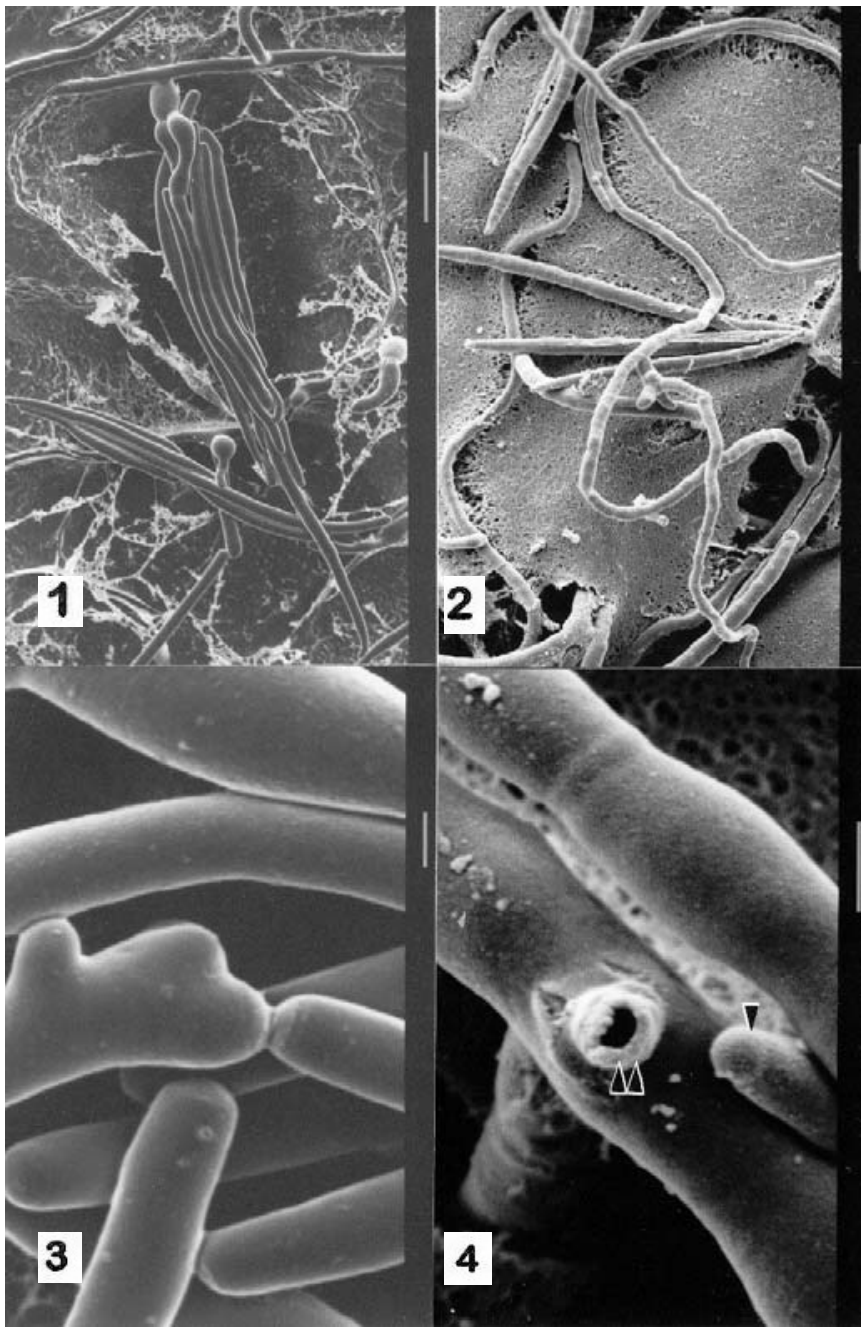

Fig.1. Conidiophore of $R$. herpotrichoides var. herpotrichoides with ampulliform conidiogenous cells giving rise to an aggregated, slimy mass of conidia.

Fig.2. Typical conidia of $P$. aestiva on SNA.

Fig.3. Conidium of $R$. herpotrichoides var. herpotrichoides attached to a polyblastic conidiogenous cell.

Fig.4. Older, swollen conidium of $P$. aestiva giving rise to a secondary conidium (arrow) via a conspicuous conidiogenous locus (double arrow). 


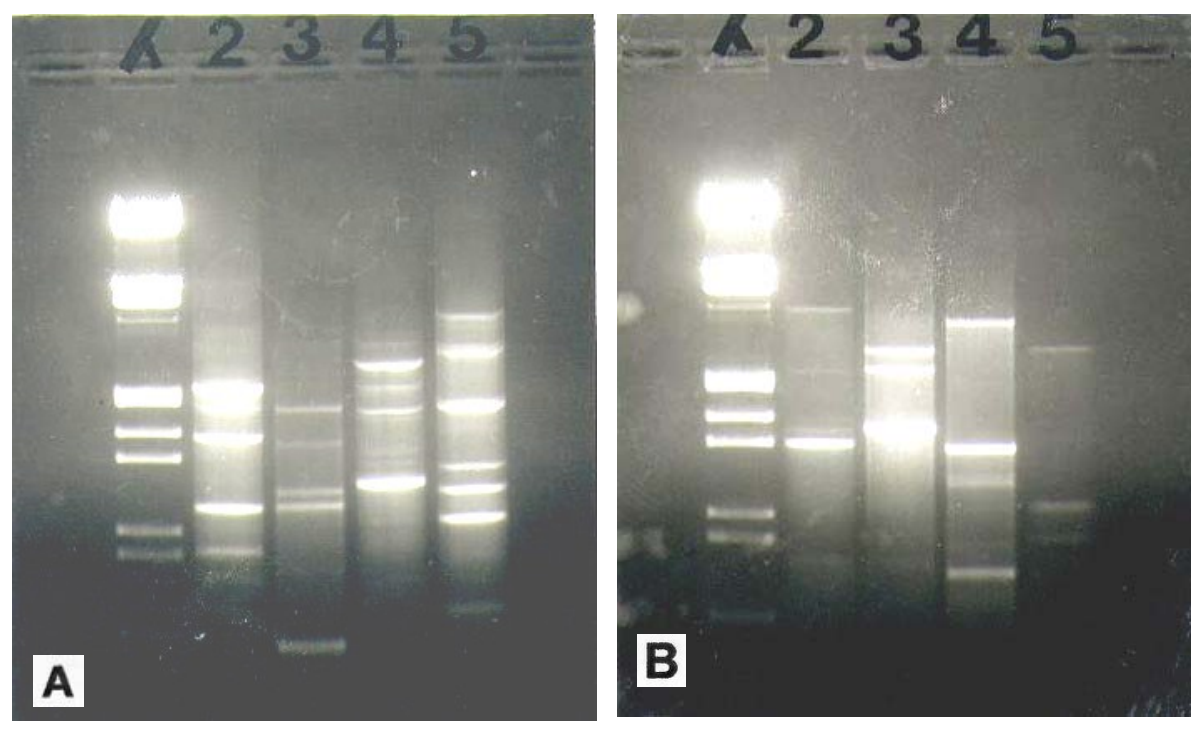

Fig.5. Polymorphic bands of amplified DNA of the three varieties of $R$. herpotrichoides and P. aestiva. Lane 1: Phage lamda DNA digested with EcoRI (A) and HindIII (B) was used as a standard. Lane 2: $R$. herpotrichoides var. herpotrichoides; Lane 3: $R$. herpotrichoides var. acuformis; Lane 3: $R$. herpotrichoides var. anguioides; Lane 4: P. aestiva.

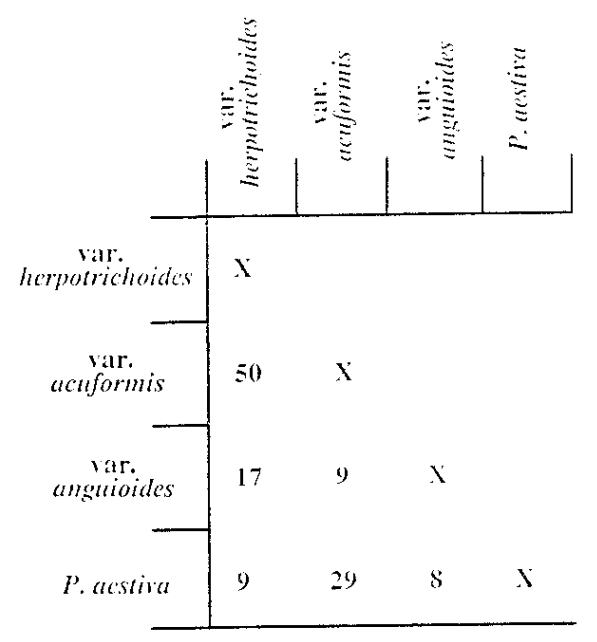

Table 1. Combined F-values (\% similarity) following RAPD- analysis ( 2 primers) of the three varieties of $R$. herpotrichoides and P. aestiva.

Using RAPDs, the present study supported these results, and also showed $P$. aestiva to be distinct from the three varieties of $R$. herpotrichoides. F-values between P.aestiva and var. herpotrichoides, var. acuformis and var. anguioides were found to be $9 \%, 29 \%$ and $8 \%$ respectively, indicating little relationship (Fig. 4, Table 1). 
In conclusion, the use of molecular techniques in Ramulispora is shown to enhance the taxonomic importance of seemingly insignificant morphological differences. Results obtained with SEM and RAPDs indicate that the four taxa studied are distinct, and that $P$. aestiva is probably not a species of Ramulispora. Furthermore, the low \% percentage similarity obtained with RAPDs, as well as other morphological differences suggest that the three varieties of $R$. herpotrichoides should be treated as three separate species.

\section{References}

Arx, von, J.A. 1983. Mycosphaerella and its anamorphs. Proceedings of the Koninklijke Nederlandse Akademie van Wettenschappen, Ser. C: Biological and Medical Sciences 86: 15-54.

Bateman G.L. 1988. Pseudocercosporella anguioides, a weakly pathogenic fungus associated with eyespot in winter wheat at a site in England. Plant Pathology 37: 291-296.

Boerema G. H., Pieters R. and Hamers M.E.C. 1992. Check- list for scientific names of common parasitic fungi. Supplement Series $2 b$ (additions and corrections): Fungi on field crops: cereal and grasses. Netherlands Journal of Plant Pathology 98: 1-32.

Braun. U. 1993. Studies on Ramularia and allied genera (VI). Nova Hedwigia 56: 423-454.

Chang E.P. and Tyler, L.J. 1964. Sporulation by Cercosporella herpotrichoides on artificial media. Phytopathology 54: 729-735.

Corlett M. 1991. An annotated list of the published names in Mycosphaerella and Sphaerella. Mycologia Memoir 18: 1-328.

Crous P.W. and Braun U. 1994. Cercospora species and similar fungi of South Africa. Mycological Research 99:31-36.

Daniels A., Lucas J.A. and Peberdy J.F. 1991. Morphology and ultrastructure of W and R pathotypes of Pseudocercosporella herpotrichoides on wheat seedlings. Mycological Research 95: 385-397.

Deighton F.C. 1973. Studies on Cercospora and its related genera. IV. Cercosporella Sacc., Pseudocercosporella gen. nov. and Pseudocercosporidium gen. nov. Mycological Papers 133: 1-62.

Fernandez F.A., Glawe D.A. and Sinclair J.B. 1991. Microcycle conidiation and nuclear behavior during conidiogenesis in Cercospora kikuchii. Mycologia 83: 752-757.

Hocart M.J. and Mc Naughton J.E. 1994. Interspecific hybridisation between Pseudocercosporella herpotrichoides and P. anguioides achieved through protoplast fusion. Mycological Research 98: 4756.

Hollins T.W., Scott P.R. and Paine J.R. 1985. Morphology, benomyl resistance and pathogenicity to wheat and rye of isolates of Pseudocercosporella herpotrichoides. Plant Pathology 34: 369-379.

Julian A.M. and Lucas J.A. 1990. Isozyme polymorphisms in pathotypes of Pseudocercosporella herpotrichoides and related species from cereals. Plant Pathology 39: 178- 190.

King A.C. 1990. First record of Tapesia yallundae as the teleomorph of Pseudocercosporella herpotrichoides var. acuformis, and its occurrence in the field in the Federal Republic of Germany. Plant Pathology 39: 44-49.

Lange-de la Camp M. 1966. Die Wirkungsweise von Cercosporella herpotrichoides Fron dem Erreger der Halmbruchkrankheit des Getreides. II. Aggressiviteit des Erregers. Phytopathologische Zeitscrift 56: $155-190$.

Nicholson P., Hollins T.W., Rezanoor H.N. and Anamthawat-Jonsson K. 1991. A comparison of cultural, morphological and DNA markers for the classification of Pseudocercosporella herpotrichoides. Plant Pathology 40: 584-594.

Nicholson P., Rezanoor H.N. and Hollins T.W. 1993. Classification of a world-wide collection of isolates of Pseudocercosporella herpotrichoides by RFLP analysis of mitochondrial and ribosomal DNA and host range. Plant Pathology 42: 58-66.

Nicholson P. and Rezanoor H.N. 1994. The use of random amplified polymorphic DNA to identify pathotype and detect variation in Pseudocercosporella herpotrichoides. Mycological Research 98(1): 13-21.

Nirenber H.I. 1981. Differenzierung der Erreger der Halmbruchkrankheit. I. Morphologie. Zeitscrift für Pflanzenkrankheiten und Pflanzenschutz 88: 241-248.

Olive L.S., Lefebvre C.L. and Sherwini H.S. 1946. The fungus that causes sooty stripe of sorghum spp. Phytopathology 36: 190-200.

Priestly R.A., Dewey F.M., Nicholson P. and Rezanoor H.N. 1992. Comparison of isoenzyme and DNA markers for differentiating W-, R- and C-pathotypes of Pseudocercosporella herpotichoides. Plant Pathology 41: 591-599.

Scott P.R., Hollins T.W. and Muir P. 1975. Pathogenicity of Cercosporella herpotrichoides to wheat, 
barley, oats and rye. Transactions of the British Mycological Society 65: 529-538.

Sprague R. 1936. Relative susceptibilty of certain species of gramineae to Cercosporella herpotrichoides. Journal of Agricultural Research 53: 659-670.

Thomas D., Maraite H. and Boutry M. 1992. Identification of rye- and wheat-types of Pseudocercosporella herpotrichoides. Journal of General Microbiology 138: 2305-2309.

Wallwork H. and Spooner B. 1988. Tapesia yallundae, the teleomorph of Pseudocercosporella herpotrichoides. Transactions of the British Mycological Society 91: 703- 705.

\title{
DIVERSITY OF COLLETOTRICHUM GLOEOSPORIOIDES PATHOGENIC TO STYLOSANTHES SP. IN TROPICAL AREAS
}

\author{
F. MunAut, N. HAMAIDE \& H. MARAite
}

Université catholique de Louvain, Faculté des Sciences agronomiques, Unité de Phytopathologie, Place Croix du Sud 2 boîte 3, B-1348 Louvain-la-Neuve, Belgium.

\begin{abstract}
Virulence spectra of Colletotrichum gloeosporioides strains isolated from Stylosanthes $\mathrm{sp}$. sampled in Africa and Australia were investigated on a set of Stylosanthes genotypes. Virulence spectra as well as aggressiveness of strains were highly variable. The distinction of strains causing type A (restricted lesions) and type B (extensive necrosis) is confirmed. Esterases extracted from both types of strain showed isozymic variation. Polymorphism was observed between and within types ; the latter can be clearly separated by specific bands. Strains isolated from Stylosanthes sp. presented esterase patterns different from those of strains isolated from other tropical plants. By Random Amplified Polymorphic DNA (RAPD) technique markers have been identified which separate strains of types A and B. Polymorphism was also observed among strains of type B from various geographical origins.
\end{abstract}

\section{Introduction}

Stylosanthes guianensis is a tropical pasture legume very interesting for its tolerance to dry conditions in tropical areas. Since 1970's, anthracnose caused by Colletotrichum gloeosporioides Penz. is an important limiting factor in South America (Grof et al. 1979), Africa (Clatworthy 1975) and Australia (O'Brien and Pont 1977). Selection of resistant genotypes is commonly used to control anthracnose, but strains able to partially or totally overcome the resistance appear sometimes rapidly.

In order to understand the mechanisms of this pathogen-host coevolution and to assist in the collection and selection of resistant Stylosanthes genotypes, the diversity in pathogenicity, DNA and isozymic patterns of C. gloeosporioides strains from various hosts and origins is investigated.

\section{Pathogenicity test}

\section{Material and methods}

Virulence spectra of C. gloeosporioides strains isolated from Stylosanthes sampled in Africa and Australia (Table 1) were investigated on a set of S. guianensis genotypes by inoculation of conidia suspensions $\left(10^{5}-10^{6}\right.$ conidia/ml $)$ on first-leaf stage plants. Plants were kept $48 \mathrm{~h}$ in the dark at $23^{\circ} \mathrm{C}$ before further incubation under a photoperiod of $14 \mathrm{~h}$. Symptom severity was quoted after eleven days. 


\section{Esterase assay}

Esterases were extracted from mycelium and conidia grown in V8 liquid cultures in a Tris buffer at pH 7.5, loaded on non-denaturing gel for electrophoresis and stained with the a-naphtyl acetate/fast red TR salt method (Shaw and Prasad 1970).

\section{DNA study}

DNA was extracted by the CTAB method (Roger and Bendich 1985). The Random Amplified Polymorphic DNA (RAPD) was developed to distinguish strains, using primers from the Kit A of Operon Technologies Inc. and the DynaZyme polymerase (Techgen International).

Table 1. Host, type, geographical origin of Colletotrichum gloeosporioides strains

\begin{tabular}{|l|l|c|c|}
\hline \multicolumn{1}{|c|}{ Host } & Type & Location \\
\hline & & & \\
HM 334 & Strain & A & Australia \\
HM 335 & Stylosanthes sp. & A & Australia \\
HM 336 & S. guianensis cv. Graham & B & Australia \\
HM 337 & S. guianensis & B & Australia \\
HM 313 & S. guianensis & B & Burundi \\
HM 312 & S. guianensis & B & Zaire \\
HM 373 & S. guianensis cv Cook & B & Zaire \\
HM 376 & S. guianensis & B & Zaire \\
HM 502 & S. guianensis cv. Cook & B & Zaire \\
HM 495 & S. guianensis cv. CIAT 184 & B & Ivory Coast \\
HM 498 & S. guianensis cv. CIAT 11366 & B & Ivory Coast \\
HM 514 & S. guianensis cv. CIAT 184 & B & Ivory Coast \\
HM 515 & S. guianensis cv. Cook & & \\
& & & \\
\hline
\end{tabular}

Type of symptom from which the strain was isolated : A, restricted clear spots with a brown margin ; B, extensive dark brown necrosis.

\section{Pathogenicity}

\section{Preliminary results and discussion}

Two types of anthracnose, previously described by Irwin and Cameron (1978), are distinguished on diseased samples and in inoculation tests. Symptoms caused on the three genotypes inoculated with the strain of type A were characterized by a clear spot surrounded by a dark margin. Susceptible genotypes infected with strains of type B presented a general necrosis of plants, starting by dark brown spots extending on the leaflets, causing their premature shedding as also on the stem. Virulence spectra as well as aggressiveness of strains appeared highly variable (Fig. 1 ), symptoms induced ranging from a few small spots to death of plants.

Evaluation of the degree of necrosis from 1 (spots less than $1 \mathrm{~mm}$ in diameter) to 5 (more than $75 \%$ necrosis of the leaf surface), is made by visual inspection of the first leaf inoculated. For each strain the results are expressed by the mean indice of the three leaflets of eighteen plants per genotype. A mean index is calculated for each genotype.

\section{Esterases}

Isozymic variation was observed for esterases extracted from both types of strain. Types could be clearly separated by specific bands. Polymorphism was also observed among strains of type B. Strains isolated from various other tropical plants 
presented esterases patterns different from the strains isolated from Stylosanthes sp. Moreover, subcultures from sector appearing on PDA cultures of a $C$. gloeosporioides strain isolated from banana, showed different isozymic patterns.

\section{DNA polymorphism}

RAPD permitted to identify markers which clearly separate type A and B. Polymorphism was also observed among type B strains which could be grouped according to geographical origin

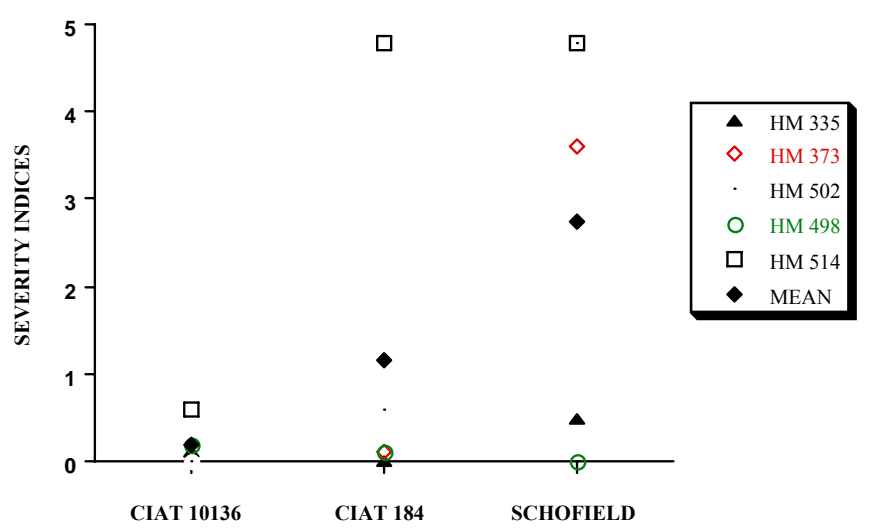

Figure 1. Anthracnose severity on the first leaf of three Stylosanthes guianensis genotypes, 11 days after inoculation with five Colletotrichum gloeosporioides strains

Strains from Australia, Burundi and Zaire were closely related while those isolated in Ivory Coast formed a second distinct group. Subcultures of strain HM 498 and from sectors of the HM 515 presented DNA polymorphism.

\section{Prospects}

Study of coevolution of Colletotrichum gloeosporioides and Stylosanthes sp. is planned in collaboration with the Universidad Nacional Autonoma Mexico and the Laboratorium voor Gentechnologie (Katholieke Universiteit Leuven) in the frame of a project coordinated by the International Board for Plant Genetic Resources (IBPGR).

\section{Acknowledgements}

The authors are grateful to IBPGR and the Belgian Administration for Development Cooperation for funding this research project.

\section{References}

Clatworthy J.N. 1975. Introduction and preliminary screening of pasture legumes at Marandellas, Rhodesia. Proceedings of the Grassland Society of Southern Africa 10: 57-63.

Grof B.R., Schultze-Kraft R.and Muller F. 1979, Stylosanthes capitata Vog., some agronomic attributes, and resistance to anthracnose (Colletotrichum gloeosporioides Penz.). Tropical grasslands 13: 28-37.

Irwijn J. A.G. and Cameron D.F. 1977. Two diseases in Stylosanthes spp. caused by Colletotrichum gloeosporioides in Australia, and pathogenic specialization within one of the causal organisms. Australian Journal of Agricultural Research 29: 305-317.

O'Brien R.G.and Pont W. 1977. Diseases of Stylosanthes in Queensland. Queensland Agricultural Research 103: 126-128. 
Rogers S.O.and Bendich A.J. 1985 Extraction of DNA from milligram amounts of fresh, herbarium and mummified plant tissues. Plant Molecular Biology 5: 69-76.

Shaw R.and Prasad R. 1970. Starch gel electrophoresis.

A compilation of recipes. Biochemical Genetics 9: 297-320.

\title{
DIVERSITY IN NON-SPECIFIC FOLIAR PATHOGENS OF WHEAT FROM NON-TRADITIONAL WARM AREAS
}

\author{
Di ZinNo T., DuveILler E. ${ }^{*}$, LONGREE H. AND MARAite H. \\ Unité de Phytopathologie, Faculté des Sciences Agronomiques, Université Catholique de Louvain (UCL), \\ Place Croix du Sud, 2, Bte 3, 1348 Louvain-la-Neuve, Belgium. \\ *Centro Internacional de Mejoramiento de Maíz y Trigo (CIMMYT), Lisboa 27, Apdo. Postal 6-641, \\ 06600 Mexico D.F., Mexico.
}

Abstract. In order to characterize the variability of the non-specific foliar pathogens of wheat from warm areas, a world-wide collection of strains is established through a collaboration between CIMMYT, Mexico and UCL, Belgium. By isolation of leaf samples sent from non-traditional warm area (South Asia, Africa, Latin America) in 1993 and 1994, we confirmed that Bipolaris sorokiniana is the most important foliar pathogen followed by Drechslera tritici-repentis, representing respectively 75 and $17 \%$ of the fungi isolated from leaf spots in 1993. This collection is the base for characterisation of the NSFP diversity by pathogenicity tests on host differentials of wheat lines developed at CIMMYT, by an analysis of the spectrum of toxins produced and by studies of genetic diversity with RAPD.

\section{Introduction}

Changes in cropping systems and progresses obtained in genetic resistance to classical diseases like rust, led to the important development of the non-specific foliar pathogens (NSFP) of wheat. With the support of the Belgian Agency of Development Cooperation, CIMMYT, Mexico and UCL, Louvain-la-Neuve collaborate to evaluate in warmer areas the sustainability of wheat production related to cropping systems and to promote research on resistance to this pathosystem.

In non-traditional warm wheat growing areas, helminthosporium blights, spot blotch and tan spot caused respectively by Bipolaris sorokiniana (Sacc. in Sorok) Shoem., teleomorph Cochliobolus sativus (Ito \& Kurib.) Drechs. ex Dastur and Drechslera tritici-repentis (Died.) Shoem., teleomorph Pyrenophora tritici-repentis (Died.) Drechs., are considered as the most important foliar pathogens, the incidence of other pathogens such as Alternaria triticina being poorly documented (various authors in Saunders 1990).

Data concerning variability among strains are useful to assist selection of resistant or tolerant cultivars. New lines being selected in "hot spot" fields where the pathogen is naturally present at a high pressure, it is important to determine if variability of pathogenicity among the strains occurring in these fields is representative of variability found in other locations. Differences in virulence among strains of $B$. sorokiniana of a same country were shown i.e. for Thailand by Hetzler (1992) and for Brazil by Mehta (1981). Concerning D. tritici-repentis, different pathotypes producing different type of lesions in correlation with toxin production were described by Lamari and Bernier (1989).

A reference collection of pathogens from diverse origins is thus the first priority. This will be achieved through a collaborative network gathering UCL, CIMMYT and National Agricultural Research Systems from the warm wheat growing area. 


\section{Material and methods}

Foliar samples were collected during 1993 and 1994 principally in the South Asian region (Bangladesh, Nepal and India) but also in South-Africa and Latin America in farmer or trial fields in characteristic locations on a great range of wheat varieties, some classical in these environments like Sonalika and Kanchan, some new resistant lines like crosses with Agropyrum curvifolium.

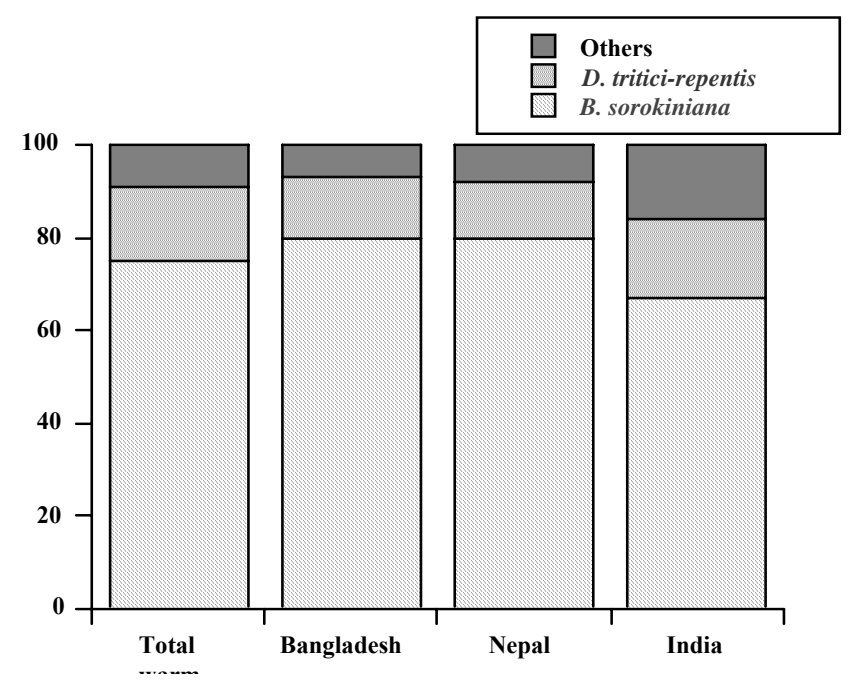

Figure 1: Distribution of the NSFP isolated from wheat in South Asian area in 1993: the number of strains isolated was 77 for all warm areas, 30 for Bangladesh, 26 for Nepal and 6 for India.

Lesions on wheat leaves and stems are observed under stereomicroscope and microscope to detect fungal fructifications.

After description, pieces of lesions are surface sterilized with $\mathrm{NaOCl}(0.5 \%$ during 3 minutes), rinsed with three changes of sterile water and placed in Petri dishes containing water agar $(1.5 \%$, streptomycin $150 \mathrm{mg} / \mathrm{l})$. After incubation in the dark at $20^{\circ} \mathrm{C}$, the plates are placed under continuous light $(15 \mathrm{~cm}$ below a white and a near-ultra-violet tube) at room temperature to develop conidiophores and then in the dark at $20^{\circ} \mathrm{C}$ to induce sporulation. Conidia are transferred to fresh water agar plates until they germinate and then on V8-PDA plates. Monoconidial cultures are conserved in V8 slants under oil.

\section{Results}

Symptoms of foliar blights range from little black points to complete chlorosis and necrosis of the leaves, besides the characteristic symptoms of helminthosporium leaf blights: oval to elongated, light brown to dark brown blotches with or without a central dark spot.

The association of Bipolaris sorokiniana with the symptoms can be easily identified by the help of a stereomicroscope by the presence of dark conidia and for 
Drechslera tritici-repentis under a light microscope by the presence of conidia showing the characteristic conical basal cell (Drechsler 1923). However if no conidia are visible, seen the diversity of pathotype-genotype combinations and of environmental conditions, a distinction between leaf symptoms of the species is almost impossible without isolation.

In 1993, a majority of Bipolaris sorokiniana (75\%) has been isolated from samples with foliar blights from warm areas. The rest is made up of $17 \%$ of Drechslera tritici-repentis and $8 \%$ of other fungi like Curvularia sp. and Alternaria sp (fig. 1). This distribution is globally the same in the particular environments analysed (Bangladesh, Nepal and India) and confirmed by the isolation of 1994.

\section{Conclusion and prospects}

This collection is the base for characterisation of the diversity of the non-specific foliar pathogens of wheat in warm areas.

Four items will particularly interest us for this characterization:

- time course evolution of the NSFP distribution in warm area by the annual visit and collect of samples in particular known locations and various cropping systems.

- variability in pathogenicity of the isolates from different location and year through inoculation test on host differentials of wheat lines developed at CIMMYT.

- variability in the spectrum of toxins produced by the isolates: non-host-specific Bipolaris toxins such as prehelminthosporal and prehelminthosporol (De Mayo et al. 1965); host-specific ones such as Drechslera toxins: necrosis and chlorosis inducing toxins (Ballance et al. 1989, Brown and Hunger 1993)

- genetic diversity with RAPD.

\section{References}

Ballance G.M., Lamari L. and Bernier C.C. 1989. Purification and characterisation of a host-selective necrosis toxin from Pyrenophora tritici-repentis. Physiological and Molecular Plant Pathology 35 : 203-213.

Brown D.A. and Hunger R.M. 1993. Production of a Chlorosis-Inducing, Host-Specific, Low-Molecular Weight Toxin by Isolates of Pyrenophora tritici-repentis, Cause of Tan Spot of Wheat. Journal of Phytopathology 137 : 221-232.

De Mayo P., William R.E. and Spencer E.Y. 1965. Terpenoids. VIII. The immediate precursor of Helminthosporal and Helminthosporol. Canadian Journal of Chemistry 43 : 1357-1365.

Drechsler C. 1923. Some graminicolous species of Helminthosporium I. J. agric. Res., 24 : 641-739.

Hetzler J., 1992. Host-pathogen interaction in population of Bipolaris sorokiniana in the Warm NonTraditional Areas. Doctoral Dissertation, Institute of Plant pathology and Plant Protection, Göttingen, $129 \mathrm{p}$.

Lamari L. and Bernier C.C. 1989. Wheat genotype that Virulence of isolates of Pyrenophora triticirepentis on 11 wheat cultivars and cytology of th differential host reactions. Canadian Journal of Plant Pathology 11 : 284-290.

Mehta Y.R. 1981. Identification of races of Helminthosporium sativum of wheat in Brasil. Pesq. Agropec. Bra. $16: 331-336$.

Saunders D.A. 1990. Wheat for the Nontraditional Warm Areas.UNDP/CIMMYT Iguaçu, Brazil, 549 pp. 


\title{
PATHOGENIC DIVERSITY IN MAGNAPORTHE GRISEA, THE RICE BLAST PATHOGEN
}

\author{
J.B.BAHAMA, J.L.NOTTEGHEM* AND H.MARAÎTE
}

Université Catholique de Louvain, Faculté des Sciences agronomiques, Unité de Phytopathologie, Place Croix du Sud 2 boîte 3, 1348 Louvain-la-neuve, Belgique

*Laboratoire de Phytopathologie CIRAD/IRAT , Av. du val de Montferrand B.P.5035, 34032 Montpellier cedex 1, France

\begin{abstract}
Rice cultivation has been introduced in high elevation swamps of Burundi in 1980. Six years after, the released cultivar, Yunnan3, was severely attacked by Magnaporthe grisea, the rice blast pathogen. In order to identify resistant genotypes, race diversity encountered in this new rice growing area was studied. The results discussed in this paper have been obtained by inoculating 25 isolates of $M$. grisea on 27 rice cultivars including 13 Japanese differentials. All the known resistance genes are broken down by Burundian isolates except Pi-ta ${ }^{2}$. A high diversity is thus established.
\end{abstract}

\section{Introduction}

Blast, caused by Magnaporthe grisea (Hebert) Barr (anamorph Pyricularia oryzae Cavara), is one the most widespread disease of rice in tropics. All the rice breeding programmes are aimed at controlling that disease by resistant cultivars.

As a preliminary to resistance breeding for blast, the pathogen variability has to be well known. Virulence spectrum of isolates collected from rice growing areas has to be determined.

In developping countries where rice is included in the subsistence crop system-a low input one- vertical resistance, which is race specific, would be avoided since the pathogen adapts to such cultivars very quickly. Moreover, when severe epidemics break out, the farmers don't have means to afford fungicides. Hence, more efforts must be done to select quantitative resistance which ensures durable protection and more stable yield.

In Burundi, rice has been introduced in high elevations in 1980 with the release of Yunnan 3. Six years after, in 1986, high damages were caused to this cultivar by neck blast. Therefore, blast is taken into account in resistance breeding.

A collection of isolates was made up and a high diversity was observed by inoculation on differentials and some reference cultivars from various rice growing areas. Results of those tests are discussed below.

\section{Symptom description}

Magnaporthe grisea infects rice at all growth stages, resulting in two types of symptoms: leaf blast and panicle blast.

The leaf spots are typically elliptical with usually grey or whitish center and a brown or reddish-brown margin. The shape and colour vary depending upon the degree of susceptibility of the cultivars and the environmental conditions. Highly resistant cultivars develop minute brown specks of pin head size, while susceptible ones growing in conducive conditions show no or very little brown margin.

Panicle blast can affect any part of the panicle but often its base, causing the rotten neck also called neck rot. Panicles often fall over and grains are empty. Panicle branches and glumes can also be attacked. This type of blast is the most detrimental to yield. 


\section{Material and methods}

Twenty five isolates of $M$. grisea including 20 from Burundi (BD) and 5 from Cameroon (CM28), Columbia (CL6), Ivory Coast (CD45), Korea (CR5) and Philippines (PH11) were grown on rice flour agar medium (1 litre of water, $15 \mathrm{~g}$ of agar, $20 \mathrm{~g}$ of rice flour) in Petri dishes at $28^{\circ} \mathrm{C} ; 60 \%$ of relative moisture and $12 \mathrm{~h}$ of light per day. After 10 days of fungal growth, a spore suspension adjusted to 25,000 conidia/ml was injected between leaf sheaths with a hypodermic syringe.

Thirteen differentials carrying different resistance genes and 13 reference cultivars were used in this experiment. Plants were grown in a greenhouse up to 4-5leaf stage in batches containing compost and watered with nutritive solution.

Seven days after inoculation, lesions were assessed using Notteghem's 6-class scale where scores 1-3 correspond to resistant cultivars, 4-6 to susceptible ones.

\section{Results}

All the resistance genes carried by differentials are broken by Burundian isolates except Pi-ta ${ }^{2}$ of $\mathrm{Pi}^{\circ} 4$ (Table1). Isolates CL6, CM28 and CR5 from respectively Columbia, Cameroon and Korea overcome Pi-ta2 gene. PH11, CL6, CM28, CR5 and CD45 are highly aggressive on IR841, IR8, L9 and Mafushi which are resistant to Burundian isolates (Table2).

BD29, BD30, BD32, BD37 and BD38 attack Tetep which is widely used as resistant donor in most of the rice breeding programmes. On IRAT13 only moderate type lesions are observed. All the isolates severely infect Yunnan 3.

\section{Discussion and Conclusion}

There is a high pathogenic diversity in the $M$. grisea population from high elevation swamps in Burundi despite the limited number of rice genotypes grown in this environment. All the known resistance genes are broken down by Burundian isolates except $\mathrm{Pi}-\mathrm{ta}^{2}$. The latter is, however, matched by isolates from other geographic areas. In addition, all the isolates have overcome five genes or more. Improving resistance strategies based on these genes separately or in combination can thus not be effective for a long time. This suggest that in Burundian context, cultivars carrying only major genes should be avoided even those carrying Pi-ta since material exchange is not rigorously controlled.

More emphasize should be put on quantitative resistance which is often durable. Cultivars with a good level of quantitative resistance such as IRAT13, L9 and Mafushi should be used as parents. Promising progenies should be tested in a large number of rice growing countries and tests would be repeated during several seasons.

Recent molecular techniques especially Restriction Fragment Length Polymorphism (RFLP), have enabled to map blast resistance genes conferring quantitative resistance (Wang et al. 1993). Using that tool should help to find out quantitatively resistant cultivars to broaden the resistance background.

Other workers (Notteghem 1993, Wang et al. 1993) propose combinating a quantitative resistance with a qualitative one to face $M$. grisea variability.

To carry out this breeding, it is essential to understand the $M$. grisea population structure. Appearance of new isolates and their spectrum should be monitored. DNA markers identification has already proved its efficiency (Wolfe 1993). 


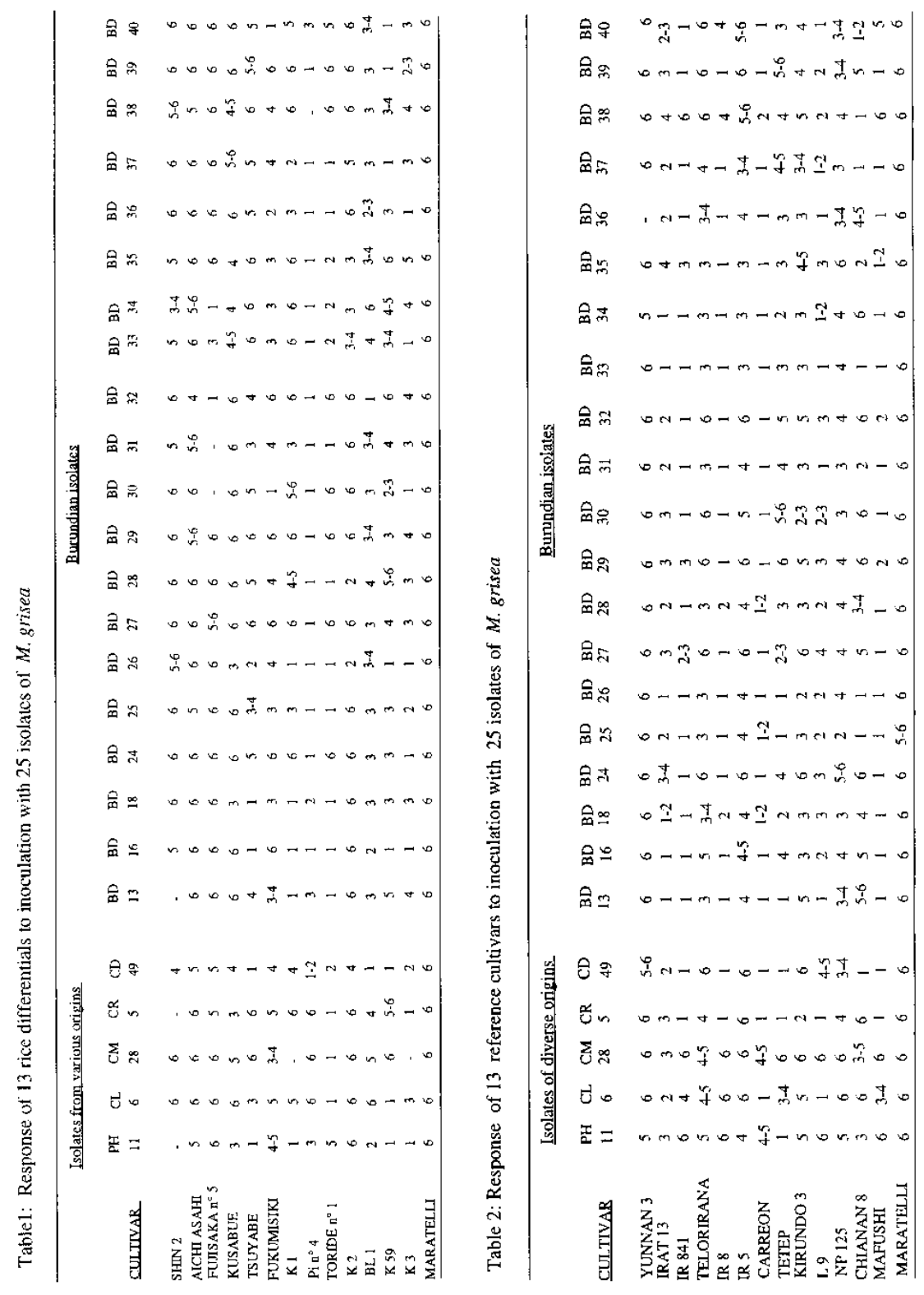




\title{
Acknowledgement
}

The authors greatly thank the European Community and the Belgian Administration for Development cooperation for their financial support.

\section{References}

Notteghem J.L. 1993. Durable resistance to rice blast disease. In Durability of Disease Resistance. Jacobs Th., Parlevliet J.E. eds., Kluwer Academic Publishers. Dordrecht/Boston/London

Wang G.L., Mackill D.J., Bonmann J.M., McCouch S.R. and Nelson R.J. 1993. RFLP mapping of genes conferring complete and partial resistance in a rice cultivar with durable resistance to blast. In Durability of Disease Resistance. Jacobs Th. and Parlevliet J.E. eds., Kluwer Academic Publishers. Dordrecht, Boston, London.

Wolfe M.S. 1993. Can the strategic use of disease resistant hosts protect their inherent durability? In Durability of Disease Resistance. Jacobs Th. and Parlevliet J.E. eds., Kluwer Academic Publishers. Dordrecht, Boston, London.

\section{CHARACTERIZATION OF POLYMYXA GRAMINIS INVOLVED IN THE TRANSMISSION OF PEANUT CLUMP VIRUS IN TROPICAL AREAS}

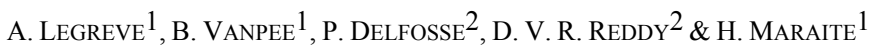 \\ ${ }^{1}$ Unité de Phytopathologie, Université catholique de Louvain, Faculté des Sciences agronomiques, Place \\ Croix du Sud 2 bte 3, B-1348 Louvain-la-Neuve, Belgium . \\ ${ }^{2}$ International Crops Research Institute for the Semi-Arid Tropics, Patancheru, Andhra Pradesh 502 324,
} India.

\begin{abstract}
The plasmodiophoraceous fungus, Polymyxa graminis Ledingham, is involved in the transmission of Peanut Clump Virus (PCV), a soil-borne virus widely distributed in India and in West Africa. This fungus is known in temperate areas to be the vector of soil-borne viruses on barley, wheat and oat. Ecological requirements of $P$. graminis isolates from various origins are possibly different. Indian and African isolates indeed grow easily at $25-30^{\circ} \mathrm{C}$, whereas those from temperate areas are favoured by temperatures between 15 and $20^{\circ} \mathrm{C}$. Host range of tropical isolates is apparently wider and less specific than that of isolates from temperate areas. Indian $P$. graminis isolates grow on monocotyledonous plants as well as on dicotyledonous plants, whereas in temperate areas, two fungal species are listed as separate species according to the host specificity; $P$. graminis growing only on graminaceous plants and Polymyxa betae Keskin on Chenopodiaceae. In order to clarify the taxonomic position and precise the requirements of $P$. graminis associated with PCV transmission, pure strains obtained from a single cystosorus are prepared from isolates of various origins. Host range, ecological requirements, such as temperature optimum and $\mathrm{pH}$, are studied. First results confirm differences in the temperature optimum, the Indian strain growing best on sorghum between 20 and $30^{\circ} \mathrm{C}$ compared to less than $20^{\circ} \mathrm{C}$ for the strains from northern origins on barley.
\end{abstract}

\section{Introduction}

Peanut clump is a soil-borne virus disease widely distributed in India and West Africa. Infected plants are stunted conspicuously and have dark green leaflets. Flowers can be produced, but any pods formed are poorly developed (Thouvenel et al. 1976; Reddy et al. 1983). The virus is transmitted with seed from groundnut, pearl millet and finger millet infected plants, but is also soil-transmitted by a plasmodiophoraceous fungus, Polymyxa graminis Ledingham, an ubiquitous obligate root parasite of graminaceous plants (Thouvenel et al., 1976; Thouvenel \& Fauquet 1981, Ratna et al. 1991). The resting spores of the fungus may contain the virus and can survive in soils for many years. In specific conditions, spores 
germinate and zoospores infect epidermic root cells of a host plant. The plasmodium, the zoosporangium, producing secondary zoospores, and the cystosorus, the survival stage of the fungus, are all formed in the infected cell.

In temperate areas, two fungal species are distinguished according to the host specificity : P. graminis growing only on Poaceae (Ledingham 1939, Barr 1979, Bastin et al. 1989, Adams 1990), and Polymyxa betae Keskin on Chenopodiaceae and some related families (Barr 1979, Barr and Asher 1992). The two species are known to be the vector of soil-borne viruses on their specific hosts. In India, $P$. graminis isolates grow on monocotyledonous plants, such as pearl millet, sorghum and wheat, as well as on different families of dicotyledonous plants including Chenopodiaceae. According to this wider and less specific host range, the taxonomic position of the $P$. graminis strains associated with peanut clump virus transmission is questionable. Is it the same species as $P$. graminis found in temperate areas or is it a third one of the same genus, able to infect and transmit virus(es) over a wider host range? Is the host specificity a relevant criterion to separate species?

The in depth understanding of the $P$. graminis populations involved in the transmission of the peanut clump virus and the acquisition of basic information for development of an integrated control of PCV at small holdings level, are the main objectives of the collaborative research project between Université catholique de Louvain - Unité de Phytopathologie and International Crops Research Institute for the Semi-Arid Tropics.

The program started in March 1993 with the isolation of $P$. graminis from various origins and the production of single cystosorus strains. The characterization of the P. graminis populations involved in the transmission of the peanut clump virus and the comparison with strains from temperate areas are in progress. The results presented here concern a first experiment on the temperature requirements for 5 strains from various origins.

\section{Single cystosorus cultures}

\section{Materials and methods}

P. graminis isolates were obtained by growing bait plants in infested soils from various origins. Pieces of rootlets containing P. graminis cystosori were soaked in sterile distilled water, cut in 1 or $2 \mathrm{~mm}$ fragments and homogenized in Omnimixer Virtis at $25000 \mathrm{rpm}$. Few drops were spread on $2 \%$ water agar in Petri dishes. The surface was carefully analysed with a Wild Macroscope at 40-100 x magnification to detect single cystosorus.S ingle cystosori were taken with a micro spear and placed adjacent to the roots of a 2-day-old pregerminated seedling planted in single tube on sterile quartz sand. Hundred to 300 plants were each inoculated with a single cystosorus for each isolates. The plants were watered with half-strength nutrient solution and grown at $15-20^{\circ} \mathrm{C}$ (night-day) or $25-30^{\circ} \mathrm{C}$ according to the temperate or tropical origin of the $P$. graminis isolates. After 2 or 3 months, each plant was removed and checked under the microscope to detect the fungus. Infected roots were dried and stored at room temperature.

Fungal multiplication was performed in an automatic immersion device. The latter was constituted by PVC tubes suspended in a container, linked with a pump to an independent nutrient solution tank. Host plants were planted in the tubes containing sterile quartz sand inoculated with root extract infested with the $P$. graminis strain. The basis of each tube was formed by a polyamid grid allowing flowing in and out of nutrient solution and zoospores. Watering is programmed 
during 6 hours each 12 hours. After 2 months, plants were removed and the roots observed to ascertain the presence of the fungus. The strains are stored in dried root fragments at room temperature.

\section{Temperature requirement}

Five single cystosorus strains were tested : strains I1-20 and I1-229 from India multiplied in sorghum roots, and the strains B1 from Belgium, $\mathrm{C} 1$ from Canada and F11 from France in barley roots.

Culture tubes filled with sterile quartz sand were inoculated with cystosori suspensions of each strain (1000 cystosori/tube). The effect of temperature on the development of temperate and tropical strains, respectively on barley or sorghum, was tested by transplanting 2-day-old seedlings in inoculated sand and growing them at the test temperatures, $10-15^{\circ} \mathrm{C}, 15-20^{\circ} \mathrm{C}, 20-25^{\circ} \mathrm{C}, 25-30^{\circ} \mathrm{C}$ or $30-35^{\circ} \mathrm{C}$ (night/12h-day/12h). Periodically, 4 plants grown at the various temperatures were removed for each strain. The roots were coloured in blue lactophenol and observed under the microscope for assessment of fungal infection.

\section{First results}

Up to now, six P. graminis strains have been obtained and multiplied: three Indian strains on sorghum and isolated from a soil sampled at Patancheru in a field infected by the peanut clump (strain I1-1, I1-20, I1-229), one Belgian strain on barley and isolated from a soil sampled at Loupoigne in a field infected by the barley yellow mosaic (strain B1), one Canadian strain on barley obtained from barley roots grown on Ottawa soil in 1987 and send by Dr D. J. S. Barr (strain C1), and one French strain on barley and isolated from a soil sampled in 1988 at Carcassonne (strain F11).

Temperature requirements for $P$. graminis infection are different according to the origin of the strains (Fig. 1). The two Indian strains have a narrow optimum temperature around $25^{\circ} \mathrm{C}$. Below $20^{\circ} \mathrm{C}$ and above $30^{\circ} \mathrm{C}$, any trace of infection was not yet observed after six weeks. Strains from temperate areas are favoured by cooler temperature: the strains $\mathrm{B} 1$ and $\mathrm{C} 1$ grew easily between 10 and $20^{\circ} \mathrm{C}$, and the strain from the south of France grew only at $15-20^{\circ} \mathrm{C}, 5$ weeks after the inoculation.

Differences in infection capacity are also evident. Belgian strain B1 showed $100 \%$ infection on barley after 2 weeks, whereas minimum 4 weeks of incubation are necessary to the strain $\mathrm{C} 1$ to reach this level. The 3 other strains seem to have a lower infection capacity, especially the strains coming from India.

\section{Discussion and prospects}

The first results demonstrate differences in the temperature requirements of $P$. graminis strains according to the origin, the Indian strains being able to develop at temperatures inhibitory to the Belgian strain. The results with the Canadian and the French strains suggest a continuous range of temperature optimum. Further repetitions of these experiments will specify the differences in response at the various growth stages of the fungus and the influence of the host in the observed differences. In view to a better understanding of the $P$. graminis populations involved in the transmission of the peanut clump virus, soil water potential and $\mathrm{pH}$ requirements will also be tested. The host ranges including dicotyledonous plants and weeds of the tropical strains will be analysed and compared to temperate $P$. betae and $P$. graminis species. A morphological analyse of the various stages of the 
fungus and a genomic analyse of the various strains on DNA fragments am plified by PCR will be done to define the taxonomic position of the fungus involved in the PCV transmission.

\section{Acknowledgements}

The authors are grateful to ICRISAT and the Belgian Administration for Development Cooperation for funding this research project.
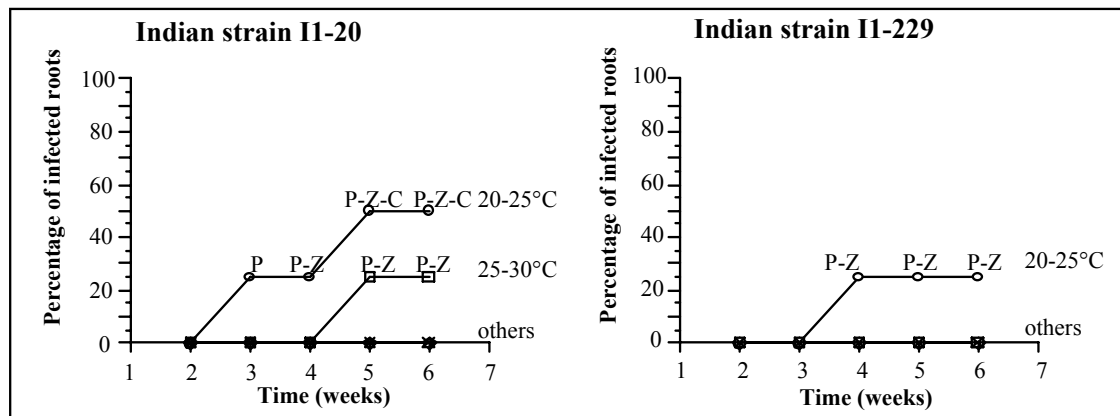

Belgian strain B1
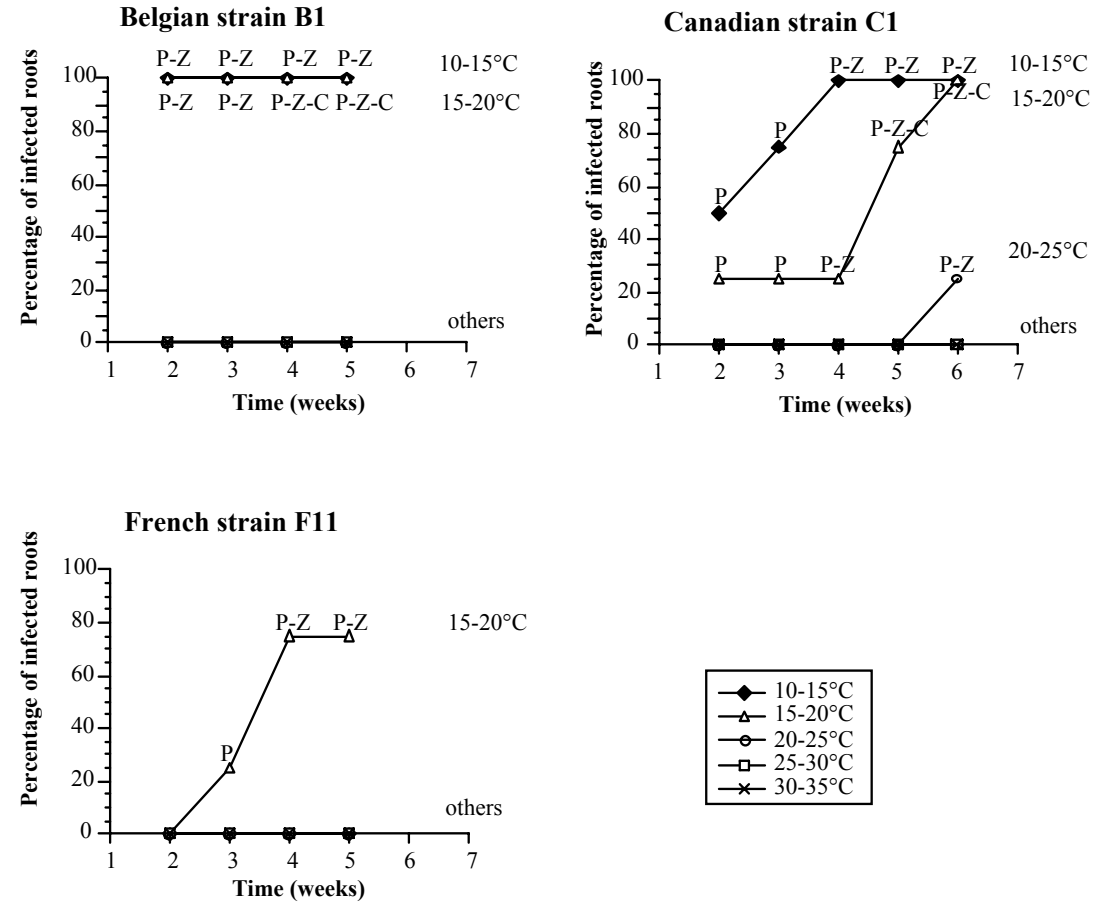

Fig. 1 Comparison of temperature requirement of Indian P. graminis strains (strains I1-20 and I1-229 on sorghum) and temperate strains (strains B1, C1 and F11 on barley). Fungal stages observed in the roots: $\mathrm{P}=$ plasmodium, $\mathrm{Z}=$ zoosporangium and $\mathrm{C}=$ cystosorus. 


\section{References}

Adams M.J. 1990. Host range and transmission of barley viruses by isolates of Polymyxa graminis. Proceedings of the first symposium of the international working group on plant viruses with fungal vectors. Schriftenreine der Deutschen Phytomedizinischen geselleschaft 1:121-123. Ed. R. Koënig. Stuttgart - Ulmer.

Barr D.J.S. 1979. Morphology and host range of Polymyxa graminis, Polymyxa betae and Ligniera pilorum from Ontario and some other areas. Canadian Journal of Plant Pathology 1: 85-94

Barr K.J. and Asher M.J.C. 1992. The host range of Polymyxa betae in Britain. Plant Pathology 41: 64-68

Bastin V., Boute C. and Maraite H. 1989. Inoculum potential and host range of Polymyxa graminis. EPPO Bulletin 19: 541-546.

Ledingham G.A. 1939. Studies on Polymyxa graminis, n. gen. n. sp., a Plasmodiophoraceous root parasite of wheat. Canadian Journal of Research C 17: 38-51.

Ratna A S., Rao A.S., Reddy A.S., Nolt B.L., Reddy D.V.R., Vijayalakshmi M. and McDonald D. 1991. Studies on the transmission of Indian peanut clump virus disease by Polymyxa graminis. Ann. appl. Biol. 118: 71-78.

Reddy D.V.R., Rajeshwari R., Iizuka N., Lesemann D.E., Nolt B.L.and Goto T. 1983. The occurrence of Indian peanut clump, a soil-borne virus disease of groundnuts (Arachis hypogea) in India. Ann. appl. Biol. 102: 305-310.

Thouvenel J.-C., Dollet M.and Fauquet C. 1976. Some properties of peanut clump, a newly discovered virus. Ann. appl. Biol. 84: 311-320.

Thouvenel J.-C.and Fauquet C. 1981. Further properties of peanut clump virus and studies on its natural transmission. Ann. Appl. Biol. 97: 99-107. 
PRACTICAL MYCOLOGY

\title{
PRACTICAL ADVICE FOR COLLECTING WOOD-ROTTING FUNGI IN THE TROPICS
}

\author{
ERAST PARMASTO
}

Institute of Zoology \& Botany, 21 Vanemuise St., EE 2400 Tartu, Estonia

Number of new species of mainly wood-rotting fungi of Aphyllophorales is increasing rapidly. Nevertheless, only a small part of the tropical territories has been mycologically explored; thousands of species have not yet found and described. Type specimens and other collections of Aphyllophorales collected earlier are in many if not most cases sterile (without basidia and spores). Their original consistency and colour have usually changed, fruit-bodies are sometimes badly damaged by insects. Comparatively very small number of species has been introduced to pure culture.

For developing modern taxonomy of this economically important group of fungi, extensive collecting of specimens is needed. Some practical advises in addition to the ones published in several handbooks are given below.

1. When sampling a specimen growing on wood, the tree species (or at least genus) must be identified if possible: there are externally similar sibling species specialized in several genera of wood-rotting fungi (Phellinus, Inonotus, Peniophora, Corticium etc.). Sample also a piece of rotten wood (substrate) or describe the rot type (white, brown, pocket rot...).

2. Describe the colour of all parts of the fresh basidiomata (fruit-bodies) as soon as possible using standard colour charts (e. g., the rather cheap "Methuen Handbook of Colour" by A. Kornerup \& J.H. Wanscher). Note colour change after bruising (handling) the basidioma.

3. Dry the specimens in the same day you collected them. When kept moist or dried slowly, basidia will collapse and spores will be damaged or destroyed (by bacteria?).

4. Separate a part of the big basidioma (or one of the basidiomata) to take a spore print. This is extremely important! If the collected basidioma is dry, put it (in the evening) for 10-30 min. into water. After some drying (basidioma must be moist but not wet) put it on a piece of black and white paper collection number written on it. Hymenophore (pores, lamellae, folds) must be facing downwards. Put the basidioma with the paper strip below it for the night in a plastic bag to avoid desiccation. Next morning you can usually get a good spore print; in several cases, sterile when collected specimens have developed new basidia and began to sporulate. Dry the basidioma used for taking a spore print, and mark it: it may be the best part of your collection. The piece of paper with spore print must be dried promptly (with no heating) and kept in a labelled envelope.

5. Spore prints when kept dry (if possible, in frigerator at $2-5^{\circ} \mathrm{C}$ ) may be used even after one or some weeks to inoculate suitable medium in your lab to get a pure culture. In some species, spores are viable up to several months.

6. When studying herbarium specimens microscopically, use the spore print to measure 25-50 randomly taken spores to calculate mean spore size and spore length/width index Q. 
7. After returning from a collecting trip, disinfect your collections in a deepfreezer. Keep them at $-18^{\circ} \mathrm{C}$ (better: at $-25^{\circ}$ or even $-30^{\circ}$ ) 1-2 days, in big fruit bodies of polypores - up to 3 days. If you put every disinfected specimen in a minigrip plastic bag, further infection by insects is surely avoided. Use of poisonous disinfectants must be avoided: some of these are almost useless (as is the frequently used in tropical herbaria naphthalene), all are dangerous for health, and many of them may disturb the use of specimens in molecular taxonomy studies later.

8. If you have found an interesting collection you consider to belong to a new species, do not hurry describing it as a new one. About 70-75\% of "new" species are based on only one collection nowadays. In many cases these are not new species but "deviating" specimens of old ones of unknown variability. Keep in mind that variation of size of spores, basidia, cystidia, pores etc. in one specimen does not give any information on variability of the species. Avoid increasing taxonomic "noise," better continue collecting in other areas to find more specimens of your fungus. 


\title{
THE INFLUENCE OF PREPARATION ON ASCOSPORE MEASUREMENTS
}

Bellis Kullman, Aivo JaKoBson, MART RAHI

Institute of Zoology and Botany of the Estonian Academy of Sciences, 21 Vanemuise St., EE2400 Tartu, Estonia

\begin{abstract}
The influence of various media (tap water (TW), 2\% potassium hydroxide solution $(\mathrm{KOH})$, cotton blue solution in lactophenol (CB), polyvinyl lacto-phenol (PVLPh), detergent solution in water (DE), fixative (FAA)) and the storage on the ascospore measurements of Byssonectria terrestris (Alb. \& Schw.:Fr.) Pfister, Neotiella vivida (Nyl.) Dennis \& Rifai and Peziza domiciliana Cooke were compared. The influence of the medium and the storage on spore dimensions of different species has the same trend but its effect may be different. The comparison of spore dimensions taken from spore print, from rehydrated herbarized material and from living material immediately or up to 45 days after preparation is not justified.

Additional keywords. Ascomycetes, spore measurements, microscopy.
\end{abstract}

\section{Introduction}

In the systematics of Ascomycetes spore measurements as quantitative characters are used for the delimitation of populations and species, for the study of geographic speciation and for the construction of phylogenetic trees (Huhtinen 1989, Fogel 1992, Möls Raitviir 1974, Schumacher 1990, Wu 1993). The natural variability of spore dimensions of a specimen may be considerable, and recorded variability always includes measurement errors. These errors have two main causes: 1). errors of the measurement procedure itself, e.g. due to the orientation of a spore in a microscopic specimen (Rahi Kullman 1993); 2) spore shape distortions (shrinkage or swelling) due to differences in the osmotic concentration (water potential) of the spore content and the medium.

There is strong experimental evidence that spore dimensions depend on the type and concentration of the fixative, medium as well on spore/specimen storage. Spore shrinkage up to $26 \%$ in linear dimensions in the Melzer reagent $(56 \%$ of the calculated volume) has been reported (Baral 1992); these data indicate that one should be extremely cautious in comparing spore measurement data when details of the measurement procedure are incomplete or missing. Dealing with data on type specimens this recommendation is essential but sometimes unrealistic.

Considering the huge amount of published data on spore dimensions it is of interest to evaluate effects on spore dimensions due to: 1) type and storage of a specimen (fresh, air dried herbarized fruitbody or spore print); 2) type of medium.

\section{Material and methods}

The influence of the following media on spore dimensions were evaluated: tap water (TW); $2 \%$ potassium hydroxide solution $(\mathrm{KOH}) ; 0.2 \%$ cotton blue solution in lactophenol (40\%) (CB); polyvinyl lactophenol containing $56 \%$ of polyvinyl alcohol, $22 \%$ of lactic acid, $22 \%$ of phenol (by volume) (PVLPh); fixative water solution containing $4 \%$ of formalin, $23 \%$ of ethanol, $1 \%$ of acetic acid (FAA); detergent

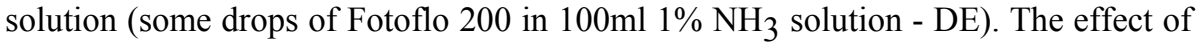
the time of taking spore measurements (immediately or up to 45 days after preparation) and the type of a microscopic specimen (made from spore print, fresh, fixed or rehydrated herbarized fruitbody) on spore dimensions were also examined. The species under study were: Byssonectria terrestris (Alb. \& Schw.:Fr.) Pfister 
(spore print from several fruitbodies of one specimen, 8 fresh and 17 herbarized fruitbody), Peziza domiciliana Cooke (spore print from one fruitbody) and Neotiella vivida (Nyl.) Dennis \& Rifai (fixed fruitbody).

From each fruitbody/spore print 25 spores were measured with an ocular micrometer (microscope "Amplival", immersion objective HI 100) with an accuracy of $\pm 0,3 \mu \mathrm{m}$. A database of spore dimensions was compiled. The mean spore length (L) and width (W), L/W ratio (Q), standard deviation (s) and the coefficient of variation $(\mathrm{V})$ were computed for each sample.

In the analysis of variance "Statgraph" procedures were applied: One- and TwoWay ANOVA for means and variances; Analysis of the Nested Design for evaluating the effects of the medium and the fruitbody. The significance level 0.05 was applied throughout the analyses.

\section{Results}

The spores of Byssonectria terrestris from one specimen measured in CB were examined. One-Way ANOVA revealed that a) samples from the same herbarized fruitbody do not differ significantly $(\mathrm{p}>0.3)$ and $b)$ spore samples from the same common spore print of several fruitbodies do not differ significantly $(p>0.9)$. Variance of spore means is the smallest in spore print (Table 1. Fig.1 A) which may serve as a "reference standard" in comparative study (Table 2).

The spore dimensions of a fixed Neotiella vivida fruitbody do not change significantly in TW, while they diminish essentially in CB.

Nested Design ANOVA revealed significant temporal differences spore dimensions measured on a fresh fruitbody and half a year later on the same dried specimen (FFinTW and HFinTW; FFinTW and HFinCB) and also significant difference spore dimensions measured in $\mathrm{CB}$ on a spore print and on the herbarized specimen (SPinCB and HFinCB).

Table 1. Spore measurements (L, W, Q) of spore print (SP), fresh (FF) and herbarized (HF) fruitbodies of one B. terrestris specimen; coefficients of variation $\left(\mathrm{V}_{\mathrm{L}}, \mathrm{V}_{\mathrm{W}}\right)$ and standard deviations $\left(\mathrm{s}_{\mathrm{L}}, \mathrm{s}_{\mathrm{W}}\right)$ of sample means $\left({ }_{2} \_\right.$); coefficients of variation of samples $\left(\mathrm{V}_{\mathrm{l}}, \mathrm{V}_{\mathrm{w}}\right)$ in different media $(\mathrm{TW}, \mathrm{CB})$. Samples from the same common spore print of several fruitbodies - *; samples from the same fruitbody $-^{\circ}$; samples from the several fruitbodies - -.; N-number of samples (of size 25)

\begin{tabular}{|lllllllllll|}
\hline & $\mathrm{N}$ & $\mathrm{L}$ & $\mathrm{V}_{\mathrm{L}}$ & $\mathrm{s}_{\mathrm{L}}$ & $\mathrm{W}$ & $\mathrm{V}_{\mathrm{W}}$ & $\mathrm{s}_{\mathrm{W}}$ & $\mathrm{V}$ & $\mathrm{V}_{\mathrm{W}}$ & $\mathrm{Q}$ \\
\hline *SPinCB & 4 & 18.8 & 0.4 & 0.1 & 8.7 & 0.3 & 0.03 & $4-5$ & $3-5$ & 2.17 \\
'HFinCB & 5 & 18.8 & 1 & 0.2 & 7.9 & 2 & 0.2 & $4-6$ & $7-11$ & 2.38 \\
-FFinTW & 7 & 20.0 & 1 & 0.1 & 8.5 & 1 & 0.1 & $4-11$ & $3-5$ & 2.35 \\
-HFinCB & 5 & 18.6 & 2 & 0.3 & 7.8 & 2 & 0.2 & $4-5$ & $6-9$ & 2.39 \\
-HFinTW & 7 & 18.5 & 3 & 0.5 & 8.0 & 3 & 0.2 & $3-4$ & $4-6$ & 2.31 \\
\hline
\end{tabular}

Tables 2-4. Mean spore measurements (L,W,Q) of spore samples $(n=25)$ and their coefficients of variation (V), standard deviations (s) in different media; differences of these measurements with respect to those measured in CB (Tables 2, 3) or in FAA (Table 4) $(\%, \mu \mathrm{m})$ and the significance level (p) of the difference.

Table 2. Byssonectria. terrestris from the same common spore print of several fruitbodies. 


\begin{tabular}{|lrrrrrrrr|}
\hline Media & & $\mu \mathrm{m}$ & $\mathrm{s}$ & $\mathrm{V}$ & $\mu \mathrm{m}$ & $\%$ & $\mathrm{p}$ & $\mathrm{Q}$ \\
\hline CB & $\mathrm{L}$ & 18.8 & 0.8 & 4 & & & & \\
& $\mathrm{~W}$ & 8.7 & 0.4 & 4 & & & & 2.17 \\
PVLPh & $\mathrm{L}$ & 18.9 & 0.7 & 4 & +0.1 & 0 & 0.64 & \\
& $\mathrm{~W}$ & 8.5 & 0.4 & 4 & -0.2 & 2 & 0.06 & 2.22 \\
FAA & $\mathrm{L}$ & 18.7 & 0.6 & 3 & -0.1 & 1 & 0.51 & \\
& $\mathrm{~W}$ & 8.6 & 0.3 & 3 & -0.2 & 1 & 0.32 & 2.18 \\
KOH & $\mathrm{L}$ & 19.0 & 1.0 & 6 & +0.2 & 1 & 0.28 & \\
& $\mathrm{~W}$ & 8.9 & 0.5 & 6 & +0.2 & 2 & 0.03 & 2.14 \\
TW & $\mathrm{L}$ & 19.5 & 1.0 & 5 & +0.6 & 3 & 0.00 & \\
& $\mathrm{~W}$ & 9.1 & 0.4 & 4 & +0.2 & 5 & 0.00 & 2.13 \\
DE & $\mathrm{L}$ & 19.5 & 1.1 & 5 & +0.7 & 4 & 0.00 & \\
& $\mathrm{~W}$ & 9.5 & 0.6 & 7 & +0.8 & 9 & 0.00 & 2.10 \\
\hline
\end{tabular}

Table 3. Peziza domiciliana from the same common spore print of one fruitbody.

\begin{tabular}{|lrrrrrrrr|}
\hline Media & & $\mu \mathrm{m}$ & $\mathrm{s}$ & $\mathrm{V}$ & $\mu \mathrm{m}$ & $\%$ & $\mathrm{p}$ & $\mathrm{Q}$ \\
\hline CB & $\mathrm{L}$ & 14.8 & 0.7 & 4 & & & & \\
& $\mathrm{~W}$ & 9.0 & 0.7 & 8 & & & & 1.65 \\
PVLPh & $\mathrm{L}$ & 14.5 & 0.6 & 4 & -0.3 & 2 & 0.07 & \\
& $\mathrm{~W}$ & 8.2 & 0.4 & 5 & -0.8 & 8 & 0.00 & 1.76 \\
TW & $\mathrm{L}$ & 14.9 & 0.6 & 4 & +0.1 & 1 & 0.89 & \\
& $\mathrm{~W}$ & 9.6 & 0.5 & 5 & +0.6 & 6 & 0.00 & 1.56 \\
\hline
\end{tabular}

Table 4. Neotiella vivida from the one fixed fruitbody

\begin{tabular}{|lrrrrrrrr|}
\hline Media & & $\mu \mathrm{m}$ & $\mathrm{s}$ & $\mathrm{V}$ & $\mu \mathrm{m}$ & $\%$ & $\mathrm{p}$ & $\mathrm{Q}$ \\
\hline FAA & $\mathrm{L}$ & 23.8 & 1.2 & 5 & & & & \\
& $\mathrm{~W}$ & 13.1 & 0.6 & 4 & 1.8 & 3 & & \\
TW & $\mathrm{L}$ & 23.6 & 0.9 & 4 & -0.2 & 1 & 0.43 & \\
& $\mathrm{~W}$ & 13.4 & 0.7 & 5 & +0.3 & 2 & 0.10 & 1.76 \\
CB & $\mathrm{L}$ & 21.8 & 0.9 & 4 & -2.0 & 8 & 0.00 & \\
& $\mathrm{~W}$ & 12.0 & 0.6 & 5 & -1.1 & 8 & 0.00 & 1.82 \\
\hline
\end{tabular}

Table 5. Analysis of Nested Designs. Variance components of the medium (ME\%), fruitbody (FB\%), and the residual (Spore\%) for spore length (L) and width (W) on the basis of the B. terrestris spore measurements of fresh (FF) and herbarized (HF) fruitbodies and the spore print (SP) as compared in different media $\mathrm{CB}, \mathrm{TW}, \mathrm{TW} \_\mathrm{CB}$. If $\mathrm{F}_{\mathrm{emp}}>\mathrm{F}_{\mathrm{kr}}$, the variance component is significant at $\mathrm{a}=0.05$.

\begin{tabular}{|lrrrrrrrrrr|}
\hline & & $\mathrm{ME} \%$ & $\mathrm{~F}_{\mathrm{emp}}$ & $\mathrm{F}_{\mathrm{kr}}$ & $\mathrm{FB} \%$ & $\mathrm{~F}_{\mathrm{emp}}$ & $\mathrm{F}_{\mathrm{k}}$ & Spore $\%$ \\
\hline FFinTW and HfinTW & $\mathrm{L}$ & 53 & 63.0 & $>$ & 4.8 & 4 & 3.5 & $>$ & 1.8 & 43 \\
& $\mathrm{~W}$ & 40 & 25.0 & $>$ & 4.8 & 9 & 5.6 & $>$ & 1.8 & 51 \\
FFinTW and HfinCB & $\mathrm{L}$ & 46 & 91.5 & $>$ & 5.3 & 0 & 1.2 & $<$ & 3.8 & 54 \\
& $\mathrm{~W}$ & 43 & 55.7 & $>$ & 5.3 & 2 & 1.8 & $<$ & 3.8 & 5 \\
HFinTW and HFin CB & $\mathrm{L}$ & 2 & 1.3 & $<$ & 5.3 & 21 & 7.9 & $>$ & 3.8 & 77 \\
& $\mathrm{~W}$ & 2 & 1.5 & $<$ & 5.3 & 14 & 5.1 & $>$ & 3.8 & 84 \\
HFinTW and HfinTW-CB & $\mathrm{L}$ & 5 & 1.7 & $<$ & 5.3 & 34 & 15.0 & $>$ & 3.8 & 61 \\
\multirow{2}{*}{ SPinCB and HfinCB } & $\mathrm{W}$ & 8 & 2.7 & $<$ & 5.3 & 23 & 7.5 & $>$ & 3.8 & 69 \\
& $\mathrm{~L}$ & 3 & 2.7 & $<$ & 6.0 & 4 & 2.1 & $<$ & 2.1 & 93 \\
& $\mathrm{~W}$ & 67 & 456 & $>$ & 6.0 & 0 & 0.4 & $<$ & 2.1 & 33 \\
\hline
\end{tabular}

The temporal changes in spore dimensions of Byssonectria terrestris are illustrated 
in Fig. 2. Changes of spore width measured in spore print in PVLPh are mixed: a small but significant decrease is followed by an increase. A significant decrease in spore dimensions was observed in a microscopic specimen of a fruitbody and a spore print mounted on PVLPh and CB respectively. Decrease is not significant up to the 6th day in spore print in CB.

\section{Discussion and recommendations}

1. Spores discharged from the ascus are significantly wider in comparison with spores in fruitbodies (Table 5: SpinCB, HFinCB. Fig. 1: $\mathrm{L}_{4}>\mathrm{L}_{E}$ or $\mathrm{L}_{\mathrm{A}}>\mathrm{L}_{B}$ ).

2. Spore dimensions decrease with the storage of a herbarized specimen. Spores in fruitbodies measured a half year later are significantly smaller in comparison with spores in fresh fruitbodies (Table 5: FFinTW and HFinTW; FFinTW and HFinCB. Fig.1: $\mathrm{E}>\mathrm{C}$ ). Weekly drying does not cause any essential change (E and 6).

3. Preliminary rehydration of a herbarized specimen in TW increases significantly the variance of means of spore dimensions in comparison with those measured on spores introduced directly into CB (Table 1. Fig. 1: C,D - B). The means of spore dimensions of fresh fruitbodies (Table 1. Fig.1: E) vary less than those of herbarized fruitbodies.

4. Variance of the spore means is the smallest in spore print (Table 1. Fig.1: A) which may serve as a "reference standard" in a comparative study. The spore discharge as a pretreatment factor probably account for this homogeneity.

5. Considering effects on spore dimensions and variability (Table 2. Fig.1: A, 1-5) on the wall structure, the media investigated can be divided as follows:

a) FAA, CB, PVLPh -impact on spore sizes and variability is minor. Preference should be given to FAA;

b) TW - causes no wall disintegration, but impact on dimension variability is greater. This may be caused by different spore viability (Stojanovic, Ristanovic and Jencic 1986);

c) $\mathrm{KOH}, \mathrm{DE}$ - due to disintegrating effects on the spore wall the impact on dimension variability is the greatest. The effects of $\mathrm{KOH}, \mathrm{TW}$ and $\mathrm{DE}$ on spore dimensions are significantly greater than that of CB.

6. The effect of various media on the length/width ratio (Q) differs, $Q$ is maximal in $\mathrm{PVLPh}$ and minimal in DE. Therefore, one must be extremely cautious when comparing data on Q (Table 2).

7. The essential increase in TW is the same for spore measurements of Byssonectria terrestris and Peziza domiciliana. Differences in its effect are probably due to different spore wall rigidity (Table 2 and 3 ).

8. The fixative seems to preserve the measurement of both dry spores and those taken from live fruitbodies. On the contrary in $\mathrm{CB}$, it dehydrates spore in live fruitbodies (see B. terrestris spore print, Table 2. and a fixed N. vivida fruitbody, Table 4).

9. In microscopic specimens from fresh fruitbodies and spore print mounted in $\mathrm{CB}$ and PVLPh temporal changes in spore dimensions may differ considerably. In microscopic specimens of spores in PVLPh the distance between glass surfaces decreases due to the medium loss by evaporation and spores may become compressed and distorted. In microscopic specimens from fruitbody these effects do not take place.

It is recommended to measure spores in $\mathrm{CB}$ in the spore print and in the herbarized fruitbody during the first six days (Fig.2-4). 


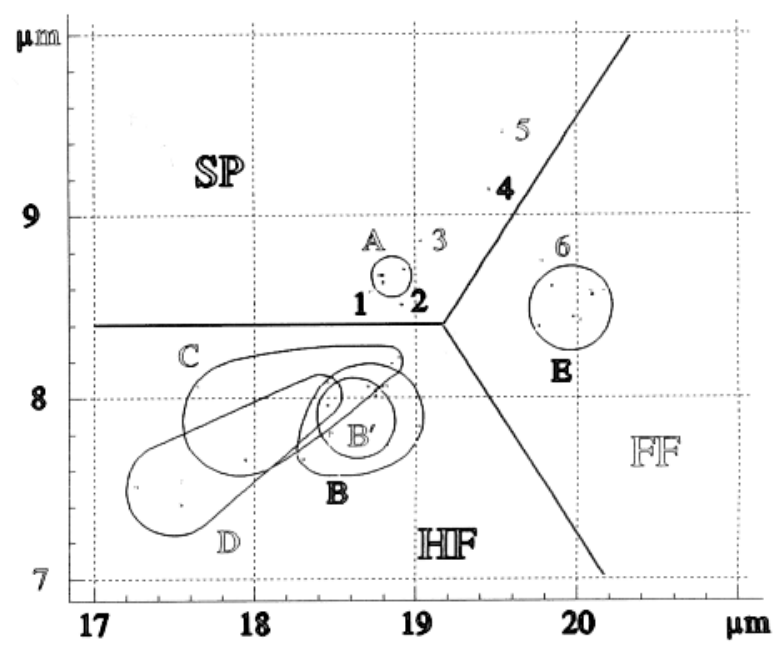

Fig.1. Mean spore length and width in one specimen of B. terrestris. SP - spore print, FF - fresh fruitbody, HF -herbarized fruitbody. Points: 1 - in fixative (FAA), 2 - in polyvinyl lacto-phenol (PVLPh), 3 - in potassium hydroxide solution (KOH), 4 - in tap water (TW), 5 - in detergent solution (DE), 6 - one week storage of the fruitbody in TW, clusters A - spore samples of the same common spore print from different fruitbodies in CB; B - samples from different fruitbodies in CB; B' - samples from the same fruitbodies in $\mathrm{CB}$; C - rehydrated samples in TW, D - rehydrated samples mounted in $\mathrm{CB}$; E -samples from the fresh fruitbody in TW.

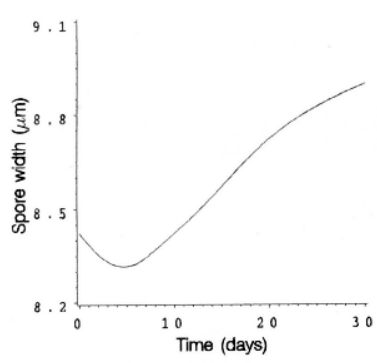

B

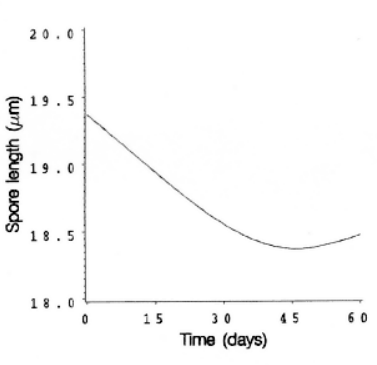

$\mathrm{C}$

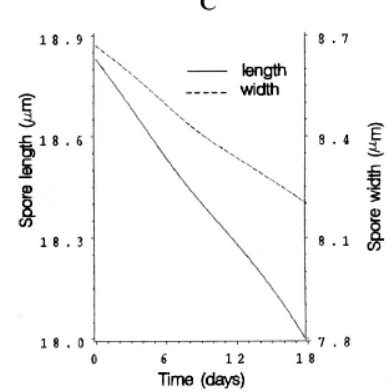

Fig. 2. Temporal changes in spore dimensions in a microscopic specimen of B. terrestris. Spore print mounted in polyvinyl lacto-phenol (A), fruitbody mounted in polyvinyl lacto-phenol (B) and spore print mounted in cotton blue solution $(\mathrm{C})$.

\section{References}

Baral H.O. 1992. Vital versus herbarium taxonomy: Morphological differences between living and dead cells of Ascomycetes, and their taxonomical implications. Mycotaxon 44(2): 333-390.

Fogel R. 1992. Utility of spore length/width ratio in separating Geopora cooperi form Cooperi and G. cooperi f. gilkeyae. Mycologia 84(1): 124-127.

Huhtinen S. 1989. A monograph of Hyaloscypha and allied genera. Karstenia 29(2): 45-252.

Rahi M. and Kullman B. 1993. Pitfalls in ascospore measurements. In Fungi and Lichenes in the Baltic Region, Abstracts: 123, 12th International Conference on Mycology and Lichenology. Vilnius. 
Möls T. and Raitviir A. 1974. Morphometrics and the taxonomiy of fungi. Tallinn (in Russian), pp. 159.

Schumacher T. 1990. The genus Scutellinia (Pyronemataceae). Opera Bot. 101: 1-107.

Stojanovic S., Ristanovic M., Jencic R. 1986. Influence of the age of cleistothecia Erysiphe graminis DC. ex Merat f. sp. tritici em. Marshal on the morphologic properties and vitality of ascospores. 5th. Jugoslovenski Simpozijum O. Zastiti Bilja. Struga (Yugoslavia). 2225 Oct. 1985. Zastita bilja 37(176): 169-174.

Wu C-G. 1993. Clomales of Taiwan: iv. A monograph of Sclerocystis (Glomaceae). Mycotaxon 49: 327349.

\title{
MICROPLATE TECHNIQUE OF PHYSIOLOGICAL TESTS FOR YEAST IDENTIFICATION
}

\author{
Piere EVRARD AND GRÉGOIRE L. HENNEBERT
}

Mycothèque de l'Université Catholique de Louvain, Unité de Microbiologie, Faculté des Sciences Agronomiques, Place Croix du Sud 3, B-1348 Louvain-la-Neuve, Belgium

Abstract. Yeasts are important in various industrial processes as producers or as contaminants. To identify yeasts quickly and reliably is crucial. Therefore exploring fast, simple and inexpensive identification methods is necessary.
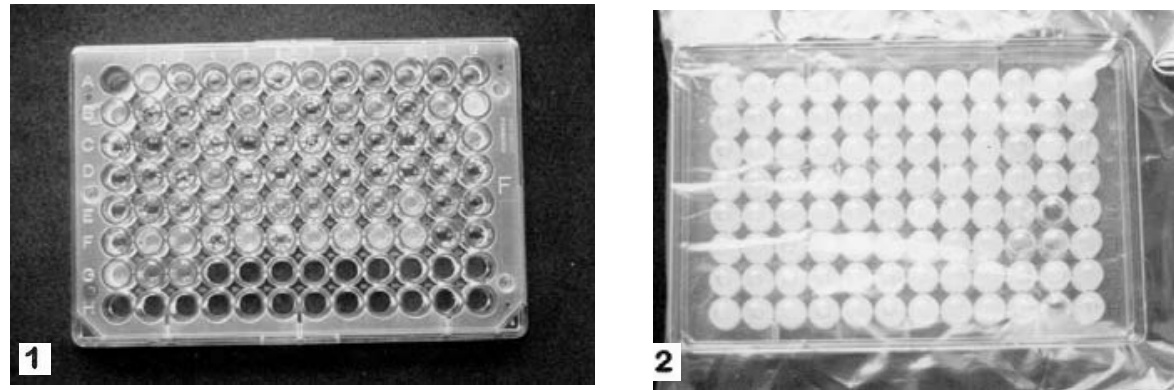

Fig. 1. Microplate 85 assimilative tests of one yeast strain. 2. Microplate grouping 12 fermentative tests of 8 strains in anaebiosis.
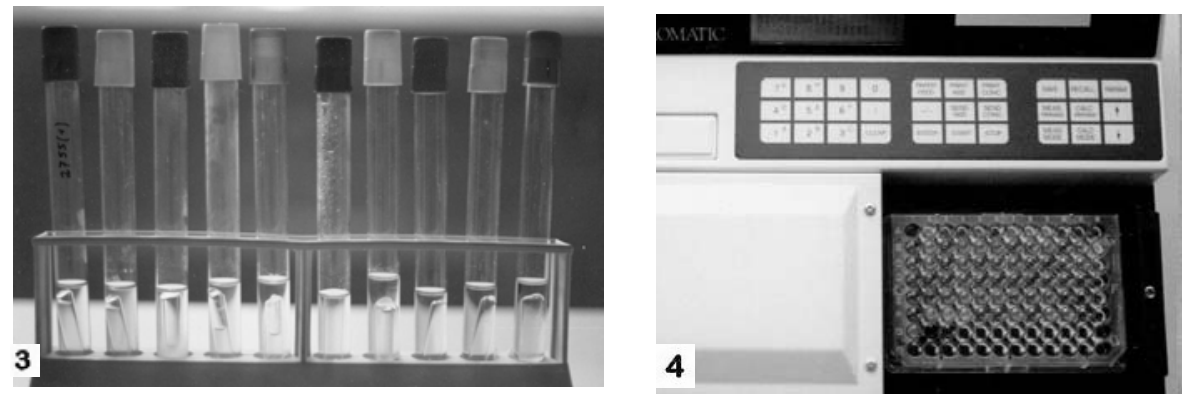

Fig. 3.Traditional optical reading of fermentative tests in tubes of one strain. 4. Automatic non-subjective reading of microplates of assimilative and fermentative tests.

Traditionally, yeasts are identified by morphological characters and physiological properties such as assimilation and fermentation tests according to the 
procedure described by Van der Walt \& Yarrow (1984) emended by Barnett et al. (1990).

Physiological tests using tubes are time consuming and expensive. We propose a new, fast and inexpensive technique for physiological tests using microplates. The oxydative microplate includes 48 carbone sources and 9 nitrogen sources for assimilation, 10 vitamines for minimum requirement, 3 cycloheximide and sodium chloride concentrations for tolerance, and the urease, the arbutin splitting and the starch formation tests. The fermentative microplate includes 12 carbon sources. The microplates are read automatically on a microplate reader. The absorbency measurements are transferred to a computer in a data matrix. A software has been created and programmed to interpret the absorbency measurement data in terms of positive, negative and weak results.

Together with morphological data, physiological data are then treated by the yeast identification expert system ALLEV for identification (Robert et al. 1994).

\section{References}

Van der Walt \& Yarrow 1984. In The Yeasts, a taxonomic study. NJW. Kreger-van Rij ed.Elsevier Amsterdam.

BarnettJ.A. 1990. The Yeasts, characteristics and identification. 3d ed. Cambridge Univ. Pr.

Robert V., De Bien J.E., Buyck B. and Hennebert G.L. 1994. "ALLEV", a new program for computerassisted identification of yeasts. Taxon, $43: 433-439$.

\section{CRYOPRESERVATION OF ASPERGILLUS REPENS}

\section{DAVID SMITH}

International Mycological Institute, Bakeham Lane, Egham, Surrey TW20 9TY, United Kingdom

Abstract. Cryogenic light microscopy has been used to develop optimum cooling protocols for fungi. The response to freezing and thawing of a fungus is demonstrated well by Aspergillus repens on cooling at $-30^{\circ} \mathrm{C}$ min -1 to $-30^{\circ} \mathrm{C}$. Ice crystalization, solute crystalization and gas bubble formation cause injury to the fungus cell. Complete evacuation of cytoplasm from the hyphae has been seen. 


\title{
CULTIVATION OF THE FUNGI
}

\section{ACTIVATED CHARCOAL-MEDIATED SPOROPHORE INITIATION IN AGARICUS AND TRICHOLOMA}

\author{
ABED PEERALLY \\ Faculty of Science, University of Mauritius, Reduit, Mauritius
}

\begin{abstract}
Agaricus bisporus is known not to produce sporophore primordia in aseptic conditions unless activated charcoal is present. Using different techniques Agaricus bisporus, A. bitorquis and Tricholoma spectabilis were grown aseptically on distilled water agar, polyethylene foam, malt extract agar and soil with and without activated charcoal. Only A. bitorquis produced ill-defined primordia in the form of mycelial aggregates on water agar and malt extract agar in the absence of activated charcoal. In the presence of activated charcoal the three species produced primordia but only $A$. bitorquis and $T$. spectabilis produced normal pins and sporophores. The best fruiting was obtained with A. bitorquis on water agar treated with activated charcoal and with $T$. spectabilis on activated charcoal treated soil. It is concluded that all three species produce similar fruiting inhibitory factors capable of being neutralised by soil bacteria and activated charcoal.
\end{abstract}

\section{Introduction}

The usefulness of activated charcoal in stimulating the fruiting process of Agaricus bisporus (Lange) Sing. was initially reported by Mader (1943). This observation led Mader to conclude that volatiles were involved in the formation of carpophores. The stimulating effects of soil bacteria in the natural initiation of fruiting in A. bisporus was first observed by Eger $(1961,1972)$ and subsequently reliable additional observation on the bacterial induction of fruiting was made by Hayes et al. (1969), Hayes (1981) and Peerally (1978, 1981).

Eger $(1961,1972)$ also reported the ability of activated charcoal to replace the effects of bacteria. Similar results were also reported by various other workers (Long and Jacobs 1974, Convy 1976, Wood 1976, Peerally 1978, Stoller 1978).

That activated charcoal could also stilmulate fruiting in Agaricus bitorquis (Sacc.) Quel. was shown by Peerally (1978). However Stoller (1978) observed that on malt extract agar, activated charcoal and lignite separately had no effects on the initiation of fruiting in $A$. bitorquis but when mixed, fruiting was profusely stimulated.

It is generally believed that activated charcoal stimulates fruiting by removal of one or more inhibitors present in the mycelium. To date it is still not understood what precise role bacteria and activated charcoal play in carpophore initiation Ingratta and Patrick (1986) suggested that the major stimulus in carpophore initiation in A. bisporus might be related to the intensified microbial activity in the casing layer which results in a reduced nutritional base in the casing and an increase of fungistasis.

The present paper reports the fruiting response in A. bisporus (strain 133), A. bitorquis (strain 1) and in Tricholoma spectabilis Peerally \& Sutra (local strain 1) under the influence of activated charcoal, in four different techniques.

\section{Methods}

Cultures were first obtaine on standard potato dewtrose agar $(\mathrm{pH} 7.5)$ and then used to onoculate autoclaved wheat grain in conical flasks. Grain spawn of $A$ bisporus was incubated at $23^{\circ} \mathrm{C}$ while that of $A$. bitorquis and $T$. spectabilis was kept at $28^{\circ} \mathrm{C}$. The three spawns were fully invaded and ready after about three weeks. 
Plates of malt 2\% agar (MEA) and double distilled water agar (DWA), both adjusted to $\mathrm{pH} 7.5$ with sodium hydroxide and calcium carbonate, were prepared and after setting, the agar surface was thoroughly covered with a thin layer of activated charcoal powder (about $0.7 \mathrm{~g}$ per plate). Plates were inoculated with single grain of spawn or with a small quantity of spawn.

Loam soil, $\mathrm{pH}$ adjusted to about $\mathrm{pH} 7.3$ with calcium hydroxide, and polyethylene foam $(8 \mathrm{~cm}$ diam, $4 \mathrm{~mm}$ thick) were placed in separate Petri dishes. Sterilisation of soil was achieved on three consecutive days for one hour. Plates with foam were autoclaved one for one hour. After sterilisation, activated charcoal powder was applied to the soil $(0.3 \mathrm{~g}$ or $1.0 \mathrm{~g}$ per plate) and to the foam $(1.0 \mathrm{~g}$ per plate). Plates were then inoculated on one side with a small quantity of grain spawn, and sterile distilled water added.
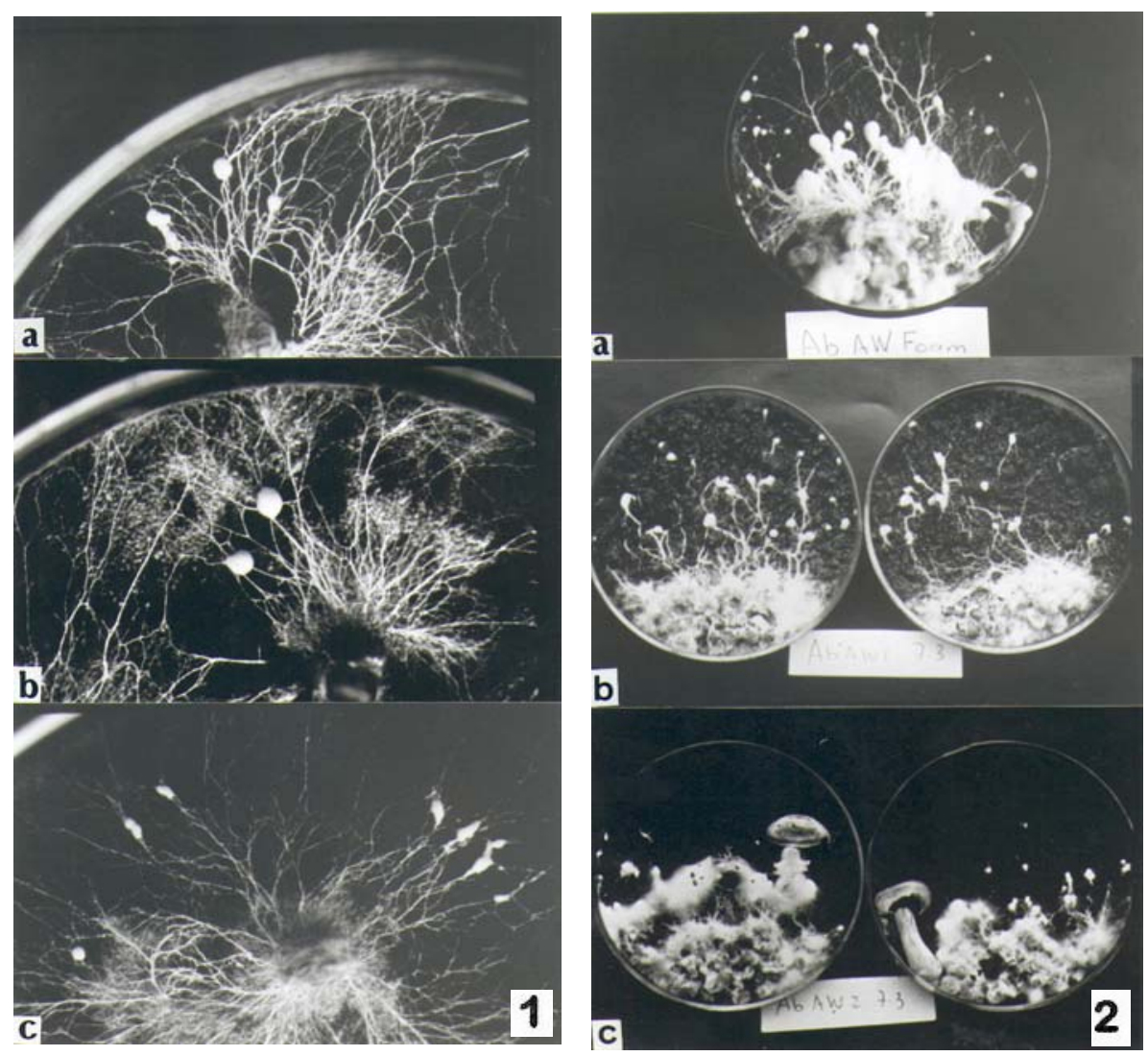

Fig. 1. Agaricus bisporus a-c Primordia production.

Fig. 2. Agaricus bitorquis a. On loam + activated charcoal. b. On soil $+0.3 \mathrm{~g}$ activated charcoal. c. On soil $+1 \mathrm{~g}$ activated charcoal.

They were at least ten replicates per treatment including the untreated control sets of plates. Experiments were repeated at least once.

Plates with $A$. bisporus were incubated for six days at $23^{\circ} \mathrm{C}$ and then transferred to an aerated room at $18-20^{\circ} \mathrm{C}$. Plates with $A$ bitorquis and T. spectabilis were incubated at $28^{\circ} \mathrm{C}$ for six days and then transferred to $21-26^{\circ} \mathrm{C}$. 


\section{Results}

Activated charcoal treated plates and control plates were observed daily. The state of mycelial growth and of fruiting initiation and development was studied over a period of two to four weeks. No attempt was made to count the number of primordia which for some treatments was indeed not feasible.

Colony growth in all untreated DWA plates was fluffy or slightly strandy. Primordia were not produced in A. bisporus and T. specatabilis. Some of the DWA plates with $A$. bitorquis produced carpophore initials but in a rather disorganized pattern, in contrast to organized, synchronous and regular development at the periphery of the colony obtained with activated charcoal. Those initials consisted of fuzzy hyphal aggregates (Wood 1978). Smooth-surfaced primordia and pins as produced in the presence of activated charcoal were never seen on untreated DWA. Initials were formed from day 7 or 8 after inoculation.

In DWA plates treated with activated charcoal colony growth was conspicuously adpressed with little aerial mycelium, in $A$. bisporus and $A$. bitorquis. Strandy growth sometimes occurred in A. bisporus and A. bitorquis conspicuously (Fig. 2a). In $T$. spectabilis strands were never observed, but instead isolated areas of fluffy mycelium were frequently produced (Fig. 1) from which well developed smooth primordia, pins and carpophores developed. Fully mature carpophores with stipe, pileus and gills were best formed by $A$. bitorquis and were profuse throughout the periphery of the colonies $A$. bisporus produced a few primordia (Fig. 3a-c) which did not develop further.

On MEA, A. bisporus and T. spectabilis did not produce any primordia whether activated charcoal was supplied or not. A. bitorquis produced a few mycelial aggregates but nothing further in presence of activated charcoal.

On foam without activated charcoal, mycelial growth of the three strains was very fluffy. In the presence of activated charcoal A. bitorquis produced very strandy mycelium, primordia, pins and mature carpophores (Fig. 2a). A. bisporus formed strandy mycelium but no primordia, while $T$. spectabilis grew to a limited extend and formed some primordias and pins.

In autoclaved soil treated with activated charcoal $A$. bitorquis (Fig. 2b, c) and $T$. spectabilis produced fluffy mycelial growth, primordia, pins and mature carpophores.

\section{Discussion}

Only A. bitorquis was capable of producing initials in the form of fuzzy myceliual aggregates on untreated DWA. In the presence of activated charcoal all three species produced pimordia, but only A. bitorquis and $T$ spectabilis formed well developed smooth primordia, pins and carpophores, similar to those produced in normal casing and in nature, respectively. Only T. spectabilis showed the peculiarity of forming fluffy aerial mycelium capable of readily producing well developed pins and carpophores. 

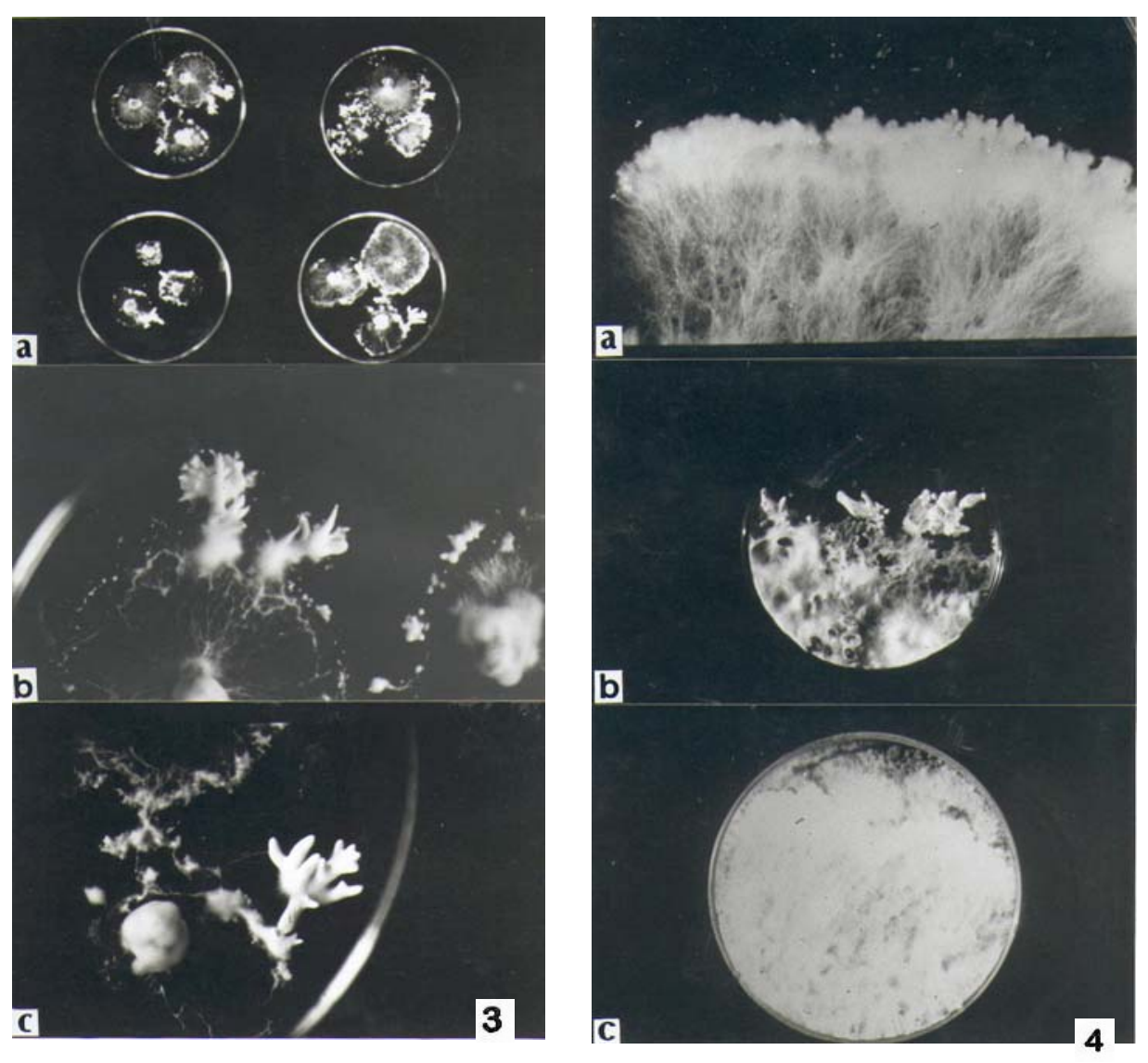

Fig. 3. Tricholoma spectabilis. a-c. fruiting on activated charcoal.

Fig. 4. Tricholoma spectabilis. a, b. mycelium and primordial on non aseptic soil. c. sterile mycelium on aseptic soil.

The fact that activated charcoal did not stimulated primordial on MEA supports the results obtained by Stolla (1978)

San Antonio and Peerally (1978) working with several strains of A. bitorquis concluded that there was no correlation between the formation of initials on agar media and productivity under normal growing conditions. This might possibly be attributed to the disactivation of activated charcoal by chemicals present in the malt agar. It is, thus, conceivable that such a correlation might exist between formation of primordias in the presence of activated charcoal and productivity of the strain.

The fact that all three species were capable of producing at least primordias in the presence of activated charcoal is interesting. Bacteria are known to be necessary for the formation of carpophores in A. bisporus, as well as for A. bitorquis and $T$. spectabilis (Peerally unpublished) (Fig. 4a-c).

It must therefore be concluded that the same inhibitory factors are present in the mycelium of these basidiomycetes and once neutralised or removed, fruiting takes 
place. The induction of fruiting in widely different terricolous basidiomycetes by bacteria and activated charcoal could well have the same mechanism but the nature of this induction still remains to be elucidatedc.

\title{
Acknowlegement
}

My thanks are due to Mr V. Aumeer for technical assistance.

\section{References}

Couvy J. 1976. La fructification d'Agaricus bisporus en milieu aseptique: un modèle experimental pour l'étude des substances impliquées dans l'initiation fructifère. Mushroom Science 9(1): 157-164.

Eger G. 1961. Untersuchungen über die Funktion der Deckschicht bei der Fruchtkörperbildung des Kultuurchampignons, Psalliota bispora Lge. Arch. Mikrobiol. 39: 313-334.

Eger G. 1972. Experiments and comments on the action of bacteria on sporophore initiation in Agaricus bisporus. Mushroom Science 8: 719-725.

Hayes W.A., Randle P.E. and Last F.T. 1969. The nature of the microbiological stimulus affecting sporophore formation in Agaricus bisporus (Lange) Sing. Annals of Applied Biology 64: 177-187.

Hayes W.A. 1981. Iterrelated studies of physical, chelmical and biological factors in casing soild and relationships with productivity in commercial culture of A. bisporus (Lange) Pilat. Mushroom Science 11 103-129.

Ingratta F.J. and Patrick Z.A. 1986. Influence of microorganisms and fungistasis in sporophore initiation in Agaricus brunnescens. Proceedings International Symposium on the Scientific and Technical Aspects of Cultivating Edible Fungi. The Penn. State Univ. Univ. Park, USA, pp. 27-40.

Long P.E. and Jacobs L. 1974. Aseptic fruiting of the cultivated mushroom, Agaricus bisporus. Trans. Brit. mycol. Soc. 63: 99-107.

Mader E.O. 1943. Some factors inhibiting the fructification and production of the cultivated mushroom. Phytoapthology 33: 1134-1145.

O'Donoghue Maguire D.C. and Ryan J.P. 1991. Influences of a wide range of bacteria, actinomycetes and fungi on mycelial growth of Agaricus bisporus (Lange) Sing. and the special fruiting requirement of A. bisporus. In Science and Cultivation of Edible Mushroom, Maher M. ed. Proc. $13^{\text {th }}$. Int. Congress, Dukin, pp. 753-759.

Peerally A. 1978. Sporophore initiation in Agaricus bisporus and Agaricus bitorquis in relation to bacteria and activated charcoal. Mushroom Science 10(1): 611-639.

Peerally A. 1981. A Petri plate agar technique for obtaining primordial in Agaricus bisporus (Lange) Sing. Mushroom Science 11:153-158.

San Antonio J.P. and Peerally A. 1978. Growth, primordial formation and fruiting of twenty-two different strains of Agaricus bitorquis. Mushroom Science 10(1): 587-594.

Stoller B.B. 1978. Synthetic casing for mushroom beds. Mushroom Science 10(2): 187-215.

Wood D.A. 1978. Studies on primordium initiation in Agaricus bisporus and A. bitorquis. Mushroom Science 10(1): 565-585.

\section{IN VITRO CULTURE OF ENDOMYCORRHIZAL FUNGI: WHY THAT INTEREST?}

\author{
S. DeClerck ${ }^{1}$, T. Diop $^{2}$, C. Plenchette ${ }^{1}$ And D.G. Strullu ${ }^{2}$.
}

${ }^{1}$ Station d'agronomie, INRA, B.V. 1540, 17 rue Sully, 21034 Dijon Cedex, France. ${ }^{2}$ Université d'Angers, 2 Bd. Lavoisier, 49045 Angers Cedex, France.

\section{Introduction}

Vesicular arbuscular mycorrhizal (VAM) fungi are found naturally in almost all tropical soils and under all terrestrial ecosystems (Sieverding 1991). Much of the interest in VAM fungi relates to their role in plant growth and phytoprotection. 
Taxonomy of these fungi has been largely based on spore characteristics (Morton, 1988). Nevertheless, accurate identification of VAM fungi in field soils is not easy as the spores may be present in different stages of their development. Mixtures of spores of different species could also lead to identification problems (Abbott and Robson 1991). Taxonomy using biochemical and genetical characterizations has also been hampered by the lack of powerful techniques of specific and sensitive detection and obtention of DNA fragments in axenic conditions. Recently, the upsurge of the polymerase chain reaction (PCR) technology has given a new impetus in biomolecular research and has widespread applications in VAM fields as diverse as phylogeny, evolutionary history and taxonomy (Simon 1993).

Mosse and Hepper (1975) were the first to propose an in vitro system based on a dual culture of spores and excised roots. Mugnier and Mosse (1987) and Becard and Fortin (1988) improved the system by using roots genetically transformed by Agrobacterium rhizogenes. Diop et al. (1992) were able to follow the entire vegetative cycle of Gigaspora margarita using Ri-TDNA transformed carrot roots. Another method of investigation was proposed by Strullu and Romand (1986, 1987 and 1991) who developed a culture technique based on the intraradical phase of the fungus i.e. the vesicles.

The continuous culture of VAM fungi means that the mycorrhizal fungi must be indefinitively subcultured in order to maintain and increase its biomass. Becard and Fortin (1988) achieved continuous cultures of G. margarita using the spores produced in vitro by this VAM fungus. Strullu and Romand (1986) proposed a method using mycorrhizal root as starting material and based on the successive reassociation of mycorrhizal roots with non mycorrhizal root systems. This system of in vitro subcultures has been applied successfully by these authors with Glomus species.

The indefinite in vitro maintenance of VAM fungal species would make it possible to maintain biodiversity through the creation of fungal libraries. Taxonomy based on morphology and molecular biology (PCR) should also lead to the characterization of these species which should become "reference species".

We propose an application of in vitro culture using the intraradical phase of the VAM fungi, applied to banana in order to obtain appropriate strain for sustainable agriculture.

Methodology
Sampling, trapping and isolation procedures
Infective VAM fungal propagules are collected in the field by soil and root
sampling (1)*. Soil samples are taken in the upper layer of the soil where most root
growth occurs. The indigenous VAM population present in soil and roots were then
multiplied and maintained on trap plants (Allium porum). The procedure of trap pot
culture is simple (2). Pots are filled with calcined clay (Terra Green) on top of wich
the field soil or root sample is placed. Another layer of Terra Green is placed above
the soil or root sample. A high mycotrophic plant with good growth and rooting e.g.
leek, is then planted in the pot. After several months, soil and root samples are taken
and screened for mycorrhizal infection. Soil and root material is then suspended in
water and after $10-15 \mathrm{~s}$ (for sedimentation of coarse sand) the suspension is
decanted over a series of soil sieves ( 2 mm - 500 - $250-125-50$ microns). Roots in
the top sieve are washed under tap water and transferred to a petri dish. Infected
roots are further separated from the noninfected roots under the stereomicroscope
(3). Infected roots are conserved at $4{ }^{\circ} \mathrm{C}$. Spores (4) are isolated from the sieves and


used as starting material for monosporal culture on leek plants (4'). Spores are also used for taxonomic description of the field isolates (4"). Collections of the VAM isolates are maintained by using the sieved soil to establish pot culture (5).

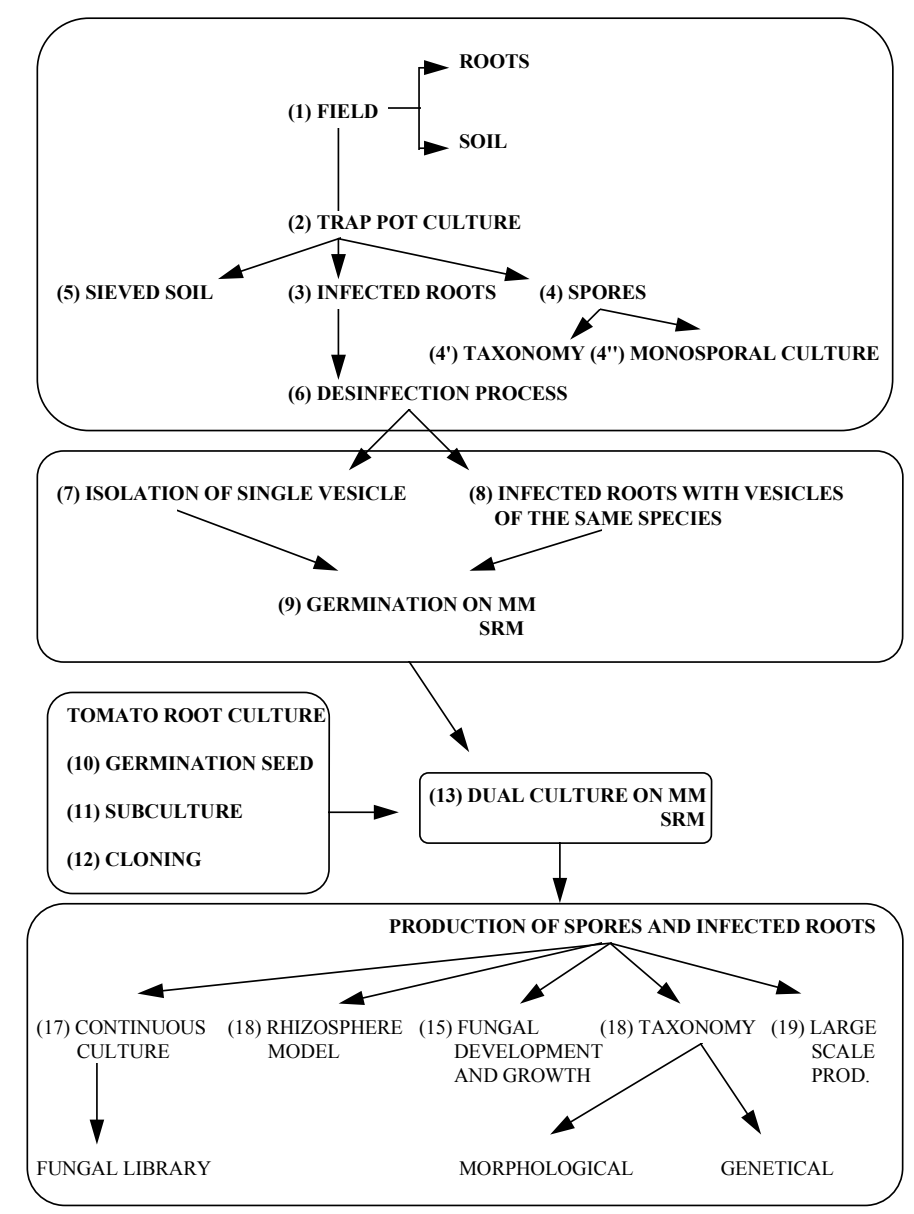

\section{In vitro culture}

Fungal inocula

Infected roots containing vesicles are desinfected using the procedure developed by Strullu and Romand (1986) (6). The roots are surface sterilized succesivelly with $95^{\circ}$ alcohol for $10 \mathrm{~s}, 6 \% \mathrm{Ca}$ hypochlorite for $2 \mathrm{~min}, 2 \%$ chloramine $\mathrm{T}$ for $10 \mathrm{~min}$ and $200 \mathrm{mg} / \mathrm{l}$ streptomycin $-100 \mathrm{mg} / \mathrm{l}$ gentamycin for $10 \mathrm{~min}$. Roots are rinsed with sterilized water after each sterilization step. Sterilized roots are conserved in Streptomycin $(200 \mathrm{mg} / \mathrm{l})$ - gentamycin $(100 \mathrm{mg} / \mathrm{l})$ at $4{ }^{\circ} \mathrm{C}$ until ready for use. Vesicles (7) are extracted from roots and germinated on Minimal (Becard and Fortin, 1988) or on Strullu -Romand (1986) medium (9). Roots containing vesicles of the same species $(8)$ are cut in pieces of $0.5 \mathrm{~cm}$ and also placed on the same mediums as described above (9).

Root organ culture 
Initiation of root culture requires seed germination under axenic conditions. Emerging radicles are then excised and cultured on White's medium (White 1943). Tomato seeds are surface sterilized by soaking in $15 \%$ hydrogene peroxide for 2 - 3 minutes. Seeds are then rinsed in sterile water and germinated (10) in the dark in sterile Petri dishes on water - agar. When radicles are $2 \mathrm{~cm}$ long, they are excised and transferred onto White's medium (White 1943) (11). Roots with the most laterals are then chosen to initiate clonal culture (12).

Dual culture

Dual culture of root and fungus is established on Minimal medium (Becard and Fortin 1988) or on Strullu - Romand medium (1986) (13). Pre-germinated single vesicles or mycorrhizal root pieces, containing vesicles of the same species, showing hyphal regrowth are used to inoculate the root system by placing them close to emerging lateral roots. Extra and intra-radical development of the fungus is eventually obtained depending on the fungus, the host and the environmental conditions.

\section{Conclusion}

The root organ culture technique for VAM fungi has numerous advantages over traditional inoculum production systems. It allows large scale production of inoculum free of pathogens (14). It permits reproducible observations of all stages of VAM fungi development (15) and can be used as a simplified rhizosphere model (16) for ecological studies i.e. effect of heavy metal toxicity, competition with other microorganisms, interactions with plant root pathogens, pesticides... The system is also useful for determining symbiotic factors provided by the root which govern fungal growth (CO_, root exudates...) (16). The continuous culture of VAM fungi would make it possible to maintain biodiversity through the creation of fungal libraries (17). Taxonomy based on morphology and molecular biology (PCR) should also lead to the characterization of these species which should become "reference species" (18).

Development of pure culture of endomycorrhizal fungi continues to represent one of the most challenging goals in fungal biology. It is accepted that understanding the mechanisms involved in the symbiotic process will lead to the development of pure culture in the near future.

\section{Acknowlegement}

This work was supported by a grant of CEE - STD3 N 92104

\section{References}

Abbott L.K. and Robson A.D.. 1991. Factors influencing ther occurrence of vesicular-arbuscular mycorrhizas. Agriculture, Ecosystems and Environment 35: 121-150.

Becard G. and Forti J.A.. 1988. Early events of vesicular-arbuscular mycorrhiza formation on Ri T-DNA transformed roots. New Phytol. 108: 211-218.

Diop T.A., Becard G. and Piche Y.. 1992. Long - term in vitro culture of an Endomycorrhizal fungus, Gigaspora margarita, on Ri T-DNA transformed roots of carrot. Symbiosis 12: 249-259.

Morton J.B.. 1988. Taxonomy of VA mycorrhizal fungi : classification, nomenclature, and identification. Mycotaxon 32: 267-324.

Mosse B. and Hepper C.M.. 1975. Vesicular-arbuscular mycorrhizal infections in root organ cultures. Physiol. Plant Pathol. 5: 215-223.

Mugnier J. and Mosse B. 1987. Vesicular-arbuscular mycorrhizal infections in transformed Ri T-DNA roots grown axenically. Phytopathology 77: 1045-1050. 
Sieverding E. 1991. Vesicular-Arbuscular Mycorrhiza Management in tropical Agrosystems. Deutsche Gesellschaft für Technische Zusammenarbeit (GTZ) Gmbh - 372 p., ISBN 3-88085-462-9.

Simon L., Bousquet J., Levesque C. and Lalonde (M. 1993. Origin and diversification of endomycorrhizal fungi and coincidence with vascular land plants. Nature 363: 67 - 69 .

Strullu D.G. and Romand C. 1986. Méthode d'obtention d'endomycorrhizes à vésicules et arbuscules en conditions axéniques. Compt. Rend. Hebd. Séance Acad. Sci. 303: 245-250.

Strullu D.G. and Romand C. 1987. Culture axénique de vésicules isolées à partir d'endomycorrhizes et réassociation in vitro à des racines de tomate. Compt. Rend. Hebd. Séance Acad. Sci. 305: 15-19.

Strullu D.G., Romand C and Plenchette C. 1991. Axenic culture and encapsulation of the intraradical forms of Glomus spp. World journal of Microbiology and Biotechnology 7: 292-297.

White P.R. 1943. A handbook of plant tissue culture. Cattel J., Lancaster, PA.

\title{
ENHANCEMENT OF CARPOPHORE PRODUCTION AND PROTEIN CONTENT OF PLEUROTUS SPP. BY CULTIVATION ON GRASS HAY.
}

\author{
KANDANDA TSHINYANGU \\ Mycothèque de l'Université Catholique de Louvain, Place Croix du Sud 3 Bte 6, B-1348 Louvain-la- \\ Neuve, Belgium
}

Abstract. Seven strains of Pleurotus mushrooms (Pleurotus ostreatus var. columbinus MUCL 28785, Pleurotus ostreatus var. columbinus MUCL 30495, P. cornucopiae MUCL 31019, P. ostreatus var. ostreatus cv. Florida MUCL 31018, P. pulmonarius MUCL 28784 and P. sajor-caju MUCL 31017) were cultivated on grass hay and wheat straw without additives. The grass hay increases both production of carpophore and protein content of all strains.

Stimulating factors of grass hay was identified by supplementing wheat straw with selected and combined chemical compounds. That study was conducted with Pleurotus ostreatus var. columbinus MUCL 28785 cultivated on all supplemented susbstrates. Several compounds (alanine, asparagine, biotin, gibberellic acid, glutamic acid, glycin, indole-acetic acid, indole-butyric acid, kinetin, manganese and serine) have been able to enhance carpophore production. The main stimulating factor of grass hay was the interaction between amino acids and manganese.

\section{IS THE RUST OF THE CREEPING THISTLE (CIRSIUM ARVENSE) THE MICROSYMBIONT OF ITS VAM ?}

\author{
HARTMUT HEILMANN \\ Birkenstrasse 10, 74592 Kirchberg/Jagst, Germany
}

\begin{abstract}
This paper investigates the causing factor of growth of the creeping thistle (Cirsium arvense) as representative of a group of plants which really bears the qualities of weeds as it can crop up anywhere and cannot be controlled by normal soil cultivation methods. On the other hand they often do not endanger the yield of the crop. Rumex crispus, Agropyron repens, Convolvulus arvense and Equisetum arvense belong to this group of weeds. After use of pesticides and fungicides the problem subsists. The failing control of the creeping thistle shows a very unsatisfactory theory of its growth. Growing factor and sprouting are obviously the same. The thistle has a VAM. Rhythm of growth and physiologic conditions of
\end{abstract}


the soil are similar to those of orchids. Sprouting from the seed does not show cotyledons but a whole plant.

The invincibility of these weeds obviously rests on an assimilatoric contribution by dissimilation in the soil.

Some Orchidaceae, Convolvulaceae and Scrophulariaceae posses VAM. In the wild a regular vegetal association of the creeping thistle can be found with orchids such as Epipactis helleborina and Platanthera bifolia, and Convolvulaceae such as Convolvulus arvense. The question arises whether exists a similar symbiotic contribution to thistle growth, also in agricultural systems.

The ecologic niche of orchids can be destroyed easily by stress on the soil microbiology e.g. by action of fertilizers or other chemicals, fungicides or pesticides. The creeping thistle is also sensitive but temporally.

The best method of creeping thistle regulation are legumes in crop rotation. Red clover (Trifolium pratense) or alfalfa (Medicago sativa) obviously have an antagonitic effect on the thistles. The action maybe due to stem rot by Sclerotinia trifoliorum, but the attacked patches in the field are very soon subjected again to growth of thistles, Agrpyron repens and/or Rumex crispus.

The root of Cirsium arvense can have a phase of dormancy for many years, this means that roots survive in soil but do not emerge. Also thousands of seeds are waiting in the soil. Do both are waiting for the action of a microsymbiont, like do species on Orchidaceae? Indeed the author interprets the fact as the possible existence of a comparable mycothrophy by VAM.

Creeping thistle is associated with a number of

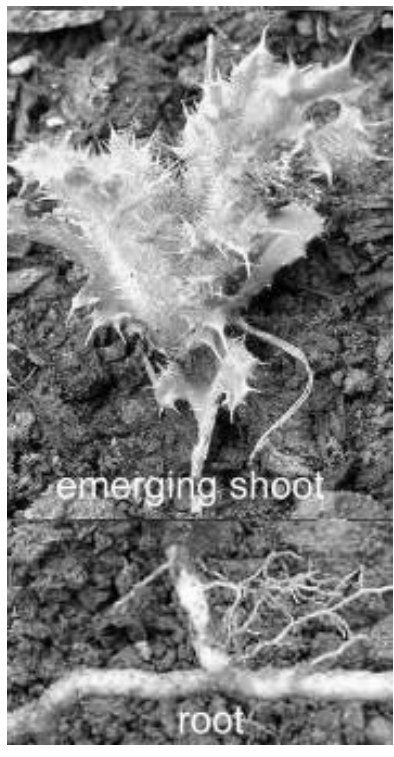
fungi, foliar pathogens such as rusts Puccinia suaveolens or Albugo trapognonis, Coelomycetes Septoria circii or Phyllosticta cirsii, stem fungi like Leptosphaeria or Ophiobolus spp. or root attack by nematodes. But none of these organisms is known to enhance growth of thistles. Then an unidentified VAM or endophytic fungus might be responsible of the weed survival and might provide the key of its control.

\title{
MORPHOLOGICAL STUDIES OF MYCELIA FROM ECTOMYCORHIZIAN FUNGI CULTIVATED IN VITRO
}

\author{
J. JEANFILS, J.-J. CUVELIER \\ Université Mons-Hainaut, Faculté de Médecine, Avenue du Champ de Mars 8, 7000 Mons
}

Abstract. Different ectomycorhizian fungi belonging at different genus were put in culture from carpophore fragments. Morphological studies of mycelium growing on different culture media were described. Chemical reactions obtained on in vitro mycelium are compared with results obtained on carpophore. 
FUNGAL BIOTECHNOLOGY

\title{
YEAST FUNGI AND ALCOHOL FROM BAMBOOS
}

\author{
FRANCIS S.S. MAGINGO \\ Applied Microbiology Unit, Department of Botany, University of Dar es Salaam, P.O. Box 35060 \\ Dar es Salaam, Tanzania.
}

\section{Introduction}

The bamboo Oxytenanthera braunii is used by the local people in southwestern Tanzania, to obtain an alcoholic beverage locally known as "ulanzi" (IBRN 1993). This is attained through fermenting sugary exudates, from young, cut and bruised shoots of the bamboo, which come out during the rainy season (Kigomo 1990). The sugary exudates are made to flow into a collecting container known as "mbeta" (Fig. $1 \& 2$ ). Exudates from many "mbeta" are then pooled together in 20 -liter plastic containers, brought into a cool corner of a house and left to ferment.

The fermentation of the bamboo exudates is a wild and natural one, as no special fermenting microorganisms are purposely inoculated into the bamboo sugary exudates. The beverage, reaches its maximum alcohol amount within 24 hours. Obtainage of an alcoholic beverage from bamboos, is practised only in the southwestern areas of Tanzania, and no where else in the world.

So far, microorganisms involved in the fermentation of the bamboo sugary exudates, have not yet been documented. This work was aimed at isolating microorganisms involved in fermenting sugary exudates of the bamboo to alcohol; and also to quantify amounts of alcohol produced.

\section{Materials and methods}

Slants of sterile medium in MacCartney bottles were brought to a bamboo growing area in southwestern Tanzania, for initiating isolation of the fermenting microorganisms. An initiation of isolation of microorganisms from plant exudates is best done on the surface of agar-containing medium (Carmo-Sousa 1969). The agar medium in the McCartney bottles was a Wickerham maintenance agar medium (Phaff et al. 1978) containing yeast extract $(0.3 \%)$, glucose $(1.0 \%)$, malt extract $(0.3 \%)$, peptone $(0.5 \%)$ and agar $(2.0 \%)$.

While working in a kitchen at the bamboo growing area, a wire loop was sterilized by heating it to red hot on a spirit lamp, and cooled in absolute alcohol. On cooling to ambient temperature, the wire loop was used to take samples from sterile membrane filters which had just been used to filter-sterilize fresh bamboo exudates for other studies. The contents on the wire loop were striken on the slants in the McCartney bottles. The bottles were then properly closed and brought to the laboratory for purification and identification of the prevalent fermenting microorganisms (Lodder 1970).

Several aliquots of fresh bamboo exudates $900 \mathrm{ml}$ in volume, were hygienically also collected in pre-sterilized 1 1iter bottles with butyl rubber stoppers and metallic caps. Two sterile hypodermic needles were injected on each bottle through the rubber stoppers, to release possible $\mathrm{CO} 2$ that could be produced during transportation. The bottles were immediately placed in well protected ice-boxes and transported to reach the laboratory in Dar es Salaam, the same day. In the laboratory, the exudates were fermented naturally, at room temperature $\left(28 \pm 1^{\circ} \mathrm{C}\right)$. 

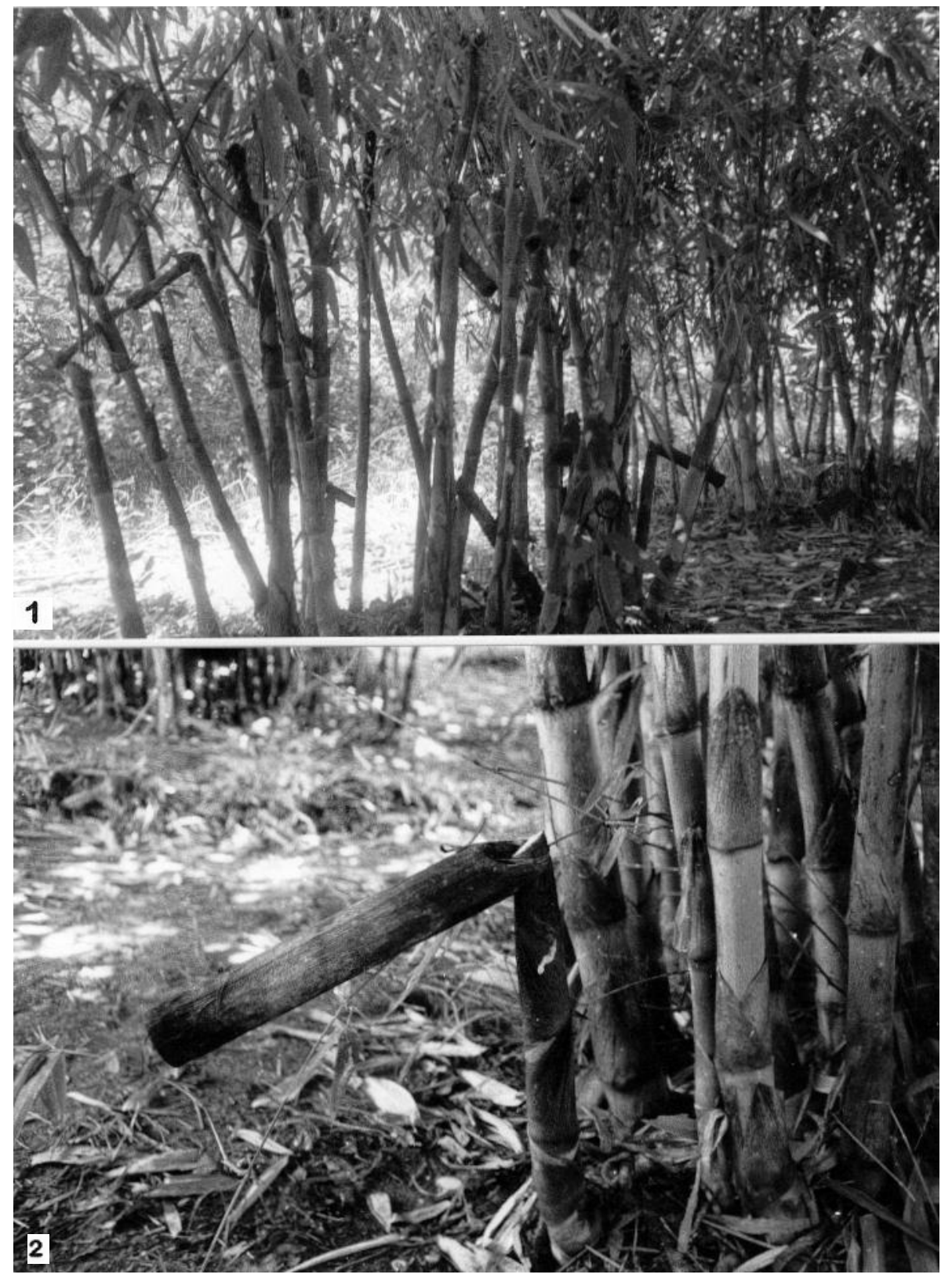

Fig.1. A stand of bamboos, having many "mbeta" hung on various young, cut bamboo shoots

Fig.2. Close up of a yound, cut bamboo shoot with a "mbeta" hung on it for collection of exudates

Samples were taken from the fermenting set-ups on daily basis for determination of types and amounts of alcohols in them on a Gas-Chromatograph (Magingo and Gijzen 1989). 

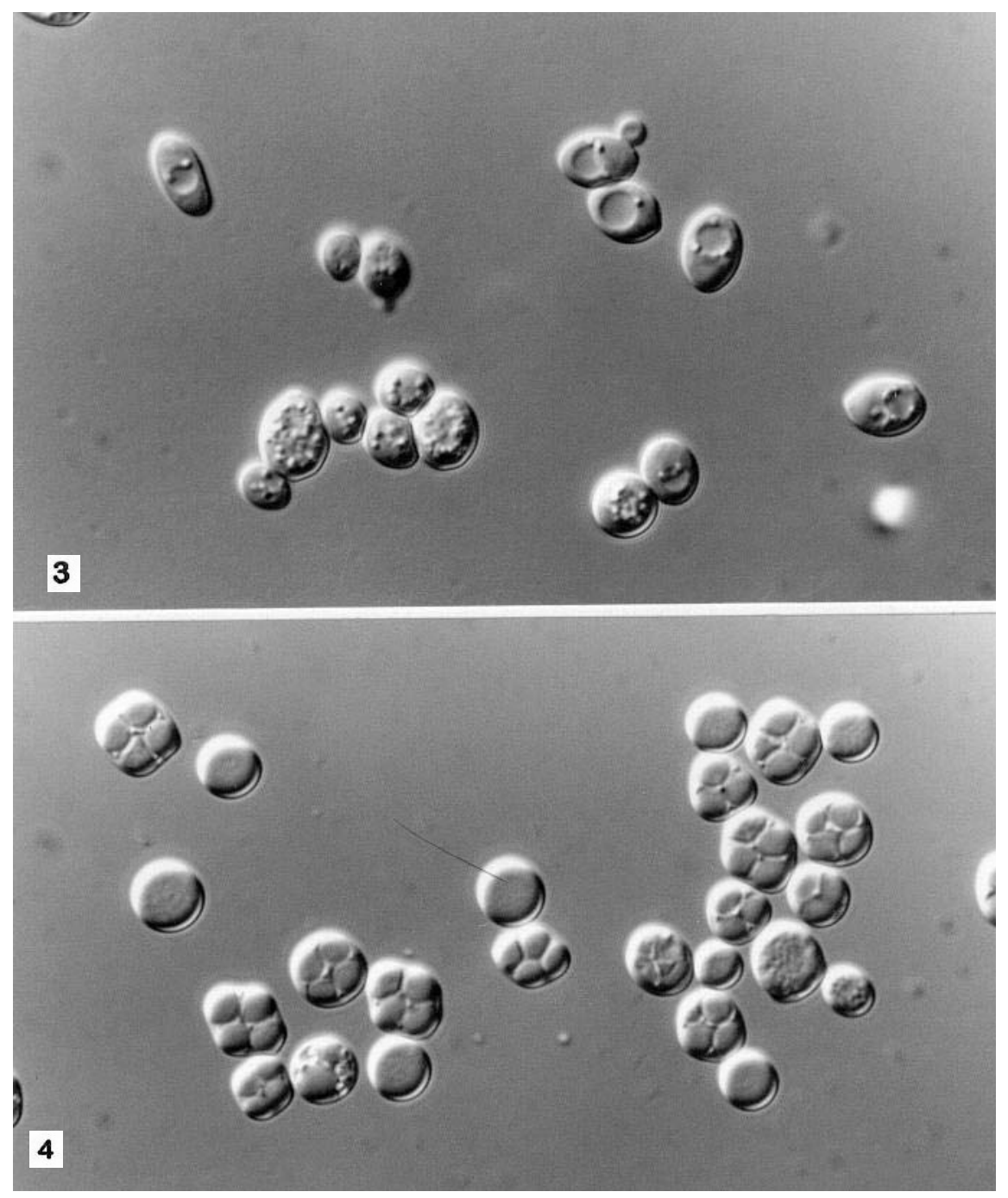

Fig.3. Asexual cells of the Saccharomyces sp. From bamboo exudates

Fig.4.Cells of the isolated Saccharomyces sp., some having ascospores

\section{Results and Discussion}

A Saccharomyces species (Fig 2-3) was the most prevalent microorganism fermenting the bamboo sugary exudates. The isolated yeast could also ferment glucose, galactose, sucrose and raffinose. The yeast isolated, could ferment $27 \%$ $\mathrm{w} / \mathrm{w}$ pure glucose, to produce and withstand $9.5 \% \mathrm{w} / \mathrm{w}$ ethanol.

In terms of alcohols, only ethanol was detected in the fermented bamboo exudates taken from one village in Iringa, Tanzania. The alcohol production reached $6.5 \% \mathrm{w} / \mathrm{w}$ on average. It was also established that, at the sampling village, one stand (clump) of the bamboo has an average of 15 young shoots, each actively producing an average of $800 \mathrm{~g}$ of the sugary exudates in one day. Such a clump, occupies an 
average area $3 \mathrm{~m}$ in diameter. It thus follows that one stand of the bamboos can produce $12 \mathrm{Kg}$ of the sugary exudates in a day which, on fermentation, will have $0.78 \mathrm{Kg}$ or 1 liter of pure ethanol. If a $2 \mathrm{~m}$ inter-stand space is allowed in a hectare of the bamboo, there will be 400 stands of the bamboo in the hectare, producing a total of $312 \mathrm{Kg}$ of pure ethanol in a day. In other words, 400 liters of pure ethanol can be produced by the bamboo, per hectare, per day. If such alcohol is distilled by solar-powered equipment, it might be a cheap source of ethanol for various uses.

The present isolated yeast from bamboo exudates, could withstand $9.5 \%$ alcohol. It is proposed here that, further work on the bamboo fermenting microorganisms, could be directed at searching for other microbial strains or types in the bamboo exudates, which can ferment higher concentrations of sugars, and/or those which can withstand higher amounts of alcohols.

\title{
References
}

Carmo-Sousa L. do 1969. Distribution of yeasts in nature. In The Yeasts. Vol. 1 Biology of yeasts. Rose A.H. and Harrison J.S. eds. Academic Press, London. pp. 79-105.

IBRN Newsletter 1993. The bamboo wine. IDRC Bamboo/Rattan Network Newsletter 14:.2 IDRC-South Asia Regional Office. New Delhi.

Kigomo B.N. 1990. Bamboo resources in the Eastern Africa Region. In Bamboos. Ramanuja Rao I.V., Gnanaharan R. and Sastry C. B. eds. Current Research. Proceedings of the International Bamboo Workshop, Cochin, India 14-18 Nov. 1988. IDRC, Canada, pp. 22-28.

Lodder J. 1970. The Yeasts. A taxonomic study. North-Holland publishing Company, Amsterdam.

Magingo F.S. and Gijzen H.J. 1989. Assessment of wine quality and state of fermentation process of Dodoma Wine Company and suggestions for improvement. Consultancy Report, Project funded by Tanzania Commission for Science and Technology, and Delegation of European Communities in Tanzania.

\section{GROWTH OF FUSIDIUM COCCINEUM ON SOLID MEDIA}

\author{
HELEN E. SMITH \& GLYN HOBBS \\ School of Biomolecular Sciences, Liverpool John Moores University, Byrom Street,Liverpool L3 3AF
}

Abstract. Growth of Fusidium coccineum on both complex and defined agar media resulted in the production of fusidic acid into the solid medium. Production of the antibiotic was found to be growth associated in both cases with production ceasing on depletion of glucose. Conidiation did not occur in any of the cultures examined indicating the absence of a temporal link between antibiotic production and conidiation in this organism.

\section{Introduction}

Fusidic acid is a clinically important antibiotic that inhibits protein synthesis at the level of translocation in both prokaryotes and eukaryotes (Tanaka et al. 1968). It exhibits potent activity against gram-positive bacteria (Godtfredsen et al. 1966) and is used extensively in the treatment of staphylococcal infections in either cream or tablet form.

Fusidic acid is produced by conventional submerged fermentation using the fungus Fusidium coccineum (Von Daehne et al. 1984). Despite the obvious clinical importance of this metabolite little information has been published on the physiology of the producing organism, for a review see Von Daehne et al. (1984). This organism differentiates in both surface and submerged culture and a link between conidiation of the fungus and fusidic acid production has been reported, 
(Navashin et al. 1981). To study this relationship further we examined the growth of the organism on agar solidified media.

\section{Organisms \\ Material and Methods}

Fusidium coccineum (CBS 197.55) was used throughout the study. This was maintained by weekly subculture on YEPG agar and incubation at $28^{\circ} \mathrm{C}$. Corynebacterium xerosis (NCTC.9755) was used in the bioassay protocol and was maintained by subculturing on CX agar slopes with incubation at $37^{\circ} \mathrm{C}$.

Media

YEPG medium contained, per litre: $10 \mathrm{~g}$ yeast extract (Difco), $20 \mathrm{~g}$ glucose, $10 \mathrm{~g}$ mycological peptone (Difco), 20g agar. Liquid starter cultures, $50 \mathrm{ml}$ in a $250 \mathrm{ml}$ conical flask, were prepared using the above medium without agar, followed by inoculation with a $5 \mathrm{~mm}$ diameter plug from a 7 day old YEPG plate. Cultures were incubated in an orbital shaker $(200 \mathrm{rpm})$, at $30^{\circ} \mathrm{C}$ for 7 days. Aliquots (100Êl) of the liquid cultures were used to inoculate plates of YEPG agar. Each plate was prepared with a cellophane membrane overlay and confluent growth was produced by spreading the inoculum across the surface of the cellophane. Plates were incubated at $30^{\circ} \mathrm{C}$ for various times as shown. Minimal medium contained, per litre final concentration: $10 \mathrm{~g}$ glucose, $3.36 \mathrm{~g}$ sodium dihydrogen orthophosphate (dihydrate), $0.85 \mathrm{~g}$ sodium nitrate, $0.75 \mathrm{~g}$ potassium chloride, $0.64 \mathrm{~g}$ sodium sulphate decahydrate, $0.26 \mathrm{~g}$ magnesium chloride hexahydrate, $2.2 \mathrm{mg}$ calcium chloride, 24Êg sodium molybdate dihydrate, $0.292 \mathrm{~g}$ EDTA. Trace salts. $2.0 \mathrm{mg}$ zinc oxide, $27 \mathrm{mg}$ ferric chloride hexahydrate, $10 \mathrm{mg}$ manganese chloride $0.85 \mathrm{mg}$ cupric chloride dihydrate, $2.4 \mathrm{mg}$ cobalt chloride hexahydrate, $0.3 \mathrm{mg}$ boric acid and $20 \mathrm{E} g$ biotin. The $\mathrm{pH}$ of the medium was adjusted to 6.8 with $1 \mathrm{M} \mathrm{NaOH}$ prior to sterilization. The trace salts solution was made up separately as a hundred fold concentrate, the $\mathrm{pH}$ was adjusted to 2.0 with concentrated hydrochloric acid and sterilised at $121 \mathrm{C}$ for $15 \mathrm{~min}$. Glucose was also sterilised separately as a ten fold concentrate.

For the physiological experiments $20 \mathrm{ml}$ of medium was dispensed into each plate and allowed to solidify prior to placing a pre-sterilised cellophane membrane on the surface. Cultures were inoculated directly on the surface of the membrane and spread with a glass spreader to achieve a confluent inoculum.

\section{Analysis of samples}

Biomass was determined by dry weight after removal of cell material from the surface of the membrane with a razor blade. The biomass was placed on a Whatman $\mathrm{N}^{\circ} 1$ filter and dried in a microwave oven for $15 \mathrm{~min}$. on the "defrost" setting.

Samples were taken from beneath the membrane using a cork borer $(5 \mathrm{~mm}$ diam.). The agar plugs were then subjected to a freeze squeeze protocol to provide liquid medium samples using the method of Tautz \& Renz (1983). Glucose was measured using a glucose oxidase kit (Boehringer-Mannheim). Fusidic acid was measured by bioassay using C. xerosis (Bywater 1975).

\section{Results}



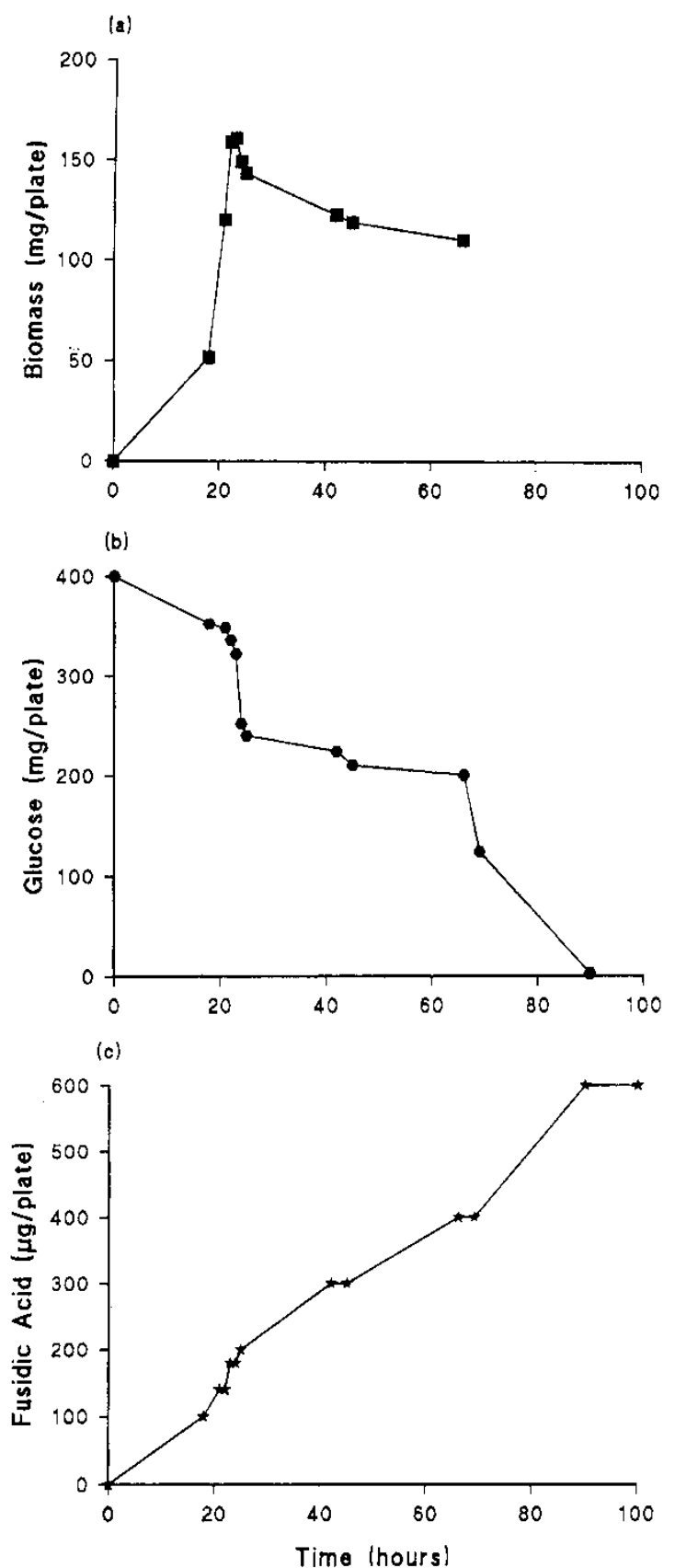

Fig.1. Growth of F. coccineum on YEPG medium. (a) biomass, (b) glucose, (c) fusidic acid. Values represent the mean of at least three determinations. 

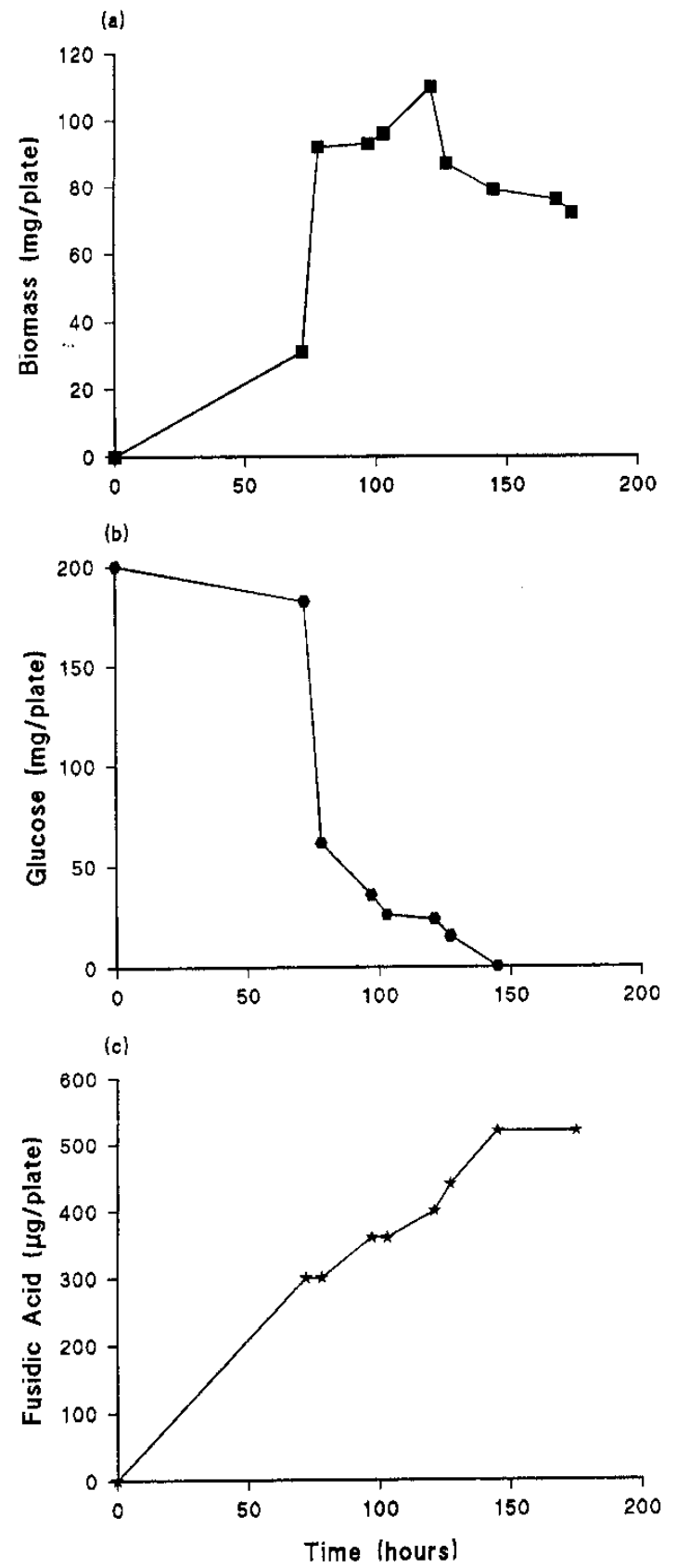

Fig.2. Growth of on minimal medium. (a) biomass, (b) glucose, (c) fusidic acid. Values represent the mean of at least three determinations.

Growth of F. coccineum on complex medium was rapid with maximum biomass levels being achieved within one day of inoculation (Fig 1a). The biomass accretion coincided with the consumption of glucose from the medium (Fig 
1b).Approximately half the available glucose was consumed during this initial growth period. After 21 hours of growth, biomass levels declined possibly due to nutrient limitation or diffusion limitation. Measurements of fusidic acid concentrations in the medium revealed a biphasic production pattern, with an exponential increase in antibiotic during the growth phase followed by a linear accumulation after biomass accumulation had ceased (Fig 1c). Glucose levels throughout the experiment were found to decrease, reaching an undetectable value after 90 hours post-inoculation. The complete removal of glucose from the medium resulted in the cessation of fusidic acid accumulation, suggesting an intimate link between antibiotic production and glucose availability. The cultures were monitored throughout for the appearance of morphological differentiation, particularly for the appearance of conidia. At no time during the experiment were conidia observed suggesting that under these conditions at least, no link between antibiotic production and conidiation could be shown.

To further investigate the relationship between glucose availability, conidiation and antibiotic production we developed a defined minimal medium that would support growth and antibiotic production. Growth of the culture on minimal medium was slower than for complex medium, reaching maximum biomass after 120 hours (Fig 2a). Biomass accumulation ceased close to the point of glucose exhaustion suggesting that growth in this culture was probably glucose limited (Fig 2b). Fusidic acid production was growth linked under these conditions and as with the complex medium the production of the metabolite ceased at the point when glucose completely disappeared from the medium (Fig 2c). Examination of the cultures for evidence of conidiation revealed a complete absence of conidia throughout the experiment, providing further evidence for the lack of association between conidiation and antibiotic production in this organism.

\section{Discussion}

Plate cultures provide a simple and convenient way of investigating the connection between morphological differentiation and metabolite consumption and production. The evidence provided herein suggests that conidiation and fusidic acid production are physiologically separate entities. This data conflicts with earlier work of Navashin et al.(1981) who describe a temporal link between conidiation and antibiotic synthesis in F. coccineum. Furthermore, our work would also suggest that the cells responsible for the production of the antibiotic are the hyphal form rather than the conidia themselves. The kinetics of production of the antibiotic on solid medium is not typical of a secondary metabolite with the greatest rate of production occurring during the rapid growth phase. The question then arises as to whether fusidic acid is an "overflow metabolite" of primary metabolism or a true secondary metabolite.

Clearly fusidic acid production is dependent upon available glucose, an efficient protocol for production therefore may be to use immobilised resting cells of the fungus which could be fed continuously with glucose.

\section{References}

Bywater M.F. 1975. Fusidic Acid. Laboratory Methods in Antimicrobial Chemotherapy. 219-221.

Godtredsen W.O., Daehne W. von, Tybring L. and Vangedal S.1966. Relationship between structure and antibacterial activity. Journal of Medical Chemistry 9:15-22.

Navashin S.M., Bartoshevich Y.E., Telesnina G.N. Zvjagilskaya R.A., Dimitriyeva S.V., Saykin Y.O. 
and Krakhmaleva I.N. 1981. Respiratory system of Fusidium coccineum and regulation of biosynthesis of fusidic acid and sporogenesis. European Journal of Applied Microbiology and Biotechnology 12:220-225

Tanaka N., Kinoshita T. and Masukawa H. 1968. Mechanism of protein synthesis inhibition by fusidic acid and related antibiotics. Biochemical and Biophysical Research Communications 30:278-283.

Tautz D. and Renz M. 1983. An optimised freeze-squeeze method for the recovery of DNA fragments from agarose gels. Analytical Biochemistry 132:14-19.

Von Daehne W., Jahnsen J., Kirk I., Larsen R. and Lorck H. 1984. Fusidic acid: Properties, biosynthesis and fermentation. Drugs and the Pharmaceutical Sciences 22:427-450.

\title{
STRAIN POLYMORPHISM IN PDR1, A TRANSCRIPTION REGULATOR OF PLEIOTROPIC DRUG RESISTANCE GENES IN SACCHAROMYCES CEREVISIAE.
}

\author{
E. CARVAJAL ${ }^{1}$, E. BALZIL ${ }^{2}$ AND A. GOFFEAU ${ }^{2}$ \\ 1 Instituto de Quimica Universidade Federal do Rio de Janeiro, Cidade Universitaria, IIha do Fundao, \\ CEP 21945, Rio de Janeiro, RJK, Brasil \\ 2 Universite Catholique de Louvain, Unite de Biochimie Physiologique, Place Croix du Sud, Louvain-la- \\ Neuve.
}

Abstract. PDR1 is a $\mathrm{Zn} 2$ Cys6 transcription factor that regulates positively PDR5 and probably other mutidrug pumps in Saccharomyces cerevisiae. (Balzi et al. 1994).

The comparison of the PDR1 DNA sequence has been carried out on the following seven so called wild type laboratory strains: IL 125-2B, D 286-2A, FM 11, GR 350, JG 200, FY 1679-28C, S288C.

Seven nucleotidic differences clearly distinguished the strains of european origin (IL1252B, D 286-2A) from those of american origine (JG200, GR 350, FM11, FY 1679-28C, S288C). Four out of the seven differences resulted in aminoacid changes, and three others were only at the nucleotide sequence. In all european strains, the amino acid residues 411,821 and 921 were found to be lysine, alanine and isoleucine instead of glutamine threonine and threonine respectively in the american strains. The fourth difference was observed in the polyasparagine strech beginning at residue 1009: in the european strains five successive asparagine residues were observed instead of ten asparagines in the american strains.

Thus the polymorphism is more pronounced in the C-terminal region of the protein.

In this family of transcription factors the C-terminus is usually involved in transactivation of transcription whereas the $\mathrm{N}$-terminus bears the zinc finger domain which binds DNA on specific consensus motif. We will construct a set of a isogenic strains carrying the different wild type alleles to investigate whether or not these modifications affect the transactivation activity of PDR1.

\section{Reference}

Balzi E., Wang M., Leterme S., Van Dyck L. and Goffeau A. 1994. PDR5, a novel yeast multidrug resistance conferring transporter controlled by the transcription regulator PDR1. J. Biol. Chem. 269: 2206-2214 


\title{
STRUCTURAL IDENTIFICATION AND BIOCHEMICAL MODE OF ACTION OF A MYCOTOXINE ISOLATED FROM COCHLIOBOLUS SATIVUS (MUCL 18528)
}

\author{
D. Vilret, P. Goblet, J. Hutschemackers, N. Cosyns, M. NoËL AND M. BRiQuet
}

Université Catholique de Louvain, Faculté des Sciences Agronomiques, Unité de Biochimie Physiologique, Place Croix du Sud 2/20, B-1348 Louvain-la-Neuve

\begin{abstract}
A mycotoxin extracted from the culture medium of Cochliobolus sativus has been purified by chromatography (TLC, HPLC) and identified by infrared, mass and NMR spectrometry. The chemical structure corresponds to the "helminthosporol", a sesquiterpene discovered in 1965 by S. Tamura.

Our results on the biochemical activity show that this compound inhibits the oxidative phosphorylation of plant mitochondria (ATP synthase) but does not inhibit the ATP phosphohydrolase (ATPase) activity. In contrast the mitochondrial ATPase activity of the yeast Saccharomyces cerevisiae is strongly inhibited by the toxin. The plasma membrane ATPase activity of Saccharomyces cerevisiae is not inhibited by helminthosporol.
\end{abstract}

\section{CHARACTERIZATION OF THE CARBOHYDRATE MOIETY ON PROTEINS SECRETED FROM DIFFERENT FUNGI}

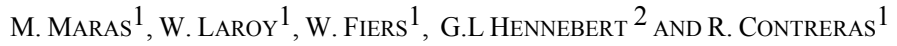 \\ ${ }^{1}$ Laboratory of Molecular Biology, State University, Ledeganckstraat 35, B-9000 Ghent, Belgium. \\ ${ }_{2}$ Mycothèque MUCL, Université Catholique de Louvain, Place Croix du Sud 3, B-1348 Louvain-la- \\ Neuve, Belgium.
}

\begin{abstract}
Many different yeasts and filamentous fungi are attractive candidates for the production of secreted, therapeutically valuable proteins. Since the glycosylation pathway in these lower eukaryots differs from that in mammalian cells, oligosaccharides different from the complex, mammalian type are synthesized. Fungi-type glycosylation, known to be mannan or (for certain species) galactomannan forms an obstacle to application of heterologous proteins as therapeutic agents. The latter proteins are rapidly cleared from the blood because of recognition and binding of fungi-like oligosaccharides to sugar binding receptor proteins (Summerfield et al., 1986). With the exception of Saccharomyces cerevisiae, detailed knowledge about glycosyl structures is very poor. Yeasts are known for their capacity to hyperglycosylate proteins. Filamentous fungi, on the other hand, seem to synthesize smaller glycans (Berka, R.M. and Barnett, C.C, 1989). This resembles more the situation in mammalian cells.

With the aim to obtain more information about the diversity of oligosaccharidestructures and about the possible existence of glycosyl structures more similar to the mammalian system, we studied glycoproteins from a collection of yeasts and filamentous fungi. Lectins were used to investigate the identity of terminal sugar residues different from mannose. In a collection of yeasts, a few species seemed to have terminal a-linked galactose residues: Binding of Bandeiraea simplicifolia I
\end{abstract}


lectin, highly specific for terminal a-linked galactose, to their secreted proteins, was demonstrated. We are now investigating a collection of filamentous fungi, to determine whether new glycosyl patterns can be found.

\title{
References
}

Summerfield J.A. and Tayler M.E. 1986. Mannose-binding proteins in human serum: identification of mannose-specific immunoglobulins and a calcium-dependent lectin, of broader carbohydrate specificity, secreted by hepatocytes Biochim. Biophys. Acta 883: 197-206.

Berka R.M. and Barnett C.C. 1989. The development of gene expression systems for filamentous fungi. Biotech. Adv. 7: 127-154.

\section{SPECIFIC AND NON-SPECIFIC INTERACTIONS IN YEAST CELL FLOCCULATION}

\author{
P.B. DENGIS, L.R. NÉLISSEN, AND P.G. ROUXHET
}

Université Catholique de Louvain, Unité de Chimie des Interfaces, Place Croix du Sud 2 Bte 18, B-1348 Louvain-la-Neuve.

\begin{abstract}
At the end of the fermentation in brewery, cell aggregates are formed which accumulate on top of the fermented wort (top strains) or which sediment to the bottom (bottom strains). The aim of this research is to understand better the mechanisms which control the yeast aggregation, in particular the importance of long range interactions (double layer interactions, van der Waals forces), short range non-specific interactions (hydrophobic interactions, salt bridges) and specific interactions (molecular recognition).

The yeast surface chemical composition is determined by X-Ray Photoelectron Spectroscopy (XPS); the hydrophobicity is evaluated by contact angles and the surface electrical properties are characterized by the zeta potential. The tendency of the cells to form aggregates is determined by turbidimetric measurements after controlled stirring and subsequent settling in defined solutions.

Top fermentation strains have been found to be more hydrophobic, to have a lower phosphate surface concentration and a less negative zeta potential. A more detailed comparison has been performed between one top and one bottom strain. For both strains aggregation takes place only at the end of the exponential growth phase and it requires the presence of calcium. The tendency of the top strain to form aggregates is maximum between $\mathrm{pH} 3$ and 4.5, i.e. close to its isoelectric point of 4 . For the bottom strain the isoelectric point is much lower than the optimum aggregation $\mathrm{pH}$ range: however the decrease of the phosphate surface concentration during the culture is in accordance with an influence of electrostatic interactions. In the adequate range of $\mathrm{pH}$, flocculation of the bottom strain requires addition of about $0.1 \mathrm{mM} \mathrm{Ca}^{++}$while the top strain flocculates without calcium addition. The two strains exhibit also different responses with respect to the addition of organic compounds in the solution. For the bottom strain, certain sugars provoke an inhibition of flocculation or a redispersion of previously formed flocks; this is a specific effect, occurring with 10 to $500 \mathrm{mM}$ glucose, mannose and maltose, but not with galactose, which points to the involvement of molecular recognition in cell aggregation. Flocculation of the top strain, on the contrary, is not influenced by these sugars; furthermore, addition of $5 \%$ ethanol broudens considerably the $\mathrm{pH}$
\end{abstract}


range (2.5-5.5) in which flocculation takes place suggesting that solvation forces play an important role.

\title{
MODIFICATION OF SUPPORT SURFACE PROPERTIES TO IMPROVE MICROORGANISM ADHESION
}

\author{
P.A. GERIN ${ }^{1}$, M.-N. Bellon-FonTAIne ${ }^{2}$, M. Asther ${ }^{3}$ AND P.G. ROUXHeT $^{1}$ \\ ${ }^{1}$ Unite de Chimie des Interfaces, UCL, Place Croix du Sud, 2/18, B-1348 Louvain-la-Neuve, \\ Belgium ${ }^{2}$ Laboratoire de Génie de l'Hygiène et des Procédés Alimentaires, INRA, 25 avenue de la \\ République, F-91300 Massy, France \\ ${ }^{3}$ Laboratoire de Biotechnologie des Champignons Filamenteux, INRA, 163 avenue de Luminy, Case \\ Postale 929, F-13288 Marseille, France
}

\begin{abstract}
For immobilisation of fungi in bioreactor, a quick adhesion and homogenous distribution of the spore inocculum on the support surface is desirable. This is expected to lead to a more uniform growth and colonisation of the support.

Polycations and ferric ions were adsorbed on the surface of a flat polycarbonate support, either as such, or pretreated by sulpho-chromic mixture. The pretreatment grafted sulphate or sulphonate groups, which lowered the isoelectric point and rendered the surface more negative. The adsorption of polycations and ferric ions increased the zeta potential and isoelectric point of these surfaces. 5-10 atomic layers of adsorbed polycations were found at the pretreated support surface; on the non-pretreated supports, less than one atomic layer was adsorbed.

The supports were then submitted to a flow of Phanerochaete chrysosporium spore suspension; these fungal spores have a hydrophobic and negatively charged surface. A few spores adhered in a patchy way on the native supports. Adsorption of polycations and ferric ions increased the amount of adhering spores and led to a homogenous distribution. This might be due to the reduction of the electrostatic repulsion between the spores and the support, which increased the chance for the spores to come in contact with the surface and to adhere.

The spores adhering to the supports were later submitted to a flow of culture medium. Neither adhesion on the support, nor the presence of adsorbed polycations inhibited or delayed the swelling, the gerrnination and the growth of the spores.
\end{abstract}




\title{
BACTERIOLOGY
}

\section{GAS CHROMATOGRAPHIC ANALYSIS OF CELLULAR FATTY ACIDS IN THE IDENTIFICATION OF MICROORGANISMS}

\author{
P. WAuthoz, M. El Lioui AND J. DeCALlONNE
}

Unité de Microbiologie, Faculté des Sciences agronomiques, Université catholique de Louvain, Place Croix du Sud, 2, bte 12, B-1348 Louvain-la-Neuve, Belgium

Since Abel et al. (1963) demonstrated that fatty acid composition can be useful to classify microorganisms into genera and species, various workers have successfully used it to characterize, differentiate and classify bacteria.

As the cellular fatty acid profile is affected by a number of parameters, rigid standardization of experimental conditions and techniques is required. The extraction and derivation procedure proposed by Miller and Berger (1985) is commonly used in bacterial analysis. It consists in the main following steps:

1. saponification $\left(\mathrm{NaOH}\right.$ in $\mathrm{MeOHaq}, 100^{\circ} \mathrm{C}, 30 \mathrm{~min}$.)

2. methyation ( $\mathrm{HCI} / \mathrm{MeOH}, 80^{\circ} \mathrm{C}, 10 \mathrm{~min}$.)

3. extraction (hexane/ethylether, $10 \mathrm{~min}$.)

4. base wash (dilute $\mathrm{NaOH}, 10 \mathrm{~min}$.).

The advantage of this method are the low time consumption, the low volume of reagents (each 1 to $2 \mathrm{ml}$ ) and the quantity of bacterial material require (about $40 \mathrm{mg}$ ).

We applied this procedure to about 300 foodborne bacteria, grown under standardized conditions. The reproducibility, from bacterial cultivation to the GC analysis, was estimated at $\mathrm{So}=94.20 \pm 2.80 \quad(n=84)$, where So is the overlap coefficient (Bousfield et al. 1985). Inside a species, the So was always greater than 79.

The great diversity of bacterial fatty acids, including saturated, monounsaturated, straight chain, branched-chain, 2- and 3-hydroxy and cyclopropane types, and the variability of their relative proportions permit one to define arge groups of strains (lactic acid bacteria, Enterobacteriaceae,...) and to separate closely related genera (Micrococcus, Staphylococcus) (Table 1).

The same extraction and derivatization procedure was also applied to characterise yeast strains. It should be noted that this procedure does not allow one to separate fatty acids from long-chain hydrocarbons, which elute after fatty acids in GC analysis.

Four stains of Saccharomyces (S. bayanus MUCL 31495; S. pastorianus MUCL 31496; S. cerevisiae MUCL 31497; S. paradoxus MUCL 31498) were investigated after grown for 72 hours, at $25^{\circ} \mathrm{C}$, in dextrose $20 \mathrm{~g} / 1$, yeast extract $5 \mathrm{~g} / 1$, meat peptone $10 \mathrm{~g} / \mathrm{l}$ broth, on a rotary shaker at $160 \mathrm{rpm}$.

The profiles obtained were rather simple with a preponderance of saturated and monounsaturated $\mathrm{C} 16$ and $\mathrm{C} 18$ acids (Table 2).

In addition, in certain genera (Bacillus, Pseudomonas), the profile So obtained may be used as characteristics in speciation (Fig. 1). 
Table 1. So value calculated between different bacterial genera.

\begin{tabular}{|c|c|c|c|c|c|c|c|c|c|c|}
\hline & $\mathrm{N}^{\circ}$ & 1 & 2 & 3 & 4 & 5 & 6 & 7 & 8 & 9 \\
\hline $\begin{array}{l}\text { Gram - } \\
\text { Enterobacteriaceae } \\
\text { Escherichia coli ATCC } 10536 \\
\text { Salmonella RL } \\
\text { Pseudomonas fluorescens UPB } \\
408\end{array}$ & $\begin{array}{l}1 \\
2 \\
3\end{array}$ & $\begin{array}{l}- \\
92 \\
71\end{array}$ & - & - & & & & & & \\
\hline $\begin{array}{l}\text { Gram+ } \\
\text { Bacillus subtilis ATCC } 12711 \\
\text { Lactic acid bacteria }\end{array}$ & 4 & 3 & 3 & 4 & - & & & & & \\
\hline $\begin{array}{l}\text { Lactobacillus plantarum DSM } \\
20174\end{array}$ & 5 & 57 & 55 & 46 & 4 & - & & & & \\
\hline $\begin{array}{l}\text { Leuconostoc mesenteroödes } \\
\text { NCIB } 3354\end{array}$ & 6 & 56 & 57 & 47 & 5 & 89 & - & & & \\
\hline Lactococcus lactis LMG 6897 & 7 & 40 & 39 & 29 & 5 & 70 & 65 & - & & \\
\hline $\begin{array}{l}\text { Pediococcus acidilactici NCIB } \\
6990\end{array}$ & 8 & 61 & 58 & 52 & 3 & 74 & 69 & 66 & - & \\
\hline $\begin{array}{l}\text { Staphylococcus aureus MBLA } \\
210\end{array}$ & 9 & 4 & 4 & 5 & 73 & 5 & 7 & 6 & 5 & - \\
\hline
\end{tabular}

So
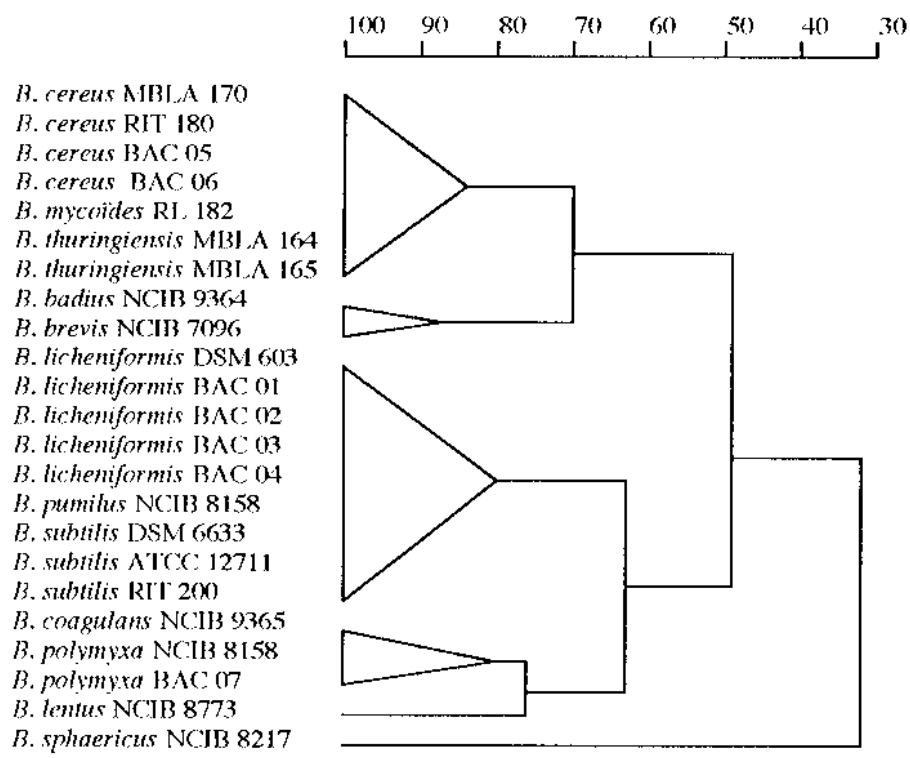

Fig.1. Dendrogram of Bacillus species based on the So values.

Table 2. Fatty acid composition of Saccharomyces strains ( $\%$ total fatty acids)

\begin{tabular}{|l|cccccccc|}
\hline Strain & \multicolumn{2}{|c|}{ Fatty acid } & & & & & & \\
& $12: 0$ & $14: 0$ & $16: 0$ & $18: 0$ & $\begin{array}{l}14: 1( \\
9 \mathrm{c})\end{array}$ & $\begin{array}{l}16: 1 \\
(9 \mathrm{c})\end{array}$ & $\begin{array}{l}18: 1 \\
(9 \mathrm{c})\end{array}$ & $\begin{array}{l}18: 1 \\
(11 \mathrm{c})\end{array}$ \\
\hline S. bayanus MUCL 31495 & 1,37 & 0,59 & 9,51 & 3,22 & 0,78 & 61,18 & 19,69 & 0,01 \\
S. pastorianus MUCL 31496 & 1,56 & 0,70 & 2,42 & 0,24 & 2,32 & 77,25 & 14,28 & 0,20 \\
S. cerevisiae MUCL 31497 & 2,33 & 0,81 & 3,32 & 0,74 & 3,64 & 63,04 & 23,50 & 2,26 \\
S. paradoxus MUCL 31498 & 0,83 & 0,93 & 5,98 & 0,45 & 1,48 & 68,28 & 19,78 & 0,95 \\
\hline
\end{tabular}


The four strains had palmitoleic acid as the major component with an occurrence of up to $76 \%$ of the total fatty acid. The proportion of oleic acid varied from 14 to $27 \%$ among strains. The palmitic acid is several folds more abundant than stearic acid. Saturated and monounsaturated $\mathrm{C} 12$ and $\mathrm{C} 14$ were present in lower proportions. Traces $(<1 \%)$ of 15:1, 15:0, 16:1 (11c) and 17:1 acids were observed. No branched-chain and cyclopropane acids, which are common components of certain bacterial lipids, appeared in the profiles.

The qualitative composition is in agreement with previous reports on Saccharomyces strains (Augustyn and Kock 1989, Kock et al. 1986, Moss et al. 1982) and is highly similar to those found for other yeast genera (Moss et al. 1982, Smit et al. 1987). Therefore, the relative simplicity of the fatty acid composition of yeasts does not seem to offer as much aid in the taxonomy of the unicellular fungi as it does for the bacteria. Betwee the four strains studied, So was greater than 82 , which is a higher value than for bacterial intra-species similarity. This does not allow one to draw conclusions on the classification of these strains in one or different species. Further experimental work is needed on more strains to evaluate the ability of using quantitative differences to composition to characterise, classify and identify yeast strains.

\section{Acknowledgements}

We thank Pr Hennebert, MUCL, for the yeast cultures. This work was supported by the Ministère de l'Agriculture de la Region Wallone and by Requasud.

\section{References}

Abel K., 1963. Classification of microorganismsby analysis of chemical composition I. Feasibility of utilizing gas chromatography. J. Bacteriol. 85: 1039-1044.

Augustyn O.P.H. and Kock L.F. 1989. Application of an adapted technique to differentiate between strains of Saccharomyces cerevisiae. J. Microbiol. Methods. 10: 9-23.

Bousfield I.J., Smith G.L, Dando T.R. and Hobbs G. Total fatty scid profiles in the identification of coryneform, nocardioform and some other bacteria. J. Gen. Microbiol. 129: 375-394.

Kock J.L.F., Cotrell M. and Lategan P.M. 1986. A rapid method to differentiate between five species of the genusSaccharomyces. Appl. Microbiol. Biotechnol. 23: 499-501.

Miller L. and Berger T. 1985. Bacteria identification by gas chromatography of whole cell fatty acids. Application Note 228-41, Hewlett Packard.

Moss C., Shinoda T and Samuels J W. 1982. Determination of cellular fatty acid compositions of various yeasts by gas-liquid chromatography. J. Clin. Microbiol. 16: 1073-1079.

Smit E. et al. 1987. Dependence of culture age on the cellular long-chain fatty acid composition of three basidiomycetes yeasts. Syst. Appli. Microbiol. 10: 38-41.

\section{IDENTIFICATION OF LACTIC ACID BACTERIA USING RAPD-PCR.}

NOËL A. AND J. DECALLONNE

Unité de Microbiologie, Université Catholique de Louvain-Faculté des Sciences agronomiques, Place Croix du Sud 2, BP 12, 1348 Louvain-La-Neuve - Belgium.

Abstract. Lactic acid bacteria play an essential role in food technology. They improve the aroma and texture in food, and inhibit the growth of spoilage bacteria. However, some strains of lactic bacteria are involved in food spoilage and few are found even pathogens.

Therefore, studies of reliable, simple and time saving identification methods are required for both basic research on lactic acid bacteria and their applications in industrial food fermentations. These methods aim to distinguish genera and species. 
Identification methods of lactic acid bacteria are conventionally based on morphological and biochemical criteria. Recently, they have highly developed by using DNA based analysis, and particularly by the Polymerase Chain Reaction (PCR) (Saïki et al. 1986).

A recent innovation is amplification fragment length polymorphism analysis by arbitrary primers (Welsh and Mac Clelland 1990). This strategy called RAPD (Random Amplified Polymorphic DNA) involves the enzymatic amplification of template DNA directed by one or more arbitrary oligonucleotide primers to produce a characteristic spectrum of products, a proportion of which can be polymorphic.

The procedure is fast, independent of prior genetic and biochemical knowledge of the organism tested.

The present work has been carried out as a contribution to this new method in the field of lactic acid bacteria identification. We describe the use of RAPD as an effective and rapid method to discriminate 12 Lactobacillus strains that were previously characterized in our laboratory by gas chromatographic analysis of cellular fatty acids (HRGC) (Wauthoz 1992).

Lactobacillus brevis NCIB 4617, NCIB 4036; Lactobacillus buchneri NCIB 8007, ENSI 9 Lactobacillus casei casei ENSI; Lactobacillus confusus NCIB 4037;Lactobacillus fermentumDSM 20052, CIP 53163, EPI 7, EPI 11; Lactobacillus plantarum DSM 20174, CIP A169.

Lactobacillus strains were grown under standardized conditions in MRS medium (Man, Rogosa and Sharpe) and total genomic DNA for RAPD was extracted from 3 $\mathrm{ml}$ of overnight culture following Cancilla (1992). RAPD was performed by the method of Mazurier (1992) using ten 10-mer primers (kit OPC-01, Operon) and the amplification products were separated according the size by agarose gel electrophoresis.

Using the oligonucleotide OPC-01 (TTCGAGCCAG) as primer, specific DNA fingerprints have been obtained for each of the studied strains (figure 1). The number of DNA bands in these fingerprints ranged from 1 to about 12 and differences in intensity were observed between these bands.

Although only a limited number of Lactobacillus strains were evaluated, these data demonstrate the ability of RAPD to differentiate between Lactobacillus strains that are not always discriminated by HRGC analysis and by conventional microbiological methods.

\section{References.}

Cancilla M.R., Powell I.B., Hillier A.J. and Davidson B.E. 1992. Rapid genomic fingerprinting of Lactococcus lactis strains by arbitrarly primed PCR with ${ }^{32} \mathrm{P}$ and fluorescent labels. Appl. and Env. Microbiol. 58: 1772-1775.

Mazurier S.I. and Wernars K. 1992. Typing of Listeria strains by random amplification of polymorphic DNA. Res. Microbiol. 143: 499-505.

Saiki R.K., Gelfand D.H., Stoffel S., Scharff S.J., Higuchi R.G., Horn G.T., Mllis K.B. and Erlich H.A. 1986. Primer directed enzymatic amplification of DNA with a thermostable DNA polymerase. Science 239: 487-491.

Wauthoz P.1992. Caractérisation et identification de la flore bactérienne des aliments par analyse chromatographique des acides gras cellulaires. $\mathrm{PhD}$ Thesis, Université Catholique de Louvain, UCL.

Welsch J. and Mac Clelland M. 1990. Fingerprinting genome using PCR with arbitrary primers. Nucleic Acids Res. 18: 7213-7218. 


\section{LAST LECTURE}

5 December 1995 


\title{
TAXONOMY AND NOMENCLATURE OF THE FUNGI: REASONS FOR A MYCOTHEQUE
}

\author{
RETROSPECTIVE ${ }^{4}$
}

\author{
G. L. HENNEBERT
}

\section{Introduction}

Philibert Biourge, in 1895, then assistant of Professor Jean-Baptiste Carnoy, studying the moulds of brewery malts, went to question the diversity of the Penicillium isolates, all being in greenish tinges close to that of Penicillium glaucum. Addressing the matter to Carnoy, he received this answer: "Then, Biourge, do a monographic study of the genus!" (Biourge in Glibert 1912, Hennebert 1979).

In 1956, Victor Estienne, my professor in plant pathology, observed with similar perplexity that "authors describe distinct species of Botrytis on the onion, Allium cepa" and asked me, "What are they, exactly? Are they not all Botrytis cinerea? You should study them." It was his suggestion for a PhD research, to solve a problem that he thought to be of host specificity: "Compare them through cross-inoculations in host plants." But crossinoculations failed.

Professor N. Fabritius Buchwald (Fig.8), author of the Studies of Sclerotiniaceae (Buchwald 1949) in the steps of Prof. Whetzel of Cornell University, said to me in Copenhagen, "The matter is primarily taxonomical. There are many names of Botrytis species in the literature. Many are not Botrytis, some are, and among these, a number are host specific, others are not. Differentiation of the species is first of all morphological. You should compare fresh collections morphologically, from nature and in culture. You should look for the perfect stage in nature and find out the correct name to apply". His impulse, in 1957, launched me into the taxonomy and the nomenclature of the fungi.

The literature from the oldest to the most recent includes more than 380 species names in Botrytis, but many have become synonymous to names of taxa as distant as Phytophthora. The remaining names, often with old descriptions in Latin, too short or lacking illustration, were investigated through examination of the original or type material, when extant, from diverse herbaria.

In the meantime, I was exploring woods, swamps, ponds, fields, vineyards, orchards and vegetable plots, flower gardens, agricultural and

\footnotetext{
${ }^{4}$ Last lecture delivered at UCL on December 5, 1995.
} 
forest soils, even caves (Hennebert 1960b, 1971) collecting specimens, and isolated Botrytis strains in pure culture.

Most of the cultures were conidial and sclerotial. I was disturbed finding in some of them small globose conidia like Penicillium conidia, even in single Botrytis conidium culture. I could not obtain their germination and concluded that they were spermatia, first discovered by De Bary (1884) in Botrytis and described by Istvanffi (1905) and Brierley (1918) (Hennebert, 1960a).

The multiplicity of forms in Botrytis cultures, conidial, sclerotial, spermatial, expressed the pleomorphy found as a quite general character of the fungi (Tulasne 1851). Fungal pleomorphy is a phenomenon that was misunderstood and confused with polymorphism and 'transformism' by Carnoy (1870) who described the transformation of a Mucor into a yeast that turned to become a Penicillium (Hennebert 1979).

The forms observed in Botrytis were said to be asexual and "imperfect". Guided by the works of Whetzel (1945), Raabe (1938) and Buchwald (1949, 1953), who observed and described the sexual state of Botrytis species, I collected apothecia on sclerotia, together with conidiophores of Botrytis, in the field under Allium ursinum, Ficaria verna and Caltha palustris, representing three different species, Botryotinia globosa Buchwald, $B$. ficariarum Hennebert and B. calthae Hennebert and Elliott (Fig. 1-3) (Hennebert 1958, 1960a, Hennebert and Groves 1963). The apothecia, the sexual, ascosporic form of reproduction, were said to be "perfect" states of the asexual Botrytis states. Apothecia were also developing spontaneously in a pure culture of Botrytis porri Buchwald from Allium porrum, confirming its identity as Botryotinia porri (van Beyma) Whetzel (Fig. 4) (Hennebert 1960a, 1963b). The species is homothallic. But in heterothallic species to produce apothecia needs crossing.

To each state of the fungi, imperfect state and perfect state, a distinct Latin binomial name was given. This is heritage of the past when mycologists considered each reproductive form a distinct species. The most common species of the genus Botrytis, thus received the following names:

Sclerotium durum Pers.: Fr., described by Persoon (1794a) for the sclerotial form, belonging to the genus Sclerotium Tode: Fries (Tode 1790).

Botrytis cinerea Pers.: Fr., described by Persoon (1797) for the conidial form, renamed from the polynomial nomenclature of Micheli (1729).

Botryotinia fuckeliana (De Bary) Whetzel, described for the ascosporic sexual ("perfect") form of reproduction, the apothecium. The apothecial state was discovered on Sclerotium echinatum Fuckel, a synonym of S. durum Pers.: Fr., by De Bary (1866), who named it Peziza fuckeliana De Bary in honour of Fuckel, a name that Fuckel (1870) himself changed into Sclerotinia fuckeliana (De Bary) Fuckel. The genus Sclerotinia was divided by Whetzel (1945) on the basis of the organic connection to diverse asexual forms and Botryotinia connected to the form Botrytis as established by De Bary $(1864,1884,1887)$. 
The spermatial form of Botrytis cinerea, discovered in this species by De Bary (1884), belongs to Myrioconium Sydow (1912) but has not been named specifically. Spermatia, so-called by Tulasne (1856), are phialoconidia which rise from both external and internal phialides on hyphae.
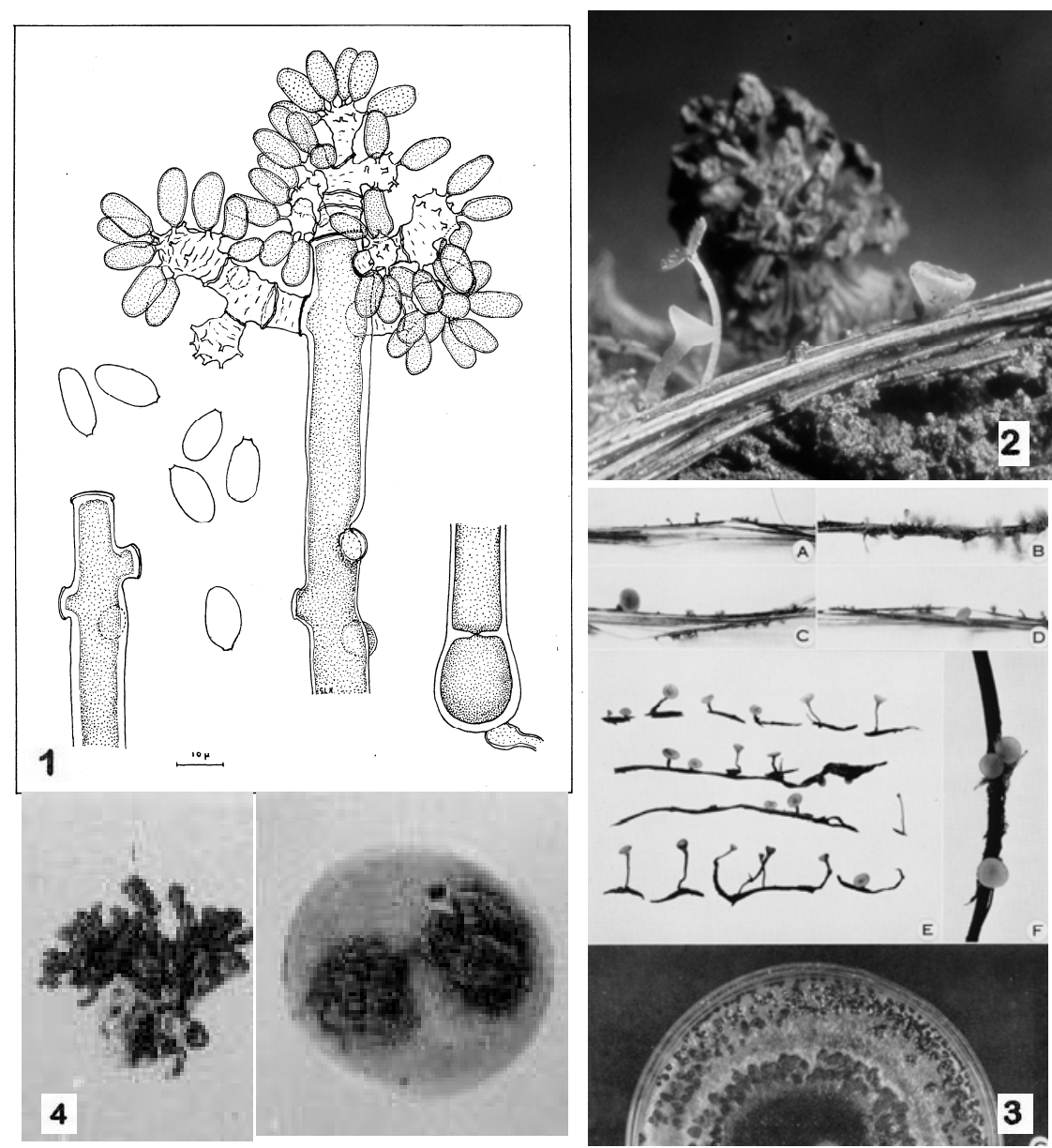

Fig. 1. Botryotinia ficariarum (Botrytis ficariarum anamorph) ; 2 and 3. Botryotinia calthae, apothecia and culture; 4. Botryotinia porri (sclerotium and apothecium in culture.

Several names for one fungus species appeared contrary to Linné's principle and that of the International Code of Botanical Nomenclature, one taxon - one name. In such cases the Code prescribes the predominance of the name of the sexual form over the other names and tolerates however the use of the other names. That peculiarity of the nomenclature of the pleomorphic fungi struck me from the early days. 
The embryo of a mycotheque

Soon I had hundreds of Botrytis isolates in pure culture beside isolates of many other fungi, an embryo of a fungus culture collection, a 'mycotheque' (Hennebert 1968d). "But why bring nature in small tubes?" questioned a relative of mine, long ago. Why a fungus culture collection? That is the question I wish to answer today, through an overview of 35 years of mycology.

Carnoy (1870) had already answered the question: "J'ai toujours cru que, pour faire sortir de l'enfance la science mycologique, il fallait cultiver les champignons, les cultiver toujours, les placer dans des milieux les plus divers, et, le microscope en main, suivre pas à pas leur développement. Lorsqu'on trouve une forme, au lieu de la décrire immédiatement comme une espèce nouvelle, n'est-il pas bien plus naturel de se demander d'abord si une culture méthodiquement variée la reproduirait toujours ou si elle ne pourrait pas en tirer autre chose? Aussi on doit savoir gré à tous ceux qui s'adonnent aux études expérimentales sur le développement et les métamorphoses de cette classe d'êtres singuliers." Here was an early promotion of the cultivation of the fungi in pure culture and of its use in experimental mycology to help fungal taxonomy.

Why a mycotheque? With Carnoy, I answer: to help elaborate a more natural taxonomy of the fungi, through demonstration and comparison in pure culture of their morphology and pleomorphy, and correlatively to resolve nomenclatural problems that pleomorphy may cause. Taxonomy and nomenclature were, to me, the prime reasons for building a mycotheque.

In those early days, I frequently visited the Centraalbureau voor Schimmelcultures, a well organized mycotheque, successively directed by Dr. Johanna Westerdijk, Dr A. van Beverwijk (Fig. 9) and Dr J.A. von Arx (Fig. 10) and where Prof. Biourge deposited Penicillium type strains in 1929.

The Mycotheque developed from that of Prof. Biourge at the University of Louvain, named MUCL, included 34,000 herbarium specimens and 24,000 living strains based upon them in $1994^{5}$.

\section{DeFINITIONS}

Taxonomy and nomenclature are too often abhorred activities and thus eliminated from training in many universities. However, I wish to let them be seen more serenely.

Taxonomy and nomenclature are at the base of all activities of man who observes, thinks and communicates. Observation leads to thought, thought to concepts. Taxonomy is the result of observation and thought. Nomenclature results from thought and concepts. Taxonomy and nomenclature is at the base of all common knowledge.

\footnotetext{
${ }^{5}$ MUCL preserves presently around 51.000 herbarium specimens and 35.000 living strains of fungi.
} 
Observation of the diversity of things induces comparison and distinction, and leads to elaboration of concepts and to formation of words. If a concept is a category, the word is a noun; if it is an attribute, the word is an epithet. Categories are genera, epithets refer to species. That is taxonomy. Nouns are generic names, nouns plus epithets are species names. That is nomenclature. Common knowledge is made of taxonomy and nomenclature.
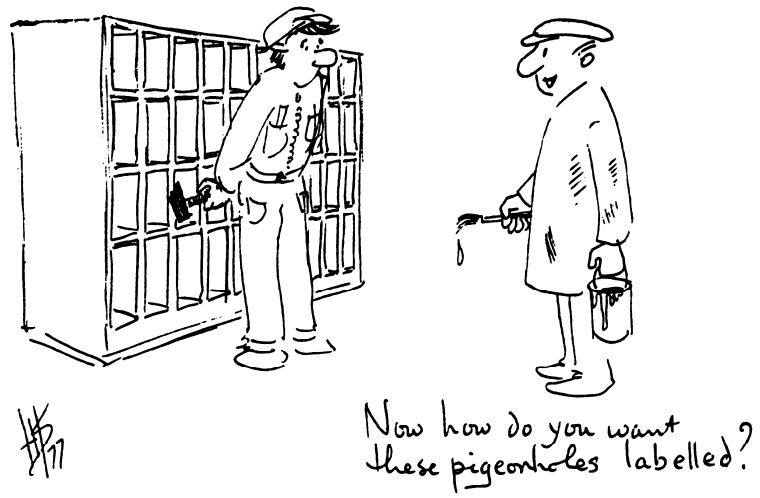

Fig. 5. Taxonomy and nomenclature in the old time (by an unknown mycologist)

The first approach of fungal biodiversity consists of taxonomy and nomenclature. The names of fungal species are, in the binomial nomenclature of Linné, a specific epithet combined with a generic name. For the stability of nomenclature, a reference specimen of the species, designated as type of its name and preserved under that name, guarantees the name application.

Classification is the direct product of taxonomy, by creation of categories of hierarchical rank: species, genera, families, orders, etc. When built on different criteria, classifications differ. Different classifications constitute different systems.

Identification is the recognition of an individual, a specimen, as belonging to a known species through its close similarity with the type or reference individual of the species, and application of the species name. If an individual does not belong to any described species, it represents a new species, perhaps a new genus, to be described, typified and named.

\section{TAXONOMY}

\section{A. Study of fungi starts in the field, by observation and collecting}

The first step of any mycological study is the observation of the fungi in the field. To teach this basic requirement, Dr Stanley J. Hughes, the first day 
after my arrival in Canada for a two-year postdoctoral stay (1960-1962), took me to the forest, in Gatineau Park, to collect microfungi, field shoes on feet, knife and basket in hands (Fig. 5).

Professor Richard P. Korf, who I visited at Cornell University in 1961 for the first time of many, was doing the same with his students: no mycology in class or laboratory before a discovery of the fungi in the field (Fig. 6). The bonnet of the car could serve as a bench to sort the collections. But take care: some fresh polypores, like Ganoderma applanatum, exude drops of so active enzymes that within one hour time you may have to let repaint the bonnet.

Nathalie Buffin, my assistant, repeatedly went collecting, whether thermophilic fungi in hot springs from coal tips in the Borinage, South of Belgium, or nematophagous Arthrobotrys species, or coprophilous or semiaquatic hyphomycetes.

Mycology starts in, and proceeds from the field. It is necessary in the field to annotate the collected specimens with indication of the substrate, host, habitat, locality, collecting date, collector's name, and any other ecological information observable in the field. Necessary also is the microscope examination and the drawing of the fungus mounted when fresh, for, as I experienced, microscope slides are much better from fresh than from dried material. Drawings, made at the same magnification, and a written description on prepared format, help to attempt a first identification and apply a name, even a provisional one. Isolation of the fungus shall be made on different culture media, preferably by transfer of single spores, conidia or mycelial threads. The fungus is then labelled and registered with number and name. Finally, specimens are dried and preserved, together with microscope slides and dried cultures, in properly sealed and labelled envelopes in the herbarium (Hennebert 1968d).

\section{B. Building a mycotheque for experimental taxonomy}

The herbarium is the first part of the mycotheque. Specimens are generally disposed by name (genus and species), either taxonomically (classes, orders, families) or alphabetically throughout. Both taxonomic and alphabetic disposition are subjected to name changes, but the former is also dependent on the classification. I adopted the alphabetical order. Packets are disposed like cards in boxes, what proved more flexible and space-saving than packets glued on herbarium sheets in folders, which I first used. Each set of specimens of the same species is separate and headed by a color card with the species name. Five suggestive colors were selected for species cards, grey for Myxomycetes, blue for Oomycetes, violet for Zygomycetes, red for Ascomycetes, brown for Basidiomycetes and green for Deuteromycetes, providing information on the class of the fungi. 


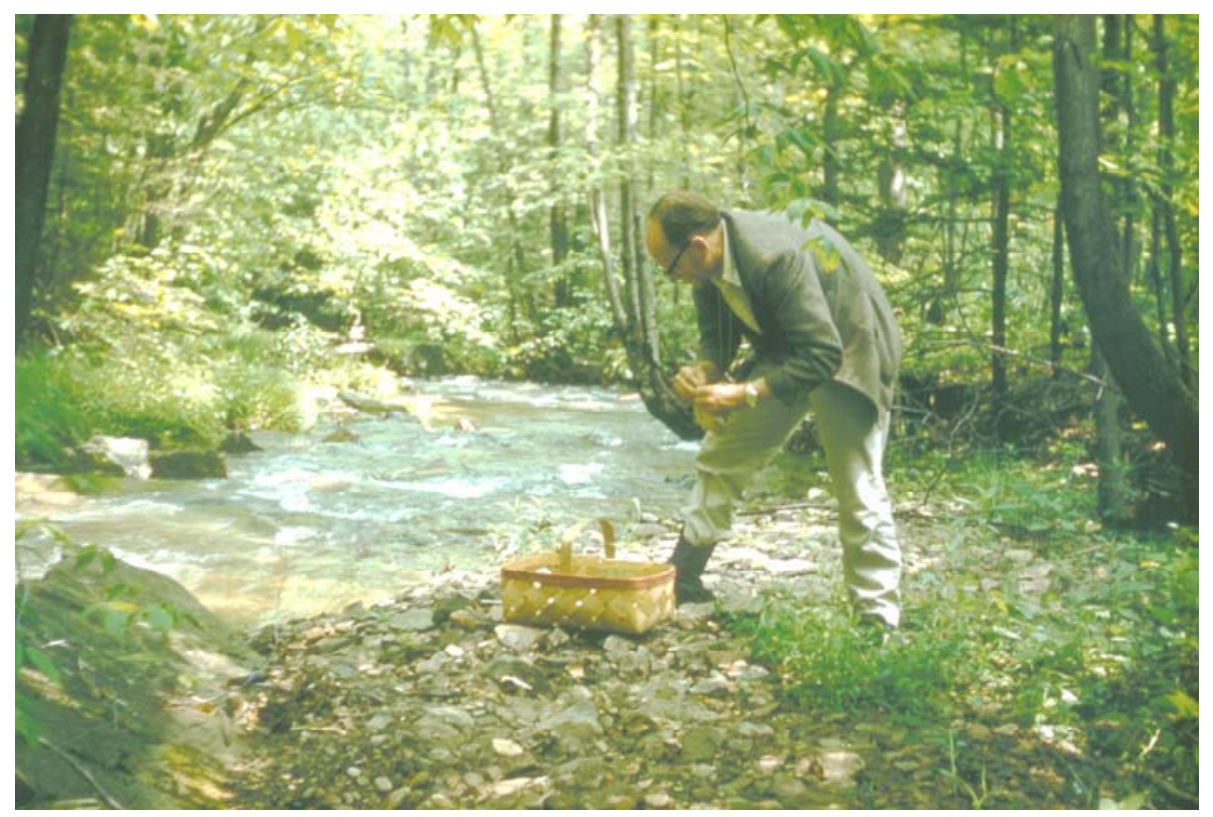

Fig. .6. Dr Stanley Hughes on collecting fungi in the Gatineau Park, near Ottawa, Canada, September 1960

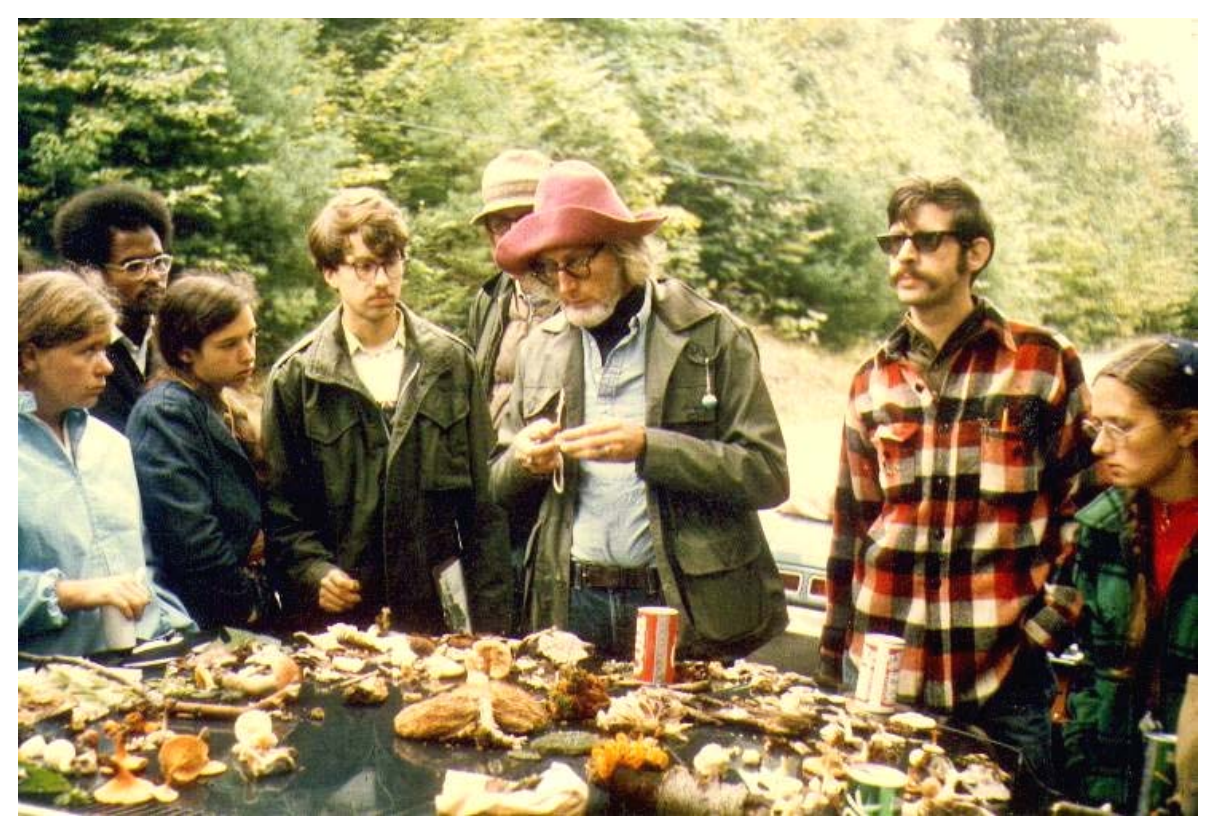

Fig. 7 Dr Richard P. Korf at the end of a collecting trip with students in a forest near Ithaka, N.Y., September 1961. 

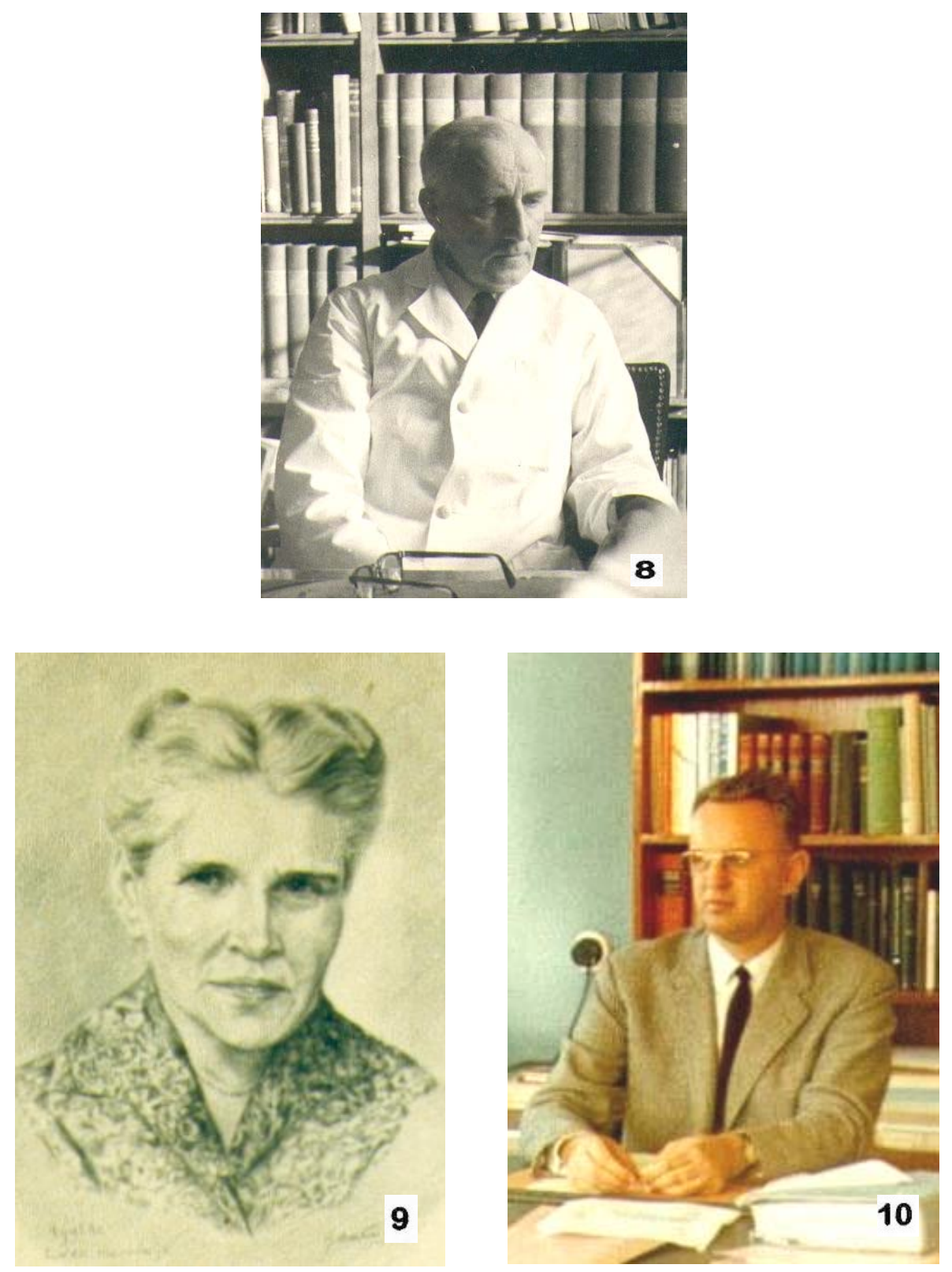

Fig. 8. Pr. Fabritius N. Buchwald, Copenhague, DK; 9. .Dr. Agatha van Beverwijk and 10. Pr. J.A. von Arx, successive directors of CBS, Baarn, NL (photo 9 of a drawing by Dr. G.A. de Vries). 
The collection of living strains is the second part of the mycotheque. Living cultures are disposed numerically and preserved in three different ways: on agar slants in two or more culture media under mineral oil, some also fresh and under water; lyophilized when sporulating; and cryopreserved at $140^{\circ} \mathrm{C}$. Much research was carried out to select the optimal cooling speed and the cryoprotectant medium in lyophilisation and cryopreservation (Hennebert 1987a, 1987c, 1987d, Berny and Hennebert 1988, 1989a, 1989b, 1990a, 1991a).

Strain record file $\quad$ Species record file Alternative record file

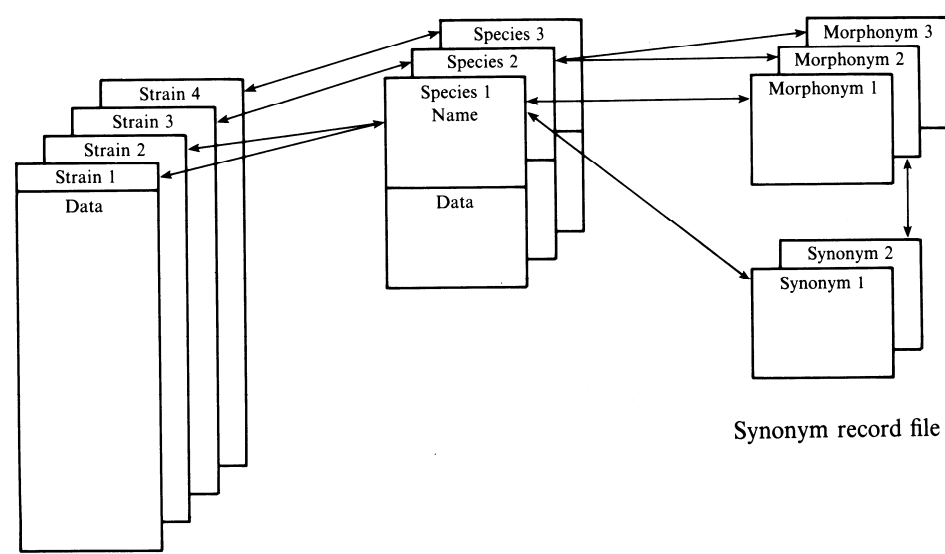

Fig. 11. The database is a set of four linked data sets, specific names and data, strain data, morphonyms and synonyms.

The register and the database make the third part of the mycotheque. All fungi collected are registered with a unique serial number. The number is applied to the specimen, the label, the slides, the drawing, the photographs, the descriptions, the living and dried cultures, the computed data set and the printed data sheet.

The numerical registration allows one to know the last number applied. Beside the numerical register, an alphabetical card system records the names and useful synonyms of the species with the numbers of the living strains represented in the collection. From 1985 onwards, computerization of the collection made the card system, not the handwritten register, obsolete. Strain and species data are recorded in the database, following a standardized scheme of data (Fig. 11) (Gams et al. 1988, Hennebert 1994a). The database allows recording and retrieval of the data, management and statistics of the mycotheque and the publication of the catalogues of strains (Hennebert et al. 1989, 1992).

The iconotheque is the fourth part of the mycotheque. It contains all drawings, descriptions and computerized datasheets of the collected fungi. The drawings are made at 1700x (Fig. 12), sometimes also $680 \times$ or $170 \times$ 
magnification from natural material and, often only, from culture material. The iconotheque includes photographs as well.

An alphabetical index of the fungal names supported by a mycological library makes a fifth part of the mycotheque. This alphabetical index with bibliographic reference was gradually compiled from diverse indexes, from Streinz's Nomenclator Fungorum (1862), Saccardo's indexes, Petrak's indexes, to the most recent $\mathrm{CAB}$ Index of fungi. The compilation greatly helped bibliographical research.

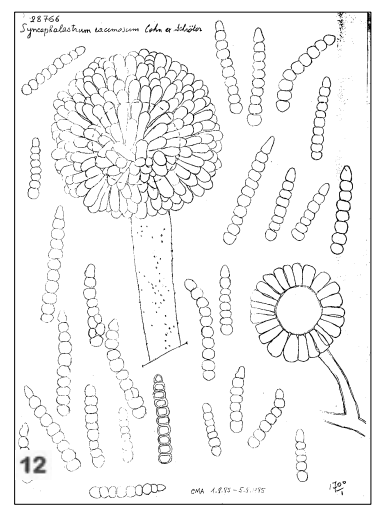

\section{C. Difficulty of interpreting old literature and tracing the type}

Many names of fungi were created during the last two and half centuries. The interpretation of the ancient authors before 1900 and of their taxonomy remains uneasy. To interpret correctly old descriptions, it is necessary to - understand Latin,

- be aware that fungi were described from nature, not in culture,

- take into account the tools of observation in current use at the time,

- know the precise meaning of descriptive terms and taxonomic criteria used at the time,

- know the value of measurement units of the period,

- remember that the rules of nomenclature applied were those of Linné until 1867, with some possible deviations peculiar to certain authors, and those of De Candolle and successors after 1867.

Tracing the type

In each case, tracing and carefully examining of the type, paratype or authentic material preserved in the author's herbarium or other herbaria is a requirement to make an enlightened interpretation of the description. Sometimes the type material is to be found, far away in a lost box above a herbarium cabinet, or covered with dust and mouse frass under the roof of a long deceased mycologist's house.

\section{Necessity of type examination}

Dr S. J. Hughes proposed to interpret adequately a two-line description like that of Wallroth's Balanium (Fig. 13) (Wallroth 1833). The type material of was necessary and made possible an adequate description in modern terms (Hughes and Hennebert 1961). At the same to justify the name Oedemium didymum (Schmidt) Hughes as correct for Botrytis didyma Schmidt 1817, required looking through one century of mycology and finding up to 5 synonymous genera and 16 synonymous species (Fig. 15, 16). Among the species 

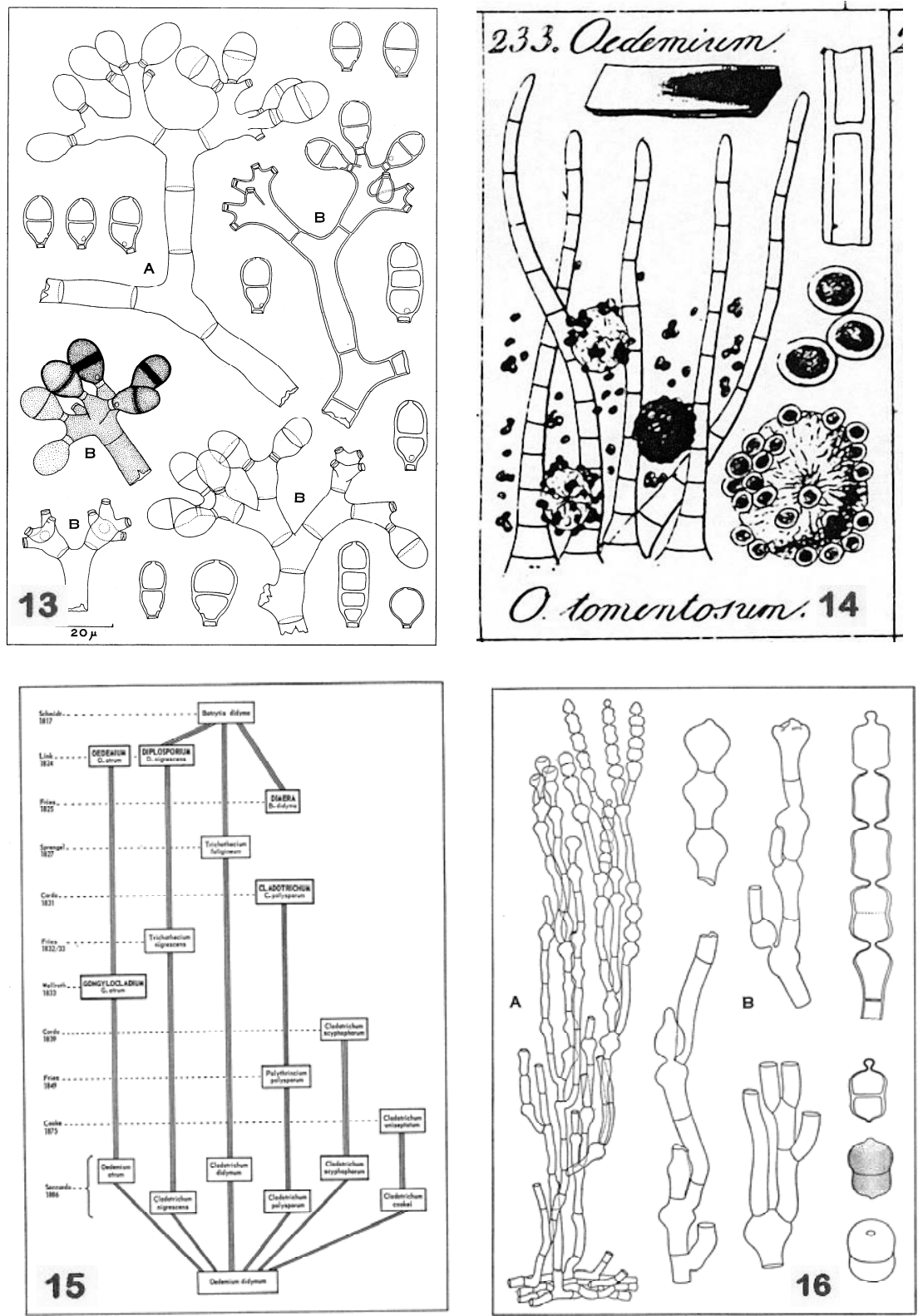

Fig. 13. Balanium stygium Wallroth; 14. Oedemium didymum, as O. tomentosum Corda after Corda 1837 and his type material; 15 . Synonymy of Oedemium. didymum: five generic and seven specific names; 16. Oedemium didymym (Schm.)Hughes. 
investigated, Oedemium atrum Corda was described and illustrated by Corda (1828) while he was only 19 years old. Corda's data could be interpreted only by examination of the type material, fortunately preserved in Prague. The specimen was composed of dematious sterile fungal setae mixed with globules that Corda (1833) recognized of heterogenous nature, being insect frass containing Penicillium conidia, three elements described by Corda as part of the fungus (Fig. 14). The decision to declare the name Oedemium atrum a nomen confusum could not have been undertaken without an examination of the type (Hughes and Hennebert 1963).

Iconotypes and neotypes

Dierckx (1901), student of Biourge, published in a short 6-page note, 22 new species of Penicillium that he had fully described and illustrated from pure culture, in a handwritten thesis. Unfortunately, when Dierckx went travelling in Indonesia, all his cultures were lost with the exception of $P$. griseo-roseum. On his return, he attempted to recover the species. Not very successful, he left notes and drawings to Biourge who succeeded to recover 20 of Dierckx 22 new species, and re-described them in his monograph of Penicillium (1923). In 1979, during an exhibition commemorating Biourge's microbiological carrier, a member of his family let us have a box from under the roof of Biourge's house containing the three plates of Dierckx's drawings (Fig. 17, 18, 19), but the handwritten thesis remains lost. The drawings are now considered as type material (iconotypes), which support Biourge's selected neotype cultures for Dierckx's species of Penicillium (Hennebert 1979, 1985).

\section{Guidance of the protologue}

If no original material can be traced, the originally published description, illustration and comments about habitat, locality etc., constituting the protologue, are the only data available to select a type. Any contemporary or later interpretation of the description of the taxon, and any application of its name by another author, should be regarded with caution.

Ostracoderma pulvinatum Fries, the only species of the genus Ostracoderma Fries 1825 (Syst. Orb. Veg. 1:150), was collected in Sweden and later recognized by Juel (1920). The fungus matched perfectly Fries's protologue of $O$. pulvinatum 1829 (Syst. mycol. 3: 214, hereunder) for which no type is available

\section{OSTRAGODERIIA pulvinatum.}

Sparstum ant gregarium, circomscriptum, rotundato - hemisphasricum, magnitudine varium, $\frac{1}{2}$ enc. circiter latnum. Peridism crustosum, sed tenuissimum et fragillimum, Jaeve, glabrum, abidum, in medio collabens et evanescens. Sporidia minuta, glowosa, aroillacea, laxe coacervata, jpsi humo incumbere videntur. In sillis, locis detsis, ail teman

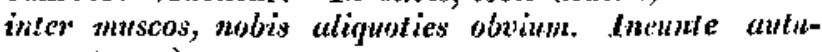
mint. (,.,.$)$ 


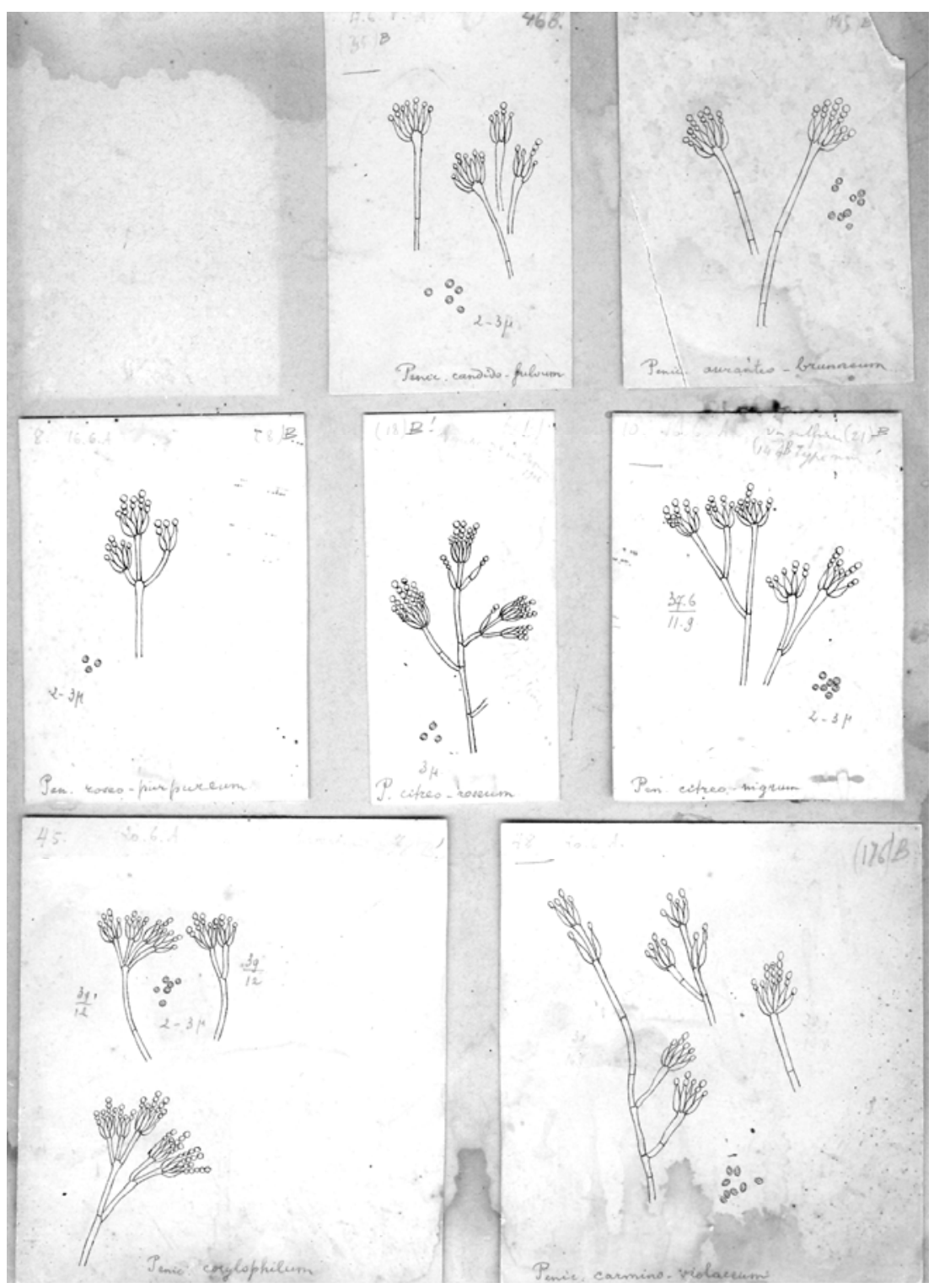

Fig. 17. Orignal Dierckx' drawings of Penicillium species (1901): from left to right, $1^{\text {st }}$ row, (first drawing lost), P. candidofulvum Dx., P. aurantiobrunneum Dx.; $2^{\mathrm{d}}$ row, P. roseopurpureum Dx., P. citreoroseum Dx., P. citreonigrum Dx.; $3^{\mathrm{d}}$ row, $P$. corylophilum Dx., P. carneoviolaceum Dx.. 


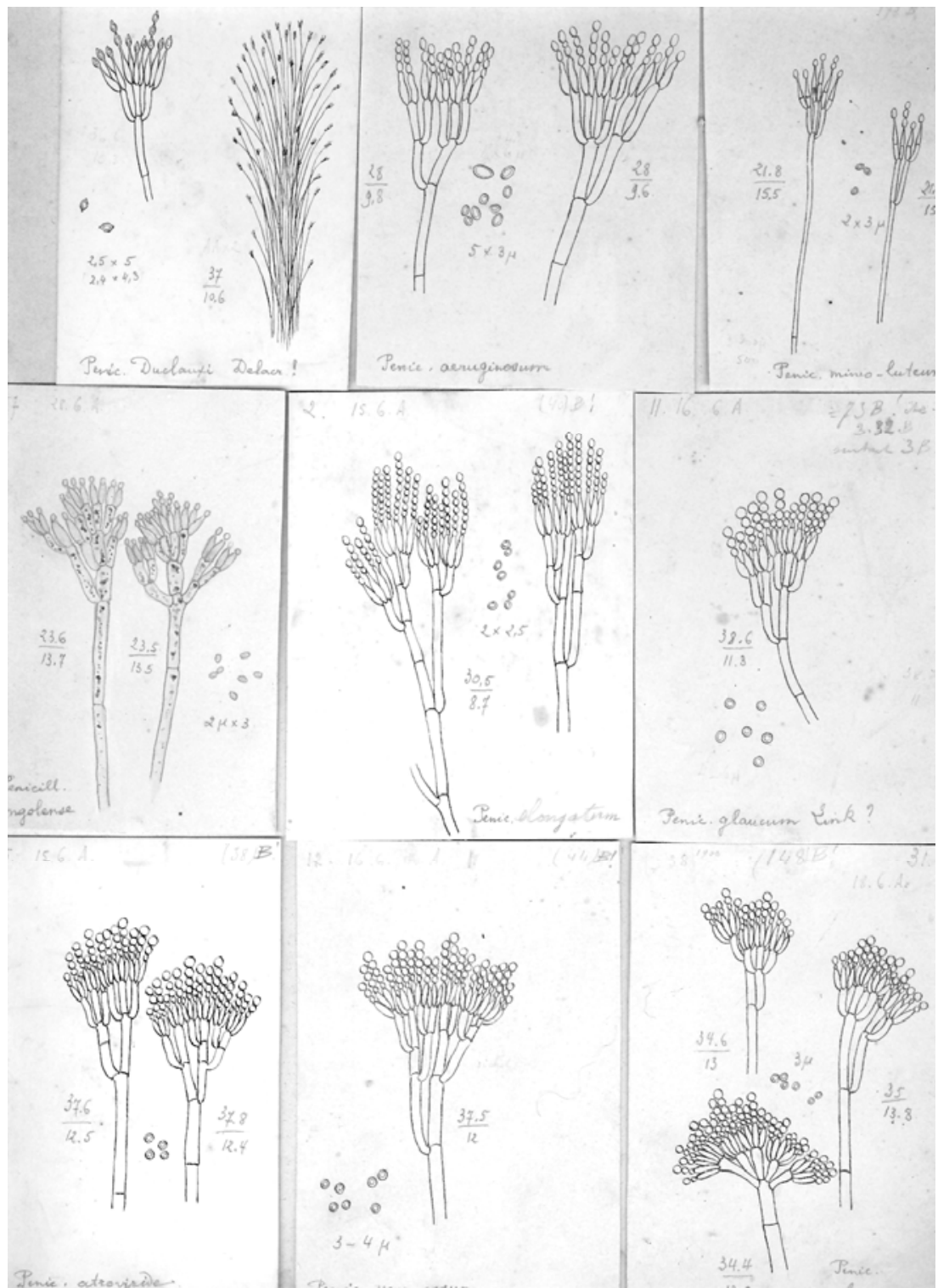

Fig. 18. Original Dierckx' drawings of Penicillium species (1901): from left to right, $1^{\text {st }}$ row, P. duclauxii Delacroix?, P. aeruginosum Dx., P. minioluteum Dx.; $2^{\mathrm{d}}$ row, $P$. congolense Dx., P. elongatum Dx., $P$. glaucum Link?; $3^{\mathrm{d}}$ row, P. atroviride Dx., P. verrucosum Dx., P. griseobrunneum Dx.. 


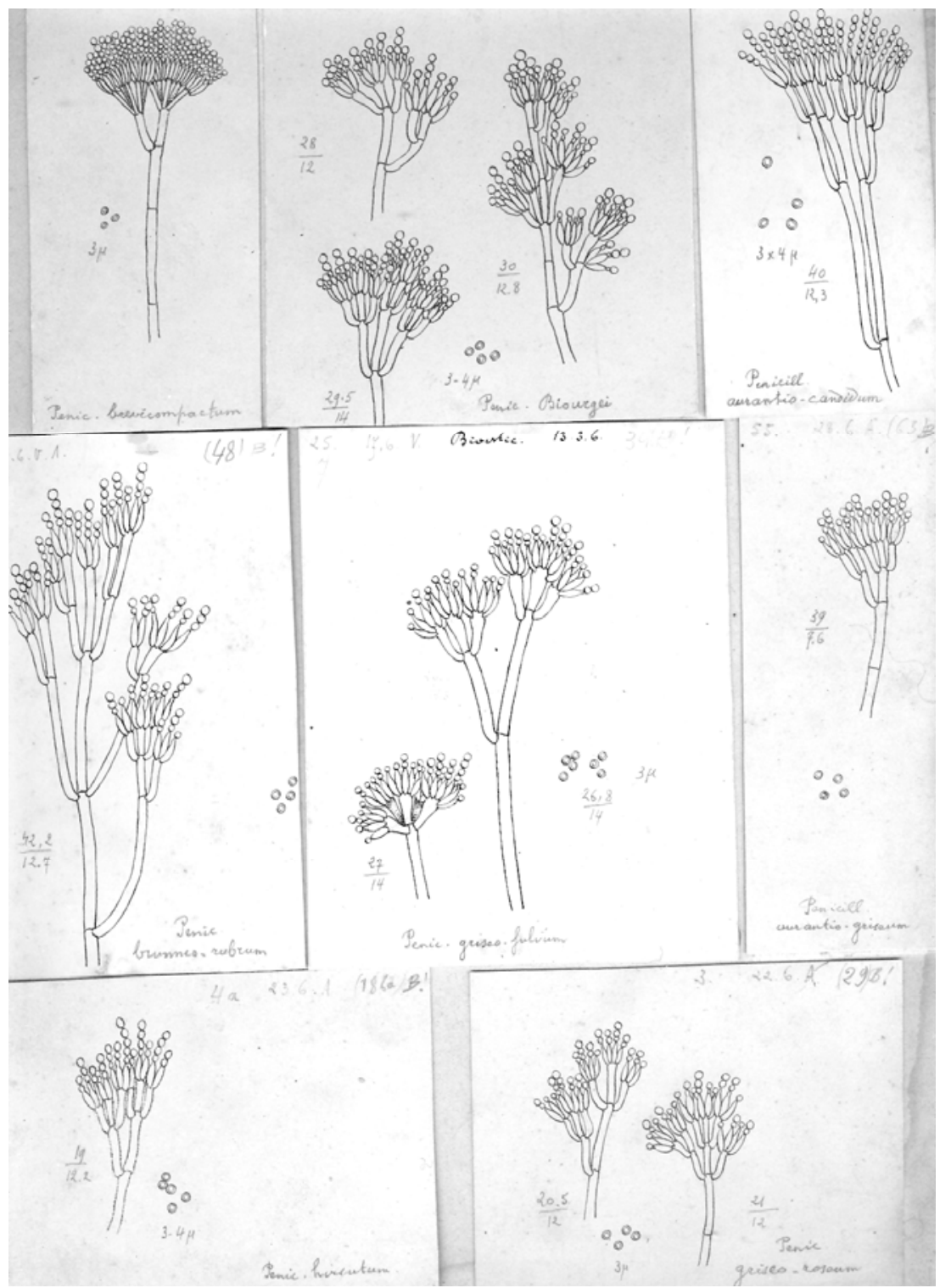

Fig. 19. Original Dierckx' drawings of Penicillium species (1901): from left to right, $1^{\text {st }}$ row, $P$. brevicompactum Dx., P. biourgei Dx., P. aurantiocandidum Dx.; $2^{\mathrm{d}}$ row, $P$. brunneorubrum Dx., $P$. griseofulvum Dx., P. aurantiogriseum Dx.; $3^{\mathrm{d}}$ row, $P$. hirsutum Dx., $P$. griseoroseum Dx.. 
Juel considered the fungus conspecific of Hyphelia terrestris Fries 1849 and therefore synonymized Ostracoderma with Hyphelia Fries 1825. But Hyphelia Fr. is not available because of misapplication of his type species name (Hughes 1958). In another hand Hughes (1958) synonymized Chromelosporium Corda 1833 with Ostracoderma Fries 1829, but did not consider the absence of a "crustose peridium" in the former, clearly indicated in Fries's protologue of Ostracoderma pulvinatum, giving the fungus the appearance of a Myxogaster ("Facile igitur crederes esse Myxogatrem") and also observed by Juel. This feature forced the rejection of Hughes' synonymy, although conidiogenesis in both genera is similar, and the distinction of Chromelosporium and Ostracoderma (Hennebert 1973).

\section{The type locality}

In absence of any original, type or authentic material of a fungus, but guided by the published protologue, one last resource is to go collecting the fungus anew in the type locality. When the locality is not destroyed, the fungus can be found unless it is of rare occurrence. I discovered Arachnophora fagicola Hennebert (Fig. 26) from a few conidiophores only, just enough to

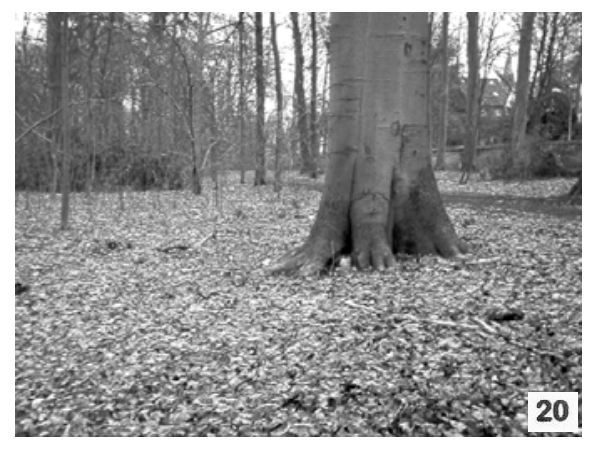
prepare a microscope slide, on a cupule under a beach tree in Parc d'Arenberg, Heverlee, Belgium (Fig. 20). Later wanting to provide sufficient type material for herbarium deposit in view of publication, I had to examine eight thousand beach cupules from the same locality to finally have eighteen minute colonies of that new fungus to serve as a type (Hennebert 1963a).

\section{Tracing the type host}

The same conduct is to be followed when a living culture is wanted for reconsideration of a rare species. Seaverinia geranii (Seaver and Horne) Whetzel (1945) had been found on Geranium maculatum in localities of New York and Wisconsin States five times in 50 years, successively by Seaver, Davis, Whetzel, and Korf. Ten years after the last collection by Korf, in the forest along the Cayuga Lake near Ithaca, I went collecting on the same spot and found the species (Fig. 21). Fresh single ascospores could cultivated and yielded the verrucose conidial state Botrytis geranii Seaver. After a proposal by Buchwald (1949), I redisposed the conidial state in the new form-genus, Verrucobotrys Hennebert, as

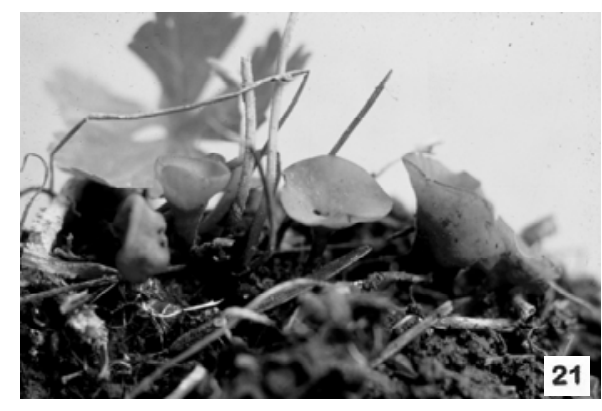


Verrucobotrys geranii (Hennebert 1973).

\section{Ex-type cultures}

Sometimes, natural type or authentic material is lost, either not deposited in herbarium, or destroyed, but ex-type cultures are preserved in culture collections. Should a taxonomist describes or re-describes a fungus from culture, he should then deposit it in a dried state in herbarium. So did I with Wardomyces columbinus (Fig. 37) and Asteromyces cruciatus (Fig. 27) (Hennebert 1962), of Custingophora olivacea (Fig. 28) (Stolk and Hennebert 1968), of Lomentospora prolificans (Fig. 40) (Hennebert and Desai 1974), and many others isolated from soil (Hennebert 1968a, 1968c, 1969). Conversely, in order to spare type material in herbarium, it is recommended to deposit living ex-type isolates in culture collections (Banno et al. 1993, 1994).

\section{Tracing earlier names}

Many taxonomists dare to describe and publish rapidly a species they consider as new, taking the risk to see it synonymized soon after. To be certain that the species has not yet been described or the name already used, before publishing a new species or a new name, a full survey of mycological literature and indexes is necessary.

A Wardomyces-like fungus isolated from soil in Belgium and considered a new species was also isolated from soil in the Netherlands. After screening the available literature for some times, I prepared a description of the new species for publication, when I received a reprint from Subramanian describing the fungus as Adoghamina ruchira Subramanian n. gen. n. sp. Two weeks later I found Barron's description of the same fungus as Gilmaniella humicola Barron n. gen. n. sp., preceding by a few weeks the publication by Subramanian. The synonymy remained to be published (Hennebert 1968b).

Nathalie Buffin collected an interesting white arbuscular hyphomycete bearing cylindrical conidia and forming white patches on semi-immersed dead leaves at a pond in the Lauzelle Forest, Louvain-la-Neuve (Fig. 23). We though the fungus to be undescribed. We composed a descriptive name, Cylindrodendrum album. Checking for a possible earlier use of the name in several indexes, we found that the name had been used already by Bonorden (1851) (Fig. 22) which description and illustration were matching our fungus exactly. There is no type material left of Bonorden's species, but a search in other herbaria revealed that the fungus had been collected twice in 120 years after Bonorden. Our collection was désignated neotype (Buffin and Hennebert 1984). 

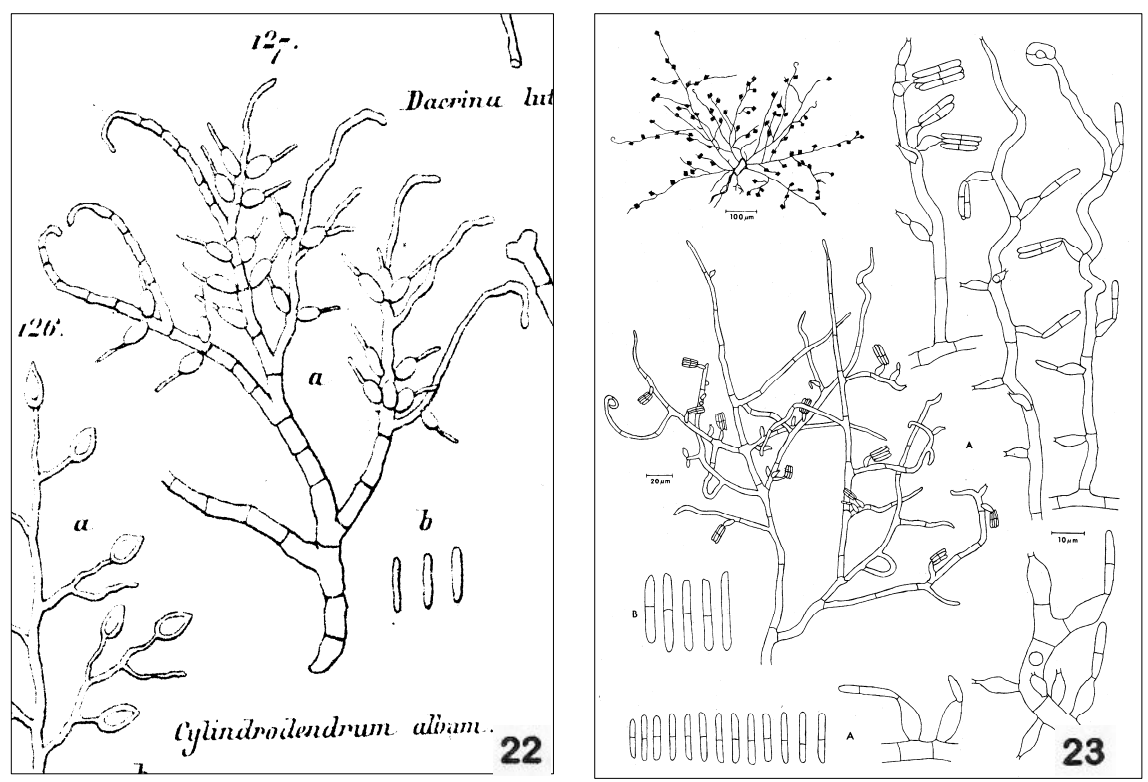

Fig. 22. Cylindrodendrum album Bonord. original drawing; 23. Drawing made by N. Buffin from fresh collection and culture.

\section{Publishing rapidly}

Even after searching for guarantee of the novelty, it is important to publish rapidly. As very few journals were editing manuscripts with a delay shorter than 6 months, Richard P. Korf and I decided to edit a new journal offering only a short delay together with quality, designed to promote the taxonomy of fungi and lichens (Hennebert and Korf 1974). Created in July 1974, Mycotaxon has published, in 1995, 67 volumes of around 540 pages each, about 36000 pages in 24 years 6 .

\section{D. Evolution of taxonomic criteria from morphology to morphogenesis}

Taxonomy of fungi evolved greatly after Linné with development of tools for a finer observation and characterization of life. New characteristics of fungi are tested as taxonomic criteria. That evolution requires constant adaptation by the taxonomist.

It does not mean that traditional criteria such as static descriptive morphology are abandoned. To the contrary, they make the basis of present taxonomy, but enriched by morphometry, morphogenesis, sporogenesis, anatomy, physiology, biochemistry and genetics.

It is possible to distinguish four periods in the evolution of taxonomical criteria: a first period of static morphology from field material, a second

${ }^{6}$ Mycotaxon published its $100^{\text {th }}$ volumes in 2007. 
period enriched by morphogenesis in experimental mycology (pleomorphism, sexuality, culturing), a third period aided by physiology and biochemistry, and a fourth, the current period, revised by genetics and phylogeny.

From Linné (1753), through Tode, Persoon, Link, Fries, Corda, Wallroth, Fresenius, Fuckel, De Bary, R. \& F. Tulasne, Saccardo and many others until the 1850-1880s, taxonomic criteria in fungi were merely morphological and static, based on the observation of natural material and evolved from macromorphology to micromorphology with the development of the microscope.

Then morphogenetic criteria were added owing repeated developmental observations in nature and lately helped by use of aseptic culture on liquid substrates by Pasteur (1866) and on solid substrates by Brefeld $(1875,1881)$

Presumed sexually functioning organs in fungi were observed by Ehrenberg (1820) in Zygomycetes, by Léveillé (1837) who described the hymenium of Basidiomycetes, by Tulasne (1856), who first described "spermatia", and De Bary (1863) who observed the "carpogone" and the "pollinode" in the Ascomycetes. Brefeld (1872) observed sexual fusion in Zygomycetes and other fungi. Stahl (1877) was the first to follow the fusion of spermatia with the ascogonial hypha he called "trichogyne" in Ascolichens. Blakeslee (1904) made the fusion of heterothallic partners in Zygomycetes. These discoveries led to the differentiation of sexual sporogenesis from conidiogenesis.

Fresenius (1850) already described the conidiogenesis of

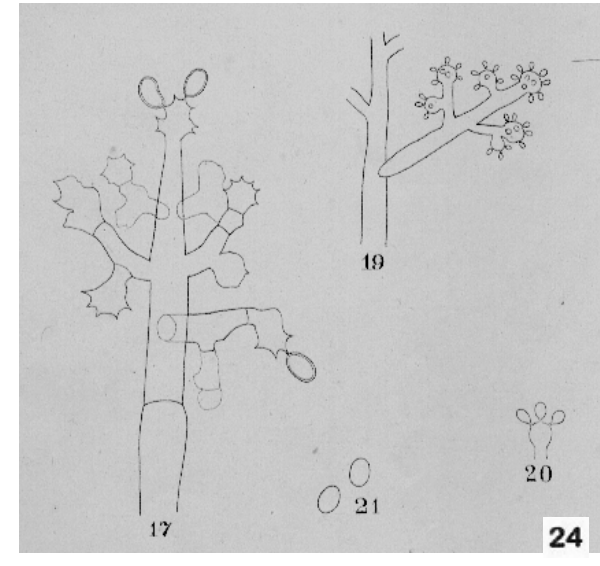
Botrytis cinerea (Fig. 24). The development of several kinds of secondary spores, or conidia, in addition to spores suspected to be sexual, led L. Tulasne (1851) to define pleomorphism in fungi. Numerous examples of this pleomorphism were illustrated in the Ascomycetes by L. Tulasne and C. Tulasne in the Selecta Fungorum Carpologia (1861-1865) and in the Basidiomycetes by De Seynes (1874) and in all groups of fungi by Brefeld (1872-1912).

Availability of the fungi in living culture greatly favoured the observation of pleomorphism and conidiogenesis and its use in taxonomy. An early example of the advantage of cultures was the morpohogenetic study of several Penicillium species made in vitro by Brefeld (1874).

More recent mycologists, Vuillemin (1910), Mason (1933), Hughes (1953) and Tubaki (1958), defined and described different types of conidiogenesis, 
and used them as criteria for generic distinction of asexual fungi. Most of them were confirmed by electron microscopy (Samson and Cole 1970).

\section{E. Development of morphogenetic criteria: the unitary parameters of conidiogenesis}

The conidiogenetic system of Hughes (1953) and Tubaki (1958) guided my descriptions and illustrations of the conidial fungi. On basis of conidiogenesis, were excluded from the genus Botrytis, species with blastic synchronous conidia but dissimilar from what was called the Botrytis cinereatype (Whetzel 1945), belonging to Chromelosporium, Haplotrichum, Mycotypha, Nematogonium and Ostracoderma, species with sympodial blastic conidia belonging to Beauveria, Calcarisporium, Cephalotrichum, Conoplea, Costantinella, Hansfordia, Nodulisporium, Rhinocladiella, Rhinotrichum and Virgaria, species with phialidic conidia referable to Verticillium, species with retrogressive conidia as in Cladobotryum and species with arthric conidia which were disposed in Geomyces (Hennebert 1960a).

At the 1st Kananaskis conference on the Taxonomy of the Fungi Imperfecti (Kendrick 1971), advances were made in the understanding of conidiogenesis. Several new terms proved to be useful: among them, "holoblastic" and "holoarthric", "enteroblastic" and "enteroarthric", that I proposed to characterize the ontogeny of the conidia in relation to the wall of the conidiogenous cell. Also emphasis was given to the ontogeny of the conidiophore itself, beside that of conidia (Pirozynski 1971). Considering the conidiophore also, I segregated several form-genera among the Botrytis-like species (Fig. 25). A first group of them is linked, or presumably so, to operculate Discomycetes, order Pezizales, distinct from another group linked to the inoperculate Discomycetes, order Helotiales, family Sclerotiniaceae. In Pezizales, the new form-genus Dichobotrys Hennebert (Fig. 25-5) was described for the conidial form of Lamprospora, Sphaerosporella, and Trichophaea species, while two species of form-genus Chromelosporium Corda are conidial states of Peziza species. Botrytis-like form-genera presumably related to operculate discomycetes, Pulchromyces Hennebert (Fig. 25-7) and Phymatotrichopsis Hennebert (Fig. 25-8) were described as new, beside the "peridial hyphomycetes" Ostracoderma Fries and Glischroderma Fuckel (Fig. 25-10) (Hennebert 1973, Hennebert and Korf 1975, Bellemère and Hennebert 1979, Sutton and Hennebert 1994, Korf 1994).

In Sclerotiniaceae, distinctive features of conidiomata were enhanced as generic criteria to discriminate, beside Botrytis sensu stricto, three distinct new form-genera, Streptobotrys Hennebert (Fig. 25-2) for the twisted conidioma of Streptotinia, Amphobotrys Hennebert (Fig. 25-3) for the dichotomous conidioma of one Botryotinia species and Verrucobotrys Hennebert (Fig. 25-4) for its verrucose conidioma of Seaverinia (Hennebert 1973). 


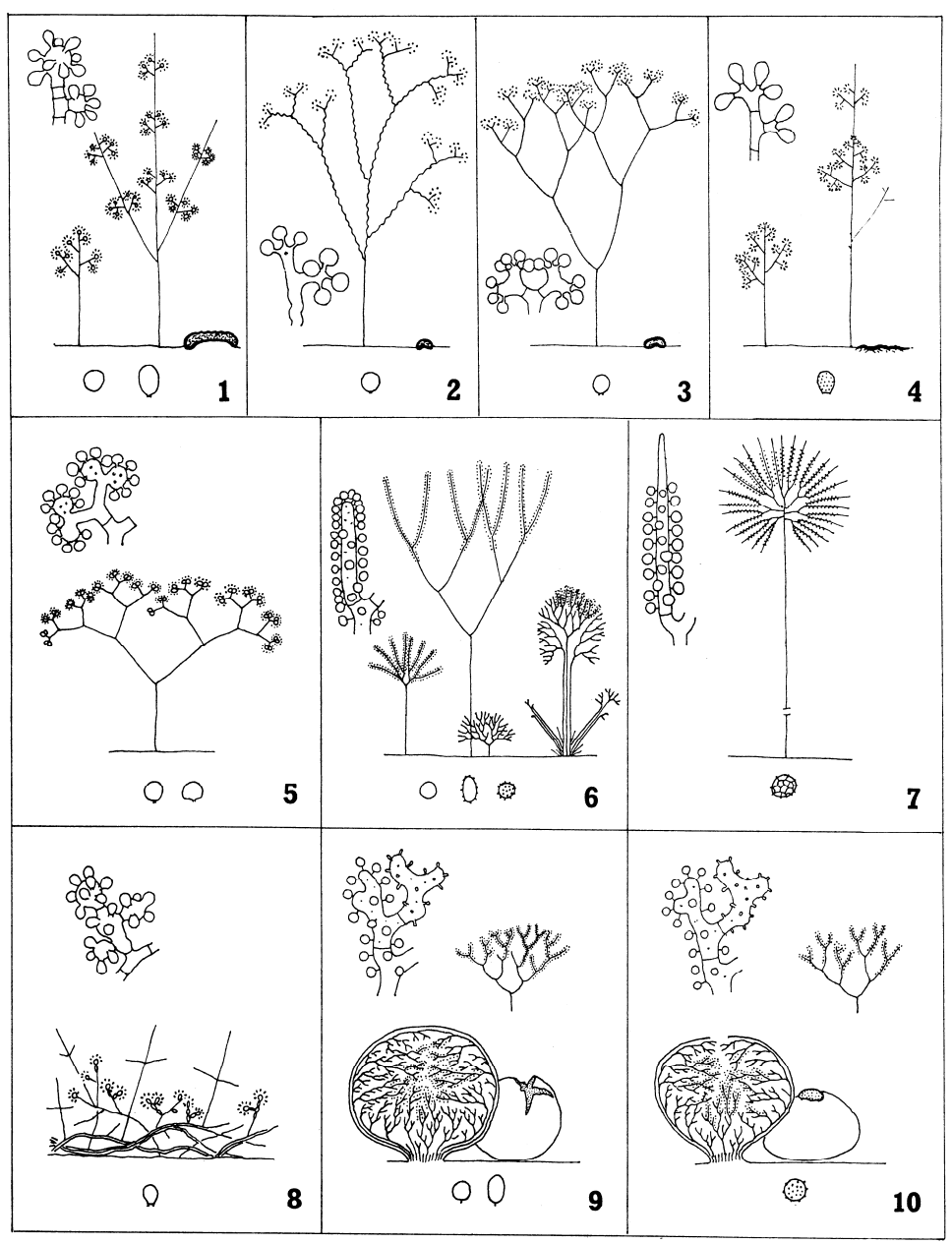

Fig. 25. Diagrammatic illustrations of coniophomata of 1. Botrytis; 2. Streptobotrys; 3. Amphobotrys; 4. Verrucobotrys; 5. Dichobotrys; 6. Chromelosporium; 7. Pulchromyces; 8. Phymatotrichopsis; 9. Ostracoderma; 10. Glischroderma.

However, the more conidial fungi were examined, the more intermediate categories of conidiogenesis were discovered: Wardomyces and Asteromyces species have solitary blastic or thalloblastic conidia born in basipetal succession on the conidiophore (Hennebert 1962), Arachnophora fagicola (Fig. 26) has solitary blastic conidia borne successively trough percurrent proliferation of the conidiophore (Hennebert 1963a), Sporoschismopsis moravica (Fig. 29) produces successive arthric conidia borne in percurrently successive phialides (Holubova-Jechova and Hennebert 1972). 

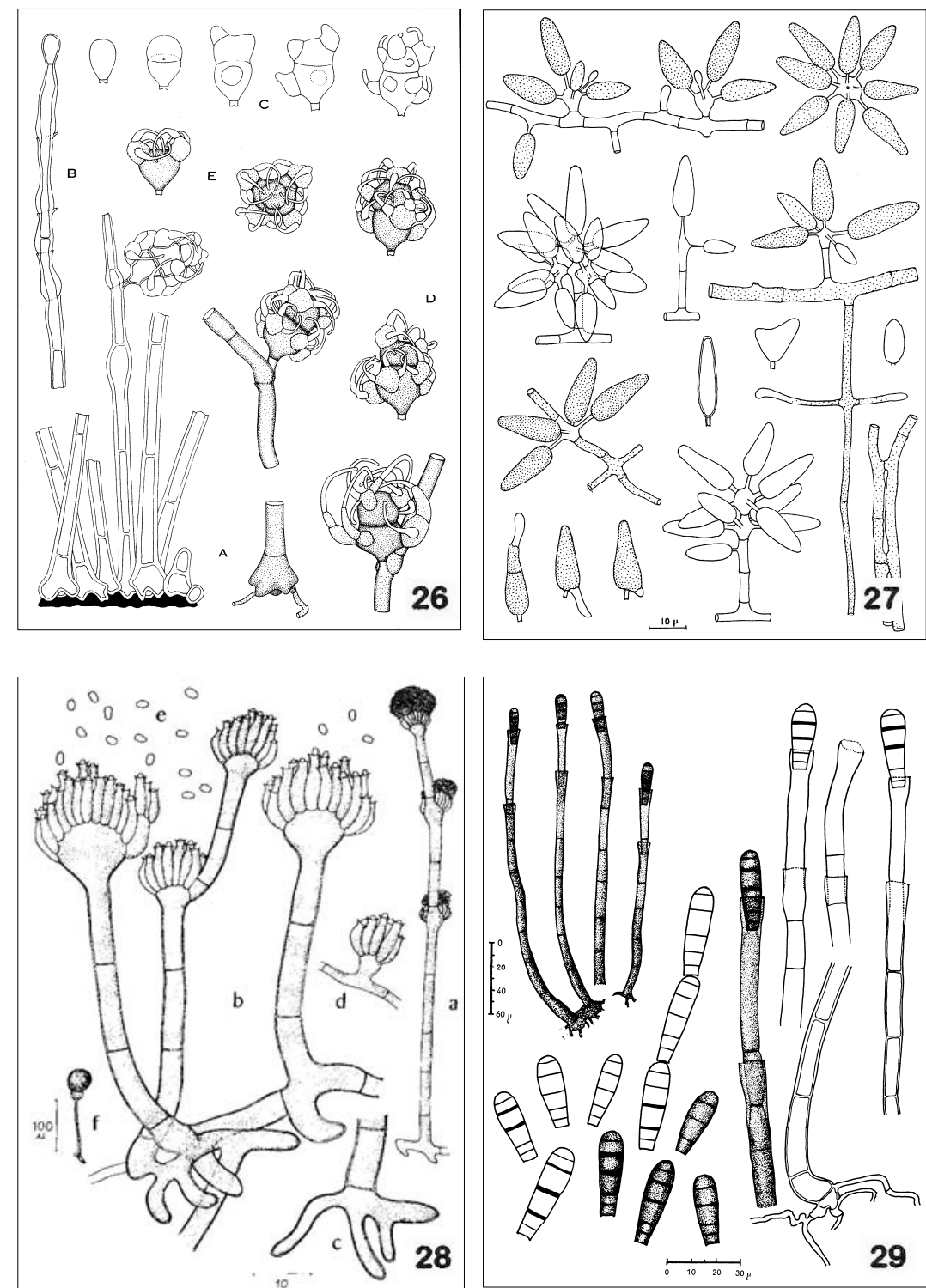

Fig. 26. Arachnophora fagicola Hennebert; 27. Asteromyces cruciatus F.-Me. Moreau ex Hennebert 28. ;Custingophora olivacea Stolk, Hennebert \& Klopotek; 29. Sporoschismopsis moravica Hol.-Jech. \& Hennebert

I thus progressively developed a more detailed, yet still unsatisfactory, scheme of conidiogenesis for my students (Hennebert 1982). Adressing the need for a finer analysis of conidiogenesis, I introduced the concept of unitary parameters acting together or successively in each specific conidiogenesis, instead of describing categories or types of conidiogenesis (Fig.30). 


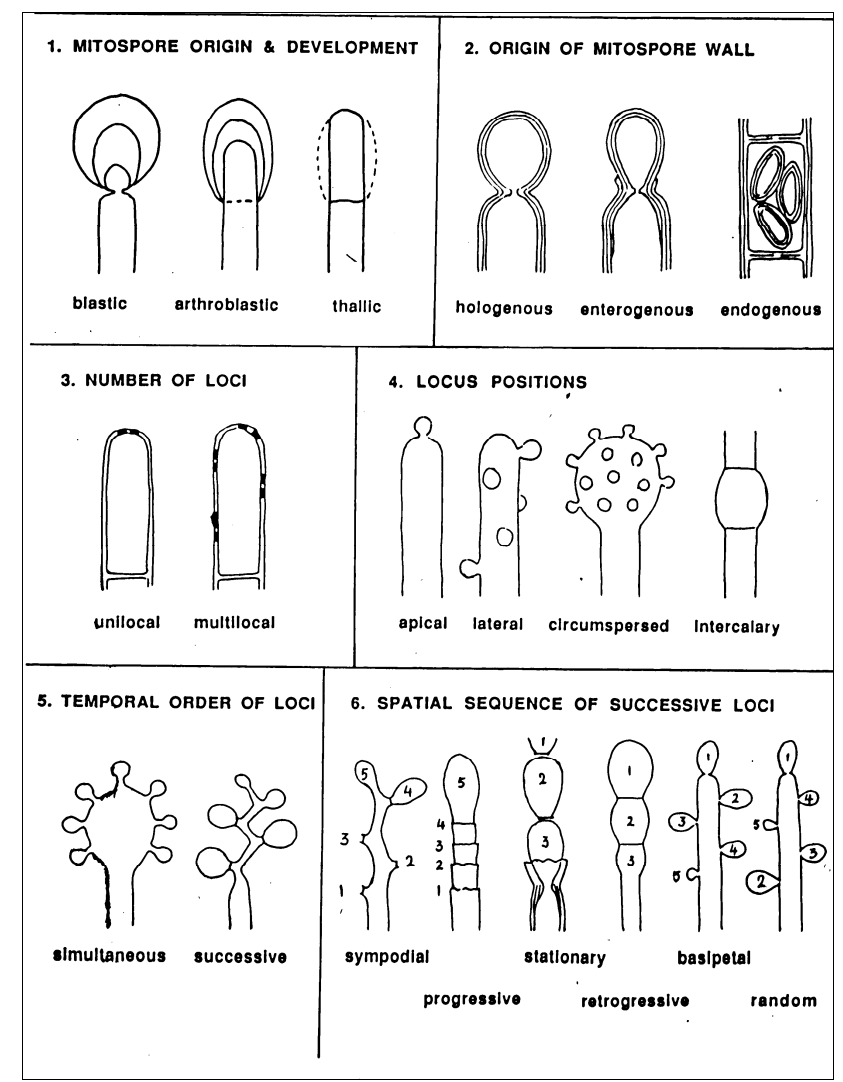

Fig. 30. One of the plates figuring some of the parameters, the combination of whichh shall define a conidiogenic pattern (Hennebert in Hawksworth 1994b)

The parameters are numerous and should be defined under a univocal descriptive terminology. A first and tentative analysis of a non-exhaustive number of unitary parameters of conidiogeneis was presented at the Ascomycetes Conference in Paris in 1993 (Hawksworth 1994b). When eventually fully refined, the unitary parameters should allow the characterization of any conidial fungus and hopefully, when computerized, serve as a basis for an expert identification system of conidial fungi (Hennebert and Sutton 1994).

\section{F. Value of cultures in demonstrating pleomorphism and variations in fungi}

Like Biourge in 1892-93 who spent a year in both Pasteur and Hansen's laboratories learning the single cell culture (Hanssen 1886), I spent two years 
in Ottawa where Dr J. W. Groves taught me the single ascospore culture and the spermatization technique.

Single spore culture has been a great help in the separation of close species. I used single spore culture currently in Penicillium, and other genera for isolation of strains. Mass conidial colonies often sectorize, showing variants. Strains received for the collection, particularly ex-type strains, were regularly checked for homogeneity from 50 single spore cultures. In an attempt to rebuild the Biourge's Penicillium collection, type strains returned from other collections were found to contain up to 4 variants. Indeed, mutations occur during maintenance and preservation of ex-type cultures and selection of the original strain has frequently been necessary. In an experiment eight different variants were isolated from a homogeneous parental strain of Penicillium expansum following exposure to diverse preservation processes (Fig. 31) (Berny and Hennebert 1990a).

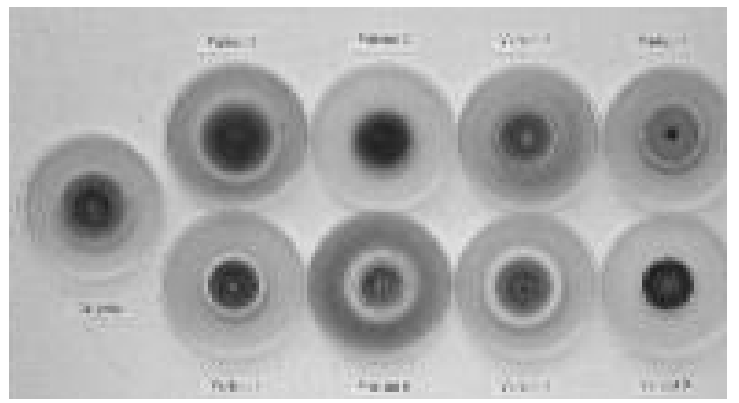

Fig. 31. Eight variants of Penicillium expansum wild strain (left) obtained from monospores after preservation processes.

Cultures help to demonstrate pleomorphism, as well as variability and mutability. Culture under diverse conditions allows the fungus to reveal itself in more facets than expected. An example is the dimorphism of many yeast and yeast-like fungi, even Saccharomyces cerevisiae, which turns filamentous under nitrogen starvation. Moniliella pollinis (Hennebert and Verachtert) De Hoog turned from arthric conidia to a sympodial budding yeast depending on the shaking speed in liquid culture (Dooms et al. 1971). Only through single spore culture, it could be demonstrated that spermatia belong to Botrytis cinerea.

Analysis of conidiogenesis In culture helped to reduce the taxonomy of the genus Botrytis to some thirty six species (Hennebert 1958, 1960a, 1963b, 1964a, 1966, 1969, 1973). Cultures also helped to demonstrate the sexualasexual connection. Through crossing single conidial isolates of Botrytis by spermatization of sclerotia, apothecia of heterothallic Botryotinia ficariarum and Botryotinia ranunculi could be obtained in pure culture (Hennebert and Groves 1963). Homothallic species like Botryotinia porri can fruit easily. Similar proof of pleomorphic connection In yeasts were also atteined through rDNA sequencing from cultures. 


\section{G. Classification of asexual fungi with sexual fungi}

With or without cultures, another way to infer taxonomic relationship of conidial fungi in absence of a sexual reproduction, is by analogy of their morphology. In Chromelosporium three species only, C. fulvum (Link: Fr.) McGinty et al.[C. ollare (Pers.) Hennebert] (Fig. 32), C. macrospermum Hennebert and C. trachycarpum Hennebert, are conidial states of Peziza species, but the type species $C$. ochraceum Corda and six other species are only presumed to be states of Operculate Discomycetes by analogy, although they had often been

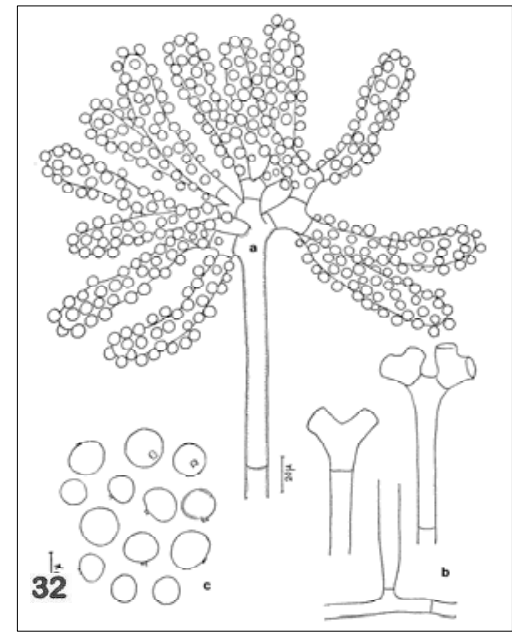
considered states of Basidiomycetes such as Hypochnus or Tomentella. At the same Phymatotrichopsis omnivora (Duggar) Hennebert is considered by some authors a state of Hydnum or Trechispora.

Ostracoderma Fr. and Glischroderma Fuck. are similarly grouped with conidial states of Operculate Discomycetes. A peridium, absent in the others, gives to Ostracoderma pulvinatum and Glischroderma cinctum Fuck. the external pulvinate smooth appearance of the myxomycete Lycogala. A closely related peridial fungus, Glischroderma cinctum Fuckel was described by Fuckel (1870) in the Gasteromycetes, and distinguished from Ostracoderma. Another 'peridial hyphomycete' with elliptical conidia, found by Bresadola, was named Lycogala torrendii as if a Myxomycete. It was renamed by Torrend (1913) Lycoperdellon torrendii and disposed in the Gasteromycetes. Heim and Malençon (1933), Heim (1934, 1949), Dissing and Lange (1962), Malençon (1960, 1964), Demoulin (1966) placed both genera in the Gasteromycetes and families were erected for them. Zeller (1948) rightly placed the fungi in the Deuteromycetes. Because of the analogy of the conidiogenesis with Chromelosporium states of Peziza species, following Donk (1962), I chose to dispose Lycoperdellon as synonym of Ostracoderma Fr. and with Glischroderma among conidial states of Operculate Discomycetes. Glischroderma cinctum and another undescribed species of Glischroderma Fuckel, with a glutinous peridium, were found by Korf and his students in the surroundings of Ithaca, NY. (Korf 1994).

The presumed position of Ostracoderma (= Lycoperdellon $)$ and Glischroderma amongst conidial forms of certified Operculate Discomycetes (Hennebert 1973) was strongly supported by the presence of ascomycetous septal pores demonstrated in SEM by Bronchart and Demoulin (1975). 
Ultrastructure is indeed another way approach relationships between conidial and sexual fungi and their phylogeny.

\section{H. Relative aid of additional criteria}

\section{Physiological characters}

After the promotion of physiological characteristics in yeast taxonomy by Guilliermond (1912), some additional characteristics, enzymatic activities, requirements in growth factors and tolerance to environmental conditions, were added. Those criteria are still in use (Berny and Hennebert, 1990b). In 1985, I initiated the miniaturization of all physiological tests of assimilation, fermentation, enzymatic activity, requirements and tolerances, using 96 well microplates read by optical densitometry. The method has greatly increased test rapidity. It was presented at the 8th International Symposium on Yeasts in Atlanta in 1992. Today, the microplate results, together with yeast morphological and genetic data, are integrated in the computer-assisted expert identification system BCCM/ALLEV-2 created by Vincent Robert at MUCL (Robert et al. 1994, 1997) and presented again as a poster at the MUCL centenary symposium (Evrard and Hennebert 1994) ${ }^{7}$.

\section{Biochemical characters}

The development of analytical methods enhanced extensive biochemical investigations on both filamentous and yeast-like fungi. However the new criteria are so far applicable to sufficiently large fresh biomass of fungi from culture, or nature. Their application to type material in herbaria is excluded. That is a handicap of the biochemical approach.

Dominant $\mathrm{Q}$ co-enzymes have become a good generic criterium and a good indicator of the generic homogeneity. Applied to Candida the genus was found heterogeneous and composed of four distinct groups (Vancanneyt et al. 1991b, 1994a).

Enzyme and protein profiles have provided taxonomic help at the species level and lower ranks (De Bertoldi and Hennebert 1977, Berny and Hennebert 1991b, Hennebert 1996b, Hennebert and Vancanneyt 1998). Whole cell protein profiles proved to be useful in confirming by maximum similarity the organic connection established otherwise between asexual and sexual strains in both ascomycete and basidiomycete yeasts. The same degree of similarity between unconnected anamorph and teleomorph species led to presume of their close relationship, if not their ontogenic identity (Vancanneyt et al. 1990, 1991a, b, c, 1992, 1994b).

\section{Genomic characters}

Genomic analysis for fungal taxonomy developed rapidly. Polymorphism of fragmented DNA (RFLP) demonstrated the relation of

\footnotetext{
${ }^{7}$ The System ALLEV later received further developments and world wide distribution as
} BIOLOMIX by the CBS, the Netherlands. 
produced mutants with the parent strain of Penicillium expansum (Cercel et al., 1998a, 1998b). Ratios in guanidine and cytosine confirmed similarities observed in protein profiles of yeasts (Vancanneyt et al. 1991c, 1994a).

Here also, genomic analysis is up to now applied only to living strains in culture, less easily to dried type material or small nature collection. The strains currently analysed are otherwise classically pre-identified. Taxonomic conclusions inferred from genomic analysis might therefore depend on the identifications of reference sequences. Since all names are obligatory linked to type material preserved in a herbarium, it is highly recommended that molecular taxonomists analyse type specimens and authenticated ex-type strains. Neglecting this basic requirement would be equivalent to ignoring 240 years of taxonomy and nomenclature (Gouliamova and Hennebert 1994a, 1994b, 1995)).

With that in mind, sequencing of nuclear rDNA or RNA associated with numerical analysis applied on ex-type strains and other reference strains provided much valuable data for both taxonomy and phylogeny of ascomycete and basidiomycete yeasts (Hendricks et al. 1989, 1991, 1992, Van de Peer et al. 1992, Wilmotte et al. 1993, Gouliamova and Hennebert 1994a, 1994b). They allowed confirmation of the anamorph-teleomorph connection in all cases tested and detection of presumed such connections in some other cases. Sequencing part of 18S RNA and the region ITS1-5.8S rDNA-ITS2 of ex-type or ex-neotype yeast strains, Saccharomyces cerevisiae and some reputedly synonymous species were compared.. S. chevalieri and $S$. ellipsoideus were found synonyms of S. cerevisiae, while S. pastorianus, S. paradoxus, S. uvarum and S. bayanus were confirmed to be distinct species (Gouliamova and Hennebert 1994b, 1998). Using the same ITS region, specific distinctions and seveal new species were established among thirteen unidentified strains of Lipomyces, otherwize detected by DNA reassociation (Gouliamova and Hennebert 1995).

\section{NOMENCLATURE}

Nomenclature Is linked intimately to taxonomy, as language is linked to concepts, and as locality names are to each destination box at the post office.

\section{A. Typification: the basis of nomenclature}

Typification of names is one of the two pillars of botanical nomenclature stability, the other being priority of names.

Automatic typification of a new name by the type of an earlier name included in the protologue was the rule until the Montreal International Code of Botanical Nomenclature (Lanjouw 1961).

Interpretation of cases differs whether names are applied to the same taxon, or to two different taxa, one designated by the earlier name, the other described under the new name. 
When a recent name is published for a taxon while an earlier legitimate and priorable name for the same taxon is cited as a synonym in the protologue and should have been used, automatic typification applies and the recent name is nomenclaturally superfluous and illegitimate. It was the rule until 1966. If the author in some way excludes the type of the cited earlier name, e.g., by designating a type of the new name for the same taxon, the new name with its type should be accepted and automatic typification should not apply. The result would be that the new name is legitimate and taxonomic synonym of the earlier name. That was the proposal made by Weresub and Hennebert (1963), accepted by the Edinburgh Code (Lanjouw 1966).

But often authors misinterpreted the description of an earlier species and therefore misapplied its name to a different taxon. There is then a conflict between the taxon represented by the earlier name cited as a synonym and its type and the taxon as described. The question in interpreting such mycological literature was: is the intention of the later author to be respected or automatic typification applied?

In such cases of misapplication of an earlier legitimate name, the Edinburgh Code (1966) maintained the automatic typification to be applied "in all circumstances" (Art. 7), no regards to the intention of the later author. The new name is then obligate synonym of the earlier one. That is the case of Hyphelia Fr.

Persoon (1794b) had described Trichoderma roseum and deposited a specimen on Populus bark in his herbarium in Leiden. Hoffmann (1795) collected the same species, named it Trichoderma rosea Pers. and described the conidia under the lens as oblong, phaseoliform and uniseptate. Later Persoon (1801) listed his Trichoderma roseum Pers. with reference to Persoon 1794 and Hoffmann 1795, adding its occurrence on Salix bark based on another specimen in his herbarium. Link (1809) erected the new genus Trichothecium Link with uniseptate conidia on the basis of Trichoderma roseum Pers.

Fries (1825) erected the new genus Hyphelia Fr., and included in the same description the name of one species "Hyphelia rosea", based on Trichoderma roseum Pers. which he explicitely propoposes as type, but commenting "not Trichothecium Link". In the same work, Fries recognized the genus Trichothecium Link, with the comment that it differs from Trichoderma roseum Pers.

. Fries (1829) in the Systema mycologicum, already written at the time of the publication of the new genus Hyphelia in 1825, indicated having seen a Persoon specimen with reference to Persoon 1801. Hughes (1958) indeed examined Persoon's three authentic specimens of Trichoderma roseum Pers. in Leiden herbarium and recognized Trichothecium roseum (Pers.) Link: Fr Fries might have had in hand a poor specimen from Persoon like the one deposited in 1801 and misinterpreted Persoon's description, consequently misapplying the name. Hyphelia Fr., based on a misapplication of the type 
species name Trichoderma rosea Pers. is therefore obligate synonym of Trichothecium Link and therefore not available for the species Fries called Hyphelia terrestris Fr. (Chromelosporium tuberculatum (Pers.) Hennebert) (Hennebert 1973)

\section{B. Pleomorphy in fungi and nomenclature}

Linné decided his taxonomy to be based on sexual reproductive structures. In the introduction of the Philosophia botanica (1753) he classified plant taxonomists according to their basic principles of classification and qualified himself as "sexualist" (Fig. 33). Consequently, fungal sexuality and pleomorphism being unknown at the time, any distinct form of reproduction in fungi was considered a distinct species and named.

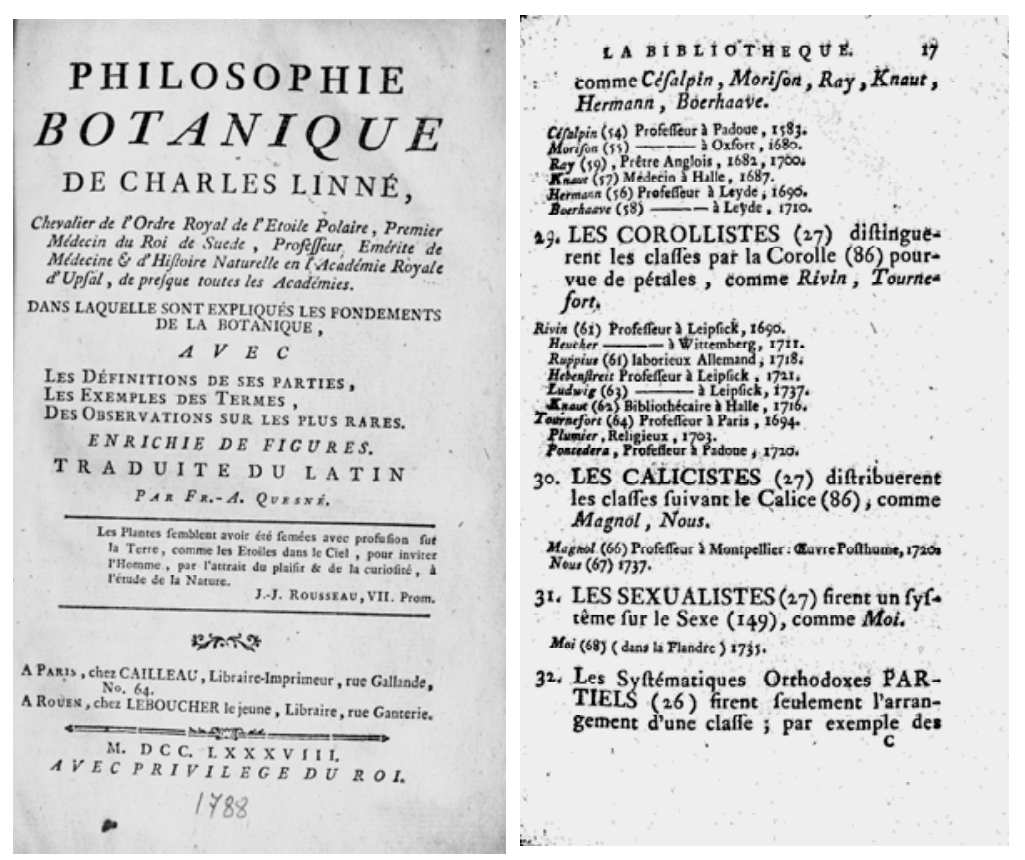

Fig. 33. Linné (1753) declares himself a "sexualist" taxonomist.

Corda (1839) described Echinobotryum parasitans as a parasite of Stysanus caput-medusae ignoring that it was another form of spores of the same fungus (Fig. 35). Even Saccardo (1882) and Penzig (1882) made the same 'mistake' when accepting Echinobotryum citri as a parasite of Stysanus monilioides, later corrected as Echinobotryum atrum(Fig. 36).

Tulasne (1851), who called pleomorphy the coexistence of several forms of reproduction in fungi, demonstrated the organic connection between ascosporic fungi and conidial fungi as the "perfect state" (which they presumed to be sexual) and the "imperfect state or states" of the same 
species, as several conidial forms of reproduction may exist in the same perfect species (Fig. 34). Number of pleomorphic fungi had therefore more than one name. Tulasne (1851), in agreement with Linne's principle, asserted the predominance of the name of the perfect sexual state upon the names of the imperfect states, to designate the whole fungus (Hennebert 1971).

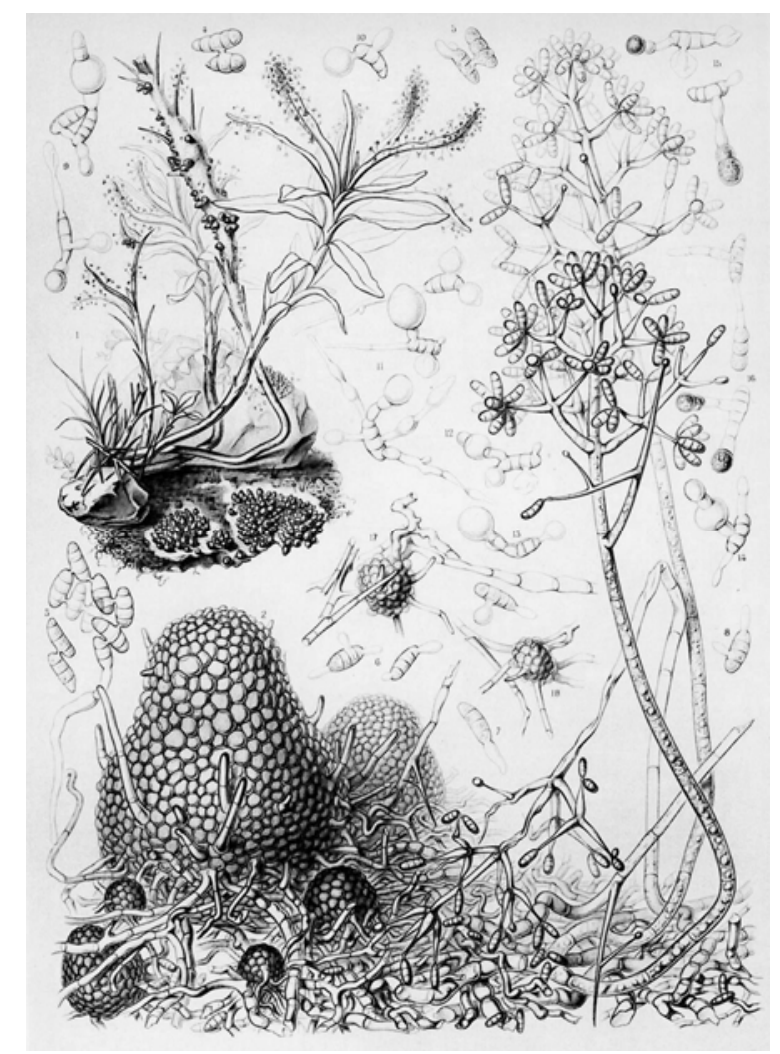

Fig. 34. Pleomorphy: Hypomyces rosellus and anamorph Cladobotryum dendroides (L.R. \& C. Tulasne, III, 1865)

However, vast amount of conidial species remained, and still remain, without connection to a sexual form. Consequently, Fuckel (1870) classified the fungi in two major groups, the Fungi Perfecti and the Fungi Imperfecti. Saccardo (1889) named them Fungi Superiores and Fungi Inferiores, the latter being "metagenetic forms" (formae metageneticae,) which he designated later Deuteromycetae or Fungi secundarii (Saccardo 1899).

Although the Lois de la Nomenclature Botanique Art. 15 by De Candolle (1867) reinforced the principle "one taxon - one name", Saccardo (1904) in his De Diagnostica et nomenclatura mycologica, recommended to classify duly demonstrated pleomorphic fungi by their appropriate names in Fungi Perfecti and Fungi Imperfecti respectively. Saccardo was supporting a tolerated use of the imperfect names beside the correct perfect name. 
The matter, submitted to the International Botanical Congress of Vienna in 1905, was left to the International Commission of Botanical Nomenclature (Briquet 1905). The proposal of the Commission (Atkinson 1909) integrating the positions of Tulasne and Saccardo, was adopted at the Botanical Congress of Brussels in 1910, and became part of the Brussels International Code of Botanical Nomenclature (Briquet 1912) as Art. 49 bis. Art. 49 bis was the first rule of nomenclature for pleomorphic fungi. It recognized the predominance of the name of the perfect state of Ascomycetes and Basidiomycetes over the names of their imperfect states, and tolerated the use of the existing names of imperfect states, to be classified in "formgenera" and assessing them "only a temporary value". A later proposal made by the British mycologists (Anonymous 1929) to extend the rule 49 bis to the Zygomycetes and the Mastigomycetes (Phycomycetes), in order to make coherent the entire nomenclature of the fungi, was defeated at the Botanical Congress of Cambridge in 1930 (Briquet 1935, ICBN Art. 57).

The Stockholm Code (Lanjouw 1952), accepted for the first time, and ruled, in Art. 69, the creation of new names for the imperfect states of already named pleomorphic fungi, while the temporary value of such names was forgotten. The rule still evolved further to a very complex Art. 59 adopted in Edinburgh Code (Lanjouw 1966) and remained unchanged until 1981. The rule intended to separate the nomenclature of the imperfect fungi from that of the perfect fungi and to preserve the predominance and stability of the names of perfect species, by subordinating taxa of imperfect states of fungi as "form-taxa", and making invalid epithets transferred from form-species to perfect species and valid but illegitimate perfect names not typified by a perfect state in accordance with their protologue. In practice this version of Art. 59, was "undoubtedly one of the thorniest articles of the Code for mycologists to apply" (Korf 1982). It was "extraordinarily complicated, so that in some instances, up to three separate conclusions could be drawn regarding names to be used. There was a need for a drastic reconsideration of some basic principles" (Hawksworth 1984). 

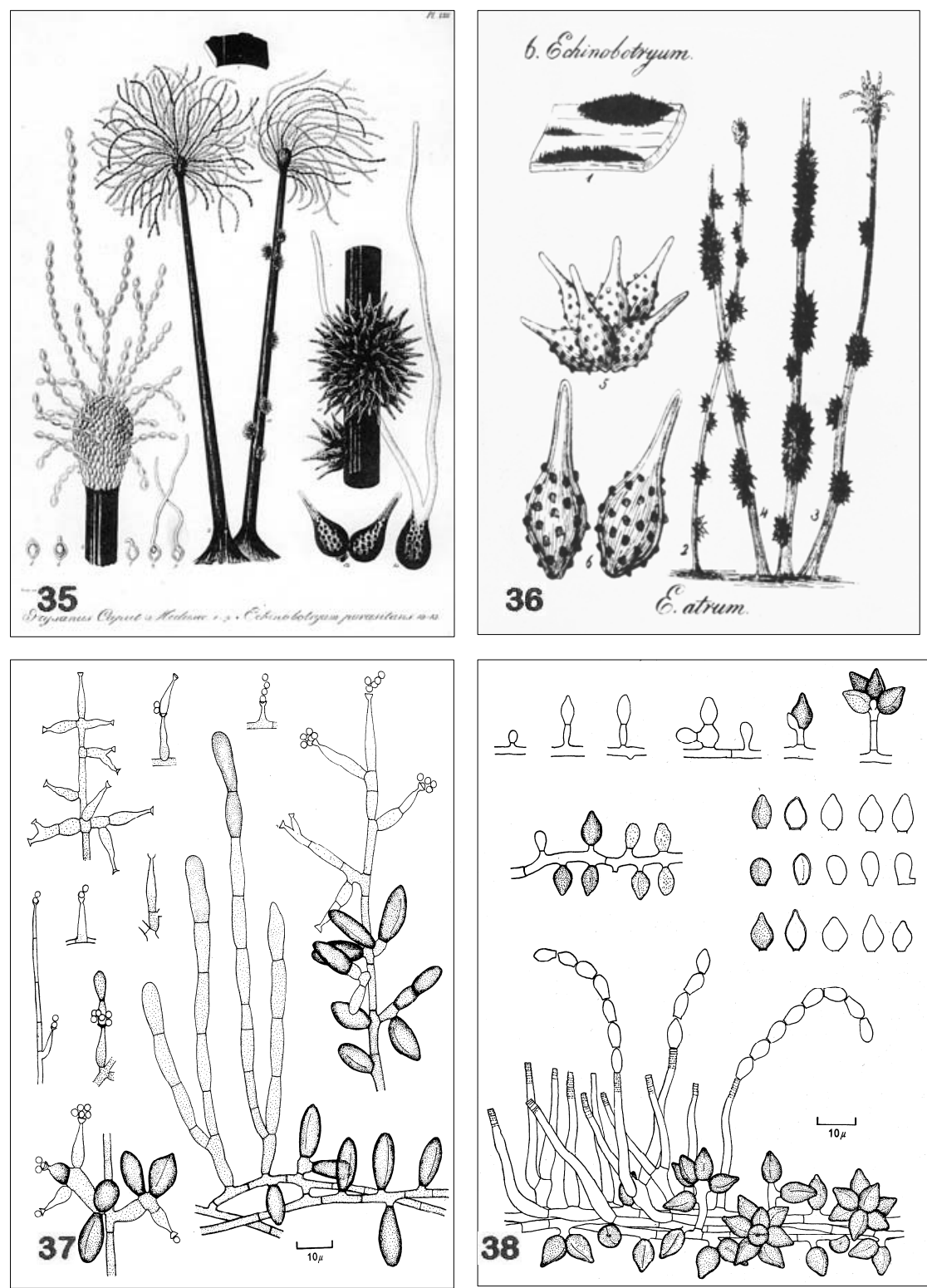

Fig. 35-38. Pleoanamorphy in fungi. 35. Echinobotryum parasitans Corda on Stysanus caputmedusae Corda after Corda, Prachtflora (1840); 36. Echinobotryum atrum Corda after Saccardo, Fungi delineati (1878); 37. Wardomyces columbinus, MUCL 6959, from culture; 38. Mammaria echinobotryoides, MUCL 7564, from culture. 

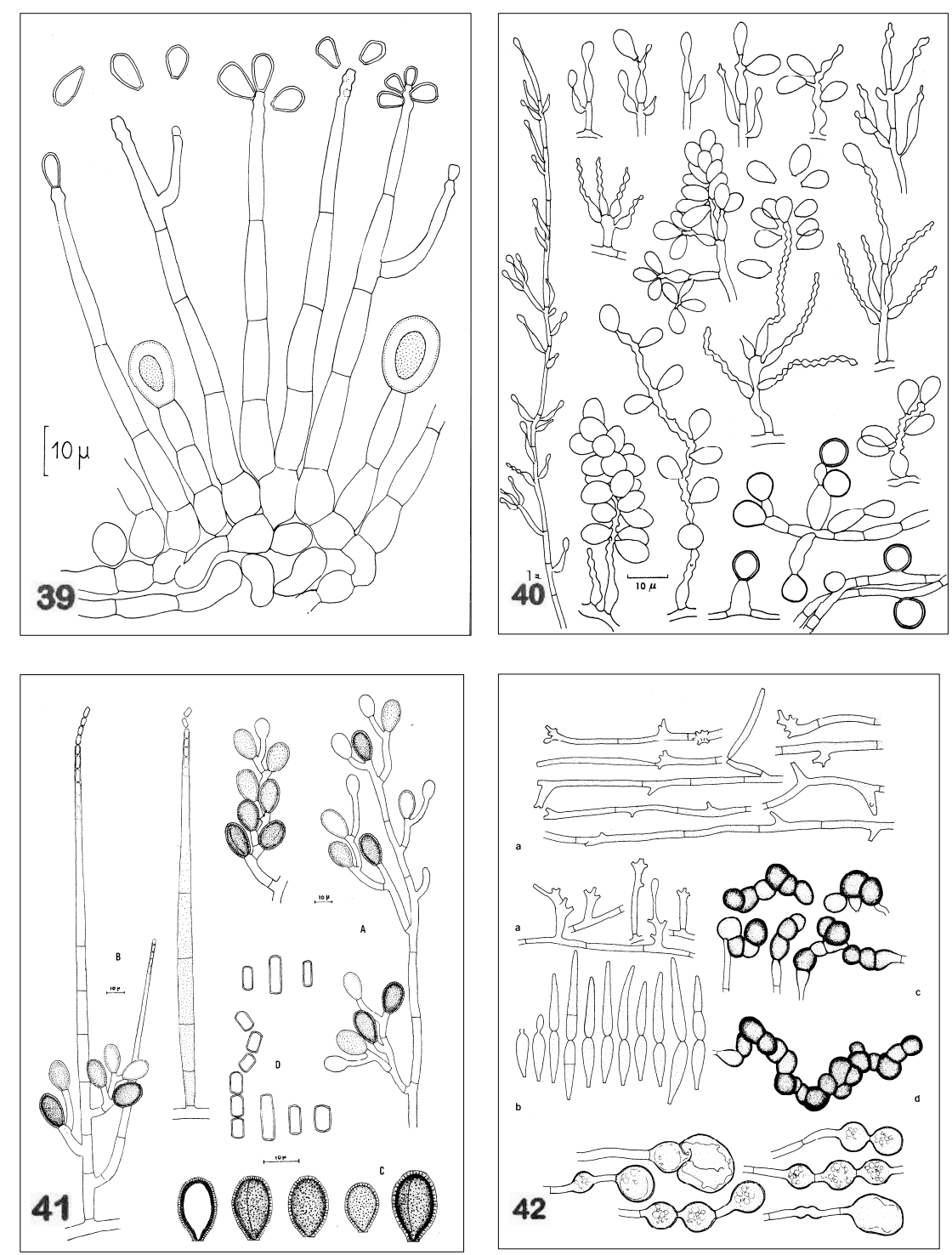

Fig. 39-42. Pleoanamorphic fungi. 39. Raffaelea ambrosiae Arx \& Hennebert: 40. Lomentospora prolificans Hennebert; 41. Chalaropsis punctulata Hennebert; 42. Isthmolongispora lanceata De Hoog \& Hennebert. 


\section{C. Revision of Art. 59 for the nomenclature of pleomorphic fungi}

Such a reconsideration of basic principles of nomenclature I attempted to offer at the First Conference of Kananaskis, Canada, on the Taxonomy of the Fungi Imperfecti in 1969 (Kendrick 1971). In a paper on the Pleomorphism in Fungi imperfecti, I analysed the fundamentals of fungal nomenclature of pleomorphic fungi and 'pleoanamorphic' fungi (a new term at the time). The matter was not easy to put into words. Supported by Richard P. Korf at Cornell University, I formulated the concepts of "phase" versus "state", "form" of a fungus, of "whole fungus" versus "perfect state" and "imperfect states", of "botanical taxon" versus "form-taxon", and of "botanical nomenclature" versus "anatomical nomenclature", in order to define adequately the divergent options applied by fungal taxonomists in naming pleomorphic fungi, to deduce the nomenclatural implications of each option (Hennebert 1971).

A "sexual phase" and an "asexual phase" were distinguished in each fungus, even when the sexual phase is not actually known by man, both phases composing together the "whole fungus". The sexual phase is represented by a unique sexual form of reproduction, while the asexual phase is possibly represented by one or several asexual forms of reproduction or by nothing else than resting organs or mycelium.

The Edinburgh Code (Lanjouw 1966) treats the fungi according two distinct systems of nomenclature.

For the Mastigomycetes and Zygomycetes, the Code applies the "botanical nomenclature", meaning that only one name is given to "the whole fungus" as known, it being either sexual, or sexual and asexual, or only asexual, in strict application of the Linnean principle "one taxon - one name", based on what I call "botanical typification" governed by Art. 7: "The nomenclatural type is not necessarily the most typical or representative element of a taxon". An example is the genus Mucor that accommodates equally either sporangiosporic species, or zygo-sporangiosporic species ('imperfect' species), or even zygo-sporangio-blastosporic species, like Mucor troglophilus Zalar et al. (1997), no matter what part of the fungus the type specimen contains.

On the other hand, for the Ascomycetes, Basidiomycetes and Deuteromycetes, the Brussels (Briquet 1912) and subsequent Codes accept the use of several names for one particular fungus, one name for the sexual phase, typified by sexual reproduction and representing the "taxon", one or several names for the asexual phase typified by asexual reproduction and which represent one or several "form-taxa". The rule prescribes the predominance of the name of the sexual phase to designate the "taxon", excluding from consideration the names of "form-taxa". That system of nomenclature applies a different principle of typification than the rest of botanical nomenclature. Governed by Art. 59, the type of Ascomycete and 
Basidiomycete names must be obligatorily the typical representative element of the taxon, i.e. the sexual fructification, while the type of Deuteromycete form-taxa is restricted to an asexual state, fructification or organ. In this system, names are clearly anatomical names, names of an anatomical part of the fungus. I called this system "anatomical nomenclature" with two distinct name statuses based on what I call here "anatomical typification". Even though the Code gives to names of sexual organs a botanical status, the resulting nomenclature of Ascomycetes or Basidiomycetes is not truly "botanical", for an obligation restricts typification, excluding from priority otherwise potentially competing names of asexual states.

Some fungal taxonomists refusing to create names for asexual states of pleomorphic fungi apply, to refer to them, an intermediate system called the "cross-reference naming system", using only the name of the form-genus associated to the sexual name (Hennebert 1971, 1983).

As a result of this analysis of the dual system of nomenclature, the First Kananaskis Conference, in 1969, decided the creation of a committee for reconsideration of Art. 59. This was formed as Subcommittee A of the International Mycological Association Nomenclature Committee at the First International Mycological Congress in Exeter, September 1971. The Subcommittee was composed of 12 members, with Dr Luella Weresub as Chairman.

\section{D. Anamorph, teleomorph, holomorph: new nomenclatural terms}

In 1977, just playing with words in a conversation with Dr Luella Weresub in Ottawa, I proposed some less 'imperfect' terms than the anthropomorphic 'perfect' and 'imperfect', hopefully terms without value judgements and possibly objective and unequivocal to designate the anatomical parts of a fungus deserving a name. Urged on by Luella Weresub who believed in those terms, we published the terms "anamorph", "teleomorph" and "holomorph" to differentiate any fungal "morph" deserving a name (Hennebert and Weresub 1977). I was inspired by the term "anamorphosis" used by Donk (1960a, 1960b) following its first use by the Vienna International Commission of Botanical Nomenclature (Briquet 1905), and by the ICBNs of 1912, 1935 and 1947, for the asexual states of pleomorphic fungi. The term was preferred to the term "forma metagenetica" used by Saccardo (1889), and to the term "deuteroform" proposed by Shear (1929) as a replacement for the German "Nebenfrucht". The term "anamorph" was proposed for any asexual state of the "anamorphosis" (the asexual phase), the term "teleomorph" for the unique sexual state of the fungus and the term "holomorph" for the "whole fungus", including even all unknown "morphs". Any asexual fungus (imperfect fungus) is therefore an "anamorphic fungus", as lacking a teleomorph, either by a gap in our knowledge or having lost the capacity for sexual reproduction, irrespectively. The anamorphosis of a fungus can be 
represented by one or several anamorphs that deserve names. When several, they were called "synanamorphs" by Hughes (1979). Fungi with synanamorphs are said "pleoanamorphic" and raise problems of nomenclature similar to those of pleomorphic fungi (Hennebert 1987c, 1991).

After several years of work, IMA Subcommittee elaborated a proposal for the modification of Art. 59 recommended by the Second International Mycological Congress in Tampa in 1977. In agreement with the proposal, the Nomenclature Session of the International Botanical Congress in Sydney in 1979 voted in favour of the use of the new terms anamorph, teleomorph and holomorph, in a new version of Art. 59. This, adopted by the Sydney Code (Stafleu 1981), simplified the rule, but reinforced the segregation of anamorphic nomenclature from teleomorphic (holomorphic) nomenclature of Ascomycetes and Basidiomycetes, based on their respective "anatomical typification". It clearly declared anamorphic names unpriorable in holomorphic nomenclature in case the anamorphic fungi were ever found to be sexual. It was the most conservative option, far from the true botanical nomenclature requiring the suppression of Art. 59, what I considered the final solution. New Art. 59 had however two advantages, to solve a serious nomenclatural knot by a clear practical line and, overall, to stimulate reconsideration of the basic principles of nomenclature of the fungi.

For plant pathologists, anamorphic names of plant pathogens often serve to designate plant diseases. Similarly, in medical mycology, names of mycoses commonly derive from the anamorphic name of the causal agent. Both practices are regrettable since they link disease names to a not infrequently changing reference basis. Using the practice as an argument in favour of an anatomical nomenclature and the maintenance of the Deuteromycetes is fallacious (De Vroey and Hennebert 1983). The true reason in maintaining Art. 59 was the fear of the major disturbance that its suppression and a return to unrestricted typification and absolute priority of names would cause in the present usual fungal nomenclature.

\section{E. Anamorph-teleomorph correspondence}

Anxious to nurture a trend toward integration of conidial fungi into the taxonomy of Ascomycetes, Bellemère and Hennebert presented before the Mycological Congress in Tampa in 1977 a compilation of the various conidiogeneses known in the Discomycetes together with their connections with teleomorphic genera (Hennebert and Bellemère 1977, 1979).

In 1979, the Second Conference of Kananaskis considered "The Whole Fungus". Enlightened by the new terminology (Hennebert and Weresub 1979) and the new rules of nomenclature for pleomorphic fungi (Weresub 1979), a wider survey of anamorph-teleomorph connections in the Ascomycetes and Basidiomycetes was elaborated (Kendrick 1979). The survey showed the intricacy of the correspondences between anamorphic and teleomorphic genera, making the dreamed integration of the 
Deuteromycetes into the Ascomycetes and Basidiomycetes an uneasy but challenging puzzle.

This is possibly due to lack of refined taxonomic criteria for the differentiation of genera at both sides. As far as anamorphs are concerned, a more detailed analysis of the unitary parameters of conidiogenesis, from the origin of the conidioma till the maturity of conidia, is needed. Hennebert and Sutton (1994) made such an attempt, which is still incomplete. A similar approach would presumably be helpful, too, in revision of teleomorphic genera.

A new survey of conidiogenesis in Ascomycete families by Sutton and Hennebert (1994) did not show any correspondence but, rather, heterogeneity between taxonomies, suggesting that reciprocal amendments might improve generic congruency. Using this approach, I obtained a strict correspondence between anamorphic and teleomorphic species of Melampsora (Hennebert 1964b). Also, through emphasis of characters discriminating new genera in the Botrytis-like complex, the anamorphic taxonomy could be appropriated to that of the correlated Discomycete teleomorphs (Hennebert 1973, Sutton and Hennebert 1994).

\section{F. Nomenclature of pleoanamorphic fungi}

Pleoanamorphic fungi (synanamorphoses) raise the same problems as those of plemorphic fungi (teleomorphosis-anamorphosis). I studied a number of pleoanamorphic fungi, beside Botrytis species: Ambrosiella xyleborii and Raffaelea ambrosiae (Fig. 39) both with two kinds of conidia (von Arx and Hennebert 1965), Chalaropsis punctulata (Fig. 41) with sympodial thalloblastic conidia and Chalara phialidic thallic conidia (Hennebert 1967), Spirosphaera species with bulbiferous conidia disintegrating into secondary arthroconidia (Hennebert 1968a), Echinobotryum atrum (Fig. 35, 36), Wardomyces columbinus (Fig. 37) and Mammaria echinobotryoides (Fig. 38) with basipetally successive blastic conidia and phialidic or annelidic conidia (Hennebert 1968c), Trichocladium and Thermomyces species with thallobastic and philidic conidia (Hennebert 1971), Lomentospora prolificans (Fig. 40) with sympodial conidia and chamydospores (Fig. 35) (Hennebert and Desai 1974) and Isthmolongispora lanceata (Fig. 42) with sympodial conidia and its Zalerion thalloblastic morph (De Hoog and Hennebert 1983). They raised the still unanswered question of a monoanamorphic versus a pleoanamorphic definition of anamorphic genera.

Here also, I distinguished three systems applied by mycologists to pleoanamorphic fungi, from the botanical (pseudo-botanical) to the anatomical system with the intermediate cross-reference system (Hennebert 1971, 1987b). The new Art 59 of the Sydney Code (Stafleu 1981), which adopts the anatomical system for pleomorphic fungi, does not require the dissection of the anamorphosis into synanamorphs with distinct names, as long as the application of any anamorphic name corresponds to its type. 
Therefore the choice of a monoanamorphic or pleoanamorphic definition of an anamorphic genus is free. It should be governed only by the perspective of a more natural classification (Weresub 1979). Examples exist in the botanical nomenclature of Entomophthorales where "one- state" and "threestate" species are disposed of and named in different genera in a unique taxonomy and nomenclature (Remaudière and Hennebert 1980).

To awaken consciousness to the nonsense of an anatomical system pushed so far as to dissect the anamorphoses of fungi and thus to mupltiply the names of synanamorphs, I took two extreme positions. Nathalie Buffin and myself decided naming provocatively the same fungus by two names, Cylindrodendrum album Bonorden and Cylindrocarpon hydrophilum Buffin and Hennebert, after it produced distinct conidiophores and conidia, although of the same phialidic conidiogenesis, under different culture conditions (Fig. 43) (Buffin and Hennebert 1984). The position actually conformed to anatomical nomenclature of anamorphic fungi. It is clear however that Cylindrocarpon hydrophilum, in a pseudo-botanical system, is a synonym of Cylindrodendrum album.

In non-conformity with that system, we described, under a unique name, Basifimbria spinosa Buffin and Hennebert, a fungus producing two kinds of conidia on the same conidiophore, and indicated, ironically, where eventually to cut the conidiophore with scissors to give the different parts different names(Fig. 44) (Buffin and Hennebert 1985, Hennebert 1987b, 1991).
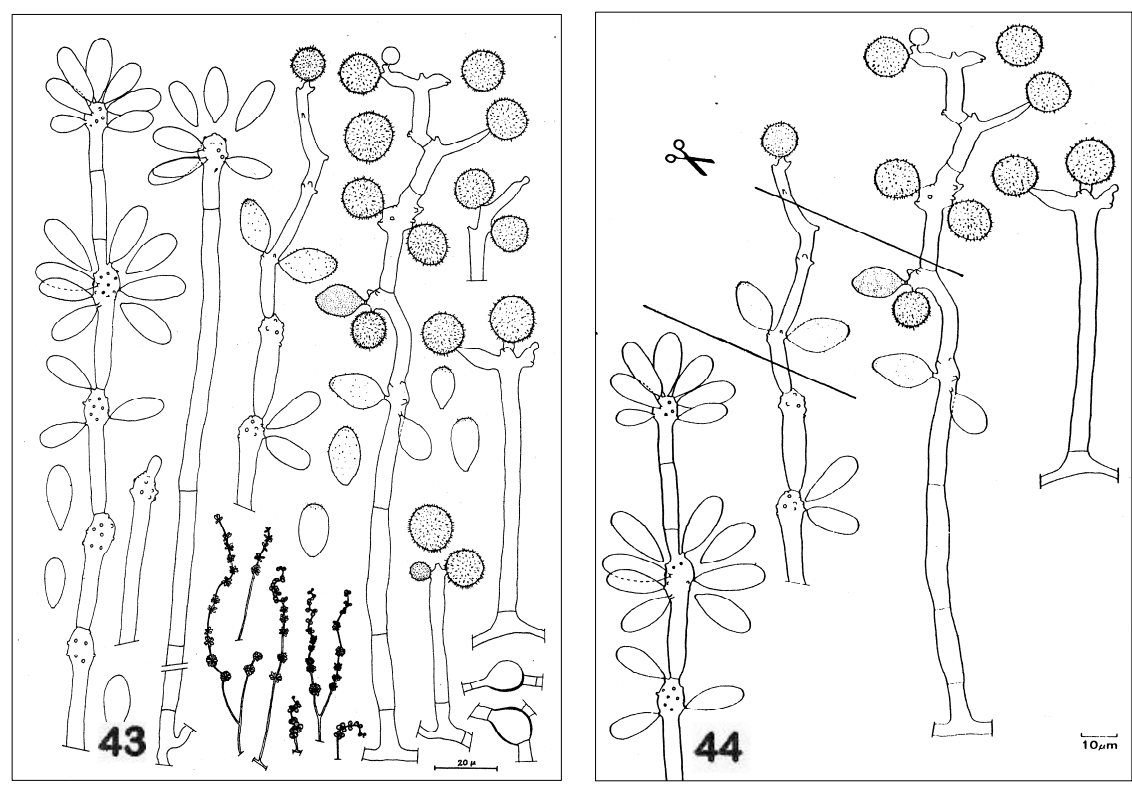

Fig. 43-44. Basifimbria spinosa Buffin \& Hennebert. 43. Variability on the same culture medium.; 44. Variability or pleoanamorphy needing sectioning into pieces for three anamorphic names? 
Obviously, good sense must prevail. The fundamental aim of naming living beings is indeed to make possible their recognition, inventory and classification, by giving each of them a single name and not a multiplicity of names (Hennebert 1991). I therefore prefer giving one name only to pleoanamorphic fungi. So I proceeded in naming and describing Botrytis, Chalaropsis, Wardomyces, Mammaria, Humicola species, using eventually a cross-reference generic denotation for what I considered the "secondary" conidiogenesis. Clearly the anatomical system of nomenclature, allowing a micro-dissection of the fungi, might "encourage les amateurs de gloriole, ceux qui se plaisent à voir leur nom imprimé" (De Candolle 1867). As asserted by mycologists 90 years ago, "names given to other states" than the teleomorph "have only a temporary value" (Briquet 1905), we should remember that all anamorphic names coined for pleomorphic fungi are by definition and typification of a subordinate status, some day in the future destined to become obsolete and to disappear (Hennebert 1987b).

\section{G. Towards a more natural classification}

My dream remains to see the day when taxonomy and nomenclature of the Deuteromycotina (anamorphs and anamorphic fungi) shall be integrated with those of the Ascomycotina and Basidiomycotina (holomorphs) with the abandonment of names for anamorphs of teleomorphic fungi, in order to use only one name per fungus, the nomen botanicum. This dream I share with others. Already Weresub and Pirozynski (1979) declared "We trust that the time will come when our grasp of the morphology, biochemistry and genetics of all fungi will enable us to classify all anamorphic fungi botanically" with correlatively the demise of the Deuteromycota. After a critical analysis of the new Art 59 of the Sydney Code, I concluded: "The morphological diversity, the ontogenetic plasticity of forms and the unity of the chemotaxonomical characters expressed by the unique gene pool in the same fungus demonstrate clearly enough the artificial nature of anatomical, monoanamorphic taxa and the need for a more synthetic concept of the species to approach a more natural taxonomy" (Hennebert 1991).

When in August 1992, Reynolds and Taylor (1993) convened a conference at Newport, Oregon, on the theme of "The Fungal Holomorph", they had, as objective, the integration of Deuteromycotina into the taxonomy of Ascomycotina and Basidiomycotina. The trend was confirmed by a large majority of mycologists at the Newport Conference (Hawksworth 1993). The arguments brought in by the speakers were mostly biomolecular: the close phylogenetic relatedness between many anamorphic and teleomorphic fungi.

The push towards integration will stimulate interactive adjustment of both taxonomies and consequently the mixing of their nomenclature so as to reach a genus - form-genus, species - form-species correspondence. The 
integration should mark different steps. The minimal step is to distribute the Deuteromycotina into two groups, the Ascodeuteromycotina and the Basidiodeuteromycotina, like Cryptococcus was segregated from Candida. A further step is to place those anamorphic genera near holomorphic genera in orders and families to which they belong: Aspergillus and Penicillium in the Eurotiales, Trichochomaceae, for instance. A next possible step would be to integrate biologically connected anamorph and teleomorph genera in unique genera, e.g., Monilia in Monilinia, Botrytis and Amphobotrys in Botryotinia; such genera should include holomorphic species and anamorphic species with their respective holomorphic or anamorphic names (Hennebert 1993).

In the integration process, what shall be the status of the anamorphic names? Two options can be conceived. One option might reject Art. 59, to anihilate anatomical typification in higher fungi; making any name of equal and concurrent status would realize a truly botanical nomenclature. Even were it applied from a future date, that option would create such a turn-over of fungal nomenclature, that it shall not be acceptable. The other option, the only feasible one, is to maintain the anatomical typification approved by Art. 59, which prescribes an obligate teleomorphic typification for holomorphic names of Ascomycotina and Basidiomycotina and a restricted anamorphic typification for anamorphic names of Asco- and Basidiodeuteromycotina, making the latter names forever non-synonymous and non-priorable, in other words "deprived of nomenclatural status by typification" in regard to holomorphic names. In such an integrated classification, the names of anamorphs of pleomorphic fungi will disappear by use of the holomorphic names. The names of those anamorphic fungi still lacking any connection to teleomorphs ("anamorphic holomorphs", according Reynolds 1993) should remain treated as "pseudo-botanical" names (Hennebert 1991) for so long as a related teleomorph remains undiscovered.

At the Newport Conference in August 1992 , the terms anamorph, and teleomorph were disputed by Sutton (1993) and Hawksworth (1993), who preferred the term "mitosporic" and "meiosporic" to qualify the fungus or parts of the ascomycete or basidiomycete fungus, the term "mitospore" replacing the 150 year-old term "conidium". After the matter was raised again at the Conference on Ascomycete Systematics in Paris in 1993, Korf and Hennebert (1993) answered the argument and emphasized the disastrous situation that such deviations in terminology already foreseen by Weresub and Hennebert (1979) would create. The terms anamorph and teleomorph are not karyological but nomenclatural terms, which adequately serve the nomenclature of higher fungi. Fungal spores are said meiotic or mitotic depending on their direct parental nuclear division, the meiotic spores being those spores containing one or more of the four meiotic nuclei. The adjectives "meiosporic" cannot correctly qualify Ascomycotina and Basidiomycotina, as spores of most Ascomycetes and numerous Basidiomycetes are mitotic, containing none of the four nuclei of meiosis. In prospect of the conceivable and hopefully predictable integration of the 
Deuteromycotina in the Ascomycotina and the Basidiomycotina, and as long as the rule of anatomical typification is maintained in these fungi, the terms anamorph and teleomorph will remain necessary.

\section{CONCLUSION}

Taxonomy and nomenclature of the fungi, reasons for a Mycotheque

Fungal taxonomy and fungal nomenclature are linked intimately. Both will really progress towards a natural classification of the fungi if they are based on an accurate and extensive knowledge of the fungi based on their morphology and development. This requires repeated collections of fungi in the field and their isolation in pure culture on various media and under diverse conditions, and on their morphological and morphogenetic description in pure culture in such media and under such conditions, using a harmonized and unequivocal terminology, and finally the application of a correct name in regard to the taxonomic literature and the rules of nomenclature.

For such achievement, a mycotheque is the basic and necessary tool for mycology. It includes a herbarium, culture collection, iconotheque, library, data bases and expert systems. Surely, fungal systematics will not be successfully completed without collaborative morphological, biological and genomic studies. But, at the least, it should first be started with field work and morphological descriptions. Genomic analysis is only an additional, currently and unfortunately luxurious, approach.

The task for fungal taxonomists is urgent:, for the undiscovered biodiversity of the fungi will not wait. Only $5 \%$ of the mycoflora consisting of a presumed 1,500,000 species is known. Much of that important and still undiscovered diversity (more than 1,400,000 species) lies in tropical zones, mainly in tropical forests (Hawksworth 1991, 1994). The actual destruction rate of the tropical forests is such that that reservoir of biodiversity shall be entirely burnt up within 100 years, by 2100 AD (Hennebert 1996a). At the current rate of 14 new species published per day by the existing mycological community, about 350 years of work by such a community will be needed if the objectives remain what they are today. Spending manpower, time and money in elaborating a genetic phylogeny, a pretended natural classification of the fungi from a non-representative sample (5\%) of the fungal world is clearly an impossible vision. The goal is not to achieve the impossible, but rather it is in the field we must spend our energies. Let's go to work. Wonders are waiting for us before being all too soon incinerated.

\section{Literature}

ANONYMOUS 1929. International Botanical Congress Cambridge (England) 1930. Nomenclature. Proposals by British Botanists. HMSO, London $220 \mathrm{p}$.

ATKINSON G.F. 1909. Motions relating to the rules for the nomenclature of the fungi proposed for action at the IIIrd International Botanical Congress at Brussels, 14-22 May 1910. Ithaca, N.Y. 14 p. BANNO I. et al. 1994. Unavailable new species. WFCC Newsletter, IUMS, 22: 18-19. 
BANNO I. et al. 1993. Unavailable new species. FEMS Microbiology letters. 108:1.

BELLEMERE A. and HENNEBERT G.L. 1977. Discomycetes and lichens as Fungi imperfecti. Second Intern. Mycol. Congress, Tampa, Florida, 1977, Abstracts 2: 760.

BERNY J-F. and HENNEBERT G.L. 1988. Alteration of Penicillium expansum through lyophilization and cryopreservation. Cryobiology 25 (6): 586.

BERNY J-F. and HENNEBERT G.L. 1989a. Improvement of the viability of yeast cells in freeze-drying. Effects of protectants and cooling rates. 13th International Specialized Symposium on Yeasts, 18-22 September 1989 (ISSY XIII), Leuven, Belgium.

BERNY J-F. and HENNEBERT G.L. 1989b. Improvement of the viability rate of yeast cells and filamentous fungus spores in freeze-drying. Effects of protectants and cooling rates. CEC, BAP, Meeting of Contractors, (S. Margharita Ligure, Genova), 8-10 November 1989, Book of Abstracts 1.14 .

BERNY J-F. and HENNEBERT G.L. 1990a. Variants of Penicillium expansum; an analysis of cultural and microscopic characters as taxonomic criteria in Samson, In R.A., and J.I. Pitt ed., Modern concepts in Penicillium and Aspergillus classification, p. 49-65. Second International Penicillium and Aspergillus Nato Workshop, 8-12 May 1989, Baarn, The Netherlands. NATO ASI Series, Series Life Sciences, vol. 185. 478p.

BERNY J.-F. and HENNEBER T G.L. 1990b. First evaluation of the kit ATB 32C of API system for the identification of Candida species. 14th Intern. Symposium on Yeasts (ISSY XIV), Abstracts p. 67.

BERNY J.F. and HENNEBERT G.L. 1991a. Viability and stability of yeast cells and filamentous fungus spores during freeze-drying: effects of protectants and cooling rates. Mycologia 83: 805-815.

BERNY J.F. and HENNEBERT G.L. 1991b. Yeast identification and classification using isoelectric focusing of whole-cell proteins. Macromolecular identification and classification of organisms. Symposium, Antwerpen, December 7, 1991. Abstracts p. 32.

BIOURGE Ph. 1923. Les moisissures du groupe Penicillium Link. Etude monographique. La Cellule 33: 5-331.

BIOURGE Ph. in GILBERT J. 1912. Assemblée générale de l'Association, à Louvain le 7-8 décembre 1912. Manifestation en l'honneur des Professeurs Biourge et Verhelst pour le 25e anniversaire de l'Ecole de Brasserie. Bull. Ass. Anc. El. Ec. Sup. Brass. Univ. Louv. 18: 129-147.

BLAKESLEE A.F. 1904. Zygospore formation a sexual process. Science 19: 864-866.

BONORDEN H.F. 1851. Handbuch der allgemeinen Mykologie als Anleitung zum Studium derselben. Stuttgart, 336 p. +12 tab.

BREFELD O. 1872-1912. Botanischer Untersuchungen über die Schimmelpilze. I.-XV. Leipzig.

BREFELD O. 1872. Botanischer Untersuchungen über die Schimmelpilze. I. Mucor mucedo, Chaetocladium jonesii, Piptocephalis freseniana, Zygomyceten. Leipzig. 64 p. 6 tab.

BREFELD O. 1874. Untersuchungen aus dem Gesammtgebiet der Mykologie. II. Botanischer Untersuchungen über die Schimmel Penicillium. Leipzig, iv $+102 \mathrm{p}$.

BREFELD O. 1875. Ueber a neue Culturmethoden für die Untersuchung der Pilze. Sitzungsber. Ges. naturf. Freunde, Berlin, p. 125.

BREFELD O. 1881. Untersuchungen aus dem Gesammtgebiet der Mykologie. IV. Botanische Untersuchungen über Schimmelpilze. Kulturmethoden zur Untersuchung der Pilze. viii +200 p.

BRIERLEY W.B. 1918. The microconidia of Botrytis cinerea. Bull. Miscellaneous information 4 : 128 146.

BRIQUET J. 1905. Texte synoptique des documents destinés à servir de base aux débats du Congrès International de Nomenclature Botanique de Vienne 1905. Commission Internationale de Nomenclature Botanique. Friedländer, Berlin. 162 p.

BRIQUET J. 1912. International Rules of Botanical Nomenclature adopted by the International Botanical Congresses of Vienna, 1905 and Brussels, 1910. G. Fischer, Jena, 110 p.

BRIQUET J. 1935. International Rules of Botanical Nomenclature adopted by the International Botanical Congresses of Vienna 1905 and Brussels 1910 revised by the International Botanical Congress of Cambridge 1930. Fischer vlg. Jena, 152 p.

BRONCHART R. and DEMOULIN V. 1975. Setum ultrastructure of Ostracoderma torrendii. Can. J. Bot. 53 (15): 1549-1553.

BUCHWALD N.F. 1949. Studies in the Sclerotiniaceae. I. Taxonomy of the Sclerotiniaceae. K. Vet.- og Landbohøjsk. Aarsskr. 1949: 75-191.

BUCHWALD N.F. 1953. Botryotinia (Sclerotinia) globosa sp. n. on Allium ursinum, the perfect stage of Botrytis globosa Raabe. Phytopathol. Zeitschr. 20: 241-254.

BUFFIN N. and HENNEBERT G.L. 1984. Cylindrodendrum album Bonorden, a pleoanamorphic semiaquatic hyphomycete. Mycotaxon 19: 323-341.

BUFFIN N. and HENNEBERT G.L. 1985. Basifimbria spinosa, a new pleoanamorphic coprophilous Hyphomycete. Proc. Indian Acad. Sci. 94, 2-(3): 259-267. 
CARNOY J.B. 1870. Recherches morphologiques et physiologiques sur les champignons. Bull. Soc. Roy. Bot. Belg. 9: 157-321.

CERCEL, M., BRYGOO Y., HENNEBERT G.L., and C. MOULLIARD 1998a. DNA Transfer of Penicillium expansum and MUCL variants to a Nylon membrane. Sixth Int. Mycol. Congress, Jerusalem Aug. 1998, Abst. p.91.

CERCEL, M., BRYGOO Y., HENNEBERT G.L., MOLLIARD C. 1998b. DNA Hybridization of Penicillium expansum and MUCL variants by using a DNA probe radioactive labelled. Sixth Int. Mycol. Congress, Jerusalem Aug. 1998, Abst. p.92.

CORDA A.C.J. 1828. Sturm's Deutschland's Flora, III (Pilze) 2 (6): 21.

CORDA A.C.J. 1837. Icones fungorum 1. Prague, 32 p. 7 tab.

CORDA A.C.J. 1833. Sturm's Deutschlands Flora. III (Pilze), Bändchen 3, Heft 13: 81

CORDA A.C.J. 1839 Pracht-Flora europaeischer Schimmelbildungen. Leipzig and Dresden. 56 p. 25 tab.

DE BARY A. 1863. Uber die Entwickelung der Ascomyceten. Leipzig. 38 p.

DE BARY A. 1864. Beiträge zur Morphologie und Physiologie der Pilze. Abh. senckenb. naturf. Ges. 5: 137.

DE BARY A. 1866. Morpohologie und Physiologie derr Pilze, Flechten und Myzomyceten. Leipzig, xii $+316 \mathrm{p}$.

DE BARY A. 1884 Vergleichende Morphologie und Biologigie der Pilze, Mycetozoen und Bacterien. Leipzig, xvi $+558 \mathrm{pp}$.

DE BARY A. 1887. Comparative morphology and biologigy of the Fungi, Mycetozoa, and Bacteria. Trad. H.E. Gransey and I.Baley Balfour, Clarendon Press, Oxford, $x x+526 \mathrm{p}$.

DE BERTOLDI M. and HENNEBERT G.L.1977. Speciation in the genus Humicola. Second Inter. Mycol. Congress, Tampa, Florida, 1977, Abstracts 1: 131.

DE CANDOLLE A. 1867. Lois de la Nomenclature Botanique adoptée par le Congrès international de Botanique, Paris, Août 1967. Genève, 64 p.

DE HOOG G.S. and HENNEBERT G.L. 1983. Taxonomy of the Dactylaria complex. III. A pleomorphic species of Isthmolongispora. Proc. Koninkl. Ned. Akad. Wetensch. C., 86 (3): 343-346.

DEMOULIN V. 1966. Un Gastéromycete remarquable de la flore belge; Glischroderma cinctum Fuck. Naturalistes Belges 47: 404-406.

DE SEYNES J. 1874. Recherches pour servir à l'histoire naturelle des végétaux inférieurs. I. Des Fistulines. Paris, 86 p. 7 tab.

DE VROEY Ch. and HENNEBERT G.L. 1983. Theoretical and practical implications of the morph terminology to the taxonomy and nomenclature of fungi of medical importance. Proceedings of the VIIIth Congress International Society for Human and Animal Mycology. Feb. 1982. M. Baxter ed., New Zealand, p. 440-445.

DIERCKX F. 1901. Essai de revision du genre Penicillium Link. Note préliminaire. Annales Soc. Scient. Brux. 25: 83-89.

DISSING H. and LANGE M. 1962. Lycoperdellaceae. Bull. Jard. Bot. 32:408-411.

DONK M.A. 1960a. Nomenclature of conventional systems. Taxon 9: 103-104.

DONK M.A. 1960b. On nomina anamorphosium.I. Taxon 9: 171-174.

DONK M.A. 1962. The generic names proposed for Hymenomycetes. XII . Deuteromycetes. Taxon 11: 75-104.

DOOMS L., HENNEBERT G.L. and VERACHTERT H.. 1971. Polyol synsthesis and taxonomic characters in the genus Moniliella. Ant. v. Leeuwenh. 37: 107-118.

EHRENBERG Ch.G. 1820. Syzygites eine neue Schimmelgattung nebst beobachtungen über sichtbare Bewegung in Schimmelen. Verhandl. Ges. Naturf. Freunde, Berlin 1: 98.

EVRAD P. and HENNEBERT G.L. 1994. Microplate technique of physiological tests for yeast identification. MUCL Centenary, Abstracts 20.

FRESENIUS G. 1850. Beiträge zur Mykologie. Heft 1: 1-38. In FRESENIUS G. 1963. Beiträge zur Mykologie, Frankfurt vi $+11 \mathrm{p}, 13$ tab.

FRIES E.M. 1825. Systema orbis vegetabilis. Pas I. Plantae homonemeae. Lund, 374 p.

FRIES E.M. 1829. Systema mycologicum. 3 (1). Gryphiswaldae, 1-260 p.

FUCKEL 1870. Symbolae mycologicae. Beiträge zur Kenntniss der rheinischen Pilze. Jb. nassau. Ver. Naturk. 24-24: 1-459.

GAMS W., HENNEBERT G.L., STALPERS J., JANSSENS D., SCHIPPER M.A., SMITH J., YARROW D. and D.L. HAWKSWORTH 1988. Structuring strain data for storage and retrieval of information on fungi and yeasts in The Microbial Information Network Europe MINE Technical Paper $n^{\circ} 1 .$, J. Gen. Microbiol. 134: 1667-1669.

GOULIAMOVA D.E. and HENNEBERT G.L. 1994a. Genomial molecular analysis for fungal taxonomy. Congress of the Microbiological Society of Greece. Volos, 4-10 mai 1994. 
GOULIAMOVA D.E. and HENNEBERT G.L. 1994b. Phylogenic relationships within the Saccharomyces cerevisiae complex species. MUCL Centenary Abstracts 25.

GOULIAMOVA D.E. and HENNEBERT G.L 1995. Diversity znf zffinities among species and strains of Lipomyces. In SAMSON R.A., STALPERS J.A., VAN DER MEI D. and A.H. STOUTHAMER Culture collection to improve the quality of life.1996, Baarn, p. 452-453.

GOULIAMOVA D.E. and HENNEBERT G.L. 1998. Phylogenetic relationships in the Saccharomyces cerevisiae complex of species. Mycotaxon 66: 337-353.

GUILLIERMOND A. 1912. Les levures. Doin ed. Paris, 566 p.

HANSEN E. Chr. 1886. Méthode pour obtenir des cultures pures de Saccharomyces et de microorganismes analogues. C. R. Lab. Carlsberg 2: 92-105.

HAWKSWORTH D.L. 1984. Recent changes in the International rules affecting the nomenclature of fungi. Microbiol. Sciences 1: 18-21.

HAWKSWORTH D.L. 1991. The fungal dimension of biodiversity: magnitude, significance, and conservation. Mycol. Res. 95: 641-655.

HAWKSWORTH D.L. 1993. Holomorphic fungi: the issues, the common ground and the way ahead. In The Fungal Holomorph. REYNOLDS D.R.and J.W. TAYLOR eds., CAB International Wallingford, p. 57-63.

HAWKSWORYH D.L. 1994a. Fungal biodiversity: a largely untapped resource. In. Aspects of African Mycology. HENNEBERT G.L ed., Proc.1st Reg. Conf. Mycology in Africa, June 1990. IMA. Louvain-la-Neuve p.5-10.

HAWKSWORTH D.L. ed. 1994b. Ascomycetessystematics. Problems and perspectives in the nineties. NATO ASI series: Life Sciences 239. Plenum Press, London 453 p.

HEIM R. 1934. Fungi Iberici. Treb. Mus. Cienc. nat. Barcelona, S. Bot.15(3):1-146.

HEIM R. 1949. Lycoperdellon et Lycoperdellaceae. Rev. de Mycol.. 14: 126-128.

HEIM R. and MALENCON G. 1933. Le genre Lycoperdellon, structure et position taxonomique. Rev. gen. Bot. 45: 53-67.

HENDRICKS L., GORIS A., NEEFS J.M., VAN DE PEER Y., HENNEBERT G.L. and DE WACHTER R. 1989. The Nucleotide sequence of the small ribosomal subunit RNA of the yeast Candida albicans and the evolutionary position of the fungi among the Eucaryotes. System. Appl. Microbiol. 12: 223229.

HENDRICKS L., A. GORIS, VAN DE PEER Y., NEEFS J.M., VANCANNEYT, M., KERSTERS K, HENNEBERT G.L. and DE WACHTER R. 1991. Phylogenetic analysis of five medically important Candida species as deduced on the basis of small ribosomal subunit RNA sequences. Journal of General Microbiology 137: 1223-1230.

HENDRICKS L., GORIS A., VAN DE PEER Y., NEEFS J.M., VANCANNEYT.M, KERSTERS K., BERNY J.F., HENNEBERT G.L. and DE WACHTER R. 1992. Phylogenetic relationships among Ascomycetes and Ascomycetes-like yeasts as deduced from small ribosomal subunit RNA sequences. System. Appl. Microbiol. 15:98-104.

HENNEBERT G.L. 1958. Botrytis globosa Raabe sur Allium ursinum en Belgique. Morphologie du conidiophore. Bull. Jard. Botan. Etat, Bruxelles, 28: 193-207.

HENNEBERT G.L. 1960a. Recherches morphologiues sur le genre Botrytis Persoon. Thèse Univ. Cath. Louvain, Inst. Agron. Heverlee, éd. p. aut., 188 p.

HENNEBERT G.L. 1960b. Note sur les Micromycetes des Cavernes. Ann. Fed. Speleol. Belg. 1: 3-13.

HENNEBERT G.L. 1962. Wardomyces and Asteromyces. Can. J. Bot. 40: 1204-1216.

HENNEBERT G.L. 1963a. Un hyphomycète nouveau Arachnophora fagicola n. gen. n. sp. Can. J. Bot. 41: 1165-1169.

HENNEBERT G.L. 1963b. Les Botrytis des Allium. Mededeelingen van de Landbouwhogeschool en de Opzoekingstations van de Staat, Gent, 28: 851-876.

HENNEBERT G.L. 1964a. Botryotinia squamosa, parasite nouveau de l'oignon en Belgique. Parasitica 20: $135-138$.

HENNEBERT G.L. 1964b. L'identification des rouilles du peuplier. Agricultura 12: 661-670.

HENNEBERT G.L. 1966. Principles of taxonomy in the form-genus Botrytis. Intern. Workshop on Botrytis, Invergrowe, ed. aut., 1-15 p.

HENNEBERT G.L. 1967. Chalaropsis punctulata, a new hyphomycete. Ant. v. Leeuwenhoek 33 (3): 333 340.

HENNEBERT G.L. 1968a. New species in Spirosphaera. Trans. Brit. Myc. Soc. 51 (1): 13-24.

HENNEBERT G.L. 1968b. Adhogamina and Gilmaniella. Trans. Brit. Myc. Soc. 51 (1): 146-147.

HENNEBERT G.L. 1968c. Echinobotryum, Wardomyces and Mammaria. Trans. Brit. Myc. Soc. 51(5): 749-762.

HENNEBERT G.L. 1968d. Le Laboratoire de mycologie systématique et appliquée. Nouvelles brèves, REUL, Louvain 24: 1-2. 
HENNEBERT G.L. 1969. Botryotinia sphaerosperma on Lilium regale. Friesia, 9 (1) : 52-57.

HENNEBERT G.L. 1971. Pleomorphism in fungi imperfecti. In Taxonomy of fungi imperfecti. KENDRICK W.B. ed., Univ. of Toronto Press, 1971, 309 p., 202-223.

HENNEBERT G.L. 1973. Botrytis and Botrytis-like genera. Persoonia 7 (2): 183-204.

HENNEBERT G.L. 1979. Philibert Biourge, microbiologiste et mycologue, 1864-1942. In Les Sciences Exactes et Naturelles à l'Université de Louvain de 1835 à 1940. IIIe Colloque d'Histoire des Sciences, Louvain-la-Neuve, 17 Mars 1977. Recueil de Travaux d'Histoire et de Philologie, Ge Série, fasc. 15, p. 61-98, 3pl. Louvain.

HENNEBERT G.L. 1982. Mycologie: systématique et biologie des champignons. Univ. Cath. Louvain, Faculté Sc. Agronomoques 205 p. (unpublished).

HENNEBERT G.L. 1983. Pleoanamormhy and its nomenclatural problems. Third Int. Mycol. Congress, Abstracts, Tokyo. 727p.

HENNEBERT G.L. 1985. Dierckx' Contribution to the Genus Penicillium, 9-21. In Advances in Penicillium and Aspergillus Systematics, by SAMSON R.A. and PITT J.I. ed., plenum Press N.Y. and London. 9-21 p., 4 fig.

HENNEBERT G.L. 1987a. Les variations thermiques à la congélation et à la sublimation dans la lyophilisation des cellules fongiques in Stabilité et conservation des microorganismes. Société française de microbiologie. 12 ème colloque, 26-27 mars 87, Institut Pasteur, Lille, 151-157, Paris

HENNEBERT G.L. 1987b. Pleoanamorphy and Its Nomenclatural Problem. In Pleomorphic Fungi, The Diversity and Its Taxonomic Implications, SUGIYAMA J. ed., p. 263-290. Elsevier Science Publishers b.v., Amsterdam.

HENNEBERT G.L. 1987c. Temperature variation in a one-step freeze-drying procedure for the long term preservation of fungi, 124-126, In Culture Collections and Genetic Engineering of Microorganisms, Ioannina, 23-25 April 87, Commission of the European Communities.

HENNEBERT G.L. 1987d. Development of improvement techniques for the preservation of fungal strains of biotechnological importance, 221-226, In Biotechnology Action Programme, Progress report 1987, Vol 2: Scientific reports from participating laboratories, ed. Magnien E., Commission of European Communities, Brussels.

HENNEBERT G.L. 1991. Art. 59 and the problem with pleoanamorphic fungi. Fourth International Mycological Congress, Regensburg, 28 August - 3 September 1990. Mycotaxon 40: 479-496.

HENNEBERT G.L. 1993. Towards a natural classification of the fungi. p.283-294. In The fungal holomorph: mitotic, meiotic and pleomorphic speciation in fungal systematics, by Eds D.R. Reynolds and J.W. Taylor. Wallingsford, CAB International. 375 pp.

HENNEBERT G.L. 1994. The Microbial Information Network, MINE. In Aspects of African Mycology, Proceedings of the First Regional Conference on Mycology in Africa, Mauritius, 13-15 June 1990, by HENNEBERT G.L. ed. IMA. Louvain-la-Neuve p.17-26.

HENNEBERT G.L. 1996a. Fungal diversity in tropical forests. In. International biodiversity Seminar. ECCO XIV Meeting. CIMERMAN A. and GUNDE-CIMERMAN N. ed., June-July 1995. Ljubljana, p. 75-93.

HENNEBERT G.L. 1996b. Whole-cell rotein electrophoresis for taxonomy and identification of Fungi., In Culture collection to improve the quality of life. by SAMSON R.A., STALPERS J.A., VAN DER MEI D. and STOUTHAMER A.H.. 1996, Baarn, p. 290-293.

HENNEBERT G.L. and A. BELLEMERE 1979. Les formes conidiennes des Discomycètes. Essai taxonomique. Revue de Mycologie 15 (3): 259-315.

HENNEBERT G.L. and B.G. DESAI 1974. Lomentospora prolificans, a new hyphomycete from greenhouse soil. Mycotaxon 1 (1): 45-50.

HENNEBERT G.L. and J.W. GROVES 1963. Three new species of Botryotinia on Ranunculaceae. Can. J. Bot. 41: 341-370.

HENNEBERT G.L. and R.P. KORF 1974. Mycotaxon, a new international journal on taxonomy and nomenclature of fungi and lichens. Mycotaxon 1(1): 1-12.

HENNEBERT G.L. and R.P KOR.F 1975. The peat mould, Chromelosporium ollare, conidial state of Peziza ostracoderma and its misapplied names, Botrytis crystallina, Botrytis spectabilis, Ostracoderma epigaeum and Peziza atrovinosa. Mycologia 67(2): 214-240.

HENNEBERT G.L., MARVEL D., MASSART P., CULOT A.B. and Ch. MEESSEN 1989. MUCL, Mycothèque de l'Université Catholique de Louvain. List of cultures 1989. Fungi and Yeasts. Publ. Belgian Coordinated Collections of Microorganisms. Science Policy Office of Belgium, Brussels. xi + $359 \mathrm{p}$.

HENNEBERT G.L., MASSART P., DECOCK C. and MOUILLARD Ch. 1992. MUCL, Mycothèque de l'Université Catholique de Louvain. Fungi and Yeasts. Publ. Belgian Coordinated Collections of Microorganisms. Science Policy Office of Belgium, DE BRABANDERE J., GILLE P. and W. KNUYT, Edits, Brussels. xxvii +364 p. 
HENNEBERT G.L. and B.C. SUTTON 1994. Unitary Parameters in Conidiogenesis. In Ascomycete Systematics: Problems and Perspectives in the Nineties, by HAWKSWORTH D.L. ed. First International Workshop on Ascomycete Systematics, Paris, 11-14 mai 1993, Plenum Press, New York.

HENNEBERT G.L. and M. VANCANNEYT1998. Proteins in fungal Taxonomy. In Methods in applied Mycology VI. Chemical Fungal Taxonomy.by .FRISVAD J., BRIDGE P and AROARA. Ed. Marcel Dekker, New York.

HENNEBERT G.L. and L.K. WERESUB 1977. Terms for states and forms of fungi, their names and types. Mycotaxon 6(1): 207-211.

HENNEBERT G.L. and L.K. WERESUB 1979. Terms for states and forms of fungi, their names and types. In The whole fungus. by KENDRICK, W.B. Ed. Waterloo, 2 vols. 794 pp., 1: 27-30.

HOFFMANN G.F. 1795. La flore de l'Allemagne. II. Cryptoganie. Göttingen.

HOLUBOVA-JECHOVA V. and G.L. HENNEBERT 1972. Sporoschismopsis, a new genus of lignicolous hyphomycetes. Bull. Jard. Bot. Nat. Belg. 42: 385-391.

HUGHES S.J. 1953. Conidiophores, conidia, and classification.. Can J. Bot. 31: 577-659.

HUGHES S.J. 1958. Revisiones hyphomycetum aloquot cum appendice de nominibus rejiiciendis. Can. J. Bot. 36: 727-836.

HUGHES S.J. 1979. New Zealand Fungi 26. Relocation of species of Endophragmia auct. with notes on relevant generic names. New Zealand J. Bot. 17: 139-188.

HUGUES S.J. and HENNEBERT G.L. 1961. Balanium Wallroth. Can. J. Bot. 39: 1505-1508.

HUGUES S.J. and HENNEBERT G.L. 1963. Oedemium, Dimera, Diplosporium, Gongylocladium and Cladotrichum. Can. J. Bot. 41: 771-810.

KENDRICK W.B. 1971. Taxonomy of Fungi imperfecti. First Kananaskis Conference. Univ. of Toronto Press, $309 \mathrm{p}$.

KENDRICK W.B. 1979. The whole fungus. Second Kananaskis conference. Waterloo. 2 vols. 794 p.

KORF R.P. 1982. Mycological and lichenological implications of changes in the Code of Nomenclature enacted in 1881. Mycotaxon 14: 476-490.

KORF R.P. 1994 Fifty years of fun with the Discomycetes and what's left to do. Symposium Mycology: past, présent and future, to honor Dr Richard P. Korf, Professor Emeritus, October 21, 1994., 18 p.

KORF R.P. and HENNEBERT G.L. 1993. A disastrous decision to suppress the terms anamorph and teleomorph. Mycotaxon. 48: 539-542.

ISTVANFFI G. de. 1905. Etudes microbiologiques et mycologiques sur le rot gris de la vigne Botrytis cinerea, Sclerotinia fuckeliana.Ann. Inst. Centr. Ampel. Roy. Hongrie, Budapest, 3: 183-360 + 211 figs.

JUEL H.O. 1920. Uber Hyphelia und Ostracoderma, zwei von Fries aufgestellte Pilzgattungen. Svensk Bot. Tidsk. 14: 212-222.

LANJOUW J. 1952. International Code of Botanical Nomenclature adopted by VIIth International Botanical Congress, Stockholm, 1950. Utrecht, 228 p.

LANJOUW J. 1961. International Code of Botanical Nomenclature adopted by Xth International Botanical Congress, Montreal, 1959.Utrecht, 372 p.

LANJOUW J. 1966. International Code of Botanical Nomenclature adopted by Xth International Botanical Congress, Edinburgh, 1964.Utrecht, 402 p.

LEVEILLE J.H. 1837. Recherches sur l'hyménium des Champignons. Ann. Sc. nat. ser. 2, 8: 321-338.

LINK H.F. 1809. Observationes in Ordines Plantarum naturales. Dissertatio 1. Magaz. Ges. naturf. Freunde, Berlin 3: 3-42.

LINNE C. 1753. Philisophia Botanica.(Philosophie Botanique de Charles Linné. Translation by F.A. Quesné, Paris, 1783, 470 p.

MALENCON G. 1960. Lycoperdellon, Hyphelia et Phymatotrichum. Bull. trimestr. Soc. mycol. Fr. 76: 217-236.

MALENCON G. 1964. Le Glischroderma cinctum Fuck., sa structure et ses affinités. Bull. trimestr. Soc. mycol. Fr. 80: 197-211.

MASON E. 1933. Annotated account of fungi received at the Imperial Mycological Institute. List II. Fascicle 2. IMI Mycol. Pap. 3: 1 - 68.

MICHELI P.A. 1729. Nova plantarum genera iuxta Tournefortii methodum disposita. Florence. 234 p., 108 tab.

PASTEUR L. 1866. Etudes sur le vin, ses maladies, causes qui les provoquent, procédé nouveaux pour le conserver et pour le vieillir. Paris.

PENZIG O. 1882. Funghi agrumicoli. Michelia 2(8): 385-508.

PERSOON C. 1794a. Nahere Bestimmun und Beschreibungen einiger sich nahe verwander Pflanzen. $P$. Usteri Ann. d. Bot. 11: 1-32.

PERSOON C. 1794b.Dispositio methodica Fungorum. J.J. Römer Neues Mag. f. d. Bot. 1: 81-128. 
PERSOON C. 1797. Tentamen Dispositionis methodicae Fungorum. Leipzig, 76 p. 4 tab.

PERSOON C.H. 1801. Synopsis methodica Fungorum. Gottingae.2vols, xxx +706 p

PIROZYNSKI K.A. 1971. Characters of conidiophores as taxonomic criteria. In Taxonomy of fungi imperfecti by KENDRICK, W.B.. Univ. of Toronto Press, 1971, 309 p., 37-49.

RAABE A. 1938. Parasitische Pilze der Unbegung von Tübingen. Ein Beitrag zur Kryptogamenflora Südwest Deutschlands. Hedwigia 78: 1-10.

REMAUDIERE G. and G.L. HENNEBERT 1980. Révision systématique de Entomophthora aphidis Hoffm. in Frés. Description de deux nouveaux pathogènes d'Aphides. Mycotaxon 11: 269-321.

REYNOLDS D.R. 1993. The fungal holomorph, an overview. In The Fungal Holomorph.by REYNOLDS D. R. and J. W.TAYLOR eds., CAB International Wallingford, p. 15-25.

REYNOLDS D.R. and J. W. TAYLOR 1993. The Fungal Holomorph: mitotic, meiotic and pleomorphic speciation in fungal systematics. Proc. Intern. Symp. Newport, 4-7 August 1992. CAB International, Wallingford, xvi + $375 \mathrm{p}$.

ROBERT V., DE BIEN J.E., BUYCK B. and G.L. HENNEBERT 1994. "ALLEV", a new program for computer-assisted identification of yeasts. Taxon $43: 433-439$.

ROBERT V. EVRARD P. and HENNEBERT G.L. 1997. BCCMtm/Allev 2.00 an automated system for the identification of Yeasts. Mycotaxon 64: 455-463.

SACCARDO P.A. 1882. Fungi italici autographice delineati. Padova. 30: 1199

SACCARDO P.A. 1889. Sylloge fungorum omnium hucusque cognitorum. VIII. Discomycetes. 1143 p.

SACCARDO P.A. 1899. Sylloge fungorum omnium hucusque cognitorum. XIV. Supplementum universale. Padova, $1316 \mathrm{p}$.

SACCARDO P.A. 1904. De Diagnostica et nomenclatura mycologica, Admonita quaedam. Ann. Mycol. 2: 195-198. Engl. transl. J. Mycology 1904: 109-114. Sylloge fungorum XVIII: iii-vii, 1906.

SAMSON R.A. and COLE G.T.1970.Patterns of Development in conidial fungi. Pitman, London, xii + $190 \mathrm{p}$.

SHEAR C.L. 1929. The problem of a natural classification of the Ascomycetes. Proc. Int. Congress Plant Sci. 2: 1618-1626.

STAFLEU F.A. 1972. International Code of Botanical Nomenclature, adopted by the XIth internationa Botanical Congress, Seattle, August 1969. Utrecht, 426 p.

STAFLEU F.A. 1981. International Code of Botanical Nomenclature adopted by XIIIIth International Botanical Congress, Sydney, 1979.Utrecht, p.

STAHL Ch.E. 1877. Beiträge zur Entwickelungsgeschchte der Flechten. Botan. Zeitg. 22: 177-180.

STOLK A.C. and HENNEBERT G.L. 1968. New species of Thysanophora and Custingophora gen. nov. Persoonia 5(2): 189-199.

STREINZ W.M. 1862. Nomenclator Fungorum. Vienne,736 p.

SUTTON B. C. 1993. Mitosporic fungi (Deuteromycetes) in the Dictionary of the Fungi. In The Fungal Holomorph. by REYNOLDS D. R.andW.J. TAYLOR eds., CAB International Wallingford, p. 27-56.

SUTTON B.C. and G.L. HENNEBERT 1994. Interconnections amongst anamorph and their possible contribution to Ascomycete systematics. p.77-100. In Ascomycete Systematics. Problems and Perspectives in the Nineties. by HAWKSWORTH D.L. ed., Plenum Press, New York, 453 pp.

SYDOW H. 1912. Mycotheca Germanica. Fasc. XXII-XXIII. Ann. Mycol. 10: 448.

TODE H. J. 1790. Fungi Mecklenburgenses selecti. 1. Nova fungorum genera complectens. Luneberg, 49 p.

TORREND C. 1913. Les basidiomycetes des environs de Lisbonne et de la région de S. Fiel. Broteria, Bot. 11:98-104.

TUBAKI K. 1958. Studies on Japanese hyphomycetes. V. Leaf and stem group with a discussion of the classification of hyphomycetes and their perfect stages. J. Hattori Bot. Lab. 20: 142-244.

TULASNE L.R. 1851. Note sur l'appareil reproducteur dans les lichens et les champignons. C. R. Acad. Sci. Paris 32: 427-430 and 470-475.

TULASNE L.R. 1856. Note sur l'appareil reproducteur multiple des Hypoxylées DC. ou Pyrénomycetes Fr. Ann. Sc. nat. ser. 4, 5: 106-118.

TULASNE L.R. and TULASNE C. 1861-1865. Selecta fungorum carpologia. Paris 3 vols. 1: xxviii + $242 \mathrm{p}$.

VANCANNEYT M., POT B., HENNEBERT G.L. and KERSTERS K. 1990. Differentiation of yeast species based on electrophoretic whole cell protein. 14th Intern. Symposium on Yeasts (ISSY XIV). Abstracts p. 69.

VANCANNEYT M., BERNY J.F., HENNEBERT G.L. and K.KERSTERS 1991a. Taxonomic study of the yeast genera Rhodosporidium, Rhodotorula and related organisms. In Macromolecular identification and classification of organisms. Symposium, Antwerpen, December 7, 1991. Abstracts p. 36. 
VANCANNEYT M., COOPMAN R, TYTGAT R,, BERNY J.F., HENNEBERT G.L. and KERSTER K.S 1991b. Taxonomic studies of basidiomycetous yeast genera Rhodosporidium Banno and Rhodotorula Harrison based on whole cell protein patterns, DNA base compositions and coenzyme Q types. J. Gen. Appl. Microbiol. 38: 363-377.

VANCANNEYT M., POT B., HENNEBERT G.L. and K. KERSTER 1991c. Differentiation of yeast species based on Electrophoretic Whole Cell Protein Patterns. Systematic and Applied Microbiology 14: 23-32.

VANCANNEYT M., VAN LEBERGE E., BERNY J.F., HENNEBERT G.L. and K. KERSTERS 1992. The application of whole-cell protein electrophoresis for the classification and identification of basidiomycetes yeast species. Antonie van Leeuwenhoek 61: 69-78.

VANCANNEYT M., COOPMAN R., TYTGAT R., HENNEBERT G.L. and K. KERSTERS 1994a Whole cell protein patterns, DNA base compositions and coenzyme Q types in the yeast genus Cryptococcus Kützing and related taxa.. System. Appl. Microbiol. 17:65-75.

VANCANNEYT M., HENNEBERT G.L. and KERSTER K. S 1994b. Identification of Basidiomycetous yeasts using whole-cell protein electrophoresis. MUCL Centenary Abstracts, 21.

VAN DE PEER Y., HENDRIKS L., GORIS A., NEEFS J.-M., VAN CANNEYT M., KERSTERS K. BERNY J.-F., HENNEBERT G.L. and R .DE WACHTER. 1992. Evolution of basidiomycetous yeasts as deduced from small ribosomal subunit RNA sequences. System. Appl. Microbiol. 15: 250 258.

VON ARX J.A. and G.L.HENNEBERT 1965. Deux champignons ambrosi25a: Ambrosiella xyleborii et Raffaelea ambrosiae. Mycopathologia et Mycologia applicata: 309-315.

VUILLEMIN P. 1910. Matériaux pour une classification rationelle des Fungi Imperfecti. C. R. Acad. Sci. Paris 150: $882-884$

WALLROTH K.F.G. 1833. Flora Cryptogamica Germaniae, 2.Algas et Fungos. Nurenberg 923 p.

WERESUB L.K., HENNEBERT G.L., CARMICHAEL J.W. and K.A. PIROZYNSKI 1979. Nomenclural Committee of Kananaskis II. On the question of naming pleomorphic anamorphic fungi. In The whole fungus.by KENDRICK W.B. ed., Waterloo, 2 vols. 794 p., 2: 689-709.

WERESUB L.K. and G.L. HENNEBERT 1963. Article 63 and the type method. Taxon 12: 218-228.

WERESUB L.K. and G.L HENNEBERT. 1979. Anamorph and teleomorph, terms for organs of reproduction rather than caryological phases. Mycotaxon 8 (1): 181-186.

WERESUB L.K.and PIROZYNSKI K.A. 1979. Pleomorphism of Fungi as treated in the history of mycology and nomenclature. In The whole fungus. by KENDRICK W.B. ed., Waterloo, 2 vols. 794 p., 1: 17-25.

WHETZEL H.H. 1945. A synopsis of the genera and species on the Sclerotiniaceae, a family of stromatic inoperculate discomycetes. Mycologia 37: 648-714.

WILMOTTE A., VAN DE PEER Y., GORIS A., CHAPELlE S., DE BAERE R., NELISSEN B., NEEFS J-M., HENNEBERT G.L. and R. DEWACHTER 1993. Evolutionary relationships among higher fungi inferred from small ribosomal subunit RNA Sequence Analysis. System. Appl. Microbiol. 16:436-444.

ZELLER S.M. 1948. Notes on certain Gasteromycetes including two new orders. Mycologia 40: 639 668.

ZALAR P., HENNEBERT G.L., GUNDE-CIMERMAN N. and CIMERMAN A. 1997. Mucor troglophilus, a new species from cave crickets. Mycotaxon 65: 507-516. 


\section{INDEX OF FUNGAL NAMES}

Species names in summaries, tables, plates and dendrograms are not indexed. Names of families, orders and classes are not indexed.

Acanthodochium

Agaricus

126,127

A. bisporus

A. bitorquis

$69,72,226$

Adoghamina ruchira

$226,227,228$

226,228

Albugo trapogonensis

Alternaria

A. triticina

206,207

Amanita zambiana

69

A. muscaria

67,71

Amauroderma

Ambrosiella

A. ferruginea

Ambrosyozima

Amphobotrys

274,294

Anthosspomella

123,125

Antrodia vaillantii

Arachnophora fagicola

$270,275,276$

Areolospora terrophila

Armillaria

$67,73,74$

Arthroascus

Ascoidea

Ashbya

A. gossypii

$41,160,161$

Aspergillus

160,161

A. clavatoflavus

$11,20,21,114$

A. flavus

175

A.niger

175

A. oryzae

$11,20,114$

A. parasiticus

175

A. repens

175

A. sojae

225

A. tamarii

175

A. zonatus

175

175

Asteromyces

A. cruciatus

275,280

Asterostroma ochroleucum

189,181

Astrocystis

Aureobasidium

Auricularia

Balanium

B. stygium 
Ballistosporomyces $\quad 43$

Basidiobotrys $\quad 123$

$\begin{array}{lr}\text { Beauveria } & 274\end{array}$

Besingtonia 43

Bipolaris sorokiniana $\quad 135,138,206,207,208$

$\begin{array}{ll}\text { Biscogniauxia } & 123,125\end{array}$

B. nummularia 125

Blastocladiella emersonii $\quad 149$

Boletus edulis $\quad 70$

Boletus expansus

$\begin{array}{lr}\text { Botryotinia 256, 274, } 294 & 256,257\end{array}$

$\begin{array}{ll}\text { B. calthae 256, } 257 & 256\end{array}$

B. globosa 256

$\begin{array}{ll}\text { B. ficariarum } & 256,257,278\end{array}$

B. fuckeliana 256

$\begin{array}{ll}\text { B. porri } & 256,257\end{array}$

B. ranunculi 278

Botrytis

B. cinerea

$21,255,293,298$

$255,259,273$

B. didyma

264

270

B. geranii

256

B. porri

Broomeia congregate

72

Byssonectria terrestris

219, 220, 222

Calcarisporium

274

Calonectria

151,152

C. clavata

C. gracilis

C. pteridis

Camillea

C. leprieurii

C tinctor

Candida

C. albicans

C. guilliermondii

C. lipolytica

C. tropicalis

Cantharellus

Cephalascus

Cephalotrichum

Ceratocystis

C. araucariae

C. piceae

Cercospora kikuchii

Cercosporella

C. herpotrichoides

Chalara

153,155

151,152

153

123

123

123

44, 148, 161, 294

44,174

45

164

45

69

40, 41

274

41,51

193

193, 194

198

196

196

291

Chalaropsis 
C. punctulata

287,291

Chlorophyllum molybdites

Chromelosporium

270,274

C. fulvum 279

C. macrospermum 279

C. ochraceum 279

$\begin{array}{lr}\text { C. ollare } & 279\end{array}$

\begin{tabular}{lr} 
C. trachycarpum & 279 \\
\hline C. tuberculam & 283
\end{tabular}

C. tuberculatum 283

$\begin{array}{ll}\text { Cladobotryum } & 274\end{array}$

C. dendroides 284

Cochliobolus 135

C. sativus

$135,139,206207,245$

Colletotrichum gloeosporioides 203, 204

Collodiscula japonica

126

Collybia aurea

Coniophora puteana

72

Coniophora marmorata

70, 183, 188, 191

188,191

Conoplea

Cookeinia

Coriolopsis

Corticium

Costantinella

Creosphaeria sassafras

126

Cryptococcus

C. albidus

C. alylolentus

43, 44, 156, 294

C. curiosus

157

157

C. curvatus

157

C. dimennae

157

C. elinovii

158

C. flavus

157

C. gastricus

157

C. heveanensis

157

C. humicola

157

C. kuetzingii

157

C. laurentii

157

C. luteolus

157

C. macerans

157

C. magnus

157

C. marinus

157

C. neoformans

174

C. skinneri

157

C. terreus

158

C. tsukubaensis

157

53

Cryphonectria parasitica

208

Curvularia

271, 276 
Cylindrocarpon hydrophilum

Cylindrocladium

$151,152,177,181$

C. camelliae

177,180

C. clavatum

C. colhounii

153,177

C. floridanum

$151,152,153,177$
177,179

C. gracile

177,179
152,153

C. hawksworthii

C. illicicola

C. infestans

C. pteridis

C. quinqueseptatum

C. theae

$151,152,153$
$153,178,179$

$151,152,153,178,179$

177,180

$151,152,153$

177,179

177

Cylindrodendrum album

275,296

43, 156

72,123

Daldinia

D. escholtzii

125

D. occidentalis

126

Debaryomyces

41, 161

D. hansenii

D. larala

161

42

Dekkera

44,45

Dematophora

126

278

Dichobotrys

Dichomitus

Dipodascus

Donkioporia expansa

41

187

Drechslera sorokiniana

Drechslera tritici-repentis

135,209

Echinobotryum atrum

206, 207, 208

Echinobotryum citri

287, 290, 295

287

Echinobotryum parasitans

287, 290

Endogone pisiformis

149

43

Eremothecium

E. ashbyii

Erythrobasidium

Euepixylon

Filobasidiella

F. neoformans var.neoformans

F. neoformans var.bacillispora

Filobasidum

Flavodon flavus

Fomitopsis widdringtoniae

Fusaroium oxysporum sp.f. cubense

161

160,161

43

123,125

156

156

156

156

72

67

27

Fusicoccum coccineum

Galactomyces

Ganoderma 
G. australe

Geomyces

Gigaspora marginata

Gilmaniella humicola

Glischroderma

274, 279

G. cinctum

Gloeophyllum trabeum

Grammothele

Grammothelopsis

Graphium

Guillermondella

Gyrodontium boveanum

Hadrotrichum

70,143

H. pyrenaicum

Hansfordia

Haplotrichum

Helminthosporium sativum

Holleya

41,162

Hormoascus

Humicola

Hymenochaeta tabacina

Hyphelia

271,282

H. rosea

$H$. terrestris

271,283

279

Hypochnus

140

Hypocreopsis lichenoides

Hypomyces rosellus

Hypoxylon

127

H. argillaceum

H. deustum

127,128

$H$. fraxinophilum

128

H. moravicum

128

H. pyrenaicum

Inonotus

Irpex

Isthmolongispora lanceata

287, 291

Kathistes

Kluyveromyces

160,161

Kondoa

156

Kretzschmaria

Lactarius
L. flammans
L. gymnocarpoides
L. gymnocarpus
L. gymnosporus
L. kabansus

$69,72,144$ 
$\begin{array}{ll}\text { L. longisporus } & 144\end{array}$

L. luteopus 144

L. medusae 144

L. pseudogymnocarpus 144

$\begin{array}{ll}\text { Lamprospora } & 274\end{array}$

Lentinellus $\quad 145$

Lentinus cladopus $\quad 69$

Leptosphaeria $\quad 235$

$\begin{array}{lr}\text { Leprieuria } & 123,125\end{array}$

Leucocoprinus $\quad 69$

Leucosporidium 156

$\begin{array}{lr}\text { Linquistia } & 123,127\end{array}$

Lomentospora prolificans $\quad 271,287,291$

Lopadostoma turgidum 126

Lycogala $\quad 72$

L. torrendii $\quad 287$

Lycoperdellon $\quad 287$

L. torrendii $\quad 287$

Lypomyces $\quad 281$

$\begin{array}{lr}\text { Macrolepiota } & 69,72\end{array}$

$\begin{array}{lr}\text { Magnaporthe grisea } & 209,210\end{array}$

Mammaria 293

M. echinobotryoides $\quad 286,291$

Megasporoporia $\quad 70$

Melampsora 291

Metschnokowia $\quad 162$

Monilia $\quad 294$

Monilinia $\quad 294$

$\begin{array}{ll}\text { Moniliella pollinis } & 278\end{array}$

Monochaeta 118

$\begin{array}{lr}\text { Mrakia 43, } 156 & 456\end{array}$

M. frigida 156

M. gelida 156

M.. nivalis 156

M. stokesi 156

Mucor racemosus 149

Mucor troglophilus 288

$\begin{array}{lr}\text { Mycosphaerella } & 196,198\end{array}$

Mycotypha 274

Mycovellosiella 198

$\begin{array}{lr}\text { Myrioconium } & 41,257\end{array}$

Nadsonia fulvescens 161

Nemania $123,125,126$

$\begin{array}{ll}N \text {. serpens } & 125\end{array}$

Nematogonium $\quad 274$

Nematospora $41,160,161$

$\begin{array}{ll}N . \text { coryli } & 160,161\end{array}$

Neolecta

149

Neotiella vivida

$220,222,224$ 
Neurospora crassa

Nodulisporium

$123,125,125,127,278$

$N$. hinnuleum

126

Nummularia viridis

Oculimacula yallundae

196

Oculimacula acuformis

198

Oedemium atrum

Oe. didymum

264, 265

Oe. tomentosum

Ophioboulus

235

Ophiostoma

O. ulmi

Ostracoderma

53

O. pulvinatum

$266,270,274,279$

266, 270, 279

126,127

Padixonia

175

Penicilliopsis

Penicillium

$P$. aeruginosum

278

P. atroviride

$P$. aurantiobrunneum

268

P. aurantiocandidum

267

P. aurantiogriseum

269

P. biourgei

269

P. brefeldianum

269

P. brevicompactum

21

268

P. brunneorubrum

268

$P$. candidofulvum

268

P. carneoviolaceaum

267

P. citreonigrum

267

P. citreoroseum

267

$P$. chrysogenum

63

$P$. congolense

268

P. corylophilum

267

P. duclauxii

268

P. elongatum

268

P. expansum

278,281

$P$. flexuosum

21

P. glaucum

255,268

P. griseobrunneum

268

P. griseofulvum

P. griseoroseum

P. hirsutum

$11,15,20,21,114,269$

P. minioluteum

269

269

\begin{tabular}{ll} 
P. roseopurpureum & 267 \\
\hline
\end{tabular}

$P$. verrucosum

268

Peniophora

Perenniporia mundula

Periconiella

Pestalotia 
Peziza

P. domiciliana

220,222

P. fuckeliana

256

Phaeosporis melasperma

Phanerochaete chrysospermum

Phellinus

$P$. rimosus

P. cryptarum

P. megaloporus

Phialophora

Phylacia

Phyllosticta circii

Phymatotrichopsis

P. omnivora

Phytophthora

Ph. infestans

Pichia

P. anomala

P. carsonii

P. etchellsii

$P$. farinosa

Pizolithus arhizus

Pleurocatera acicularis

140,141

Pleurotus

P. cornucopiae

$P$. ostreatus

$P$. ostreatus var. columbinus

P. ostreatus cv.Florida

P. pulmonarius

P. sajor-caju

Pneumocystis

$50,96,149$

P. carinii

Podaxis pistillaris

Polymyxa betae

213

Polymyxa graminis

Polyporus squamosus

Polyporus tenuiculus

Poria expansa

Poria megalopora

Poronia

Porosordaria

Protomyces

Pseudocercosporella

$50,96,149$

$P$. aestiva

$P$. anguioides

196, 198

P. herpotrichoides var. herpotrichoides

$P$. herpotrichoides var. acuformis

196,198

Puccinia gentiana

196, 198

P. suaveolens 
Pulchromyces

Pycnoporus

Pyricularia oryzae

209,210

Pyrenophora tritici-repentis

Pyxidiophora

$51,140,14$

Raffaelea ambrosiae

$50,287,291$

Ramulispora

$R$. herpotrichoides var. herpotrichoides

196,198

$R$. herpotrichoides var. acuformis

196,198

$R$. herpotrichoides var. anguioides

196, 198

$R$. sorghi

199

198

Rhizopus oryzae

Rhinocladiella

125,274

Rhinotrichum

Rhodosporidium

40, 156

$R$. spaerocarpum

$R$. diobovatum

157

R. kratochvilovae

157

$R$. paludigenum

157

$R$. toruloides

40

Rhodotorula

43, 156

R. acheniorum

157

$R$. araucariae

157

R. armeniaca

156

R. aurantiaca

157

R. auriculariae

156

R. bacarum

156

$R$. bogoriensis

156

R. buffonii

44

$R$. diffluens

156

$R$. foliorum

157

R. fragaria

156

R. glutinis

157

R. graminis

157

$R$. hordea

156

R. hylophila

156

$R$. ingeniosa

156

$R$. javanica

156

$R$. lactosa

156

$R$. minuta

157

R. mucilaginosa

157

$R$. muscorum

156

R. philyla

156

R. pustula

156

$R$. sonckii

156

Rhopalostroma

123

Rosellinia

$123,125,126$

Russula

69,72

Saccharomyces

$40,51,160,161,167,170,172,176,238,244-48,281$ 
S. bayanus

$167,172,174,248,281$

S. carlsbergensis

S. cerevisiae

S. chevalieri

$40,167,172,174,248$

S. ellipsoideus

281

281

S. paradoxus

S. pastorianus

S. uvarum

(174, 248, 281

Saitoella $167,172,174,248,281$

248,281

96,149

Saturnospora

Schizosaccharomyces

S. pombe

Saccharomycopsis lypolitica

$41,42,50,96,149$

161,174

164

Schwanniomyces

S. occidentalis

Sclerotium durum

Sclerotium echinatum

Scorias spongiosa

145

Seaverinia

S. geranii

Septoria circii

Serpula lacrymans

183

Sphaerosporella

274

Spirosphaera

Sporobolomyces

43

Sporoschismopsis moravica

275,276

Sporothrix

125

Streptobotrys

274

Streptotinia

274

Stysanus caput-medusae

283,286

Stysanus monilioides

283

Subbaromyces

Suillus cothurnatus

51

S. granulatus

117

Symbiotaphrina

70

Tapesia

T. yallundae

T. yallundae var. acuformis

51

196

196

T. acuformis

198

198

Taphrina

Termitomyces

$50,95,149$

Thermomyces

69

Torulaspora

291

Trametes

Trametes versicolor

Tremetes versicolor

156

T. aurantia

158

T. brasiliensis

158

T. coalescens 
$\begin{array}{ll}\text { T. encephala } & 158\end{array}$

$\begin{array}{lr}\text { T. foliacea } & 158\end{array}$

$\begin{array}{ll}\text { T. fuciformis } & 158\end{array}$

$\begin{array}{lr}\text { T. globospora } & 158\end{array}$

$\begin{array}{lr}\text { T. mesenterica } & 158\end{array}$

$\begin{array}{lr}\text { T. samoensis } & 158\end{array}$

$\begin{array}{lr}\text { T. subanomala } & 158\end{array}$

Trichocladium 291

$\begin{array}{lr}\text { Trichoderma harzianum } & 193,194\end{array}$

Trichoderma roseum 282

Tricholoma 226

$\begin{array}{ll}\text { T. spectabilis } & 226,227,228\end{array}$

Trichomonascus 41

$\begin{array}{ll}\text { Trichophaea } & 274\end{array}$

Trichothecium $\quad 282$

$\begin{array}{lr}\text { T. roseum } & 282\end{array}$

Tseuchiyaea $\quad 43$

Ustilago maydis $\quad 43$

Ustulina $\quad 128$

$\begin{array}{lr}\text { Verrucobotrys } & 270,274\end{array}$

V. geranii 271

Verticillium $\quad 274$

$\begin{array}{ll}\text { Virgaria } & 274 \\ \text { Virgariella } & 2725\end{array}$

Virgariella 125

Wardomyces $\quad 275,293$

W. columbinus $\quad 271,286,291$

Williopsis $\quad 42$

Wyngea 42

W. robertsii 41

Xylaria $72,114,128,131$

$\begin{array}{ll}X . \text { allantoidea } & 127,134\end{array}$

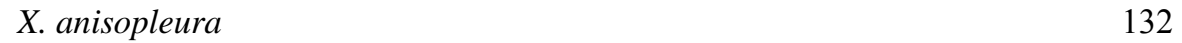

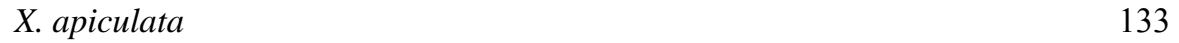

X. arbuscula 133

$\begin{array}{ll}X \text {. cubensis } & 127,132,134\end{array}$

$X$. feejeensis 132

$X$. furcata $\quad 126,131$

X. grammica 133

$\begin{array}{lr}X . \text { longipes } & 126\end{array}$

X. luteostromata 132

X. magnoliae $\quad 95,121$

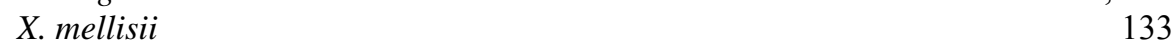

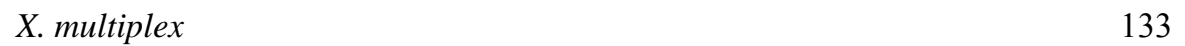

$\begin{array}{ll}X . p a l l i d a & 133\end{array}$

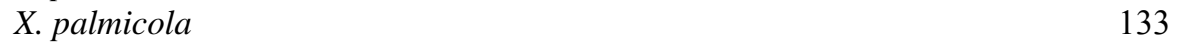

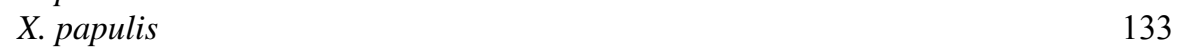

X. poitei 134

$\begin{array}{ll}X \text {. polymorpha } & 21,132\end{array}$

X. schreuderiana 133 
$X$. schweinitzii

$X$. scruposa

132

Xylocoremium flabelliforme

127

Xylocladium

123

Yarrowia lipolytica

164

Zygosaccharomyces

176 
